HYDROLOGIC DATA FROM AN AREA SOUTHWEST OF

TALLAHASSEE, FLORIDA, WHERE MUNICIPAL

WASTEWATER EFFLUENT IS APPLIED BY

SPRAY IRRIGATION

By Michael c. Yurewicz

U.S. GEOLOGICAL SURVEY

Open-File Report 83-769

Prepared in cooperation with the

CITY OF TALLAHASSEE

Tallahassee, Florida

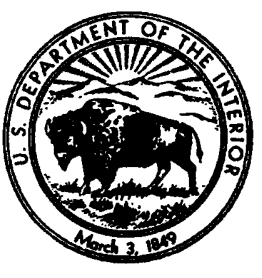


UNITED STATES DEPARTMENT OF THE INTERIOR

JAMES G. WATT, Secretary

GEOLOGICAL SURVEY

Dallas L. Peck, Director

For additional information write to:

District Chief

U.S. Geological Survey Suite 3015

227 North Bronough Street

Tallahassee, Florida 32301
Copies of this report can be purchased from:

Open-File Services Section Western Distribution Branch U.S. Geological Survey Box 25425, Federal Center Denver, Colorado 80225 (Telephone: (303) 234-5888) 


\section{CONTENTS}

Page

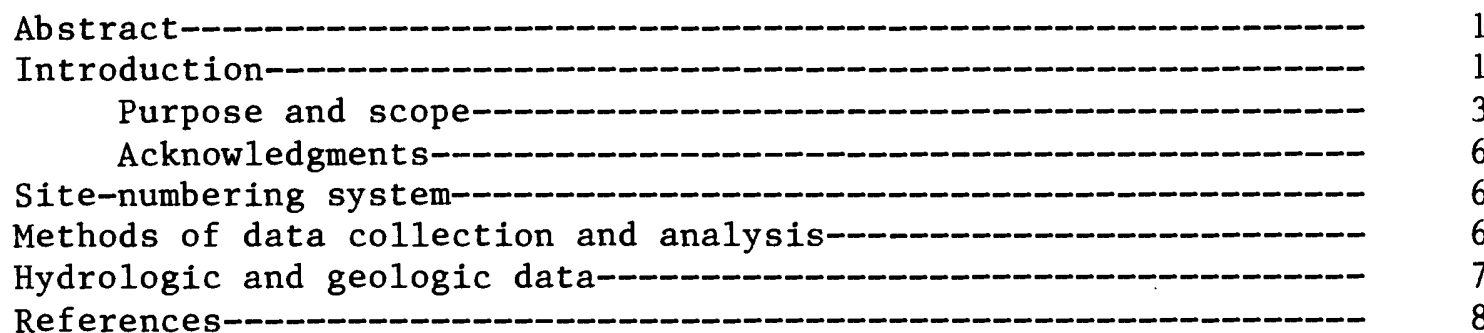

ILLUSTRATIONS

Page

Figure 1. Map showing area of investigation and locations of the southwest and southeast spray fields near

Tallahassee, Florida-_-

2. Map showing area of investigation as related to Leon and Wakulla Counties and locations of selected sampling sites for hydrologic data------

3. Map showing southwest spray field and location of selected sampling sites-

4-8. Graphs showing natural gamma logs for:

4. Site 24- 9

5. Site 25-_- 10

6. Site 26-_ 11

7. Site 28-_- 12

8. Site 29-_ 13

9. Graph showing monthly minimum and maximum ground-water levels for site 1 from 0ctober 1972 through May 1981-_- 
TABLES

Page

Table 1. Locations and descriptions of data-collection sites---- 15

2. Lithologic descriptions of sites 27,29 , and 33-_-_-- 19

3. Monthly spray-irrigation volumes for the southwest spray field, 1966-81- 22

4. Annual spray-irrigation volumes for the southwest spray field, 1966-81- 28

5. Nitrifier and denitrifier bacteria in soil samples, September 1977 to June 1978-__-

6. Physical and chemical characteristics of well and water at sites 1 through 70:

Site No. Page Site No. Page Site No. Page

$\begin{array}{rccccc}1 & 30 & 25 & 102 & 48 & 127 \\ 2 & 36 & 26 & 106 & 49 & 128 \\ 3 & \text { dry hole } & 27 & 108 & 50 & 128 \\ 4 & 37 & 28 & 109 & 51 & 129 \\ 5 & 43 & 29 & 111 & 52 & 129 \\ 6 & 49 & 30 & 114 & 53 & 130 \\ 7 & 56 & 31 & 115 & 54 & 130 \\ 8 & 59 & 32 & 116 & 55 & 132 \\ 9 & 60 & 33 & 118 & 56 & 136 \\ 10 & 62 & 34 & 120 & 57 & 138 \\ & & & & & \\ 11 & 62 & 35 & 121 & 58 & 142 \\ 12 & 64 & 36 & 121 & 59 & 142 \\ 13 & 65 & 37 & 122 & 60 & 143 \\ 14 & 69 & 38 & 122 & 61 & 143 \\ 15 & 73 & 39 & 123 & 62 & 144 \\ & & & & & \\ 16 & 74 & 40 & 123 & 63 & 145 \\ 17 & 75 & 41 & 124 & 64 & 146 \\ 18 & 80 & 42 & 124 & 65 & 147 \\ 19 & 84 & 43 & 125 & 66 & 149 \\ 20 & 87 & 44 & 126 & 67 & 149 \\ & & & & & \\ 21 & 89 & 45 & 126 & 68 & 150 \\ 22 & 94 & 46 & 127 & 69 & 150 \\ 23 & 98 & 47 & 127 & 70 & 151 \\ 24 & 98 & & & & \end{array}$




\title{
HYDROLOGIC DATA FROM AN AREA SOUTHWEST OF \\ TALLAHASSEE, FLORIDA, WHERE MUNICIPAL WASTEWATER EFFLUENT IS APPLIED BY SPRAY IRRIGATION
}

\author{
By Michael C. Yurewicz
}

\begin{abstract}
Water quality and ground-water levels were monitored in the area of a field where municipal wastewater is applied by spray irrigation. The area irrigated is 4 miles southwest of Tallahassee, Florida, on land covered in part by pine forest and in part by selected grasses and forage crops. Hydrologic and geologic data were collected from March 1972 through June 1981. Hydrologic data include ground-water levels, water quality, and spray-irrigation rates. Measurements of ground-water levels were made at 60 wells. Water-quality samples were collected at 1 municipal wastewater effluent site, 3 springs, 17 soil sites, and 64 wells. Water-quality data include physical, chemical, and bacteriological analyses. Geologic data include geophysical logs and lithologic descriptions of 34 wells. Monthly and annual volumes of sprayed effluent are included for July 1966 through September 1981 .
\end{abstract}

\section{INTRODUCTION}

The City of Tallahassee, Fla., has been spray irrigating pine forest as well as selected grasses and forage crops with municipal wastewater effluent since 1966 at the southwest spray irrigation field and wastewater treatment plant (fig. 1). Initially, 20.5 acres were sprayed at an approximate rate of $0.5 \mathrm{Mgal}$ per day. The spray field reached its maximum size in 1977 when it was expanded to 119 acres. The volume of effluent sprayed was a maximum of $45 \mathrm{Mgal}$ per month from March 1972 through January 1973. Although spray irrigation was initially done on an experimental basis, it subsequently was adopted as a routine procedure so that discharge of wastewater effluent to Munson Slough could be decreased.

Spray irrigation at the southwest field will be limited in the future for two reasons: (1) a larger spray field has been constructed 8 miles southeast of Tallahassee (fig. 1); and (2) much of the land being spray irrigated at the southwest field will be used for treatment plant expansions such as sludge handling facilities, sludge pits, and maintenance buildings. These changes are the result of implementation of the Tallahassee-Leon County 201 Facilities Plan, as outlined by William M. Bishop Consulting Engineers, Inc. (1976), and the Tallahassee-Leon County 208 Areawide Waste Treatment Management Plan, as reported by the Tallahassee-Leon County Planning Department (1978). 


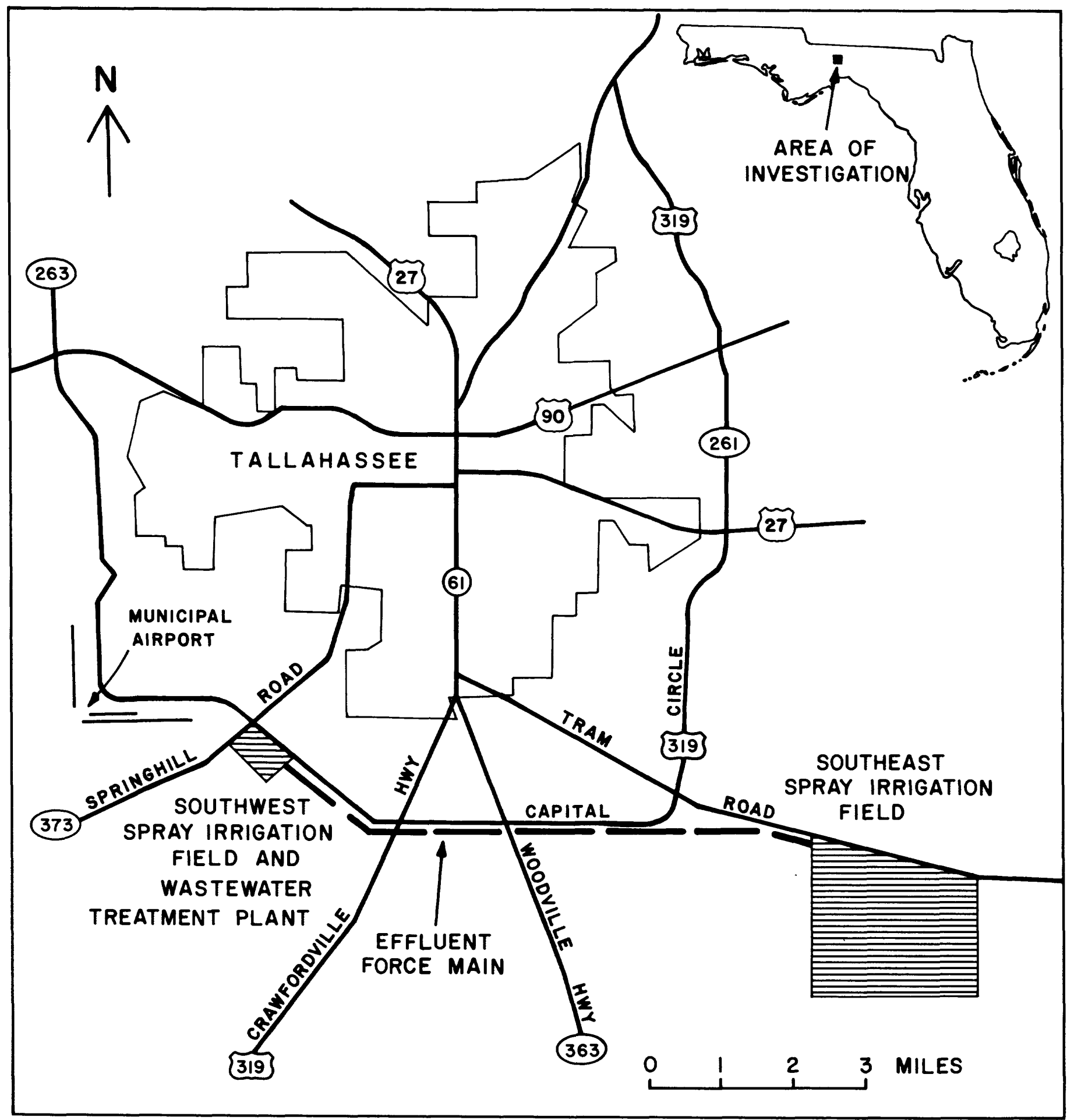

Figure 1.--Area of investigation and locations of the southwest and southeast spray fields near Tallahassee, Florida. 
The switch from the southwest field to the southeast field began in November 1980. Plans are to enlarge the 1,086 acres of the southeast field now available for spray irrigation by an additional 750 acres. By mid-1981, construction of four sludge pits had begun on the southwest field. The completed sludge pits occupy 9.7 acres and are lined to prevent percolation of soluble sludge waste into the ground-water system. Additional sludge pits will be constructed as the need arises, thus further decreasing the area of the southwest field available for spray irrigation.

\section{Purpose and Scope}

The purpose of this report is to present hydrologic and geologic data that will aid in the assessment of the effects of spray irrigation of secondary-treated municipal wastewater on a ground-water system (in particular, on Floridan aquifer water). It is intended that the data not only will enable better management of the southwest spray-irrigation operation, but also--together with the experience gained through the investigation--assist city, state, and federal officials in planning the future size and operational needs of disposal facilities for municipal wastewater in Tallahassee.

This report presents data collected from March 1972 through June 1981. The area of investigation covers approximately 83 square miles (fig. 2). Water samples from wells and springs were collected in the immediate area of the southwest spray field (fig. 3) and as much as 11 miles from the spray field to ascertain the subsurface area affected by spray irrigation. Physical, chemical, and bacteriological analyses were made of ground-water and municipal wastewater effluent samples. Data on ground-water levels, geophysical logs, and lithologic samples were collected and analyzed. The investigation was carried out in cooperation with the City of Tallahassee.

Interpretive results of the investigation were presented by slack (1975) for data collected from July 1972 through June 1974. Data collected from July 1966 through June 1981 are interpreted in a report in preparation. In addition, the effects of spray irrigation with municipal wastewater effluent in terms of crop uptake, changes in soil characteristics, and shallow ground-water quality have been reported by Overman (1979) in a study of effluent irrigation of coastal bermuda grass and other forage crops.

The investigation of the effects of spray irrigation with municipal wastewater effluent on the ground-water system are expected to continue with the major effort directed toward the southeast spray field. Baseline hydrologic data were obtained at the southeast spray field before spray irrigation began, and the installation of multiple-depth wells will permit the assessment of the effects on the ground-water system both horizontally and vertically. This new hydrologic information will permit a more extensive evaluation than was possible at the southwest spray field, where the investigation began 6 years after irrigation was initiated. A limited data-collection program is planned at the southwest spray field to assess future changes resulting from application of wastewater effluent. 


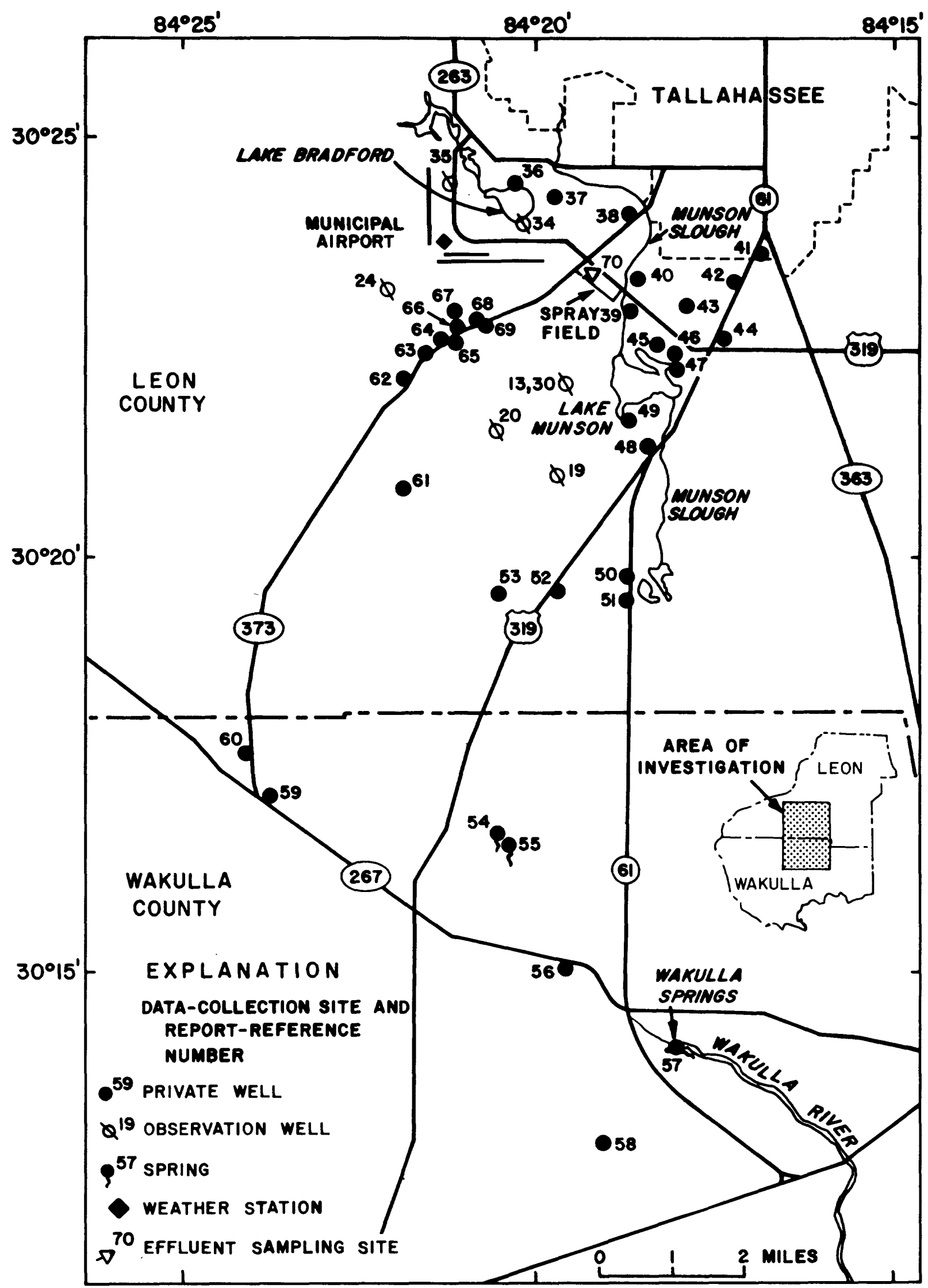

Figure 2.--Area of investigation as related to Leon and Wakulla Counties and locations of selected sampling sites for hydrologic data. 


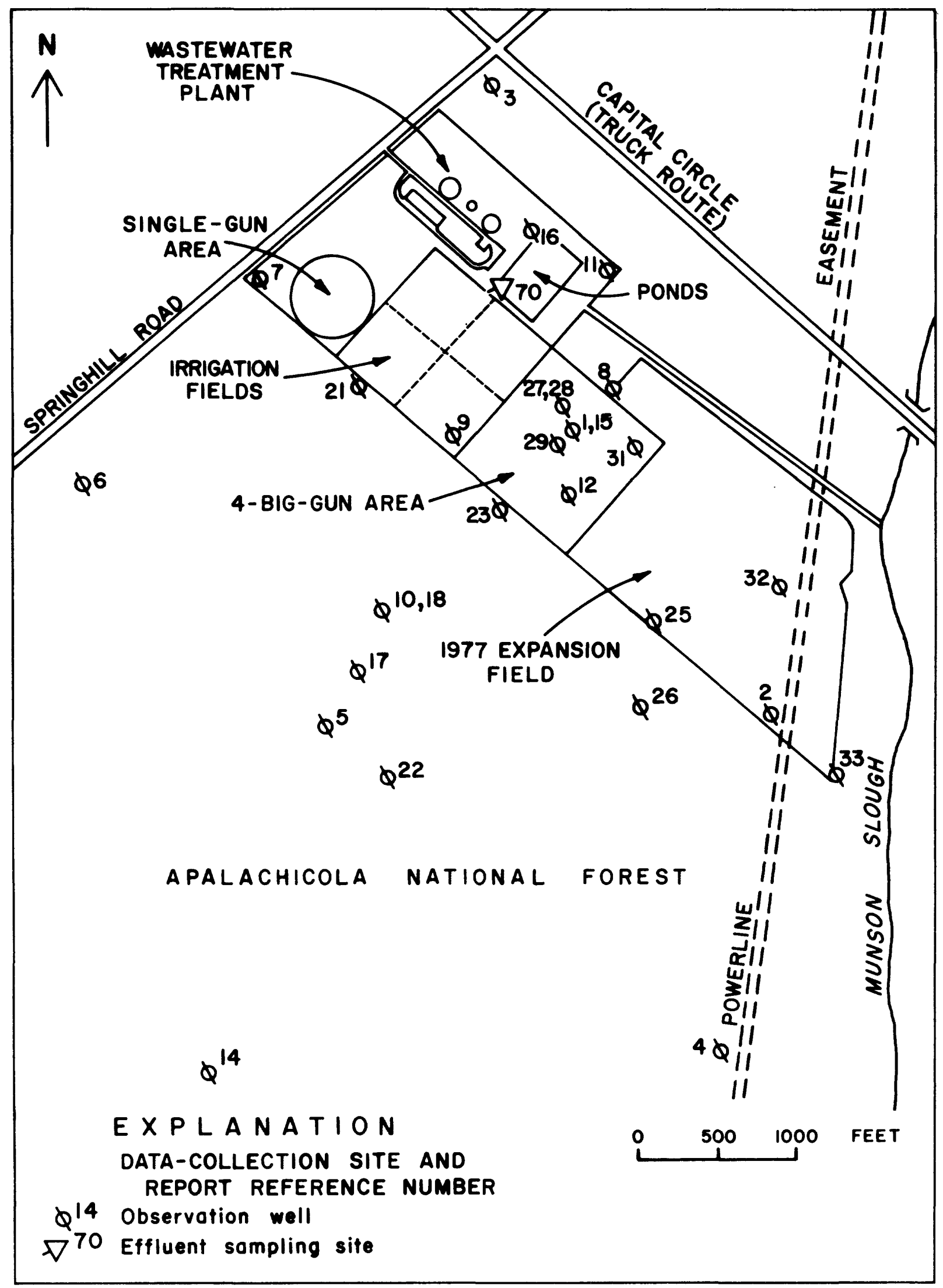

Figure 3.--Southwest spray field and location of selected sampling sites. 


\section{Acknowledgments}

The assistance of many individuals is acknowledged. William G. Leseman, laboratory supervisor, and Keith Turner, chief plant operator, of the Thomas P. Smith Waste Water Renovation Plant, Tallahassee, provided water-quality analyses and monthly application volumes of wastewater effluent sprayed at the southwest field. Tom Kwader of the Northwest Florida Water Management District obtained geophysical logs. Finally, many individuals allowed access to their land for the collection of ground-water samples and measurement of water levels.

\section{SITE-NUMBERING SYSTEM}

Three numbering systems are used in this report to identify and locate data-collection sites. The first is the map site number (sites 1 through 70, figs. 2, 3, and table 1), which is the primary means of identification and location of sites used throughout the report; the second is an 8-digit number that is usually applied to stream gaging and stream-quality sampling sites; and the third is the site-ID number of the U.S. Geological Survey which consists of 15 digits that signify the latitude and longitude of a point believed to represent the location of the site. The first 6 digits of the site-ID number denote the degrees, minutes, and seconds of latitude; the next 7 digits denote the degrees, minutes, and seconds of longitude; the last 2 digits provide a sequential numbering system for sites located within a 1-second grid. Once assigned, this number does not change, even if the latitude or longitude initially taken as representing that site has changed. Hence, although the site-ID number is a unique number, it may not be an accurate latitude-longitude location indicator. The number can be used both to enter data into and to retrieve data from the National Water Data Storage and Retrieval System. Consequently, any available historlc data can be obtained from computer files by using either or both the 8- or 15-digit Survey numbers. Inquiries regarding the availability of such data may be made to the U.S. Geological Survey, Suite 3015, 227 N. Bronough Street, Tallahassee, FL 32301.

\section{METHODS OF DATA COLLECTION AND ANALYSIS}

Whenever applicable, standard methods were used for data collection and analysis. Such methods are described in a series of manuals published by the U.S. Geological Survey entitled "U.S. Geological Survey Techniques of Water-Resources Investigations" (Goerlitz and Brown, 1972; Greeson and others, 1977; and Skougstad and others, 1979).

Most of the water-quality samples were bottled appropriately and sent to a laboratory for analysis immediately following collection; proper preservation methods were employed (Greeson and others, 1977; Friedman and Beetem, 1979). Analyses were made at one of three laboratories: the City of Tallahassee Water-Quality Laboratory in Tallahassee, Fla.; the U.S. Geological Survey Atlanta Central Laboratory in Doraville, Ga;; or the U.S. Geological Survey Quality of Water Service Un1t in Ocala, Fla. Analytical methods for both U.S. Geological Survey laboratories are described in Goerlitz and Brown (1972), Greeson and others (1977), and Skougstad and others (1979). Analytical methods used by the City of Tallahassee laboratory are described in American Public Health Association and others (1975). 
Although the analytical methods differ, they are compatible; quality control is maintained by a quality-assurance program established between the U.S. Geological Survey and City of Tallahassee laboratories.

\section{HYDROLOGIC AND GEOLOGIC DATA}

Figures 4 through 8 show natural gamma logs for selected sites in the area being sprayed. Lithologic logs and gamma logs obtained earlier in the investigation for 27 other sites in or near the southwest spray field are given in an earlier report (Slack, 1975). Such logs are used to provide geohydrologic information of the subsurface. Figure 9 shows that monthly maximum and minimum ground-water levels for site 1 varied significantly throughout the period of record: the maximum was 30.46 feet above NGVD of 1929 (April 1973), and the minimum was 17.47 feet (December 1972), a difference of 12.99 feet.

Table 1 presents physical descriptions and names of data-collection sites. Many of the data-collection sites are residential wells near the southwest spray field. Verification of the total well depths, casing depths, and other well construction information for most of these sites was not possible. Additional well construction information for the wells given in table 1 are available from the Ground-Water Site Inventory File which is a U.S. Geological Survey computer system for storing ground-water information.

Table 2 gives 1ithologic descriptions of the materials penetrated by the drilling of three wells. Similar lithologic descriptions obtained earlier in the investigation for 21 other sites are given in an earlier report (Slack, 1975). Lithologic descriptions plus geophysical logs such as those presented in figures 5-9 are needed to develop geohydrologic cross sections of the subsurface in and near the southwest spray field.

Data-collection sites equipped with continuous (digital) water-level recorders are: site 1 , equipped with a continuous water-level recorder from 1972 through 1981; site 34 from 1972 through 1979; and site 35 from 1977 through 1981. Tables 3 and 4 list monthly and annual application volumes of wastewater effluent for the southwest field, respectively. The application volumes are presented for each of the four general areas shown on figure 3: the single-gun area, the 16-acre irrigation fields area, the 4-big-gun area, and the 1977 expansion field. Monthly spray irrigation volumes were highly variable for each area throughout the period of investigation. A volume exceeding 3,000 million gallons was sprayed from July 1966 through September 1981. Because of missing data, this figure is less than the actual volume sprayed.

Table 5 lists the nitrifier and denitrifier bacteria found in soil samples. Table 6 presents water-quality data for 70 sites; these include wells, springs, and effluent. Well depths and discharge are included for some sites. Seventeen data sampling sites are identified as in or near the four spray areas or the unlined holding ponds. Additional records are on file with the U.S. Geological Survey in Tallahassee, Fla. 


\section{REFERENCES}

American Public Health Association and others, 1975, Standard methods for the examination of water and wastewater, 14th edition: New York, American Public Health Association, Inc., 1193 p.

Friedman, L. C., and Beetem, W. A., 1979, 1980 Water quality laboratory services catalog: U.S. Geological Survey Open-File Report 79-697, $186 \mathrm{p}$.

Goerlitz, D. F., and Brown, Eugene, 1972, Methods for analysis of organic substances in water: U.S. Geological Survey Techniques of Water-Resources Investigations, Book 5, Chapter A3, 40 p.

Greeson, P. E., Ehlke, T. A., Irwin, G. A., Lium, B. W., and Slack, K. V., 1977, Methods for collection and analysis of aquatic biological and microbiological samples: U.S. Geological Survey Techniques of Water-Resources Investigations, Book 5, Chapter A4, 332 p.

Overman, A. R., 1979, Effluent irrigation of coastal bermuda grass: Journal of the Environmental Engineering Division, American Society of Civil Engineers, v. 105, no. EE1, Proceedings Paper 14359, p. 55-60.

Skougstad, M. W., Fishman, M. J., Friedman, L. C., Erdmann, D. E., and Duncan, S. S., 1979, Methods for determination of inorganic substances in water and fluvial sediments: U.S. Geological Survey Techniques of Water-Resources Investigations, Book 5, Chapter A1, $626 \mathrm{p}$.

Slack, L. J., 1975, Hydrologic environmental effect of sprayed sewage effluent, Tallahassee, Florida: U.S. Geological Survey WaterResources Investigations 55-75, $73 \mathrm{p}$.

Tallahassee-Leon County Planning Department, 1978, Final plan report, Tallahassee-Leon County 208 Areawide Waste Treatment Management Plan study: Tallahassee, Fla., 336 p.

William M. Bishop Consulting Engineers, Inc., 1976, Tallahassee-Leon County Florida 201 Facilities Plan: Consultant's report to the Tallahassee-Leon County Planning Commission, in files of Tallahassee-Leon County Planning Commission, Tallahassee, Fla. 


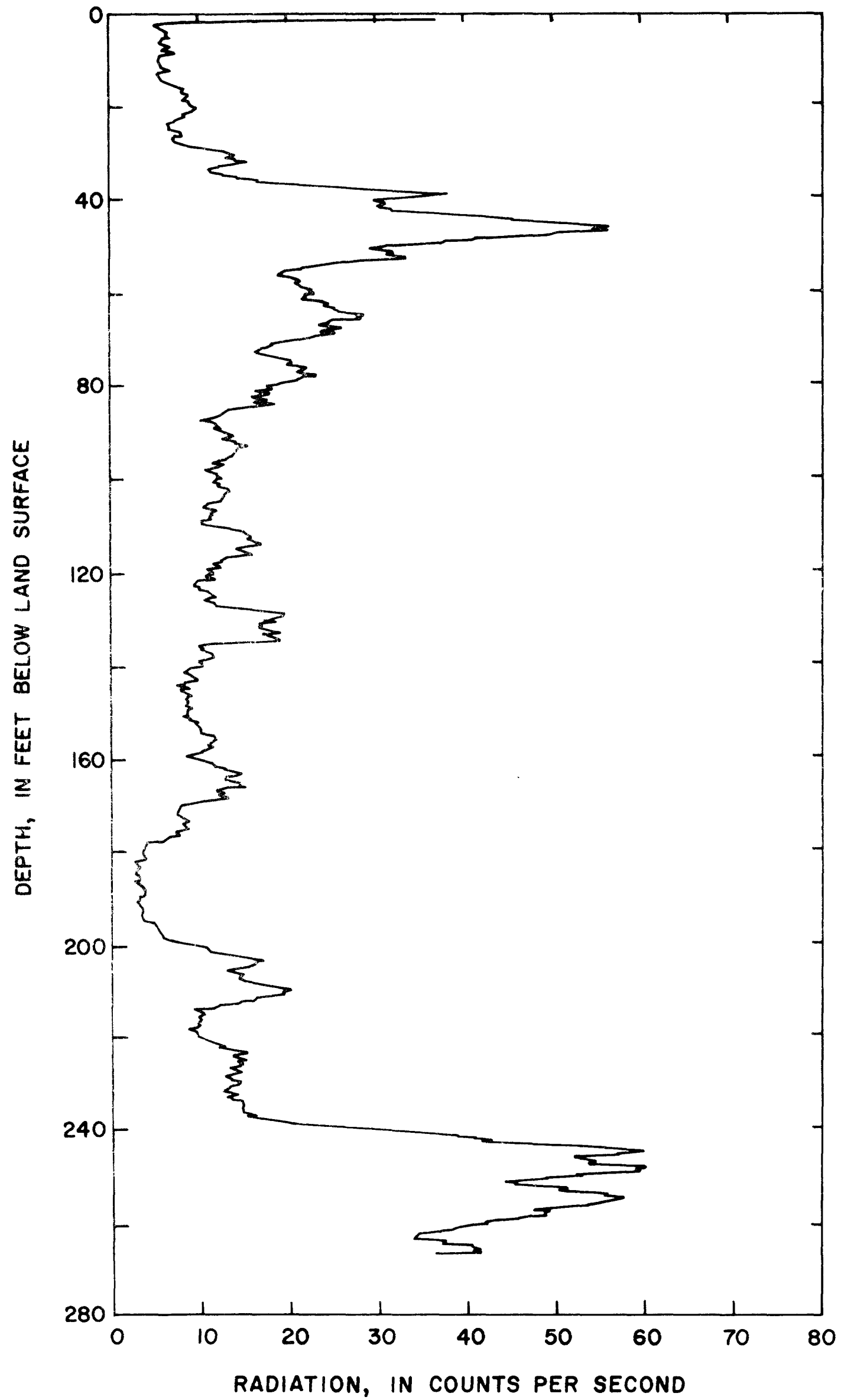

Figure 4.--Natural gamma $\log$ for site 24 . 


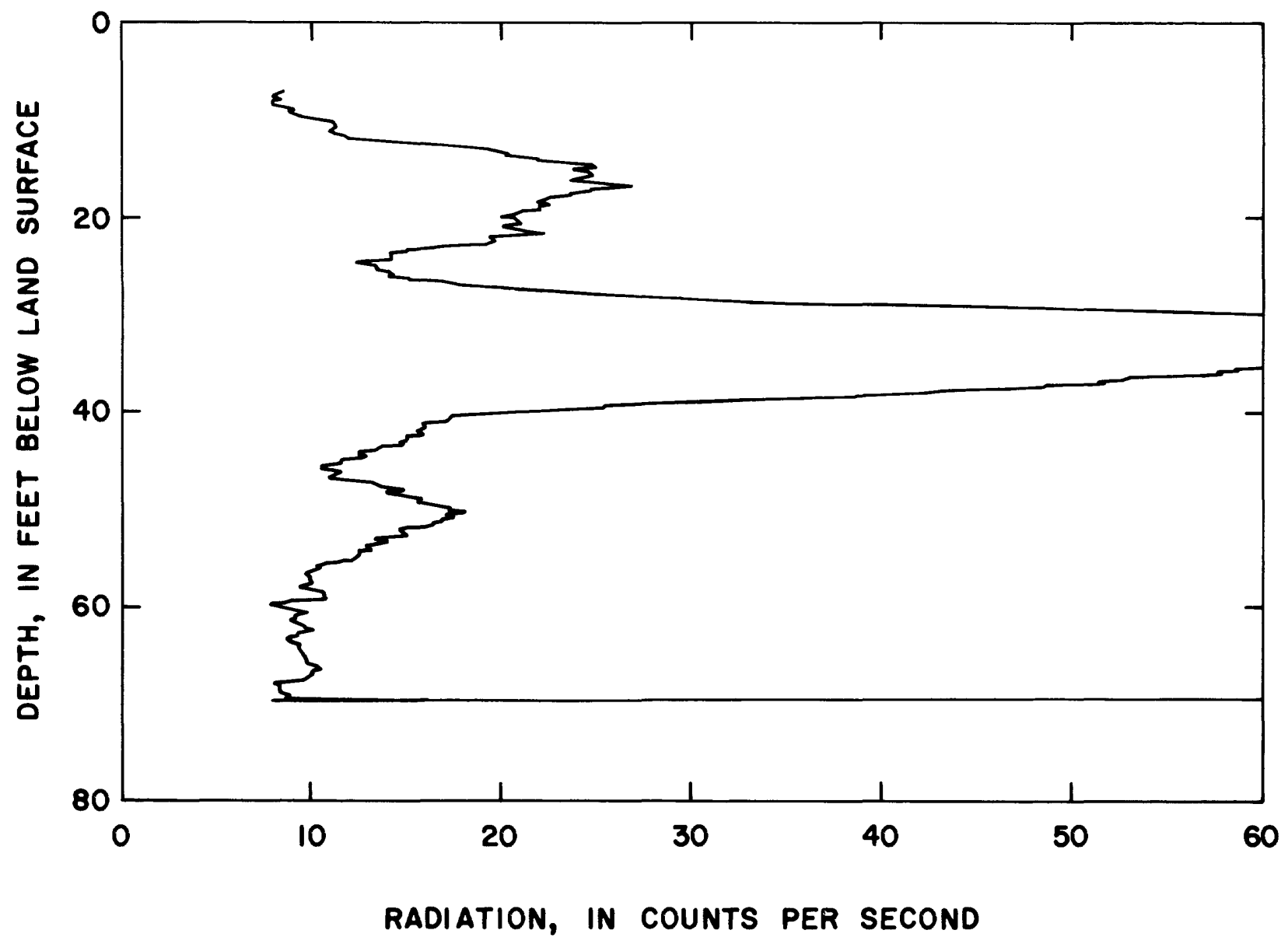

Figure 5.--Natural gamma $\log$ for site 25. 


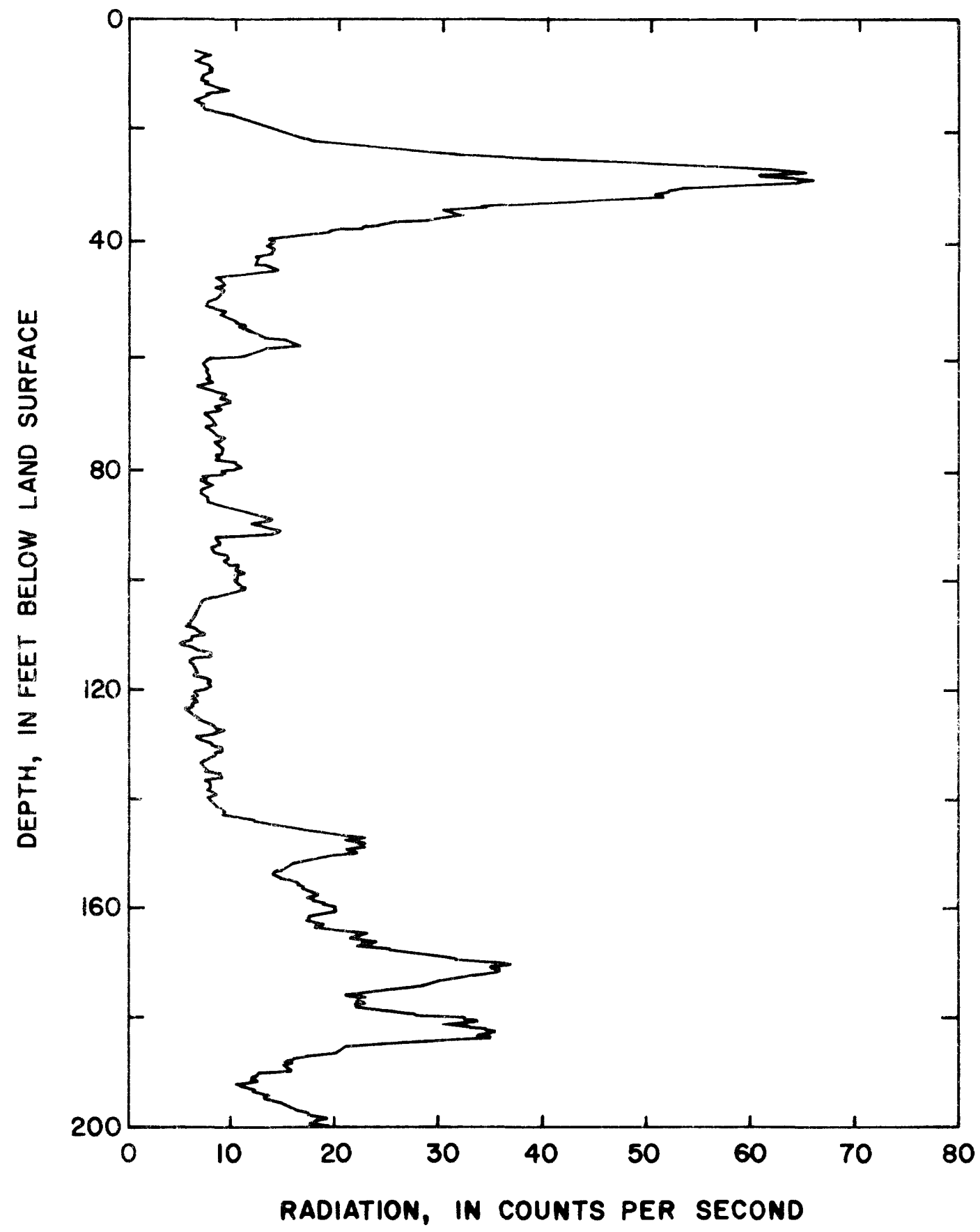

Figure 6.--Natural gamma log for site 26. 


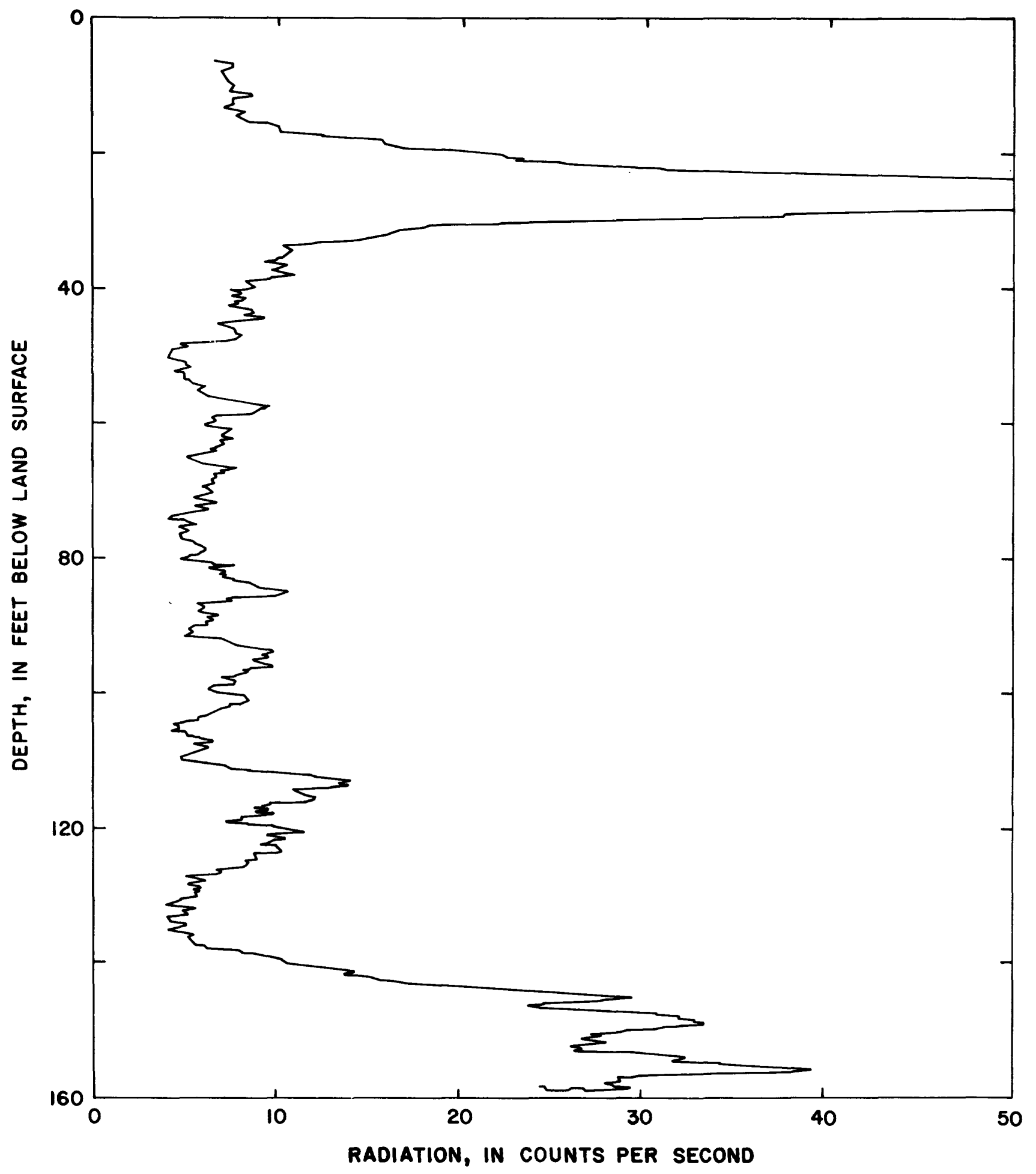

Figure 7.--Natural gamma log for site 28. 


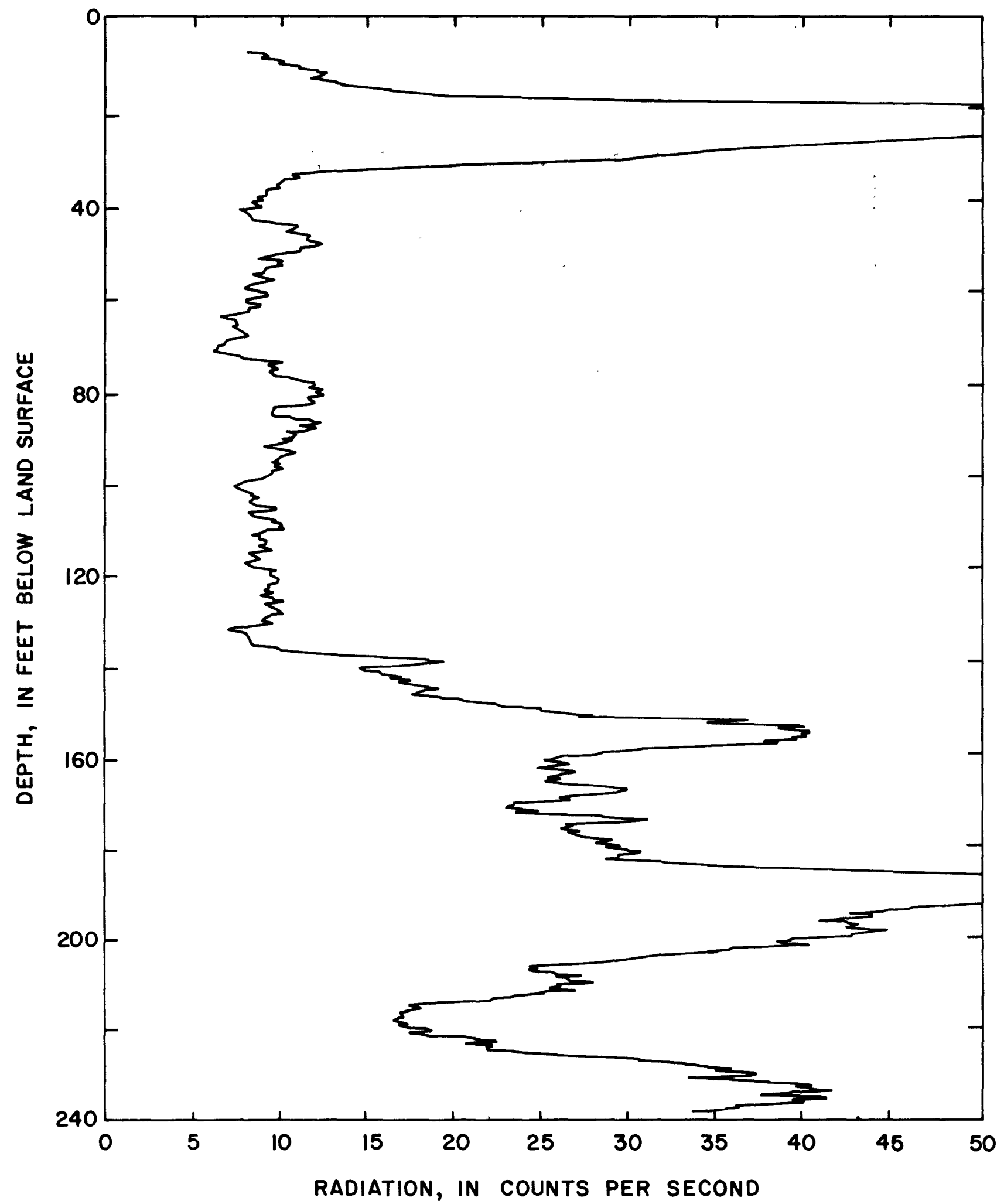

Figure 8.--Natural gamma log for site 29. 


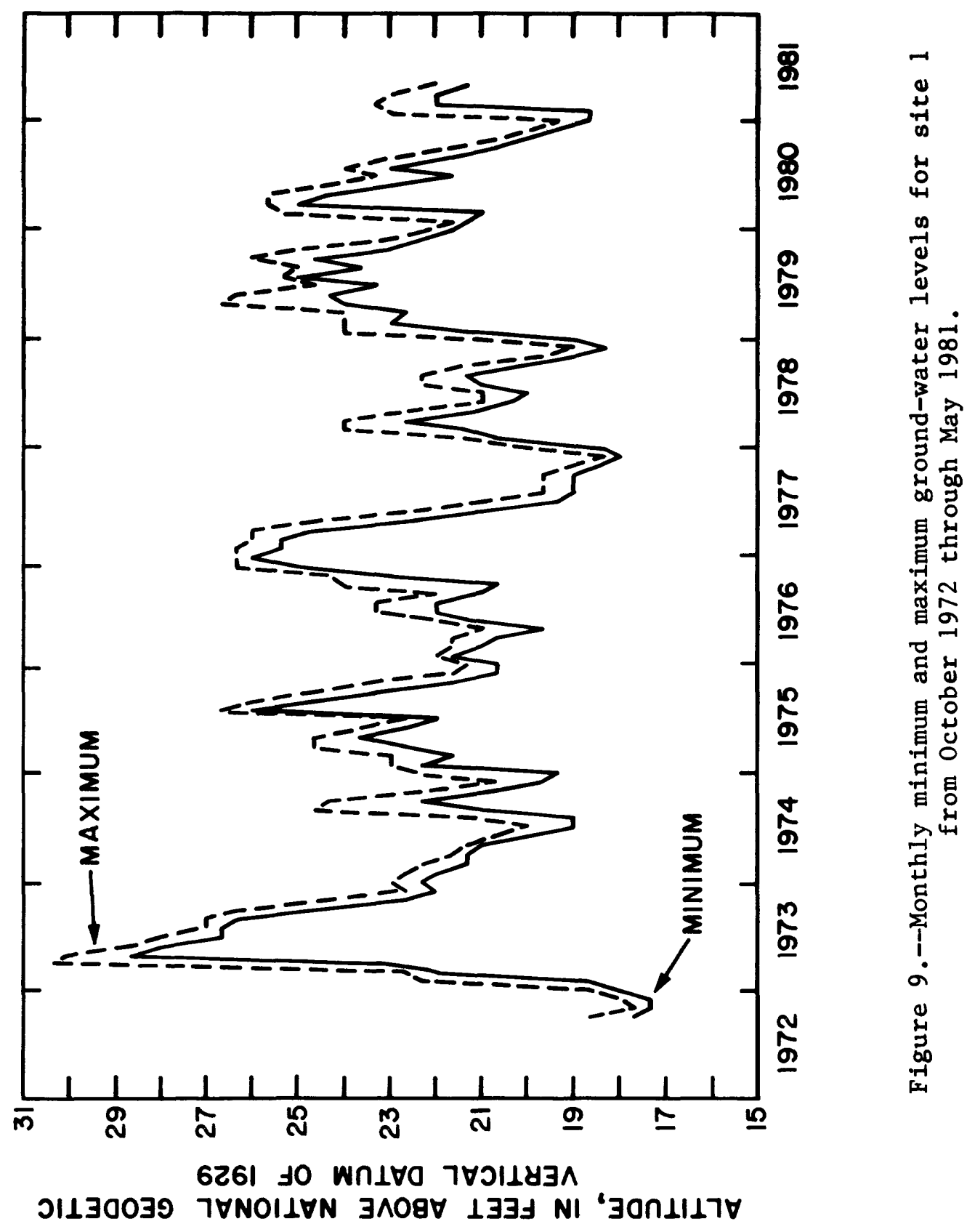




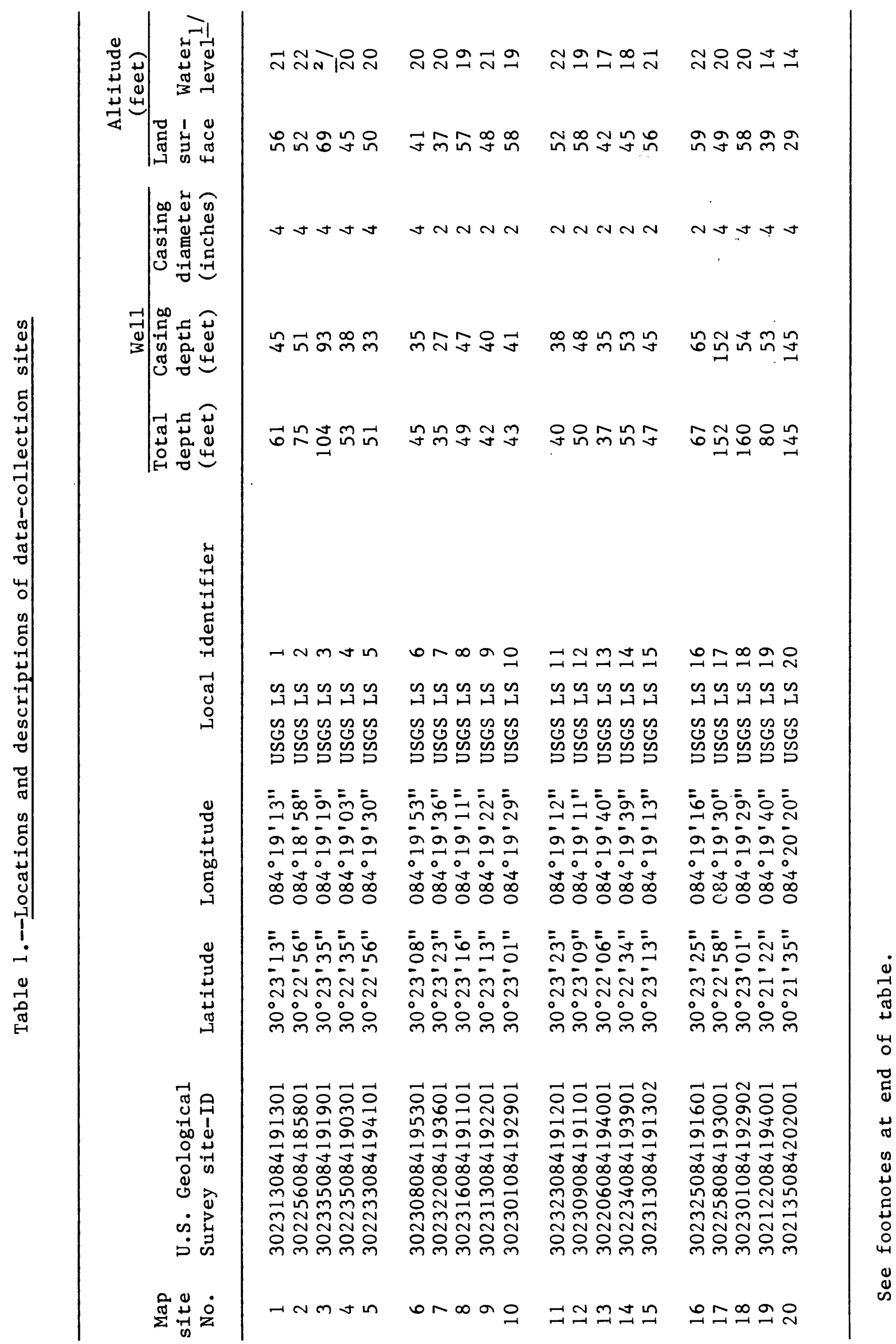




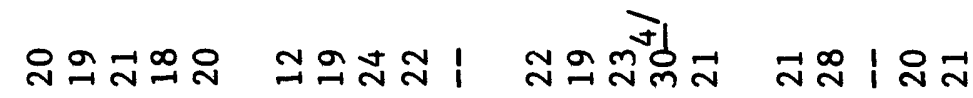

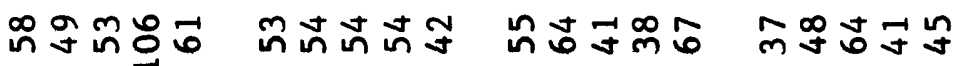

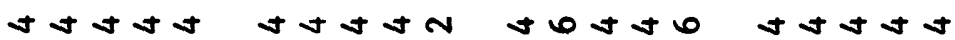

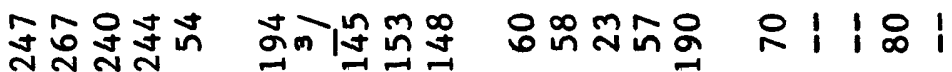

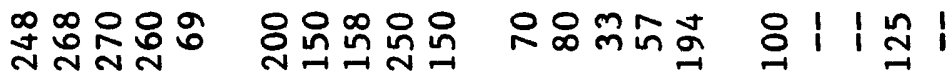

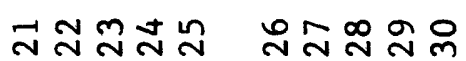

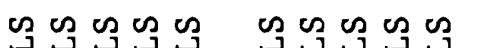

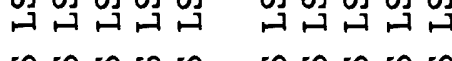

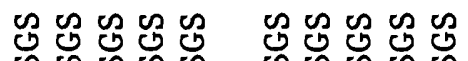

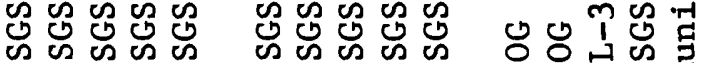

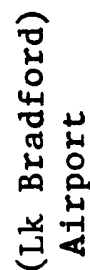
๑ロロロロ

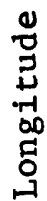

ㅎํㅊㅎㅎㅇㅎㅇ

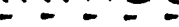

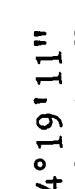

$=\overline{i n}=\overline{0}$ - 0 - 0

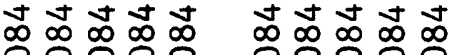

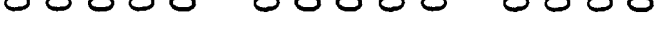

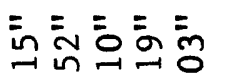

$\bar{i} \bar{j} \overline{\bar{c}} \overline{0}$

in $\rightarrow \pi 0$

สท่⿰幺幺ं

กิสกิกิก

o. 000

$\sin 4 \pi$

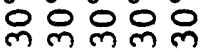

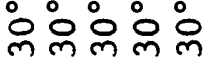

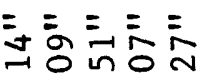

的旅方

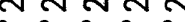

0000

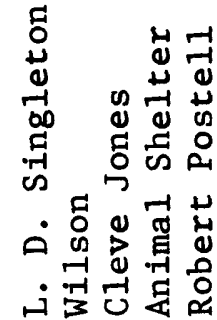

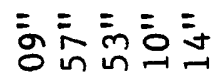

a $\infty \overline{0}=$

$\pi-7$ -

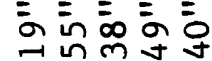

-

ก.-1

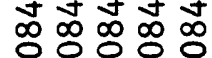

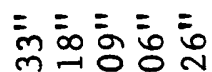

ììn

4n $y$ n

ㅇํㅇ:

जีन चีतี

응영융요 으윤융ㅇㅇ

สำㅇํㅇ

윽ำำ

कानव के

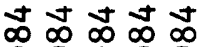

잉ㅇㅇㅇㅇㅇ

는 그응

寸古牙古

\% ㅇㅇㅇ 용

엉

กี่

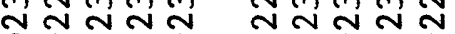

ㄱํำำำ

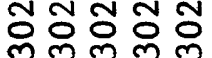

응오응응을

엄 $\infty$

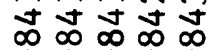

: $0^{\infty} 0^{\infty} 0^{\infty}$

긍 그을

mำ

สงกสบ

유유유유

홍융ㅇㅇㅇㅇㅇㅇㅇㅇ

约方

워고-

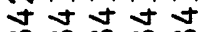

○

m

ปี่

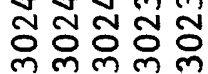

乎离京

ㄲํำน

ํํำ 워ำ

$\vec{m} \tilde{m} m \ddot{n}$

ํำ 


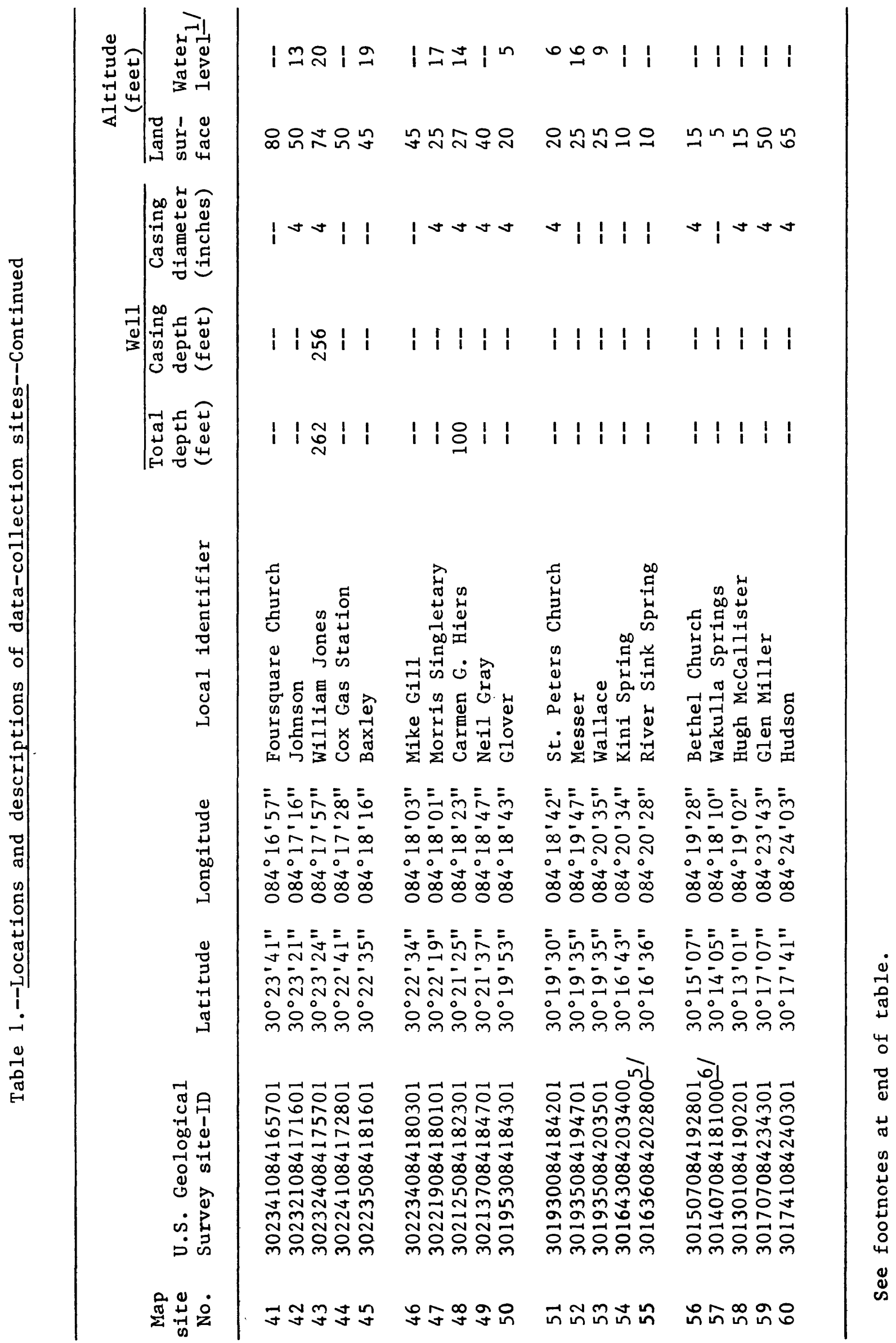




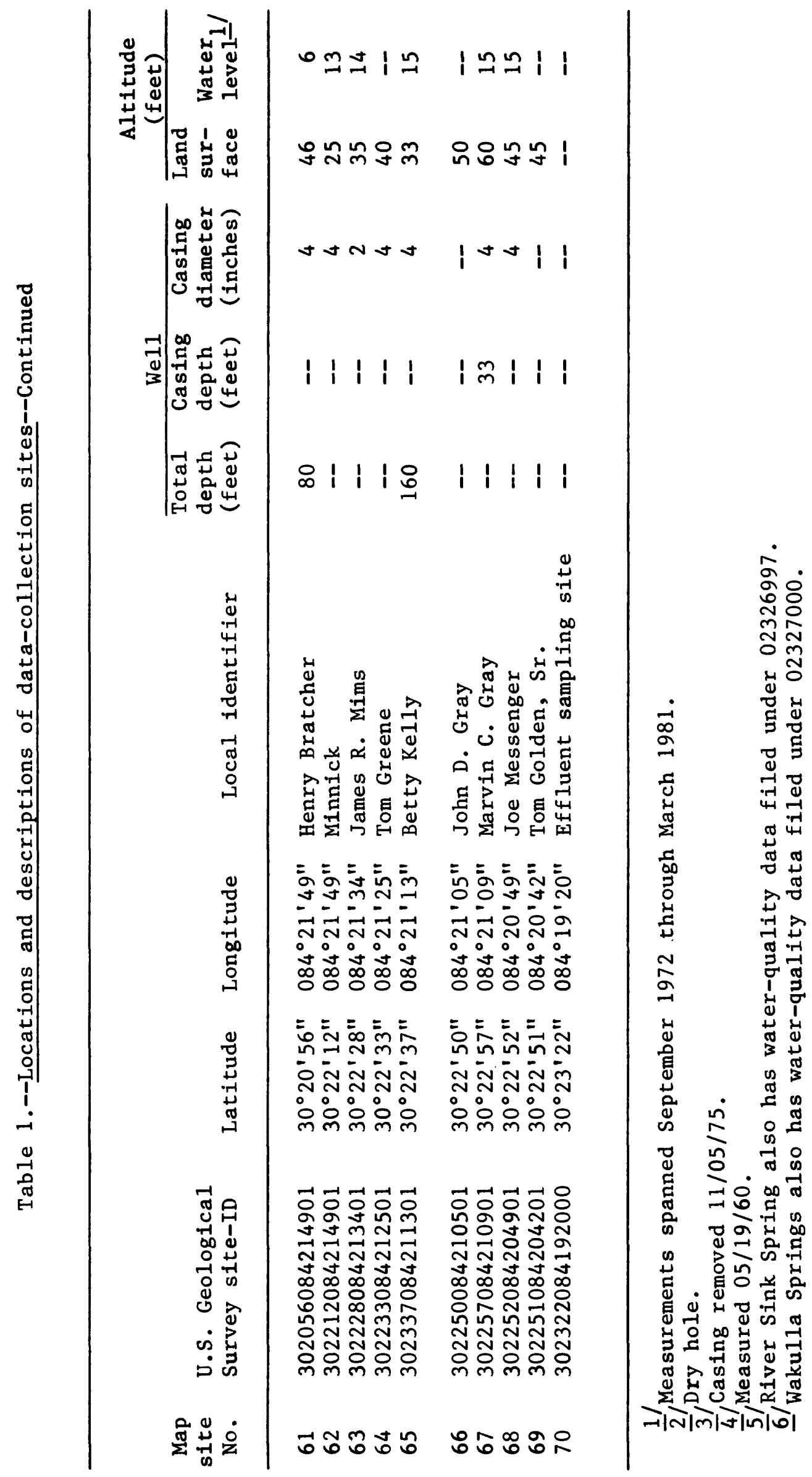


Table 2.--Lithologic descriptions of sites 27,29 , and 33

\section{Depth}

$(\mathrm{ft})$

\section{Site $27 \underline{1 /}$}

0-22 SAND, clear to frosted, stain pale yellowish orange, fine to coarse, angular to subrounded, very argillaceous.

22-27 CLAY, dark yellowish orange, very sandy; clay, white, very dense; sand, same as above, 50 percent.

27-35 CLAY, grayish orange, sandy, 60 percent; sand, clear to frosted, subangular to rounded, medium to coarse (mostly coarse), argillaceous; limestone, white, finely granular, hard, black mineral inclusions, chalky, poor to medium porosity, nonfossiliferous, 5 percent.

35-45 SAND, clear to frosted, stained very pale orange, fine to coarse (mostly medium), angular to rounded, argillaceous; phosphorite.

45-60 SAND, same as above, but mostly coarse.

60-80 MARL, white to yellowish gray, very (finely) sandy, calcareous, silty, nonfossiliferous.

80-90 LIMESTONE, white to yellowish gray, hard, slightly sandy, slightly moldic, porous, nonfossiliferous.

90-100 SAND, clear to frosted, stained very pale orange, argillaceous, coarse, subangular to rounded, 60 percent; limestone, yellowish gray, crystalline to granular, hard, moldic, porous, slightly sandy, nonfossiliferous.

100-106 CLAY, grayish orange, very sandy, 80 percent; 1imestone, white to yellowish gray, granular, moldic, hard, rubbly, chalky, sandy, very porous, no identifiable fossils except echinoid spine fragment (?); limestone, pinkish gray, crystalline, hard, "smooth," medium porosity, slightly moldic, nonfossiliferous; sand, same as above.

106-110 LIMESTONE, same as above, 75 percent rubbly type.

See footnotes at end of table. 
Table 2.--Lithologic descriptions of sites 27, 29, and 33--Continued

\section{Depth}

(ft)

\section{Lithology}

\section{Site 27 니}

110-120 LIMESTONE, same as above, but also fossiliferous (bryozoan, echinoid spines, forams, shell fragments), 50 percent; sand, frosted, coarse, subrounded to rounded, 50 percent.

120-130 CLAY, moderate yellowish brown, very dense, slightly sandy, 75 percent; sand, clear to frosted, stained yellowish brown, medium to coarse (mostly coarse), argillaceous.

130-140 LIMESTONE, very pale orange to white granular, soft to hard, extremely moldic, porous, sandy, conglomeratic appearance, chalky, fossiliferous.

$$
\text { Site } 29 \underline{1,2 /}
$$

32-35 SAND, clear to frosted, fine to coarse (mostly medium), very argillaceous, angular to subangular, 55 percent; clay, light brown.

35-50 LIMESTONE, white, micritic, finely sandy, chalky, very porous, moldic, hard to soft, cancellaria, 90 percent; clay, grayish yellow, sandy; clear to frosted, fine, angular to subangular.

50-70 LIMESTONE, white "clastic," crystalline (possibly recrystallized), very porous, hard, miliolids (?).

70-80 LIMESTONE, same as above; finely broken miliolids (?), 90 percent; clay, grayish yellow (40) to grayish olive (47).

80-100 SAND, fine to coarse (mostly medium), clear to frosted, angular to subrounded, 90 percent; limestone, white, same as above; phosphorite (very small quantity).

100-110 LIMESTONE, white, crystalline, hard, dense, 85 percent, no fossils found; sand, clear to frosted, medium, angular to subrounded. 
Table 2.--Lithologic descriptions of sites 27,29 , and 33--Continued

Depth

(ft)

Lithology

\section{Site $29 \underline{1,2 /}$}

110-135 LIMESTONE, white, crystalline, hard, very porous, slightly moldic, some fragments, sandy, fossiliferous--operculinoides (abundant), also shell fragments; sand, medium, same as above.

135-141 SAND, fine to coarse, mostly medium, clear to frosted, angular to subrounded, 60 percent, limestcne, same as above, abundance of microfossils--also few shell fragments; Lepidocyclina, Operculinoides, nummulites (?).

Site $33^{3 /}$

0-10 SAND, gray.

10-25 CLAY, gray, plastic.

25-36 SAND, yellow; clay, with little limestone.

$1 /$ Lithologic description by Jeffrey Wagner (written commun., 1981), Northwest Florida Water Management District (formerly with U.S. Geological Survey). feet.

2/ Lithologic descriptions are not available for depths below 141

3/ Driller's notes. 
Table 3.--Monthly spray-irrigation volumes for the southwest spray field, 1966-81

[Volumes are in million gallons. Records prior to 1977 are approximate. Sums are not computed for periods of no record. Months with no record are indicated by --]

\begin{tabular}{|c|c|c|c|c|c|c|}
\hline Month & Year & $\begin{array}{l}\text { Single } \\
\text { gun } \\
\text { area }\end{array}$ & $\begin{array}{c}\text { Irrigation } \\
\text { fields } \\
\text { area }\end{array}$ & $\begin{array}{l}\text { 4-big } \\
\text { gun } \\
\text { area }\end{array}$ & $\begin{array}{c}1977 \\
\text { expansion } \\
\text { area }\end{array}$ & $\begin{array}{l}\text { Monthly } \\
\text { sum, } \\
\text { a11 areas }\end{array}$ \\
\hline July & 1966 & 7.2 & 8 & 0 & 0 & 15.2 \\
\hline August & 1966 & 7.2 & 8 & 0 & 0 & 15.2 \\
\hline September & 1966 & 7.2 & 8 & 0 & 0 & 15.2 \\
\hline October & 1966 & 7.2 & 8 & 0 & 0 & 15.2 \\
\hline November & 1966 & 7.2 & 8 & 0 & 0 & 15.2 \\
\hline December & 1966 & 7.2 & 8 & 0 & 0 & 15.2 \\
\hline January & 1967 & 7.2 & 8 & 0 & 0 & 15.2 \\
\hline February & 1967 & 7.2 & 8 & 0 & 0 & 15.2 \\
\hline March & 1967 & 7.2 & 8 & 0 & 0 & 15.2 \\
\hline April & 1967 & 7.2 & 8 & 0 & 0 & 15.2 \\
\hline May & 1967 & 7.2 & 8 & 0 & 0 & 15.2 \\
\hline June & 1967 & 7.2 & 8 & 0 & 0 & 15.2 \\
\hline Ju1y & 1967 & 7.2 & 8 & 0 & 0 & 15.2 \\
\hline August & 1967 & 7.2 & 8 & 0 & 0 & 15.2 \\
\hline September & 1967 & 7.2 & 8 & 0 & 0 & 15.2 \\
\hline October & 1967 & 7.2 & 8 & 0 & 0 & 15.2 \\
\hline November & 1967 & 7.2 & 8 & 0 & 0 & 15.2 \\
\hline December & 1967 & 7.2 & 8 & 0 & 0 & 15.2 \\
\hline January & 1968 & 7.2 & 8 & 0 & 0 & 15.2 \\
\hline February & 1968 & 7.2 & 8 & 0 & 0 & 15.2 \\
\hline March & 1968 & 7.2 & 8 & 0 & 0 & 15.2 \\
\hline April & 1968 & 7.2 & 8 & 0 & 0 & 15.2 \\
\hline May & 1968 & 7.2 & 8 & 0 & 0 & 15.2 \\
\hline June & 1968 & 7.2 & 8 & 0 & 0 & 15.2 \\
\hline Ju1y & 1968 & 7.2 & 8 & 0 & 0 & 15.2 \\
\hline August & 1968 & 7.2 & 8 & 0 & 0 & 15.2 \\
\hline September & 1968 & 7.2 & 8 & 0 & 0 & 15.2 \\
\hline October & 1968 & 7.2 & 8 & 0 & 0 & 15.2 \\
\hline November & 1968 & 7.2 & 8 & 0 & 0 & 15.2 \\
\hline December & 1968 & 7.2 & 8 & 0 & 0 & 15.2 \\
\hline
\end{tabular}


Table 3.--Monthly spray-irrigation volumes for the southwest spray field, 1966-81--Continued

\begin{tabular}{|c|c|c|c|c|c|c|}
\hline Month & Year & $\begin{array}{l}\text { Single } \\
\text { gun } \\
\text { area }\end{array}$ & $\begin{array}{c}\text { Irrigation } \\
\text { fields } \\
\text { area }\end{array}$ & $\begin{array}{l}\text { 4-big } \\
\text { gun } \\
\text { area }\end{array}$ & $\begin{array}{c}1977 \\
\text { expansion } \\
\text { area }\end{array}$ & $\begin{array}{l}\text { Monthly } \\
\text { sum, } \\
\text { al1 areas }\end{array}$ \\
\hline January & 1969 & 7.2 & 8 & 0 & 0 & 15.2 \\
\hline February & 1969 & 7.2 & 8 & 0 & 0 & 15.2 \\
\hline March & 1969 & 7.2 & 8 & 0 & 0 & 15.2 \\
\hline April & 1969 & 7.2 & 8 & 0 & 0 & 15.2 \\
\hline May & 1969 & 7.2 & 8 & 0 & 0 & 15.2 \\
\hline June & 1969 & 7.2 & 8 & 0 & 0 & 15.2 \\
\hline July & 1969 & 7.2 & 8 & 0 & 0 & 15.2 \\
\hline August & 1969 & 7.2 & 8 & 0 & 0 & 15.2 \\
\hline September & 1969 & 7.2 & 8 & 0 & 0 & 15.2 \\
\hline October & 1969 & 7.2 & 8 & 0 & 0 & 15.2 \\
\hline November & 1969 & 7.2 & 8 & 0 & 0 & 15.2 \\
\hline December & 1969 & 7.2 & 8 & 0 & 0 & 15.2 \\
\hline January & 1970 & 7.2 & 8 & 0 & 0 & 15.2 \\
\hline February & 1970 & 7.2 & 8 & 0 & 0 & 15.2 \\
\hline March & 1970 & 7.2 & 8 & 0 & 0 & 15.2 \\
\hline April & 1970 & 7.2 & 8 & 0 & 0 & 15.2 \\
\hline May & 1970 & 7.2 & 8 & 0 & 0 & 15.2 \\
\hline June & 1970 & 7.2 & 8 & 0 & 0 & 15.2 \\
\hline July & 1970 & 7.2 & 8 & 0 & 0 & 15.2 \\
\hline August & 1970 & 7.2 & 8 & 0 & 0 & 15.2 \\
\hline September & 1970 & 7.2 & 8 & 0 & 0 & 15.2 \\
\hline October & 1970 & 7.2 & 8 & 0 & 0 & 15.2 \\
\hline November & 1970 & 7.2 & 8 & 0 & 0 & 15.2 \\
\hline December & 1970 & 7.2 & 8 & 0 & 0 & 15.2 \\
\hline January & 1971 & 7.2 & 8 & 0 & 0 & 15.2 \\
\hline February & 1971 & 7.2 & 8 & 0 & 0 & 15.2 \\
\hline March & 1971 & 7.2 & 8 & 0 & 0 & 15.2 \\
\hline April & 1971 & 7.2 & 8 & 0 & 0 & 15.2 \\
\hline May & 1971 & 7.2 & 8 & 0 & 0 & 15.2 \\
\hline June & 1971 & 7.2 & 8 & 0 & 0 & 15.2 \\
\hline July & 1971 & 7.2 & 8 & 0 & 0 & 15.2 \\
\hline August & 1971 & 7.2 & 8 & 0 & 0 & 15.2 \\
\hline September & 1971 & 7.2 & 8 & 0 & 0 & 15.2 \\
\hline October & 1971 & 7.2 & 8 & 0 & 0 & 15.2 \\
\hline November & 1971 & 7.2 & 8 & 0 & 0 & 15.2 \\
\hline December & 1971 & 7.2 & 8 & 0 & 0 & 15.2 \\
\hline
\end{tabular}


Table 3.--Monthly spray-irrigation volumes for the southwest spray field, 1966-81--Continued

\begin{tabular}{|c|c|c|c|c|c|c|}
\hline Month & Year & $\begin{array}{l}\text { Single } \\
\text { gun } \\
\text { area }\end{array}$ & $\begin{array}{c}\text { Irrigation } \\
\text { fields } \\
\text { area }\end{array}$ & $\begin{array}{c}\text { 4-big } \\
\text { gun } \\
\text { area }\end{array}$ & $\begin{array}{c}1977 \\
\text { expansion } \\
\text { area }\end{array}$ & $\begin{array}{c}\text { Monthly } \\
\text { sum, } \\
\text { al1 areas }\end{array}$ \\
\hline January & 1972 & 7.0 & 8 & 0 & 0 & 15.0 \\
\hline February & 1972 & 7.0 & 8 & 0 & 0 & 15.0 \\
\hline March & 1972 & 7.0 & 8 & 30 & 0 & 45.0 \\
\hline April & 1972 & 7.0 & 8 & 30 & 0 & 45.0 \\
\hline May & 1972 & 7.0 & 8 & 30 & 0 & 45.0 \\
\hline June & 1972 & 7.0 & 8 & 30 & 0 & 45.0 \\
\hline July & 1972 & 7.0 & 8 & 30 & 0 & 45.0 \\
\hline August & 1972 & 7.0 & 8 & 30 & 0 & 45.0 \\
\hline September & 1972 & 7.0 & 8 & 30 & 0 & 45.0 \\
\hline October & 1972 & 7.0 & 8 & 30 & 0 & 45.0 \\
\hline November & 1972 & 7.0 & 8 & 30 & 0 & 45.0 \\
\hline December & 1972 & 7.0 & 8 & 30 & 0 & 45.0 \\
\hline January & 1973 & 7.0 & 8 & 30 & 0 & 45.0 \\
\hline February & 1973 & 0.0 & 9 & 30 & 0 & 39.0 \\
\hline March & 1973 & 0.0 & 9 & 30 & 0 & 39.0 \\
\hline April & 1973 & 0.0 & 9 & 30 & 0 & 39.0 \\
\hline May & 1973 & 0.0 & 9 & 30 & 0 & 39.0 \\
\hline June & 1973 & 0.0 & 9 & 30 & 0 & 39.0 \\
\hline July & 1973 & 0.0 & 9 & 30 & 0 & 39.0 \\
\hline August & 1973 & 0.0 & 9 & 30 & 0 & 39.0 \\
\hline September & 1973 & 0.0 & 9 & 30 & 0 & 39.0 \\
\hline October & 1973 & 0.0 & 9 & 30 & 0 & 39.0 \\
\hline November & 1973 & 0.0 & 9 & 30 & 0 & 39.0 \\
\hline December & 1973 & 0.0 & 9 & 30 & 0 & 39.0 \\
\hline January & 1974 & 0.0 & 9 & 30 & 0 & 39.0 \\
\hline February & 1974 & 0.0 & 9 & 30 & 0 & 39.0 \\
\hline March & 1974 & 0.0 & 9 & 30 & 0 & 39.0 \\
\hline April & 1974 & 0.0 & 9 & 30 & 0 & 39.0 \\
\hline May & 1974 & 0.0 & 9 & 30 & 0 & 39.0 \\
\hline June & 1974 & 0.0 & 9 & 30 & 0 & 39.0 \\
\hline July & 1974 & 0.0 & 9 & 30 & 0 & 39.0 \\
\hline August & 1974 & 0.0 & - & 0 & 0 & - \\
\hline September & 1974 & 0.0 & -- & 0 & 0 & - \\
\hline October & 1974 & 0.0 & -- & 0 & 0 & - \\
\hline November & 1974 & 0.0 & - & 0 & 0 & -- \\
\hline December & 1974 & 0.0 & - & 0 & 0 & -- \\
\hline
\end{tabular}


Table 3.--Monthly spray-irrigation volumes for the southwest spray field, 1966-81--Continued

\begin{tabular}{|c|c|c|c|c|c|c|}
\hline Month & Year & $\begin{array}{l}\text { Single } \\
\text { gun } \\
\text { area }\end{array}$ & $\begin{array}{l}\text { Irrigation } \\
\text { fields } \\
\text { area }\end{array}$ & $\begin{array}{l}\text { 4-big } \\
\text { gun } \\
\text { area }\end{array}$ & $\begin{array}{c}1977 \\
\text { expansion } \\
\text { area }\end{array}$ & $\begin{array}{c}\text { Monthly } \\
\text { sum, } \\
\text { a11 areas }\end{array}$ \\
\hline January & 1975 & 0.0 & -- & 0 & 0 & -- \\
\hline February & 1975 & 0.0 & -- & 0 & 0 & -- \\
\hline March & 1975 & 0.0 & -- & 0 & 0 & -- \\
\hline April & 1975 & 0.0 & -- & 0 & 0 & -- \\
\hline May & 1975 & 0.0 & -- & 0 & 0 & -- \\
\hline June & 1975 & 0.0 & -- & 0 & 0 & -- \\
\hline July & 1975 & 0.0 & -- & 0 & 0 & - \\
\hline August & 1975 & 0.0 & -- & 0 & 0 & -- \\
\hline September & 1975 & 0.0 & -- & 0 & 0 & -- \\
\hline October & 1975 & 0.0 & -- & 0 & 0 & -- \\
\hline November & 1975 & 0.0 & -- & 0 & 0 & -- \\
\hline December & 1975 & 0.0 & -- & 0 & 0 & -- \\
\hline January & 1976 & 0.0 & -- & 0 & 0 & -- \\
\hline February & 1976 & 0.0 & - & 0.218 & 0 & - \\
\hline March & 1976 & 0.0 & -- & 0.252 & 0 & -- \\
\hline April & 1976 & 0.0 & 16.98 & 18.310 & 0 & 35.29 \\
\hline May & 1976 & 0.0 & - & 0.00 & 0 & - \\
\hline June & 1976 & 0.0 & -- & 0.332 & 0 & -- \\
\hline July & 1976 & 0.0 & -- & -- & 0 & -- \\
\hline August & 1976 & 0.0 & -- & - & 0 & - \\
\hline September & 1976 & 0.0 & -- & -- & 0 & - \\
\hline October & 1976 & 0.0 & -- & -- & 0 & -- \\
\hline November & 1976 & 0.0 & -- & -- & 0 & -- \\
\hline December & 1976 & 0.0 & -- & -- & 0 & -- \\
\hline January & 1977 & 0.0 & -- & -- & 0 & -- \\
\hline February & 1977 & 0.0 & -- & -- & 0 & -- \\
\hline March & 1977 & 0.0 & 1.30 & 3.390 & 0 & 4.69 \\
\hline April & 1977 & 0.0 & 6.98 & 13.520 & 0 & 20.50 \\
\hline May & 1977 & 0.0 & 4.58 & 7.410 & 0 & 11.99 \\
\hline June & 1977 & 0.0 & 5.08 & 6.110 & 0 & 11.19 \\
\hline July & 1977 & 0.0 & 3.44 & 9.760 & 0 & 13.20 \\
\hline August & 1977 & 0.0 & 4.09 & 7.670 & 0 & 11.76 \\
\hline September & 1977 & 0.0 & 4.00 & 7.000 & 0 & 11.00 \\
\hline October & 1977 & 0.0 & 3.57 & 9.650 & 0 & 13.22 \\
\hline November & 1977 & 0.0 & 3.07 & 5.390 & 0 & 8.46 \\
\hline December & 1977 & 0.0 & 3.82 & 4.060 & 4.59 & 12.47 \\
\hline
\end{tabular}


Table 3.--Monthly spray-irrigation volumes for the southwest spray fie1d, 1966-81--Continued

\begin{tabular}{|c|c|c|c|c|c|c|}
\hline Month & Year & $\begin{array}{l}\text { Single } \\
\text { gun } \\
\text { area }\end{array}$ & $\begin{array}{c}\text { Irrigation } \\
\text { fields } \\
\text { area }\end{array}$ & $\begin{array}{l}\text { 4-big } \\
\text { gun } \\
\text { area }\end{array}$ & $\begin{array}{c}1977 \\
\text { expansion } \\
\text { area }\end{array}$ & $\begin{array}{l}\text { Monthly } \\
\text { sum, } \\
\text { all areas }\end{array}$ \\
\hline January & 1978 & 2.430 & 4.330 & 5.750 & 3.990 & 16.500 \\
\hline February & 1978 & 2.250 & 3.970 & 3.870 & 0.000 & 10.090 \\
\hline March & 1978 & 1.360 & 2.120 & 3.400 & 7.720 & 14.600 \\
\hline April & 1978 & 1.410 & 1.620 & 3.230 & 7.950 & 14.210 \\
\hline May & 1978 & 1.530 & 1.790 & 3.110 & 8.260 & 14.690 \\
\hline June & 1978 & 1.300 & 1.600 & 4.180 & 19.300 & 26.380 \\
\hline Ju1y & 1978 & 1.130 & 1.340 & 1.640 & 9.860 & 13.970 \\
\hline August & 1978 & 1.160 & 1.810 & 1.580 & 4.600 & 9.150 \\
\hline September & 1978 & 1.690 & 2.100 & 5.010 & 16.900 & 25.700 \\
\hline October & 1978 & 4.850 & 4.740 & 5.560 & 26.380 & 41.530 \\
\hline November & 1978 & 4.750 & 4.440 & 5.850 & 23.780 & 38.820 \\
\hline December & 1978 & 4.150 & 4.790 & 6.250 & 23.600 & 38.790 \\
\hline January & 1979 & 1.330 & 2.630 & 4.750 & 18.200 & 27.910 \\
\hline February & 1979 & 2.070 & 2.650 & 3.780 & 17.490 & 25.990 \\
\hline March & 1979 & 4.320 & 4.330 & 5.420 & 23.380 & 37.450 \\
\hline April & 1979 & 2.280 & 2.370 & 3.210 & 13.800 & 21.660 \\
\hline May & 1979 & 1.830 & 0.000 & 3.100 & 7.400 & 12.330 \\
\hline June & 1979 & 3.830 & 0.000 & 4.740 & 17.900 & 26.470 \\
\hline July & 1979 & 4.450 & 0.000 & 4.730 & 10.350 & 19.530 \\
\hline August & 1979 & 4.250 & 0.000 & 2.300 & 7.610 & 14.160 \\
\hline September & 1979 & 0.300 & 0.000 & 0.000 & 0.930 & 1.230 \\
\hline October & 1979 & 2.100 & 0.000 & 0.000 & 9.970 & 12.070 \\
\hline November & 1979 & .450 & 0.000 & 0.000 & 3.440 & 3.890 \\
\hline December & 1979 & .450 & 0.000 & 0.000 & 5.170 & 5.620 \\
\hline January & 1980 & 1.260 & 0.000 & 0.000 & 7.330 & 8.590 \\
\hline February & 1980 & 0.000 & 0.000 & 0.000 & 0.000 & 0.000 \\
\hline March & 1980 & 0.148 & 0.816 & 6.610 & 17.980 & 25.554 \\
\hline April & 1980 & 1.048 & 0.000 & 2.640 & 12.330 & 16.018 \\
\hline May & 1980 & 2.730 & 2.329 & 4.200 & 11.100 & 20.359 \\
\hline June & 1980 & 2.070 & 2.359 & 5.030 & 5.680 & 15.139 \\
\hline July & 1980 & 0.298 & 0.000 & 0.169 & 5.560 & 6.027 \\
\hline August & 1980 & 0.890 & 0.746 & 1.510 & 7.070 & 10.216 \\
\hline September & 1980 & 1.820 & 2.055 & 3.070 & 3.980 & 10.925 \\
\hline October & 1980 & 2.580 & 1.510 & 2.470 & 4.510 & 11.070 \\
\hline November & 1980 & 1.860 & 1.171 & 1.610 & 7.340 & 11.981 \\
\hline December & 1980 & 0.920 & 0.000 & 2.260 & 12.320 & 15.500 \\
\hline
\end{tabular}


Table 3.--Monthly spray-irrigation volumes for the southwest spray fie1d, 1966-81--Continued

\begin{tabular}{lccccrr}
\hline \multicolumn{1}{c}{ Month } & Year & $\begin{array}{c}\text { Single } \\
\text { gun } \\
\text { area }\end{array}$ & $\begin{array}{c}\text { Irrigation } \\
\text { fields } \\
\text { area }\end{array}$ & $\begin{array}{c}\text { 4-big } \\
\text { gun } \\
\text { area }\end{array}$ & $\begin{array}{c}1977 \\
\text { expansion } \\
\text { area }\end{array}$ & $\begin{array}{c}\text { Monthly } \\
\text { sum, } \\
\text { a11 areas }\end{array}$ \\
\hline January & 1981 & 0.000 & 0.799 & 5.510 & 29.740 & 36.049 \\
$\begin{array}{l}\text { February } \\
\text { March }\end{array}$ & 1981 & 0.000 & 0.000 & 5.154 & 27.680 & 32.834 \\
Apri1 & 1981 & 0.000 & 0.000 & 3.816 & 9.900 & 13.716 \\
May & 1981 & 0.000 & 0.000 & 0.000 & 5.210 & 5.210 \\
June & 1981 & 0.347 & 0.000 & 0.000 & 6.600 & 6.947 \\
& 1981 & 0.000 & 0.000 & 0.000 & 6.600 & 6.600 \\
July & 1981 & 2.917 & 0.000 & 0.000 & 7.792 & 10.709 \\
August & 1981 & 1.625 & 0.000 & 0.000 & 2.132 & 3.757 \\
September & 1981 & 4.258 & 0.000 & 0.000 & 16.020 & 20.278 \\
& & & & & & \\
\hline
\end{tabular}


Table 4.--Annual spray-irrigation volumes for the southwest spray field, 1966-81

[Volumes are in million gallons. Records prior to 1977 are approximate. Sums are not computed for periods of no record. Years with no record are indicated by --]

\begin{tabular}{|c|c|c|c|c|c|}
\hline Year & $\begin{array}{l}\text { Single } \\
\text { gun } \\
\text { area }\end{array}$ & $\begin{array}{c}\text { Irrigation } \\
\text { fields } \\
\text { area }\end{array}$ & $\begin{array}{l}\text { 4-big } \\
\text { gun } \\
\text { area }\end{array}$ & $\begin{array}{l}1977 \\
\text { expansion } \\
\text { area }\end{array}$ & $\begin{array}{c}\text { Yearly } \\
\text { sum, } \\
\text { a11 areas }\end{array}$ \\
\hline 1966 & 43.200 & 48.000 & 0.000 & 0.000 & 91.200 \\
\hline 1967 & 86.400 & 96.000 & 0.000 & 0.000 & 182.400 \\
\hline 1968 & 86.400 & 96.000 & 0.000 & 0.000 & 182.400 \\
\hline 1969 & 86.400 & 96.000 & 0.000 & 0.000 & 182.400 \\
\hline 1970 & 86.400 & 96.000 & 0.000 & 0.000 & 182.400 \\
\hline 1971 & 86.400 & 96.000 & 0.000 & 0.000 & 182.400 \\
\hline 1972 & 84.000 & 96.000 & 300.000 & 0.000 & 480.000 \\
\hline 1973 & 7.000 & 107.000 & 360.000 & 0.000 & 474.000 \\
\hline 1974 & 0.000 & 63.000 & 210.000 & 0.000 & 273.000 \\
\hline 1975 & 0.000 & -- & 0.000 & 0.000 & - \\
\hline 1976 & 0.000 & 17.980 & 19.112 & 0.000 & 36.092 \\
\hline 1977 & 0.000 & 39.930 & 73.960 & 4.590 & 118.480 \\
\hline 1978 & 28.010 & 34.650 & 49.430 & 152.340 & 264.430 \\
\hline 1979 & 28.660 & 11.980 & 32.030 & 135.640 & 208.310 \\
\hline 1980 & 15.624 & 10.986 & 29.569 & 95.200 & 151.379 \\
\hline 1981 & 9.147 & 0.799 & 14.480 & 111.674 & 136.100 \\
\hline
\end{tabular}


Table 5.--Nitrifier and denitrifier bacteria in soil samples, September 1977 to June 1978

[Samples were collected next to sites indicated. Analyses for samples collected in July 1973 are given in Slack (1975). The results were obtained using the multiple-tube technique and are reported per $100 \mathrm{ml}$. e = estimated values]

\begin{tabular}{|c|c|c|c|}
\hline \multirow{2}{*}{$\begin{array}{l}\text { Report } \\
\text { reference } \\
\text { No. }\end{array}$} & \multicolumn{2}{|c|}{ Nitrifiers } & \multirow{2}{*}{ Denitrifiers } \\
\hline & Nitrosomonas & Nitrobacter & \\
\hline
\end{tabular}

September $12-15,1977$

$\begin{array}{rrrr}1 & 1,100,000 & 14,000 & 2,400,000 \\ 3 & 15,000 & \mathrm{e} 2,000 & 7,500 \\ 5 & 3,000 & 2,400,000 & 3,000 \\ 9 & 24,000 & \mathrm{e} 2,800 & - \\ 20 & 30 & 640 & 40 \\ 25 & 30 & 1,200 & 30 \\ 28 & 430 & \mathrm{e} 1,100 & 240,000\end{array}$

November $1-4,1977$

400,000
2,300
15,000
$2,400,000$
900
300
300
300
240,000

$1,500,000$ 300 300

3,000

$\mathrm{e} 1,100$

2,100

15,000

240,000

6,400
$1,100,000$ 300
2,300
460,000 300 300 300 240,000 240,000

June $12-16,1978$

$\begin{array}{rr}1 & 240,000 \\ 3 & 240,000 \\ 5 & 300 \\ 6 & 300 \\ 19 & 2,300 \\ 20 & 9,300 \\ 25 & 2,300 \\ 26 & 300 \\ 28 & 240,000\end{array}$

$$
\begin{array}{r}
300 \\
3,900 \\
240,000 \\
110,000 \\
300 \\
300 \\
300 \\
9,300 \\
2,300
\end{array}
$$$$
\begin{array}{r}
240,000 \\
1,400 \\
900 \\
300 \\
900 \\
300 \\
1,100 \\
400 \\
240,000
\end{array}
$$ 
Site 1 in 4-big-gun area

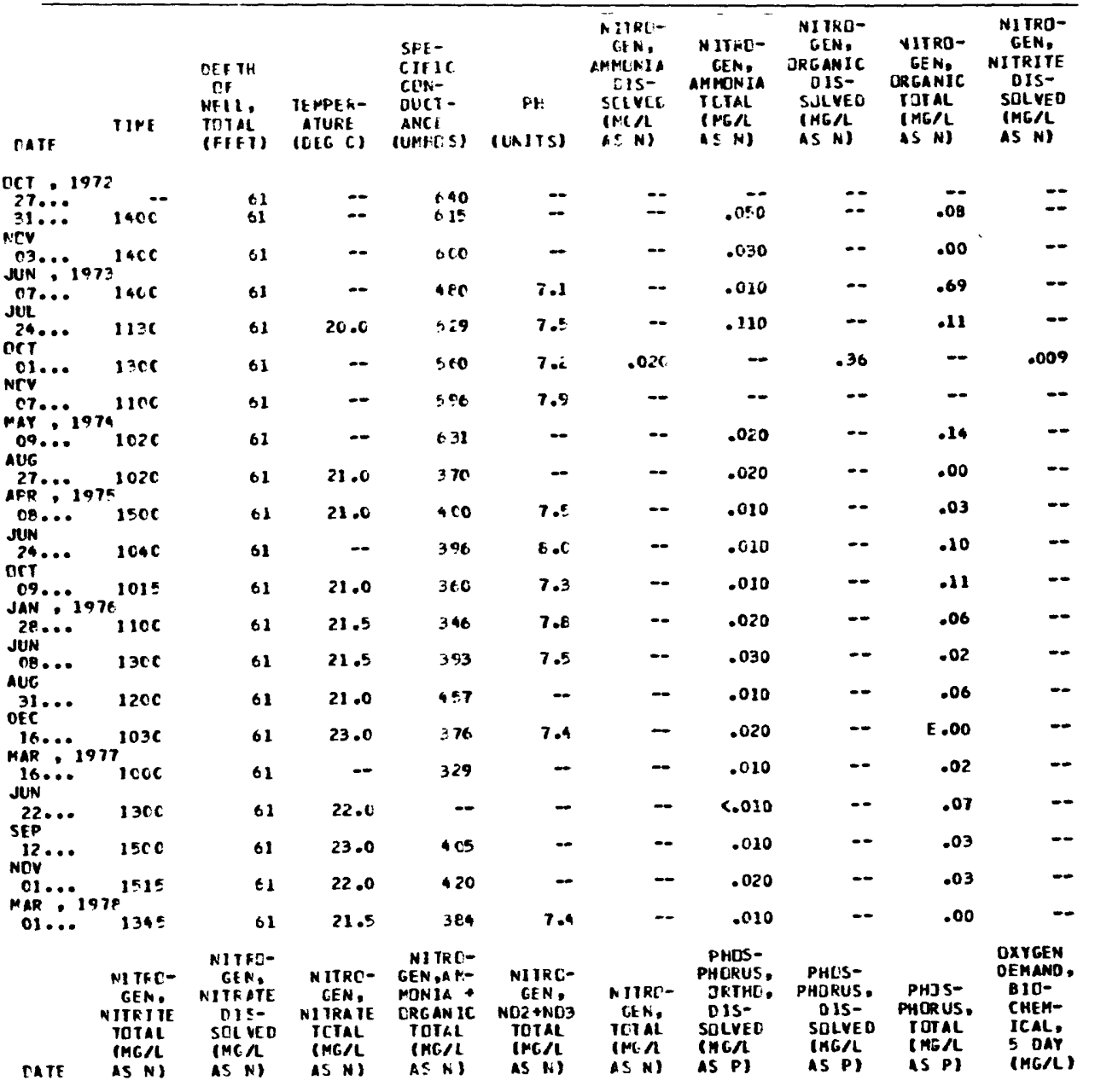

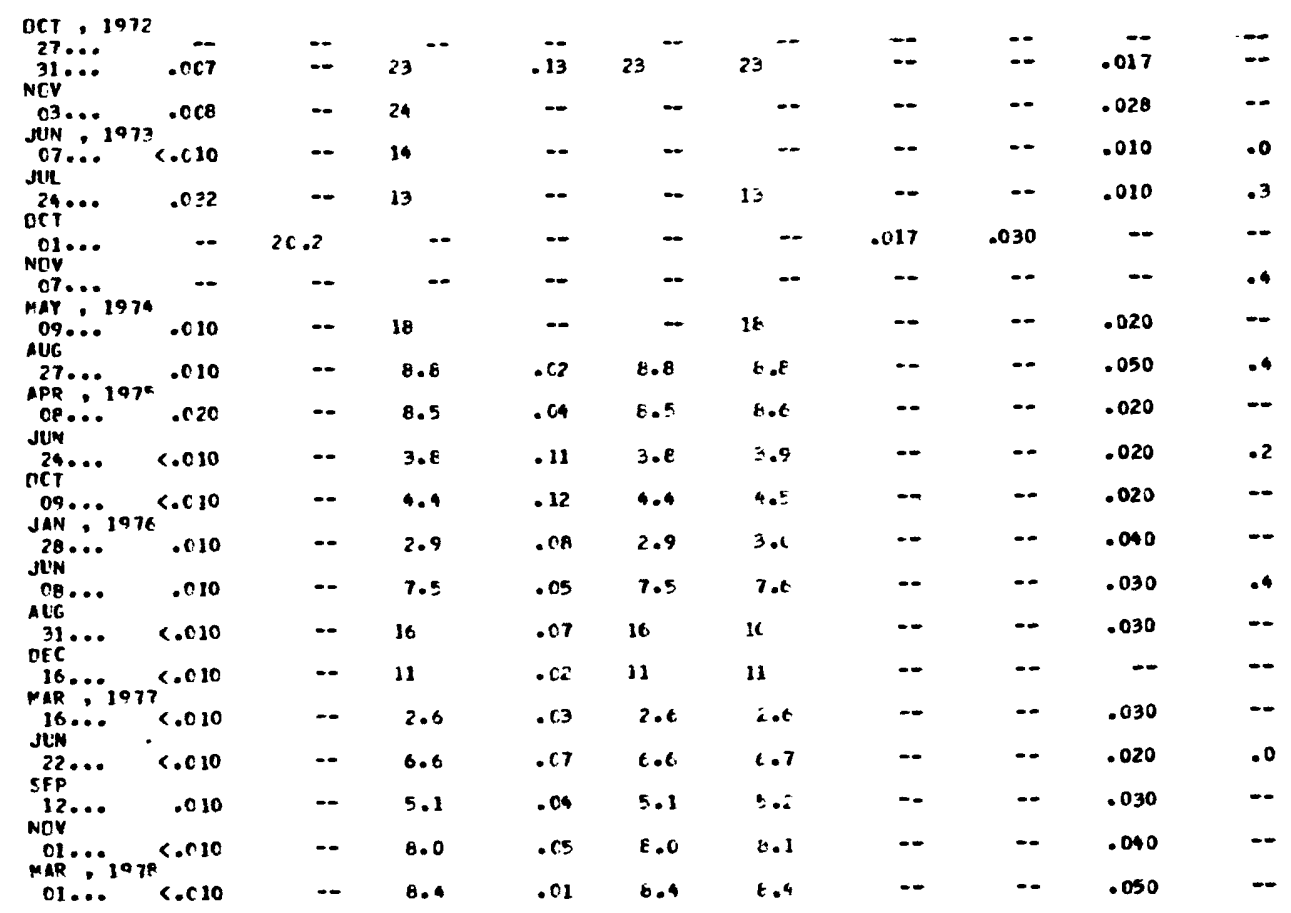


Site 1 in 4-big-gun area--Continued

\begin{tabular}{|c|c|c|c|c|c|c|c|c|c|}
\hline & $\begin{array}{l}\text { CXYGEA } \\
\text { OEMARE. } \\
\text { CHEY- } \\
\text { ICAL } \\
\text { IHICH } \\
\text { IEVELI } \\
\text { (YC/L) }\end{array}$ & $\begin{array}{l}\text { CARELIN, } \\
\text { INCR- } \\
\text { GAAIC, } \\
\text { TUTAL } \\
\text { PMCAL } \\
\text { AS (I) }\end{array}$ & $\begin{array}{l}\text { CARBEN, } \\
\text { CRGATIC } \\
\text { TCIAL } \\
\text { IMGA } \\
\text { AS C) }\end{array}$ & $\begin{array}{l}\text { CERECN, } \\
\text { TOTAL } \\
\text { PR C R } \\
\text { AS C) }\end{array}$ & $\begin{array}{l}\text { ALKA- } \\
\text { LINI TY } \\
\text { FIELL } \\
\text { (MEA } \\
\text { AS } \\
\text { CACE }\end{array}$ & $\begin{array}{l}\text { HAE- } \\
\text { NFSS } \\
\text { ACNCAR- } \\
\text { ECNATE } \\
\text { THC/L } \\
\text { CACCOS }\end{array}$ & $\begin{array}{l}\text { HAKJ)- } \\
N E S S \\
\text { (MG/L } \\
A S \\
\left(A C()_{3}\right)\end{array}$ & $\begin{array}{l}\text { CALCIUM } \\
\text { UIS- } \\
\text { SULVES } \\
\text { (MCAL } \\
\text { AS CA) }\end{array}$ & $\begin{array}{l}\text { MAGNE- } \\
\text { SIUH, } \\
\text { OIS- } \\
\text { SOLVED } \\
\text { (HG/L } \\
\text { AS MG) }\end{array}$ \\
\hline
\end{tabular}

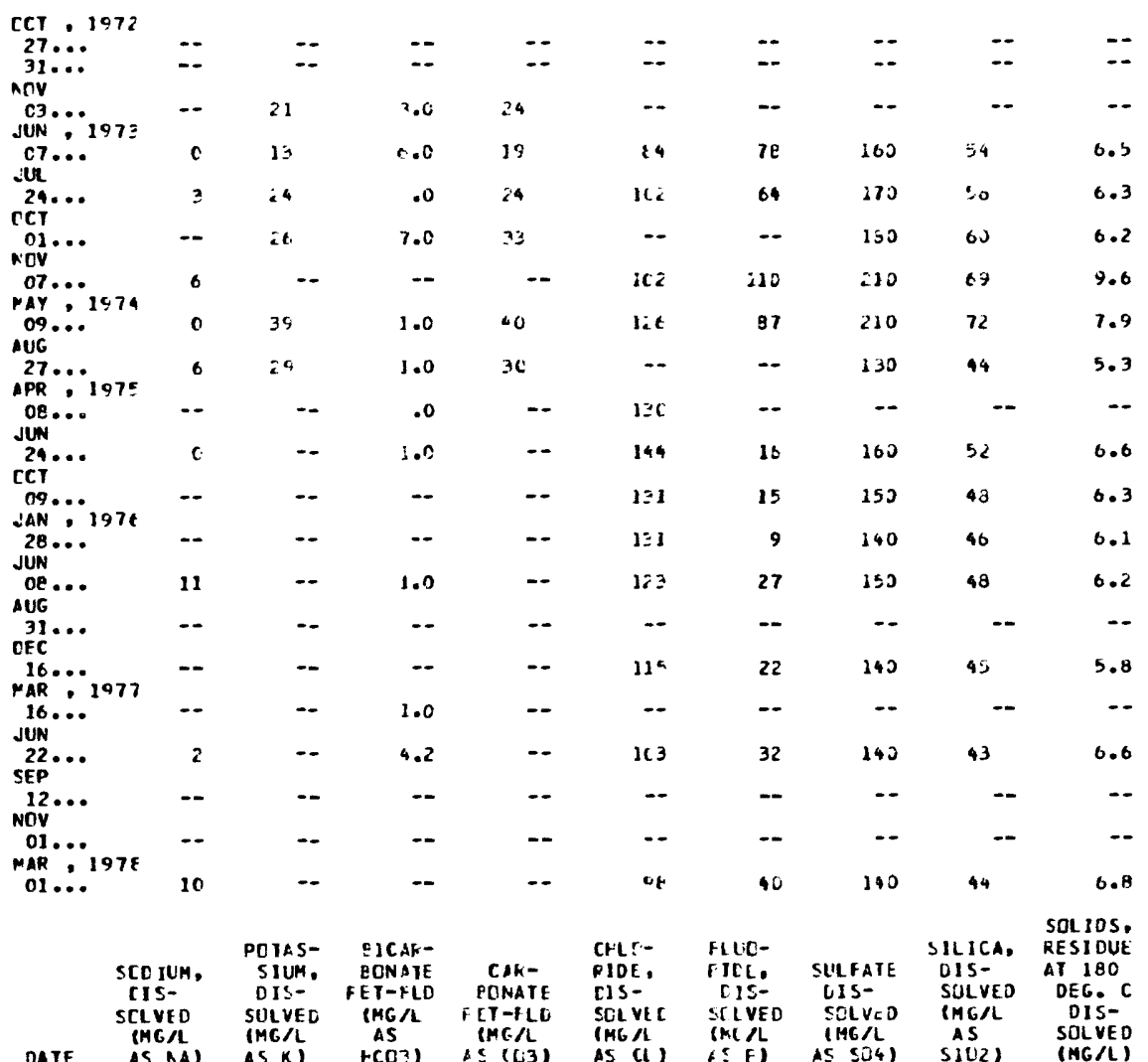

CCT 1972

\begin{tabular}{|c|c|c|c|c|c|c|c|c|c|}
\hline $\begin{array}{c}\mathrm{CCT} .1972 \\
27 . . .\end{array}$ & -- & $\cdots$ & -- & -- & 63 & -- & -- & $\cdots$ & -- \\
\hline $\operatorname{NOV}_{31} \cdots$ & - & -- & -- & -- & 67 & -- & -- & 11 & $\cdots$ \\
\hline JUN:1973 & $\cdots$ & - & -- & - & 65 & -- & -- & 12 & -- \\
\hline $\begin{array}{l}07 . . . \\
\text { JUL }\end{array}$ & $3 c$ & 1.6 & 102 & $c$ & 53 & .1 & II & 9.9 & 357 \\
\hline $\mathrm{CCI}^{24} \cdots$ & 32 & 2.1 & 124 & 0 & 48 & .1 & 13 & 8.7 & 325 \\
\hline NCV $01 .$. & 34 & 3.6 & $\because$ & 0 & 52 & -- & $\cdots$ & $\cdots$ & $\cdots$ \\
\hline PAY : 1974 & 32 & 4.5 & 124 & 0 & 57 & -- & -- & -- & - \\
\hline$\underset{\text { AUG }}{09} \ldots$ & 44 & 3.5 & 153 & c & $5 C$ & -- & 32 & -- & 462 \\
\hline$\underset{A P R}{27} \cdots 1975$ & $2 C$ & 2.3 & $\cdots$ & $\cdots$ & 20 & $\cdots$ & $\cdots$ & - & $\cdots$ \\
\hline$\underset{\text { JUN }}{C 8 \ldots}$ & -- & $=$ & 158 & 0 & 23 & $<.1$ & 13 & $\cdots$ & 224 \\
\hline $\mathrm{CCT}^{24} \cdots$ & $2 \mathrm{C}$ & 2.1 & 176 & c & 24 & .1 & 13 & 7.3 & 232 \\
\hline JAN 1976 & If & 1.8 & 160 & $c$ & $2 \mathrm{C}$ & $\bullet 1$ & 11 & 7.1 & 212 \\
\hline$\underset{\text { JUN }}{28} \cdots$ & 15 & 1.5 & $16 C$ & 0 & $1 \div$ & <.1 & 10 & 6.7 & 199 \\
\hline$\underset{\text { AUG }}{\text { OB.... }}$ & 21 & 2.3 & 150 & $c$ & 24 & .1 & 18 & 7.4 & 230 \\
\hline DEC $31 . \cdots$ & $3 c$ & $\cdots$ & -- & -- & 33 & $\cdots$ & $\cdots$ & -- & -- \\
\hline MAR $\because 1977$ & 21 & 2.5 & 140 & $c$ & 24 & .1 & -- & 7.4 & 234 \\
\hline$\underset{\text { JUN }}{16}$ & 16 & $\cdots$ & -- & $\cdots$ & 17 & $\cdots$ & $\cdots$ & $\cdots$ & -- \\
\hline$\underset{\text { SEP }}{22} \cdots$ & 21 & 2.3 & 125 & c & 22 & $<.1$ & 14 & 7.5 & 244 \\
\hline $\operatorname{NOV}^{12} \cdots$ & 21 & $\cdots$ & - & $\cdots$ & 28 & - & $\cdots$ & $\because$ & $\ldots$ \\
\hline MAR $\because 1978$ & $2 C$ & -- & $\cdots$ & $\cdots$ & 25 & - & - & $\cdots$ & 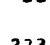 \\
\hline $01 \ldots$ & 26 & $2 \cdot 4$ & 320 & $c$ & 24 & •] & 1. & 7.3 & 223 \\
\hline
\end{tabular}




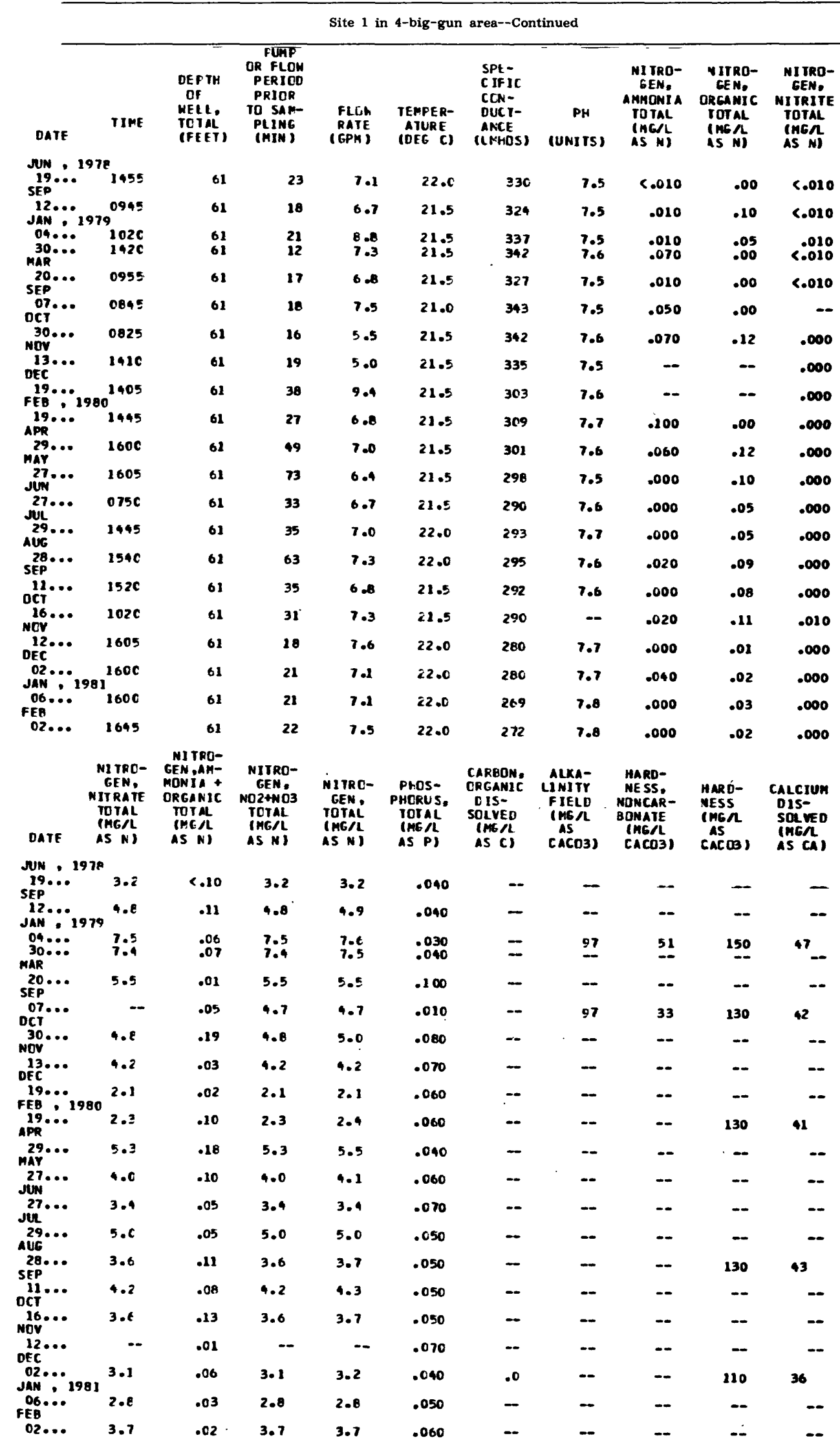


Site 1 in 4-big-gun area--Continued

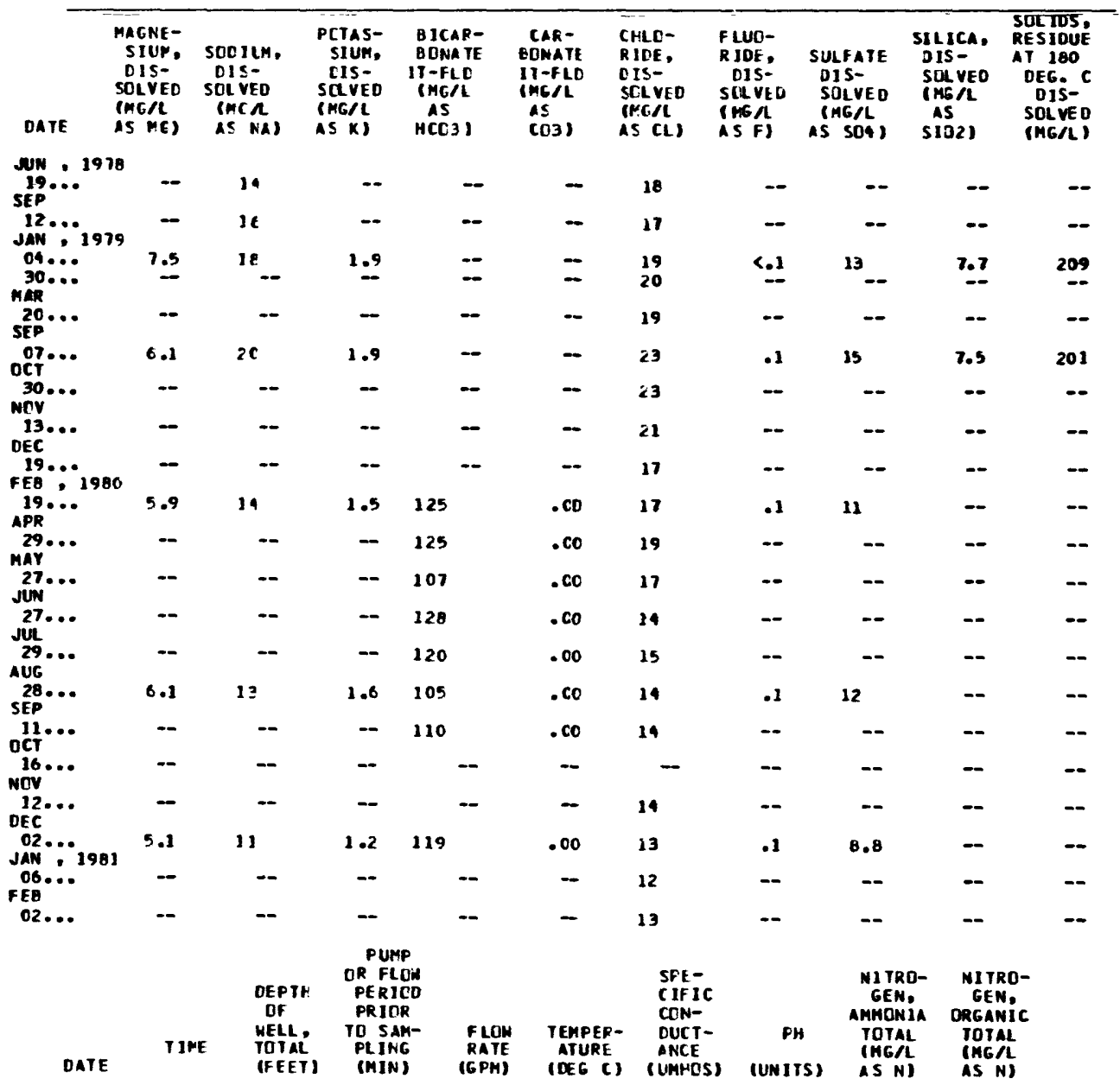
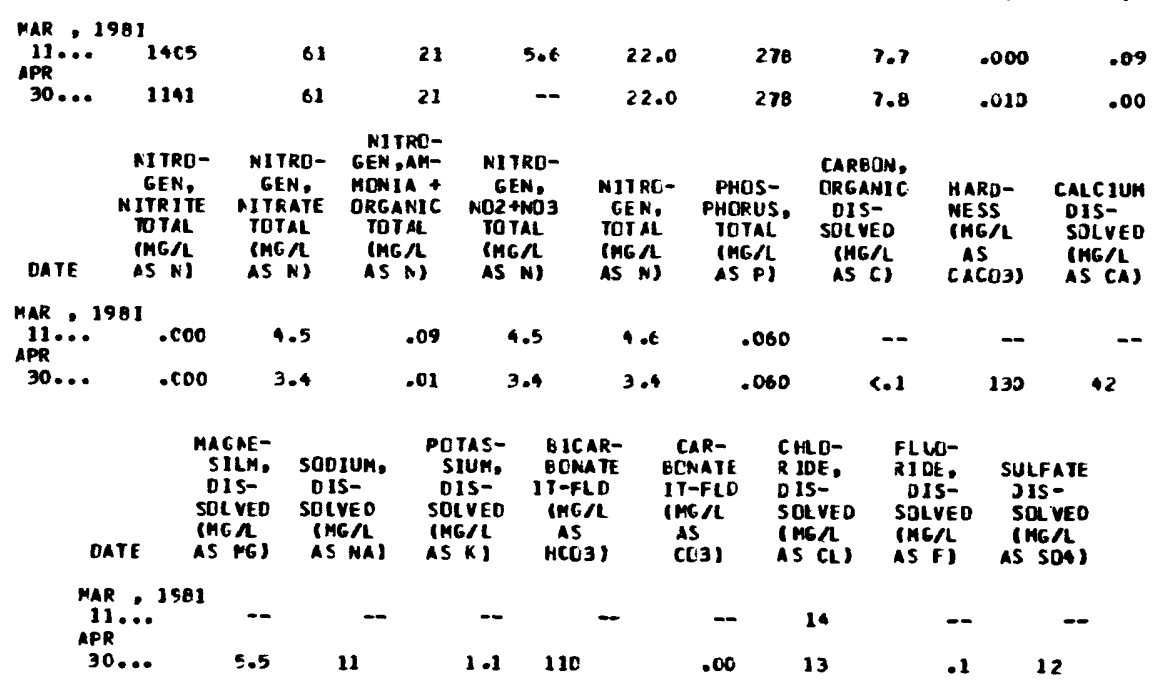
Site 1 in 4-big-gun area--Continued

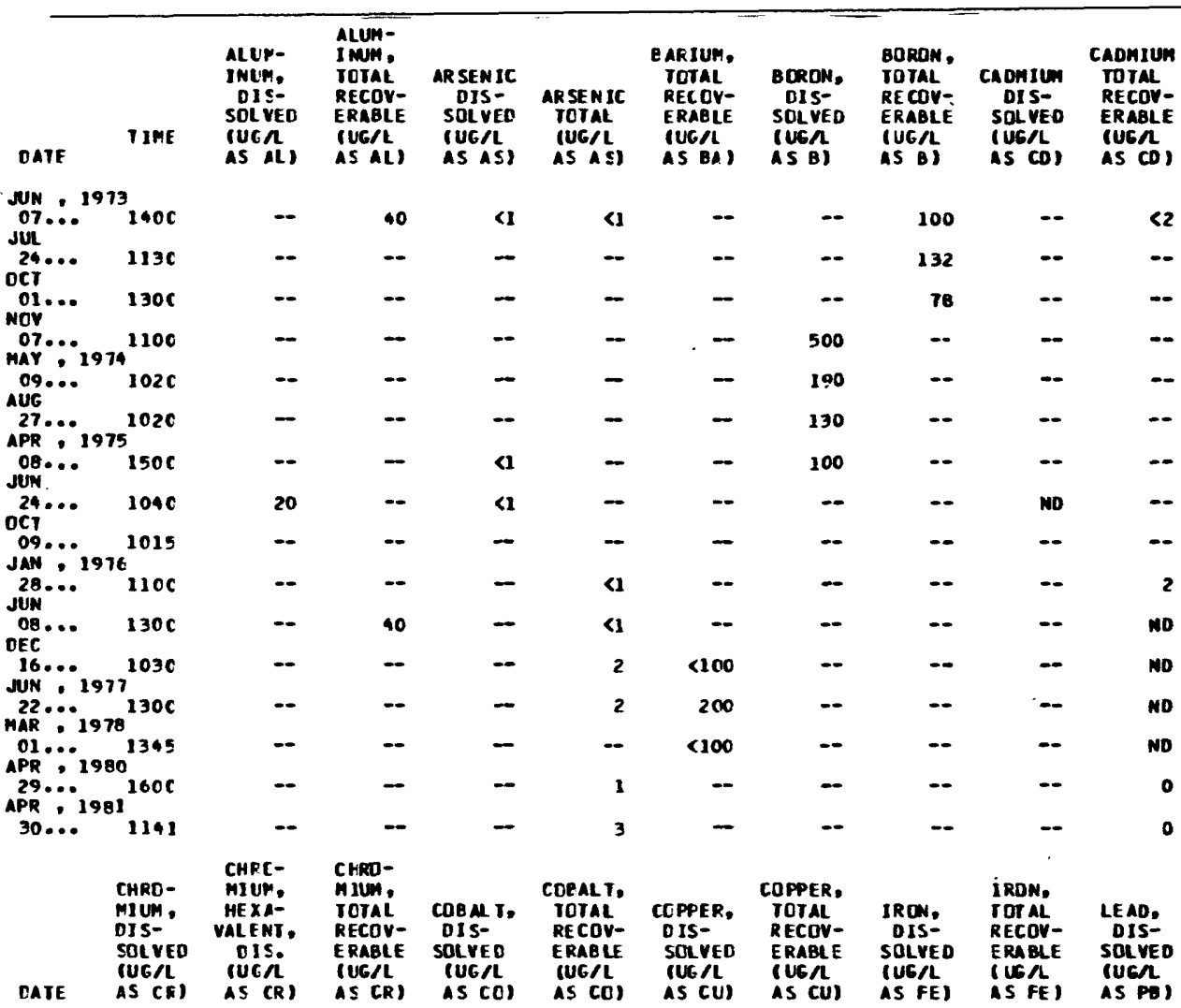

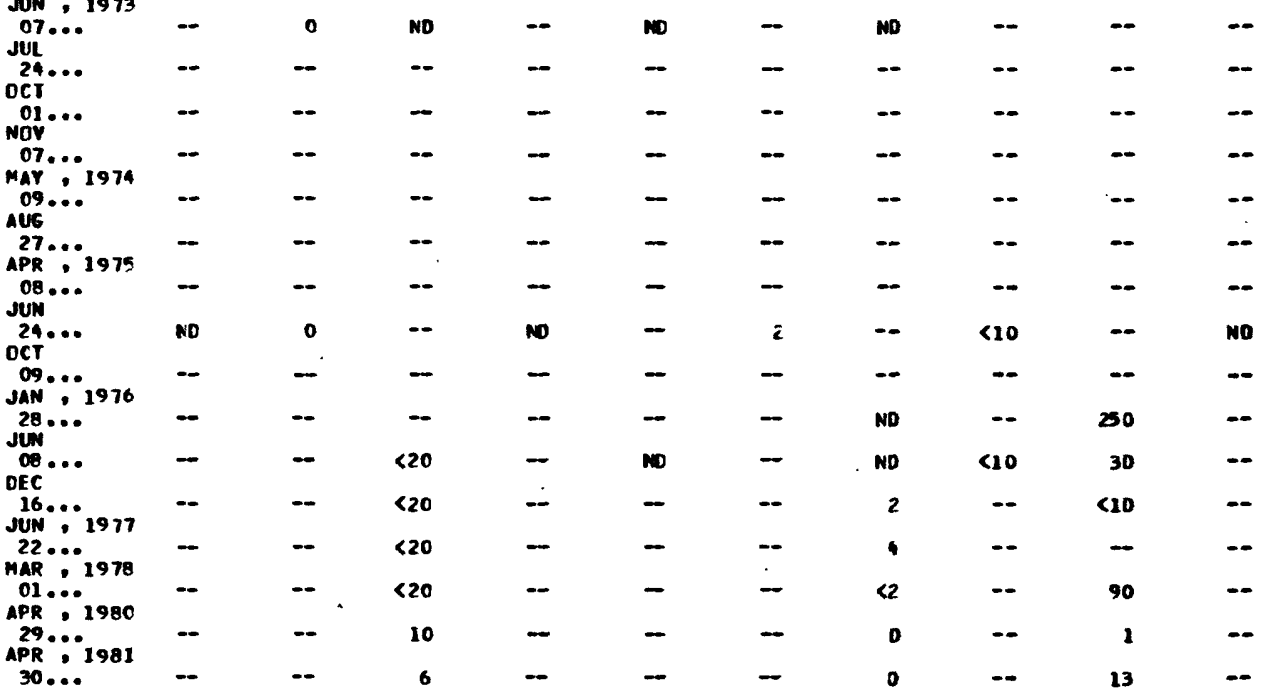


Table 6.--Physical and chemical characteristics of well and water at sites 1 through $70--$ Continued

Site 1 in 4-big-gun area--Continued

\begin{tabular}{|c|c|c|c|c|c|c|c|c|c|c|}
\hline ATE & $\begin{array}{l}\text { LEAD, } \\
\text { TOTAL } \\
\text { RECDV- } \\
\text { ER AELE } \\
\text { (UG/L } \\
\text { AS PE) }\end{array}$ & $\begin{array}{c}\text { LITHIUM } \\
\text { DIS- } \\
\text { SOEVED } \\
\text { IUGA } \\
\text { AS LII }\end{array}$ & $\begin{array}{l}\text { LITHIV } \\
\text { TOTAL } \\
\text { RECOY- } \\
\text { ERABLE } \\
\text { (UG/L } \\
\text { AS LI) }\end{array}$ & $\begin{array}{l}\text { MANGA- } \\
\text { NESE, } \\
\text { TOTAL } \\
\text { RECBV- } \\
\text { ERABLF } \\
\text { (UGAL } \\
\text { AS MA) }\end{array}$ & $\begin{array}{l}\text { MANGA- } \\
\text { NESE, } \\
\text { DIS- } \\
\text { SOLVEO } \\
\text { (UGAL } \\
\text { AS RN) }\end{array}$ & $\begin{array}{c}\text { MERCURY } \\
\text { OIS- } \\
\text { SCIVCO } \\
\text { (UG } \\
\text { AS HE) }\end{array}$ & $\begin{array}{l}\text { ME RCURY } \\
\text { TUTAL } \\
\text { RECOY- } \\
\text { ERABLE } \\
\text { (UG/L } \\
\text { IS HE) }\end{array}$ & $\begin{array}{l}\text { MOLYB- } \\
\text { DENUA, } \\
\text { OIS- } \\
\text { SOLVEO } \\
\text { (UG/L } \\
\text { AS MO) }\end{array}$ & $\begin{array}{l}\text { OOL YB- } \\
\text { JENUH, } \\
\text { TOTAL } \\
\text { ZECOV- } \\
\text { ERABLE } \\
\text { (UGA } \\
\text { (S MO) }\end{array}$ & $\begin{array}{l}\text { NICKEL, } \\
\text { DIS- } \\
\text { SOLVED } \\
\text { (UGA } \\
\text { AS NI, }\end{array}$ \\
\hline
\end{tabular}

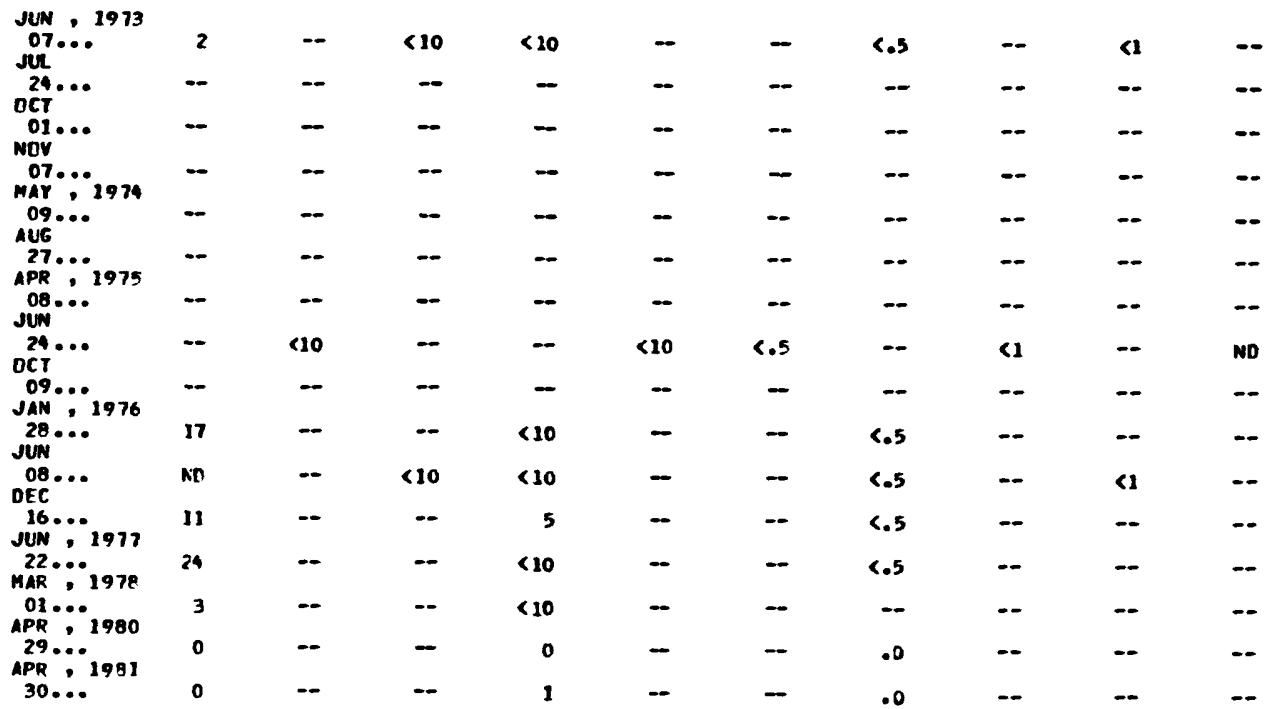

\begin{tabular}{|c|c|c|c|c|c|c|c|c|c|}
\hline DATE & $\begin{array}{l}\text { NICKEL. } \\
\text { TOTAL } \\
\text { RE COV- } \\
\text { ERAQLE } \\
\text { (UG/L } \\
\text { AS NI) }\end{array}$ & $\begin{array}{l}\text { SELE- } \\
\text { NIUM, } \\
\text { OIS- } \\
\text { SOLVED } \\
\text { (UG/L } \\
\text { AS SE ) }\end{array}$ & $\begin{array}{l}\text { SELE- } \\
\text { NIUM. } \\
\text { TOTAL } \\
\text { (UG/L } \\
\text { AS SE) }\end{array}$ & $\begin{array}{l}\text { SILVER, } \\
\text { TOTAL } \\
\text { RECEV- } \\
\text { ERABLE } \\
\text { (UGAL } \\
\text { AS AG) }\end{array}$ & $\begin{array}{l}\text { STRCN- } \\
\text { TIUA. } \\
\text { OIS- } \\
\text { SOLVED } \\
\text { (UG } \\
\text { AS SR) }\end{array}$ & $\begin{array}{l}\text { VANA- } \\
\text { DIUH. } \\
\text { DIS- } \\
\text { SOLVED } \\
\text { (UEA } \\
\text { AS VI }\end{array}$ & $\begin{array}{l}\text { VANA- } \\
\text { DIUHA } \\
\text { TJTAL } \\
\text { lUGAL } \\
\text { AS VI }\end{array}$ & $\begin{array}{l}\text { ZINC, } \\
\text { DIS- } \\
\text { SOLVED } \\
\text { (UG/L } \\
\text { AS ZN) }\end{array}$ & $\begin{array}{l}\text { ZINC, } \\
\text { TOTAL } \\
\text { RECOV- } \\
\text { ERABLE } \\
\text { (UG RL } \\
\text { AS ZN) }\end{array}$ \\
\hline
\end{tabular}

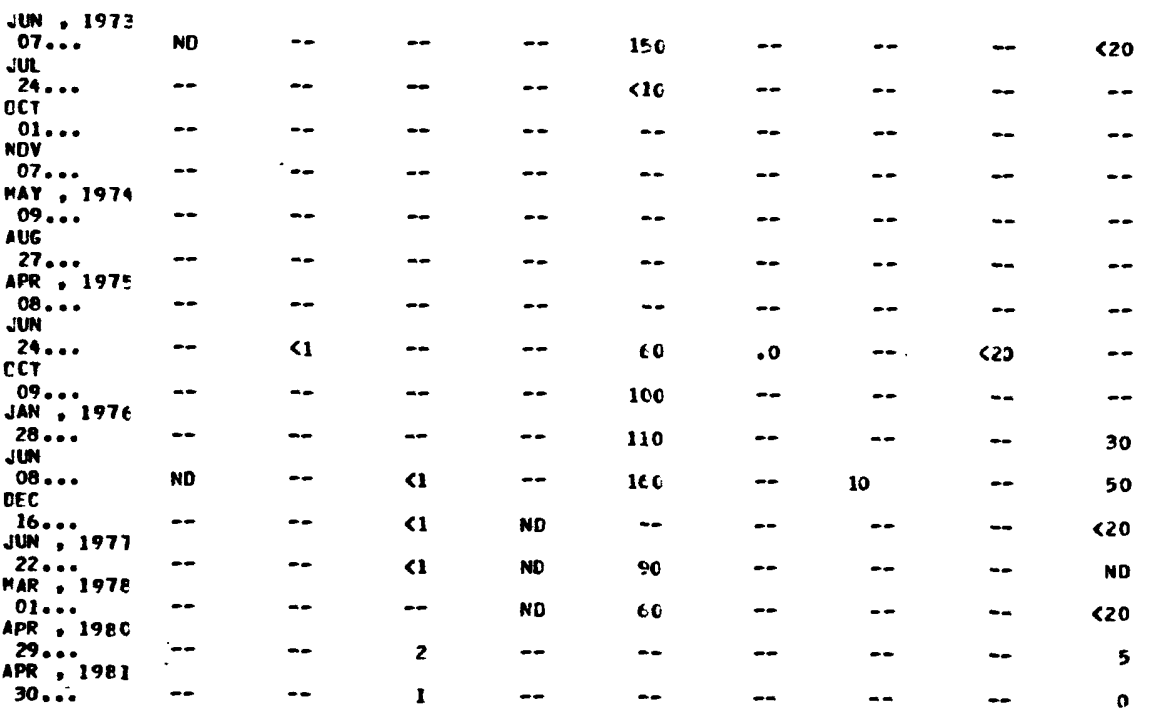

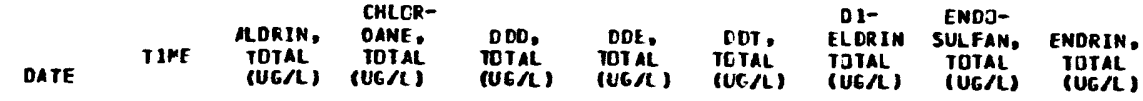

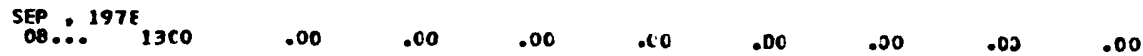




\begin{tabular}{|c|c|c|c|c|c|c|c|c|}
\hline \multicolumn{9}{|c|}{ Site 1 in $4-b i g-g u n$ area--Continued } \\
\hline DATE & $\begin{array}{l}\text { HEP TA- } \\
\text { CHLCR, } \\
\text { TOTAL } \\
\text { (UG L) }\end{array}$ & $\begin{array}{l}\text { HEPT A- } \\
\text { CHLDR } \\
\text { EPCXI DE } \\
\text { TOTAL } \\
\text { (UG/L) }\end{array}$ & $\begin{array}{l}\text { LINDANE } \\
\text { TOTAL } \\
\text { (UC/L) }\end{array}$ & $\begin{array}{l}\text { METH- } \\
\text { DXY- } \\
\text { CHLOR, } \\
\text { TOTAL } \\
\text { (UG/L) }\end{array}$ & $\begin{array}{l}\text { IREX, } \\
\text { TETAL } \\
\text { (UE } / L \text { ) }\end{array}$ & $\begin{array}{c}\text { TOX- } \\
\text { AP HENE, } \\
\text { TOTAL } \\
\text { (UG/L) }\end{array}$ & $\begin{array}{l}\text { PCB, } \\
\text { IOTAL } \\
\text { (UG } \Omega \text { ) }\end{array}$ & $\begin{array}{l}\text { TIAPH- } \\
\text { THA- } \\
\text { LENES. } \\
\text { POLY- } \\
\text { CHLDR. } \\
\text { IOJAL } \\
\text { (JG/LI) }\end{array}$ \\
\hline $\operatorname{SEP}_{\text {OB. }}$ & .00 & .00 &.$\infty 0$ & .00 &.$\infty 0$ & 0 & .00 & .00 \\
\hline
\end{tabular}

Site 2 in 1977 expansion field

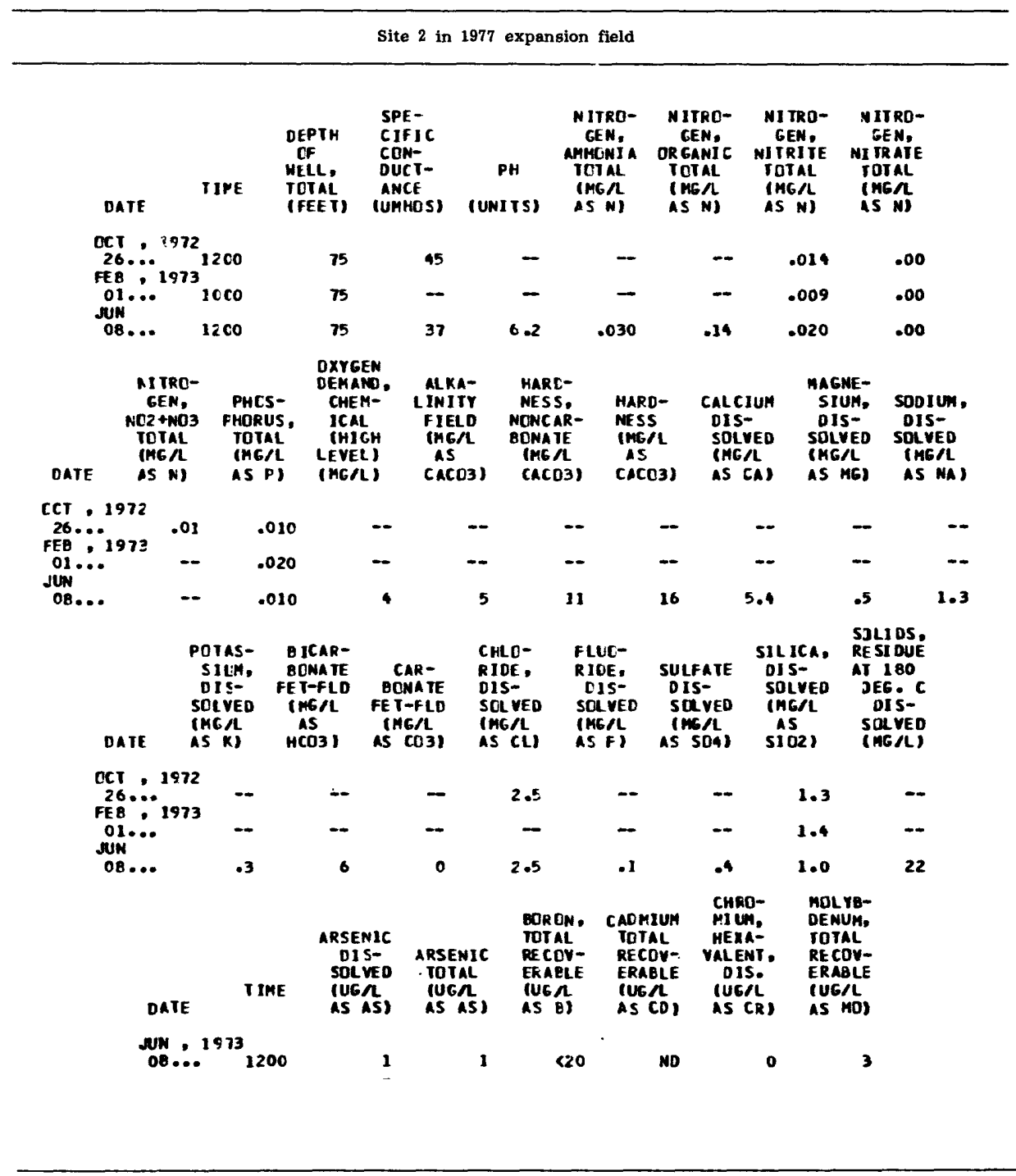


Site 4

\begin{tabular}{|c|c|c|c|c|c|c|c|c|c|c|}
\hline DATE & TIME & $\begin{array}{l}\text { DEFIH } \\
\text { EF } \\
\text { HELL, } \\
\text { TCTAL } \\
\text { (FEET) }\end{array}$ & $\begin{array}{l}\text { TEPPE E- } \\
\text { A TUFE } \\
\text { (DEG C) }\end{array}$ & $\begin{array}{l}\text { SPI- } \\
\text { CIFIC } \\
\text { CON- } \\
\text { DUCI- } \\
\text { ANCE } \\
\text { (UNHOS) }\end{array}$ & $\begin{array}{c}P H \\
\text { (UMITS) }\end{array}$ & $\begin{array}{l}\text { AIIRO- } \\
\text { CEND } \\
\text { AMPCINIA } \\
\text { CIS- } \\
\text { SCLVEO } \\
\text { (MEA } \\
\text { AS N) }\end{array}$ & $\begin{array}{l}\text { NITRO- } \\
\text { GEN, } \\
\text { AMRONIA } \\
\text { TITAL } \\
\text { (MG/L } \\
\text { AS N) }\end{array}$ & $\begin{array}{l}\text { NITRO- } \\
\text { GLN, } \\
\text { ORGANIC } \\
\text { DIS- } \\
\text { SOLVED } \\
\text { (MGAL } \\
\text { AS N) }\end{array}$ & $\begin{array}{l}\text { NITRD- } \\
\text { GEN, } \\
\text { ORGANIC } \\
\text { TOTAL } \\
\text { ( MGNL } \\
\text { IS NI) }\end{array}$ & $\begin{array}{l}\text { NITRD- } \\
\text { GEN, } \\
\text { NITRITE } \\
\text { DIS- } \\
\text { SOLVED } \\
\text { (HGAL } \\
\text { AS NI }\end{array}$ \\
\hline NCV . 1 & $140 \mathrm{C}$ & 53 & - & - & -- & -- & .020 & $\cdots$ & .05 & -- \\
\hline $\begin{array}{l}\text { DEC } \\
15 \ldots \ldots \\
\text { FEB } \because 1\end{array}$ & - & 53 & -- & -- & -- & -- & -- & -- & - & - \\
\hline MAR & $100 \mathrm{C}$ & 53 & 20.0 & - & -- & - & .08 .0 & -- & .10 & - \\
\hline$\underset{A P R}{17} \ldots$ & - & 53 & -- & 60 & - & - & -- & $\cdots$ & -- & - \\
\hline$\underset{\operatorname{MAY}}{12} \cdots$ & $134 \mathrm{C}$ & 53 & - & 65 & 7.6 & - & .010 & $\cdots$ & .15 & -- \\
\hline JUN & $130 \mathrm{C}$ & 53 & 21.5 & 65 & 7.1 & - & $<.010$ & -- & .17 & - \\
\hline JuL & $120 \mathrm{C}$ & 53 & - & 68 & 7.9 & - & .030 & $\cdots$ & .05 & -- \\
\hline $\begin{array}{l}18 \ldots \ldots \\
24 \ldots \\
A \cup G\end{array}$ & $\begin{array}{l}123 C \\
1045\end{array}$ & $\begin{array}{l}53 \\
53\end{array}$ & 21.5 & $\begin{array}{l}58 \\
62\end{array}$ & $\begin{array}{l}7.3 \\
7.3\end{array}$ & $=$ & $\begin{array}{l}<.010 \\
.010\end{array}$ & $=$ & $\begin{array}{l}.21 \\
.14\end{array}$ & - \\
\hline OCT $^{28} \ldots$ & 1500 & 53 & 22.0 & $m$ & - & .020 & - & $\cdots$ & -- & .005 \\
\hline $\operatorname{Nov}^{01} \cdots$ & 1000 & 53 & 22.0 & 62 & 6.8 & .010 & - & .02 & -- & .008 \\
\hline $\begin{array}{l}07 \ldots \\
\text { DEC }\end{array}$ & $093 \mathrm{C}$ & 53 & 20.5 & 61 & 7.2 & - & -- & .01 & -- & -- \\
\hline JAN $12 \ldots$ & $974^{1200}$ & 53 & 21.5 & $n$ & 7.5 & .010 & - & .18 & -- & .010 \\
\hline FEB & $120 \mathrm{C}$ & 53 & 22.0 & 62 & 7.0 & $.0<0$ & - & - & + & .010 \\
\hline & $093 \mathrm{C}$ & 53 & 22.0 & 67 & 6.7 & .050 & $\cdots$ & .08 & -- & $<.010$ \\
\hline APR & $090 \mathrm{C}$ & 53 & 22.0 & 66 & 7.9 & .030 & $m$ & .28 & -- & $<.010$ \\
\hline$\underset{\text { JUN }}{03} \cdot \cdots$ & $090 \mathrm{C}$ & 53 & 22.0 & 68 & 7.5 & .010 & - & $\cdots$ & -- & $<.010$ \\
\hline FEB & $975^{090 \mathrm{C}}$ & 53 & 22.0 & 65 & - & - & .030 & - & .00 & - \\
\hline$\underset{A P R}{03} \cdots$ & $103 C$ & 53 & 22.0 & 62 & 8.3 & - & .020 & $\cdots$ & .00 & -- \\
\hline JUN & 0900 & 53 & 22.0 & 55 & 8.3 & -- & .010 & - & .00 & -- \\
\hline $25 \ldots$ & 1340 & 53 & 21.5 & 62 & 7.9 & - & .020 & - & .00 & - \\
\hline DATE & $\begin{array}{l}\text { NITRC- } \\
\text { GEN, } \\
\text { NITRI IE } \\
\text { TOTAL } \\
\text { (HG } \\
\text { AS N) }\end{array}$ & $\begin{array}{l}\text { NIIRO- } \\
\text { GEN, } \\
\text { NITRATE } \\
\text { DIS- } \\
\text { SOL VED } \\
\text { (MGA } \\
\text { AS N) }\end{array}$ & $\begin{array}{l}\text { NIIRQ- } \\
\text { GEN: } \\
\text { NI IRATE } \\
\text { ICTAL } \\
\text { (MGN } \\
\text { AS N) }\end{array}$ & $\begin{array}{l}\text { NITRC- } \\
\text { GEN,AY- } \\
\text { MONIA + } \\
\text { GRGANIC } \\
\text { TOTAL } \\
\text { (MGNL } \\
\text { AS N) }\end{array}$ & $\begin{array}{l}\text { NIIRD- } \\
\text { GEN, } \\
\text { NO2+ND3 } \\
\text { TOTAL } \\
\text { (MGNL } \\
\text { AS N) }\end{array}$ & $\begin{array}{l}\text { NITRD- } \\
\text { GEN, } \\
\text { ICTAL } \\
\text { (MEN } \\
\text { AS N) }\end{array}$ & $\begin{array}{l}\text { PHOS- } \\
\text { PHCRUS, } \\
\text { JRTHE, } \\
\text { DIS- } \\
\text { SDLVED } \\
\text { (HCA } \\
\text { AS P) }\end{array}$ & $\begin{array}{l}\text { PHOS- } \\
\text { PHORUS, } \\
\text { DIS- } \\
\text { SOLVEO } \\
\text { IMG/L } \\
\text { AS PI }\end{array}$ & $\begin{array}{l}\text { PHJS- } \\
\text { PHORUS, } \\
\text { TOTAL } \\
\text { IMG } \\
\text { AS PI }\end{array}$ & $\begin{array}{l}\text { DXYGEN } \\
\text { DEMAND, } \\
\text { BID- } \\
\text { CHEM- } \\
\text { ICAL, } \\
5 \text { DAY } \\
\text { (MG/L) }\end{array}$ \\
\hline
\end{tabular}

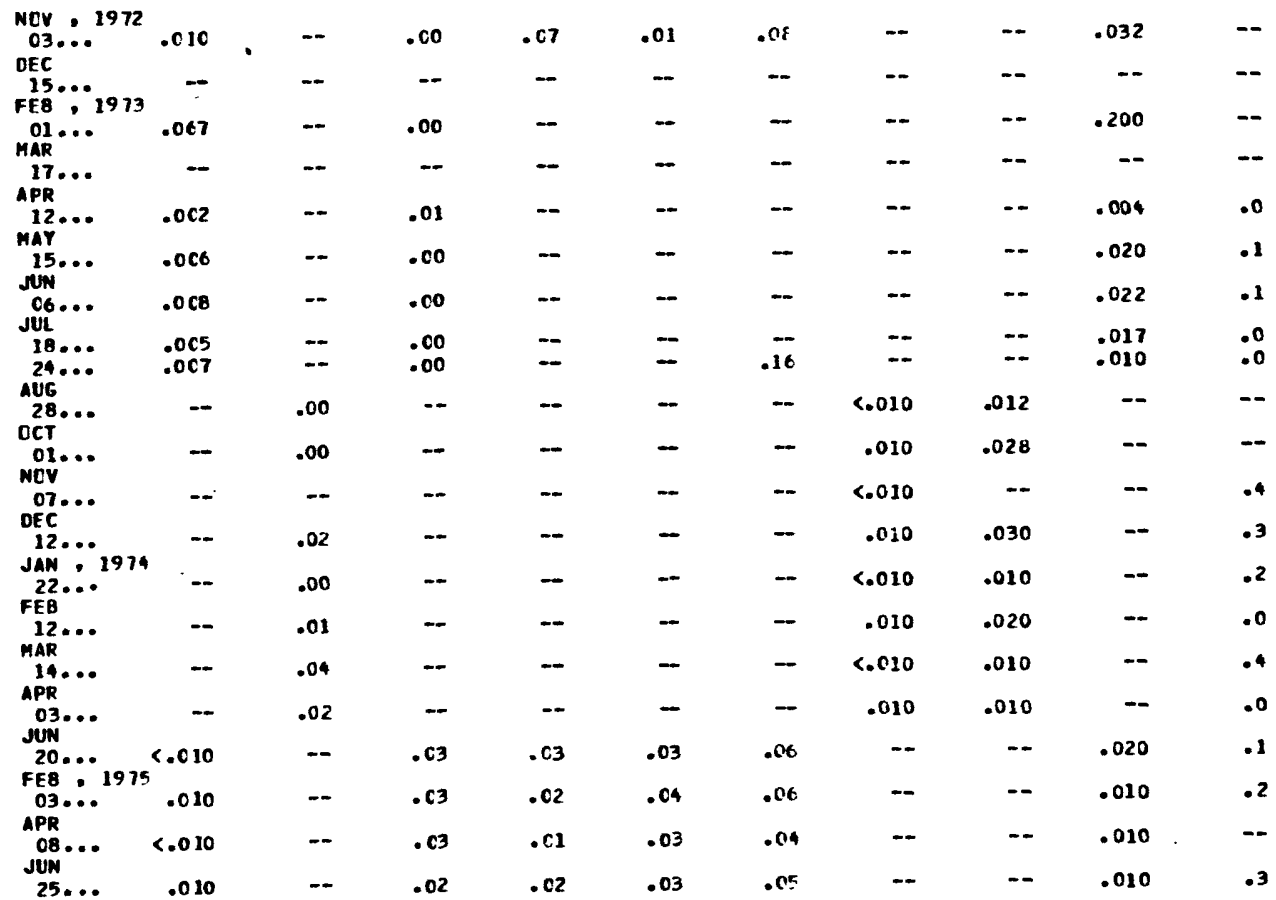




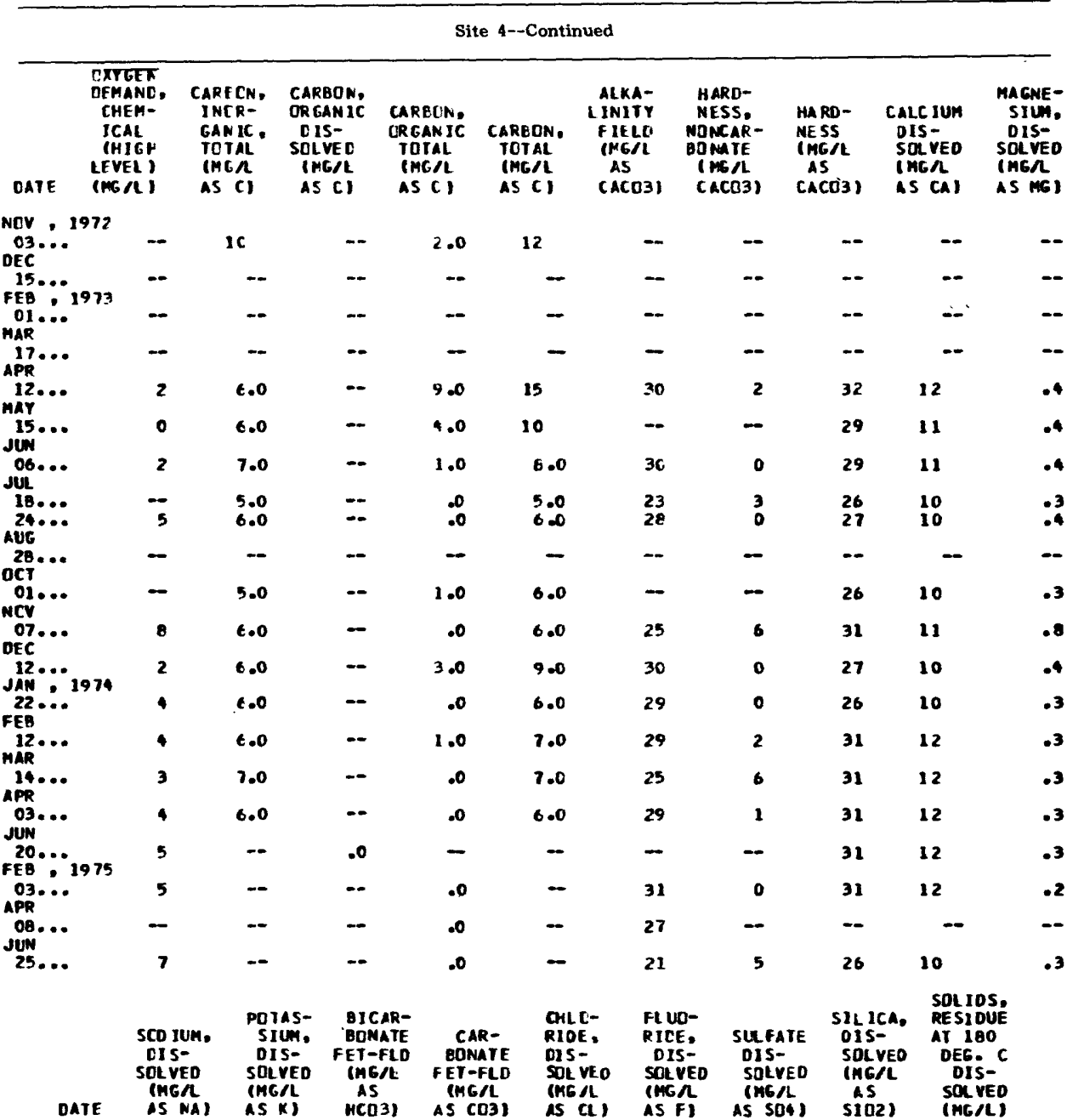

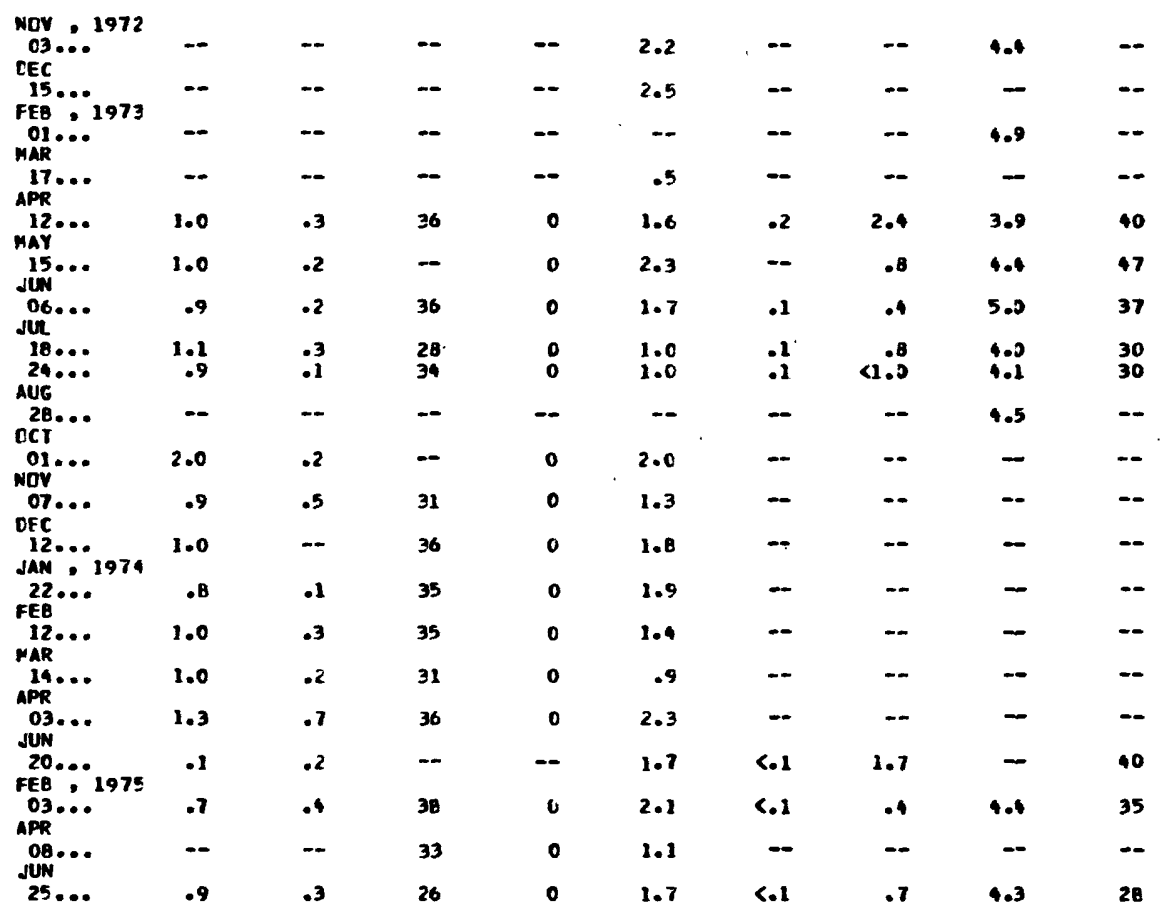


Site 4--Continued

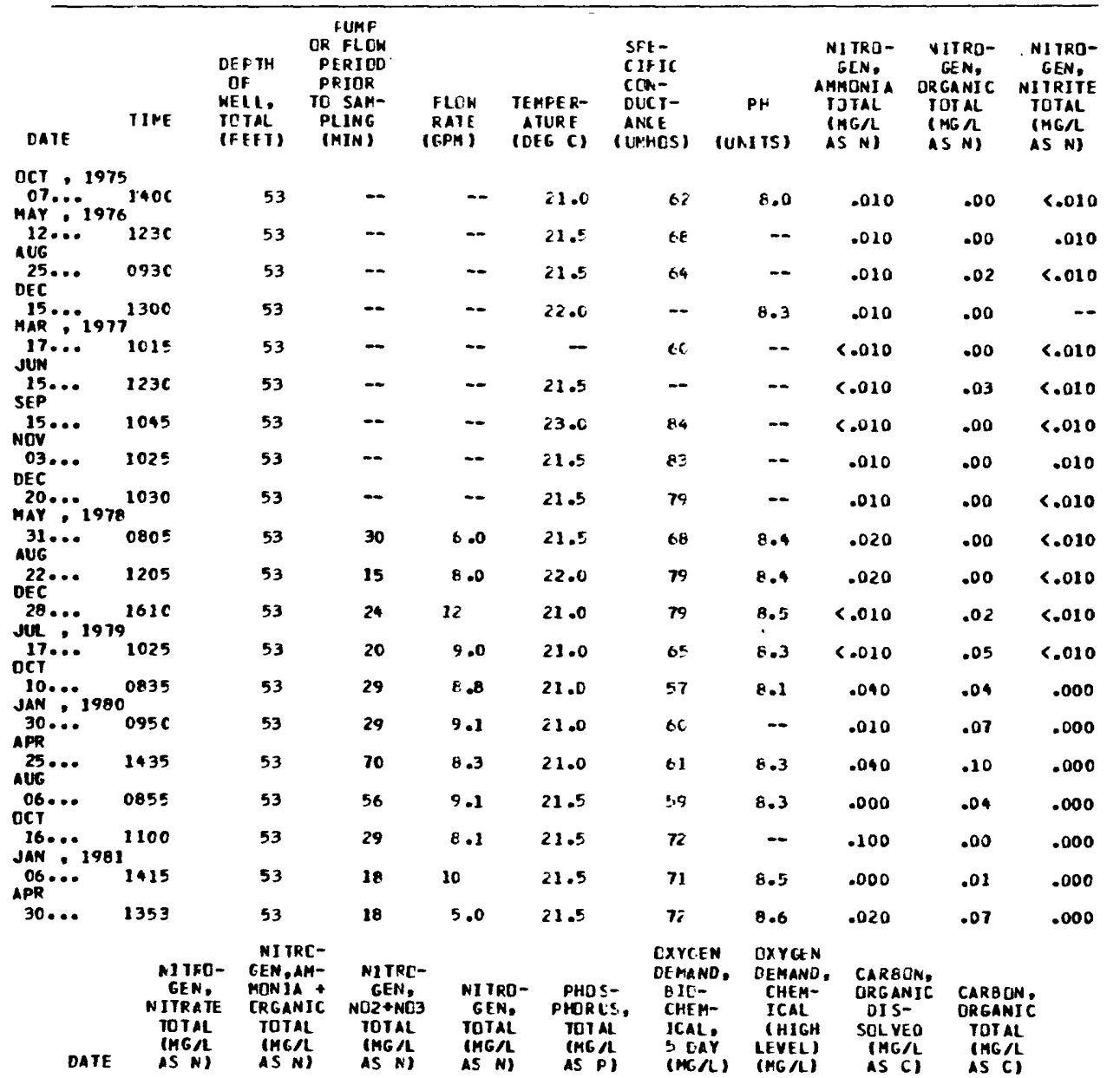

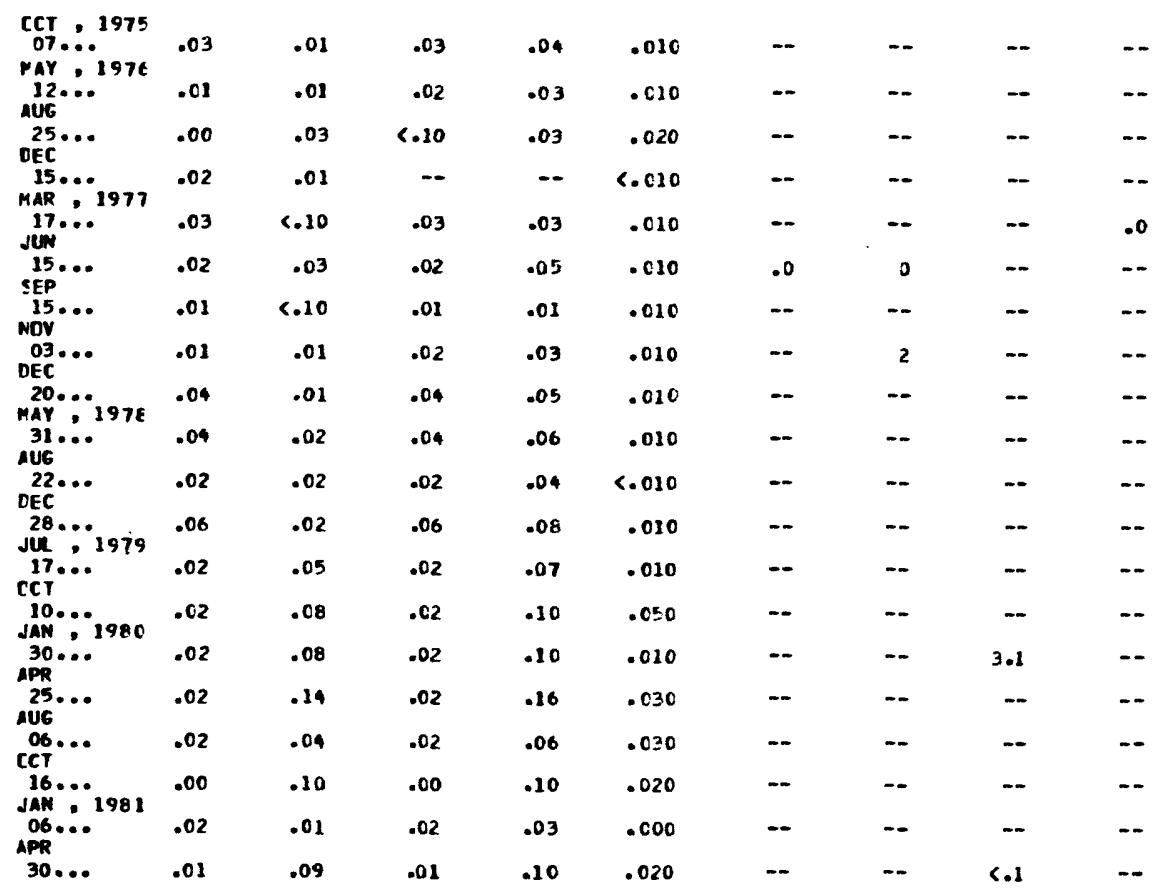


Table 6.--Physical and chemical characteristics of well and water at sites 1 through 70 --Continued

Site 4--Continued

\begin{tabular}{|c|c|c|c|c|c|c|c|c|c|}
\hline DATE & $\begin{array}{l}\text { ALKA- } \\
\text { IINITY } \\
\text { FIELO } \\
\text { IHGA } \\
\text { AS } \\
\text { CACO3) }\end{array}$ & $\begin{array}{l}\text { HARO- } \\
\text { NESS, } \\
\text { NONCAR- } \\
\text { ECNATE } \\
\text { (MCR } \\
\text { CACO3) }\end{array}$ & $\begin{array}{l}\text { HARS- } \\
\text { MESS } \\
\text { (HG } \\
\text { AS } \\
\text { CACS3) }\end{array}$ & $\begin{array}{l}\text { CAL C IUH } \\
\text { DI S- } \\
\text { SOL VED } \\
\text { (MGAL } \\
\text { AS CA) }\end{array}$ & $\begin{array}{l}\text { MAGNE- } \\
\text { SIUA. } \\
\text { DIS- } \\
\text { SLL VED } \\
\text { (MG AL } \\
\text { AS MG) }\end{array}$ & $\begin{array}{l}\text { SODIUH, } \\
\text { DIS- } \\
\text { SOLVED } \\
\text { (MG/L } \\
\text { AS MA) }\end{array}$ & $\begin{array}{l}\text { POTAS- } \\
\text { SIUN, } \\
\text { DIS- } \\
\text { SOLVED } \\
\text { (MG K } \\
\text { AS K) }\end{array}$ & $\begin{array}{l}\text { BICAR- } \\
\text { BDNATE } \\
\text { FET FLL } \\
\text { (MG/L } \\
\text { AS } \\
\text { HCO3) }\end{array}$ & $\begin{array}{l}\text { BICAR- } \\
\text { BONATE } \\
\text { IT-FLD } \\
\text { IHGAL } \\
\text { AS } \\
\text { HCO3) }\end{array}$ \\
\hline
\end{tabular}

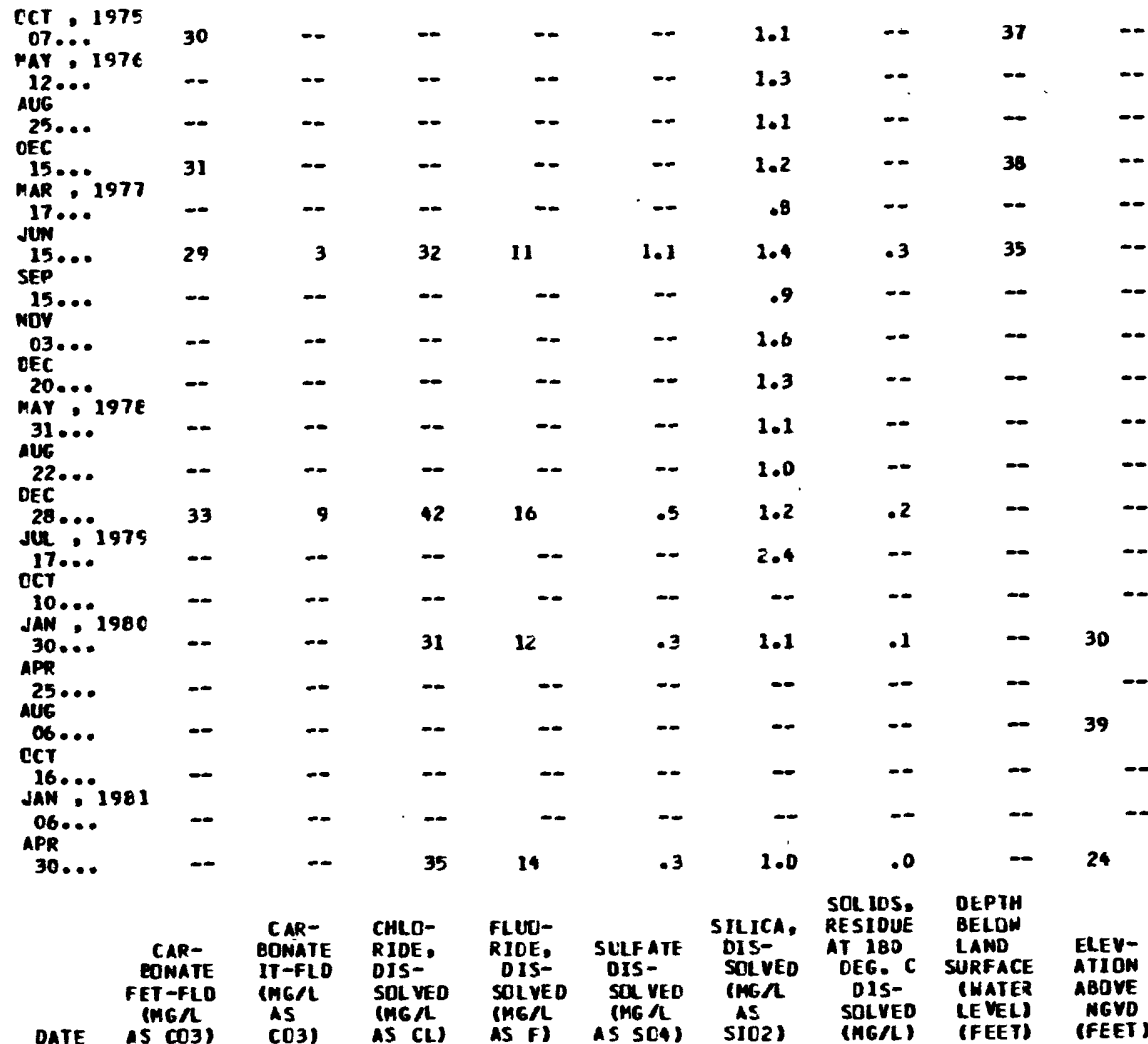

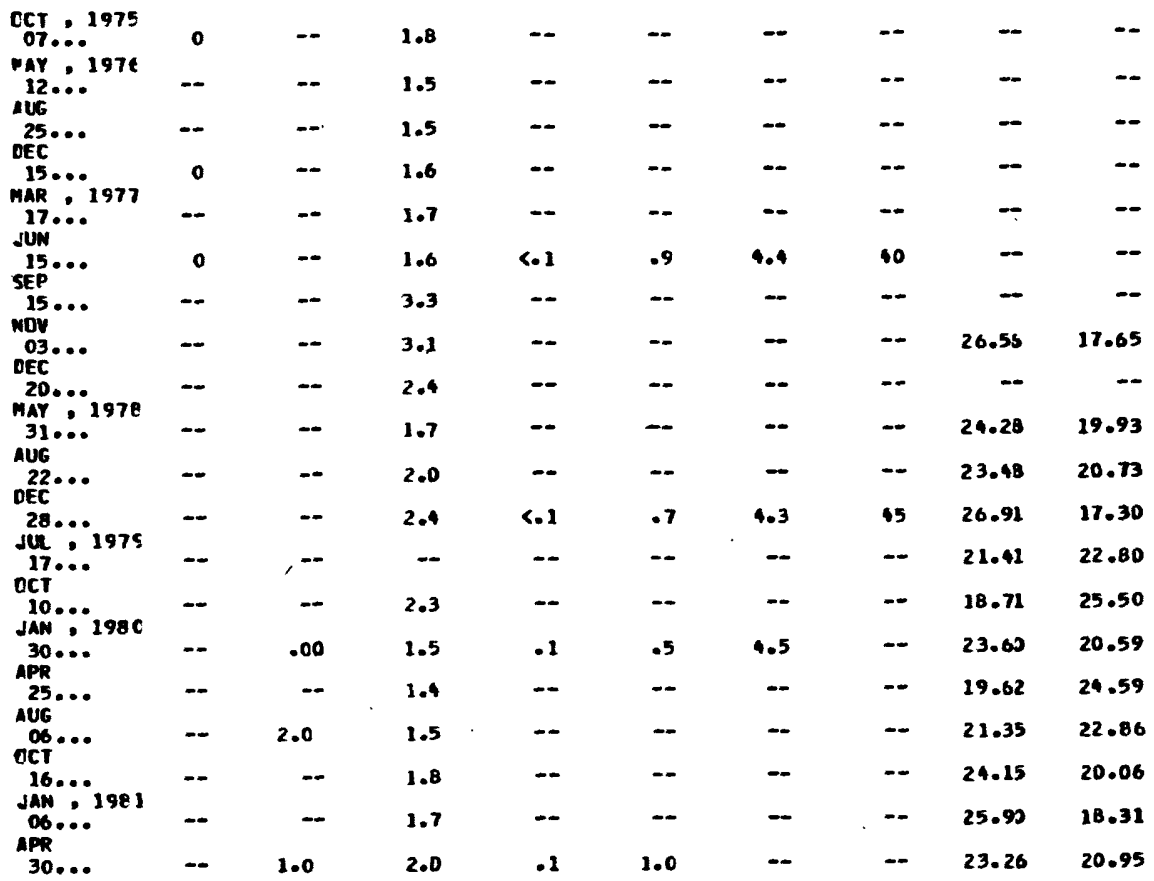


Site 4--Continued

\begin{tabular}{|c|c|c|c|c|c|c|c|c|c|c|c|}
\hline DATE & TIME & $\begin{array}{l}\text { DLUF- } \\
\text { INUH, } \\
\text { DIS- } \\
\text { SOLVED } \\
\text { (UG/L } \\
\text { ES IL) }\end{array}$ & $\begin{array}{c}\text { ARSENIC } \\
\text { DIS- } \\
\text { SOLVEO } \\
\text { (UGAL } \\
\text { AS AS) }\end{array}$ & $\begin{array}{l}\text { ARSEMIC } \\
\text { TOTM } \\
\text { (UG/L } \\
\text { AS AS) }\end{array}$ & $\begin{array}{l}\text { EORON, } \\
\text { DIS- } \\
\text { SOL VED } \\
\text { (UGR } \\
\text { AS E) }\end{array}$ & $\begin{array}{l}\text { EOKCH, } \\
\text { TOIAL } \\
\text { RECCV- } \\
\text { ERAELE } \\
\text { (UGA } \\
\text { AS E) }\end{array}$ & $\begin{array}{l}\text { CAOHIUA } \\
\text { DIS- } \\
\text { SCLVED } \\
\text { WUE A } \\
\text { AS CDS }\end{array}$ & $\begin{array}{l}\text { CAOMIUH } \\
\text { TOIAL } \\
\text { RECOY- } \\
\text { ERABLE } \\
\text { TUG/L } \\
\text { AS COS }\end{array}$ & $\begin{array}{l}\text { CHRD- } \\
\text { MI UM. } \\
\text { DIS- } \\
\text { SOLVED } \\
\text { (UG/L } \\
\text { AS CR) }\end{array}$ & $\begin{array}{l}\text { CHRO- } \\
\text { MIUH, } \\
\text { HEXA- } \\
\text { VALENT. } \\
\text { DIS. } \\
\text { IUG AL } \\
\text { AS CR }\end{array}$ & $\begin{array}{l}\text { CHRO- } \\
\text { MIUM. } \\
\text { TOTAL } \\
\text { RECOV- } \\
\text { ERABLE } \\
\text { (UGA } \\
\text { AS (R) }\end{array}$ \\
\hline $\begin{array}{l}\text { IPR } \\
12 .: . \\
\text { MAY }\end{array}$ & 1340 & 20 & $<1$ & $<1$ & -- & -- & ND & $-\infty$ & ND & 0 & -- \\
\hline IVN & 1300 & 30 & <1 & 3 & -- & is & ND & -- & - & 0 & -- \\
\hline $\begin{array}{l}06 . . . \\
\text { JUL }\end{array}$ & 1200 & -- & $<1$ & $<1$ & -- & $<20$ & - & ND & -- & D & NO \\
\hline cCl $18 \ldots$ & $\begin{array}{l}1230 \\
1045\end{array}$ & $=$ & $=$ & $=$ & $\ddot{--}$ & $\begin{array}{r}12 \\
<20\end{array}$ & $\overline{--}$ & $=$ & $=$ & -- & 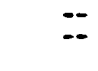 \\
\hline $\operatorname{mov}^{01}$ & 1000 & -- & -- & -- & - & $\boldsymbol{E}$ & - & - & - & -- & $\cdots$ \\
\hline DEC $07 . .$. & 0930 & - & -- & $\cdots$ & 50 & -- & -- & - & - & -- & -- \\
\hline $12 \ldots$ & $4^{1200}$ & -- & $<1$ & -- & - & -- & -- & 9 & - & -- & -- \\
\hline FEQ $22 \ldots$ & 1200 & -- & -- & - & $<20$ & -- & -- & - & - & -- & -- \\
\hline $12 \ldots$ & 0930 & -- & -- & -- & $<20$ & -- & -- & -- & - & -- & -- \\
\hline$\underset{A P R}{14} \ldots$ & 0900 & -- & $\cdots$ & $\cdots$ & $<20$ & -- & -- & -- & - & -- & -- \\
\hline JUN... & 0900 & -- & 3 & -- & 6 & -- & -- & ND & - & -- & -- \\
\hline FEE 20. & $75^{0900}$ & -- & 4 & -- & $<20$ & -- & -- & -- & - & -- & -- \\
\hline $\begin{array}{l}03 \ldots . . . \\
\text { JUN }\end{array}$ & 1030 & 20 & $<1$ & - & 5 & -- & 2 & - & NJ & 0 & - \\
\hline JUN $25 .$. & 1340 & 20 & <1 & - & - & $\cdots$ & ND & -- & NJ & 0 & $-\infty$ \\
\hline $15 \ldots$ & 1230 & -- & -- & -- & -- & -- & -- & -- & $\cdots$ & -- & -- \\
\hline DATE & $\begin{array}{l}\text { COBALT, } \\
\text { DIS- } \\
\text { SCLVED } \\
\text { (UG/L } \\
\text { IS CO) }\end{array}$ & $\begin{array}{l}\text { CCEALT, } \\
\text { TOTAL } \\
\text { RE COV- } \\
\text { ERABLE } \\
\text { CUGA } \\
\text { IS COS }\end{array}$ & $\begin{array}{l}\text { COPPER, } \\
\text { DIS- } \\
\text { SDLVED } \\
\text { (UG/L } \\
\text { AS CU) }\end{array}$ & $\begin{array}{l}\text { COPPER, } \\
\text { TOTAL } \\
\text { RECCV- } \\
\text { ERAELE } \\
\text { (UGA } \\
\text { AS CUS }\end{array}$ & $\begin{array}{l}\text { IRON, } \\
\text { DIS- } \\
\text { SOLVED } \\
\text { (UGA } \\
\text { AS FE) }\end{array}$ & $\begin{array}{l}\text { LEAD, } \\
\text { DIS- } \\
\text { SOL VED } \\
\text { (UGA } \\
\text { AS PE) }\end{array}$ & $\begin{array}{l}\text { LEAD, } \\
\text { TOTAL } \\
\text { RECOV- } \\
\text { ERAELE } \\
\text { (UE RL } \\
\text { AS PB) }\end{array}$ & $\begin{array}{c}\text { LITHIUN } \\
\text { DIS- } \\
\text { SDLVED } \\
\text { PUG/L } \\
\text { AS LI) }\end{array}$ & $\begin{array}{l}\text { LITHIUS } \\
\text { TOTAL } \\
\text { RECOV- } \\
\text { ERABLE } \\
\text { (UG/L } \\
\text { AS LBS }\end{array}$ & $\begin{array}{l}\text { MANGA- } \\
\text { MESE, } \\
\text { IOTAL } \\
\text { RECOV- } \\
\text { ERABLE } \\
\text { (UG LL } \\
\text { AS HN) }\end{array}$ & $\begin{array}{l}\text { MANGA- } \\
\text { NESE, } \\
\text { DIS- } \\
\text { SOLVED } \\
\text { (UG/L } \\
\text { AS IN) }\end{array}$ \\
\hline $\begin{array}{r}A P R \\
12 \ldots \\
M A Y\end{array}$ & NO & -- & $<20$ & -- & $<10$ & ND & - & $<10$ & -- & -- & $<10$ \\
\hline JU: $15 . .$. & ND & -- & ND & - & $<10$ & $<2$ & - & $<10$ & - & -- & $<10$ \\
\hline JuL 06 & -- & NO & - & ND & -- & $\cdots$ & 5 & - & $<10$ & $<10$ & -- \\
\hline $\begin{array}{l}18 \ldots \\
24 \cdots \\
\text { cCT }\end{array}$ & 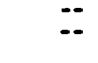 & $=$ & $=$ & $\cdots$ & $=$ & $=$ & $=$ & 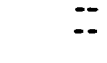 & $=$ & $=$ & 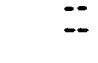 \\
\hline $01 . .$. & -- & -- & - & - & -- & -- & -- & - & -- & -- & - \\
\hline CEC & -- & -- & $-\infty$ & -- & -- & -- & -- & -- & - & -- & -- \\
\hline $12 \ldots$ & -- & -- & $<2$ & -- & $<10$ & 4 & -- & -- & -- & -- & $<10$ \\
\hline $22 \ldots$ & -- & -- & -- & - & $\cdots$ & - & -- & -- & - & -- & -- \\
\hline MAR & -- & -- & -- & -- & -- & -- & -- & -- & -- & -- & - \\
\hline$\underset{A P R}{14} \ldots$ & -- & -- & - & -- & -- & -- & $\cdots$ & -- & - & -- & -- \\
\hline JUW & -- & -- & $<2$ & $\cdots$ & $<10$ & 5 & -- & -- & - & -- & $<10$ \\
\hline FEB... & $\cdots$ & -- & $\cdots$ & -- & $\cdots$ & -- & -- & -- & -- & -- & -- \\
\hline JUW & No & -- & NO & - & $<10$ & 3 & -- & $<10$ & - & -- & (10 \\
\hline $\begin{array}{r}25 \ldots \\
\text { JUN } \bullet\end{array}$ & $<2$ & - & $<2$ & - & $<10$ & NO & - & C10 & - & -- & (10 \\
\hline $15 \ldots$ & -- & -- & -- & - & -- & $\cdots$ & -- & $\cdots$ & - & -- & - \\
\hline
\end{tabular}




\begin{tabular}{|c|c|c|c|c|c|c|c|c|c|c|}
\hline \multicolumn{11}{|c|}{ Site 4--Continued } \\
\hline $\begin{array}{l}\text { MERCURY } \\
\text { OIS- } \\
\text { SOLVED } \\
\text { (UG/L } \\
\text { AS HG) }\end{array}$ & $\begin{array}{c}\text { MERCURY } \\
\text { YOIAL } \\
\text { RECCV- } \\
\text { ERABLE } \\
\text { TUGA } \\
\text { OS HG }\end{array}$ & $\begin{array}{l}\text { HOLYB- } \\
\text { DENUM, } \\
\text { DIS- } \\
\text { SOLVEO } \\
\text { (UGAL } \\
\text { AS MO) }\end{array}$ & $\begin{array}{l}\text { MOL YE- } \\
\text { DENUH, } \\
\text { TOT MA. } \\
\text { RECCV- } \\
\text { ERAELE } \\
\text { (UGA } \\
\text { AS MO) }\end{array}$ & $\begin{array}{l}\text { NICKEL, } \\
\text { OIS- } \\
\text { SOLVEO } \\
\text { (UG/L } \\
\text { AS NI) }\end{array}$ & $\begin{array}{l}\text { NICKEL, } \\
\text { TOTAL } \\
\text { RECDV- } \\
\text { ERAELE } \\
\text { IUGA } \\
\text { AS NI) }\end{array}$ & $\begin{array}{l}\text { SELE- } \\
\text { NIUM, } \\
\text { OIS- } \\
\text { SOLVED } \\
\text { (UG/L } \\
\text { AS SE) }\end{array}$ & $\begin{array}{l}\text { STRON- } \\
\text { TIUN, } \\
\text { DIS- } \\
\text { SDLVED } \\
\text { (UG/L } \\
\text { AS SR) }\end{array}$ & $\begin{array}{l}\text { YANA- } \\
\text { DIUA, } \\
\text { DIS- } \\
\text { SOLVED } \\
\text { IUG/L } \\
\text { AS VI }\end{array}$ & $\begin{array}{l}\text { ZINC, } \\
\text { DIS- } \\
\text { SOLVED } \\
\text { (UG/L } \\
\text { AS ZN) }\end{array}$ & $\begin{array}{l}\text { Z INC, } \\
\text { TOTAL } \\
\text { RECON- } \\
\text { ERABLE } \\
\text { (UGA } \\
\text { AS ZN) }\end{array}$ \\
\hline
\end{tabular}

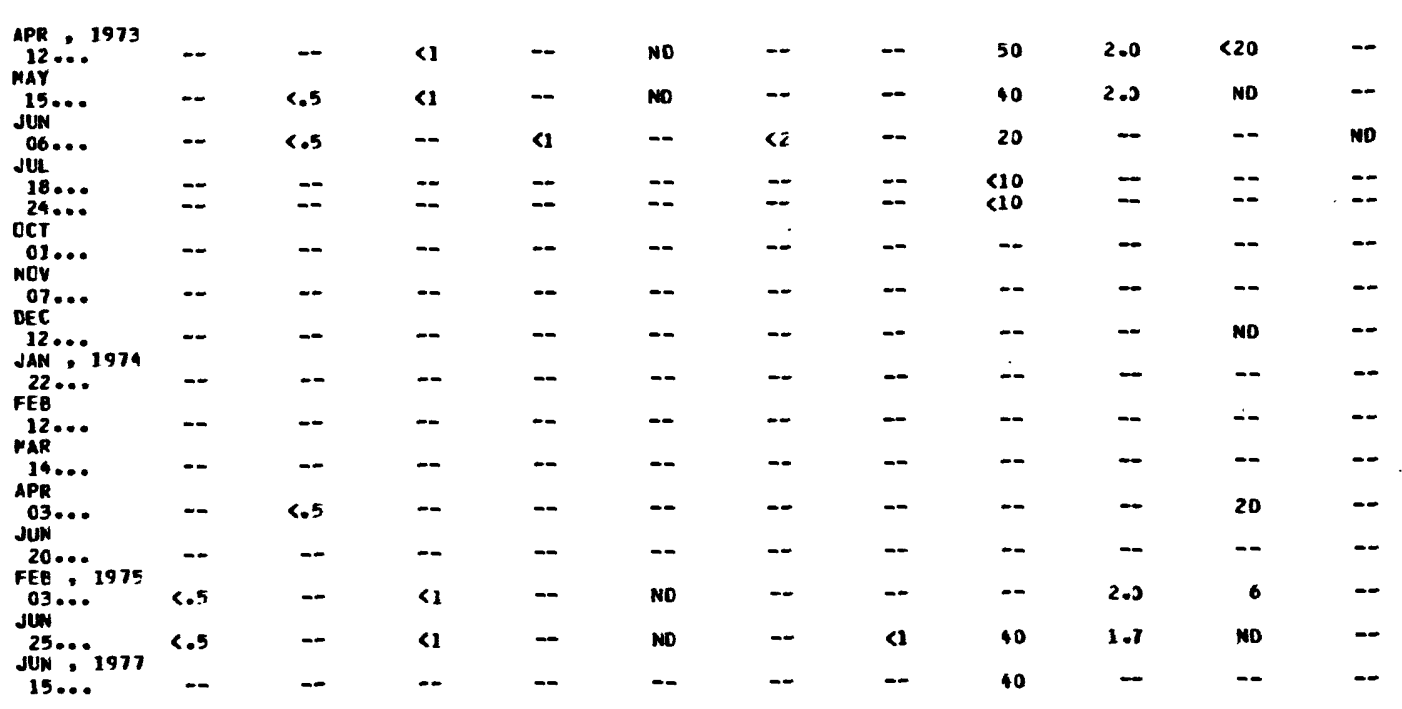

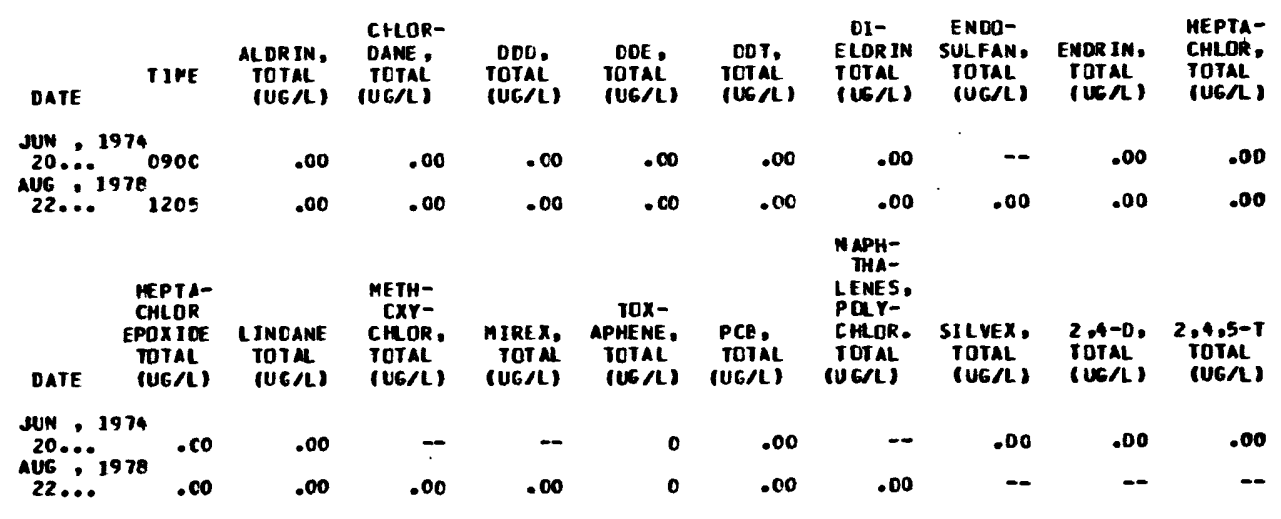


Site 5

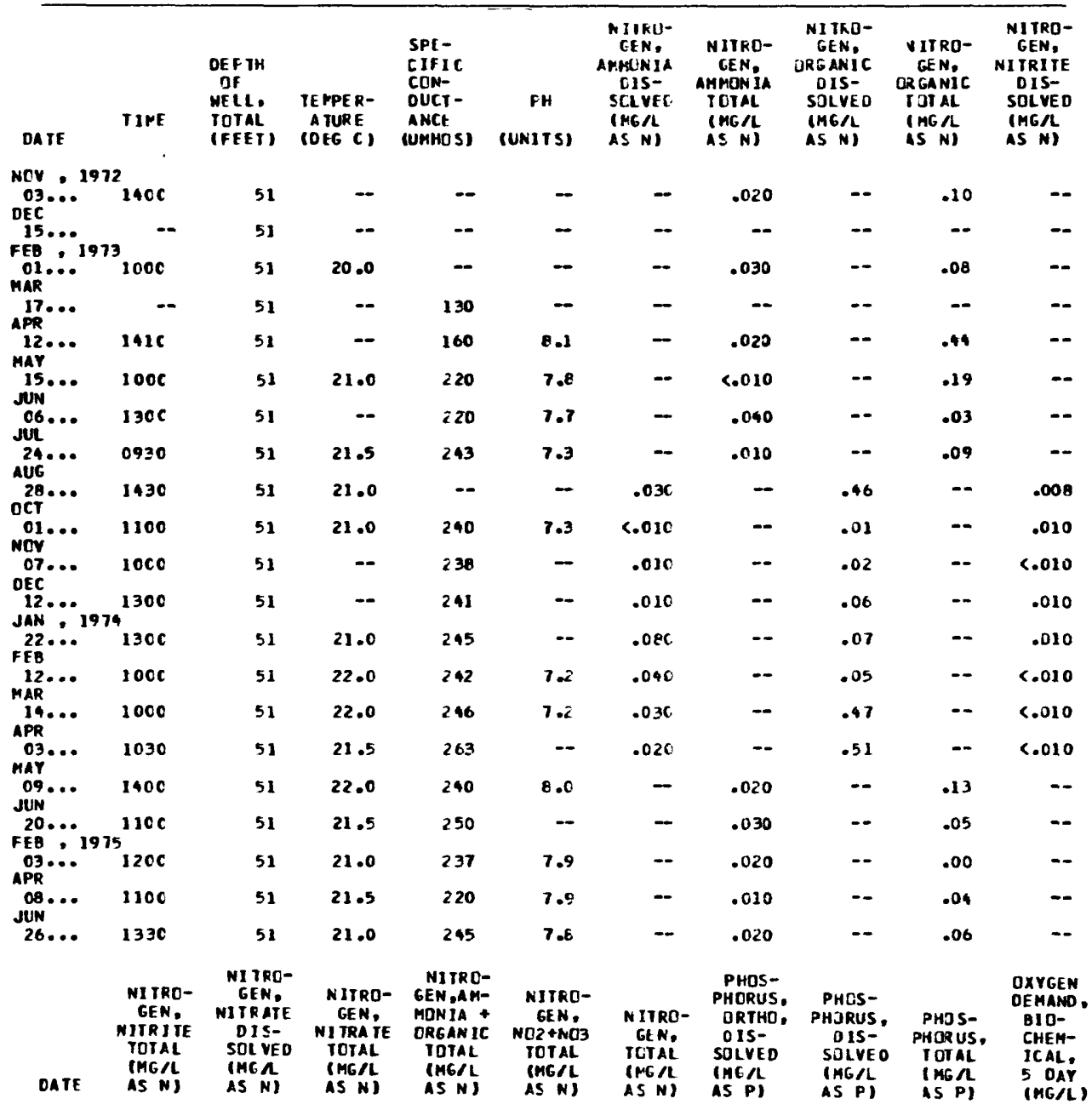

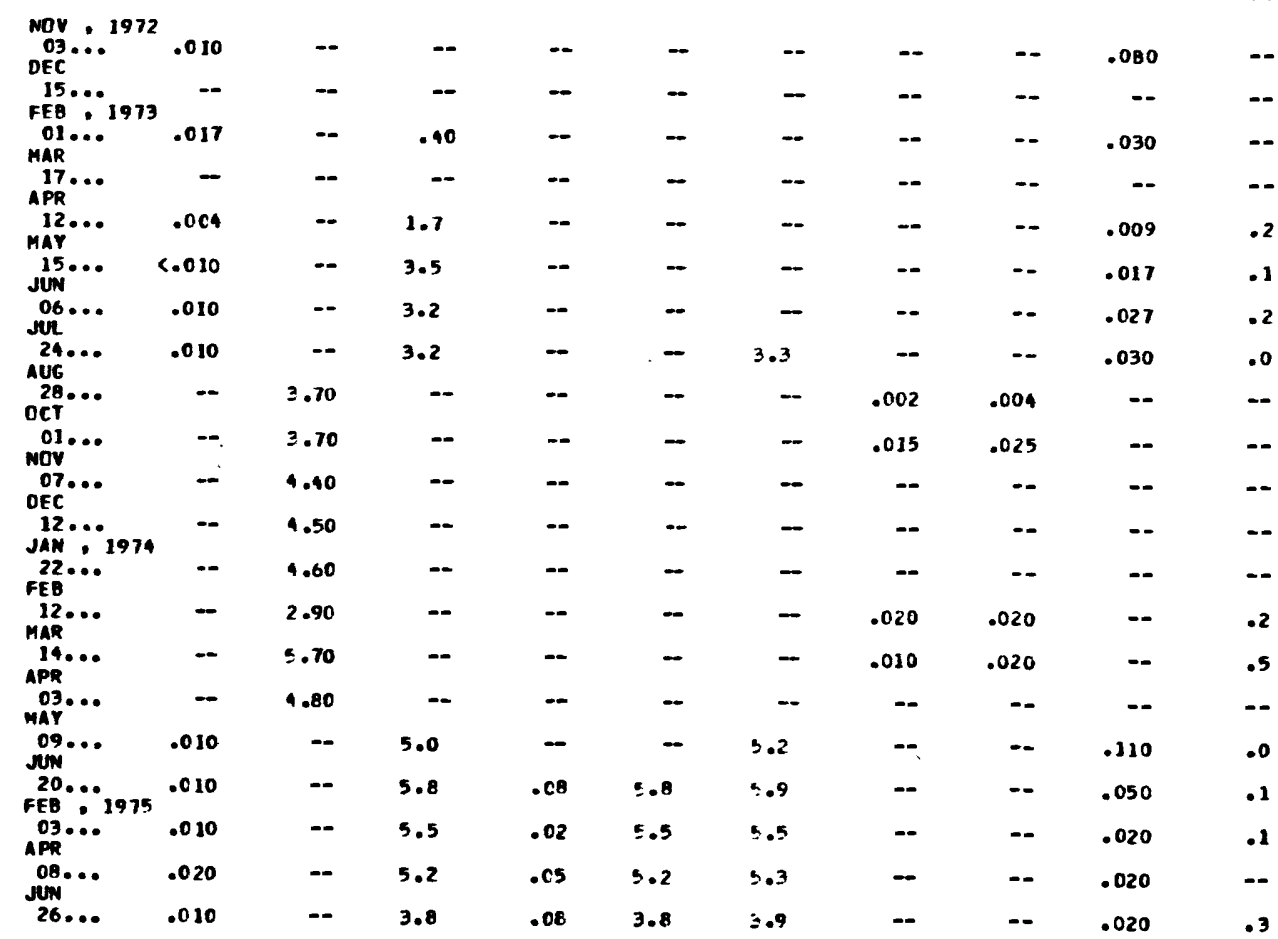


Site 5--Continued

\begin{tabular}{|c|c|c|c|c|c|c|c|c|c|}
\hline DATE & $\begin{array}{l}\text { OYYGEN } \\
\text { DEMAND. } \\
\text { CHEY- } \\
\text { ICAL } \\
\text { IHIGH } \\
\text { IEVEL) } \\
\text { (YGAL) }\end{array}$ & $\begin{array}{l}\text { CAR BDN, } \\
\text { INOR- } \\
\text { GANIC, } \\
\text { TOTAL } \\
\text { (MGAL } \\
\text { AS C) }\end{array}$ & $\begin{array}{l}\text { CARBGN, } \\
\text { DRGANIC } \\
\text { TOTAL } \\
\text { (HGA } \\
\text { AS C) }\end{array}$ & $\begin{array}{l}\text { CAREON. } \\
\text { TOTAL } \\
\text { (MGR } \\
\text { AS C) }\end{array}$ & $\begin{array}{l}\text { MKA- } \\
\text { LINIIY } \\
\text { FIELD } \\
\text { IMG } R \\
\text { AS } \\
\text { CACES) }\end{array}$ & $\begin{array}{l}\text { HARD- } \\
\text { M SS, } \\
\text { NONCAR- } \\
\text { EONATE } \\
\text { (ME-AL } \\
\text { CACO3) }\end{array}$ & $\begin{array}{l}\text { HARO- } \\
\text { NESS } \\
\text { (HGA } \\
\text { AS } \\
\text { CACD3) }\end{array}$ & $\begin{array}{l}\text { CALCIUA } \\
\text { DIS- } \\
\text { SOLVED } \\
\text { (MG/L } \\
\text { AS CA) }\end{array}$ & $\begin{array}{l}\text { MAGNE- } \\
\text { SIUH, } \\
\text { DIS- } \\
\text { SOLVED } \\
\text { (NG/L } \\
\text { AS MG) }\end{array}$ \\
\hline
\end{tabular}

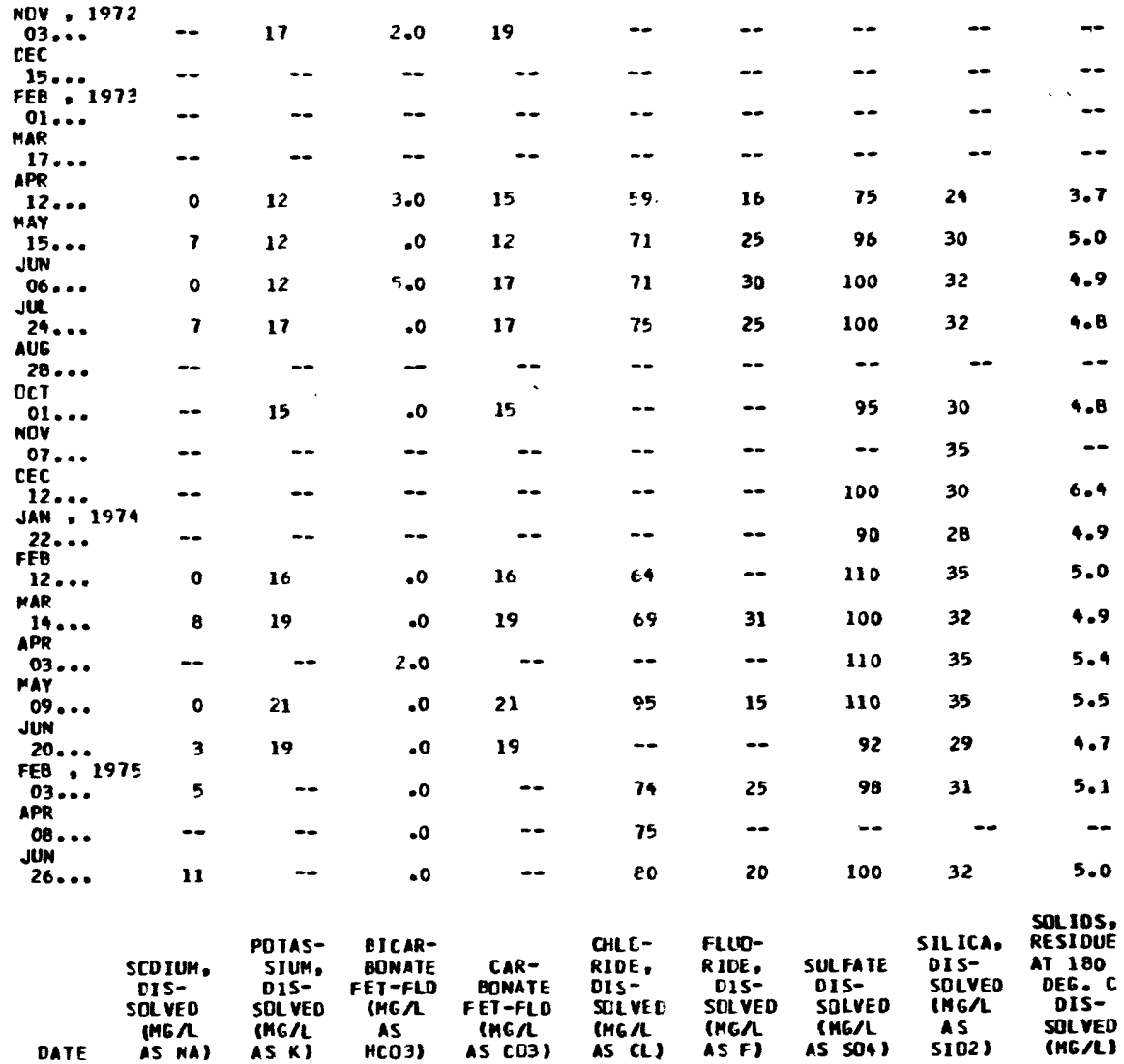

\begin{tabular}{|c|c|c|c|c|c|c|c|c|c|}
\hline $\begin{array}{l}\text { NOV }, 1972 \\
\text { O3.... } \\
\text { OEC }\end{array}$ & $\cdots$ & -- & $\cdots$ & -- & 1.5 & $\cdots$ & $\cdots$ & 6.5 & $\cdots$ \\
\hline FEB $\because 1973$ & - & $\cdots$ & - & -- & $<.1$ & -- & -- & - & $\cdots$ \\
\hline MAR & -- & -- & -- & -- & - & - & -- & 3.4 & $\cdots$ \\
\hline $17 \ldots$ & - & $\cdots$ & - & -- & 7.0 & - & -- & $\cdots$ & -- \\
\hline $\operatorname{maY}_{12} \cdots$ & 4.0 & $\cdot 3$ & 72 & 0 & -- & $\cdot 2$ & 1.6 & 5.5 & 101 \\
\hline JUM & 6.0 & .4 & 86 & 0 & 14 & $\cdots$ & .8 & 6.3 & 135 \\
\hline JUL... & 6.1 & $\cdot 4$ & 86 & 0 & 15 & $\boldsymbol{I} I$ & 2.0 & 7.0 & 115 \\
\hline$\underset{A \cup G}{24 \ldots . .}$ & 7.1 & .3 & 91 & 0 & 15 & .1 & 1.6 & 6.6 & 145 \\
\hline $\mathrm{OCT}^{28} \cdots$ & -- & $\cdots$ & $=$ & -- & -- & - & - & 6.5 & $\cdots$ \\
\hline$\underset{\text { NOV }}{01} \cdots$ & 6.5 & .4 & -- & 0 & 16 & -- & -- & - & $\cdots$ \\
\hline OEC & 7.0 & .7 & -- & $\cdots$ & 16 & $\cdots$ & $=$ & - & - \\
\hline JAN : 1974 & 7.7 & .5 & $\cdots$ & -- & 16 & - & $\cdots$ & $=$ & $\cdots$ \\
\hline FEB & 7.4 & .4 & -- & $\cdots$ & 17 & - & -- & - & - \\
\hline $\operatorname{MAR}_{12 \cdots}$ & 7.9 & .5 & 78 & 0 & 16 & -- & -- & - & $\cdots$ \\
\hline${ }_{A P R}^{14} \cdots$ & 8.0 & .4 & 84 & 0 & 16 & $\cdots$ & $\cdots$ & - & $=-$ \\
\hline par:.. & 9.2 & .7 & - & -- & 19 & $\cdots$ & $\cdots$ & - & $\cdots$ \\
\hline juw & 9.2 & .5 & 116 & 0 & 18 & $\cdots$ & 3.6 & $\cdots$ & 170 \\
\hline FEB : 197 E & 8.3 & .4 & $\cdots$ & -- & 17 & .1 & 4.3 & - & 179 \\
\hline$\underset{A P R}{03 \cdots}$ & $\cdots$ & .2 & 90 & 0 & 15 & .1 & 3.2 & 6.9 & 132 \\
\hline jun & $\cdots$ & $\cdots$ & 92 & 0 & 13 & - & $\cdots$ & $\cdots$ & - \\
\hline & e.1 & .6 & 98 & 0 & 14 & .1 & 4.3 & 6.5 & 50 \\
\hline
\end{tabular}


Site 5--Continued

\begin{tabular}{|c|c|c|c|c|c|c|c|c|c|c|c|c|}
\hline DATE & IIME & $\begin{array}{l}\text { DEPTH } \\
\text { OF } \\
\text { WELL, } \\
\text { TOTAL } \\
\text { (FEE I) }\end{array}$ & $\begin{array}{l}\text { PLIMP } \\
\text { OR FLLW } \\
\text { PEF IDD } \\
\text { PR ICR } \\
\text { TO SIM- } \\
\text { PL ING } \\
\text { (I IN) }\end{array}$ & $\begin{array}{r}\text { FLOW } \\
\text { RATE } \\
\text { (CPM) }\end{array}$ & $\begin{array}{l}\text { TE MPE R- } \\
\text { A TUKE } \\
\text { (DEG C) }\end{array}$ & $\begin{array}{l}\text { SPE- } \\
\text { CIFIC } \\
\text { CCN- } \\
\text { DUCT- } \\
\text { ANCE } \\
\text { (UMHOS) }\end{array}$ & $\begin{array}{c}\text { FH } \\
\text { (LNIIS) }\end{array}$ & $\begin{array}{l}\text { NITRD- } \\
\text { GEN, } \\
\text { AMRONIA } \\
\text { TCTAL } \\
\text { (MGA } \\
\text { MS N) }\end{array}$ & $\begin{array}{l}\text { NITRO- } \\
\text { GEN, } \\
\text { ORGANIC } \\
\text { TJTAL } \\
\text { (MGNL } \\
\text { AS NI }\end{array}$ & $\begin{array}{l}\text { NITRO- } \\
\text { GEN, } \\
\text { NI TRITE } \\
\text { TJT AL } \\
\text { I MG } \\
\text { IS N) }\end{array}$ & $\begin{array}{l}\text { NITRO- } \\
\text { GEN, } \\
\text { NITRATE } \\
\text { TOTAL } \\
\text { (MG/L } \\
\text { AS N] }\end{array}$ & $\begin{array}{l}\text { MIIRL- } \\
\text { GENOAM- } \\
\text { MONIA } \\
\text { ORGANIC } \\
\text { TOTAL } \\
\text { IMGNL } \\
\text { AS NI }\end{array}$ \\
\hline JUL, & 1975 & & . & & & & & & & & & \\
\hline oct & 1015 & 51 & -- & - & 21.5 & 230 & 7.7 & .010 & .08 & C.010 & 4.0 & .09 \\
\hline JAT... & $1976^{1200}$ & 51 & -- & - & $21 \cdot 0$ & 240 & 7.6 & .020 & .01 & C.010 & 1.6 & .02 \\
\hline mar & 1330 & 51 & - & - & 21.5 & 236 & 8.2 & .020 & .00 & .010 & 3.2 & .02 \\
\hline $12 \ldots$ & 1410 & 51 & -- & -- & 21.0 & 240 & - & .010 & .00 & .010 & 4.0 & .01 \\
\hline DEC & 1530 & 51 & -- & - & 21.0 & 245 & -- & .010 & .04 & C.010 & 4.2 & .05 \\
\hline MAR $15 \ldots$ & $1977^{1130}$ & 51 & -- & - & 21.0 & 225 & 7.7 & .020 & $t .00$ & c.010 & 5.3 & .02 \\
\hline JUN $17 . .$. & 1130 & 51 & -- & - & $\cdots$ & 241 & - & .010 & .00 & C.010 & 2.4 & .01 \\
\hline $\operatorname{sEP}_{22}$ & 1430 & 51 & -- & -- & 20.0 & - & - & C.010 & .20 & <. 010 & 2.4 & .20 \\
\hline Nov & 1030 & 51 & -- & - & 21.0 & 260 & $-\infty$ & $<.010$ & .00 & c. 010 & 1.8 & c.10 \\
\hline OEC & 1015 & 51 & - & - & 21.5 & 264 & - & .010 & .02 & $<.010$ & 4.0 & .03 \\
\hline FEQ $19 .$. & $1978^{1245}$ & 51 & -- & -- & 21.5 & 263 & $m$ & .010 & .00 & <.010 & 3.6 & .01 \\
\hline พAY & 1200 & 51 & - & -- & 21.0 & 245 & 7.6 & .030 & .04 & C.010 & 3.1 & .05 \\
\hline aUg & 0905 & 51 & 26 & 6.7 & 21.0 & 250 & 7.7 & C.010 & .00 & $<.010$ & 3.6 & $<.10$ \\
\hline DEC & 1250 & 51 & 25 & 8.0 & 21.5 & 250 & 7.7 & .020 & .00 & .010 & 3.4 & .02 \\
\hline$\underset{\operatorname{MAR}}{12}, 1$ & $1979^{1545}$ & 51 & 15 & 10 & 19.5 & 255 & 7.8 & .010 & .02 & $<.010$ & 2.9 & .03 \\
\hline $21 \cdots$ & 1340 & 51 & 15 & 12 & 20.5 & 239 & 7.2 & .010 & .12 & $<.010$ & 3.0 & .13 \\
\hline Mov & 1350 & 51 & 15 & 12 & 20.5 & 246 & 7.7 & C.010 & .13 & C.010 & 3.0 & .13 \\
\hline FEB 14 & $1980^{0910}$ & 51 & 28 & 12 & 20.5 & 233 & 7.8 & .020 & .04 & .000 & 2.9 & .06 \\
\hline JUN & 1020 & 51 & 38 & 9.6 & 21.0 & 244 & 7.9 & .010 & .03 & .000 & 2.7 & .04 \\
\hline$\underset{\text { Aug }}{09} \cdots$ & 1420 & 51 & 78 & 8.3 & 20.5 & 228 & 7.8 & .020 & .02 & .000 & 3.0 & .04 \\
\hline & 1325 & 51 & 35 & 9.1 & 21.0 & 231 & 7.9 & .000 & .05 & .000 & 3.0 & .05 \\
\hline DATE & $\begin{array}{l}\text { NITRC- } \\
\text { GEN, } \\
\text { NO2+NC3 } \\
\text { TOTAL } \\
\text { (RG/ } \\
\text { AS N) }\end{array}$ & $\begin{array}{l}\text { NIJRE- } \\
\text { GEN, } \\
\text { TOTAE } \\
\text { (MG LL } \\
\text { AS N) }\end{array}$ & $\begin{array}{l}\text { PHCS- } \\
\text { PHORUS, } \\
\text { YOTAL } \\
\text { (MCA } \\
\text { AS F) }\end{array}$ & $\begin{array}{l}\text { OX YGEN } \\
\text { DE MAND. } \\
\text { B TO- } \\
\text { CHEN- } \\
\text { ICAL: } \\
5 \text { DAY } \\
\text { (FG/L) }\end{array}$ & $\begin{array}{l}\text { OXYGEN } \\
\text { DEMAND, } \\
\text { CHEK- } \\
\text { ICAL } \\
\text { (HIGH } \\
\text { LEVEL) } \\
\text { (MGR) }\end{array}$ & $\begin{array}{l}\text { CAREON, } \\
\text { ORGANIC } \\
\text { TOTAL } \\
\text { (MG/L } \\
\text { AS C) }\end{array}$ & $\begin{array}{l}\text { ALXA- } \\
\text { LINITY } \\
\text { FIfLO } \\
\text { I HGA } \\
\text { AS } \\
\text { CACO3) }\end{array}$ & $\begin{array}{l}\text { HARD- } \\
\text { MESS, } \\
\text { NONCAR- } \\
\text { BONATE } \\
\text { (MGAL } \\
\text { CACD3) }\end{array}$ & $\begin{array}{l}\text { HARO- } \\
\text { NESS } \\
\text { ING/L } \\
\text { AS } \\
\text { CACO3) }\end{array}$ & $\begin{array}{l}\text { CALCIUH } \\
\text { DIS- } \\
\text { SOLVED } \\
\text { (ME } \\
\text { AS CA) }\end{array}$ & $\begin{array}{l}\text { MAGME- } \\
\text { SIUN, } \\
\text { DIS- } \\
\text { SOLVED } \\
\text { (MGA } \\
\text { AS MG) }\end{array}$ & $\begin{array}{l}\text { SODIUM, } \\
\text { DIS- } \\
\text { SOLVED } \\
\text { (HG/L } \\
\text { AS NA) }\end{array}$ \\
\hline
\end{tabular}

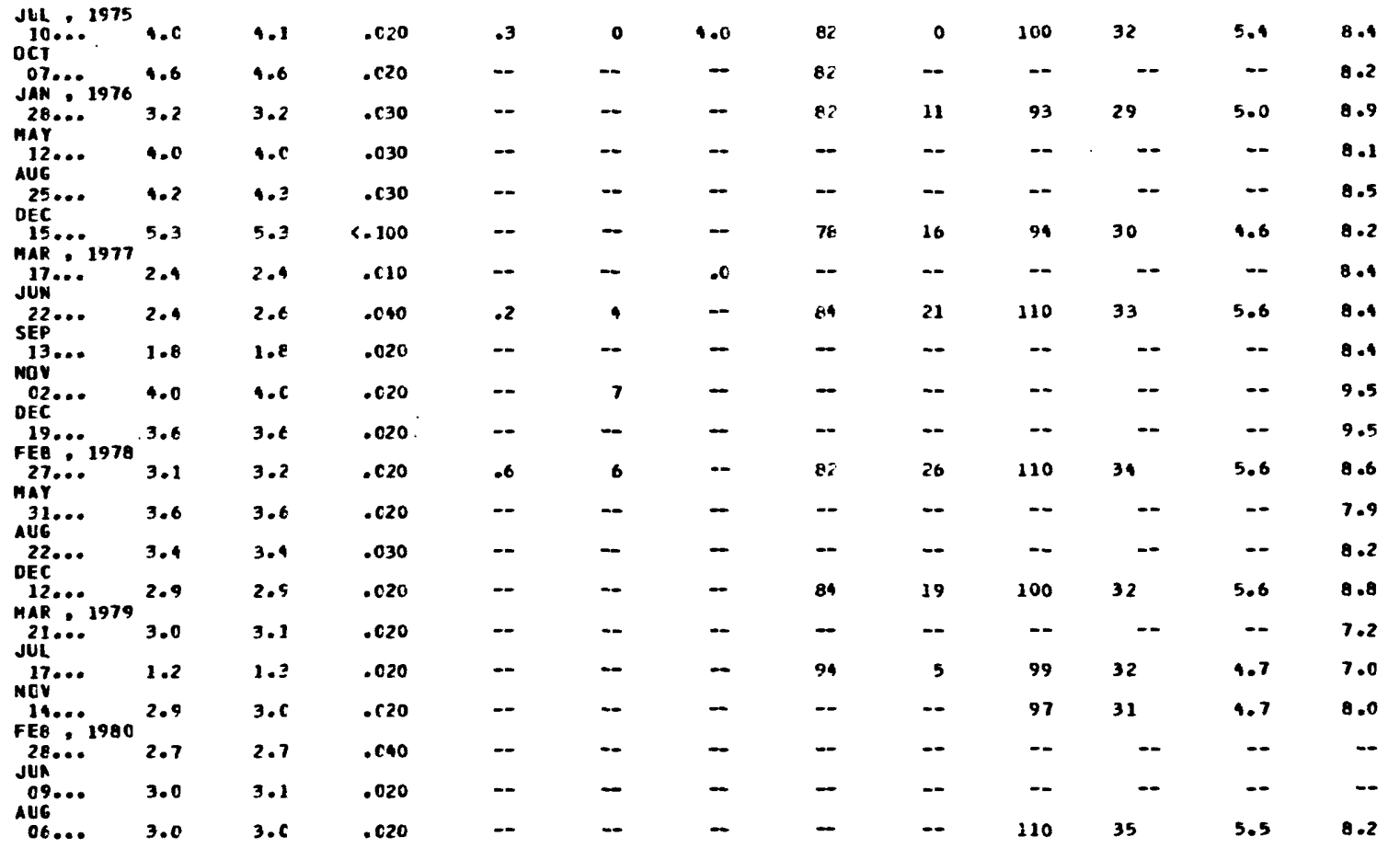


Site 5--Continued

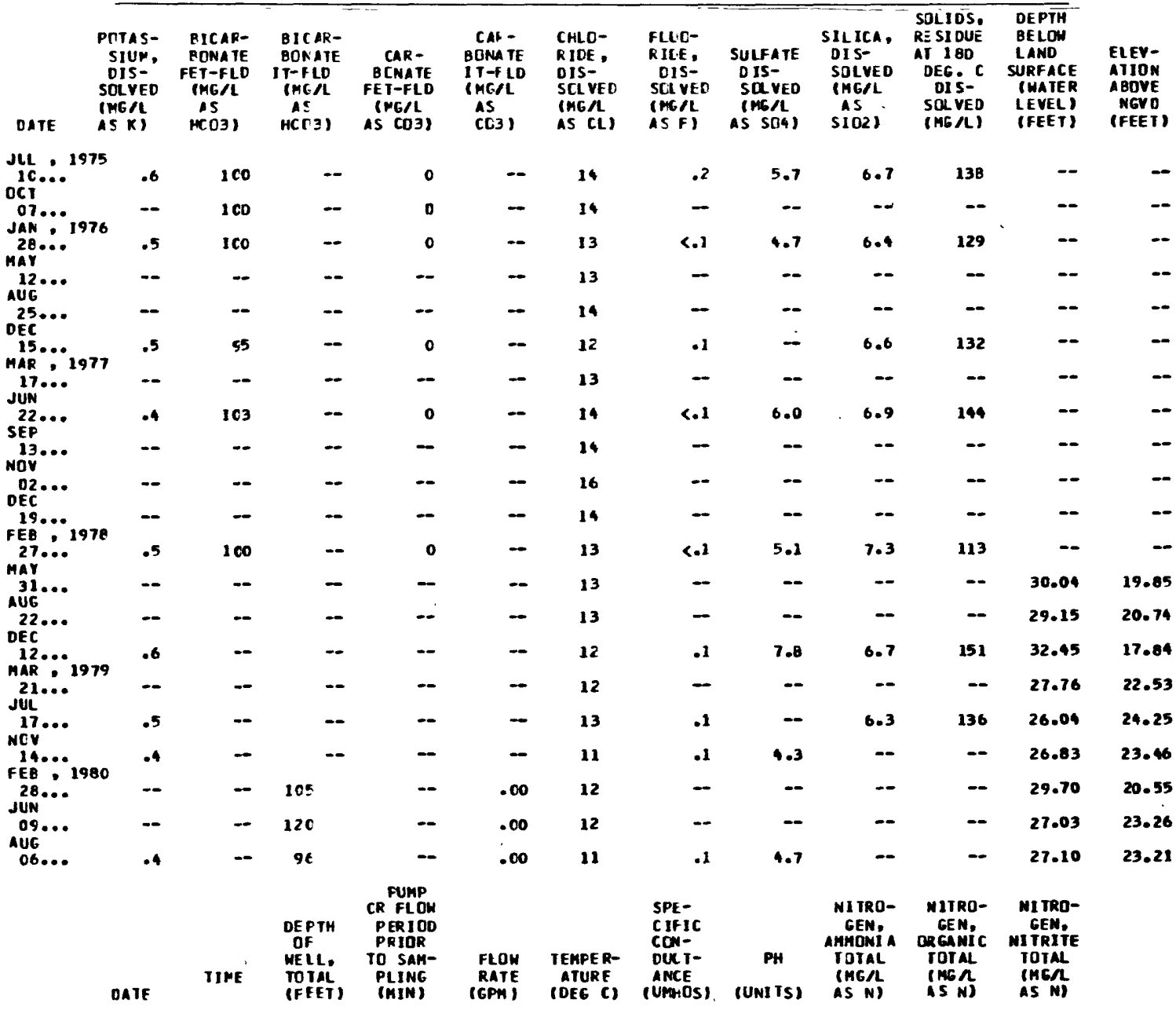

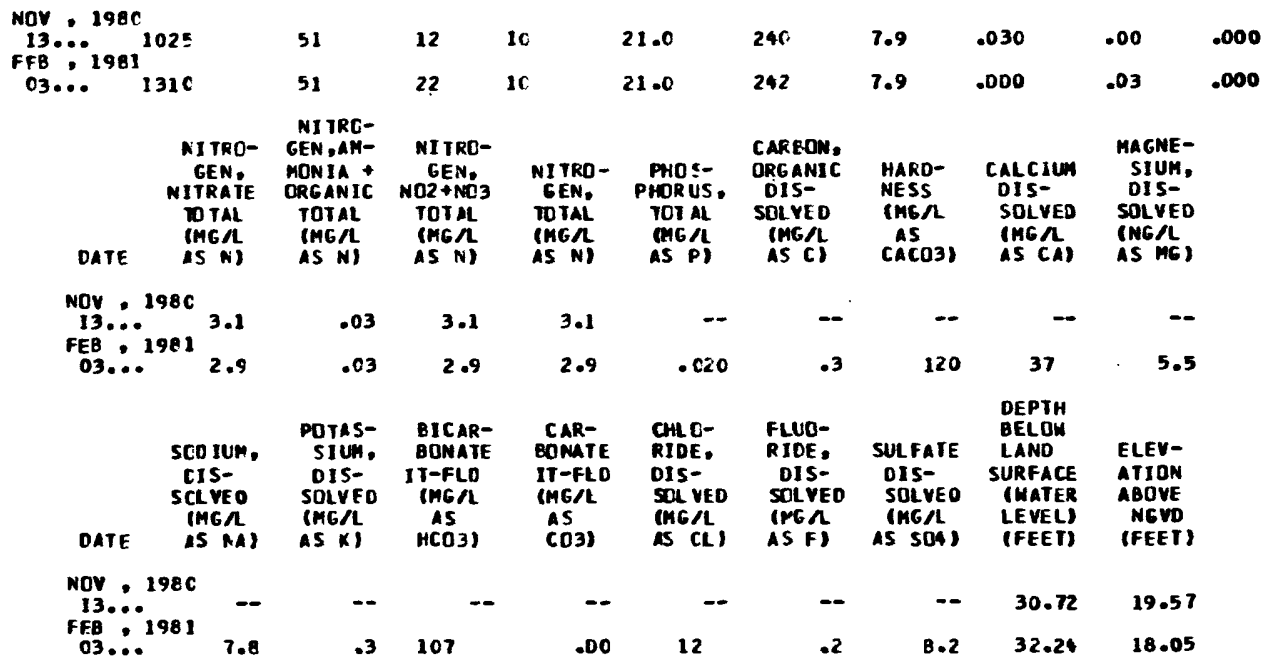


Site 5--Continued

APR 1973

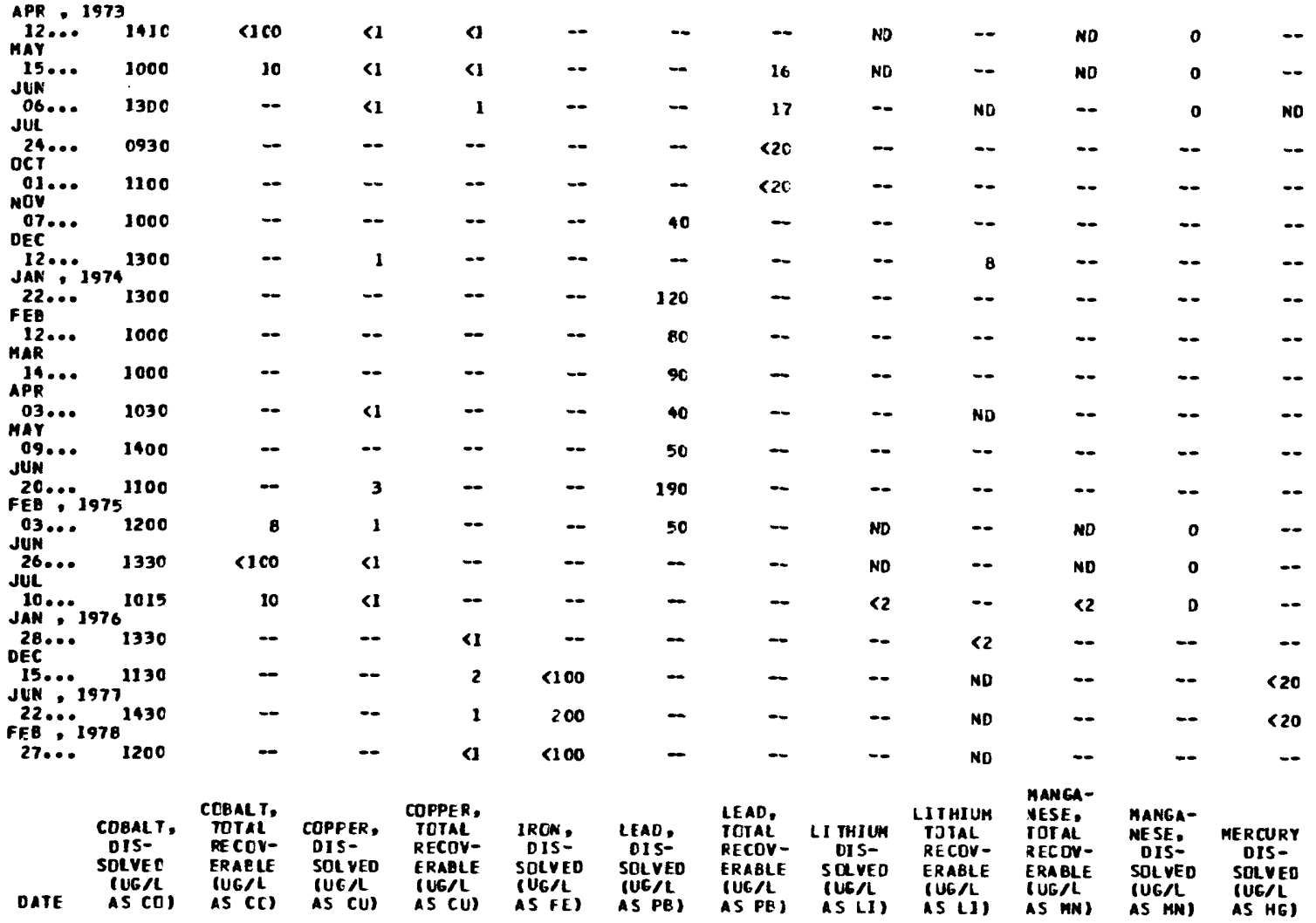

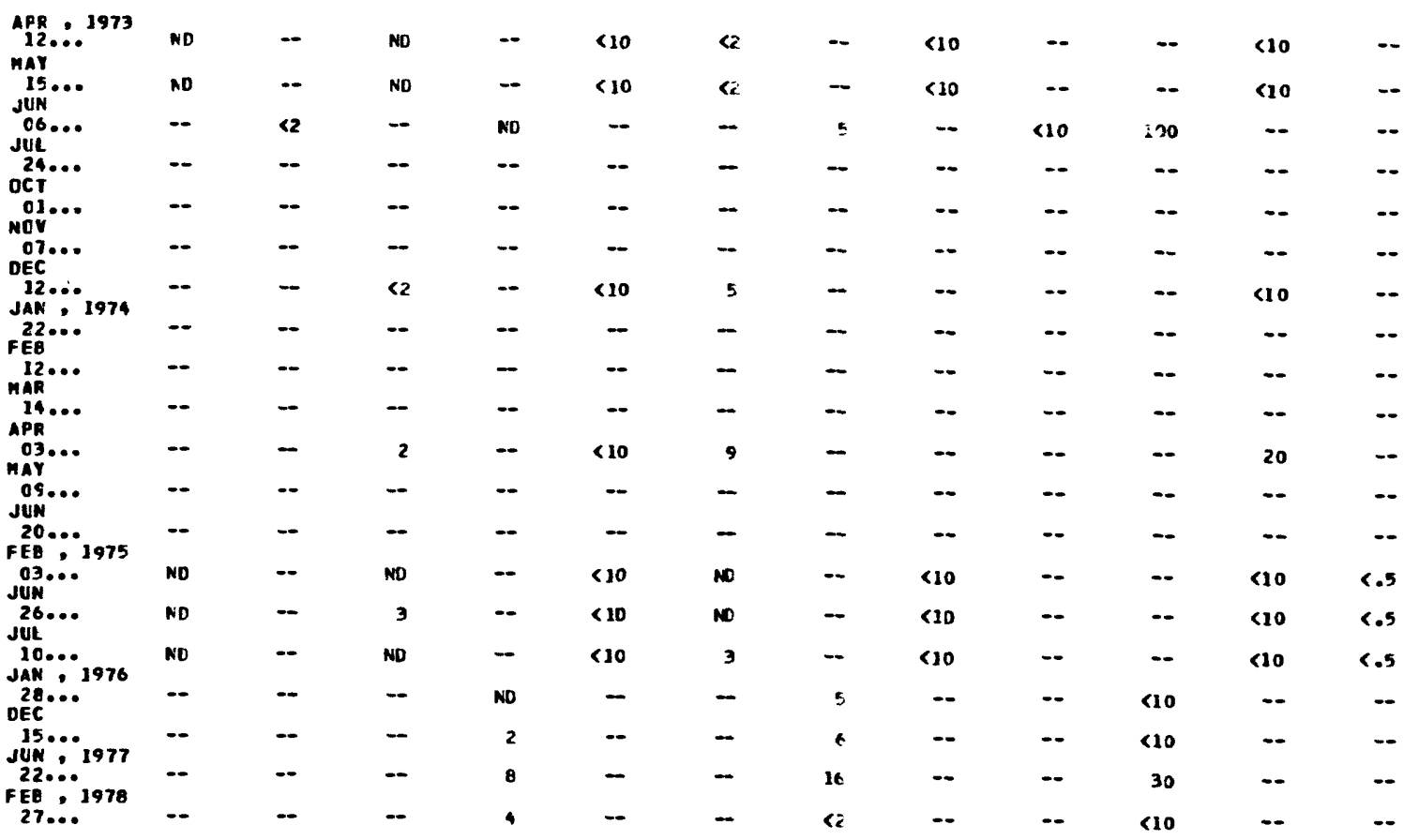


Table 6.--Physical and chemical characteristics of well and water at sites 1 through 70--Continued

Site 5--Continued

\begin{tabular}{|c|c|c|c|c|c|c|c|c|c|c|c|}
\hline & $\begin{array}{l}\text { MERCURY } \\
\text { TOTAL } \\
\text { RECOV- } \\
\text { ERABLE } \\
\text { (UG/L } \\
\text { AS HG) }\end{array}$ & $\begin{array}{l}\text { MDLYE- } \\
\text { DENUF. } \\
\text { DIS- } \\
\text { SOLYED } \\
\text { (UG } \\
\text { AS MC) }\end{array}$ & $\begin{array}{l}\text { DEP UM, } \\
\text { TOTAL } \\
\text { RECCV- } \\
\text { ER ALE } \\
\text { (UGAL } \\
\text { AS MO) }\end{array}$ & $\begin{array}{l}\text { NI CKEL, } \\
\text { OIS- } \\
\text { SOLVED } \\
\text { (UG/L. } \\
\text { AS NI) }\end{array}$ & $\begin{array}{l}\text { NICKEL, } \\
\text { TETAL } \\
\text { RECEV- } \\
\text { ERABLE } \\
\text { TUCAL } \\
\text { IS NII }\end{array}$ & $\begin{array}{l}\text { SELE- } \\
\text { NIUM, } \\
\text { CIS- } \\
\text { SCLYED } \\
\text { (UE/L } \\
\text { AS SE) }\end{array}$ & $\begin{array}{l}\text { SELE- } \\
\text { AIUM, } \\
\text { ICIAL } \\
\text { (UE/L } \\
\text { AS SE) }\end{array}$ & $\begin{array}{l}\text { SI (VER, } \\
\text { TCTAL } \\
\text { R ECOV- } \\
\text { ERABLE } \\
\text { (UG/L } \\
\text { AS AG) }\end{array}$ & $\begin{array}{l}\text { SIRON- } \\
\text { TIUH, } \\
\text { DIS- } \\
\text { SOLVED } \\
\text { (UG/L } \\
\text { AS SR ) }\end{array}$ & $\begin{array}{l}\text { VANA- } \\
\text { DIUM, } \\
\text { DIS- } \\
\text { SOLVED } \\
\text { (UGR } \\
\text { AS VI) }\end{array}$ & $\begin{array}{l}\text { ZINC, } \\
\text { DIS- } \\
\text { SOLVED } \\
\text { (UGA } \\
\text { AS IN) }\end{array}$ \\
\hline
\end{tabular}

AFR 1973

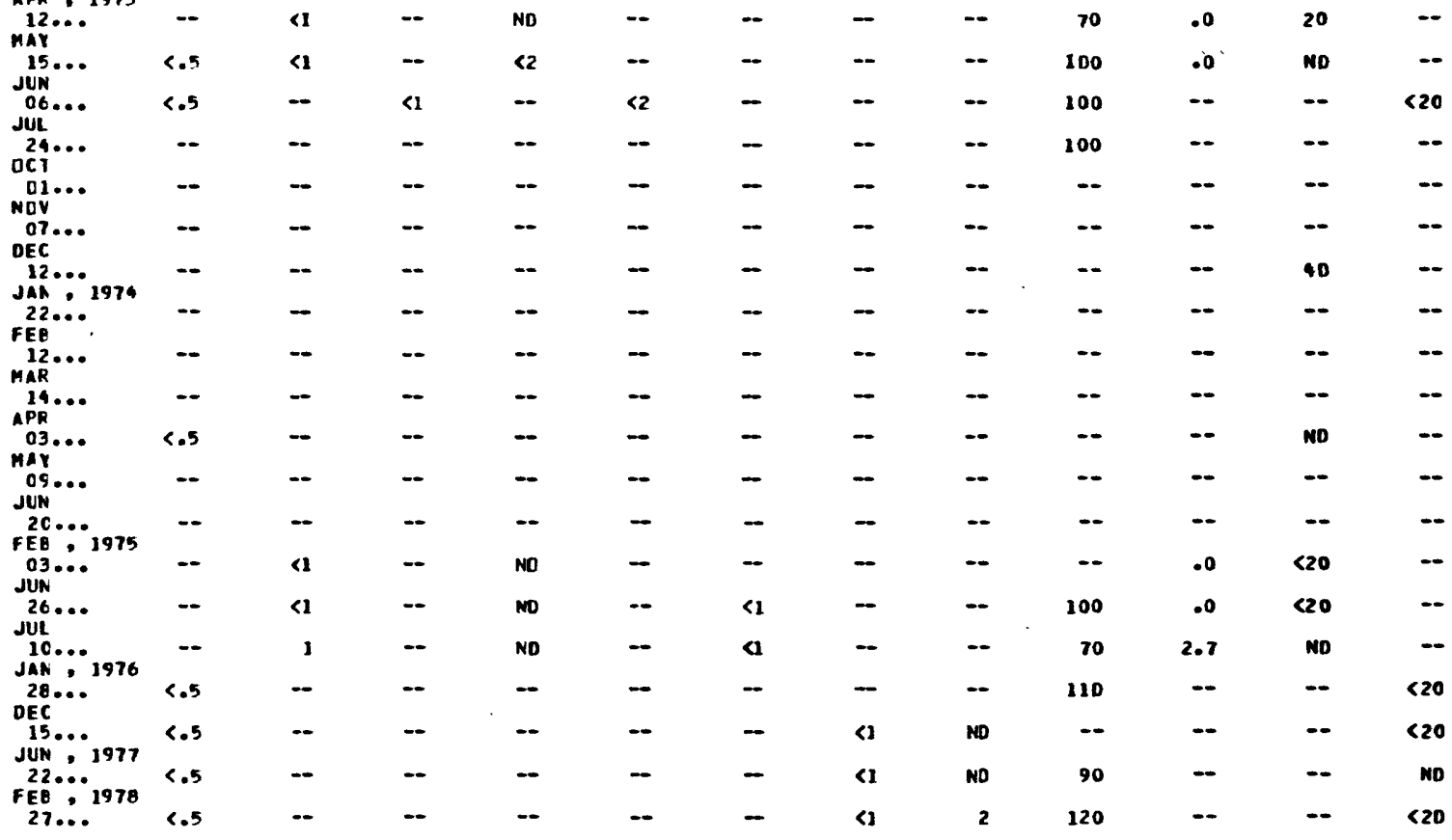

\begin{tabular}{|c|c|c|c|c|c|c|c|c|c|c|}
\hline DATE & TIME & $\begin{array}{l}\text { ALOR IN, } \\
\text { TOTAL } \\
\text { (UGAL) }\end{array}$ & $\begin{array}{l}\text { CHLOR- } \\
\text { DANE : } \\
\text { TOTAL } \\
\text { (UG/L) }\end{array}$ & $\begin{array}{l}\text { DOC, } \\
\text { TOTAL } \\
\text { (UG/L) }\end{array}$ & $\begin{array}{l}\text { DOE, } \\
\text { JOTAL } \\
\text { (UG/L) }\end{array}$ & $\begin{array}{l}\text { DOT, } \\
\text { TCTAL } \\
\text { (UG/L) }\end{array}$ & $\begin{array}{l}\text { OI- } \\
\text { ELORIN } \\
\text { TOTAL } \\
\text { (UG/L) }\end{array}$ & $\begin{array}{l}\text { ENDO- } \\
\text { SULFAN, } \\
\text { TJTAL } \\
\text { (UG/L) }\end{array}$ & $\begin{array}{l}\text { ENDRIN, } \\
\text { TOT AL } \\
\text { (UG几) }\end{array}$ & $\begin{array}{l}\text { HEPTA- } \\
\text { CHLOR, } \\
\text { TOTAL } \\
\text { (UGA) }\end{array}$ \\
\hline JUN ... & $974110 \mathrm{C}$ & .00 & .00 & .00 & .00 & .00 & .00 & $\cdots$ & .00 & .00 \\
\hline AUG : & 978 & .00 & .00 & - $\mathrm{CO}$ & .00 & .00 & .00 & .00 & .00 & .00 \\
\hline DATF & $\begin{array}{l}\text { HEPT A- } \\
\text { CHLCR } \\
\text { EPOXIOE } \\
\text { TOTAL } \\
\text { (UG/L) }\end{array}$ & $\begin{array}{l}\text { IINCANE } \\
\text { TOTAL } \\
\text { (UT/L) }\end{array}$ & $\begin{array}{l}\text { METH- } \\
\text { CXY- } \\
\text { CHEOR, } \\
\text { TOTAL } \\
\text { (UG/L) }\end{array}$ & $\begin{array}{l}\text { MIRt } x_{\text {, }} \\
\text { TOT AL } \\
\text { (UG/L) }\end{array}$ & $\begin{array}{l}\text { TOX- } \\
\text { APHENE, } \\
\text { TOTAL } \\
\text { (UG/L) }\end{array}$ & $\begin{array}{l}\text { PCB, } \\
\text { TOTAL } \\
\text { (UG/L) }\end{array}$ & $\begin{array}{l}\text { NAPH- } \\
\text { THA- } \\
\text { LENES. } \\
\text { PQY- } \\
\text { CHLOR. } \\
\text { TOTAL: } \\
\text { (UGAL) }\end{array}$ & $\begin{array}{l}\text { SILVEX, } \\
\text { TDTAL } \\
\text { (UGAL) }\end{array}$ & $\begin{array}{l}2,4-0, \\
\text { rótal } \\
\text { (UERl) }\end{array}$ & $\begin{array}{l}2,4,5-1 \\
\text { Total } \\
\text { (U6n) }\end{array}$ \\
\hline $\begin{array}{l}\text { JUN } \\
20 .: \\
\text { AUG }\end{array}$ & 974.00 & .00 & - & -- & 0 & .00 & - & .00 & .00 & .00 \\
\hline $22 \ldots$ &. $\mathrm{co}$ & .00 & .00 & .00 & 0 & .00 & .00 & - & -- & - \\
\hline
\end{tabular}


Site 6

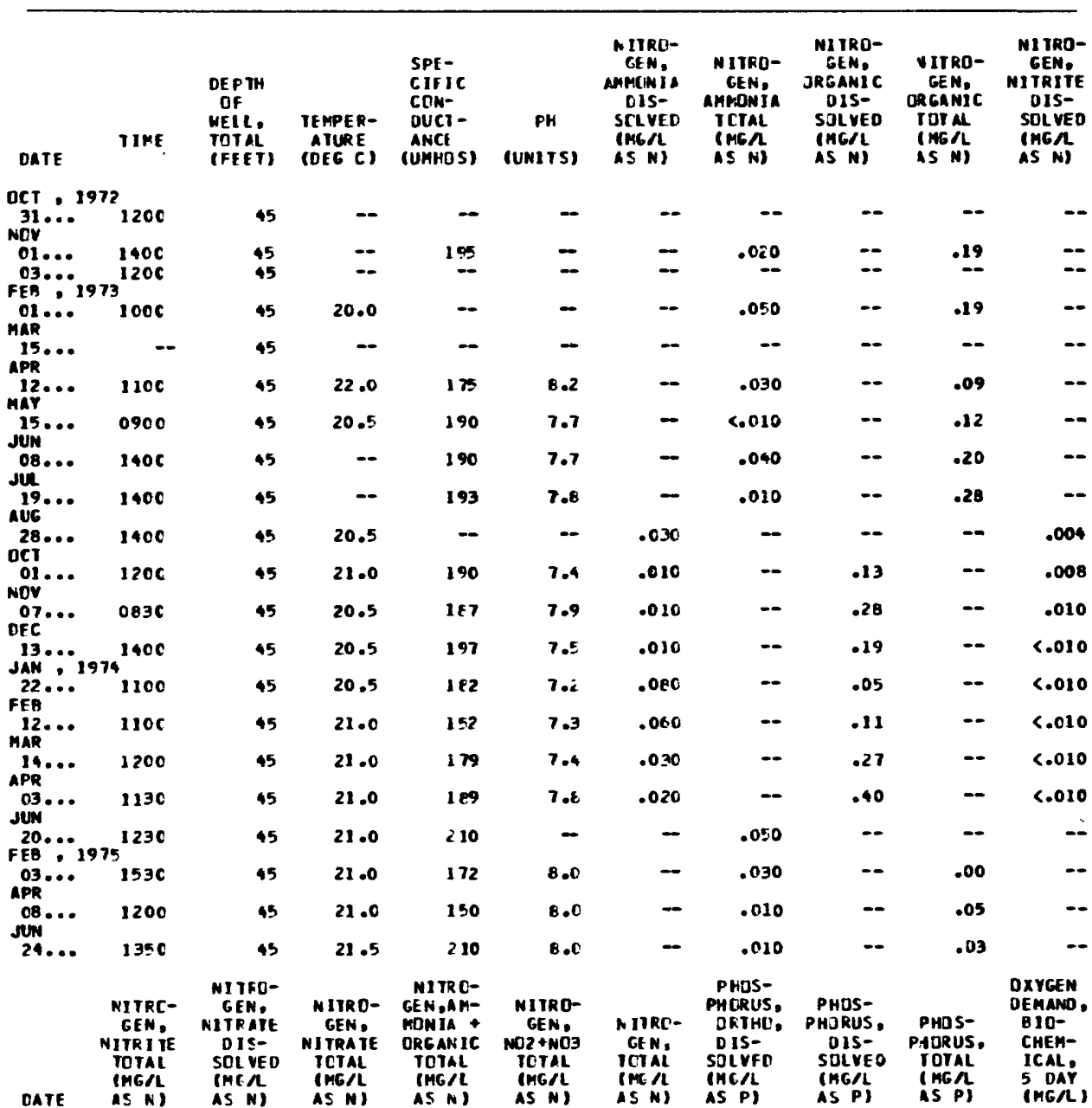

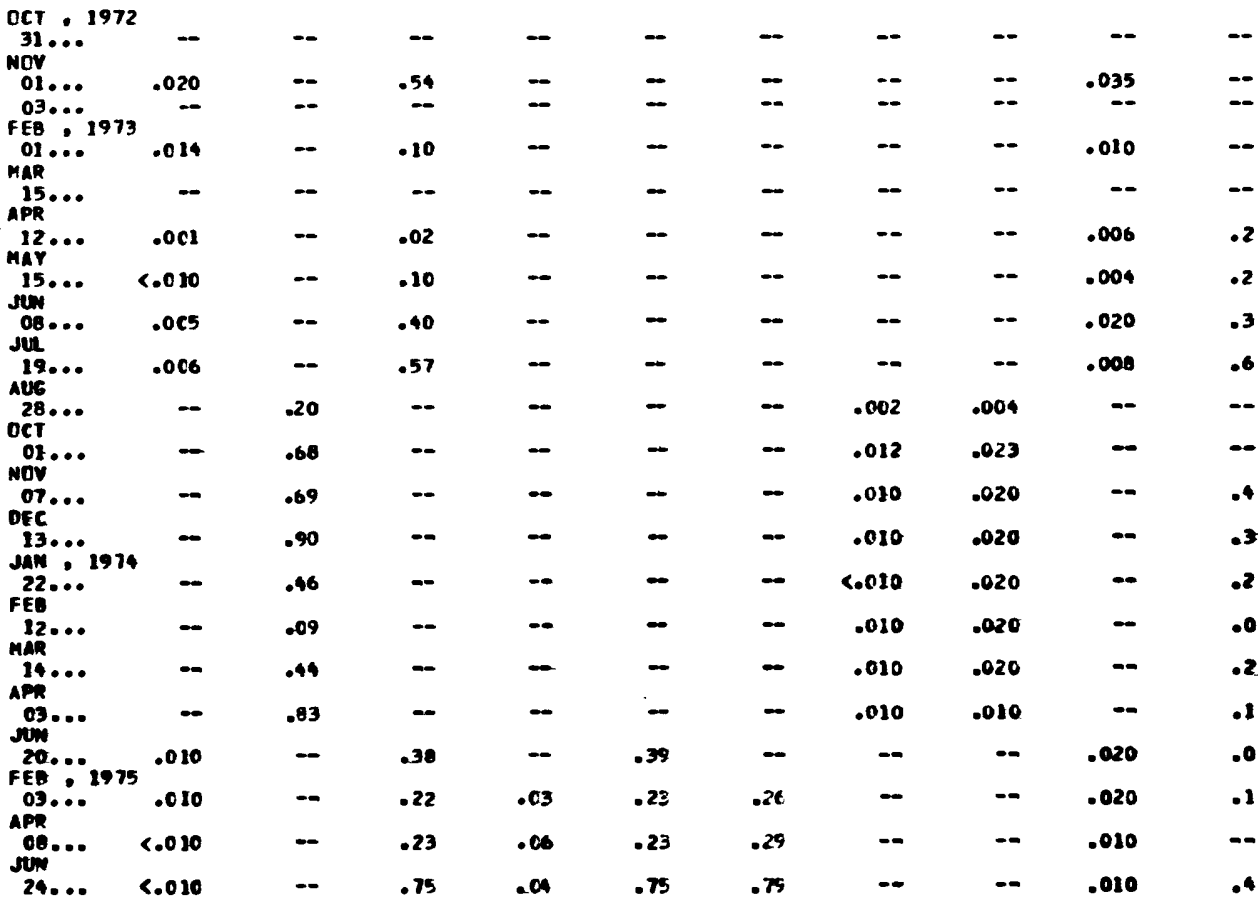


Site 6--Continued

\begin{tabular}{|c|c|c|c|c|c|c|c|c|c|c|}
\hline DATE & $\begin{array}{l}\text { DXY } \\
\text { DEM } \\
\text { CH } \\
\text { IC } \\
\text { IH } \\
\text { IEV } \\
\text { ING }\end{array}$ & & $\begin{array}{l}\text { CARBON, } \\
\text { INCR- } \\
\text { GANIC, } \\
\text { IUTAL } \\
\text { IMGAL } \\
\text { AS CI }\end{array}$ & $\begin{array}{l}\text { CARBCN, } \\
\text { ORGANIC } \\
\text { TUTAL } \\
\text { IMGA } \\
\text { AS C) }\end{array}$ & $\begin{array}{l}\text { CAPBON, } \\
\text { TOTAL } \\
\text { INGA } \\
\text { AS C) }\end{array}$ & $\begin{array}{l}\text { AKA- } \\
\text { INITY } \\
\text { FIELI } \\
\text { IMG } A \\
\text { AS } \\
\text { CACD3) }\end{array}$ & $\begin{array}{l}\text { HAFD- } \\
\text { NESS, } \\
\text { NONCAR- } \\
\text { BONATE } \\
\text { (MCA } \\
\text { CACQ3) }\end{array}$ & $\begin{array}{l}\text { HARD- } \\
\text { NESS } \\
\text { (MGAL } \\
\text { AS } \\
\text { CACO3) }\end{array}$ & $\begin{array}{l}\text { CALCIUY } \\
\text { DIS- } \\
\text { SOLVEO } \\
\text { (MG/L } \\
\text { AS CA) }\end{array}$ & $\begin{array}{l}\text { MAGNE- } \\
\text { SIUA, } \\
\text { DIS- } \\
\text { SOLVED } \\
\text { (MGIL } \\
\text { AS MG) }\end{array}$ \\
\hline \multicolumn{2}{|c|}{ CCT, 1972} & -- & -- & -- & -- & - & - & - & - & - \\
\hline $01 \ldots$ & 4973 & $=$ & 19 & 1.0 & 20 & $=$ & $=$ & $=$ & $=$ & $=$ \\
\hline$\underset{\text { MAR }}{01}$ & & - & - & - & - & -- & - & -- & -- & $=$ \\
\hline$\underset{A P R}{15} \cdots$ & & -- & -- & - & -- & -- & -- & -- & - & - \\
\hline $\operatorname{mar}_{12} \cdots$ & & 2 & 14 & 8.0 & 22 & $E 7$ & 4 & 91 & 34 & 1.4 \\
\hline JUN & & 1 & 18 & 2.0 & 20 & E9 & $\mathbf{I 1}$ & 99 & 36 & 2.3 \\
\hline$\underset{\text { Jur }}{08 . . .}$ & & 1 & 19 & 1.0 & 20 & es & B & 97 & 35 & 2.2 \\
\hline$\underset{A \cup G}{19} \cdots$ & & - & 22 & .0 & 22 & $e 6$ & 8 & 94 & 34 & 2.2 \\
\hline${ }_{\mathrm{DCT}}^{28} \cdots$ & & - & - & - & 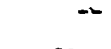 & - & - & - & - & -- \\
\hline$\underset{\text { NOV }}{01 . \cdots}$ & & - & 20 & .0 & 20 & 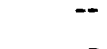 & $\cdots$ & 89 & 32 & 2.2 \\
\hline OEC & & 0 & 18 & .0 & 18 & 77 & 14 & 91 & 31 & 3.4 \\
\hline JAN 13 & 1974 & -- & - & - & -- & $e 2$ & 2 & 84 & 28 & 3.5 \\
\hline FEB & & 0 & 19 & .0 & 19 & 81 & 0 & 79 & 28 & 2.2 \\
\hline${ }_{M A R}^{12} \cdots$ & & 0 & 16 & .0 & 16 & 71 & 8 & 79 & 30 & 1.0 \\
\hline$\underset{A P R}{14} \cdots$ & & 8 & 18 & 2.0 & 20 & 71 & 14 & 85 & 31 & 1.8 \\
\hline$\underset{\text { JuN }}{03} \ldots$ & & 3 & 21 & .0 & 21 & -- & -- & 95 & 33 & 3.0 \\
\hline FEB 20 & 1975 & 2 & 21 & .0 & 21 & - & - & 84 & 30 & 2.1 \\
\hline$\underset{A P R}{03} \ldots$ & & 0 & -- & .0 & -- & $e 5$ & 7 & 92 & 34 & 1.7 \\
\hline$\underset{\text { JUN }}{08 \cdots}$ & & -- & -- & .0 & $\cdots$ & 82 & - & -- & - & $\cdots$ \\
\hline $24 \ldots$ & & 6 & $\cdots$ & .0 & $\cdots$ & $\varepsilon 3$ & 10 & 93 & 33 & 2.5 \\
\hline DATE & $\begin{array}{c}\text { sco } \\
\text { c1 } \\
\text { sct } \\
\text { in } \\
\text { is }\end{array}$ & & $\begin{array}{l}\text { POTAS- } \\
\text { SIUH, } \\
\text { DIS- } \\
\text { SOLVED } \\
\text { (MG/L } \\
\text { ASK) }\end{array}$ & $\begin{array}{l}\text { ELCAR- } \\
\text { BONATE } \\
\text { FET-FLD } \\
\text { (MG/L } \\
\text { AS } \\
\text { HCO3) }\end{array}$ & $\begin{array}{c}\text { CAR- } \\
\text { BDNATE } \\
\text { FET-FLD } \\
\text { (MGR } \\
\text { AS CO3) }\end{array}$ & $\begin{array}{l}\text { CHLO- } \\
\text { RIDE, } \\
\text { OIS- } \\
\text { SOL VEO } \\
\text { IMG /L } \\
\text { AS CL) }\end{array}$ & $\begin{array}{l}\text { FLUD- } \\
\text { RIDE } \\
\text { DIS- } \\
\text { SOLVED } \\
\text { (HE/L } \\
\text { AS F) }\end{array}$ & $\begin{array}{l}\text { SULFATE } \\
\text { DIS- } \\
\text { SOLVED } \\
\text { (MGR } \\
\text { AS SO4) }\end{array}$ & $\begin{array}{l}\text { SILICA, } \\
\text { DIS- } \\
\text { SOLVEO } \\
\text { (NG/L } \\
\text { AS } \\
\text { SIO2) }\end{array}$ & $\begin{array}{l}\text { SOLIDS, } \\
\text { RESI DUE } \\
\text { AT IBO } \\
\text { DEG. C } \\
\text { DIS- } \\
\text { SOL VED } \\
\text { (HG/L) }\end{array}$ \\
\hline
\end{tabular}

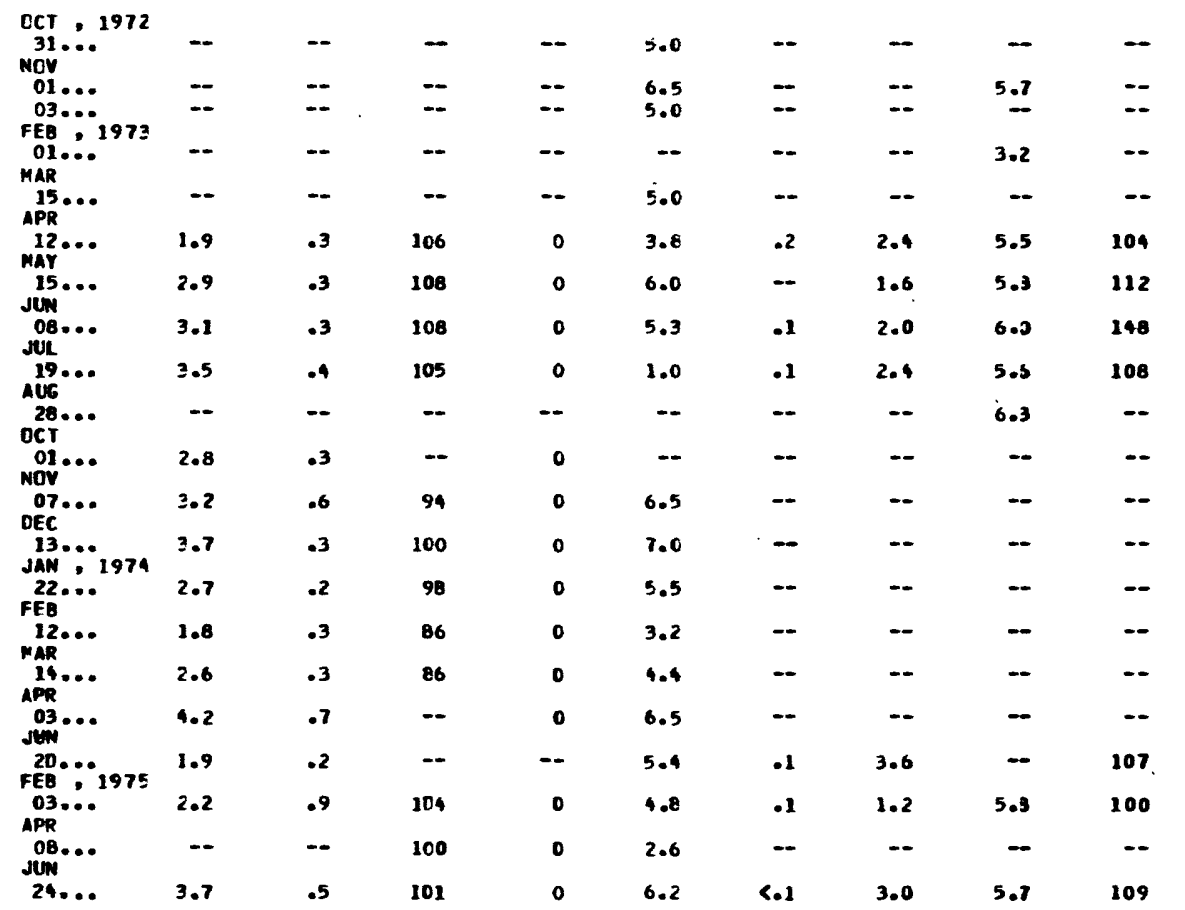


Table 6.--Physical and chemical characteristics of well and water at sites 1 through 70--Continued

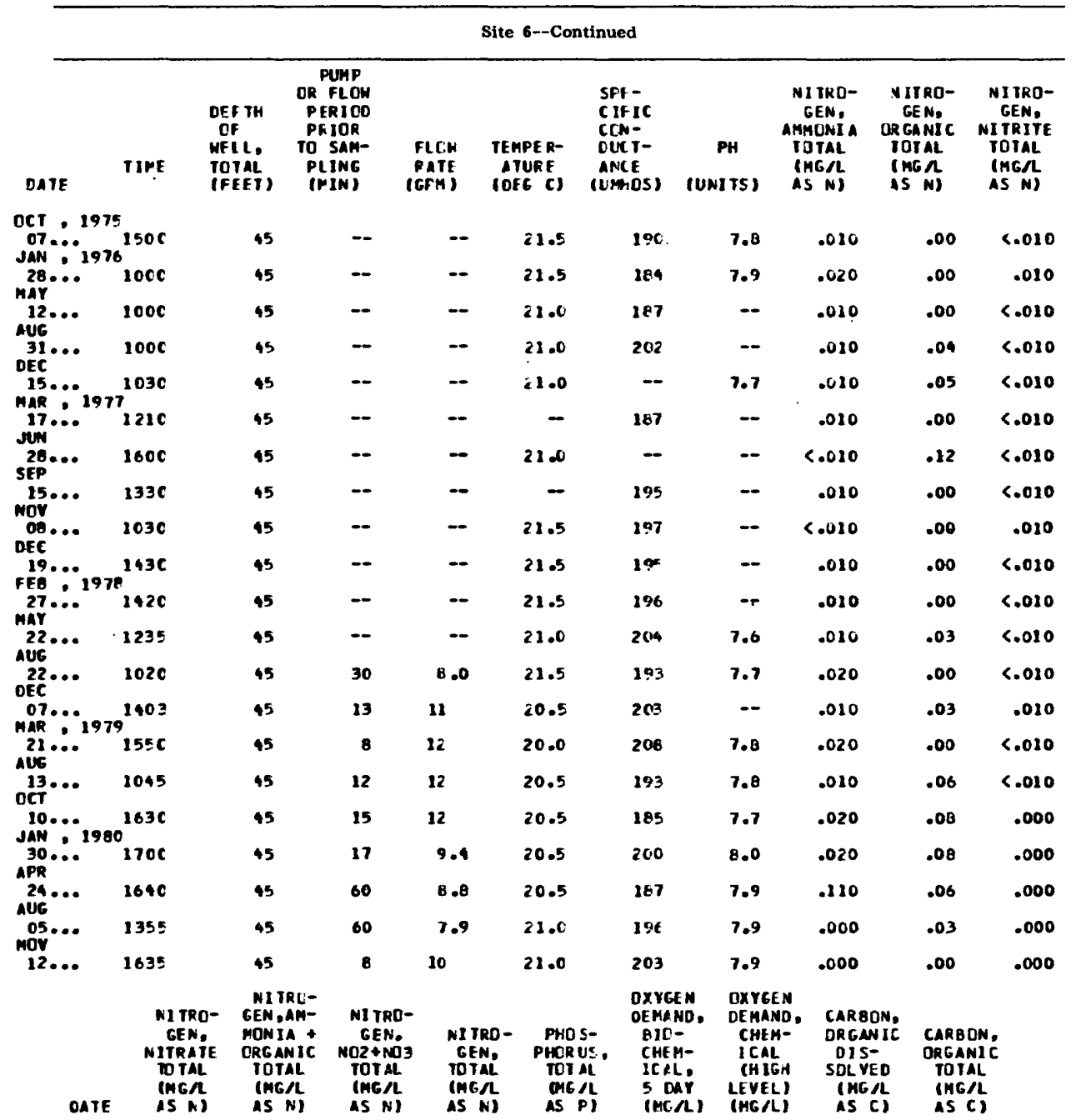

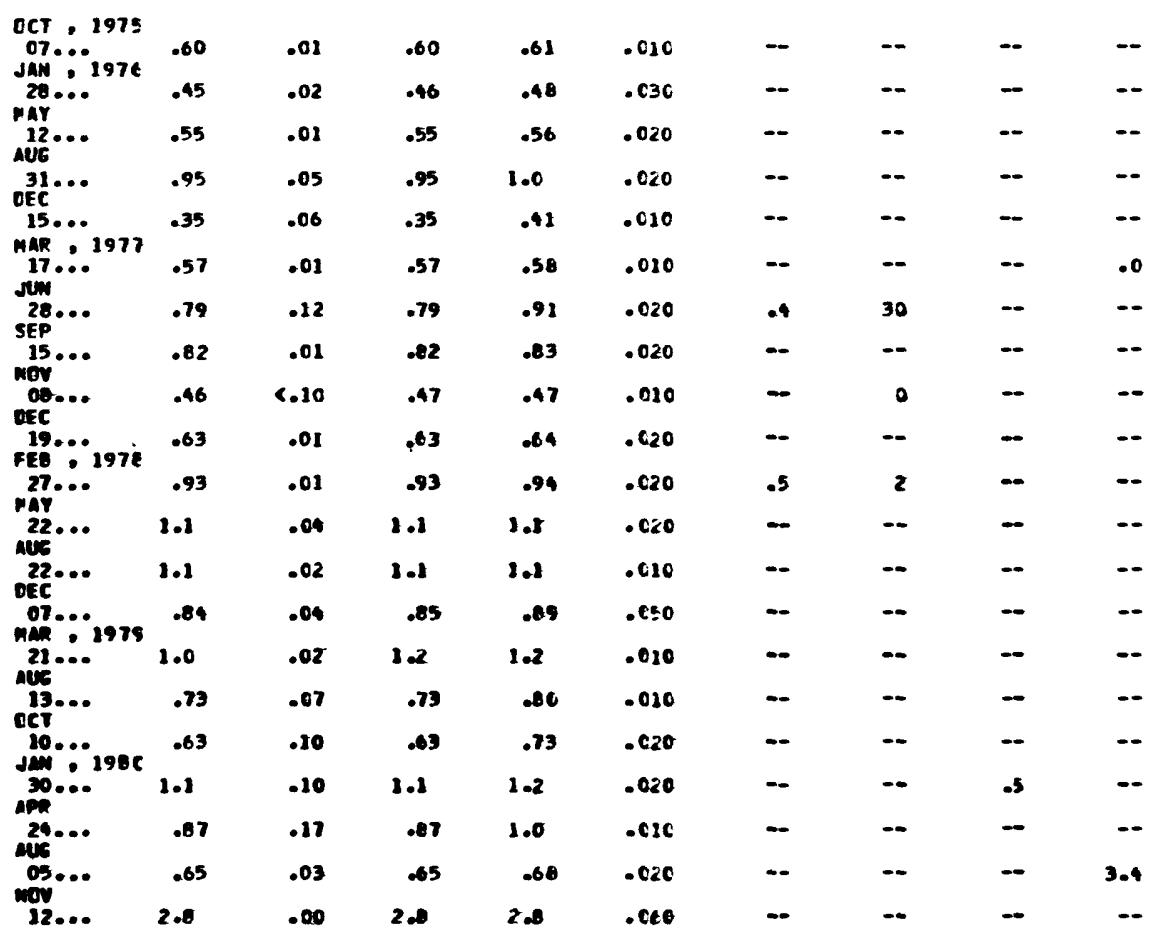




\begin{tabular}{|c|c|c|c|c|c|c|c|c|}
\hline \multicolumn{9}{|c|}{ Site 6--Continued } \\
\hline $\begin{array}{l}\text { ALKA- } \\
\text { LINITY } \\
\text { FIEID } \\
\text { IMGIL } \\
\text { AS } \\
\text { CACCB) }\end{array}$ & $\begin{array}{l}\text { HAFD- } \\
\text { NE SS } \\
\text { RCNCAR- } \\
\text { PONATE } \\
\text { (MG/L } \\
\text { CACQ3) }\end{array}$ & $\begin{array}{l}\text { HAR:- } \\
\text { NESS } \\
\text { (MG/L } \\
\text { AS } \\
\text { CAC! } 3 \text { ) }\end{array}$ & $\begin{array}{l}\text { CALCIUM } \\
\text { TIS- } \\
\text { SOLYED } \\
\text { IMGA } \\
\text { AS (A) }\end{array}$ & $\begin{array}{l}\text { MAGNE - } \\
\text { SIUL, } \\
\text { DIS- } \\
\text { SCLVEL } \\
\text { (MG AL } \\
\text { AS MC) }\end{array}$ & $\begin{array}{l}\text { SCOIUH, } \\
\text { DIS- } \\
\text { SCIVED } \\
\text { (HEA } \\
\text { AS IMA) }\end{array}$ & $\begin{array}{l}\text { PUTAS- } \\
\text { SIUM, } \\
\text { DIS- } \\
\text { SDLVED } \\
\text { (HG/L } \\
\text { AS R) }\end{array}$ & $\begin{array}{l}\text { BICAR- } \\
\text { BONATE } \\
\text { FET-FLD } \\
\text { (HGA } \\
\text { AS } \\
\text { HCD3) }\end{array}$ & $\begin{array}{l}\text { BICAR- } \\
\text { BONATE } \\
\text { IT-FLD } \\
\text { IAG/L } \\
\text { AS } \\
\text { HCOB) }\end{array}$ \\
\hline
\end{tabular}

DATE (ACC3) CACO3) CAC(3) AS (A) AS MC) AS MA) ASK) HCO3) HCO3)

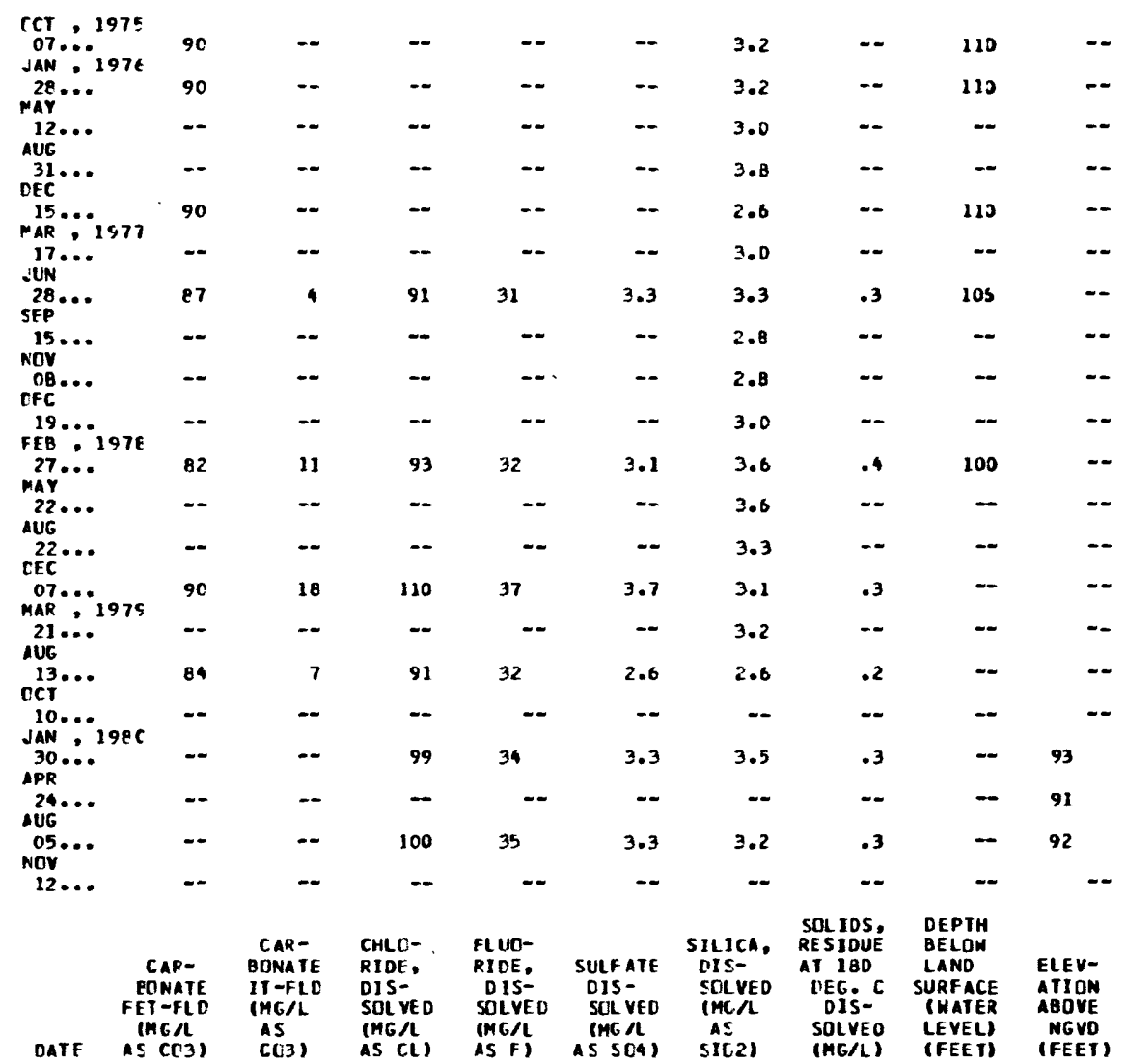

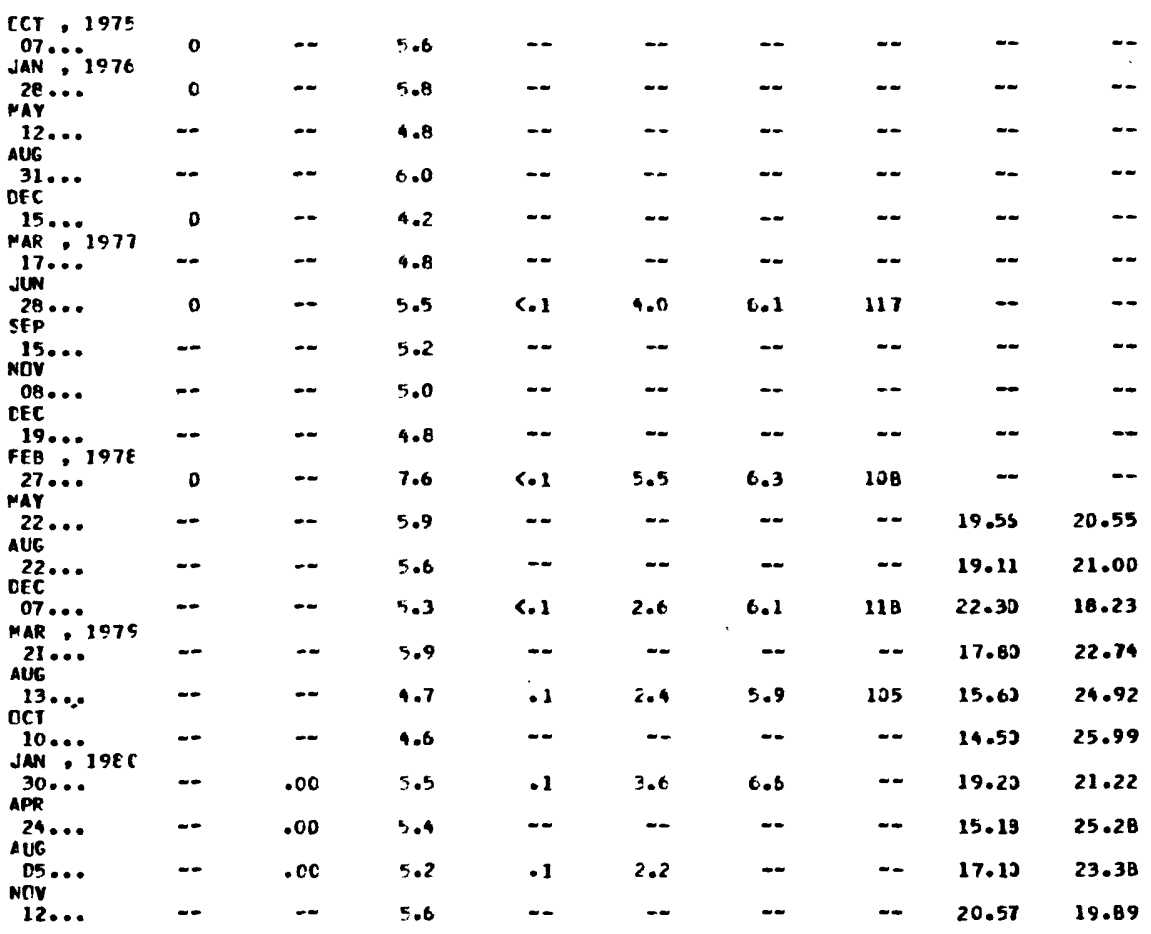




\begin{tabular}{|c|c|c|c|c|c|c|c|c|}
\hline \multicolumn{9}{|c|}{ Site 6--Continued } \\
\hline DAIE & TIPE & $\begin{array}{l}\text { DEPIH } \\
\text { LF } \\
\text { WELL, } \\
\text { TOTAL } \\
\text { (FEE I) }\end{array}$ & $\begin{array}{l}\text { PUPP } \\
\text { QR FL OW } \\
\text { PERIOD } \\
\text { PRILIR } \\
\text { TO SAM- } \\
\text { PLING } \\
\text { (MIN) }\end{array}$ & $\begin{array}{l}\text { FLOH } \\
\text { RATH } \\
\text { (GPH) }\end{array}$ & $\begin{array}{l}\text { TEHF EP- } \\
\text { ATLRE } \\
\text { (OEG C) }\end{array}$ & $\begin{array}{l}\text { SIE- } \\
\text { CIFJC } \\
\text { CEN- } \\
\text { DUCY- } \\
\text { ANCE } \\
\text { (UMHSS) }\end{array}$ & $\begin{array}{c}\text { PH } \\
\text { (UNITS) }\end{array}$ & $\begin{array}{l}\text { YITRO- } \\
\text { GEN. } \\
\text { AMMUNIA } \\
\text { TOIAL } \\
\text { (HG/L } \\
\text { IS N] }\end{array}$ \\
\hline
\end{tabular}

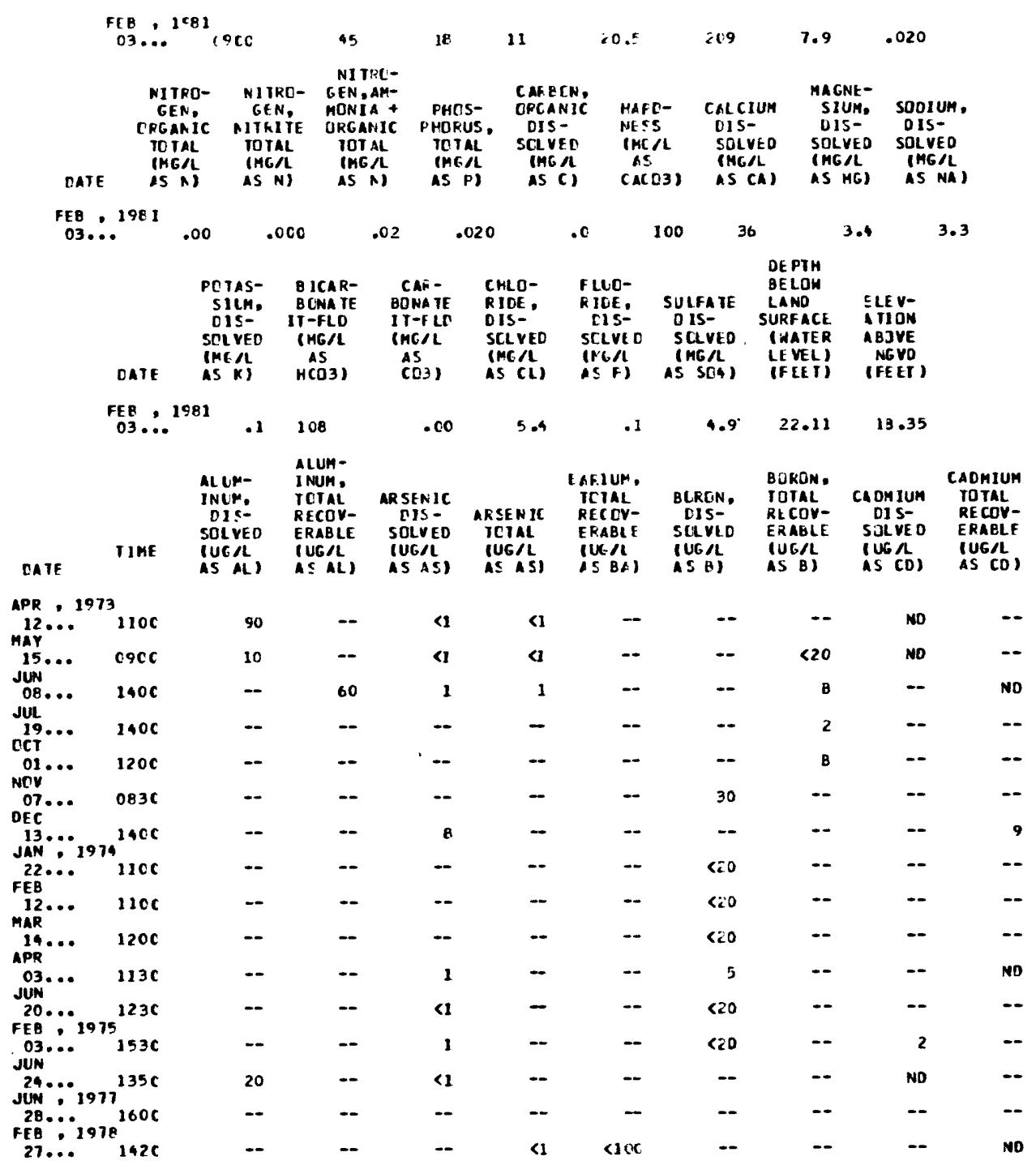


Site 6--Continued

\begin{tabular}{|c|c|c|c|c|c|c|c|c|c|c|}
\hline EATE & $\begin{array}{l}\text { CHRC } \\
\text { PIUI } \\
\text { CIS } \\
\text { nit } \\
\text { iUG } \\
\text { AS }\end{array}$ & 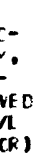 & $\begin{array}{l}\text { CHKD- } \\
\text { MIUM, } \\
\text { HEXA- } \\
\text { VALENT, } \\
\text { OIS. } \\
\text { (UGAL } \\
\text { AS CR) }\end{array}$ & $\begin{array}{l}\text { CHR::- } \\
\text { MIUIT, } \\
\text { TOTAL } \\
\text { RECEV- } \\
\text { ERAIILE } \\
\text { (UGA } \\
\text { AS CR) }\end{array}$ & $\begin{array}{l}\text { (CEALT, } \\
\text { CIS- } \\
\text { SCLVED } \\
\text { CUEAL } \\
\text { AS COS }\end{array}$ & 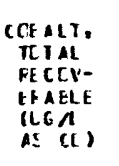 & 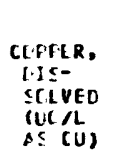 & $\begin{array}{l}\text { CCPFER, } \\
\text { TOTAL } \\
\text { RECJV- } \\
\text { IRABLE } \\
\text { (UGAL } \\
\text { is CUS }\end{array}$ & $\begin{array}{l}\text { IRGN, } \\
\text { DIS- } \\
\text { SULVED } \\
\text { (UG/L } \\
\text { AS FE) }\end{array}$ & $\begin{array}{l}\text { LEAD: } \\
\text { DIS- } \\
\text { SOLVED } \\
\text { (UG } \\
\text { AS PB) }\end{array}$ \\
\hline \multicolumn{11}{|r|}{ ND } \\
\hline $15 . .$. & & NE & 0 & -- & ND & -- & No & -- & $<13$ & ND \\
\hline dUN & & - & -- & ND & No & $\mathrm{NO}$ & - & NO & - & -- \\
\hline $19 . .$. & & - & -- & -- & -- & -- & -- & -- & - & -- \\
\hline $01 . .$. & & - & -- & -- & -- & -- & - & -- & - & - \\
\hline rEC... & & -- & -- & -- & -- & -- & $\cdots$ & -- & - & $\cdots$ \\
\hline $13 \ldots$ & & -- & -- & - & -- & $\cdots$ & $<2$ & $\cdots$ & <1) & 9 \\
\hline $22 \ldots$ & & -- & -- & -- & -- & -- & -- & -- & -- & $\cdots$ \\
\hline $12 \ldots$ & & -- & -- & -- & -- & -- & -- & -- & - & -- \\
\hline$\underset{A P R}{14} \ldots$ & & - & -- & -- & -- & - & $\cdots$ & -- & -- & - \\
\hline sum... & & -- & - & -- & -- & -- & $<2$ & -- & $<10$ & 5 \\
\hline FEB 20. & & -- & -- & -- & -- & -- & -- & -- & -- & -- \\
\hline $\operatorname{JUN}_{\text {JUN }}^{03 . .}$ & & ND & 0 & -- & $<2$ & $\cdots$ & ND & -- & 《ा & 7 \\
\hline JUN $24:$ & & ND & 0 & -- & ND & -- & $<2$ & -- & 13 & ND \\
\hline FEB 28 & & -- & -- & -- & -- & -- & -- & -- & -- & -- \\
\hline $27 \ldots$ & & -- & -- & - & -- & -- & -- & 2 & - & -- \\
\hline CATE & $\begin{array}{l}\text { IEA } \\
\text { TOY } \\
\text { REC } \\
\text { ERA } \\
\text { IUE } \\
\text { IS }\end{array}$ & 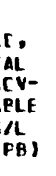 & $\begin{array}{c}\text { IITHIUM } \\
\text { DIS- } \\
\text { SOLVED } \\
\text { (UGA } \\
\text { AS LI) }\end{array}$ & $\begin{array}{l}\text { L YTHILM } \\
\text { TOTAL } \\
\text { RECCV- } \\
\text { ERABLL } \\
\text { (UG } \\
\text { AS LII }\end{array}$ & $\begin{array}{l}\text { MANGA- } \\
\text { NESE, } \\
\text { IOTAL } \\
\text { RECOV- } \\
\text { ERABLE } \\
\text { (UGA } \\
\text { AS MNI }\end{array}$ & $\begin{array}{l}\text { MANGA- } \\
\text { NESEE: } \\
\text { OI S- } \\
\text { SOLVED } \\
\text { WG LL } \\
\text { AS MNI }\end{array}$ & $\begin{array}{c}\text { MEFCURY } \\
\text { DIS- } \\
\text { SCL VED } \\
\text { (US } / \\
\text { AS HGS }\end{array}$ & $\begin{array}{l}\text { MERCURY } \\
\text { TOTAL } \\
\text { RECDV- } \\
\text { ERABLE } \\
\text { (UGA } \\
\text { AS HG) }\end{array}$ & $\begin{array}{l}\text { MULYB- } \\
\text { DENUM, } \\
\text { DIS- } \\
\text { SULVED } \\
\text { (UG/L } \\
\text { AS MO) }\end{array}$ & $\begin{array}{l}\text { MOL YB- } \\
\text { DENUH, } \\
\text { TOTAL } \\
\text { RECOV- } \\
\text { ERABLE } \\
\text { (UG/L } \\
\text { AS MO) }\end{array}$ \\
\hline $\begin{array}{l}\text { APR } \\
12 . \therefore\end{array}$ & & -- & $<10$ & - & -- & $\$ 10$ & - & -- & 《I & -- \\
\hline $15 . .$. & & -- & $<10$ & -- & -- & $<10$ & -- & $<.5$ & $<$ & -- \\
\hline 08... & & ND & -- & $<10$ & $<10$ & -- & -- & $<.5$ & -- & 1 \\
\hline $19 . .$. & & -- & -- & -- & -- & -- & - & -- & -- & -- \\
\hline $01 . .$. & & -- & -- & -- & -- & -- & -- & -- & - & -- \\
\hline $07 . .$. & & -- & - & -- & - & -- & -- & -- & -- & $-\cdot$ \\
\hline $13 . .$. & 974 & -- & -- & -- & -- & 14 & -- & -- & - & $\cdots$ \\
\hline $22 . .$. & & -- & -- & -- & -- & -- & - & -- & - & -- \\
\hline $12 \ldots$ & & $\cdots$ & -- & -- & -- & -- & -- & -- & -- & -- \\
\hline $14 . .$. & & -- & -- & -- & -- & -- & -- & -- & -- & $\cdots$ \\
\hline JUN... & & -- & $\cdots$ & -- & $-\cdot$ & 20 & -- & $<.5$ & -- & -- \\
\hline $20 \ldots$ & & $\cdots$ & -- & -- & -- & -- & -- & -- & -- & $-\cdot$ \\
\hline JuW & & - & $<10$ & -- & -- & 13 & C. 5 & -- & a & -- \\
\hline $24 \ldots$ & & -- & $<10$ & -- & -- & $<10$ & C.5 & -. & I & - \\
\hline 28. & & -- & -- & -- & - & -- & -- & -- & -- & -- \\
\hline 27 & & ND & -- & -- & $<10$ & -- & - & $<.5$ & -- & - \\
\hline
\end{tabular}


Site 6--Continued

\begin{tabular}{|c|c|c|c|c|c|c|c|c|c|}
\hline DATE & $\begin{array}{l}\text { NICKEL, } \\
\text { CIS- } \\
\text { SOLVED } \\
\text { (UCA } \\
\text { ASNI) }\end{array}$ & $\begin{array}{l}\text { MCAEL, } \\
\text { WUTAL } \\
\text { QECEV- } \\
\text { ERAFLE } \\
\text { (UG/L } \\
\text { AS NI) }\end{array}$ & $\begin{array}{l}\text { SELL- } \\
\text { NIUN, } \\
\text { OIS- } \\
\text { SOL VED } \\
\text { (UGA } \\
\text { AS SE I }\end{array}$ & $\begin{array}{l}\text { SELE- } \\
\text { PI'M, } \\
\text { YCTAL } \\
\text { (UE, } \\
\text { ES SE) }\end{array}$ & $\begin{array}{l}\text { SILVEH, } \\
\text { ILIAL } \\
\text { REC:L- } \\
\text { LKAELE } \\
\text { (LEA } \\
\text { AI AC) }\end{array}$ & $\begin{array}{l}\text { SIFCN- } \\
\text { IIUH. } \\
\text { IIE- } \\
\text { SLVEC } \\
\text { IULAL } \\
\text { AS SR) }\end{array}$ & $\begin{array}{l}\text { VARA- } \\
\text { DILM. } \\
\text { JIS- } \\
\text { YJLVEU } \\
\text { (UGA } \\
\text { OS V) }\end{array}$ & $\begin{array}{l}\text { ZINWD } \\
\text { UIS- } \\
\text { SOLVEO } \\
\text { (UG/L } \\
\text { AS ZN) }\end{array}$ & $\begin{array}{l}\text { ZINC, } \\
\text { TOIAL } \\
\text { RECOV- } \\
\text { ERABLE } \\
\text { (UG/L } \\
\text { AS ZN) }\end{array}$ \\
\hline
\end{tabular}

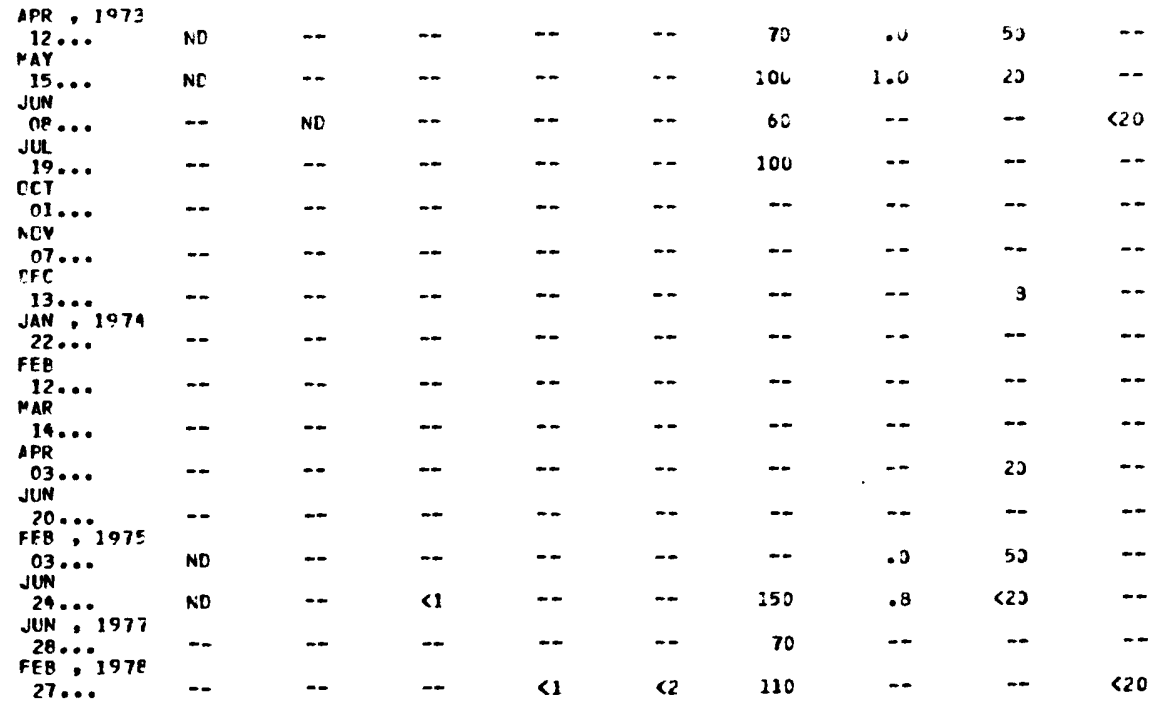

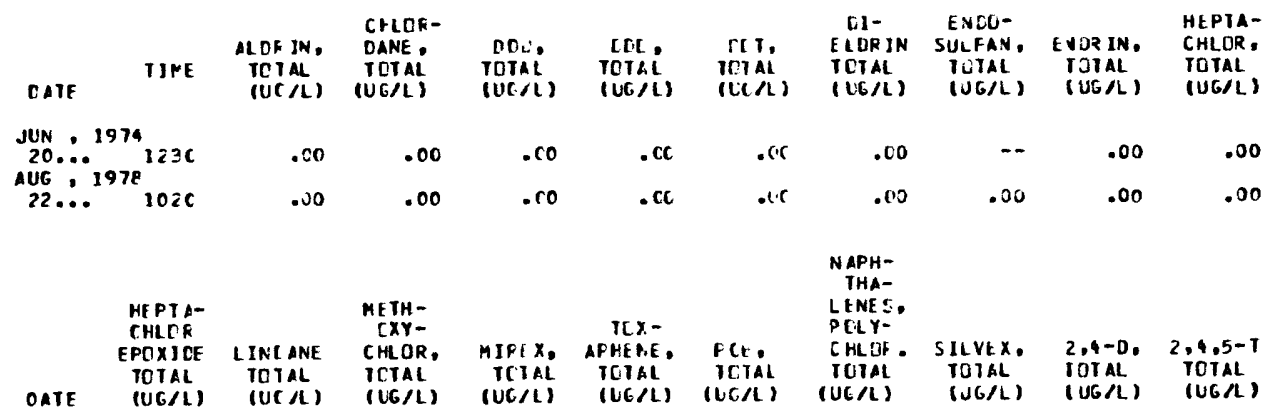

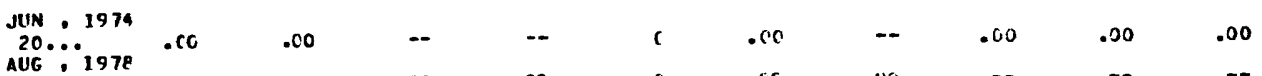

$\begin{array}{ccccccccccc}\text { AUG } & .1978 \\ 22 . .0 & .00 & .00 & .00 & .00 & \text { c } & .00 & .00 & \ldots & \ldots\end{array}$ 
Site 7 near single-gun area

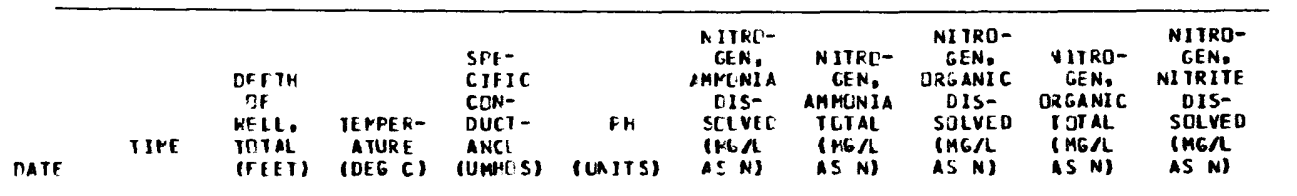

DEC , 1972

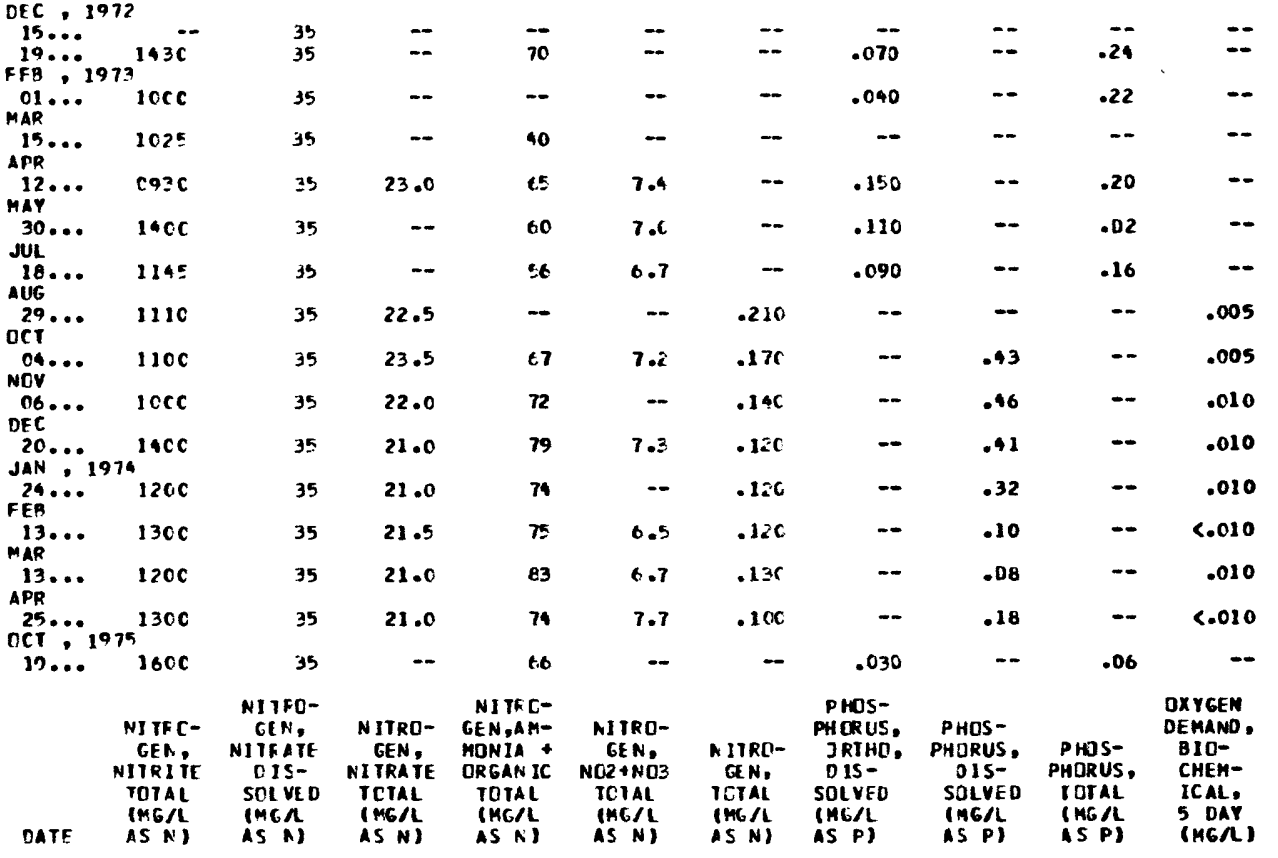

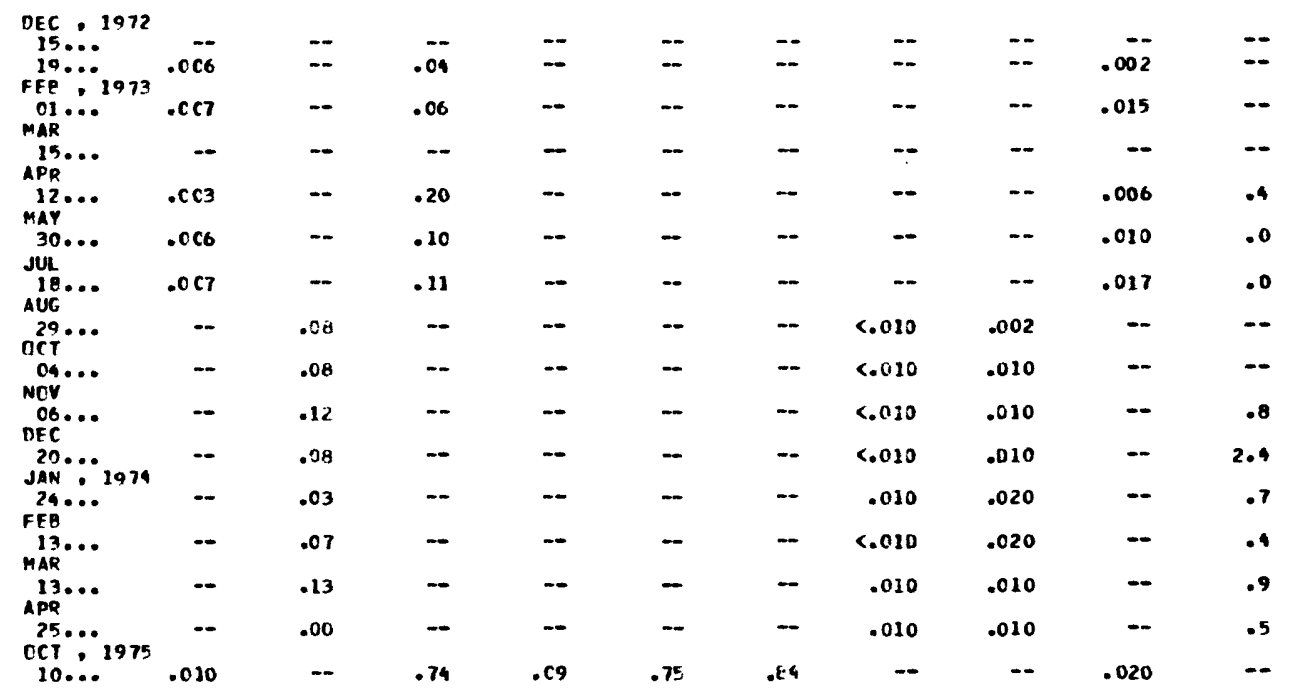


Site 7 near single-gun area--Continued

\begin{tabular}{|c|c|c|c|c|c|c|c|c|c|}
\hline CATE & $\begin{array}{l}\text { CXYCEN } \\
\text { CEMAND, } \\
\text { CHEY- } \\
\text { ICAL } \\
\text { (HICH } \\
\text { LEVEL) } \\
\text { IPEAI) }\end{array}$ & $\begin{array}{l}\text { CAREIIN. } \\
\text { INOE- } \\
\text { GANIC, } \\
\text { TOTAL } \\
\text { IMG/L } \\
\text { AS CI }\end{array}$ & $\begin{array}{l}\text { CAKBLN, } \\
\text { OFGAL IC } \\
\text { ICTAL } \\
\text { IMGA } \\
\text { IS (C) }\end{array}$ & $\begin{array}{l}\text { CAPFCN, } \\
\text { TOTAL } \\
\text { (MG/L } \\
\text { AS C) }\end{array}$ & 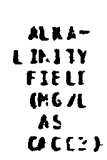 & $\begin{array}{l}\text { MAKC- } \\
\text { NESS } \\
\text { MLNCAR- } \\
\text { BLIRATE } \\
\text { IHC/L } \\
\text { CACE3) }\end{array}$ & $\begin{array}{l}\text { HAKO- } \\
\text { NESS } \\
\text { (MEAL } \\
\text { AS } \\
\text { CACDZS) }\end{array}$ & $\begin{array}{l}\text { CALCIUA } \\
\text { UIS- } \\
\text { SULVES } \\
\text { (MGR } \\
\text { AS CAS }\end{array}$ & $\begin{array}{l}\text { MAGNE- } \\
\text { SIUA, } \\
\text { DIS- } \\
\text { SDLVEO } \\
\text { (AGG } \\
\text { AS MG) }\end{array}$ \\
\hline
\end{tabular}

\begin{tabular}{|c|c|c|c|c|c|c|c|c|c|c|}
\hline $15 \ldots$ & & -- & -- & $\cdots$ & $-\infty$ & - & - & $\cdots$ & - & $=-$ \\
\hline FEB & 973 & $\cdots$ & - & $\cdots$ & -- & $\cdots$ & $\cdots$ & $\cdots$ & - & - \\
\hline$\underset{\text { MAR }}{01}$ & & - & -- & -- & -- & $\cdots$ & -- & -- & - & -- \\
\hline$\underset{A P R}{15} \cdots$ & & -- & $\cdots$ & $\cdots$ & -- & -- & $\cdots$ & $\cdots$ & $\cdots$ & $\cdots$ \\
\hline $\operatorname{maY}_{12} \cdots$ & & 5 & 9.0 & 7.0 & 18 & Zb & 2 & 23 & 9.7 & .5 \\
\hline sut & & 10 & 9.0 & $\ldots 0$ & 11 & $\therefore 3$ & 1 & 24 & 3.3 & .4 \\
\hline$\underset{\text { NUE }}{18} \ldots$ & & -- & 9.0 & .0 & 9.0 & (1) & 0 & 21 & 7.3 & .3 \\
\hline${ }_{\text {nCt }}^{29} \ldots$ & & -- & $\cdots$ & $\cdots$ & $\cdots$ & $\cdots$ & $\cdots$ & $\cdots$ & $\cdots$ & $-\infty$ \\
\hline $\operatorname{Nov}^{04} \cdot \cdots$ & & 20 & 9.5 & 3.5 & 13 & $\approx 0$ & $b$ & 32 & 12 & .4 \\
\hline & & IE & $1 \mathrm{~J}$ & $\therefore 0$ & 12 & $\cdots$ & - & 32 & 12 & .5 \\
\hline SON & 974 & 8 & B.C & 4.0 & 12 & $\equiv 1$ & $\cdots$ & $\cdots$ & $\cdots$ & $\cdots$ \\
\hline FEB & & 4 & 9.0 & 3.0 & 12 & $\cdots$ & - & 26 & 10 & .3 \\
\hline${ }_{\text {MAR }}^{13} \cdots$ & & $c$ & 10 & $\therefore 0$ & 12 & 20 & 4 & 34 & 13 & .4 \\
\hline$\underset{A P R}{13} \cdots$ & & 8 & 11 & 1.0 & 12 & 30 & 7 & 37 & 14 & .4 \\
\hline СCT & 1975 & 10 & 8.0 & 1.5 & 9.5 & 29 & 9 & 37 & 14 & .5 \\
\hline $10 \ldots$ & & - & $\cdots$ & $\cdots$ & $\cdots$ & ¿1 & - & $\cdots$ & -- & - \\
\hline OATE & & & $\begin{array}{l}\text { POIAS- } \\
\text { SIUH, } \\
\text { DIS- } \\
\text { SOLVEO } \\
\text { (MG/L } \\
\text { AS K) }\end{array}$ & $\begin{array}{l}\text { BICAK- } \\
\text { EONATE } \\
\text { FET-FLO } \\
\text { (MG/L } \\
\text { AS } \\
\text { HCO3) }\end{array}$ & $\begin{array}{r}\text { CAK- } \\
\text { RONATE } \\
\text { ET }+L U \\
\text { TMC/L } \\
\text { AS COS) }\end{array}$ & $\begin{array}{l}\text { CrLC- } \\
\text { RIDF, } \\
\text { OIS- } \\
\text { SLL VEL } \\
\text { (KG AL } \\
\text { AS CL) }\end{array}$ & $\begin{array}{l}\text { FED- } \\
\text { FIEE } \\
\text { OIS- } \\
\text { SILVED } \\
\text { IMCA } \\
\text { SEI }\end{array}$ & $\begin{array}{l}\text { SULFAIE } \\
\text { DIS- } \\
\text { SOLVEO } \\
\text { (MG/L } \\
\text { AE SOG) }\end{array}$ & $\begin{array}{l}\text { SILICA. } \\
\text { OIS- } \\
\text { SOLVEO } \\
\text { IMG/L } \\
\text { AS } \\
\text { SIOLI }\end{array}$ & $\begin{array}{l}\text { SOLICS, } \\
\text { RESIDUE } \\
\text { AT } 180 \\
\text { OEG. C } \\
\text { OIS- } \\
\text { SOLVED } \\
\text { (HG/L) }\end{array}$ \\
\hline
\end{tabular}

\begin{tabular}{|c|c|c|c|c|c|c|c|c|c|}
\hline $\begin{array}{l}\text { CFC }, 1972 \\
15 \ldots .\end{array}$ & 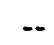 & $=$ & $\cdots$ & $=$ & 3.6 & $\rightarrow$ & $\cdots$ & - & - \\
\hline FEE & -- & -- & -- & $\cdots$ & 3.2 & .2 & 6.7 & .8 & - \\
\hline O1... & - & - & $\cdots$ & $\ldots$ & $\cdots$ & $\cdots$ & - & 1.5 & -- \\
\hline$\underset{\triangle P R}{15} \cdots$ & $=$ & -- & -- & c & 2.0 & $-\infty$ & -- & $=$ & - \\
\hline$\underset{\operatorname{maY}}{12} \cdots$ & 1.5 & .3 & 30 & 0 & 2.9 & .1 & 1.6 & 3.1 & 42 \\
\hline jel & 1.5 & .2 & 28 & 0 & 2.5 & .1 & .4 & 3.3 & 38 \\
\hline$\underset{A \cup G}{18}$ & 1.4 & .2 & 26 & 0 & 1.0 & .1 & .8 & 3.2 & 25 \\
\hline סст & $\cdots$ & -- & - & -- & $\cdots$ & - & - & 4.2 & - \\
\hline $\operatorname{Nov}_{\text {Nov... }}$ & 1.5 & .2 & 31 & 0 & 2.5 & $\cdots$ & $=$ & $\cdots$ & $\cdots$ \\
\hline TEC & 1.3 & .5 & $\cdots$ & 0 & 2.5 & $\cdots$ & $\cdots$ & - & - \\
\hline JAN 20.1974 & $\cdots$ & -- & 38 & 0 & 2.0 & $\cdots$ & - & $-\infty$ & $\cdots$ \\
\hline$\underset{\text { FEE }}{24 \cdots \cdots}$ & 1.0 & .1 & $\cdots$ & 0 & 2.4 & $\cdots$ & - & -- & $\cdots$ \\
\hline$\underset{N R}{13}$ & 1.4 & .2 & 36 & $c$ & 1.7 & $\cdots$ & $\cdots$ & $\cdots$ & $\cdots$ \\
\hline 13. & 1.6 & -1 & 37 & 0 & 1.9 & 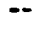 & - & -- & $\because$ \\
\hline CCT $25 \cdot 1975$ & 1.2 & .6 & 35 & 0 & 2.9 & -- & -- & - & $\cdots$ \\
\hline 10 & 1.07 & $\cdots$ & 26 & 0 & 1.2 & - & $\cdots$ & $\cdots$ & - \\
\hline
\end{tabular}


Site 7 near single-gur area--Continued

\begin{tabular}{|c|c|c|c|c|c|c|c|c|c|c|}
\hline OATE & TINE & $\begin{array}{l}\text { ALCY- } \\
\text { INLM, } \\
\text { DIS- } \\
\text { SOLVED } \\
\text { (UCAL } \\
\text { AS AL) }\end{array}$ & $\begin{array}{l}\text { ARSENIC } \\
\text { EIS- } \\
\text { SCLVED } \\
\text { (LGIL } \\
\text { AS ASI }\end{array}$ & $\begin{array}{l}\text { ARSENIC } \\
\text { TOTAL } \\
\text { (UG/L } \\
\text { AS S S }\end{array}$ & $\begin{array}{l}\text { ECKGN, } \\
\text { DIS- } \\
\text { SOLVFE } \\
\text { IUT /L } \\
\text { AS EI }\end{array}$ & $\begin{array}{l}\text { PCKCN, } \\
\text { TCIAL } \\
\text { RCCOV- } \\
\text { LEABLE } \\
\text { (LCA } \\
\text { AS B) }\end{array}$ & 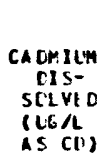 & $\begin{array}{l}\text { CADMIUM } \\
\text { IJTAL } \\
\text { RECOV- } \\
\text { ERABLE } \\
\text { (UG/L } \\
\text { AS CO) }\end{array}$ & $\begin{array}{l}\text { CHRD- } \\
\text { YIUH, } \\
\text { JIS- } \\
\text { SULVED } \\
\text { (UGR } \\
\text { AS CR) }\end{array}$ & $\begin{array}{c}\text { CHRD- } \\
\text { MIUM, } \\
\text { HEXA- } \\
\text { VALENT, } \\
\text { DIS. } \\
\text { IUGAL } \\
\text { AS CR I }\end{array}$ \\
\hline $\begin{array}{l}\text { DEC } \\
\text { MAR : }\end{array}$ & $12143 \mathrm{C}$ & $\cdots$ & (1 & $\cdots$ & $\cdots$ & $\cdots$ & -- & -- & $\cdots$ & $\cdots$ \\
\hline$\underset{A P R}{C 5} \cdots$ & $140 C$ & 20 & -- & - & - & -- & $<2$ & - & ND & 0 \\
\hline $12 \ldots$ & $093 \mathrm{C}$ & 160 & $<1$ & - & - & -- & ND & -- & No & 0 \\
\hline $\begin{array}{c}30 \\
\text { JuL }\end{array}$ & $14 \mathrm{CC}$ & $\cdots$ & -- & 3 & - & -- & $\cdots$ & No & $\cdots$ & - \\
\hline & 1145 & - & -- & -- & -- & 13 & - & $=-$ & $\cdots$ & $\cdots$ \\
\hline NeV & $11 \mathrm{Cc}$ & $\cdots$ & $\cdots$ & $\cdots$ & - & 6 & $\cdots$ & - & $\cdots$ & $\cdots$ \\
\hline JAN... & $4^{10 C C}$ & - & $\cdots$ & -- & 40 & - & -- & -- & - & $\cdots$ \\
\hline FEP 24 & 1200 & $\cdots$ & - & - & $<20$ & -- & $\cdots$ & -- & -- & - \\
\hline $\operatorname{MAR}_{13}^{13} \cdots$ & $13 \mathrm{CC}$ & $=$ & $\cdots$ & $-\infty$ & $<2 C$ & $=-$ & - & $\cdots$ & $=$ & -- \\
\hline${ }_{A P R}^{13} \ldots$ & $120 C$ & -- & -- & - & $\angle 2 C$ & - & -- & $=$ & $-\infty$ & - \\
\hline $25 \ldots$ & $130 \mathrm{C}$ & -- & 1 & - & $<20$ & -- & - & ND & - & 0 \\
\hline DATE & $\begin{array}{l}\text { CHPE- } \\
\text { YIUY, } \\
\text { TOIAL } \\
\text { PECCV- } \\
\text { FRAELE } \\
\text { TUG } \\
\text { AS CRI }\end{array}$ & $\begin{array}{l}\text { COBALI, } \\
\text { OIS- } \\
\text { SOLVED } \\
\text { (UCAL } \\
\text { AS CO) }\end{array}$ & $\begin{array}{l}\text { COBALT, } \\
\text { TOTAL } \\
\text { RECOV- } \\
\text { EFABLE } \\
\text { (UG/L } \\
\text { AS CO) }\end{array}$ & $\begin{array}{l}\text { CDPFEP, } \\
\text { DIS- } \\
\text { SOLYED } \\
\text { (UC/L } \\
\text { AS CL) }\end{array}$ & $\begin{array}{l}\text { COFFFR. } \\
\text { JOIAL } \\
\text { RECOV- } \\
\text { ERAFLE } \\
\text { (UEAL } \\
\text { AS CU) }\end{array}$ & $\begin{array}{l}\text { IFGN } \\
\text { DIS- } \\
\text { SOLVES } \\
\text { IUG L } \\
\text { IS FES }\end{array}$ & $\begin{array}{l}\text { LEAD, } \\
\text { DIS- } \\
\text { SUVED } \\
\text { (UG/L } \\
\text { AS PE) }\end{array}$ & $\begin{array}{l}\text { LEAD, } \\
\text { IOTAL } \\
\text { RECOV- } \\
\text { ERABLE } \\
\text { (UG/L } \\
\text { AS PB) }\end{array}$ & $\begin{array}{l}\text { LI IHIUN } \\
\text { DIS- } \\
\text { SJLVED } \\
\text { (UG/L } \\
\text { AS II) }\end{array}$ & $\begin{array}{l}\text { LITHRU } \\
\text { TOTAL } \\
\text { RECOY- } \\
\text { ERABLE } \\
\text { (UGAL } \\
\text { AS LI) }\end{array}$ \\
\hline
\end{tabular}

DEC, 1972

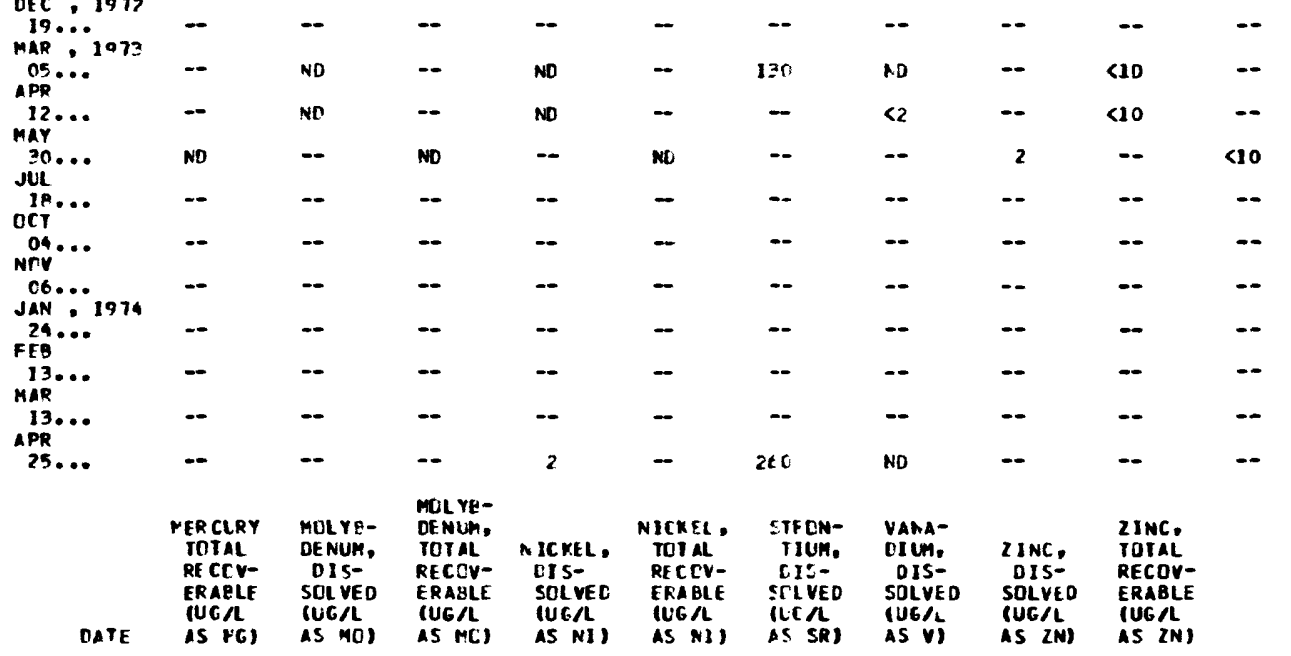

\begin{tabular}{|c|c|c|c|c|c|c|c|c|c|}
\hline $19 \ldots 1912$ & -- & -- & $-\infty$ & $\cdots$ & $=$ & - & $\cdots$ & - & -- \\
\hline$\underset{\text { OPR }}{P A R}: 1973$ & $\cdots$ & $<1$ & - & NO & -- & -- & .0 & 40 & $=$ \\
\hline $\operatorname{maY}_{\operatorname{mar}}$ & -- & $<1$ & - & NO & -- & 20 & 2.0 & 63 & - \\
\hline$\underset{\text { JuL }}{30 \ldots . . .}$ & 1.5 & -- & $<1$ & -- & NO & 60 & - & - & BD \\
\hline $\mathrm{CCT}^{18}$ & -- & -- & - & -- & -- & $<10$ & -- & - & $-\infty$ \\
\hline Nov & -- & -- & - & $\cdots$ & -- & -- & - & - & $\cdots$ \\
\hline $\begin{array}{l}\text { O6... } \\
\text { JAN } 1974\end{array}$ & $=$ & -- & - & -- & $=-$ & $-\infty$ & - & - & - \\
\hline FEB & -- & - & -- & $-\infty$ & $-\infty$ & -- & -- & - & -- \\
\hline $\operatorname{MaR} \cdots$ & $-\infty$ & - & -- & $\cdots$ & -- & 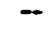 & -- & - & $\cdots$ \\
\hline APR & -- & -- & 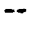 & $\cdots$ & -- & -- & -- & - & 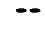 \\
\hline $25 \ldots$ & Q.5 & -- & - & -- & -- & -- & - & 43 & \\
\hline
\end{tabular}




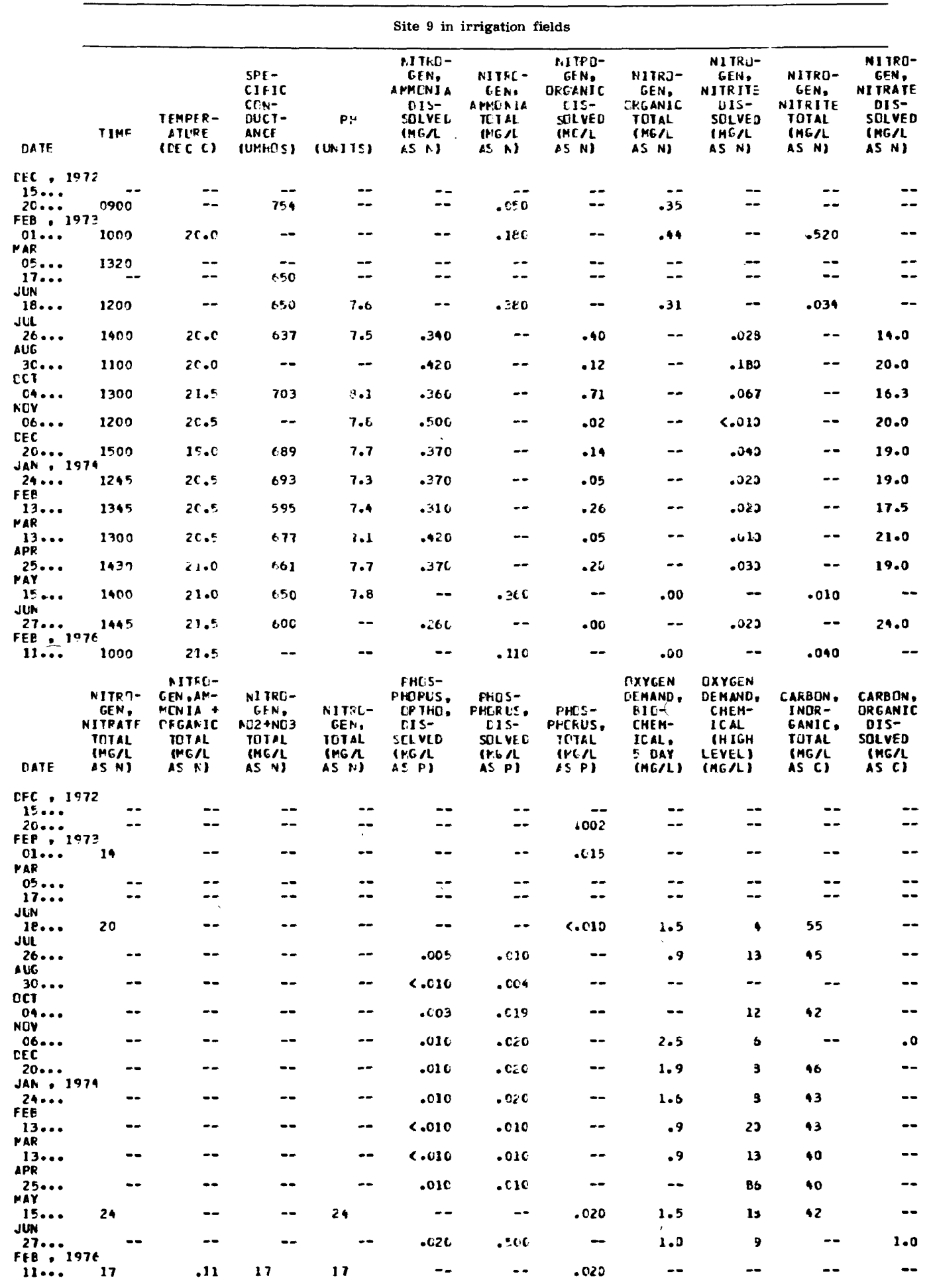


Table 6.--Physical and chemical characteristics of well and water at sites 1 through 70--Continued

Site 9 in irrigation fields--Conitnued

\begin{tabular}{|c|c|c|c|c|c|c|c|c|c|c|}
\hline $\begin{array}{l}\text { CAREON, } \\
\text { CRGANIC } \\
\text { TOT AL } \\
\text { (MGAL } \\
\text { AS C) }\end{array}$ & $\begin{array}{l}\text { CARBCN, } \\
\text { TCTAL } \\
\text { (HGAL } \\
\text { AS C) }\end{array}$ & $\begin{array}{l}\text { ALKA- } \\
\text { LINITY } \\
\text { FIELD } \\
\text { (MG/L } \\
\text { AS } \\
\text { CACIB) }\end{array}$ & $\begin{array}{l}\text { SCOILM, } \\
\text { DIS- } \\
\text { SCLVEC } \\
\text { (MGAL } \\
\text { AS WA) }\end{array}$ & $\begin{array}{l}\text { IOIAS- } \\
\text { SIUH, } \\
\text { DIS- } \\
\text { SOIVEL } \\
\text { (ME/L } \\
\text { AS HI) }\end{array}$ & $\begin{array}{l}\text { PICAK- } \\
\text { ECNAIL } \\
\text { FIT-FLL } \\
\text { IYEA } \\
\text { AS } \\
\text { MCOZI }\end{array}$ & 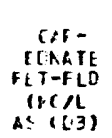 & $\begin{array}{l}\text { CHLJ- } \\
\text { FICE. } \\
\text { TIS- } \\
\text { SJIVED } \\
\text { (HC/L } \\
\text { OS CL) }\end{array}$ & $\begin{array}{l}\text { FLUI- } \\
\text { RIOE, } \\
\text { UIS- } \\
\text { SULVED } \\
\text { (HG/ } \\
\text { ASFI }\end{array}$ & $\begin{array}{l}\text { SULFAIL } \\
\text { DIS- } \\
\text { SOLVED } \\
\text { (HG/L } \\
\text { AS SO4) }\end{array}$ & $\begin{array}{l}\text { SILICA, } \\
\text { DLS- } \\
\text { SULVED } \\
\text { IMG/ } \\
\text { AS } \\
\text { SIO2) }\end{array}$ \\
\hline
\end{tabular}

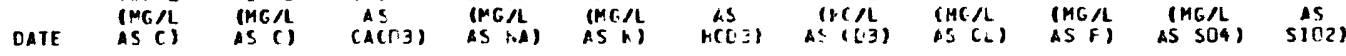

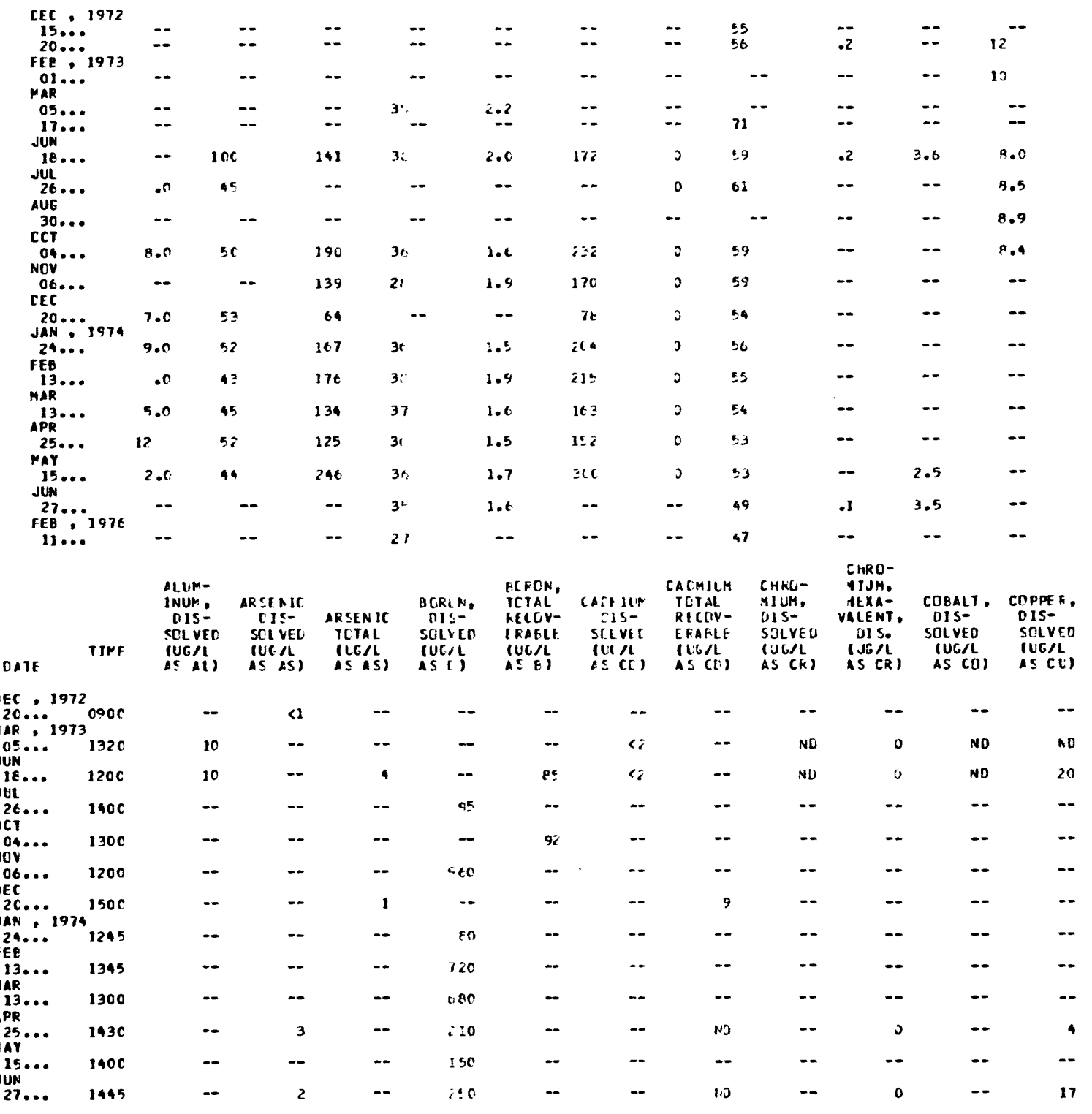


Site 9 in irrigation fields--Conitnued

\begin{tabular}{|c|c|c|c|c|c|c|c|c|c|c|c|c|c|}
\hline DATE & $\begin{array}{l}\text { COF } \\
\text { TC } \\
\text { RE } \\
\text { EF } \\
\text { IC } \\
\text { AS }\end{array}$ & $\begin{array}{l}\text { ER, } \\
A L \\
O V- \\
\text { PLE } \\
\text { CL } \\
\text { CU) }\end{array}$ & $\begin{array}{l}\text { JRCN, } \\
\text { DIS:- } \\
\text { SOLVED } \\
\text { (UEIL } \\
\text { AS FE) }\end{array}$ & $\begin{array}{l}\text { LEAL, } \\
\text { DIS- } \\
\text { SOLVED } \\
\text { (UCA } \\
\text { AS FB) }\end{array}$ & $\begin{array}{c}\text { LIJHILA } \\
\text { CIS- } \\
\text { SOLVED } \\
\text { (UG/L } \\
\text { AS (I) }\end{array}$ & $\begin{array}{l}\text { MANGA- } \\
\text { NE SE, } \\
\text { TOTAL } \\
\text { RECIV- } \\
\text { ERABLE } \\
\text { (UG/L } \\
\text { AS MA) }\end{array}$ & $\begin{array}{l}\text { MANGA- } \\
\text { NESE. } \\
\text { DIS- } \\
\text { SOLVED } \\
\text { (UG/L } \\
\text { AS MN) }\end{array}$ & $\begin{array}{l}\text { MEFCURY } \\
\text { TOTAL } \\
\text { RECOV- } \\
\text { ERABLE } \\
\text { IUG/L } \\
\text { AS HE) }\end{array}$ & $\begin{array}{l}\text { HOL YB- } \\
\text { DENUH, } \\
\text { OIS- } \\
\text { SOL VED } \\
\text { (UG/L } \\
\text { AS MO) }\end{array}$ & $\begin{array}{l}\text { NICKEL. } \\
\text { DIS- } \\
\text { SOLVED } \\
\text { SUGIL: } \\
\text { AS NI) }\end{array}$ & $\begin{array}{l}\text { STRON- } \\
\text { TIUN. } \\
\text { DIS- } \\
\text { SDLVED } \\
\text { IUG } \\
\text { SS SRI }\end{array}$ & $\begin{array}{l}\text { VANA- } \\
\text { DIUN, } \\
\text { DIS- } \\
\text { SOLVED } \\
\text { IUGA } \\
\text { AS VI }\end{array}$ & $\begin{array}{l}\text { ZINC, } \\
\text { DIS- } \\
\text { SOLVEO } \\
\text { (UG/L } \\
\text { AS ZN) }\end{array}$ \\
\hline $\begin{array}{l}\text { DEC : } \\
2 C . \\
\text { MAR }\end{array}$ & $\begin{array}{l}1972 \\
1973\end{array}$ & $\cdots$ & -- & -- & $\cdots$ & -- & - & -- & -- & -- & -- & -- & - \\
\hline JUN... & & $\cdots$ & 20 & No & $<10$ & -- & 40 & - & 4 & ND & -- & .0 & 20 \\
\hline Je... & & -- & $<$ io & 6 & $<10$ & -- & 30 & $<.5$ & $<1$ & 2 & 200 & $\cdots$ & 130 \\
\hline 2E.... & & $=-$ & -- & -- & - & - & - & - & -- & -- & $\cdots$ & $-\infty$ & - \\
\hline nov... & & -- & -- & - & -- & - & - & -- & $-\infty$ & -- & -- & $\cdots$ & - \\
\hline DEC & & - & - & $\cdots$ & $\cdots$ & $\cdots$ & - & -- & $\cdots$ & $\cdots$ & $\cdots$ & $\cdots$ & - \\
\hline JAN $20 .$. & 1974 & 9 & $\cdots$ & $\cdots$ & -- & 100 & - & - & - & $\cdots$ & $\cdots$ & $\cdots$ & $\cdots$ \\
\hline FEB $^{24} \cdots$ & & $\cdots$ & - & $\cdots$ & $-\infty$ & - & - & - & $\cdots$ & -- & $\cdots$ & -- & $\cdots$ \\
\hline${ }_{\text {MAR }}^{13} \cdots$ & & $\cdots$ & $-\infty$ & - & $-\infty$ & - & - & - & $-\infty$ & - & - & $\cdots$ & $\cdots$ \\
\hline${ }_{A P R}^{13} \cdots$ & & $\cdots$ & -- & -- & -- & -- & - & -- & $\cdots$ & -- & -- & -- & - \\
\hline MAY & & -- & $<10$ & 2 & - & - & 50 & $<.5$ & - & -- & - & $\cdots$ & 40 \\
\hline JUN & & -- & - & -- & -- & - & - & - & - & $\cdots$ & $\cdots$ & $\cdots$ & $\cdots$ \\
\hline $27 \ldots$ & & $\cdots$ & $<10$ & NO & - & -- & 33 & $<.5$ & $\cdots$ & -- & $\cdots$ & $\cdots$ & 190 \\
\hline
\end{tabular}

Site 10

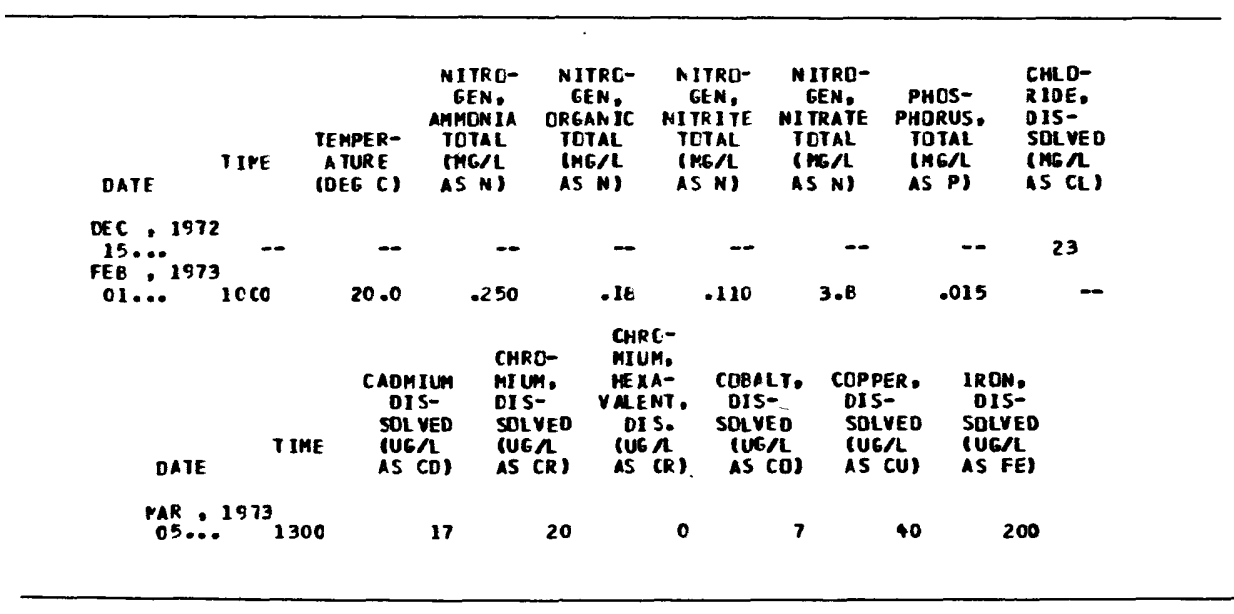

Site 11 near holding ponds

\begin{tabular}{|c|c|c|c|c|c|c|c|c|c|c|c|}
\hline DATE & IIME & $\begin{array}{l}\text { TEMPER- } \\
\text { ATURE } \\
\text { (CEG C) }\end{array}$ & $\begin{array}{l}\text { SPE- } \\
\text { CIFIC } \\
\text { CON- } \\
\text { DUCT- } \\
\text { ANCE } \\
\text { (UMHOS) }\end{array}$ & $\begin{array}{c}\text { PH } \\
\text { (UNITS) }\end{array}$ & $\begin{array}{l}\text { NITRO- } \\
\text { GEN, } \\
\text { A HMONIA } \\
\text { DIS- } \\
\text { SOLVEC } \\
\text { (MG G } \\
\text { AS N) }\end{array}$ & $\begin{array}{l}\text { NITRO- } \\
\text { GEN, } \\
\text { AwDNIA } \\
\text { TOTAL } \\
\text { (MG A } \\
\text { AS N) }\end{array}$ & $\begin{array}{c}\text { NITRO- } \\
\text { GEN, } \\
\text { ORGANIC } \\
\text { DIS- } \\
\text { SOLVED } \\
\text { (MG/L } \\
\text { AS N) }\end{array}$ & $\begin{array}{l}\text { MITRO- } \\
\text { GEN, } \\
\text { ORGANIC } \\
\text { TOTAL } \\
\text { (NG/L } \\
\text { AS N) }\end{array}$ & $\begin{array}{l}\text { MITRO- } \\
\text { GEN, } \\
\text { NITRITE } \\
\text { DIS- } \\
\text { SOLVED } \\
\text { (MG/L } \\
\text { AS N) }\end{array}$ & $\begin{array}{l}\text { MITRD- } \\
\text { GEN口 } \\
\text { MITRITE } \\
\text { TOTAL } \\
\text { (NG } \\
\text { AS M) }\end{array}$ & $\begin{array}{c}\text { MITRO- } \\
\text { GEN, } \\
\text { NIIRATE } \\
\text { DIS- } \\
\text { SOLVED } \\
\text { (MGR } \\
\text { AS NI }\end{array}$ \\
\hline $\begin{array}{l}\text { OEC : } \\
\text { I5: } \\
\text { FEB }\end{array}$ & - & -- & -- & -- & -- & -- & - & -- & $\cdots$ & $\cdots$ & $\cdots$ \\
\hline MAR & 1000 & -- & -- & -- & -- & .550 & -- & 1.4 & - & .026 & - \\
\hline $05 \ldots$ & 1210 & $=$ & 290 & $\overline{--}$ & 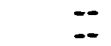 & -- & $=$ & $\because$ & $=$ & 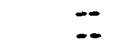 & $\because$ \\
\hline APR & -- & -- & 370 & $=$ & -- & - & - & -- & - & $\cdots$ & - \\
\hline $\begin{array}{l}12 \ldots \\
\text { JUL }\end{array}$ & 1630 & -- & 245 & - & $\therefore$ & .090 & - & .35 & -- & .110 & - \\
\hline$\underset{A \cup 6}{25} \cdots$ & 1500 & 23.0 & 275 & 7.8 & .530 & - & .72 & -- & .190 & - & 1.00 \\
\hline $\mathrm{CCT}^{29}$ & 1400 & 22.5 & -- & $\cdots$ & .270 & -- & .32 & - & .004 & - & 1.10 \\
\hline$\underset{\text { NOV }}{\text { OA... }}$ & 1200 & 22.0 & 265 & 8.1 & .210 & -- & .76 & -- & .009 & -- & .91 \\
\hline 06. & 1015 & 21.5 & 235 & 3.2 & .200 & - & .40 & -- & .013 & -- & .91 \\
\hline
\end{tabular}


Site 11 near holding ponds--Continued

\begin{tabular}{|c|c|c|c|c|c|c|c|c|c|}
\hline $\begin{array}{l}\text { N1 IRC- } \\
\text { GEN, } \\
\text { NITR IE } \\
\text { IOTAL } \\
\text { (HGAL } \\
\text { AS NI) }\end{array}$ & $\begin{array}{l}\text { PHCS- } \\
\text { PHOFUS, } \\
\text { DQ THC, } \\
\text { DIS- } \\
\text { SOL VED } \\
\text { IHG AL } \\
\text { AS P) }\end{array}$ & $\begin{array}{l}\text { PHOS- } \\
\text { PHORUS, } \\
\text { CIS- } \\
\text { SCLVED } \\
\text { (HG/L } \\
\text { AS PI }\end{array}$ & $\begin{array}{l}\text { PHCS- } \\
\text { PHORUS, } \\
\text { TOTAL } \\
\text { (MG/L } \\
\text { AS P) }\end{array}$ & $\begin{array}{l}\text { DXYCEA } \\
\text { DEMAND, } \\
\text { BIL- } \\
\text { CHEH- } \\
\text { ICAL } \\
\text { S DAY } \\
\text { (MGLI) }\end{array}$ & $\begin{array}{l}\text { CXYCEN } \\
\text { CENANE, } \\
\text { CHEM- } \\
\text { ICAL } \\
\text { (HIGH } \\
\text { LEVEL) } \\
\text { (MG/L) }\end{array}$ & $\begin{array}{l}\text { CAKEON, } \\
\text { I NOR- } \\
\text { G ANIC, } \\
\text { TOTAL } \\
\text { (HG/L } \\
\text { AS C) }\end{array}$ & $\begin{array}{l}\text { CAREON, } \\
\text { ORGANIC } \\
\text { TOTAL } \\
\text { (MG/L } \\
\text { IS C) }\end{array}$ & $\begin{array}{l}\text { CARBDN, } \\
\text { TOTAL } \\
\text { (MG/L } \\
\text { IS C) }\end{array}$ & $\begin{array}{l}\text { ALKA- } \\
\text { LINITY } \\
\text { FIELD } \\
\text { IAG/ } \\
\text { AS } \\
\text { CACO3) }\end{array}$ \\
\hline
\end{tabular}

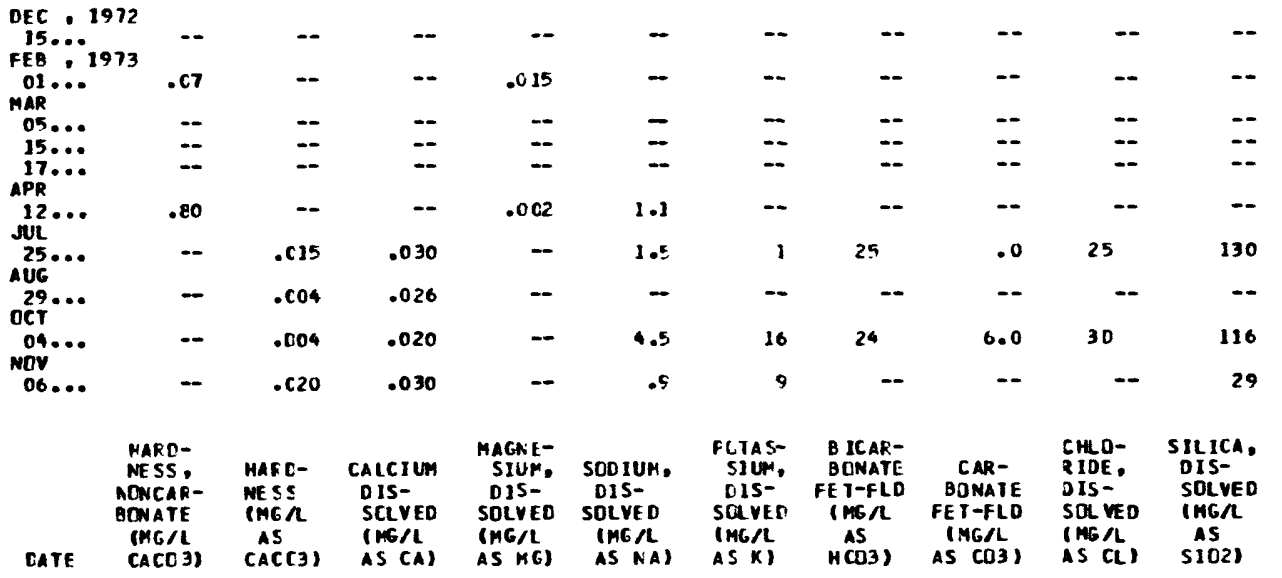

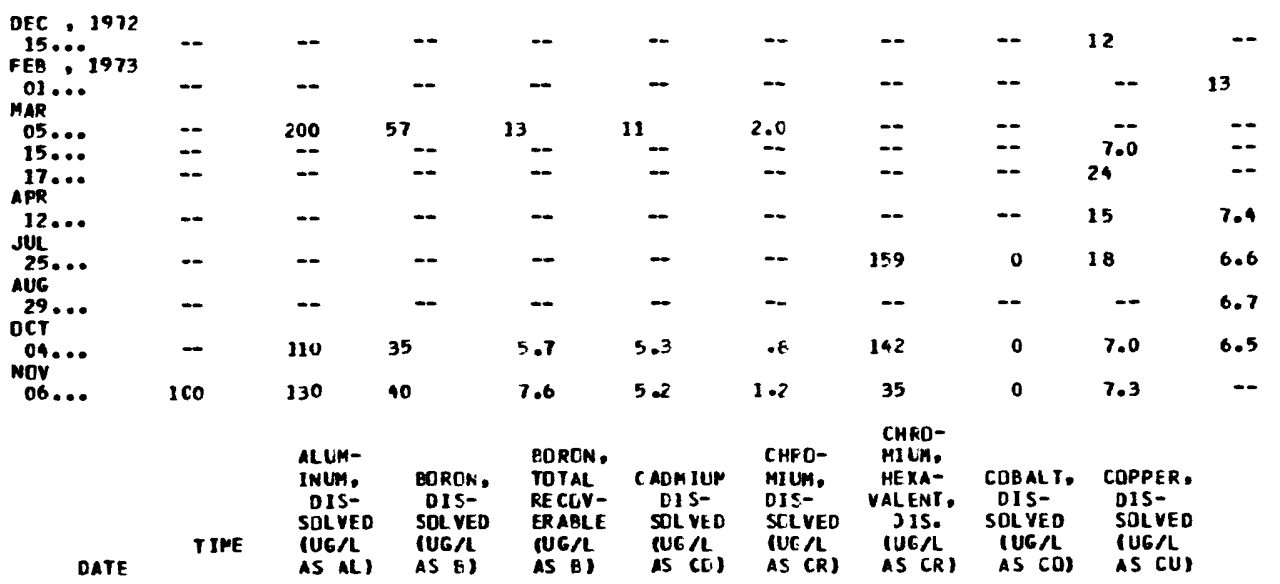

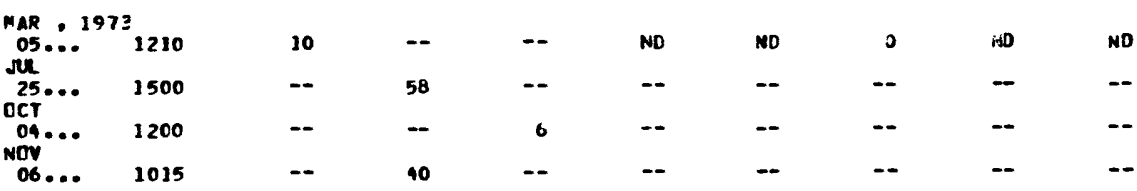

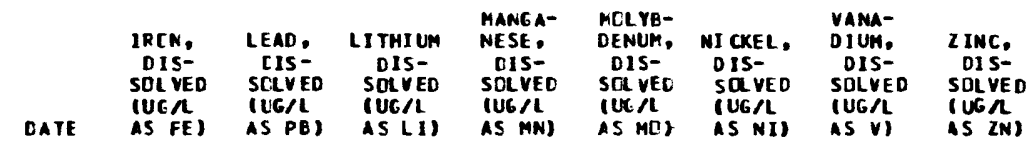

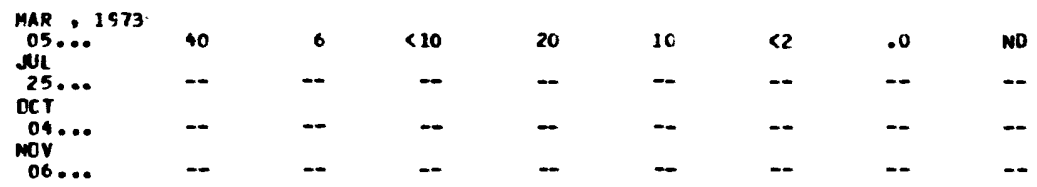


Site 12 in 4-big-gun area

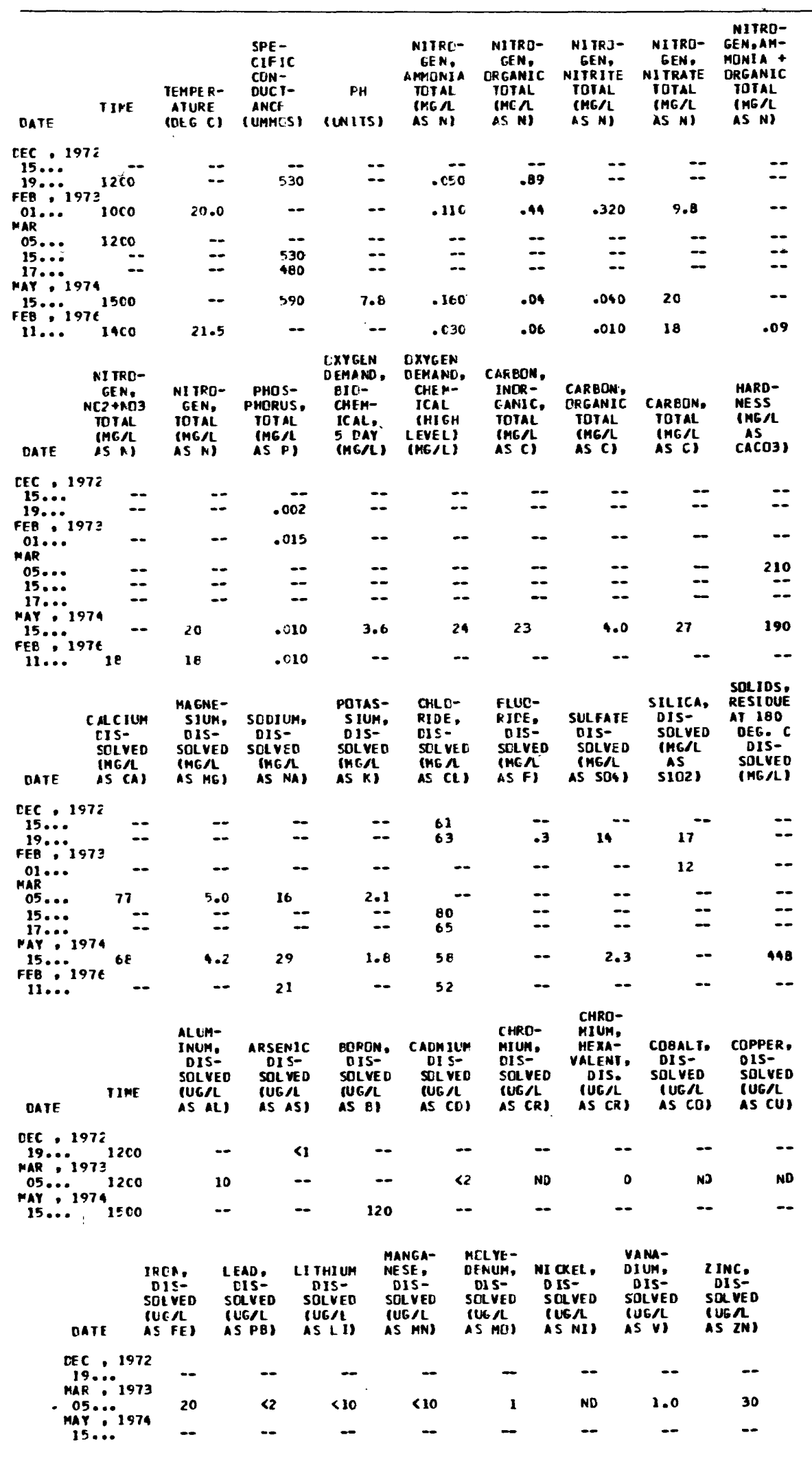




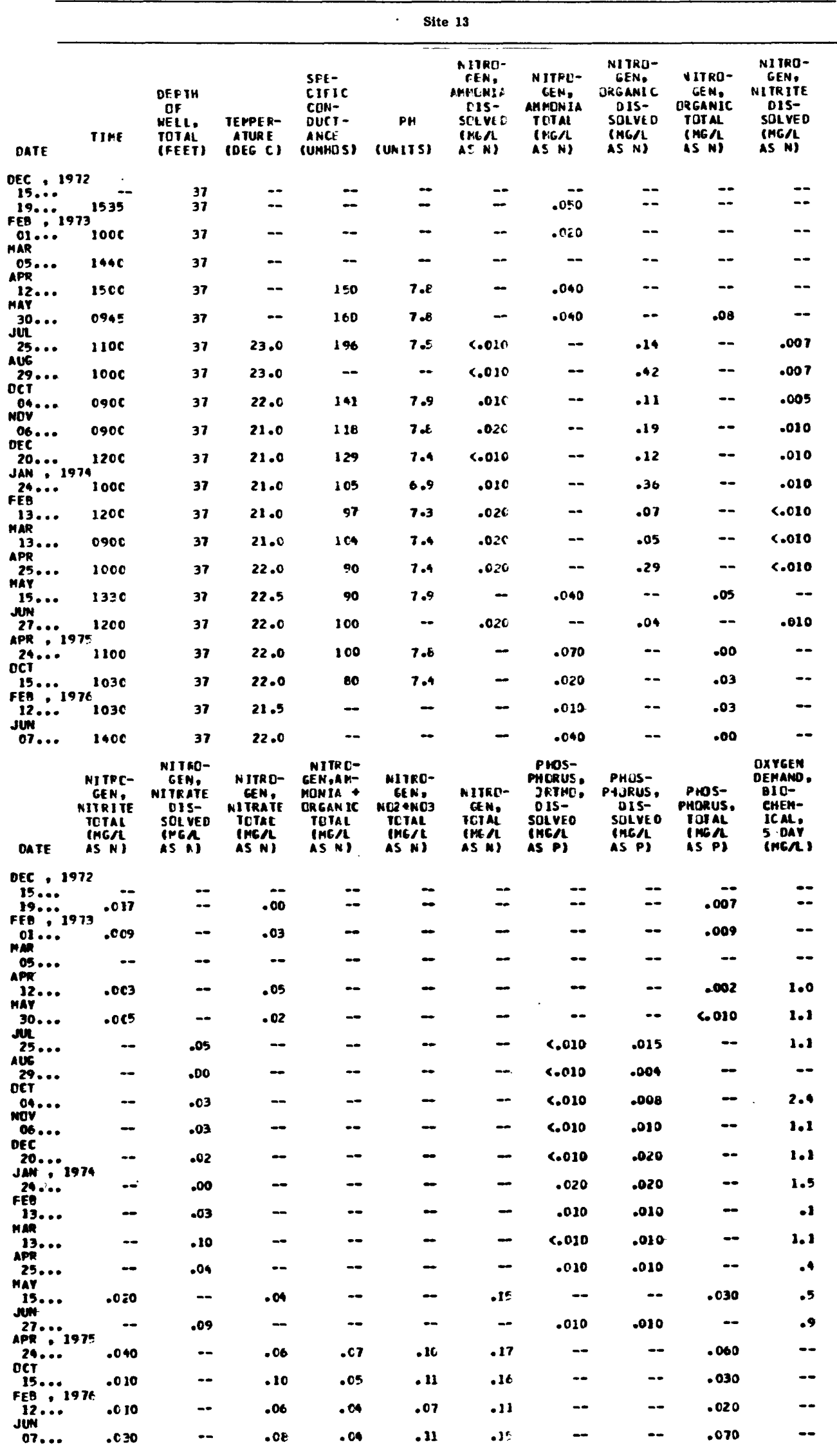




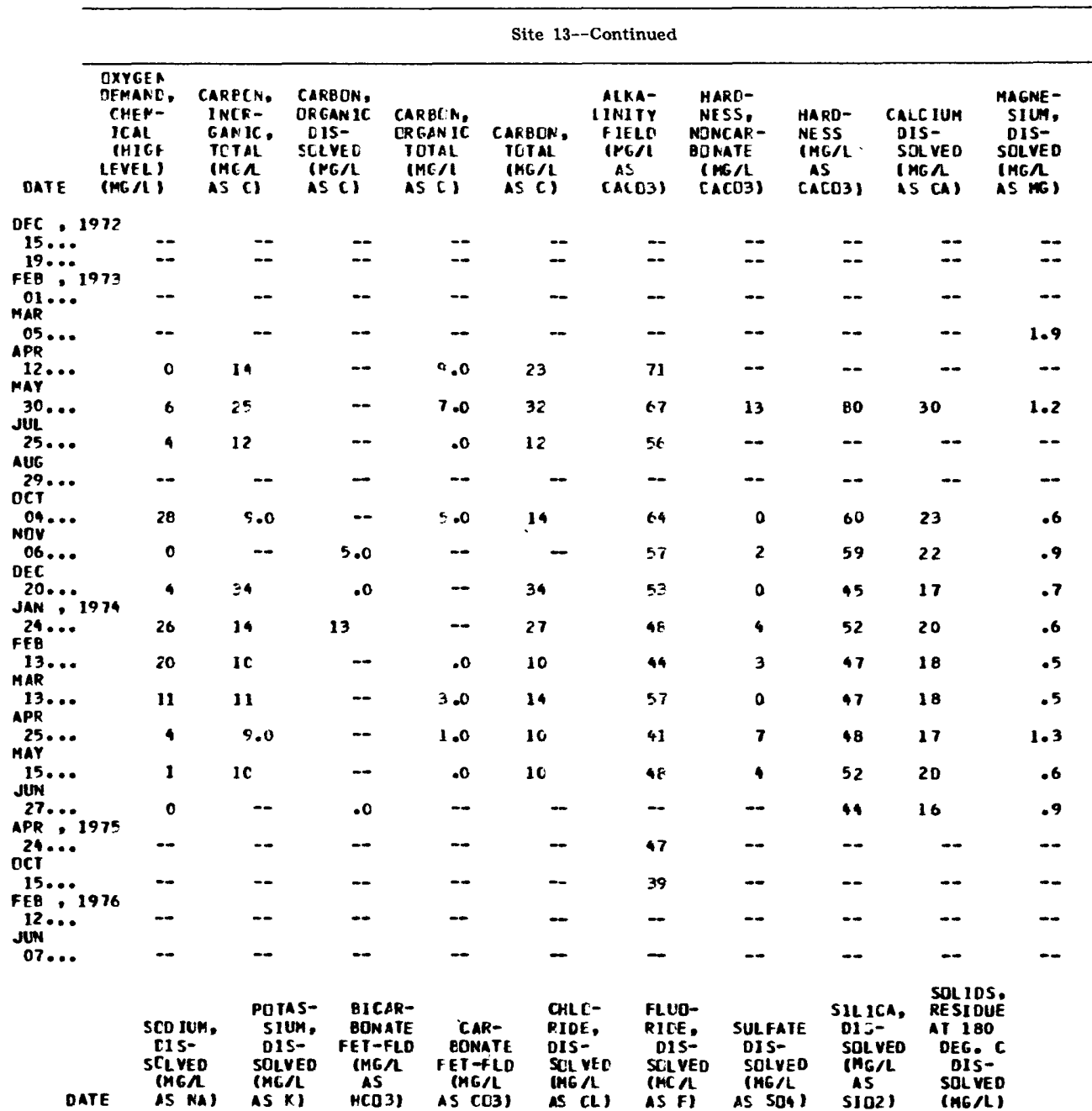

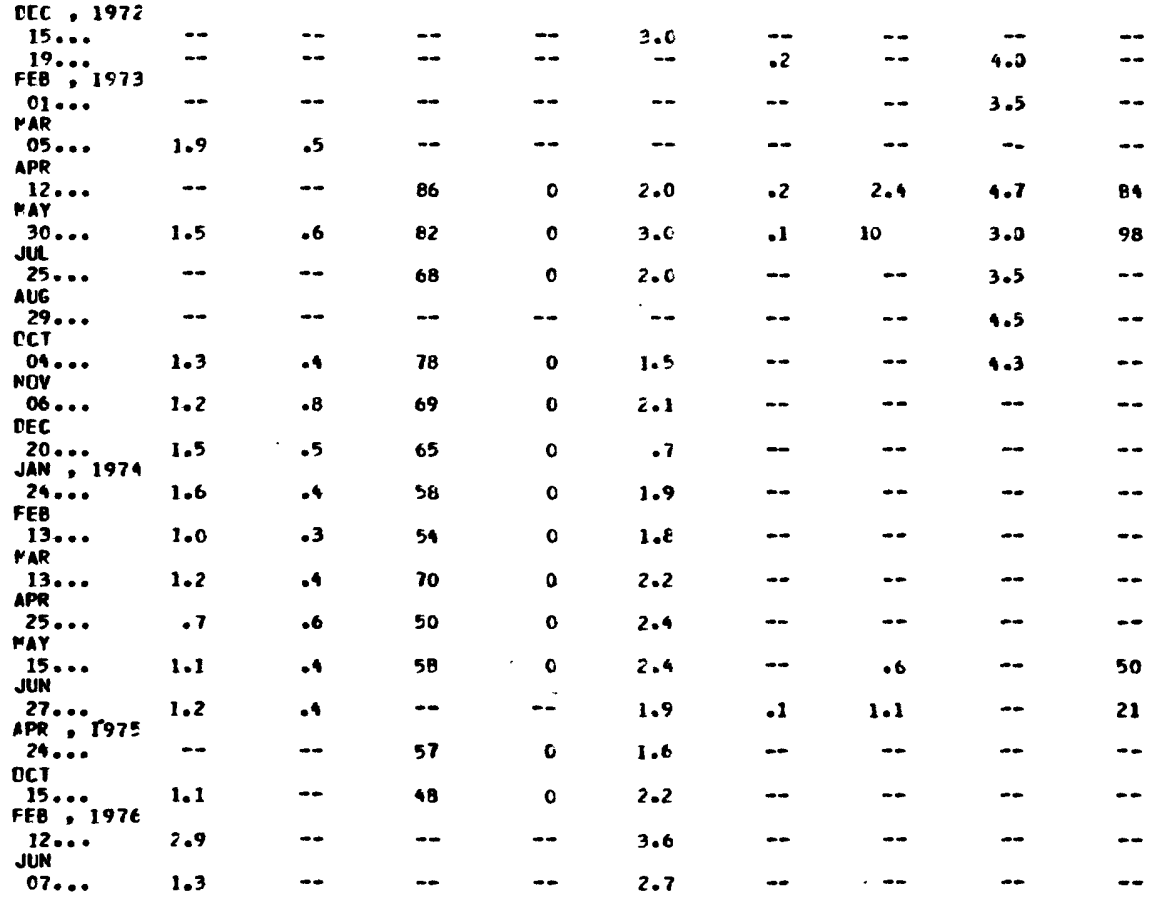


Site 13--Continued

\begin{tabular}{|c|c|c|c|c|c|c|c|c|c|}
\hline DATE & TINE & $\begin{array}{l}\text { OH PTH } \\
\text { OF } \\
\text { WELL. } \\
\text { IDTAL } \\
\text { (FEET) }\end{array}$ & $\begin{array}{l}\text { TEMPEF- } \\
\text { ATURE } \\
\text { (DE C C) }\end{array}$ & $\begin{array}{l}\text { SPI- } \\
\text { CIFIC } \\
\text { CON- } \\
\text { WUCT- } \\
\text { ANCE } \\
\text { IUMHLSI }\end{array}$ & $\begin{array}{c}P H \\
\text { IUNIISI }\end{array}$ & $\begin{array}{l}\text { NITKO- } \\
\text { CEN, } \\
\text { AMM(NIA } \\
\text { TCTAL } \\
\text { IMC } \\
\text { AS NS }\end{array}$ & $\begin{array}{l}\text { NIIRJ- } \\
\text { GEN. } \\
\text { ERGANIC } \\
\text { TOTAL } \\
\text { (NG/L } \\
\text { AS N) }\end{array}$ & $\begin{array}{l}\text { NITKG- } \\
\text { GLN, } \\
\text { NITKIIE } \\
\text { IUTAL } \\
\text { ING/L } \\
\text { AS NN) }\end{array}$ & $\begin{array}{l}\text { NITRO- } \\
\text { GEN, } \\
\text { NITRAIE } \\
\text { IOTAL } \\
\text { (MG/L } \\
\text { AS N) }\end{array}$ \\
\hline
\end{tabular}

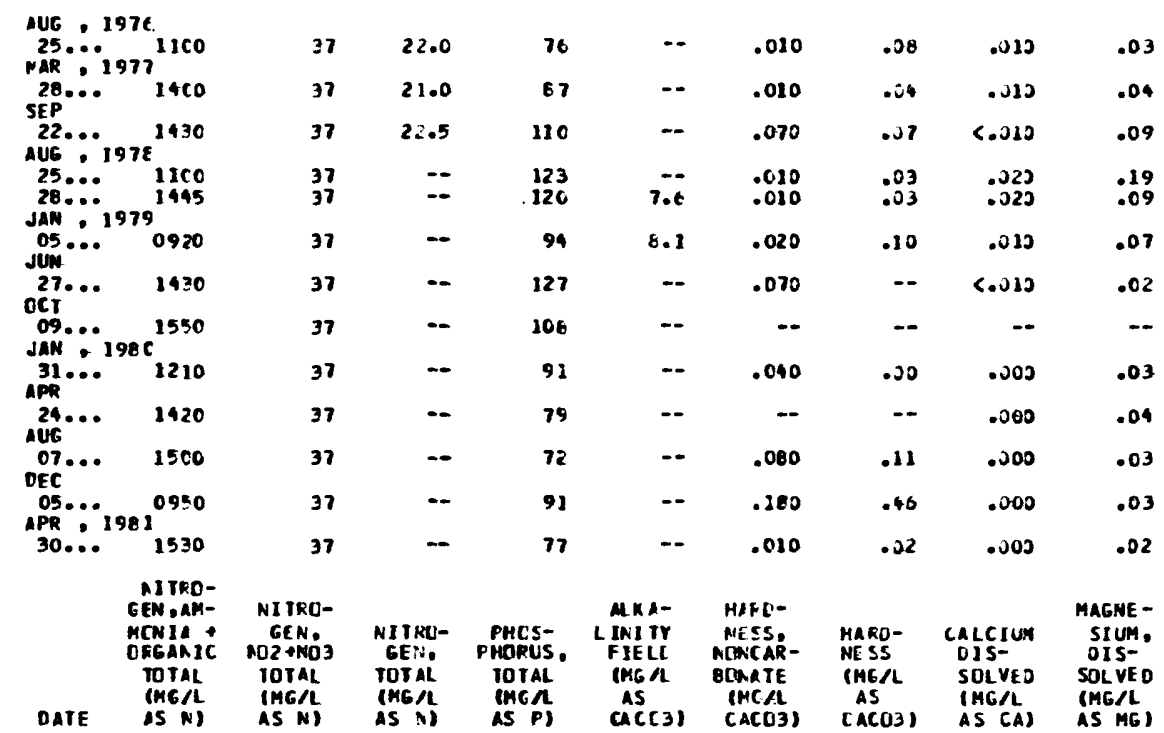

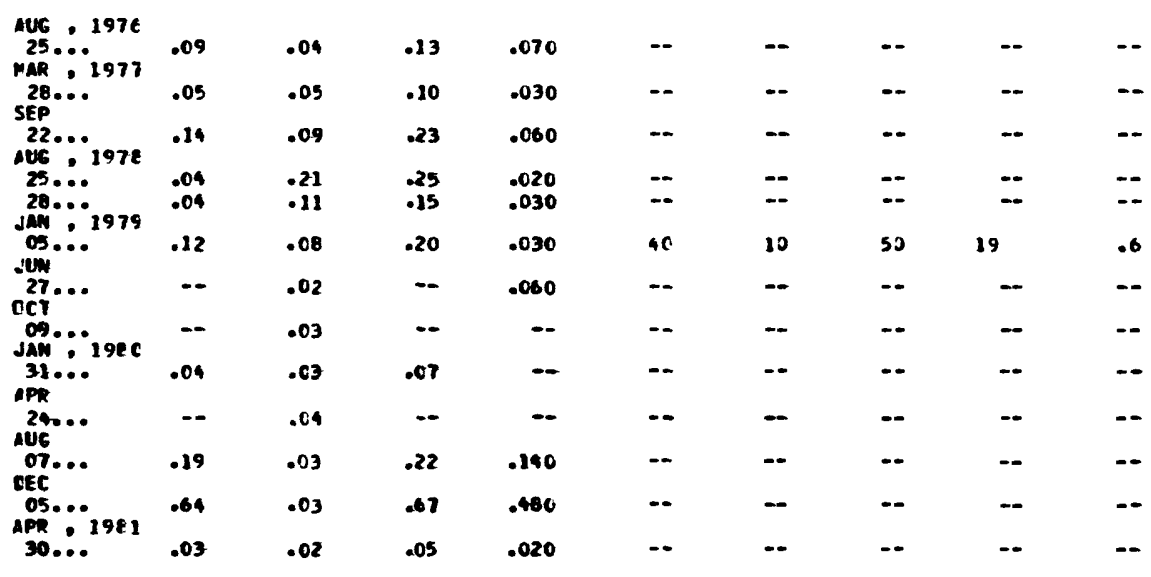

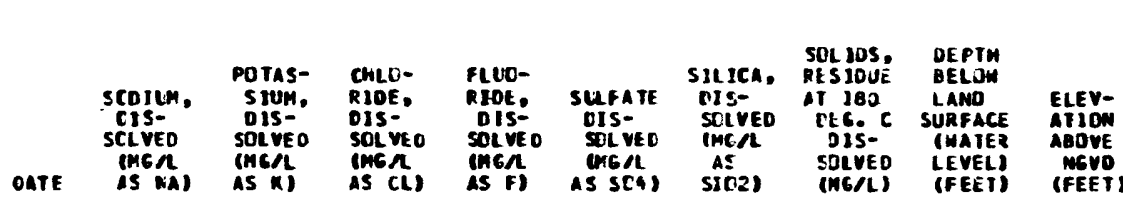

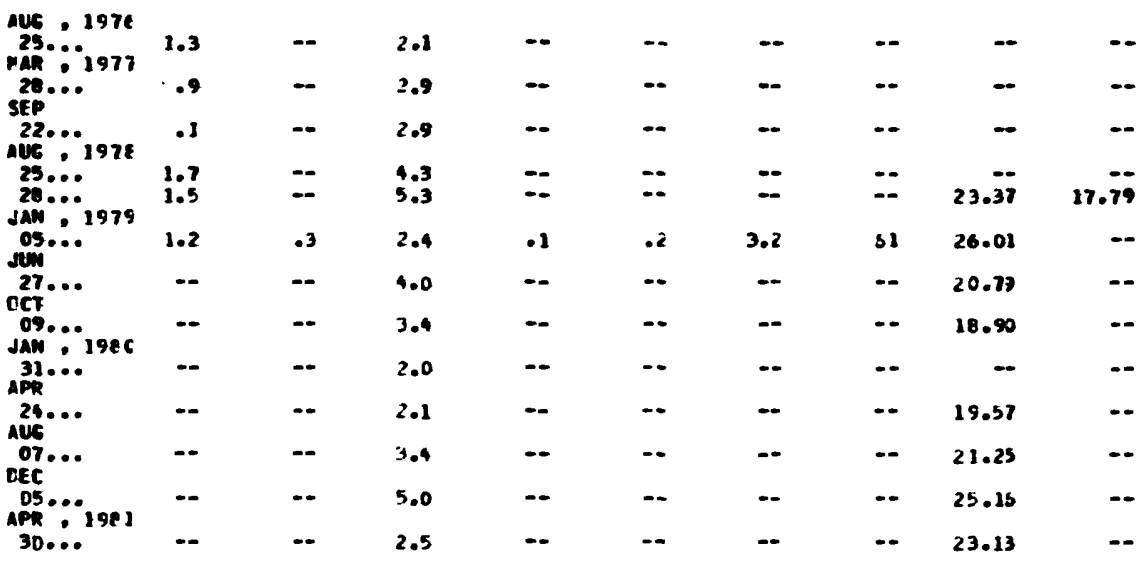


Site 13--Continued

\begin{tabular}{|c|c|c|c|c|c|c|c|c|c|c|}
\hline DATE & TIME & $\begin{array}{l}\text { ALCF- } \\
\text { INCF, } \\
\text { DIS- } \\
\text { SOLVED } \\
\text { (UCA } \\
\text { AS AL) }\end{array}$ & $\begin{array}{c}\text { ARSENIC } \\
\text { CIS- } \\
\text { SCLVEO } \\
\text { (UG/L } \\
\text { AS ASI }\end{array}$ & $\begin{array}{l}\text { AR SEN IC } \\
\text { TOTAL } \\
\text { (UG/L } \\
\text { AS ASI }\end{array}$ & $\begin{array}{l}\text { ECRON, } \\
\text { CIS- } \\
\text { SCLVEO } \\
\text { (UG/ } \\
\text { AS E) }\end{array}$ & $\begin{array}{l}\text { ECKON, } \\
\text { TCTAL } \\
\text { RECOV- } \\
\text { ERABLE } \\
\text { IUE IL } \\
\text { AS EI }\end{array}$ & $\begin{array}{c}\text { CACMIUN } \\
\text { DIS- } \\
\text { SOLVED } \\
\text { (U6/L } \\
\text { AS CO) }\end{array}$ & $\begin{array}{l}\text { CAOMIUM } \\
\text { TOTAL } \\
\text { RECOY- } \\
\text { ERABLE } \\
\text { IUG/L } \\
\text { AS COI }\end{array}$ & $\begin{array}{l}\text { EHRO- } \\
\text { IIUH, } \\
\text { JIS- } \\
\text { SQLVE O } \\
\text { (UGNL } \\
\text { IS CR) }\end{array}$ & $\begin{array}{l}\text { CHRO- } \\
\text { HIUH, } \\
\text { HEXA- } \\
\text { VALENT, } \\
\text { DIS. } \\
\text { (UGA } \\
\text { AS CR) }\end{array}$ \\
\hline DEC , & & & & & & & & & & \\
\hline MAR :. & $73^{153 E}$ & $=$ & - & $<1$ & - & -- & -- & -- & -- & - \\
\hline MAY 05 & $14 \bullet C$ & 10 & -- & - & - & -- & $<2$ & - & NO & 0 \\
\hline $30 \ldots$ & 0945 & - & - & 1 & - & - & -- & $<2$ & -- & - \\
\hline 25... & 1100 & -- & -- & -- & $<20$ & -- & - & -- & -- & -- \\
\hline Non... & $090 \mathrm{C}$ & -- & - & - & $\cdots$ & 6 & - & $=$ & -- & - \\
\hline OEC & $09 C \mathrm{C}$ & -- & - & - & $5 C$ & - & -- & -- & -- & -- \\
\hline JAN $20 . \cdots$ & $74^{120 C}$ & - & $<1$ & -- & - & - & -- & 9 & -- & - \\
\hline FEB & $10 \mathrm{cC}$ & -- & - & $\cdots$ & $<20$ & -- & -- & -- & - & $=$ \\
\hline $\operatorname{MAR}_{\text {AR }}^{13} \ldots$ & 1200 & $\cdots$ & -- & $\cdots$ & $<2 c$ & -- & - & $\cdots$ & -- & -- \\
\hline$\underset{A P R}{13 \ldots}$ & $090 \mathrm{C}$ & -- & -- & -- & $<20$ & - & -- & -- & -- & -- \\
\hline MAY 25 & $100 C$ & -- & $<1$ & - & $<20$ & -- & $\ldots$ & $<2$ & - & 0 \\
\hline JUN & $133 c$ & - & -- & - & 5 & - & -- & -- & -- & -- \\
\hline $27 \ldots$ & $\begin{array}{l}\text { 12C: } \\
\text { CHRC- }\end{array}$ & -- & $<1$ & - & $\boldsymbol{\varepsilon}$ & - & -- & 3 & -- & 0 \\
\hline DATE & $\begin{array}{l}\text { MIUM, } \\
\text { TOTAL } \\
\text { RE COV- } \\
\text { FRAB LE } \\
\text { (UG } \\
\text { AS (R) }\end{array}$ & $\begin{array}{l}\text { COE ALT, } \\
\text { OIS- } \\
\text { SOLVED } \\
\text { CUE L } \\
\text { AS COI }\end{array}$ & $\begin{array}{l}\text { COEAL 1, } \\
\text { TOTAL } \\
\text { RECQV- } \\
\text { ERABLE } \\
\text { (UGAL } \\
\text { AS CD) }\end{array}$ & $\begin{array}{l}\text { CQPPER, } \\
\text { DIS- } \\
\text { SOLVED } \\
\text { (UC,L } \\
\text { AS CU) }\end{array}$ & $\begin{array}{l}\text { COPPER, } \\
\text { TOIAL } \\
\text { RECQV- } \\
\text { ERABLF } \\
\text { (UG/L } \\
\text { AS CUY }\end{array}$ & $\begin{array}{l}\text { IRLIN, } \\
\text { CIS- } \\
\text { SCLYEO } \\
\text { (UEN } \\
\text { AS FE) }\end{array}$ & $\begin{array}{l}\text { LEAD, } \\
\text { OIS- } \\
\text { SUVED } \\
\text { (UG } \\
\text { AS PB) }\end{array}$ & $\begin{array}{l}\text { LEAD, } \\
\text { TOYAL } \\
\text { RECDV- } \\
\text { ERABLE } \\
\text { (UG/L } \\
\text { AS PB) }\end{array}$ & $\begin{array}{c}\text { LITHIUM } \\
\text { OI S- } \\
\text { SOL VEO } \\
\text { (UG } \\
\text { AS LI) }\end{array}$ & $\begin{array}{l}\text { LI THIUM } \\
\text { TOTAL } \\
\text { RECDY- } \\
\text { ERABLE } \\
\text { (UGA } \\
\text { AS LI) }\end{array}$ \\
\hline OEC : & $\cdots$ & -- & $\cdots$ & $=$ & - & - & -- & -- & -- & -- \\
\hline $\begin{array}{c}\text { MAR } \\
05 . .\end{array}$ & - & NO & -- & MO & - & 20 & $<2$ & -- & $<10$ & - \\
\hline $\begin{array}{l}30 \ldots \\
\text { JUL }\end{array}$ & 20 & - & No & - & $3 c$ & -- & -- & 38 & -- & <10 \\
\hline${ }_{0 C T}^{25} \cdots$ & -- & -- & - & -- & - & - & -- & -- & - & -- \\
\hline NO4... & - & -- & -- & - & -- & - & -- & -- & -- & - \\
\hline OEC & -- & -- & - & -- & - & - & - & -- & -- & -- \\
\hline JAN 20 & -- & -- & -- & 2 & - & $<1 C$ & 13 & -- & -- & - \\
\hline FEB $24 \cdots$ & -- & -- & -- & - & -- & - & -- & -- & -- & - \\
\hline MAR & -- & $=-$ & - & -- & - & - & - & -- & - & - \\
\hline${ }_{A P R}^{13} \cdots$ & -- & -- & -- & - & - & -- & -- & -- & -- & - \\
\hline $\operatorname{mar} \cdots$ & $\cdots$ & -- & - & 2 & $\cdots$ & $<10$ & ND & -- & -- & - \\
\hline $\begin{array}{l}15 \ldots . . . \\
\text { JUN }\end{array}$ & - & -- & -- & - & - & -- & -- & -- & -- & - \\
\hline $27 \ldots$ & $\cdots$ & - & -- & 7 & - & $<10$ & 5 & -- & - & - \\
\hline DATE & $\begin{array}{l}\text { MANGA- } \\
\text { NESE, } \\
\text { TOTAL } \\
\text { RECEY- } \\
\text { FRABLE } \\
\text { (UGAL } \\
\text { AS MA) }\end{array}$ & $\begin{array}{l}\text { MARGA- } \\
\text { NESE. } \\
\text { DIS- } \\
\text { SOL VEO } \\
\text { (UGAL } \\
\text { AS MN) }\end{array}$ & $\begin{array}{l}\text { ME RCURY } \\
\text { TOTAL } \\
\text { RECUV- } \\
\text { ERABLE } \\
\text { (UG/L } \\
\text { AS HG) }\end{array}$ & $\begin{array}{l}\text { MCIYE- } \\
\text { DENUM, } \\
\text { DIS- } \\
\text { SOLVEO } \\
\text { (UG/L } \\
\text { AS MO) }\end{array}$ & $\begin{array}{l}\text { MOL YR- } \\
\text { DE NUM, } \\
\text { TOTAL } \\
\text { RECOV- } \\
\text { ERABLE } \\
\text { (UG /L } \\
\text { AS MO) }\end{array}$ & $\begin{array}{l}\text { NICKEL, } \\
\text { DIS- } \\
\text { SQ VED } \\
\text { (U6/L } \\
\text { AS N1) }\end{array}$ & $\begin{array}{l}\text { MI CKEL, } \\
\text { TOTAL } \\
\text { RECDV- } \\
\text { ERABLE } \\
\text { (UG/L } \\
\text { AS NI) }\end{array}$ & $\begin{array}{l}\text { SIRON- } \\
\text { IIUH, } \\
\text { DIS- } \\
\text { SOLYED } \\
\text { (UG/L } \\
\text { AS SR) }\end{array}$ & $\begin{array}{l}\text { YANA- } \\
\text { DIUM, } \\
\text { OIS- } \\
\text { SOLVED } \\
\text { (UGA } \\
\text { S VI) }\end{array}$ & $\begin{array}{l}\text { ZINC, } \\
\text { DIS- } \\
\text { SOLVED } \\
\text { (USA } \\
\text { AS IN) }\end{array}$ \\
\hline
\end{tabular}

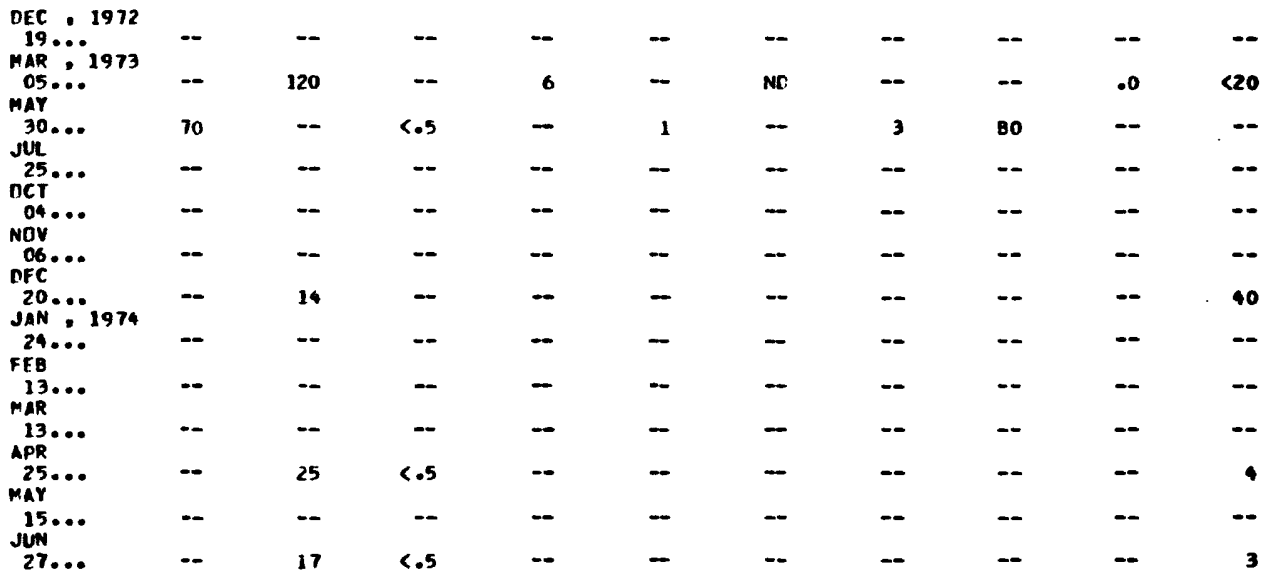


Site 14

\begin{tabular}{|c|c|c|c|c|c|c|c|c|c|c|}
\hline DATE & TIME & $\begin{array}{l}\text { DEFTH } \\
\text { OF } \\
\text { WELL, } \\
\text { TOIAL } \\
\text { (FEET) }\end{array}$ & $\begin{array}{l}\text { TEPPER- } \\
\text { A TURE } \\
\text { (OEG C) }\end{array}$ & $\begin{array}{l}\text { SPI- } \\
\text { CIf IC } \\
\text { CON- } \\
\text { DUCT- } \\
\text { ANCE } \\
\text { IUMHOSI }\end{array}$ & $\begin{array}{c}\text { Fh } \\
\text { (UAITS) }\end{array}$ & $\begin{array}{l}\text { MIIKC- } \\
\text { GEN: } \\
\text { APMINIA } \\
\text { EIS- } \\
\text { SCLVES } \\
\text { (ME/L } \\
\text { ASN) }\end{array}$ & $\begin{array}{l}\text { N JTKO- } \\
\text { CEN, } \\
\text { AMMONIA } \\
\text { TLTAL } \\
\text { (NG/L } \\
\text { AS NI }\end{array}$ & $\begin{array}{l}\text { NITRO- } \\
\text { GEN, } \\
\text { JRGANIC } \\
\text { UIS- } \\
\text { SQLVED } \\
\text { (MGN } \\
\text { AS N) }\end{array}$ & $\begin{array}{l}\text { VITRO- } \\
\text { GEN, } \\
\text { ORGANIC } \\
\text { TOIAL } \\
\text { IAGAL } \\
\text { IS N }\end{array}$ & $\begin{array}{l}\text { NITRO- } \\
\text { GEN, } \\
\text { NI IRITE } \\
\text { DIS- } \\
\text { SDLVED } \\
\text { (MG/L } \\
\text { AS NI }\end{array}$ \\
\hline $\begin{array}{l}\text { OEC } . . \\
15 . .\end{array}$ & 972 & 55 & $\cdots$ & -- & $\cdots$ & -- & $\cdots$ & $\cdots$ & $\ldots$ & $\cdots$ \\
\hline FEB & $973^{102 C}$ & 55 & -- & - & - & -- & .040 & $\cdots$ & -- & - \\
\hline MAR $\cdots$ & $10 \mathrm{cc}$ & 55 & 20.0 & - & $\cdots$ & - & .030 & -- & $\cdots$ & - \\
\hline $05 \ldots$ & $143 C$ & 55 & - & -- & - & - & - & -- & -- & -- \\
\hline$\underset{A F R}{17 \ldots}$ & -- & 55 & - & 110 & - & - & - & -- & - & -- \\
\hline $12 \ldots$ & $145 \mathrm{C}$ & 55 & -- & 108 & 7.4 & -- & .040 & -- & -- & -- \\
\hline JUL. & 1015 & 55 & - & - & 7.7 & - & .040 & $\cdots$ & .06 & -- \\
\hline $25 \ldots$ & $140 \mathrm{C}$ & 55 & 22.0 & -- & -- & .cor & + & .37 & -- & .006 \\
\hline סCT & $103 C$ & 55 & 21.5 & -- & -- & $\cos c$ & - & .54 & -- & .006 \\
\hline $04 \cdots$ & $100 \mathrm{C}$ & 55 & 23.0 & - & $7 \mathcal{E}$ & .036 & - & .63 & - & .009 \\
\hline DEC & $093 \mathrm{C}$ & 53 & -- & P7 & 8.0 & $.02 \mathrm{C}$ & - & .D1 & - & .010 \\
\hline JAN". & $974^{1300}$ & 55 & 20.0 & -- & 7.9 & S.01C & $\cdots$ & .25 & - & .010 \\
\hline $24 \ldots$ & $110 \mathrm{C}$ & 55 & 20.5 & 68 & 7.1 & .010 & - & .07 & - & .010 \\
\hline MAR $13 .$. & $110 \mathrm{C}$ & 55 & 20.5 & 77 & 7.9 & .030 & - & .71 & -- & $<.010$ \\
\hline 13... & $100 \mathrm{C}$ & 35 & 20.0 & $E 7$ & 7.4 & $.02 \mathrm{C}$ & - & .06 & -- & $<.010$ \\
\hline WAY 25 & 1100 & 55 & 20.5 & EI & 7.0 & $.03 C$ & - & .32 & -- & $<.010$ \\
\hline JUN & 1200 & 5.5 & 21.0 & 100 & $\cdots$ & - & .000 & -- & .17 & -- \\
\hline APR & $975^{140 \mathrm{C}}$ & 53 & 21.5 & -- & -- & - & .050 & -- & .01 & -- \\
\hline OCT & $100 \mathrm{C}$ & 25 & 20.5 & 100 & 7.5 & -- & .000 & -- & .00 & -- \\
\hline $14 \ldots .$. & $\begin{array}{l}141 C \\
143 C\end{array}$ & $\begin{array}{l}55 \\
55\end{array}$ & $=$ & $\begin{array}{l}87 \\
83\end{array}$ & $=$ & $\overline{-}$ & $\begin{array}{l}.070 \\
.030\end{array}$ & $=$ & $\begin{array}{l}.01 \\
.00\end{array}$ & $=$ \\
\hline JAN".. & $97 t^{1600}$ & 55 & - & 83 & - & - & .090 & -- & .08 & -- \\
\hline $23 \ldots$ & $150 \mathrm{C}$ & 55 & 20.0 & -- & - & - & .060 & -- & .00 & -- \\
\hline DATE & $\begin{array}{l}\text { NITRC- } \\
\text { GEN, } \\
\text { NITRI TE } \\
\text { TOTAL } \\
\text { (MG/L } \\
\text { SS N ) }\end{array}$ & $\begin{array}{l}\text { NIIFD- } \\
\text { GEN, } \\
\text { NITEAIE } \\
\text { OIS- } \\
\text { SOLVED } \\
\text { (MEA } \\
\text { AS R) }\end{array}$ & $\begin{array}{l}\text { NITRO- } \\
\text { GEN, } \\
\text { NIIRAIE } \\
\text { ICTAL } \\
\text { (MGAL } \\
\text { ASNI }\end{array}$ & $\begin{array}{l}\text { NITFC- } \\
\text { GENAAM- } \\
\text { MONIA + } \\
\text { ORGANIC } \\
\text { TCTAL } \\
\text { (KCAL } \\
\text { OSN) }\end{array}$ & $\begin{array}{l}\text { N1 IRO- } \\
\text { GEN, } \\
\text { ND2 +NO3 } \\
\text { TOTAL } \\
\text { (MG/L } \\
\text { AS N) }\end{array}$ & $\begin{array}{l}\text { NIIRC- } \\
\text { CEN, } \\
\text { TLTAL } \\
\text { IMEAL } \\
\text { IS N) }\end{array}$ & $\begin{array}{l}\text { PHOS- } \\
\text { PHORUS, } \\
\text { ORIHO, } \\
\text { D IS- } \\
\text { SOIVEO } \\
\text { (MGA } \\
\text { AS P) }\end{array}$ & $\begin{array}{l}\text { PHCS- } \\
\text { PHJRUS, } \\
\text { DIS- } \\
\text { SJLVED } \\
\text { (MG/L } \\
\text { AS P) }\end{array}$ & $\begin{array}{l}\text { PHOS- } \\
\text { PHORUS. } \\
\text { TUI AL } \\
\text { (MO AL } \\
\text { AS P) }\end{array}$ & $\begin{array}{l}\text { DXYGEN } \\
\text { DEMAND. } \\
\text { BIO- } \\
\text { CHEM- } \\
\text { ICAL, } \\
5 \text { DAY } \\
\text { IMG/L) }\end{array}$ \\
\hline
\end{tabular}

DEC , 1972

\begin{tabular}{|c|c|c|c|c|c|c|c|c|c|c|}
\hline $\begin{array}{l}15 \ldots \\
20 \ldots \ldots \\
F E \theta\end{array}$ & .012 & -- & .00 & $=$ & $=$ & $=$ & $=$ & $=$ & .007 & $\overline{--}$ \\
\hline MAR & . C 11 & $=-$ & .03 & -- & - & - & -- & -- & .009 & -- \\
\hline $05 \ldots$ & $=$ & -- & $=$ & $=$ & $\overline{-}$ & - & - & - & -- & -- \\
\hline APR & -- & -- & -- & - & - & - & -- & - & -- & -- \\
\hline$\underset{\operatorname{MAY}}{12} \cdots$ & .013 & -- & .07 & -- & - & - & $=-$ & -- & .002 & -- \\
\hline$\underset{\text { JUL }}{30 \ldots}$ & .005 & -- & $.0 t$ & -- & - & -- & -- & -- & .010 & .5 \\
\hline$\underset{\text { aUG }}{25} \cdots$ & $-\infty$ & .00 & $\cdots$ & -- & - & -- & $<.010$ & .005 & -- & .6 \\
\hline${ }_{\mathrm{OCT}}^{29} \cdots$ & - & .00 & -- & - & - & -- & $<.010$ & .008 & -- & -- \\
\hline $\begin{array}{l}04 \\
\text { NกY }\end{array}$ & -- & .09 & -- & -- & - & -- & $<.010$ & .004 & -- & 2.2 \\
\hline DEC & - & .02 & - & - & - & - & $<.010$ & .010 & $\cdots$ & .5 \\
\hline $\operatorname{JAN}_{20}^{20}, 1974$ & - & .06 & -- & - & - & - & $<.010$ & .010 & $\cdots$ & 3.7 \\
\hline$\underset{F E B}{24} \cdots$ & - & .02 & -- & -- & - & -- & .020 & .020 & - & 1.2 \\
\hline$\underset{\operatorname{MAR}}{13} \cdots$ & $\cdots$ & .04 & -- & $-\infty$ & - & - & $<.010$ & $<.010$ & $\cdots$ & 1.4 \\
\hline$\underset{A P R}{13} \ldots$ & $\cdots$ & .07 & -- & -- & - & - & C.D10 & .010 & -- & 1.3 \\
\hline MaY 25 & -- & .06 & -- & -- & - & - & $<.010$ & $<.010$ & -- & .6 \\
\hline JiN & .030 & $\cdots$ & .04 & - & $\cdots$ & - & -- & -- & .020 & .7 \\
\hline 27PR : 1975 & .030 & -- & .09 & .06 & .12 & .18 & - & -- & .050 & .4 \\
\hline OCT & .050 & -- & .04 & .06 & .09 & $\cdot 15$ & - & -- & .020 & -- \\
\hline $\begin{array}{l}14 \ldots \ldots \\
14 \ldots \ldots \\
14 \ldots \ldots 1976\end{array}$ & $\begin{array}{l}.040 \\
.030 \\
.070\end{array}$ & $=$ & $\begin{array}{l}.06 \\
.06 \\
.02\end{array}$ & $\begin{array}{l}.08 \\
.03 \\
.17\end{array}$ & $\begin{array}{l}.10 \\
.09 \\
.09\end{array}$ & $\begin{array}{l}.18 \\
.18 \\
.26\end{array}$ & $=$ & $=$ & $\begin{array}{l}.040 \\
.030 \\
.060\end{array}$ & $\because$ \\
\hline 23. & .040 & -- & .02 &. $\mathrm{Cb}$ & .06 & .12 & -- & -- & .100 & - \\
\hline
\end{tabular}


Site 14--Continued

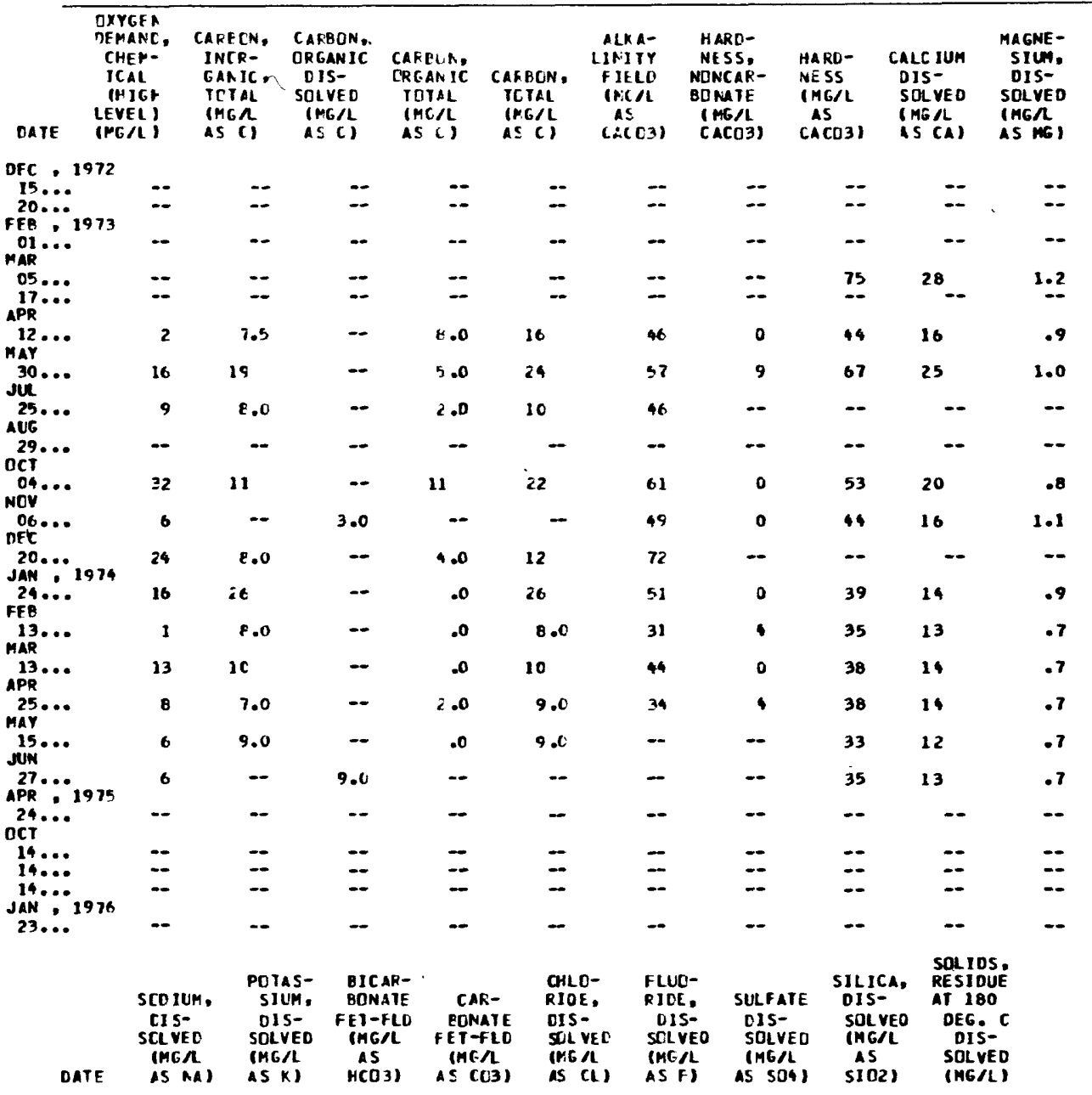

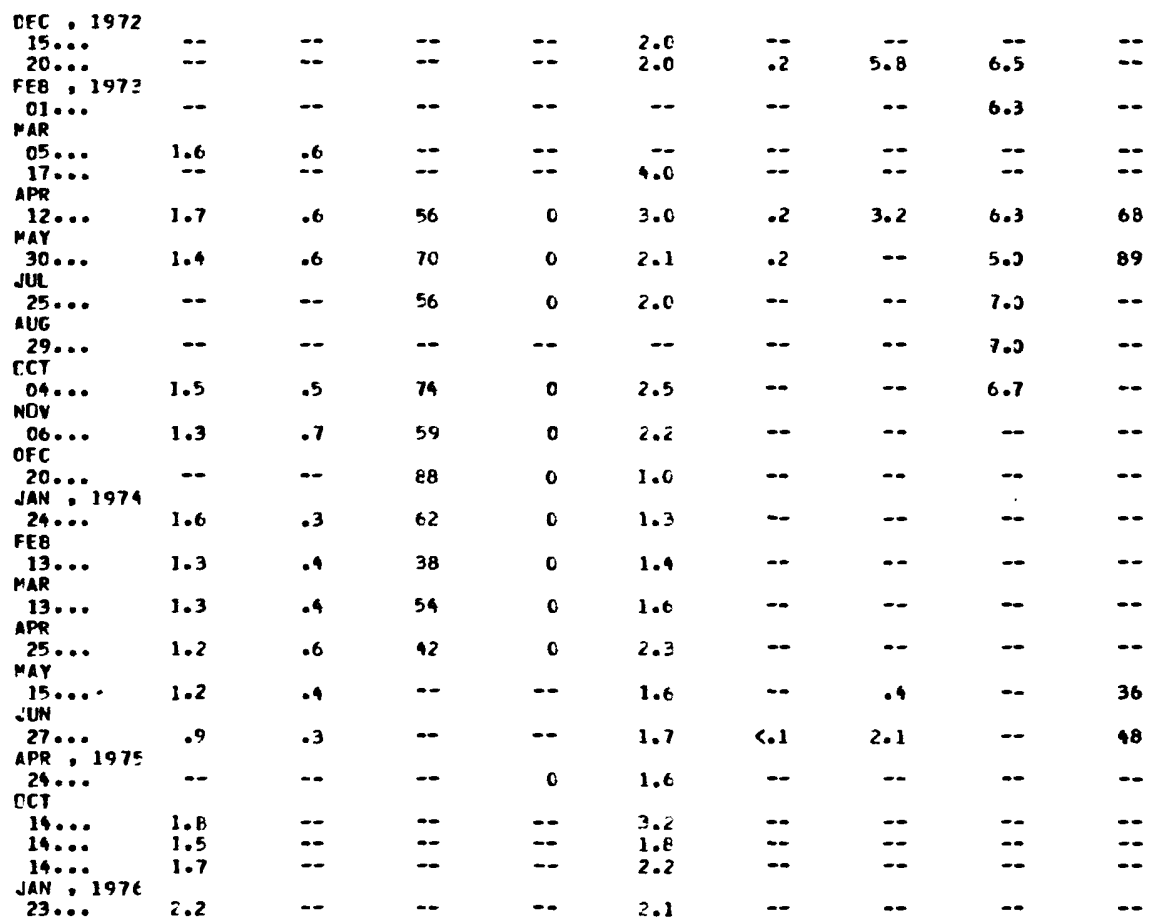


Site 14--Continued

\begin{tabular}{|c|c|c|c|c|c|c|c|c|c|}
\hline DATE & TIVE & $\begin{array}{l}\text { DEFTH } \\
\text { CF } \\
\text { WELL. } \\
\text { TDIAL } \\
\text { (FIET) }\end{array}$ & $\begin{array}{l}\text { TEMPCR- } \\
\text { ATURE } \\
\text { (DEG () }\end{array}$ & $\begin{array}{l}\text { SPF- } \\
\text { CIFIC } \\
\text { CON- } \\
\text { CUCT- } \\
\text { ANCE } \\
\text { I LAHCS) }\end{array}$ & (LN1 is) & $\begin{array}{l}\text { NITKD- } \\
\text { CIND } \\
\text { AMHCNIA } \\
\text { ICTLL } \\
\text { (MT } \\
\text { AS MI) }\end{array}$ & $\begin{array}{l}\text { WIIRJ- } \\
\text { GLN. } \\
\text { WHENIC } \\
\text { TOTAL } \\
\text { (ME/L } \\
\text { AS N) }\end{array}$ & $\begin{array}{l}\text { NITRU- } \\
\text { GEII, } \\
\text { NITRITE } \\
\text { IUT AL } \\
\text { (HG/L } \\
\text { AS N) }\end{array}$ & $\begin{array}{l}\text { NITRO- } \\
\text { GEN. } \\
\text { NITRAIE } \\
\text { TJTAL } \\
\text { IMG/L } \\
\text { AS NI }\end{array}$ \\
\hline JUN & $197 t$ & & & & & & & & \\
\hline AUG & $15 \mathrm{CO}$ & 55 & 21.5 & -- & -- & .050 & .00 & .023 & .05 \\
\hline FEB 26 & $1977^{1300}$ & 55 & 21.0 & 85 & -- & .040 & .03 & .013 & .04 \\
\hline $16 \ldots$ & 1200 & 55 & 20.0 & - & -- & .010 & .23 & .023 & .02 \\
\hline$\underset{\text { SEP }}{28} \cdots$ & 1000 & 55 & 22.5 & 74 & -- & .010 & .60 & .023 & .03 \\
\hline AUG $27 .$. & $1978^{1500}$ & 55 & 22.0 & -- & $\cdots$ & .020 & .19 & .060 & .15 \\
\hline $\begin{array}{l}22 \ldots \\
24 \ldots \ldots \\
25 \ldots \ldots \\
28 \ldots \\
. A N\end{array}$ & $\begin{array}{r}1325 \\
1130 \\
0825 \\
1335 \\
1979^{1325}\end{array}$ & $\begin{array}{l}55 \\
55 \\
55 \\
55\end{array}$ & $\begin{array}{l}-- \\
-- \\
--\end{array}$ & $\begin{array}{l}86 \\
79 \\
78 \\
76\end{array}$ & $\begin{array}{l}-- \\
-- \\
\varepsilon .6\end{array}$ & $\begin{array}{r}-- \\
.080 \\
.080 \\
. \therefore 00\end{array}$ & $\begin{array}{l}.- \\
.00 \\
.00 \\
.00\end{array}$ & $\begin{array}{r}-0 \\
.035 \\
.035 \\
.053\end{array}$ & $\begin{array}{l}.5 \\
.01 \\
.01 \\
.00\end{array}$ \\
\hline $\begin{array}{l}\text { OA..... } \\
\text { WAR }\end{array}$ & $\begin{array}{l}1530 \\
1230\end{array}$ & $\begin{array}{l}55 \\
55\end{array}$ & -- & $\begin{array}{l}76 \\
79\end{array}$ & $\begin{array}{l}8.0 \\
8.5\end{array}$ & $\begin{array}{r}.010 \\
.040\end{array}$ & .02 & $\begin{array}{l}<.013 \\
<.010\end{array}$ & $\begin{array}{l}.04 \\
.02\end{array}$ \\
\hline${ }_{P P R}^{19} \ldots$ & 1520 & 55 & - & 86 & 8.4 & $<.010$ & .18 & $<.013$ & .06 \\
\hline JUW & $14 \mathrm{CO}$ & 55 & - & 81 & $\varepsilon . \Sigma$ & -- & -- & -- & .05 \\
\hline сCT $27 .$. & $14 c 0$ & 55 & -- & 82 & 8.3 & .050 & .01 & C.v1s & .05 \\
\hline NOV & 1440 & 55 & - & 79 & 8.4 & -- & -- & -- & $\cdots$ \\
\hline JAN $13 . \cdots$ & $198 \mathrm{C}^{1540}$ & 55 & $-\infty$ & 78 & 8.4 & -- & -- & .000 & .03 \\
\hline $\begin{array}{l}31 \\
\text { FF8 }\end{array}$ & 1120 & 55 & $-\infty$ & 77 & -- & -- & -- & .003 & .04 \\
\hline $28 \ldots$ & 1530 & 55 & $-\infty$ & 76 & -- & - & -- & .000 & .03 \\
\hline$\underset{\text { JUW }}{24} \cdots$ & 1310 & 55 & -- & 70 & -- & .100 & .13 & .013 & .02 \\
\hline$\underset{A \cup E}{27 \ldots}$ & 1000 & 55 & - & 93 & -- & .040 & .23 & .000 & .04 \\
\hline 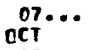 & 1315 & 55 & - & 95 & -- & -- & -- & .000 & .05 \\
\hline $\operatorname{CEC}_{22} \cdots$ & 1515 & 55 & $-\infty$ & 87 & -- & .010 & .22 & .000 & .05 \\
\hline $05 . .$. & 0915 & 55 & - & 77 & -- & -- & -- & .000 & .03 \\
\hline DATE & $\begin{array}{l}\text { NITFO- } \\
\text { CEN,AM- } \\
\text { PONIA } \\
\text { CRGANIC } \\
\text { IOTAL } \\
\text { IMCN } \\
\text { IS AI }\end{array}$ & $\begin{array}{l}\text { NITRO- } \\
\text { GEN. } \\
\text { NC2+NO3 } \\
\text { IOTAL } \\
\text { (MEAL } \\
\text { IS N) }\end{array}$ & $\begin{array}{l}\text { NIIRC- } \\
\text { GEA, } \\
\text { TOTAL } \\
\text { (MG/L } \\
\text { AS A) }\end{array}$ & $\begin{array}{l}\text { PHOS- } \\
\text { FHOPUS, } \\
\text { TOTAL } \\
\text { (MGA } \\
\text { AS P) }\end{array}$ & $\begin{array}{l}\text { ALKA- } \\
\text { LINIIY } \\
\text { FIELC } \\
\text { IRG } A \\
\text { AS } \\
\text { CACEZ) }\end{array}$ & $\begin{array}{l}\text { HAKC- } \\
\text { NESS, } \\
\text { NONCAR- } \\
\text { BOMATE } \\
\text { (KCAL } \\
\text { CACD3) }\end{array}$ & $\begin{array}{l}\text { HAKO- } \\
\text { NE SS } \\
\text { (MG/L } \\
\text { AS } \\
\text { CACOS) }\end{array}$ & $\begin{array}{l}\text { CALCIUA } \\
\text { OIS- } \\
\text { SOLLEO } \\
\text { (MG/L } \\
\text { AS CA) }\end{array}$ & $\begin{array}{l}\text { HAGNE - } \\
\text { SIUM. } \\
\text { OIS- } \\
\text { SOL VED } \\
\text { (MG/L } \\
\text { AS MG) }\end{array}$ \\
\hline
\end{tabular}

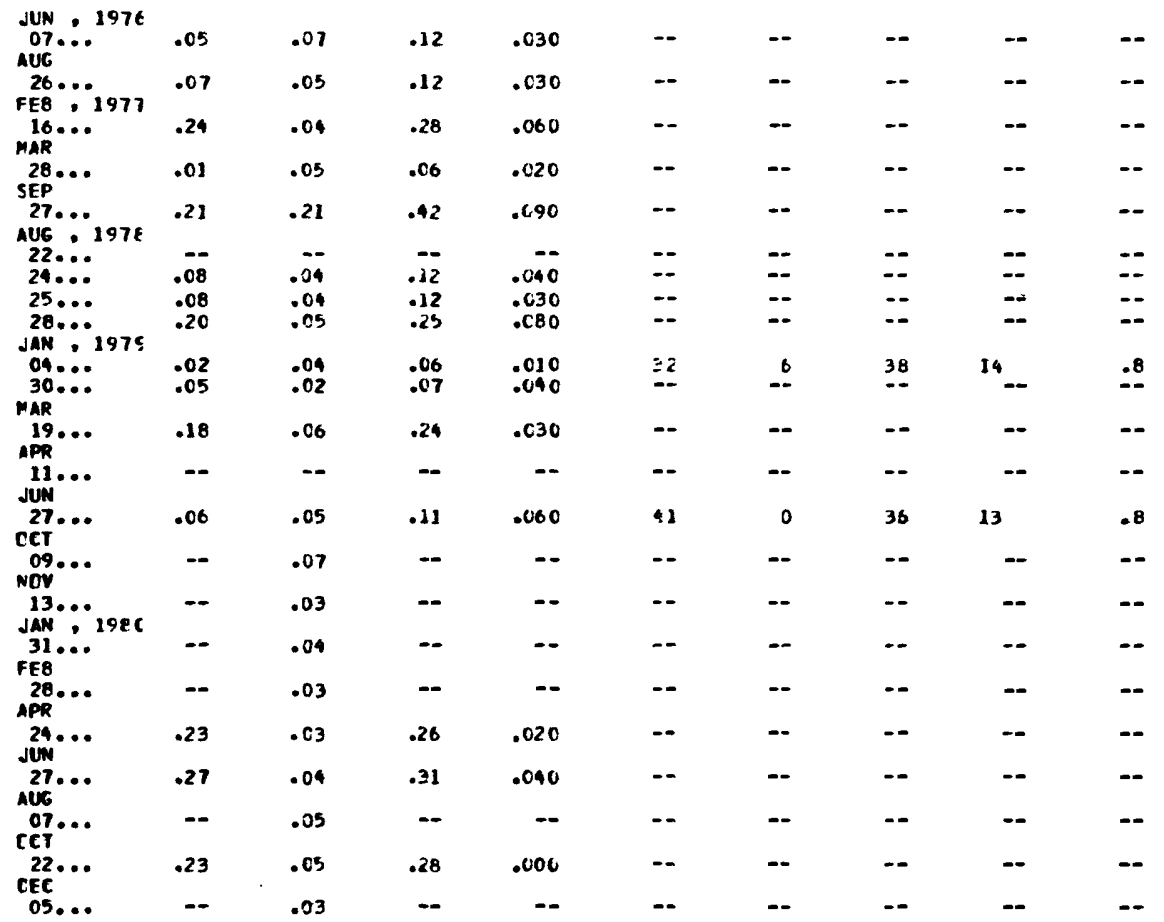


Site 14--Continued

\begin{tabular}{|c|c|c|c|c|c|c|c|c|c|}
\hline ATE & $\begin{array}{l}\text { SCOILP, } \\
\text { CIS- } \\
\text { SCLVED } \\
\text { (MGA } \\
\text { IS NAS }\end{array}$ & $\begin{array}{l}\text { PUTAS- } \\
\text { SIUH, } \\
\text { DIS- } \\
\text { SOLVED } \\
\text { IMG/L } \\
\text { ASKI) }\end{array}$ & $\begin{array}{l}\text { CHLU- } \\
\text { RIDE, } \\
\text { CIS- } \\
\text { SOL VED } \\
\text { IHG / } \\
\text { AS CL) }\end{array}$ & $\begin{array}{l}\text { FLUE- } \\
\text { FI DE } \\
\text { DIS- } \\
\text { SOLVEO } \\
\text { (MGRL } \\
\text { AS F) }\end{array}$ & $\begin{array}{l}\text { SULFATE } \\
\text { DIS- } \\
\text { SCL VEO } \\
\text { IYGA } \\
\text { AS S(4) }\end{array}$ & $\begin{array}{l}\text { SILICA, } \\
\text { DIS- } \\
\text { SOLVEO } \\
\text { IHE/L } \\
\text { AS } \\
\text { SICIZ) }\end{array}$ & $\begin{array}{l}\text { SOLIDS, } \\
\text { RESIDUE } \\
\text { AT IBO } \\
\text { OEG. C } \\
\text { DIS- } \\
\text { SOLVED } \\
\text { (MG/L) }\end{array}$ & $\begin{array}{l}\text { OEPTH } \\
\text { BELOH } \\
\text { LAND } \\
\text { SURFACE } \\
\text { (WATER } \\
\text { LEVEL) } \\
\text { (FEE I) }\end{array}$ & $\begin{array}{l}\text { ELEV- } \\
\text { AIION } \\
\text { ABOVE } \\
\text { NGVD } \\
\text { (FEET) }\end{array}$ \\
\hline
\end{tabular}

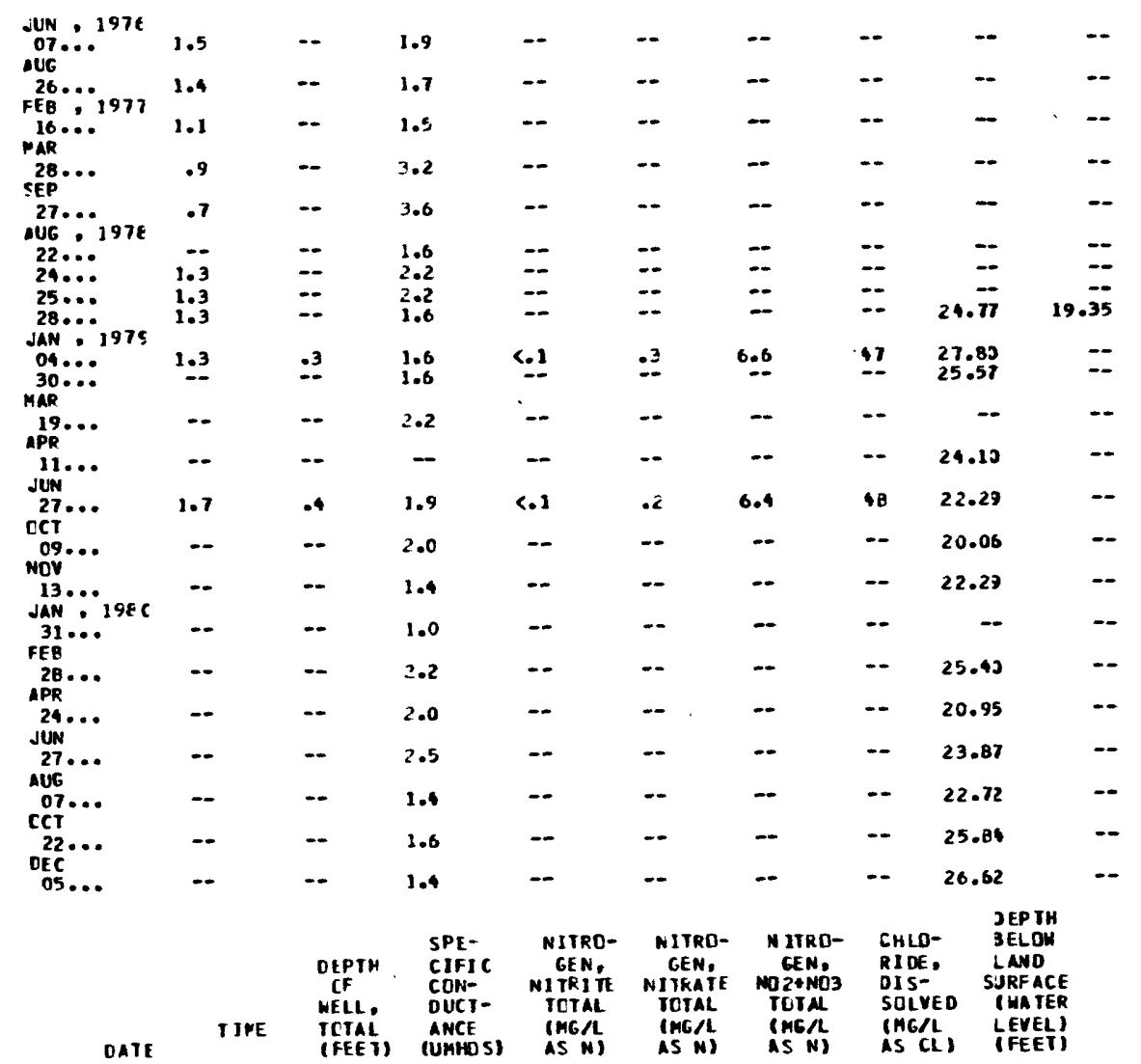

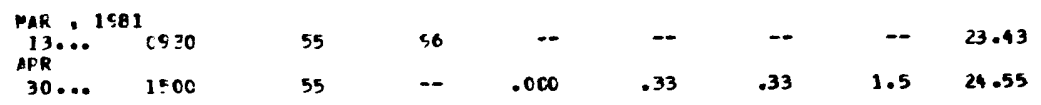

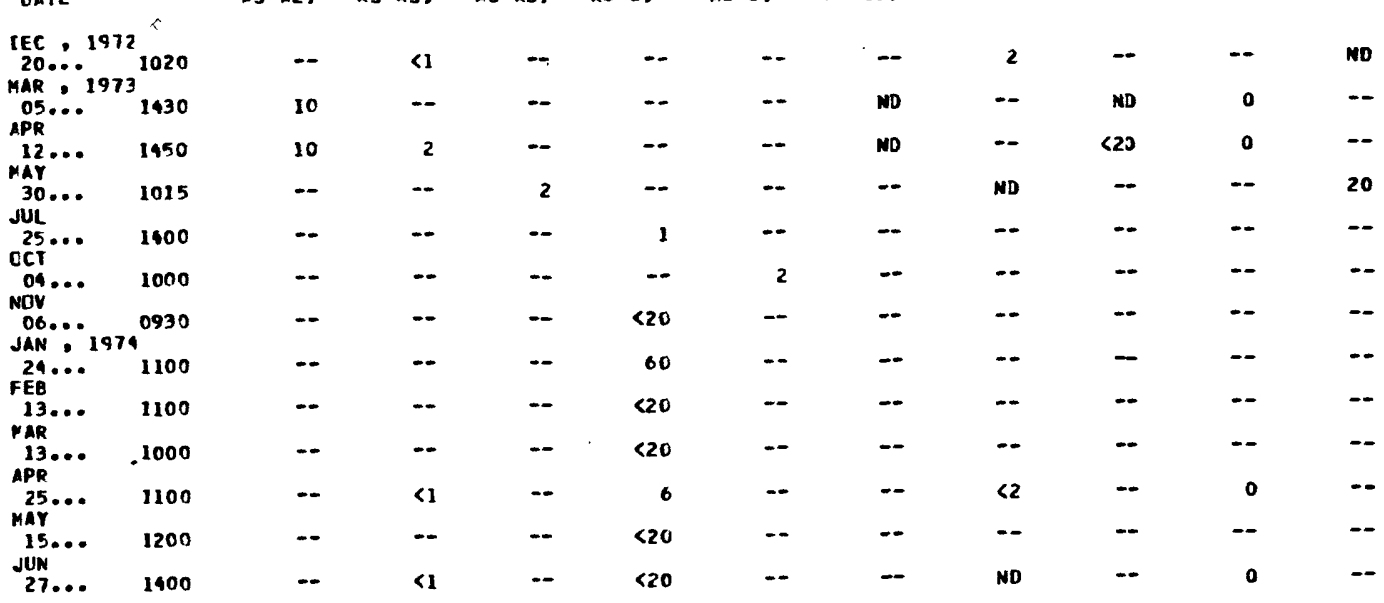


Site 14--Continued

\begin{tabular}{|c|c|c|c|c|c|c|c|c|c|c|}
\hline DATE & $\begin{array}{c}\text { COBALI. } \\
\text { OIS- } \\
\text { SREVE } \\
\text { (UIG/L } \\
\text { AS CC) }\end{array}$ & $\begin{array}{l}\text { COBALP- } \\
\text { TOTOL } \\
\text { RECEV- } \\
\text { ERAPLE } \\
\text { IUCAL } \\
\text { AS CD) }\end{array}$ & $\begin{array}{l}\text { COPPER, } \\
\text { DIS- } \\
\text { SCIVED } \\
\text { (LG/L } \\
\text { AS (U) }\end{array}$ & $\begin{array}{l}\text { COPFEP. } \\
\text { TOTAL } \\
\text { RECCV- } \\
\text { ERALLE } \\
\text { (UG/L } \\
\text { AS CLU }\end{array}$ & $\begin{array}{l}\text { IPCN, } \\
\text { CIS: } \\
\text { SCLVEC } \\
\text { (UG/L } \\
\text { AS FE) }\end{array}$ & $\begin{array}{l}\text { IEAD } \\
\text { CIS- } \\
\text { SrLVEO } \\
\text { (UAN } \\
\text { ASPES }\end{array}$ & $\begin{array}{l}\text { LIAD. } \\
\text { TOTAL } \\
\text { RECOY- } \\
\text { ERABLE } \\
\text { IUGAL } \\
\text { AS PES }\end{array}$ & $\begin{array}{c}\text { LITHIUM } \\
\text { OIS- } \\
\text { SJLVEO } \\
\text { IUGL } \\
\text { AS LIS }\end{array}$ & $\begin{array}{l}\text { LITH IUM } \\
\text { IOTML } \\
\text { RECOV- } \\
\text { ERABLE } \\
\text { IUEAL } \\
\text { OS LII }\end{array}$ & $\begin{array}{l}\text { MANGA- } \\
\text { MESE, } \\
\text { TOTAL } \\
\text { RECON- } \\
\text { ERABLE } \\
\text { IUGA } \\
\text { AS MNI }\end{array}$ \\
\hline DEC . 1 & 972 & $<2$ & - & $\cdots$ & - & - & 52 & $\cdots$ & $<10$ & 30 \\
\hline $\begin{array}{l}\text { MAR } \\
\text { OS } \\
\text { APR }\end{array}$ & AD & -- & ND & $\cdots$ & 30 & $<2$ & 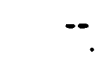 & $<10$ & - & $\cdots$ \\
\hline $\operatorname{mav}_{12} \ldots$ & no & $=$ & no & - & $<10$ & $<$ & $\cdots$ & $<10$ & - & - \\
\hline jull & - & ND & - & $<20$ & $\cdots$ & - & 24 & $\because$ & $<10$ & 60 \\
\hline OCT & - & -- & -- & - & - & - & - & - & $\cdots$ & $\cdots$ \\
\hline $\mathrm{NCV}^{\mathrm{O}} \ldots$ & -- & $\cdots$ & - & - & - & 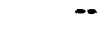 & $-\infty$ & 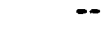 & $\cdots$ & $\cdots$ \\
\hline$\underset{\operatorname{JAN}}{\text { DAN... }}$ & 974 & $\cdots$ & $\cdots$ & - & - & $\cdots$ & $\cdots$ & $\cdots$ & $\cdots$ & $\Rightarrow$ \\
\hline FEB $24 \cdots$ & $\approx$ & - & - & $\cdots$ & - & - & -- & $\cdots$ & $\cdots$ & $\cdots$ \\
\hline MAR & $\cdots$ & $\cdots$ & 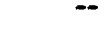 & $\cdots$ & - & - & $\cdots$ & $\cdots$ & $\infty$ & $\cdots$ \\
\hline$\underset{A P R}{13} \cdots$ & - & -- & $\cdots$ & $\cdots$ & - & $\cdots$ & $\cdots$ & $=$ & $\cdots$ & \\
\hline MAY & $\cdots$ & $\cdots$ & 7 & $\cdots$ & $<10$ & NE & $\cdots$ & $\cdots$ & $=$ & - \\
\hline JUW & $\cdots$ & -- & $\cdots$ & $\cdots$ & - & - & $\cdots$ & $\cdots$ & $\cdots$ & \\
\hline $27 \ldots$ & $\cdots$ & $\cdots$ & 5 & $\cdots$ & stc & $\mathrm{HI}$ & - & $\rightarrow$ & $\cdots$ & - \\
\hline DATE & $\begin{array}{l}\text { MANGA- } \\
\text { HESF: } \\
\text { CIS- } \\
\text { SOLVED } \\
\text { HUG/L } \\
\text { AS MAI }\end{array}$ & $\begin{array}{l}\text { MERE IRV } \\
\text { TOIAL } \\
\text { RE CIV- } \\
\text { ER APLE } \\
\text { (UT AL } \\
\text { AS HG) }\end{array}$ & $\begin{array}{l}\text { MCLYE- } \\
\text { OENUH, } \\
\text { EIS- } \\
\text { SCLVEO } \\
\text { (U6/L } \\
\text { AS MC) }\end{array}$ & $\begin{array}{l}\text { MOLYF- } \\
\text { OENLY. } \\
\text { YOTAL } \\
\text { RECIY- } \\
\text { ERAGLE } \\
\text { TUC } \\
\text { AS KCS }\end{array}$ & $\begin{array}{l}\text { MICKEL. } \\
\text { OIS- } \\
\text { Solvet } \\
\text { lugel } \\
\text { as Nil }\end{array}$ & $\begin{array}{l}\text { HCHEL, } \\
\text { ICTAL } \\
\text { ACEV- } \\
\text { ERABLE } \\
\text { (UGCL } \\
\text { AS NI) }\end{array}$ & $\begin{array}{l}\text { S JRCN- } \\
\text { TIUN } \\
\text { OIS- } \\
\text { SELVE } \\
\text { (LGA } \\
\text { RS SO) }\end{array}$ & $\begin{array}{l}\text { VANA- } \\
\text { OIUH, } \\
\text { DIS- } \\
\text { SJLVED } \\
\text { WUG/L } \\
\text { AS VI }\end{array}$ & $\begin{array}{l}\text { ZINC. } \\
\text { OIS- } \\
\text { SOLVEO } \\
\text { (U⿺R } \\
\text { AS IN) }\end{array}$ & $\begin{array}{l}\text { ZINC, } \\
\text { TOTAL } \\
\text { RECQY- } \\
\text { ERABtE } \\
\text { (UGA } \\
\text { AS IN) }\end{array}$ \\
\hline
\end{tabular}

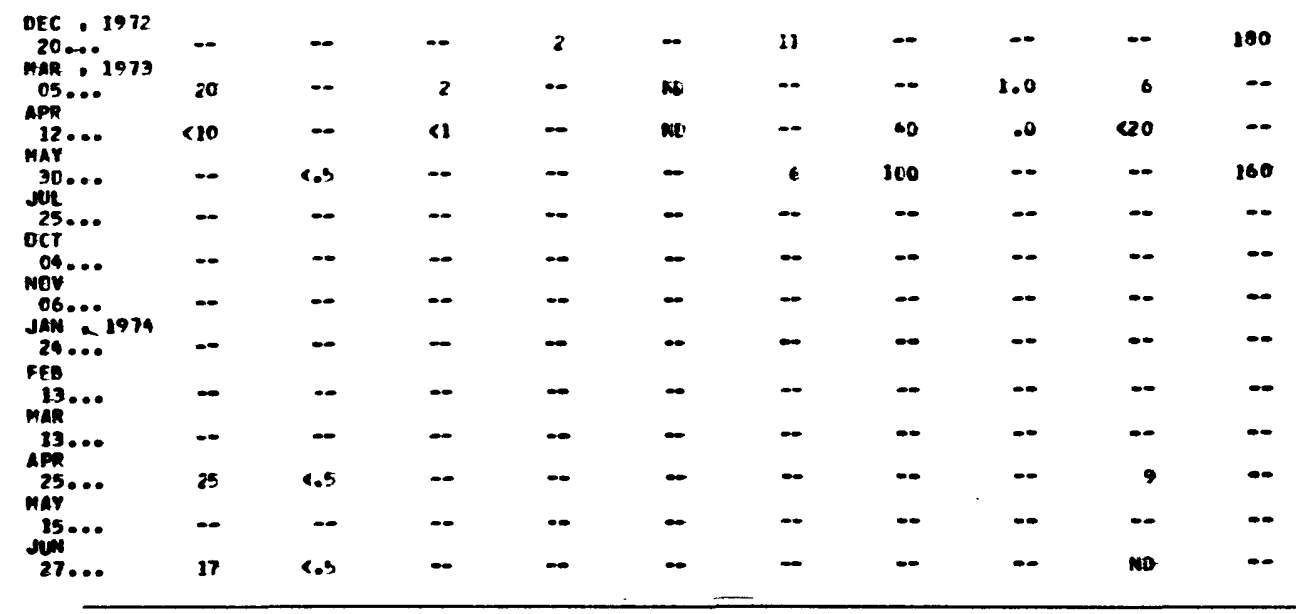

Site 15 in 4-big-gun area

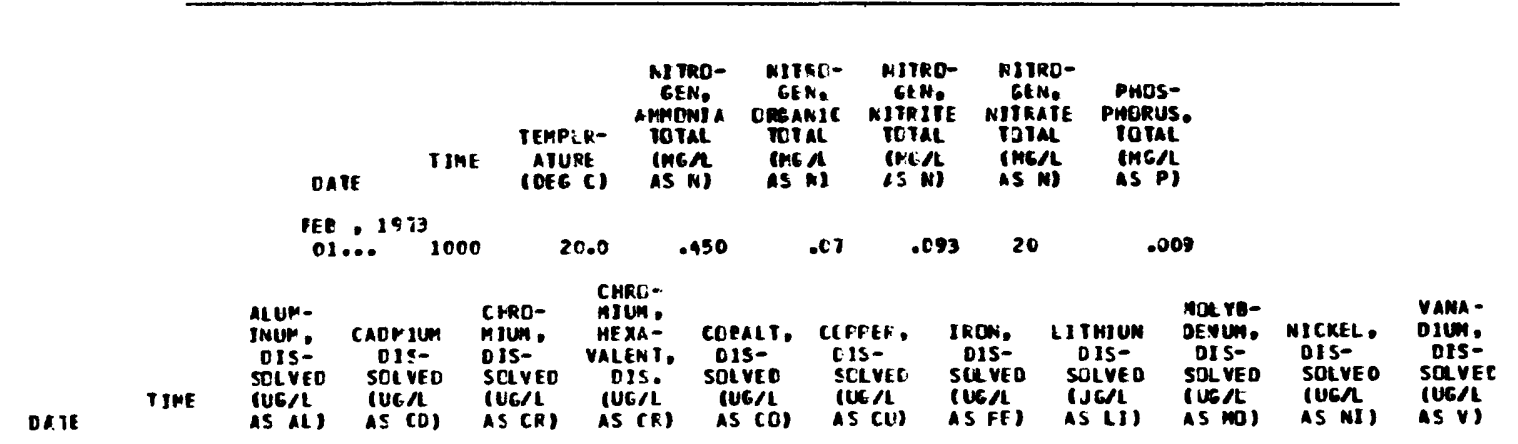

MAR 1973

05

20

30

\begin{abstract}
0
\end{abstract}
(O) 
Site 16 near holding ponds

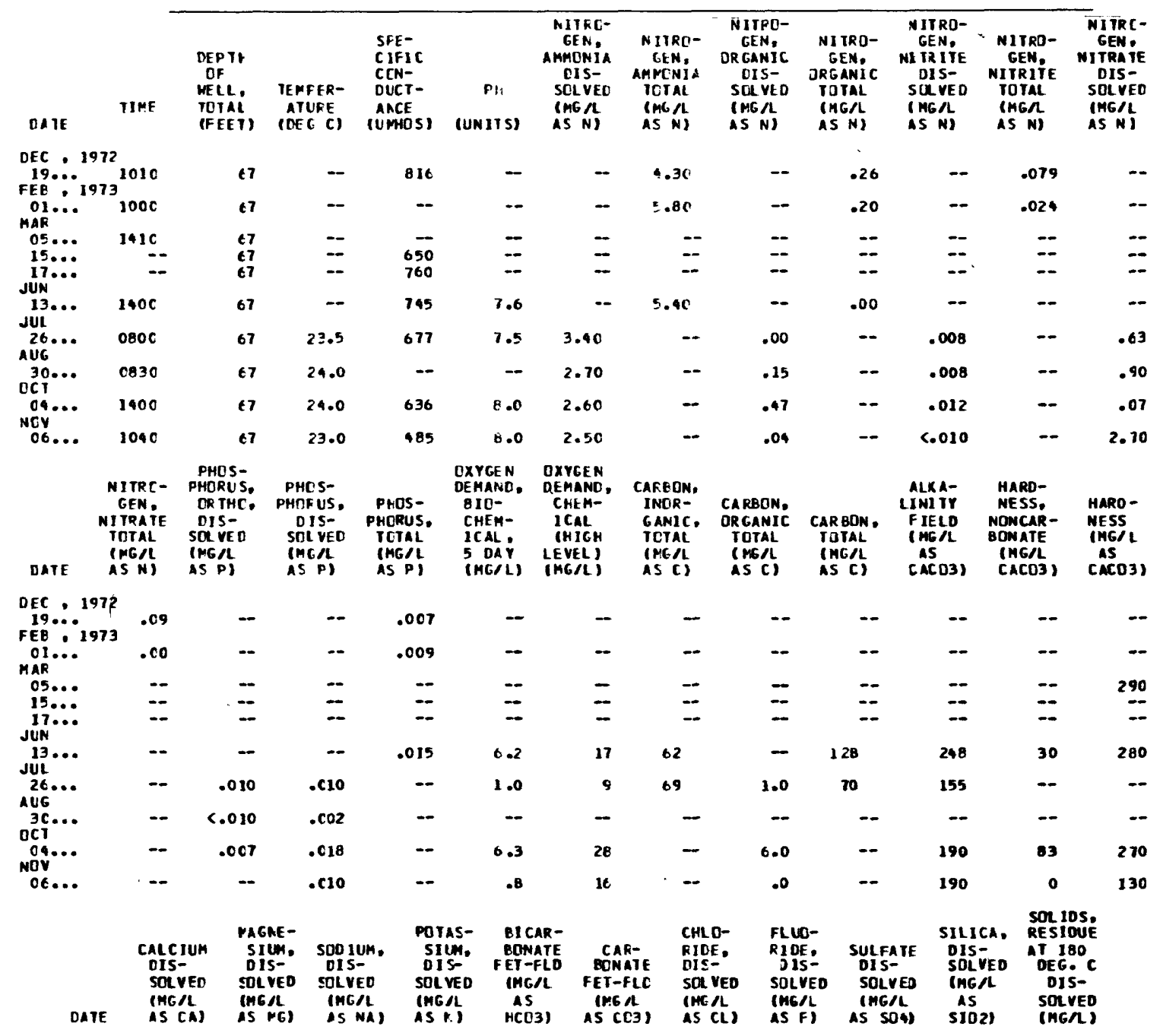

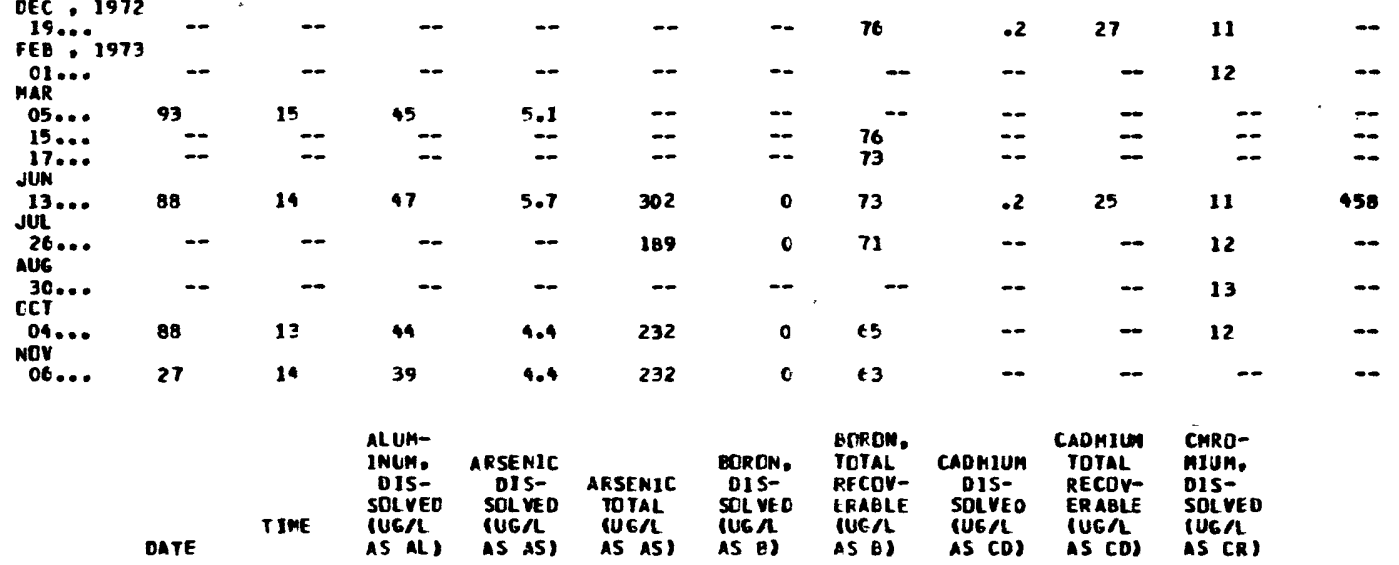

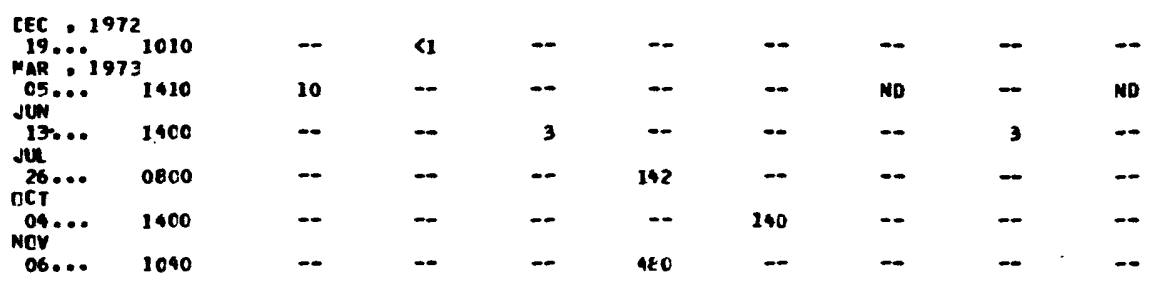


Site 16 near holding ponds---Continued

\begin{tabular}{|c|c|c|c|c|c|c|c|c|c|}
\hline DATE & $\begin{array}{l}\text { CHRC- } \\
\text { VIUY, } \\
\text { YEXA- } \\
\text { VALENI, } \\
\text { DIS. } \\
\text { CUCA } \\
\text { AS (R) }\end{array}$ & $\begin{array}{l}\text { CHAD- } \\
\text { MILW, } \\
\text { TOTAL } \\
\text { RECBV- } \\
\text { ERABLE } \\
\text { IUGAL } \\
\text { AS (R) }\end{array}$ & $\begin{array}{l}\text { COQALI, } \\
\text { DIS- } \\
\text { SOLVED } \\
\text { (UGA } \\
\text { AS CO) }\end{array}$ & $\begin{array}{l}\text { CEAALI, } \\
\text { TOTAL } \\
\text { RE COY- } \\
\text { ER ABLE } \\
\text { CUGAL } \\
\text { AS CO) }\end{array}$ & $\begin{array}{l}\text { CCPPEF, } \\
\text { to1s- } \\
\text { SCL VEC } \\
\text { (UGA } \\
\text { as (L) }\end{array}$ & $\begin{array}{l}\text { IELW: } \\
\text { CIS- } \\
\text { SCLVED } \\
\text { (UC } / L \\
\text { AS FE) }\end{array}$ & $\begin{array}{l}\text { LEAD, } \\
\text { DIS- } \\
\text { SOLVEO } \\
\text { IUG/L } \\
\text { AS PO) }\end{array}$ & $\begin{array}{l}\text { IIHIUY } \\
\text { DIS- } \\
\text { SOLVEO } \\
\text { IUGJL } \\
\text { AS LIS }\end{array}$ & $\begin{array}{l}\text { LI IHIUH } \\
\text { TOTAL } \\
\text { RECON- } \\
\text { ERABLE } \\
\text { IUG } \\
\text { AS LII }\end{array}$ \\
\hline
\end{tabular}

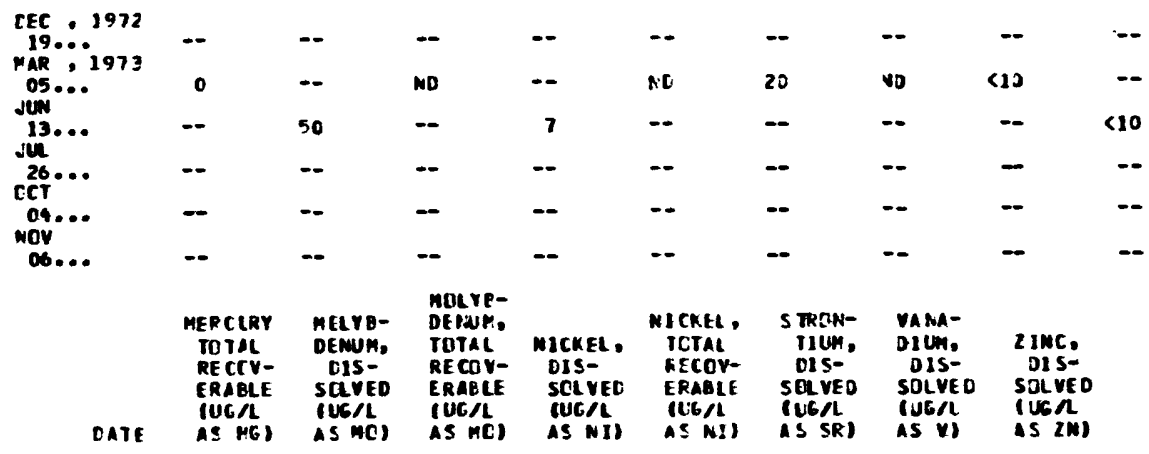

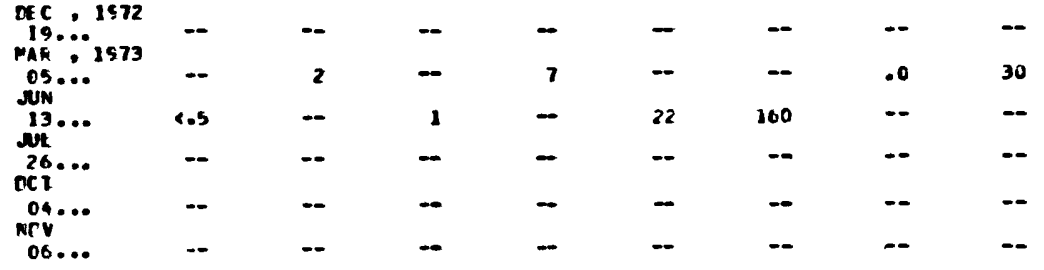

Site 17

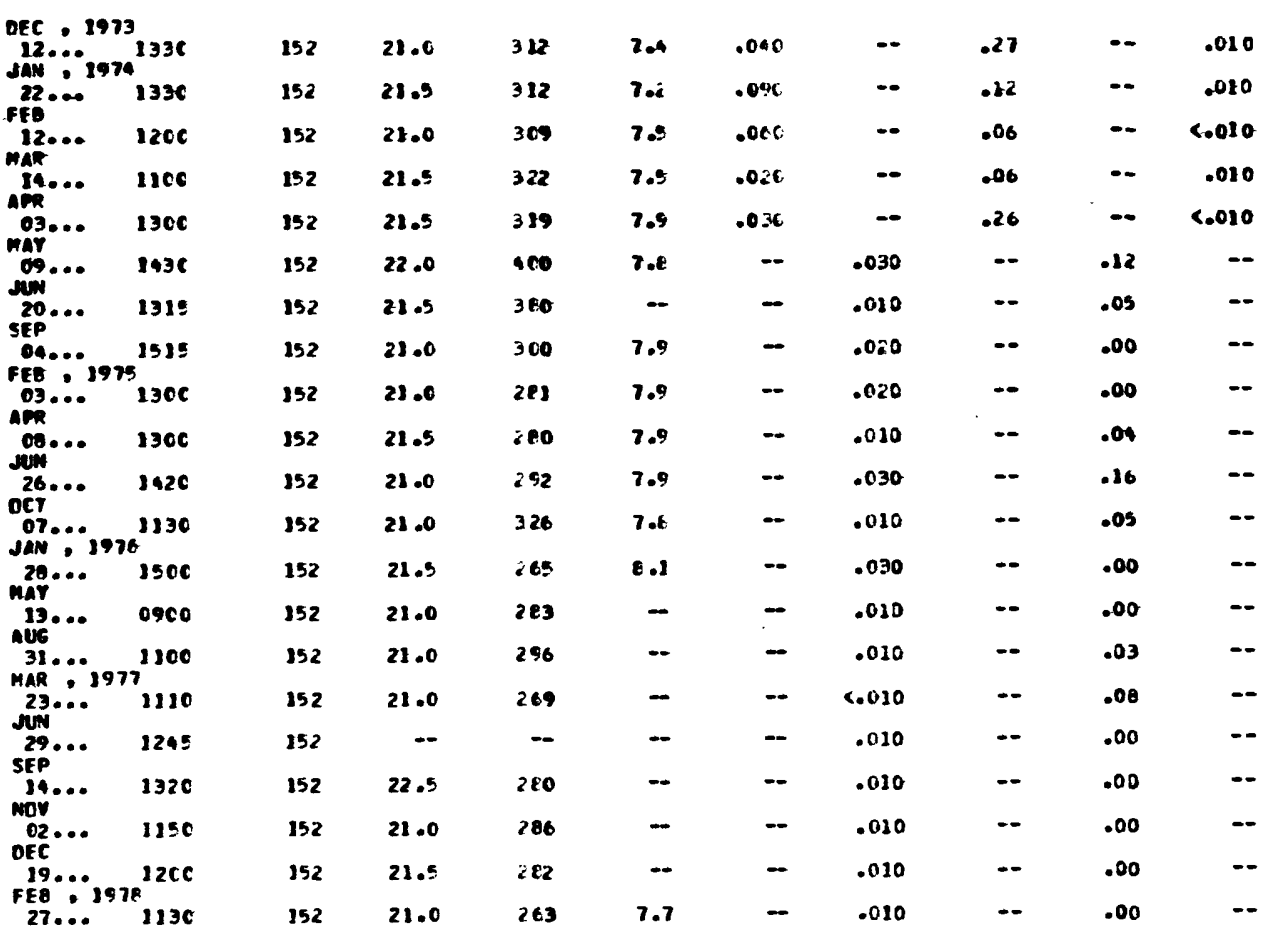


Site 17--Continued

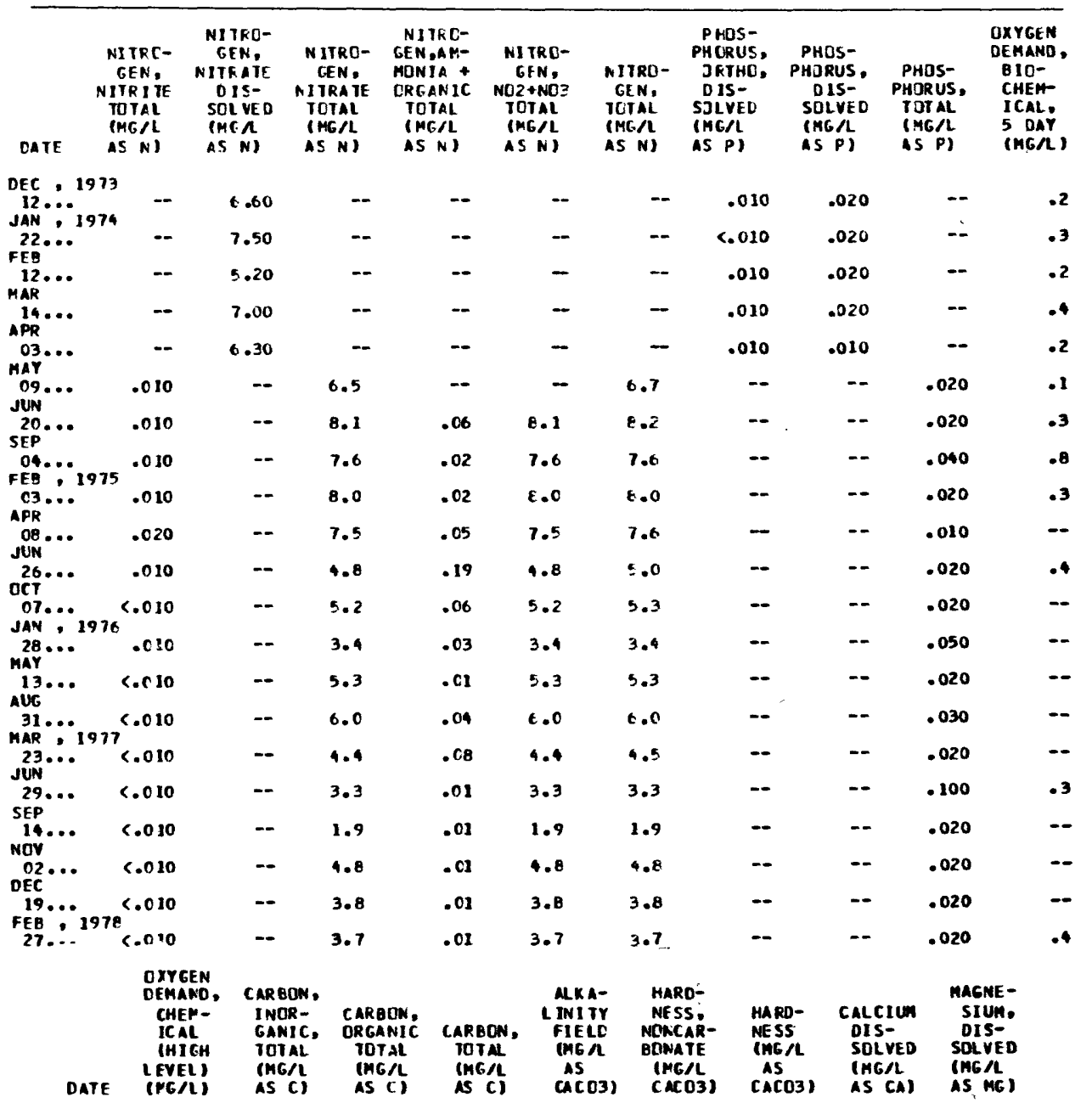

\begin{tabular}{|c|c|c|c|c|c|c|c|c|c|}
\hline $\begin{array}{l}\text { OEC } 1973 \\
\text { JAN }\end{array}$ & 21 & 20 & .0 & 20 & e4 & 36 & 120 & 34 & 9.2 \\
\hline FEB & 22 & 20 & .0 & 20 & 85 & 35 & 120 & 39 & 6.4 \\
\hline $\operatorname{MAR}_{\operatorname{MAR}}^{12}$ & 18 & 17 & .0 & 17 & 78 & 42 & 120 & 37 & 6.5 \\
\hline$\underset{A P R}{14} \cdots$ & B & 21 & .0 & 21 & 83 & 37 & 120 & 38 & 6.0 \\
\hline $03 \ldots$ & 8 & 19 & 1.0 & $2 C$ & 26 & 44 & 130 & 41 & 6.6 \\
\hline JuN & 0 & 22 & .0 & 22 & e9 & 99 & 140 & 44 & 6.7 \\
\hline$\underset{\text { SEP }}{20} \cdots$ & 1 & 25 & .0 & 25 & -- & -- & 120 & 30 & 6.6 \\
\hline FFA : 1975 & 5 & 20 & .0 & 20 & e5 & 35 & 120 & 39 & 6.4 \\
\hline APR & 4 & - & .0 & $-\infty$ & $E 2$ & 37 & 120 & 37 & 6.4 \\
\hline Jun & $\cdots$ & $\cdots$ & .0 & -- & 90 & - & - & - & - \\
\hline $\mathrm{CCT}^{26} \cdots$ & 4 & -- & .0 & $\cdots$ & E9 & 21 & 110 & 35 & 6.1 \\
\hline JAN 07.1976 & $\cdots$ & $\cdots$ & - & -- & -- & $\cdots$ & - & $\cdots$ & $\cdots$ \\
\hline MAY & - & -- & -- & -- & $9 \mathrm{E}$ & 10 & 110 & 34 & 5.0 \\
\hline$\underset{\operatorname{aug}}{13} \cdots$ & $\cdots$ & $\cdots$ & $\cdots$ & -- & - & $\cdots$ & $\cdots$ & $\cdots$ & -- \\
\hline MAP $=1977$ & -- & - & $\cdots$ & $\cdots$ & $\cdots$ & $\cdots$ & $\cdots$ & - & - \\
\hline $23 \ldots$ & $\cdots$ & $\cdots$ & 6.0 & -- & $\cdots$ & $\cdots$ & $\cdots$ & $\cdots$ & $\cdots$ \\
\hline SEP & 2 & $\cdots$ & $\cdots$ & $\cdots$ & 99 & 21 & 120 & 36 & 6.3 \\
\hline $\operatorname{NaV}^{14} \cdot$. & $\cdots$ & $\cdots$ & $\cdots$ & $\cdots$ & -- & $\cdots$ & $\cdots$ & $\cdots$ & $\cdots$ \\
\hline DEC & 1 & $\cdots$ & - & $\cdots$ & $\cdots$ & $\cdots$ & $\cdots$ & - & $\cdots$ \\
\hline $\begin{array}{l}19 \ldots \\
27 \ldots \ldots\end{array}$ & 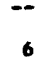 & - & $\cdots$ & $-\infty$ & 90 & 23 & 110 & 35 & 6.2 \\
\hline
\end{tabular}




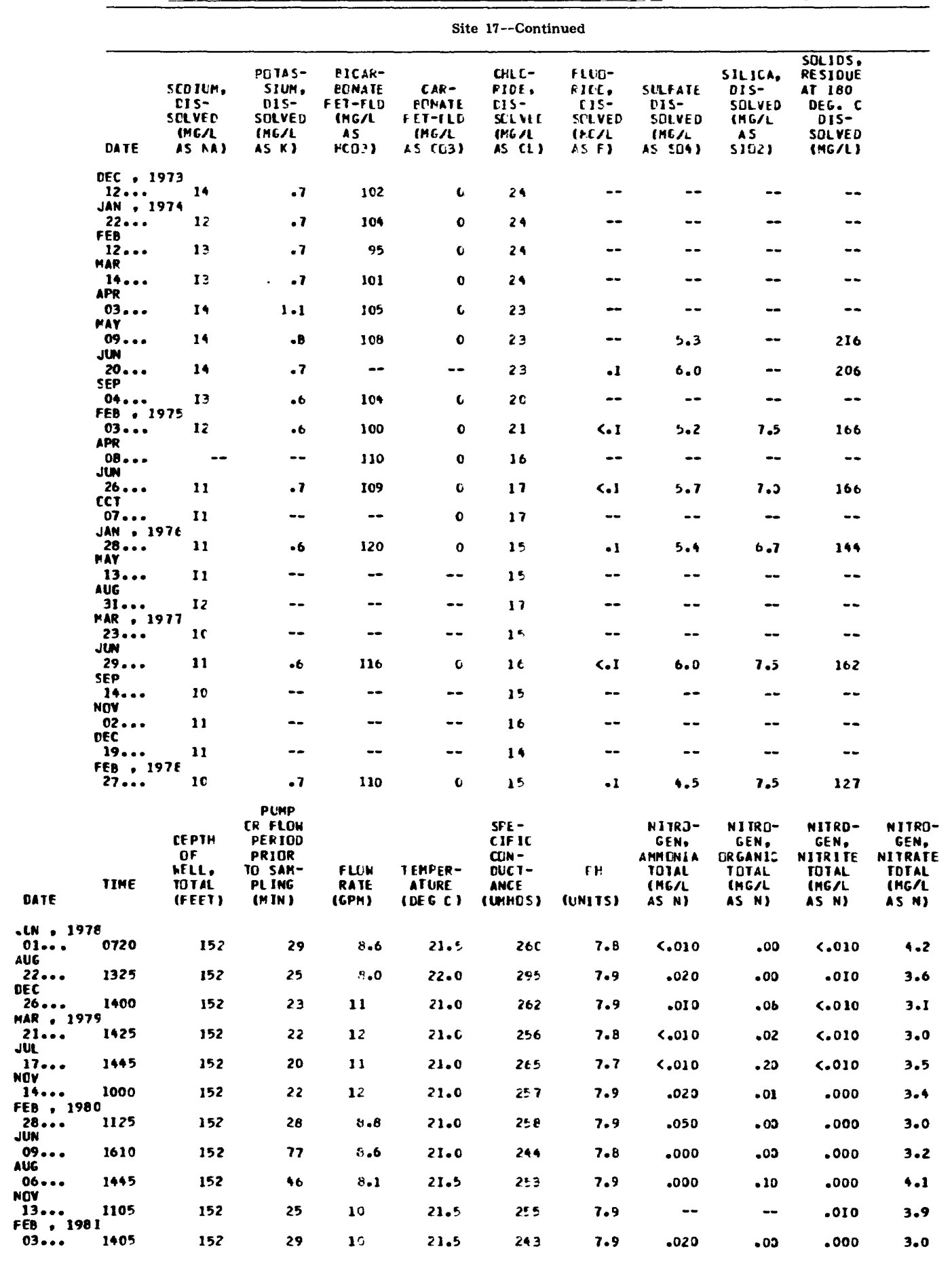




\begin{tabular}{|c|c|c|c|c|c|c|c|c|c|c|}
\hline \multicolumn{11}{|c|}{ Site 17--Continued } \\
\hline $\begin{array}{l}\text { NITRO- } \\
\text { GEN,AM- } \\
\text { MONIA + } \\
\text { ORGANIC } \\
\text { TMTAL } \\
\text { (MGNL } \\
\text { IS N) }\end{array}$ & $\begin{array}{l}\text { ATTRD- } \\
\text { GEN, } \\
\text { NC2 +ND3 } \\
\text { TOTAL } \\
\text { IMGA } \\
\text { AS NI }\end{array}$ & $\begin{array}{l}\text { N1 TRO- } \\
\text { GEN, } \\
\text { IOIAL } \\
\text { IMG/L } \\
\text { IS N) }\end{array}$ & $\begin{array}{l}\text { PHOS- } \\
\text { PHORUS, } \\
\text { IOTAL } \\
\text { IMG } \\
\text { AS PI }\end{array}$ & $\begin{array}{l}\text { CARBLN, } \\
\text { ORGANIC } \\
\text { DIS- } \\
\text { SOLVED } \\
\text { (MGAL } \\
\text { AS C) }\end{array}$ & $\begin{array}{l}\text { AKA- } \\
\text { LINITY } \\
\text { FIELD } \\
\text { IMGA } \\
\text { AS } \\
\text { CACE3) }\end{array}$ & $\begin{array}{l}\text { HAFD- } \\
\text { ME SS, } \\
\text { NCNCAR- } \\
\text { BQNATE } \\
\text { (HC AL } \\
\text { CACO3) }\end{array}$ & $\begin{array}{l}\text { HARD- } \\
\text { NESS } \\
\text { (AGA } \\
\text { AS } \\
\text { CACO3) }\end{array}$ & $\begin{array}{l}\text { CALCIUH } \\
\text { DIS- } \\
\text { SDLVED } \\
\text { (MC/L } \\
\text { AS CA) }\end{array}$ & $\begin{array}{l}\text { MAGNE- } \\
\text { SIUH. } \\
\text { DIS- } \\
\text { SOLVED } \\
\text { (MG/L } \\
\text { AS MG) }\end{array}$ & $\begin{array}{l}\text { SODIUN, } \\
\text { DIS- } \\
\text { SOLVED } \\
\text { (MGAL } \\
\text { AS MA) }\end{array}$ \\
\hline
\end{tabular}

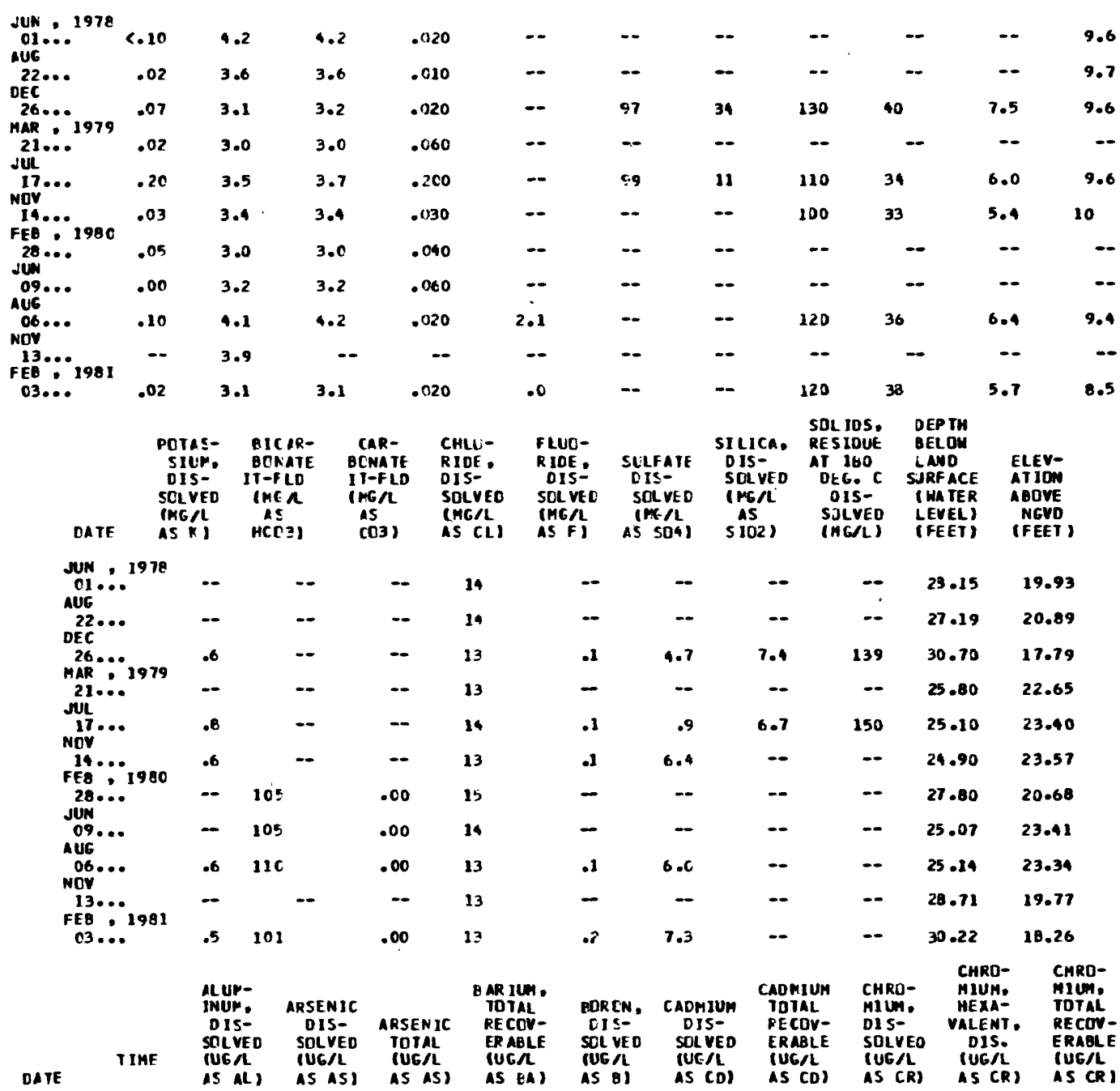

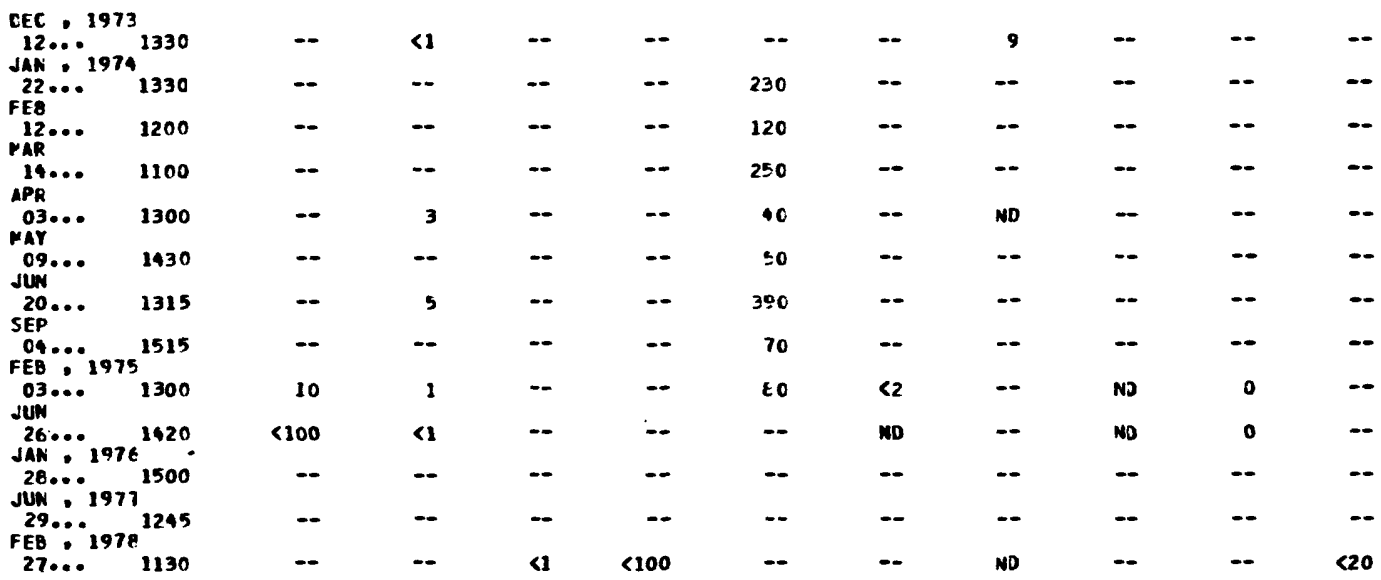


Site 17--Continued

\begin{tabular}{|c|c|c|c|c|c|c|c|c|c|c|c|}
\hline CATE & $\begin{array}{l}\text { CCE } \\
\text { nI } \\
\text { snL } \\
\text { lu } \\
\text { as }\end{array}$ & $\begin{array}{l}\text { LT, } \\
\text { Ec } \\
\text { (L) } \\
\text { (C) }\end{array}$ & $\begin{array}{l}\text { COPFER, } \\
\text { DIS- } \\
\text { SOIVEO } \\
\text { luGR } \\
\text { AS (U) }\end{array}$ & $\begin{array}{l}\text { COFPER, } \\
\text { TCTAL } \\
\text { RECOV- } \\
\text { ERABLE } \\
\text { (UG/L } \\
\text { AS CUS }\end{array}$ & $\begin{array}{l}\text { IRON } \\
\text { DIS- } \\
\text { SOLVEO } \\
\text { (UGAL } \\
\text { ASFE) }\end{array}$ & 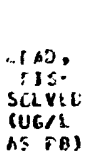 & $\begin{array}{l}\text { LFAD, } \\
\text { TCTAL } \\
\text { HCCOY- } \\
\text { EFBBLE } \\
\text { IUL } / L \\
\text { AS PE) }\end{array}$ & $\begin{array}{c}\text { LJ THILM } \\
\text { OIS- } \\
\text { SOLVED } \\
\text { (U6/L } \\
\text { ASLI) }\end{array}$ & $\begin{array}{l}\text { MANGA- } \\
\text { NESL, } \\
\text { IJTAL } \\
\text { RLCOV- } \\
\text { ERABLE } \\
\text { (UGAL } \\
\text { AS MN) }\end{array}$ & $\begin{array}{l}\text { IANGA- } \\
\text { VESE, } \\
\text { OIS- } \\
\text { SOLVED } \\
\text { (USAL } \\
\text { AS MNI }\end{array}$ & $\begin{array}{c}\text { MERCURY } \\
\text { DIS- } \\
\text { SOLVED } \\
\text { (UG/L } \\
\text { AS HG) }\end{array}$ \\
\hline $\begin{array}{l}\text { DEC } \because 1 \\
12 \ldots \cdots \\
\text { JAN }: 1\end{array}$ & $\begin{array}{l}973 \\
974\end{array}$ & -- & $<2$ & $\cdots$ & $<10$ & $\boldsymbol{\varepsilon}$ & $\cdots$ & -- & $\cdots$ & 14 & - \\
\hline FEB & & - & -- & -- & - & - & - & - & -- & - & -- \\
\hline $12 \ldots$ & & $\cdots$ & -- & - & - & - & -- & -- & $\cdots$ & $\cdots$ & $\cdots$ \\
\hline APR & & $\cdots$ & $\cdots$ & - & $\cdots$ & -- & - & $\cdots$ & -- & -- & -- \\
\hline $\begin{array}{l}03 \ldots . . . \\
\text { MAY }\end{array}$ & & - & NO & - & 40 & 7 & -- & -- & $\cdots$ & $<10$ & -- \\
\hline JLN & & - & -- & - & $\cdots$ & - & - & -- & $\cdots$ & -- & $\cdots$ \\
\hline$\underset{\text { SEP }}{20} .$. & & - & -- & - & $\cdots$ & - & - & $\cdots$ & - & $\cdots$ & $\cdots$ \\
\hline$\underset{F E \theta}{04 \cdots}$ & 975 & - & - & -- & - & - & - & - & -- & -- & - \\
\hline JUN & & AD & NO & - & $<10$ & $\bar{z}$ & - & $<10$ & -- & $<10$ & $<.5$ \\
\hline JAN 261 & 976 & AD & $<2$ & - & $<10$ & 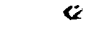 & $\cdots$ & $<10$ & -- & $<10$ & $<.5$ \\
\hline JUW $\because 1$ & 977 & - & - & - & - & - & -- & $\cdots$ & - & $\cdots$ & -- \\
\hline $\begin{array}{c}29 \ldots \\
27 \ldots 1\end{array}$ & 978 & $\cdots$ & $-\infty$ & 3 & - & - & - & - & $<10$ & - & - \\
\hline DATE & $\begin{array}{l}\text { MER } \\
\text { TC } \\
\text { RE } \\
\text { ER } \\
\text { IU } \\
\text { AS }\end{array}$ & $\begin{array}{l}\text { CURY } \\
\text { AL } \\
\text { CY- } \\
\text { eLE } \\
\text { Hel } \\
\text { Hel }\end{array}$ & $\begin{array}{l}\text { MOL IB- } \\
\text { DERLA, } \\
\text { DIS- } \\
\text { SDL VEO } \\
\text { (UCA } \\
\text { AS MD) }\end{array}$ & $\begin{array}{l}\text { AICKEL, } \\
\text { DIS- } \\
\text { SOLVED } \\
\text { (UG/L } \\
\text { ASNI) }\end{array}$ & $\begin{array}{l}\text { SELL- } \\
\text { NILIA, } \\
\text { DIS- } \\
\text { SOLVED } \\
\text { (UC/L } \\
\text { AS SE) }\end{array}$ & $\begin{array}{l}\text { SELE- } \\
\text { NIUR, } \\
\text { TOTAL } \\
\text { (UETL } \\
\text { AS SE) }\end{array}$ & $\begin{array}{l}\text { SILVEF, } \\
\text { ICTAL } \\
\text { RECOV- } \\
\text { EFABLE } \\
\text { (UG/L } \\
\text { AS AG) }\end{array}$ & $\begin{array}{l}\text { S TRON- } \\
\text { TIUM. } \\
\text { UIS- } \\
\text { SUVED } \\
\text { (UG/L } \\
\text { AS SF) }\end{array}$ & $\begin{array}{l}\text { VANA- } \\
\text { DIUM, } \\
\text { DIS- } \\
\text { SOLVED } \\
\text { IUG/L } \\
\text { AS VI }\end{array}$ & $\begin{array}{l}\text { ZINC, } \\
\text { OIS- } \\
\text { SOLVED } \\
\text { (UGR } \\
\text { IS ZN) }\end{array}$ & $\begin{array}{l}\text { ZINC, } \\
\text { IOTAL } \\
\text { RE COV- } \\
\text { ERABLE } \\
\text { (UG/L } \\
\text { AS } 2 N \text { ) }\end{array}$ \\
\hline OFC 1 & 973 & $\cdots$ & $\cdots$ & - & - & $\cdots$ & $\cdots$ & $\cdots$ & -- & 50 & -- \\
\hline $\begin{array}{l}J_{22} \cdots 1 \\
F E 8\end{array}$ & 974 & - & - & $\cdots$ & - & - & - & $\cdots$ & - & $\cdots$ & $\cdots$ \\
\hline MAR & & - & -- & - & $\cdots$ & - & - & -- & -- & $\cdots$ & $\cdots$ \\
\hline$\underset{A P R}{14} \cdots$ & & - & -- & $\cdots$ & - & - & - & -- & $\cdots$ & $\cdots$ & $\cdots$ \\
\hline $03 \ldots$ & & $<.5$ & $\cdots$ & $\cdots$ & $\cdots$ & - & - & $-\infty$ & $-\infty$ & 40 & $\cdots$ \\
\hline $\begin{array}{l}\text { MAY } \\
\text { O9.... } \\
\text { JUN }\end{array}$ & & - & -- & $\cdots$ & $\cdots$ & $\cdots$ & $\cdots$ & $\cdots$ & $\cdots$ & $=-$ & $\cdots$ \\
\hline$\underset{\text { SEP }}{20} \cdots$ & & - & $=$ & -- & - & -- & - & -- & $\cdots$ & $\cdots$ & - \\
\hline FEB $\because$ & 975 & $\cdots$ & $\cdots$ & -- & $\cdots$ & -- & - & -- & -- & -- & -- \\
\hline JUN & & - & 1 & ND & - & -- & - & $\cdots$ & 3.0 & 20 & $\cdots$ \\
\hline JAN 26 & 976 & -- & $<1$ & ND & $<1$ & $\cdots$ & - & 100 & .0 & 40 & -- \\
\hline SUN $\because 1$ & 977 & - & - & $\cdots$ & - & - & $\cdots$ & 120 & $\cdots$ & $\cdots$ & - \\
\hline FEB 29,1 & 978 & -- & - & $\cdots$ & - & $\cdots$ & $\cdots$ & 100 & - & $\cdots$ & -- \\
\hline $27 \ldots$ & & C.5 & $\cdots$ & - & - & $\mathbf{\alpha}$ & $<$ & 130 & $-\infty$ & $\cdots$ & $<20$ \\
\hline
\end{tabular}

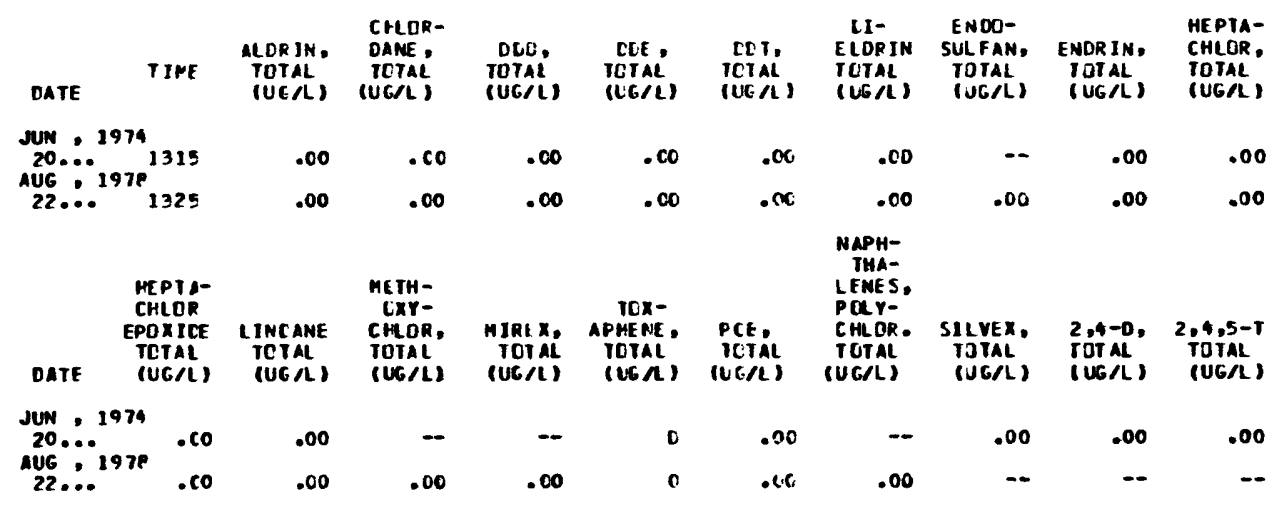


Table 6.--Physical and chemical characteristics of well and water at sites 1 through 70--Continued

\begin{tabular}{|c|c|c|c|c|c|c|c|c|c|c|}
\hline \multicolumn{11}{|c|}{ Site 18} \\
\hline TIME & $\begin{array}{l}\text { TEPTH } \\
\text { OF } \\
\text { HELL, } \\
\text { TOTAL } \\
\text { (FEET) }\end{array}$ & $\begin{array}{l}\text { PLMP } \\
\text { CR FLLOH } \\
\text { PERIOD } \\
\text { PRIOR } \\
\text { PO SAM- } \\
\text { PLING } \\
\text { (MIN) }\end{array}$ & $\begin{array}{l}\text { FLDM } \\
\text { RATE } \\
\text { (GPM) }\end{array}$ & $\begin{array}{l}\text { TEMPER- } \\
\text { ATURE } \\
\text { (EEG C) }\end{array}$ & $\begin{array}{l}\text { SPE - } \\
\text { CIF IC } \\
\text { CON- } \\
\text { OUC T- } \\
\text { ANCE } \\
\text { (UKHOS) }\end{array}$ & $\begin{array}{c}\text { FH } \\
\text { (UNITS) }\end{array}$ & $\begin{array}{l}\text { MIIRJ- } \\
\text { GEN, } \\
\text { AMMONIA } \\
\text { JIS- } \\
\text { SJLVEO } \\
\text { (HG/L } \\
\text { AS N) }\end{array}$ & $\begin{array}{l}\text { NITRO- } \\
\text { GEN. } \\
\text { AMMONIA } \\
\text { TOTAL } \\
\text { (MGR } \\
\text { AS N) }\end{array}$ & $\begin{array}{l}\text { MIIRD- } \\
\text { GEN, } \\
\text { ORGANIC } \\
\text { OIS- } \\
\text { SOLVED } \\
\text { (MGA } \\
\text { IS N) }\end{array}$ & $\begin{array}{l}\text { NITRO- } \\
\text { GEN, } \\
\text { ORGANIC } \\
\text { TOTAL } \\
\text { (HGN } \\
\text { AS N) }\end{array}$ \\
\hline
\end{tabular}

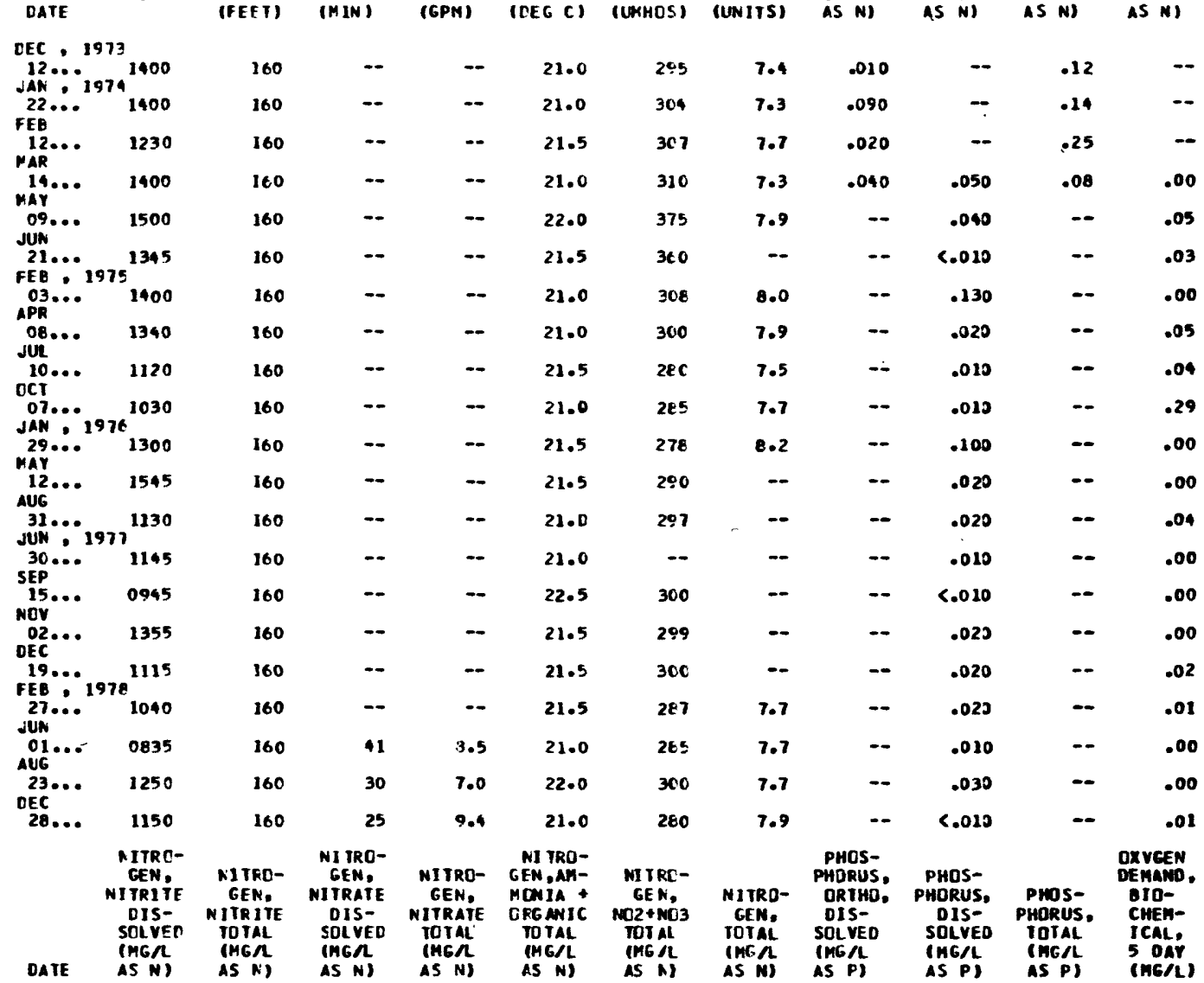

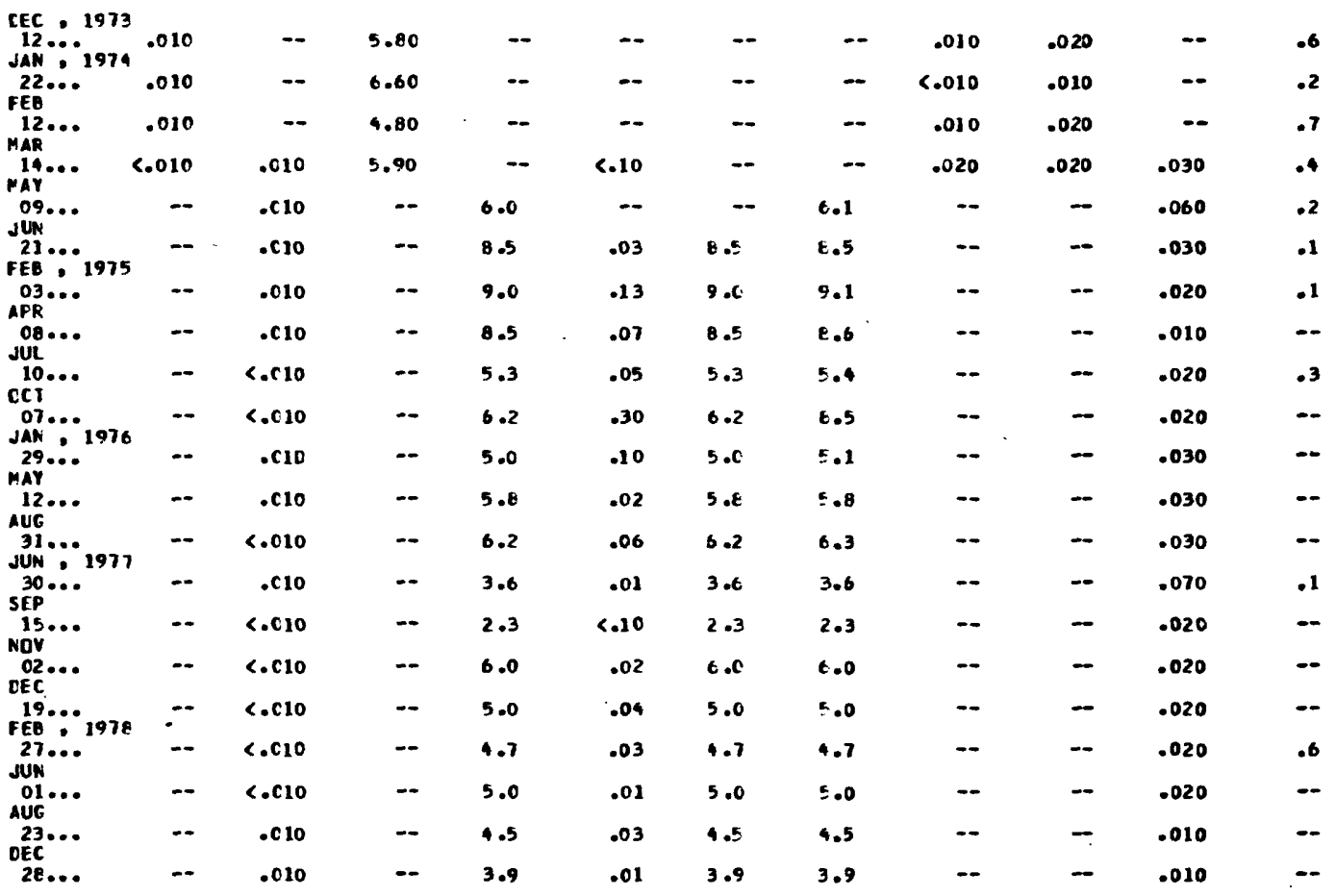




\begin{tabular}{|c|c|c|c|c|c|c|c|c|c|c|c|}
\hline & \multicolumn{11}{|c|}{ Site 18--Continued } \\
\hline DATE & $\begin{array}{l}\text { Dxy } \\
\text { off } \\
\text { CH } \\
\text { IC } \\
\text { in } \\
\text { LFV } \\
\text { img }\end{array}$ & & $\begin{array}{l}\text { CARE CN. } \\
\text { INCR- } \\
\text { GARIC. } \\
\text { IOTM } \\
\text { IMGA } \\
\text { AS Cl }\end{array}$ & $\begin{array}{l}\text { CARBON, } \\
\text { ORGANIC } \\
\text { TCTAL } \\
\text { IPGA } \\
\text { ASCI }\end{array}$ & $\begin{array}{l}\text { CAREGR, } \\
\text { IOTAL } \\
\text { (MGG/ } \\
\text { AS C) }\end{array}$ & $\begin{array}{l}\text { ALKA- } \\
\text { Lifity } \\
\text { FIELO } \\
\text { (PG/L } \\
\text { AS } \\
\text { CACO3) }\end{array}$ & 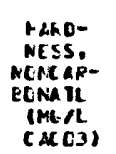 & $\begin{array}{l}\text { HARO- } \\
\text { NESS } \\
\text { (nG/L } \\
\text { AS } \\
\text { CACO3) }\end{array}$ & $\begin{array}{l}\text { CALCIUH } \\
\text { OIS- } \\
\text { SOLVEO } \\
\text { (MGLL } \\
\text { AS CA) }\end{array}$ & 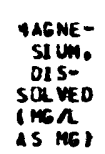 & $\begin{array}{c}\text { SODIUN, } \\
\text { DIS- } \\
\text { SOLVED } \\
\text { IMG/L } \\
\text { AS MA I }\end{array}$ \\
\hline & 973 & o & 25 & 2.0 & 27 & $\approx$ & $\because$ & - & $\cdots$ & - & $\cdots$ \\
\hline & & 0 & 22 & .0 & $2 z$ & 79 & 41 & 120 & 29 & 6.4 & 11 \\
\hline & & 16 & 21 & .0 & 21 & ge & 35 & 120 & 30 & 6.6 & 12 \\
\hline 34. & & 12 & $: 2$ & .0 & 22 & $\boldsymbol{x}$ & 34 & 120 & 36 & 6.8 & 13 \\
\hline$\because$ & & 4 & 21 & .0 & 21 & 89 & 4 & 340 & 43 & 0.7 & 12 \\
\hline & & 6 & $i z$ & .0 & 23 & -- & - & 120 & 36 & 0.5 & 12 \\
\hline 03. & & 4 & - & - & - & $x$ & 3 & 130 & 39 & 7.0 & 12 \\
\hline & & $\cdots$ & $\cdots$ & .0 & -- & 90 & - & -- & - & - & - \\
\hline 10. & & - & -- & .0 & - & $\boldsymbol{*}$ & 26 & 110 & 35 & 0.3 & 11 \\
\hline 07. & & -- & - & -- & - & $x$ & $\cdots$ & -- & - & $\cdots$ & 11 \\
\hline 29. & & - & - & - & - & $\%$ & 19 & 120 & 37 & 0.0 & 11 \\
\hline 12 . & & $-\infty$ & - & -- & $\cdots$ & - & - & - & - & $\cdots$ & 12 \\
\hline 31. & דות & - & - & - & - & - & - & - & $\cdots$ & - & 12 \\
\hline 30. & & 10 & - & 5.7 & -- & $\boldsymbol{x}$ & 23 & 120 & 37 & 6.2 & 11 \\
\hline 15. & & $\cdots$ & $\cdots$ & - & -- & - & - & - & - & $\ldots$ & 13 \\
\hline & & 2 & - & - & - & - & - & - & $\ldots$ & -- & 13 \\
\hline & & - & - & - & - & - & - & - & - & $\cdots$ & 12 \\
\hline & & 2 & - & - & - & 90 & $3 i$ & 120 & 30 & 6.5 & 12 \\
\hline & & - & - & - & - & - & -. & - & $\cdots$ & $\ldots$ & 11 \\
\hline & & $\ldots$ & - & - & - & - & - & $\cdots$ & $\cdots$ & $\cdots$ & 11 \\
\hline 28. & & - & - & $\infty$ & - & 98 & 35 & 130 & 4 & 2.3 & $\|$ \\
\hline SAIE & & & 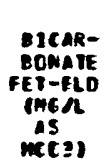 & 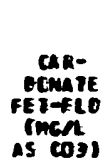 & 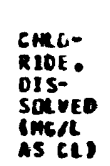 & 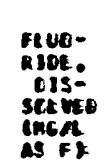 & $\begin{array}{l}\text { sulfate } \\
\text { ols- } \\
\text { satues } \\
\text { imede } \\
\text { as soss }\end{array}$ & 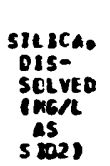 & 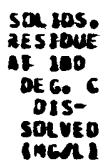 & 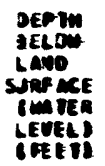 & 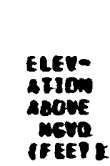 \\
\hline & 733 & .- & 98 & 0 & 22 & - & $\rightarrow$ & $\ldots$ & $\ldots$ & $\infty$ & $\infty$ \\
\hline & & .7 & 96 & 0 & 23 & $\infty$ & $\cdots$ & -- & $\cdots$ & $\cdots$ & .. \\
\hline & & $\infty$ & 104 & c & 22 & $\infty$ & - & - & $\ldots$ & - & $\cdots$ \\
\hline & & . & 105 & - & 23 & - & 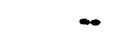 & $\ldots$ & $\cdots$ & $\ldots$ & $\cdots$ \\
\hline & & .7 & 108 & 0 . & 23 & - & 4.0 & - & 214 & - & -- \\
\hline & & .7 & - & - & 20 & .2 & 5.2 & -- & 241 & - & -. \\
\hline & & .6 & 110 & 0 & 22 & <.\} & 4.9 & 7.6 & 181 & $\ldots$ & $\cdots$ \\
\hline & & -- & 110 & 0 & 19 & - & - & $\cdots$ & - & - & -. \\
\hline 0 & & .7 & 107 & 0 & 18 & 2 & 0.6 & 7.3 & 160 & - & 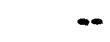 \\
\hline & & - & 110 & 0 & 20 & - & - & -- & - & -- & $\ldots$ \\
\hline & & .8 & 120 & 0 & 17 & $<.1$ & 5.6 & 6.6 & 153 & - & - \\
\hline 12 & & - & $\cdots$ & - & 17 & - & - & -- & $\cdots$ & - & - \\
\hline 31 & & - & -- & - & 19 & - & - & -- & -- & - & - \\
\hline 3 & & .6 & 116 & 0 & 17 & $<.1$ & 0.0 & 7.5 & 162 & -- & - \\
\hline 15. & & -- & - & -- & 19 & - & - & -- & -- & - & - \\
\hline & & - & -- & - & 19 & -- & - & - & - & - & -. \\
\hline & & - & $\ldots$ & - & 16 & - & -- & - & - & - & -- \\
\hline & & . & 110 & 0 & 18 & $<.2$ & 7.6 & 7.6 & 146 & -- & - \\
\hline & & -- & - & - & 16 & - & - & - & - & 37.03 & 20.07 \\
\hline & & - & - & - & 17 & - & - & - & -. & 36.81 & 21.09 \\
\hline${ }_{2}$ & & .7 & -- & - & 15 & $<.1$ & 5.4 & 7.4 & 163 & 40.30 & 17.98 \\
\hline
\end{tabular}




\section{Site 18--Continued}

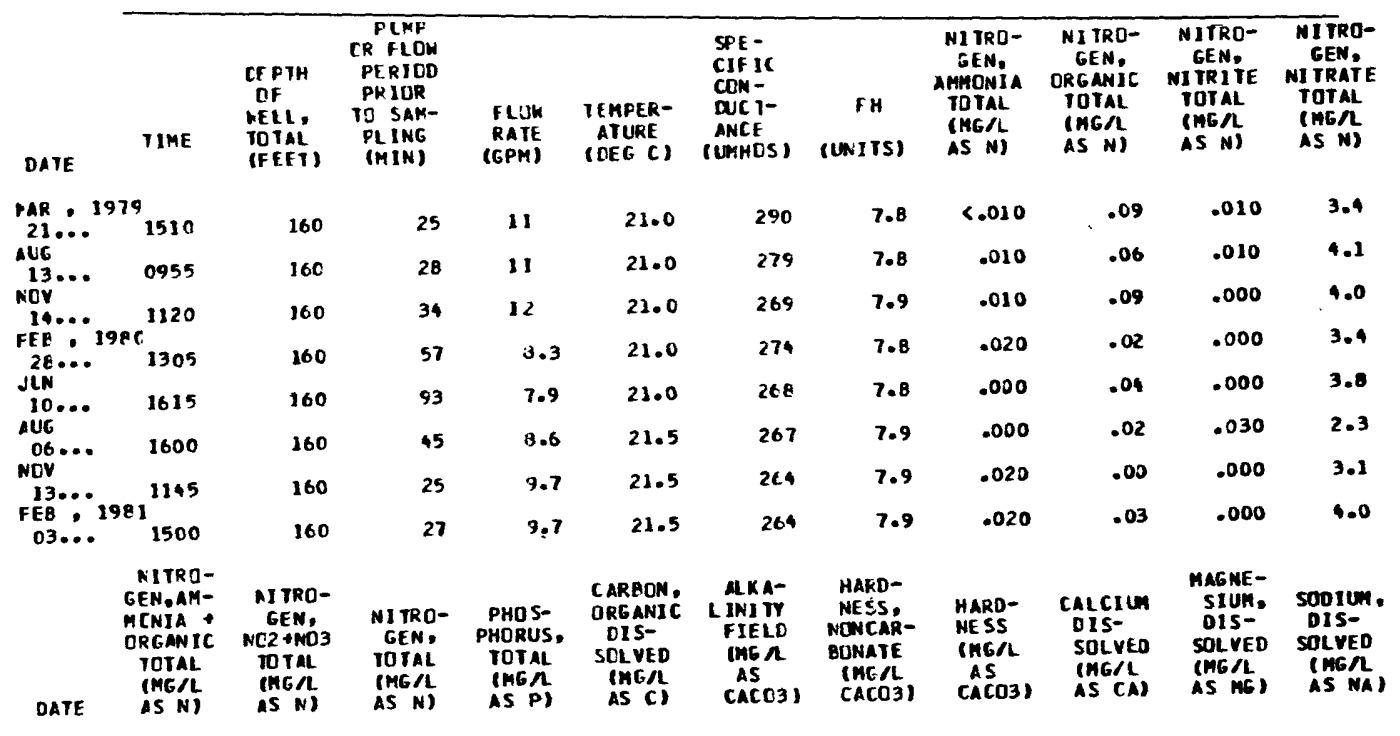

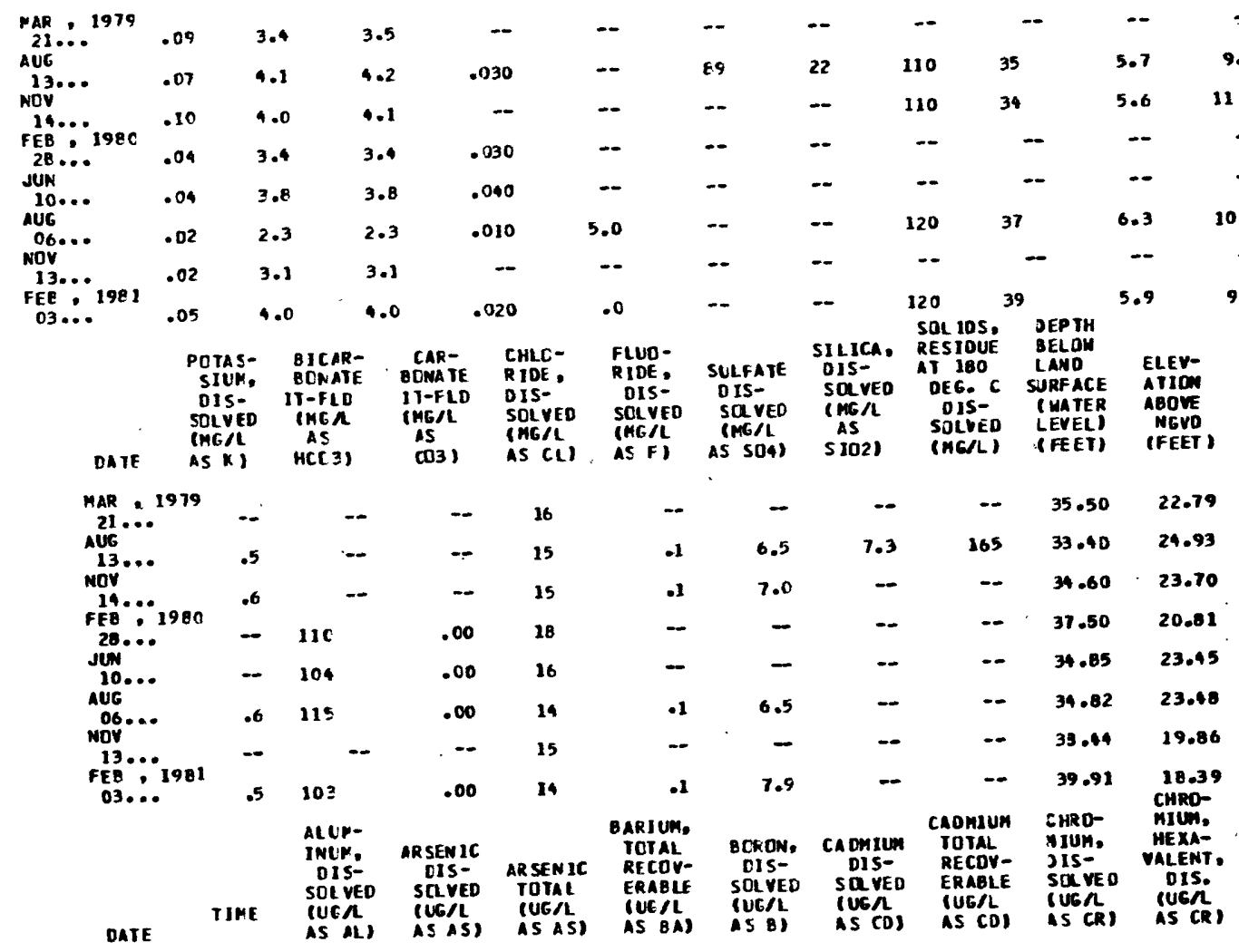

DEC 1973 12. $140 \mathrm{C}$

J2. 1974 140C

FEB $12 . .123 C$

MAR $14 . . .140 \mathrm{C}$

MaY $09 . .2150 \mathrm{C}$

JUW 134 5

FEB.. 1975 1400

JUL

JAN 19761120

$29.0130 \mathrm{C}$

JN : 19771145

FFB: 197e 


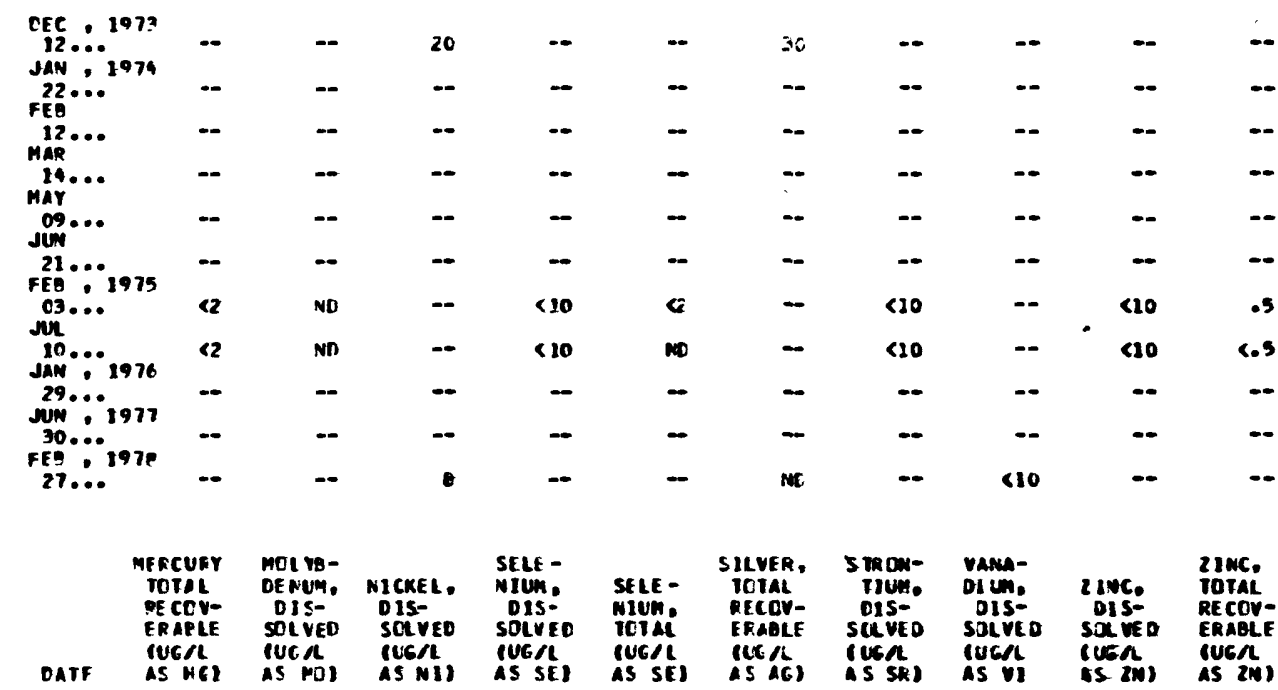

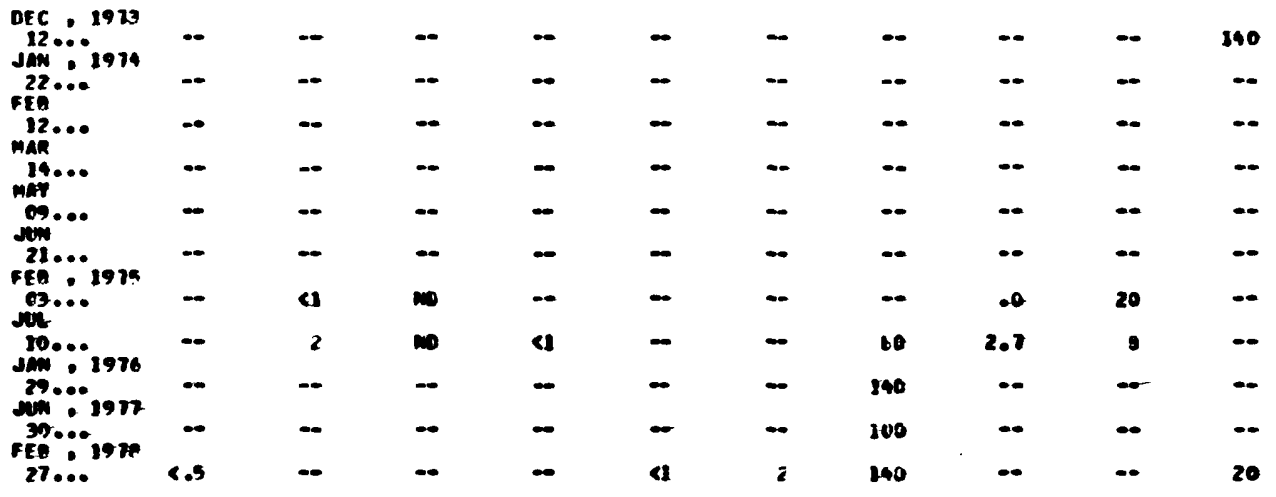

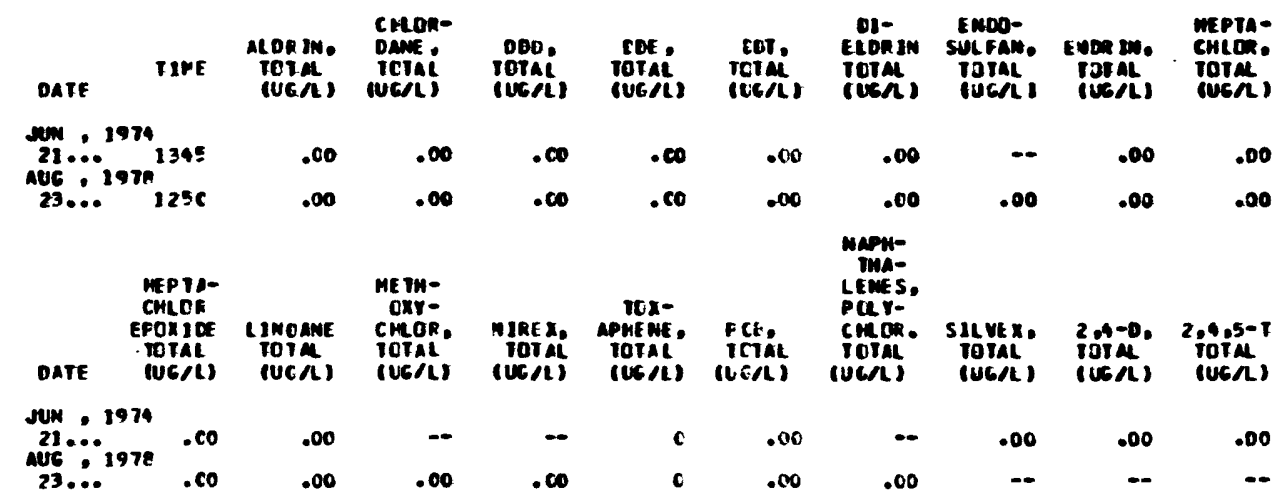


Site 19

\begin{tabular}{|c|c|c|c|c|c|c|c|c|c|c|c|}
\hline DATE & TIME & $\begin{array}{l}\text { CEPTH } \\
\text { OF } \\
\text { MELL. } \\
\text { IOTAL } \\
\text { (FEFT) }\end{array}$ & $\begin{array}{l}\text { PUMP } \\
\text { CR FLIOH } \\
\text { PERIDO } \\
\text { PRIDR } \\
\text { TU SAM- } \\
\text { PLING } \\
\text { (MIN) }\end{array}$ & $\begin{array}{l}\text { FLUM } \\
\text { RATE } \\
\text { (GPM) }\end{array}$ & $\begin{array}{l}\text { TEMPER- } \\
\text { ATURE } \\
\text { (IEEG C) }\end{array}$ & $\begin{array}{l}\text { SPE - } \\
\text { CIF IC } \\
\text { CON- } \\
\text { CUC I- } \\
\text { ANCE } \\
\text { (UAHCS) }\end{array}$ & $\begin{array}{c}\text { PH } \\
\text { (UNits) }\end{array}$ & $\begin{array}{l}\text { NIIRJ- } \\
\text { GEN, } \\
\text { AHMONIA } \\
\text { OIS- } \\
\text { SOLVED } \\
\text { (MG L } \\
\text { AS N) }\end{array}$ & $\begin{array}{l}\text { MITRO- } \\
\text { GEN, } \\
\text { AMHONIA } \\
\text { TOTAL } \\
\text { (MG/L } \\
\text { AS N) }\end{array}$ & $\begin{array}{l}\text { MIIRO- } \\
\text { GEN, } \\
\text { ORGANIC } \\
\text { OIS- } \\
\text { SOLVEO } \\
\text { (HGAL } \\
\text { AS N) }\end{array}$ & $\begin{array}{l}\text { NITRD- } \\
\text { GEN, } \\
\text { ORGANIC } \\
\text { TOTAL } \\
\text { (MG/L } \\
\text { AS N) }\end{array}$ \\
\hline $\begin{array}{l}\text { CEC : } \\
19 . .\end{array}$ & 73400 & 80 & - & $-\infty$ & 21.0 & 155 & 7.9 & .020 & -- & .22 & - \\
\hline FEB & $75^{0900}$ & 80 & -- & - & 22.0 & 125 & - & .020 & -- & .00 & - \\
\hline OPR & 1000 & ec & - & -- & 21.0 & IE I & 7.9 & $\cdots$ & .020 & -- & .00 \\
\hline JUN & 1430 & 80 & -- & - & 21.5 & 150 & 7.6 & -- & .030 & -- & .00 \\
\hline $24 \ldots$ & 1530 & 80 & - & -- & 21.5 & 145 & 6.1 & $\cdots$ & .020 & -- & .00 \\
\hline JAN... & $770^{1300}$ & 80 & - & $\rightarrow$ & 21.0 & 126 & 7.7 & - & .013 & $\cdots$ & .00 \\
\hline $29 . \cdots$ & 1515 & 80 & - & $m$ & 21.5 & 152 & e.2 & - & .030 & -- & .00 \\
\hline$\underset{\text { AUG }}{12} \cdots$ & 1200 & 80 & $\cdots$ & -- & 21.5 & 156 & - & -- & .010 & $\cdots$ & .00 \\
\hline DEC & 1100 & 80 & $\cdots$ & -- & $21: 5$ & 150 & $-\infty$ & - & .010 & -- & .04 \\
\hline$\underset{\text { MAR }}{15 \ldots}$ & $77^{1530}$ & 80 & - & - & 21.5 & -- & 7.6 & -- & .010 & - & .02 \\
\hline JUN $17 .$. & 0930 & 80 & $\cdots$ & -- & -- & 150 & -- & -- & $<.013$ & - & .03 \\
\hline$\underset{\text { SEP }}{15} \ldots$ & 1100 & 80 & $\cdots$ & $\cdots$ & 21.5 & - & - & $m$ & $<.010$ & -- & .14 \\
\hline nov 15 & 1530 & 80 & -- & - & - & 170 & - & - & .010 & -- & .00 \\
\hline OEC & 1045 & 80 & - & - & 21.0 & 150 & - & $\cdots$ & .010 & -- & .00 \\
\hline ray... & $778^{0945}$ & 80 & - & $\cdots$ & 22.0 & 173 & - & -- & .020 & -- & .00 \\
\hline$\underset{A U G}{23} \cdots$ & 1010 & 80 & 38 & 3.5 & 21.0 & $15 \mathrm{C}$ & 7.6 & - & .010 & $m$ & .01 \\
\hline OEC & 1120 & $8 C$ & 20 & 8.0 & 21.5 & $1+9$ & 7.8 & - & .030 & -- & .00 \\
\hline AUE & $779^{1345}$ & 80 & 25 & 3.0 & 20.0 & $3 \in]$ & 7.7 & - & .012 & -- & .01 \\
\hline $13 \ldots$ & 1925 & 80 & 19 & 12 & 21.0 & 155 & 7.6 & -- & -- & $\cdots$ & - \\
\hline 15... & $8 c^{0910}$ & 80 & 29 & 12 & 21.0 & 147 & 7.9 & -- & .010 & -- & .01 \\
\hline $20 \ldots$ & 1420 & 80 & 28 & 8.8 & 20.5 & 172 & 7.8 & -- & - & -- & $\cdots$ \\
\hline DATE & $\begin{array}{l}\text { NITRO- } \\
\text { GEN, } \\
\text { NITRITF } \\
\text { OIS- } \\
\text { SOLVED } \\
\text { (MG/L } \\
\text { AS N) }\end{array}$ & $\begin{array}{l}\text { MITRO- } \\
\text { GER, } \\
\text { NIRRIIE } \\
\text { YOTAL } \\
\text { IMGA } \\
\text { IS MI }\end{array}$ & $\begin{array}{l}\text { N1JPO- } \\
\text { GEN, } \\
\text { MITRATE } \\
\text { DIS- } \\
\text { SOLVEO } \\
\text { (HCN } \\
\text { AS N) }\end{array}$ & $\begin{array}{l}\text { NITRO- } \\
\text { GEN, } \\
\text { NITRATE } \\
\text { IOTAL } \\
\text { (MGA } \\
\text { AS N) }\end{array}$ & $\begin{array}{l}\text { NITKO- } \\
\text { CEN \&AM- } \\
\text { KONIA + } \\
\text { CRGANIC } \\
\text { IOIAL } \\
\text { (MGIL } \\
\text { AS NI }\end{array}$ & $\begin{array}{l}\text { NIT RL- } \\
\text { GE R, } \\
\text { NO2 }+ \text { NO3 } \\
\text { TOI AL } \\
\text { (MG A } \\
\text { AS N) }\end{array}$ & $\begin{array}{l}\text { NITRO- } \\
\text { GEN, } \\
\text { TOTAL } \\
\text { (NG/L } \\
\text { AS N) }\end{array}$ & $\begin{array}{l}\text { PHOS- } \\
\text { PHORUS, } \\
\text { ORTHJ, } \\
\text { OIS- } \\
\text { SOLVED } \\
\text { (MG } \\
\text { AS P) }\end{array}$ & $\begin{array}{l}\text { PHOS- } \\
\text { PHDRUS, } \\
\text { DIS- } \\
\text { SOLVED } \\
\text { (NG/L } \\
\text { AS P) }\end{array}$ & $\begin{array}{l}\text { PHOS- } \\
\text { PHORUS, } \\
\text { TOTAL } \\
\text { (MGIL } \\
\text { AS P) }\end{array}$ & $\begin{array}{l}\text { OXYGEN } \\
\text { DEMAMO. } \\
\text { BIO- } \\
\text { CHEM- } \\
\text { ICAL. } \\
5 \text { DAY } \\
\text { (MGR) }\end{array}$ \\
\hline
\end{tabular}

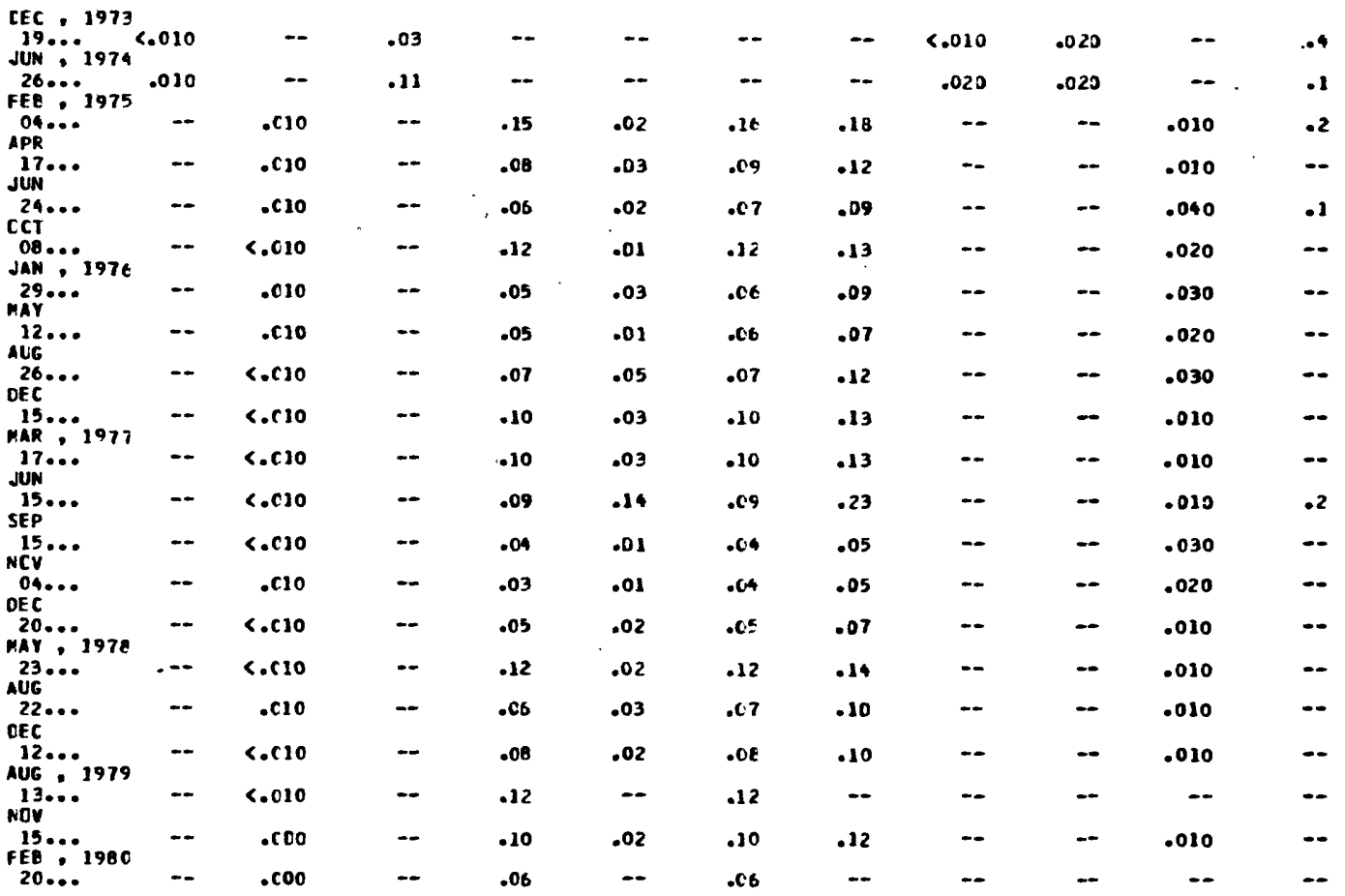


Site 19--Continued

\begin{tabular}{|c|c|c|c|c|c|c|c|c|c|c|}
\hline $\begin{array}{l}\text { CXYGEN } \\
\text { OEYAND. } \\
\text { CHEM- } \\
\text { ICAL } \\
\text { IHIGH } \\
\text { LEVEL) } \\
\text { IMGAL) }\end{array}$ & $\begin{array}{l}\text { CARECN, } \\
\text { INOR- } \\
\text { CANIC, } \\
\text { IOIAL } \\
\text { (MCAL } \\
\text { IS C) }\end{array}$ & $\begin{array}{l}\text { CAREUN, } \\
\text { CRGANIC } \\
\text { TUTAL } \\
\text { (HCAL } \\
\text { AS C) }\end{array}$ & $\begin{array}{l}\text { CARBLN, } \\
\text { IOTAL } \\
\text { (MGA } \\
\text { AS C) }\end{array}$ & $\begin{array}{l}\text { ALYA- } \\
\text { I INITY } \\
\text { FIFLD } \\
\text { CMEIL } \\
\text { AS } \\
\text { CAC(S) }\end{array}$ & $\begin{array}{l}\text { HAE C- } \\
\text { NESS, } \\
\text { NCNC AK- } \\
\text { ECNA If } \\
\text { (N:G A } \\
\text { CACE:) }\end{array}$ & $\begin{array}{l}\text { HAEU- } \\
\text { NHSS } \\
(M C / L \\
\text { AS } \\
(A C D 3)\end{array}$ & $\begin{array}{l}\text { CALCIUM } \\
\text { DIS- } \\
\text { SJIVED } \\
\text { (HG/L } \\
\text { AS CA) }\end{array}$ & $\begin{array}{l}\text { MAGNE- } \\
\text { SIUH, } \\
\text { UIS- } \\
\text { SOLVES } \\
\text { (MEIL } \\
\text { AS ME) }\end{array}$ & $\begin{array}{l}\text { SODIUM, } \\
\text { DIS- } \\
\text { SOLVED } \\
\text { (MGA } \\
\text { AS NA) }\end{array}$ & $\begin{array}{l}\text { PUTAS- } \\
\text { SIUN, } \\
\text { DIS- } \\
\text { SOL VEO } \\
\text { IMG/L } \\
\text { AS KI }\end{array}$ \\
\hline
\end{tabular}

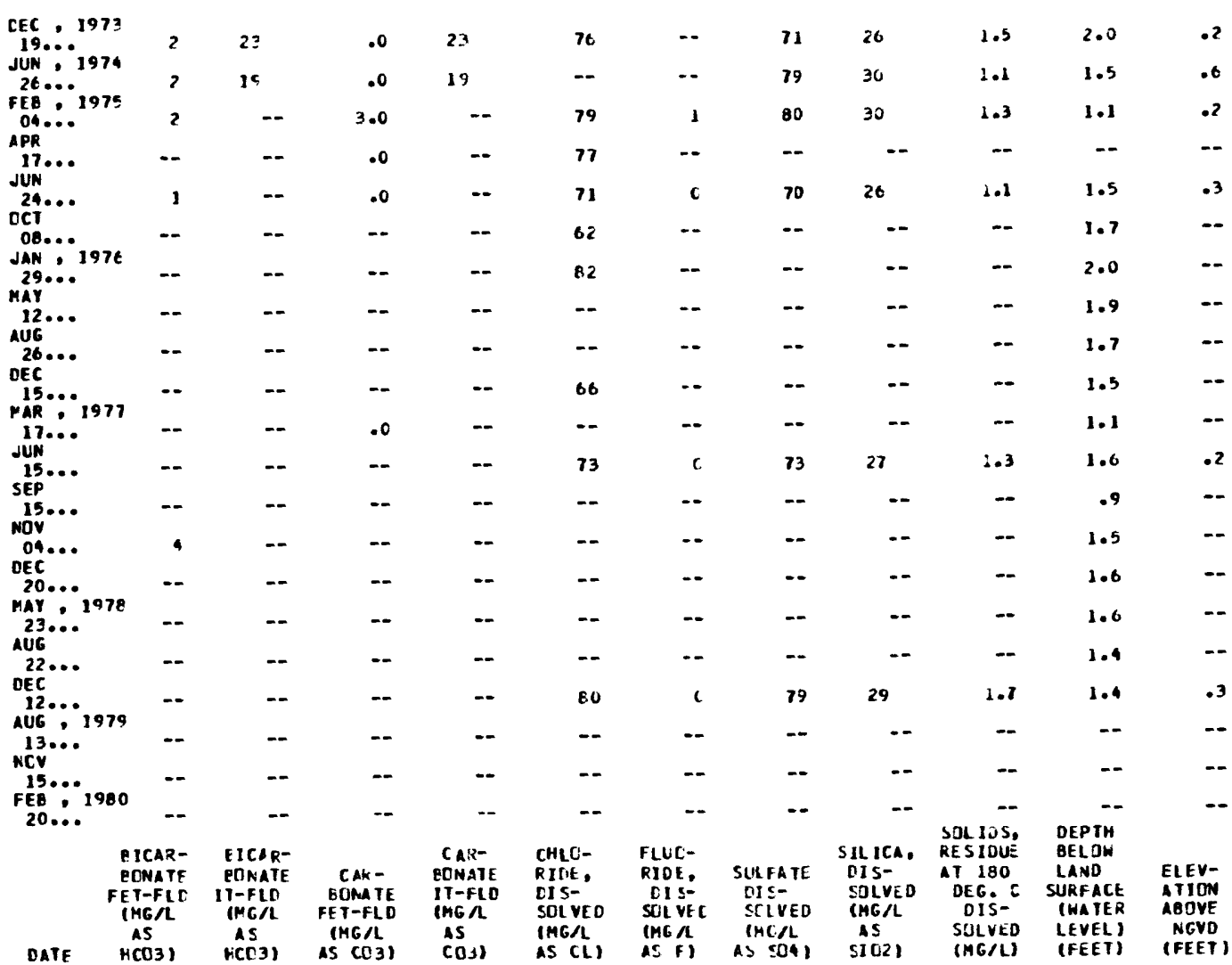

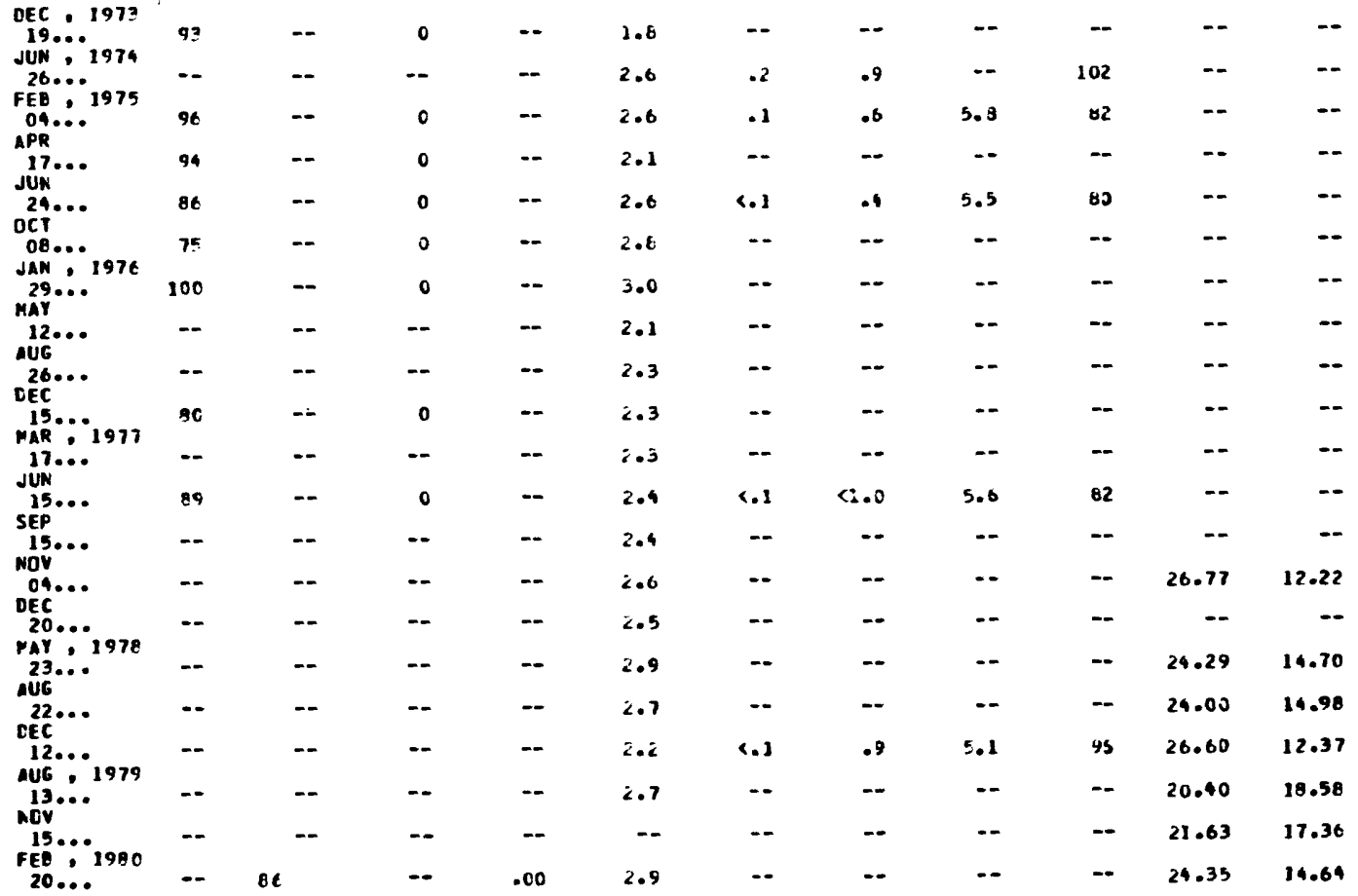


Site 19--Continued

\begin{tabular}{|c|c|c|c|c|c|c|c|c|c|}
\hline DATE & TIME & $\begin{array}{l}\text { DLFYH } \\
\text { OF } \\
\text { WELL, } \\
\text { TUTAL } \\
\text { (FEET) }\end{array}$ & $\begin{array}{l}\text { PUMP } \\
\text { OF FLOA } \\
\text { PERIOD } \\
\text { PRIOR } \\
\text { IC SAM- } \\
\text { PLING } \\
\text { (MIN) }\end{array}$ & $\begin{array}{l}\text { FLCW } \\
\text { RAIE } \\
\text { (GPM) }\end{array}$ & $\begin{array}{l}\text { TEMFEF- } \\
\text { ATUEE } \\
\text { (DEG C) }\end{array}$ & $\begin{array}{l}\text { SPE- } \\
\text { CIF IT } \\
\text { CCA- } \\
\text { DUCT- } \\
\text { ANCE } \\
\text { (UMHOS) }\end{array}$ & $\begin{array}{c}\text { FH } \\
\text { (unsts) }\end{array}$ & $\begin{array}{l}\text { NIIRU- } \\
\text { GEN. } \\
\text { AMHUNIA } \\
\text { TOTAL } \\
\text { (HG/L } \\
\text { AS NI }\end{array}$ & $\begin{array}{l}\text { NIIRO- } \\
\text { GEN, } \\
\text { ORGANIC } \\
\text { TOTAL } \\
\text { (HGAL } \\
\text { AS N) }\end{array}$ \\
\hline
\end{tabular}

\begin{tabular}{|c|c|c|c|c|c|c|c|c|c|}
\hline $\begin{array}{c}26 . \\
\text { SFP. }\end{array}$ & $134 c$ & 60 & 53 & $8 \cdot k$ & 21.5 & 168 & 7.7 & .010 & \\
\hline $09 \ldots$ & 1130 & 80 & 51 & 9.1 & 22.0 & 173 & 1.7 & -- & . \\
\hline & $\begin{array}{l}\text { MITPO- } \\
\text { GER: } \\
\text { NITRIIE } \\
\text { IOTAL } \\
\text { IMGA } \\
\text { AS M) }\end{array}$ & $\begin{array}{l}\text { NIIRO- } \\
\text { CEN, } \\
\text { AITRATE } \\
\text { TOIAL } \\
\text { IMGAL } \\
\text { AS NI }\end{array}$ & $\begin{array}{l}\text { NITRO- } \\
\text { GEN,AM- } \\
\text { MONIA } \\
\text { ORGANIC } \\
\text { TCTAL } \\
\text { (MGA } \\
\text { AS NI }\end{array}$ & $\begin{array}{l}\text { NI TKO- } \\
\text { GEN, } \\
\text { MOZ +NO3 } \\
\text { TOIAL } \\
\text { (MGNL } \\
\text { AS N) }\end{array}$ & $\begin{array}{l}\text { NIIRL- } \\
\text { GEA, } \\
\text { TEIAL } \\
\text { (MG AL } \\
\text { AS A) }\end{array}$ & $\begin{array}{l}\text { PHCS- } \\
\text { PHLFUS, } \\
\text { ICTAL } \\
\text { (MG/L } \\
\text { OS P) }\end{array}$ & $\begin{array}{l}\text { HAED- } \\
\text { NESS } \\
\text { (MG/L } \\
\text { AS } \\
\text { CACOS) }\end{array}$ & $\begin{array}{l}\text { CALCIUH } \\
\text { DIS- } \\
\text { SOLVED } \\
\text { (MG/L } \\
\text { AS CA) }\end{array}$ & $\begin{array}{l}\text { SIU } \\
\text { DIS } \\
\text { SOLVE } \\
\text { IAG } \\
\text { AS MC }\end{array}$ \\
\hline
\end{tabular}

\begin{tabular}{|c|c|c|c|c|c|c|c|c|c|c|}
\hline & &. $\operatorname{coO}$ & .07 & .04 & .07 & .31 & .040 & 80 & 30 & 1.3 \\
\hline 09. & & .000 & .08 & -- & .08 & -- & $\cdots$ & -- & - & \\
\hline & $\begin{array}{r}\text { SCD } \\
\text { CI } \\
\text { SCL } \\
\text { I } \\
\text { AS }\end{array}$ & $\begin{array}{l}1 \mathrm{ILM} \\
\text { S- } \\
\text { YED } \\
G / 2 \\
M A 1\end{array}$ & $\begin{array}{l}\text { POTAS- } \\
\text { SIUM. } \\
\text { DIS- } \\
\text { SDLVED } \\
\text { IMG/L } \\
\text { ASKI }\end{array}$ & $\begin{array}{c}\text { EICAR- } \\
\text { BONATE } \\
\text { II-FLO } \\
\text { IMG/L } \\
\text { AS } \\
\text { HCO3) }\end{array}$ & $\begin{array}{l}\text { CAR- } \\
\text { PONATE } \\
\text { IT-FLC } \\
\text { (MGAL } \\
\text { AS } \\
\text { CU,3) }\end{array}$ & $\begin{array}{l}\text { CHLC- } \\
\text { RIDE, } \\
\text { DIS- } \\
\text { SLL VEO } \\
\text { (MG IL } \\
\text { AS CLI }\end{array}$ & $\begin{array}{l}\text { FLUQ- } \\
\text { FIEE: } \\
\text { FIE- } \\
\text { SRLVEO } \\
\text { IHEA } \\
\text { AS FI }\end{array}$ & $\begin{array}{l}\text { SULFAIE } \\
\text { DIS- } \\
\text { SOLVED } \\
\text { (MG/L } \\
\text { AS SOA) }\end{array}$ & $\begin{array}{l}\text { DEPIH } \\
\text { BELDH } \\
\text { LAND } \\
\text { SUKFACE } \\
\text { (HAIER } \\
\text { LEVEL) } \\
\text { IFEET) }\end{array}$ & $\begin{array}{l}\text { ELEV- } \\
\text { ATION } \\
\text { ABJVE } \\
\text { MGVD } \\
\text { (FEET) }\end{array}$ \\
\hline
\end{tabular}

\begin{tabular}{|c|c|c|c|c|c|c|c|c|c|c|c|}
\hline & $\begin{array}{l}\text { JUN } \ldots 1 \\
26 \ldots \\
\text { SEP } \\
\text { O9... }\end{array}$ & $\begin{array}{c}1.5 \\
\ldots\end{array}$ & $\begin{array}{l}-2 \\
--\end{array}$ & 86 & $\begin{array}{l}.00 \\
.00\end{array}$ & $\begin{array}{l}2.4 \\
3.0\end{array}$ & -0 & $\begin{array}{l}.7 \\
--\end{array}$ & $\begin{array}{l}23.27 \\
23.40\end{array}$ & $\begin{array}{l}15.72 \\
15.62\end{array}$ & \\
\hline DATE & TIME & $\begin{array}{l}\text { HLUF- } \\
\text { INUF, } \\
\text { DIS- } \\
\text { SOLVED } \\
\text { OUGAL } \\
\text { IS ALI }\end{array}$ & $\begin{array}{c}\text { ARSENIC } \\
\text { DIS- } \\
\text { SOLVED } \\
\text { (UG/L } \\
\text { AS AS) }\end{array}$ & $\begin{array}{l}\text { BCRON, } \\
\text { OI S- } \\
\text { SCL YED } \\
\text { IUG /L } \\
\text { AS B) }\end{array}$ & $\begin{array}{c}\text { CADMIUM } \\
\text { DIS- } \\
\text { SOLVEO } \\
\text { CUG/L } \\
\text { AS CDS }\end{array}$ & $\begin{array}{l}\text { CACMIUH } \\
\text { TCTAL } \\
\text { RECEY- } \\
\text { EFAELE } \\
\text { CUGAL } \\
\text { AS CEI }\end{array}$ & $\begin{array}{l}\text { CHFO- } \\
\text { MUUH. } \\
\text { OIS- } \\
\text { STLVED } \\
\text { (UC/L } \\
\text { AS CR) }\end{array}$ & $\begin{array}{l}\text { CHKD- } \\
\text { MIUH, } \\
\text { HEXA- } \\
\text { VALENT, } \\
\text { OIS. } \\
\text { (UG/L } \\
\text { AS CR) }\end{array}$ & $\begin{array}{l}\text { COBALT, } \\
\text { DIS- } \\
\text { SOLVED } \\
\text { IUG/L } \\
\text { AS CD) }\end{array}$ & $\begin{array}{l}\text { COPPER, } \\
\text { OIS- } \\
\text { SOIVED } \\
\text { (UGA } \\
\text { AS CU) }\end{array}$ & $\begin{array}{l}\text { IRON, } \\
\text { DIS- } \\
\text { SOLVED } \\
\text { (UG/L } \\
\text { AS FE) }\end{array}$ \\
\hline CEC & 7321400 & -- & 0 & -- & -- & $<20$ & $\cdots$ & $=$ & $\cdots$ & $<2$ & $<10$ \\
\hline FEE... & $7^{0900}$ & - & 2 & B & -- & NO & $\cdots$ & 0 & $\cdots$ & $<2$ & $<10$ \\
\hline JUN & 1000 & 10 & 1 & $<20$ & $<2$ & -- & ND & 0 & MD & MD & $<10$ \\
\hline $\operatorname{Jun}_{\text {Jü }}^{24}$ & $777^{1530}$ & $<100$ & $<1$ & -- & NC & -- & ND & 0 & $<2$ & ND & 20 \\
\hline $15 \ldots$ & 1100 & - & -- & $\cdots$ & -- & $\cdots$ & $\cdots$ & -- & -- & $\cdots$ & $\cdots$ \\
\hline OATE & $\begin{array}{l}\text { LEAD, } \\
\text { DIS- } \\
\text { SOLVEC } \\
\text { (UE/L } \\
\text { AS PB) }\end{array}$ & $\begin{array}{l}\text { ITHIUH } \\
\text { DIS- } \\
\text { SOLVED } \\
\text { IUGA } \\
\text { AS LII }\end{array}$ & $\begin{array}{l}\text { FANGA- } \\
\text { NESE. } \\
\text { DIS- } \\
\text { SULVEO } \\
\text { (UG/L } \\
\text { AS MN) }\end{array}$ & $\begin{array}{l}\text { MERCURY } \\
\text { DIS- } \\
\text { SULVED } \\
\text { (UGA } \\
\text { AS HG) }\end{array}$ & $\begin{array}{l}\text { MFPCURY } \\
\text { TOTAL } \\
\text { RE COV- } \\
\text { ERAGLE } \\
\text { (UGAL } \\
\text { AS HG) }\end{array}$ & $\begin{array}{l}\text { MOL YE- } \\
\text { RENUM, } \\
\text { EI I - } \\
\text { SCL VEC } \\
\text { (UG A } \\
\text { AS MC) }\end{array}$ & $\begin{array}{l}\text { NICKEL, } \\
\text { DIS- } \\
\text { SCLVEO } \\
\text { (UCAL } \\
\text { AS NI) }\end{array}$ & $\begin{array}{l}\text { SELE- } \\
\text { NIUM, } \\
\text { OIS- } \\
\text { SOLVED } \\
\text { (UG /A } \\
\text { AS SE) }\end{array}$ & $\begin{array}{l}\text { SIRON- } \\
\text { IIUH, } \\
\text { DIS- } \\
\text { SOLVED } \\
\text { (UG/L } \\
\text { AS SR) }\end{array}$ & $\begin{array}{l}\text { VANA- } \\
\text { OIUH, } \\
\text { DIS- } \\
\text { SOLVED } \\
\text { (UG/L } \\
\text { AS V) }\end{array}$ & $\begin{array}{l}\text { ZINC, } \\
\text { DIS- } \\
\text { SOLVED } \\
\text { (UG/L } \\
\text { AS ZN) }\end{array}$ \\
\hline CEC, & 28 & $\cdots$ & 14 & -- & -- & -- & - & $\cdots$ & - & $-\infty$ & ND \\
\hline $\begin{array}{l}\text { JUN, } \\
26 . \cdots \\
\text { FEE }\end{array}$ & 74 & - & 50 & - & $<.5$ & -- & -- & -- & $=$ & -- & ND \\
\hline$\underset{\text { JUN }}{04 . \cdots}$ & 2 & $<10$ & $<10$ & $<.5$ & -- & $<1$ & ND & -- & -- & .0 & 6 \\
\hline JUN & Nr & $<10$ & $<10$ & $<.5$ & $\cdots$ & $<1$ & No & $<1$ & 60 & - B & $<20$ \\
\hline $15 \ldots$ & -- & -- & 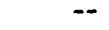 & -- & $\cdots$ & - & -- & $\cdots$ & 30 & -- & - \\
\hline
\end{tabular}

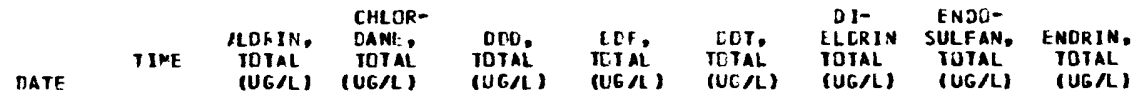

$$
\begin{aligned}
& \text { AUG - } 1978 \\
& \text { 22.: 1978 } \\
& .00 \\
& .00 \\
& \text { UGAL) } \\
& \text { U. } \\
& \text { NAPH- }
\end{aligned}
$$

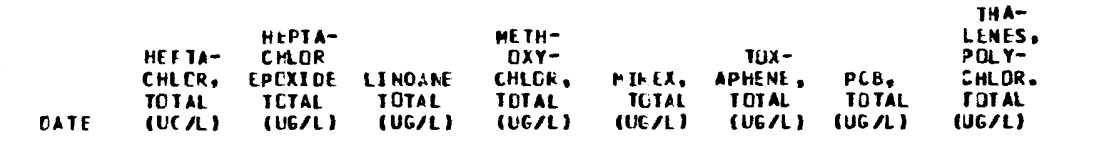

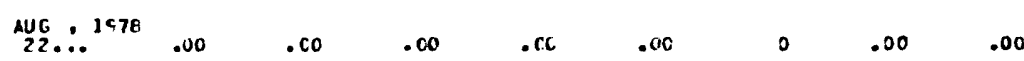


Site 20

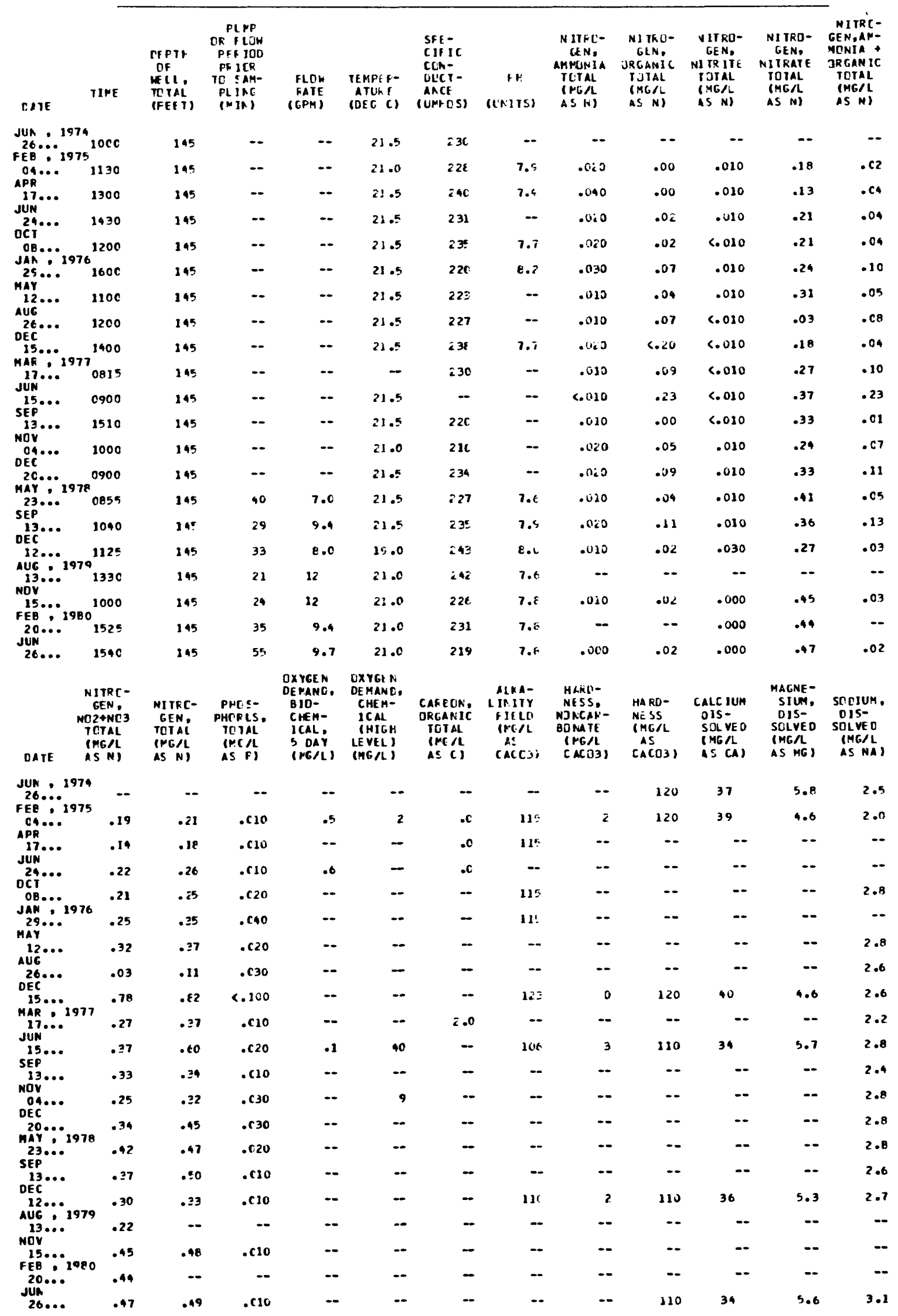


Site 20--Continued

\begin{tabular}{|c|c|c|c|c|c|c|c|c|c|c|c|}
\hline ITE & $\begin{array}{l}\text { PCTAS- } \\
\text { SIUM, } \\
\text { DIS- } \\
\text { SOLVED } \\
\text { (MG/L } \\
\text { ASKI) }\end{array}$ & $\begin{array}{l}\text { PICAF- } \\
\text { PCNAIE } \\
\text { FFT-FLD } \\
\text { (MG/ } \\
\text { AS } \\
\text { HCOZ) }\end{array}$ & $\begin{array}{l}\text { EICAK- } \\
\text { BCAATE } \\
\text { IT-FIO } \\
\text { IMCAL } \\
\text { AS } \\
\text { HCC 3) }\end{array}$ & $\begin{array}{l}\text { CAR - } \\
\text { BCNA IE } \\
\text { FET-FL } \\
\text { (FGL } \\
\text { AS CC3) }\end{array}$ & $\begin{array}{l}\text { CAK- } \\
\text { BONATE } \\
\text { IT }-F L D \\
\text { IMG/L } \\
\text { AS } \\
\text { CO2) }\end{array}$ & $\begin{array}{l}\text { CHLD- } \\
\text { RIEE. } \\
\text { DIS- } \\
\text { SCIVED } \\
\text { (MG/L } \\
\text { AS CL) }\end{array}$ & $\begin{array}{l}\text { FLLO } \\
\text { RICE. } \\
\text { CIS- } \\
\text { SOLVE } \\
\text { IFG/L } \\
\text { ASFI }\end{array}$ & $\begin{array}{l}\text { SULFATE } \\
\text { DIS- } \\
\text { SQLVIO } \\
\text { (MGA } \\
\text { AS SOA) }\end{array}$ & $\begin{array}{l}\text { SILICA. } \\
\text { OIS- } \\
\text { SJLVEO } \\
\text { IMG/L } \\
\text { AS } \\
\text { SIDZ) }\end{array}$ & $\begin{array}{l}\text { SJLIOS, } \\
\text { RE SIOUE } \\
\text { AI } 180 \\
\text { JEG C } \\
\text { DIS- } \\
\text { SOLVED } \\
\text { (MG L) }\end{array}$ & $\begin{array}{l}\text { DEPIH } \\
\text { BELON } \\
\text { LAND } \\
\text { SURFACE } \\
\text { (HATER } \\
\text { LEVEL) } \\
\text { (FEET) }\end{array}$ \\
\hline
\end{tabular}

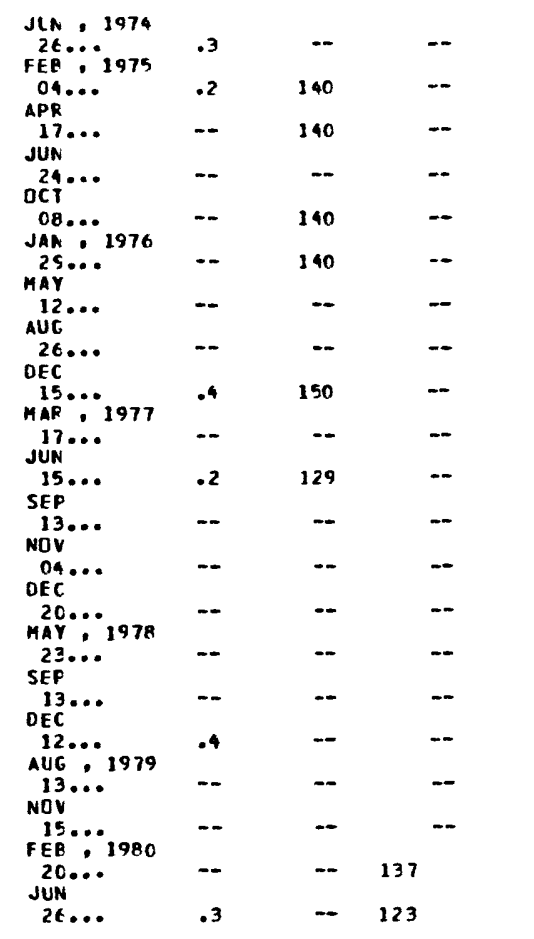

$\begin{array}{cc}-- & - \\ 0 & - \\ 0 & - \\ -- & - \\ 0 & - \\ 0 & - \\ -- & - \\ -- & - \\ 0 & - \\ -- & - \\ 0 & - \\ -- & - \\ -- & - \\ -- & - \\ -- & - \\ -- & - \\ -- & - \\ -- & - \\ -- & - \\ -- & .00 \\ -- & .10\end{array}$

- 4

$-$

$\therefore$

2.2

(20)

(FEE T 
Site 20--Continued

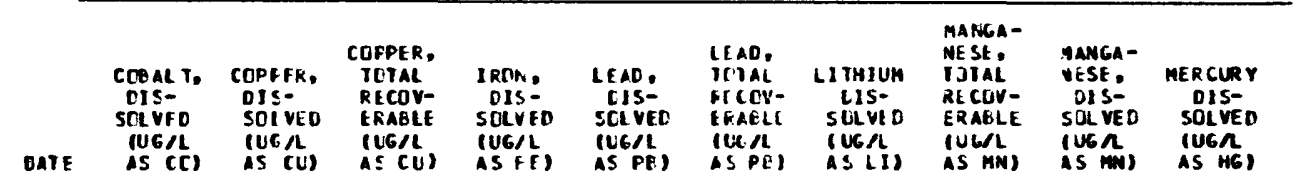

\begin{tabular}{|c|c|c|c|c|c|c|c|c|c|c|c|}
\hline$\cdots$ & 75 & $\cdots$ & $<2$ & -- & C 10 & Nu & -- & $\cdots$ & -- & - & $\cdots$ \\
\hline & & 12 & NO & $\cdots$ & $<10$ & 3 & - & $<10$ & -- & $<10$ & $<.5$ \\
\hline & 976 & $<2$ & No & -- & 10 & No & - & $<10$ & $\cdots$ & $<10$ & $<.5$ \\
\hline 15... & 1977 & - & $\cdots$ & 2 & $\cdots$ & $\cdots$ & 3 & -- & 20 & $\because$ & - \\
\hline $15 \ldots$ & & $\cdots$ & -- & -- & $\cdots$ & $\cdots$ & - & - & - & -- & 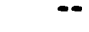 \\
\hline DATE & $\begin{array}{l}\text { MFR } \\
\text { TO } \\
\text { RE } \\
\text { IR } \\
\text { IE } \\
\text { IS }\end{array}$ & 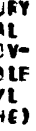 & $\begin{array}{l}\text { MOLYB- } \\
\text { DEALM. } \\
\text { OLS- } \\
\text { SOL VEO } \\
\text { CUEA } \\
\text { AS FD) }\end{array}$ & $\begin{array}{l}\text { NI CKEL } \\
\text { DIS- } \\
\text { SCLVED } \\
\text { (UG/L } \\
\text { AS NIS }\end{array}$ & $\begin{array}{l}\text { SELE- } \\
\text { NIUH. } \\
\text { DIS- } \\
\text { SQLVED } \\
\text { (UEIL } \\
\text { AS SE) }\end{array}$ & $\begin{array}{l}\text { SELE - } \\
\text { NWUA, } \\
\text { TETAL } \\
\text { IUETL } \\
\text { AS SES }\end{array}$ & $\begin{array}{l}\text { SILVER, } \\
\text { ICIAL } \\
\text { RECOV- } \\
\text { FEABLF } \\
\text { IUE IL } \\
\text { IS ACS }\end{array}$ & 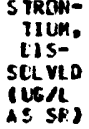 & $\begin{array}{l}\text { YALA- } \\
\text { OI UM. } \\
\text { DUS- } \\
\text { SCLVEO } \\
\text { IUGRL } \\
\text { AS VI }\end{array}$ & $\begin{array}{l}\text { ZINC. } \\
\text { DIS- } \\
\text { SOL VED } \\
\text { IUSA } \\
\text { AS ZN) }\end{array}$ & $\begin{array}{l}\text { ZINC, } \\
\text { TOTAL } \\
\text { RECOU- } \\
\text { ERABLE } \\
\text { (UGA } \\
\text { AS ZN) }\end{array}$ \\
\hline
\end{tabular}

\begin{tabular}{|c|c|c|c|c|c|c|c|c|c|c|}
\hline WN 26.1974 & $<.5$ & - & -- & $\cdots$ & - & - & $-\infty$ & $\cdots$ & 80 & 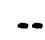 \\
\hline$\therefore$ & - & $\mathbf{I}$ & 2 & - & $\cdots$ & -- & - & .5 & 6 & $\infty$ \\
\hline OEC $\because 1976$ & $-\infty$ & 2 & mo & 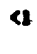 & - & -- & - & .0 & $\omega$ & - \\
\hline $15 . .$. & $<.5$ & - & - & - & e1 & AC: & $\cdots$ & 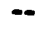 & $\cdots$ & $<20$ \\
\hline 1 & -- & $-\infty$ & - & $\cdots$ & - & - & 00 & $\cdots$ & $\cdots$ & \\
\hline
\end{tabular}

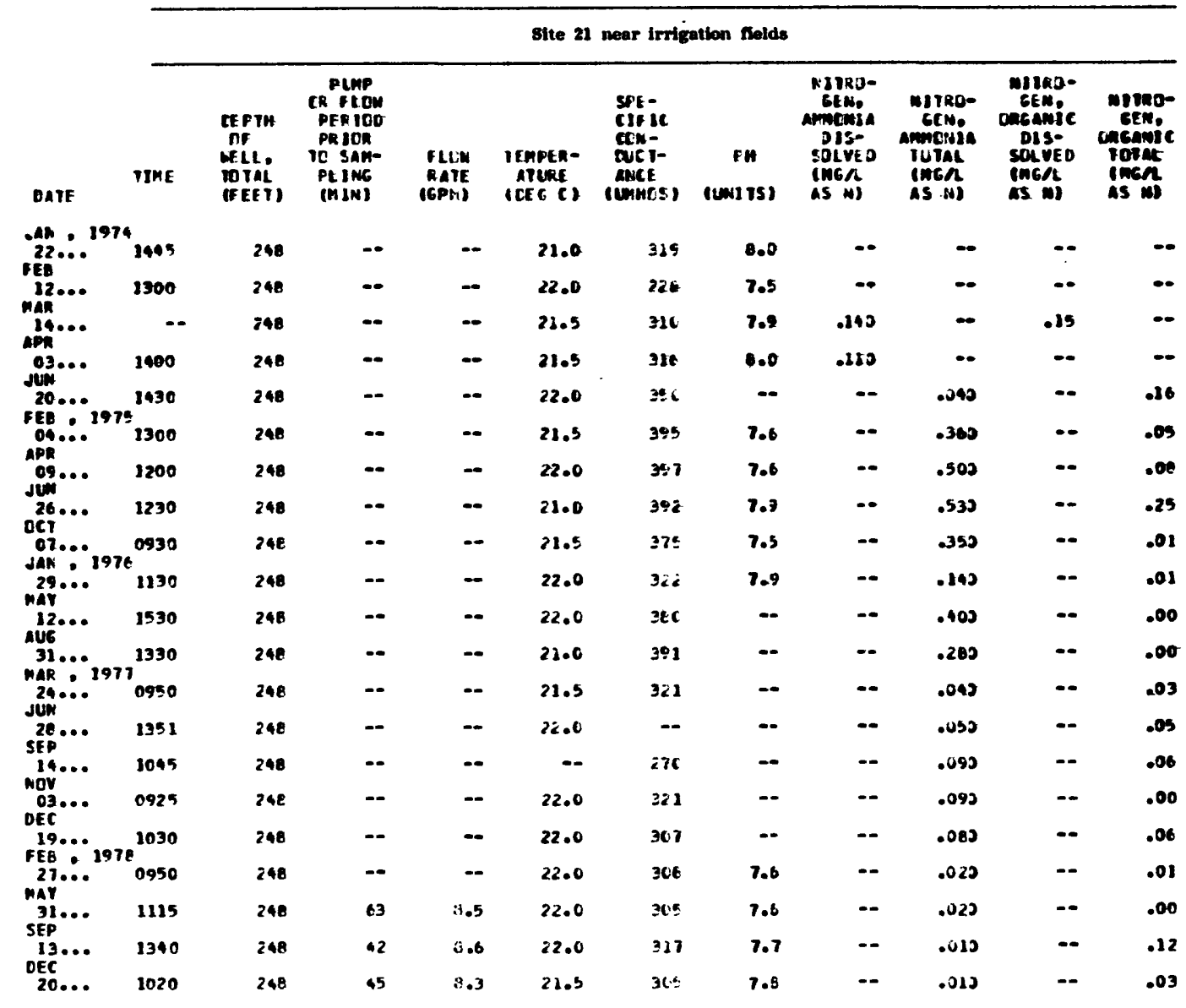


Table 6.--Physical and chemical characteristics of well and water at sites 1 through 70--Continued

Site 21 near irrigation fields--Continued

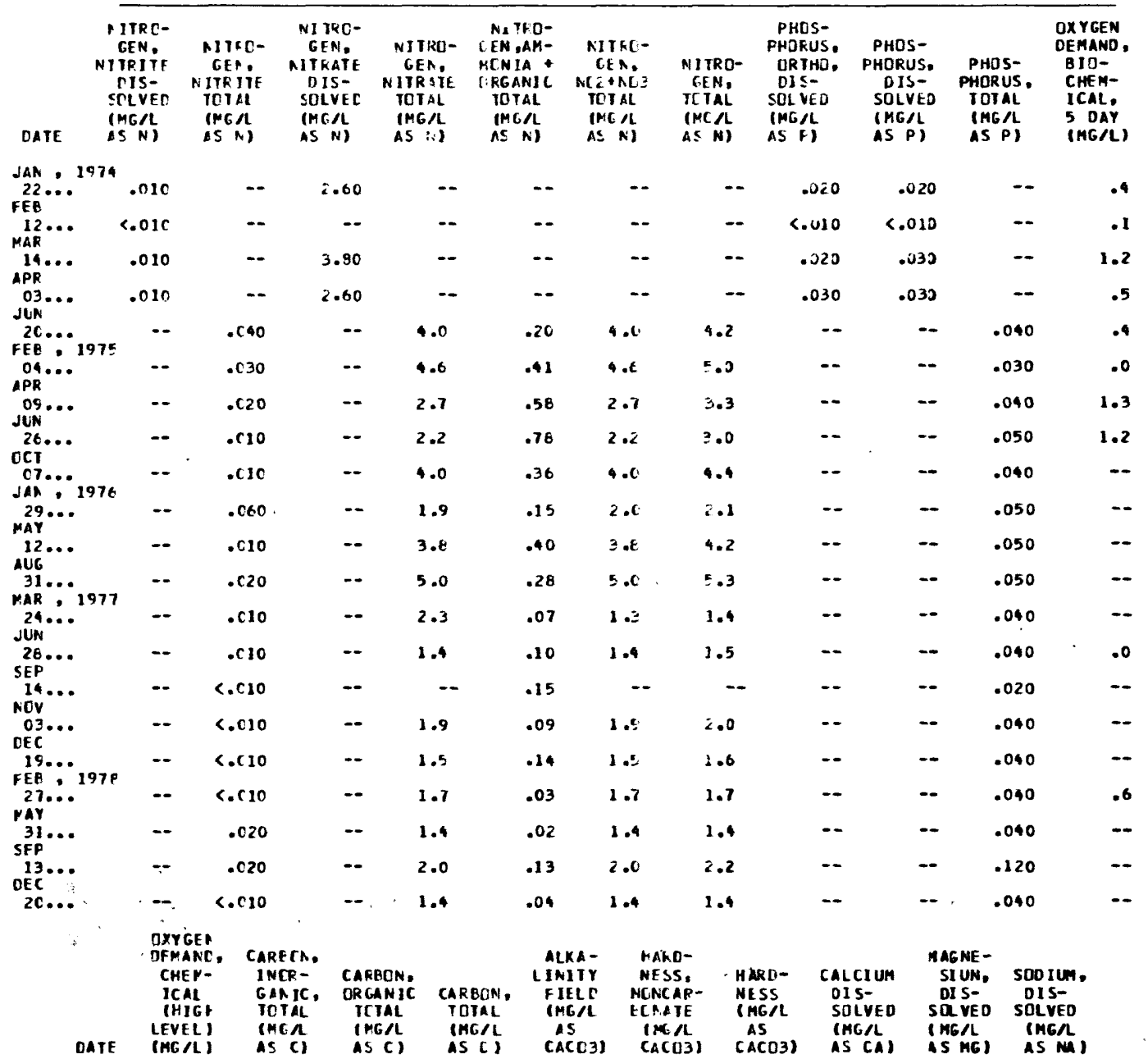

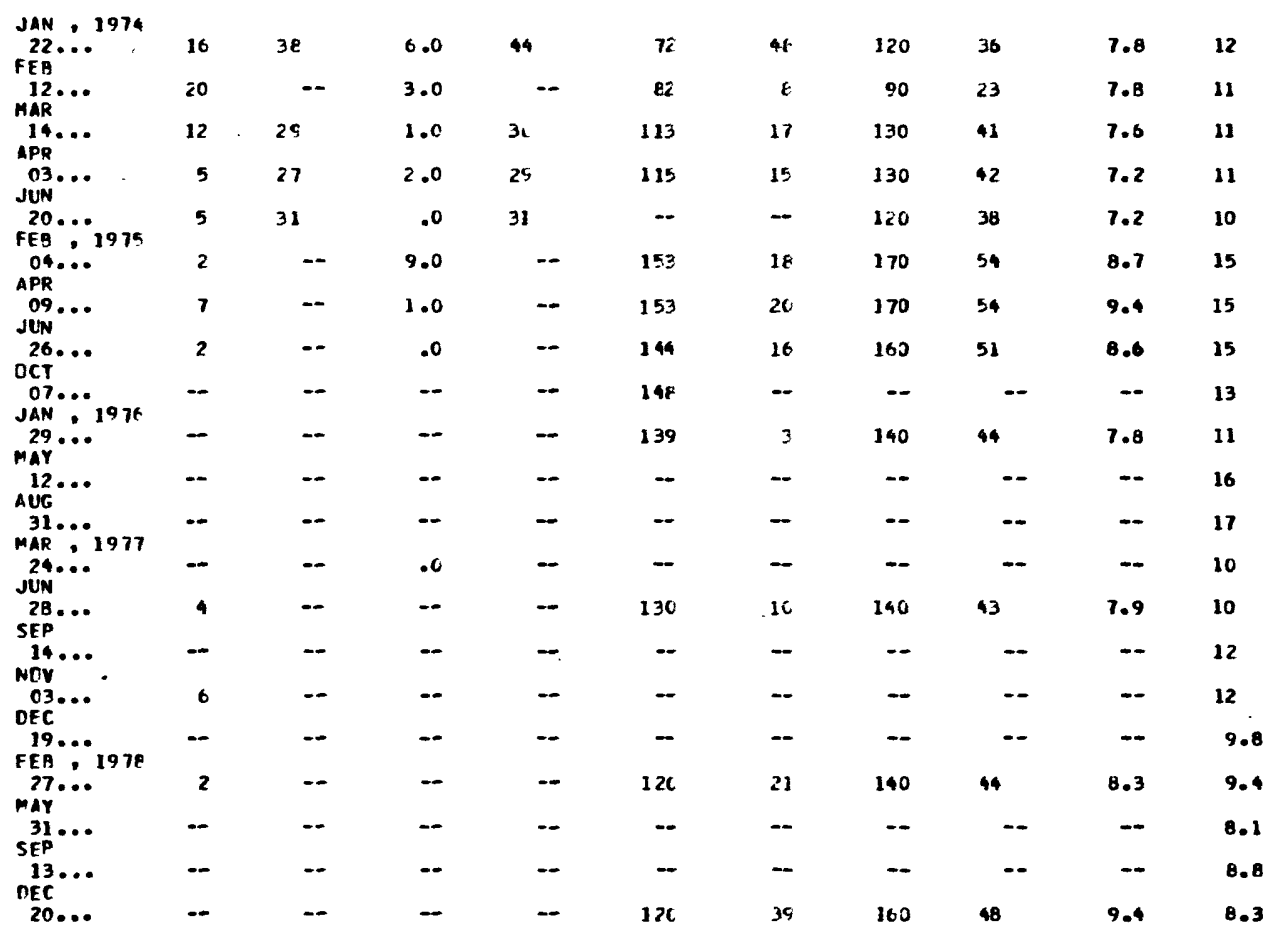


Site 21 near irrigation fields--Continued

\begin{tabular}{|c|c|c|c|c|c|c|c|c|c|c|}
\hline ATE & 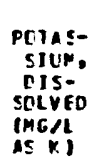 & $\begin{array}{l}\text { BICAR- } \\
\text { BCNATE } \\
\text { FI-FLLO } \\
\text { IMGA } \\
\text { AE } \\
\text { HCT } 3)\end{array}$ & $\begin{array}{c}\text { CAR - } \\
\text { ECNAIE } \\
\text { FE T-FLO } \\
\text { (MGN } \\
\text { AS COS }\end{array}$ & 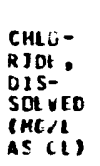 & $\begin{array}{l}\text { FLCO- } \\
\text { RIGE: } \\
\text { cis: } \\
\text { sctvet } \\
\text { irc } \\
\text { as is }\end{array}$ & 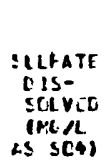 & $\begin{array}{l}\text { SIIICA, } \\
\text { OIS- } \\
\text { SCLYED } \\
\text { (REAL } \\
\text { RS } \\
\text { SIOZ) }\end{array}$ & $\begin{array}{l}\text { SOL LOS, } \\
\text { RESICUE } \\
\text { AI } 180 \\
\text { OEG. } \\
\text { JIS- } \\
\text { SOLVEO } \\
\text { (MGA) }\end{array}$ & $\begin{array}{l}\text { DEP IH } \\
\text { BELOW } \\
\text { LAND } \\
\text { SURFACE } \\
\text { (WATER } \\
\text { LEVEL) } \\
\text { (FEET) }\end{array}$ & $\begin{array}{l}\text { ELEV- } \\
\text { AIION } \\
\text { AHQVE } \\
\text { NGVO } \\
\text { (FEET) }\end{array}$ \\
\hline
\end{tabular}

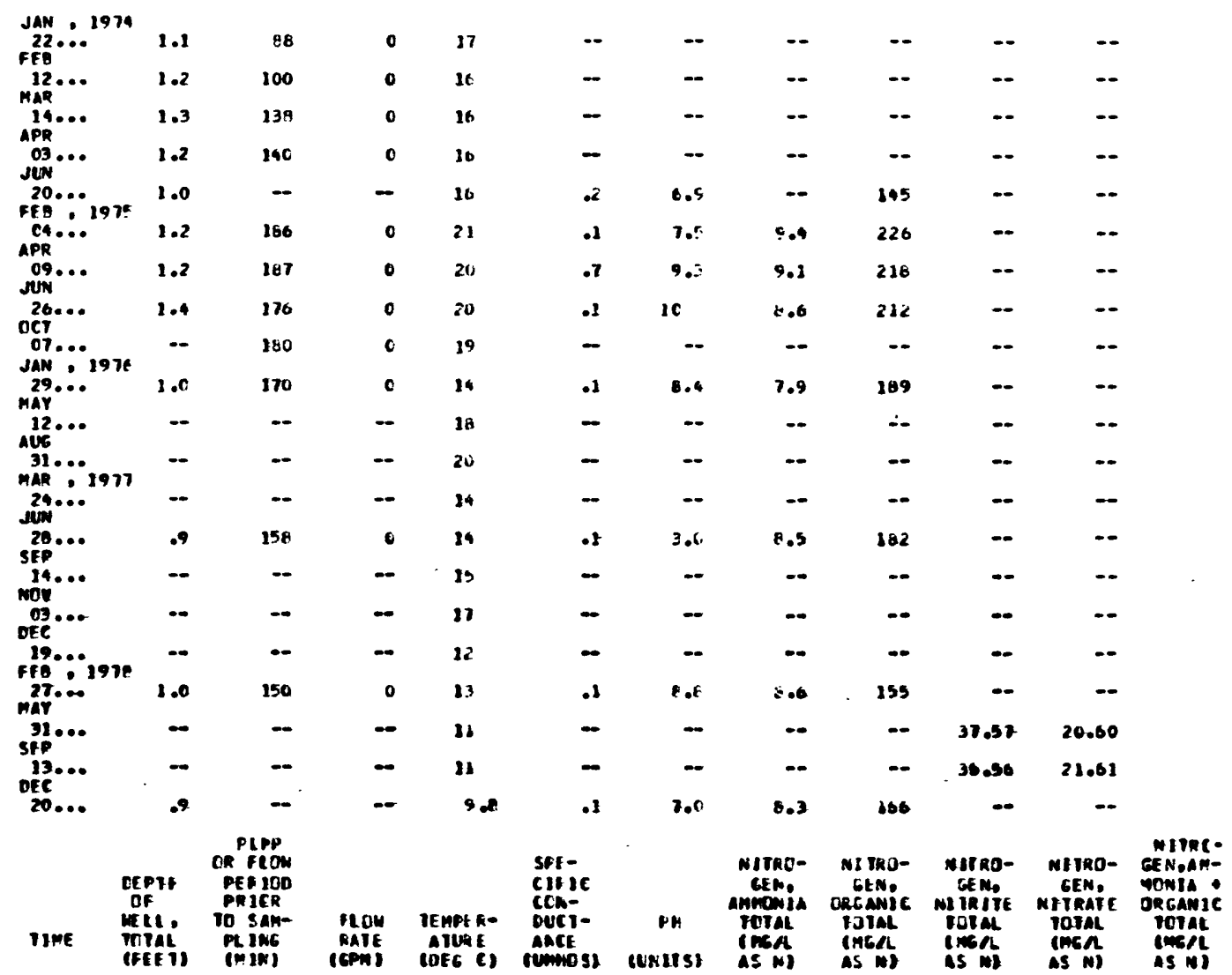

\begin{tabular}{|c|c|c|c|c|c|c|c|c|c|c|c|c|}
\hline $28 . .$. & 1515 & 240 & 32 & 6.3 & 22.0 & $2 \pi$ & 1.6 & -- & - & -- & .19 & - \\
\hline & 1309 & 240 & 92 & B. & 21.5 & 297 & 2.5 & $\cdots$ & $\cdots$ & -- & - & $\ldots$ \\
\hline & $1093 \mathrm{C}$ & 24 & 32 & $\Delta$ & 21.5 & 297 & 7.6 & -- & -- & -- & -. & 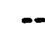 \\
\hline & 1040 & 248 & 58 & 7.1 & 23.5 & 286 & 1.8 & .2110 & .16 & .000 & 1.4 & .32 \\
\hline & 0920 & 240 & 59 & 6.5 & 28.0 & $\therefore 7$ & -- & .690 & .20 & .000 & .33 & .23 \\
\hline & 1400 & 240 & 51 & 8.1 & 200 & 280 & 1.8 & .000 & .01 & .000 & 2.2 & .01 \\
\hline$\because 1901$ & 1010 & 240 & 36 & 8.1 & 22.0 & $2 x$ & 7.6 & .020 & .06 & .000 & 1.1 & .00 \\
\hline & 1128 & 240 & 41 & 7.7 & 2200 & $2 x$ & T.E & .010 & .02 & .000 & 1.1 & .03 \\
\hline & 1005 & 240 & 40 & 8.3 & $2: .0$ & 27 & 7.8 & $.0 \% 0$ & .00 & .000 & 1.1 & .02 \\
\hline & 1110 & 248 & 42 & 6.8 & 21.8 & 279 & 7.8 & .010 & .07 & .000 & .32 & .01 \\
\hline 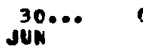 & 0900 & 248 & 40 & 9.1 & 21.5 & $2 E C$ & 3.7 & .0010 & .00 & .000 & 1.1 & .00 \\
\hline $17 \ldots . .$. & $\begin{array}{l}0955 \\
1120\end{array}$ & $\begin{array}{l}246 \\
240\end{array}$ & 10 & 8.8 & 220 & $=$ & 7.7 & $\begin{array}{l}.010 \\
.012\end{array}$ & $\begin{array}{l}.04 \\
.04\end{array}$ & $\begin{array}{l}.000 \\
.000\end{array}$ & $\begin{array}{l}.54 \\
.54\end{array}$ & $\begin{array}{l}.05 \\
.05\end{array}$ \\
\hline
\end{tabular}


Site 21 near irrigation fields--Continued

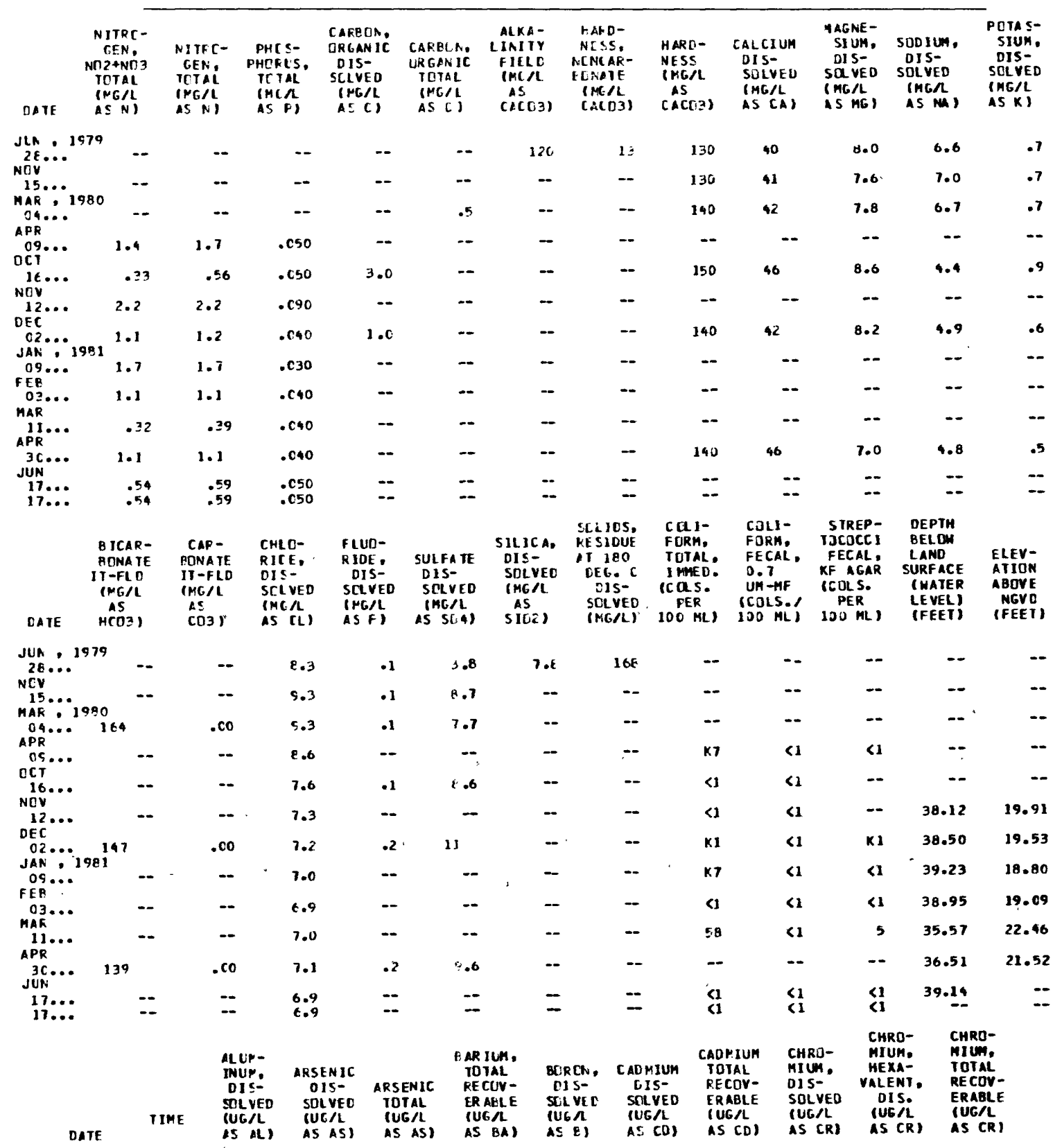

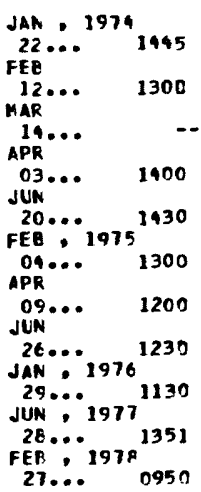

$\begin{array}{ccc}-- & -- & - \\ -- & -- & - \\ -- & -- & - \\ -- & 2 & - \\ -- & 5 & -- \\ 8 & 1 & - \\ 20 & 1 & - \\ 20 & <1 & - \\ -- & -- & - \\ -- & -- & - \\ -- & -- & <1\end{array}$

$\begin{array}{rr}-- & 2 c c \\ -- & 30 \\ -- & 8 c \\ -- & 3 \\ -- & 90 \\ -- & 60 \\ -- & 60 \\ -- & -- \\ -- & -. \\ -- & -- \\ 1100 & --\end{array}$

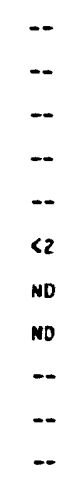

--
-
-
--
--
--
--
-
-
-
-

$\begin{array}{ccc}-- & -- & - \\ -- & -- & - \\ - & -- & - \\ - & -- & -- \\ - & -- & - \\ <2 & 0 & -- \\ \text { N3 } & 0 & -- \\ 3 & 0 & -- \\ -- & -- & -- \\ -- & -- & -- \\ -- & -- & <20\end{array}$ 
Table 6.--Physical and chemical characteristics of well and water at sites 1 through 70--Continued

Site 21 near irrigation fields--Continued

\begin{tabular}{|c|c|c|c|c|c|c|c|c|c|c|}
\hline $\begin{array}{l}\text { CCBALT, } \\
\text { OIS- } \\
\text { SCLVED } \\
\text { (UG/L } \\
\text { AS CO) }\end{array}$ & $\begin{array}{l}\text { CCPPEF, } \\
\text { DIS- } \\
\text { SDLVED } \\
\text { (UG/L } \\
\text { AS (U) }\end{array}$ & $\begin{array}{l}\text { CCPFER } \\
\text { IOIAL } \\
\text { RECUV- } \\
\text { ERABLE } \\
\text { UUG } \\
\text { AS CUI }\end{array}$ & $\begin{array}{l}\text { IRDF: } \\
\text { DI:- } \\
\text { SOLVEO } \\
\text { (UG/L } \\
\text { AS IE) }\end{array}$ & $\begin{array}{l}\text { IH.GN, } \\
\text { TOTAL } \\
\text { RECEV- } \\
\text { TRAELE } \\
\text { (UEAL } \\
\text { IS FE) }\end{array}$ & $\begin{array}{l}\text { IEAL, } \\
\text { CIS- } \\
\text { SCLVEL } \\
\text { (UEA } \\
\text { AS FE ) }\end{array}$ & $\begin{array}{l}\text { LEAD, } \\
\text { TOTAL } \\
\text { RFCOV- } \\
\text { EFAELE } \\
\text { ILEAL } \\
\text { AS PB) }\end{array}$ & $\begin{array}{l}\text { LITHIUH } \\
\text { DIS- } \\
\text { SLLVED } \\
\text { IUEN } \\
\text { AS LII }\end{array}$ & $\begin{array}{l}\text { MANGA- } \\
\text { NE SE. } \\
\text { TOTAL } \\
\text { RECOV- } \\
\text { ERABLE } \\
\text { (UG/L } \\
\text { IS MN) }\end{array}$ & $\begin{array}{l}\text { RANGA- } \\
\text { NESE. } \\
\text { DIS- } \\
\text { SOLVED } \\
\text { (UG/L } \\
\text { AS MN) }\end{array}$ & $\begin{array}{c}\text { MERCURY } \\
\text { DIS- } \\
\text { SOLVED } \\
\text { (UG/L } \\
\text { AS HG) }\end{array}$ \\
\hline
\end{tabular}

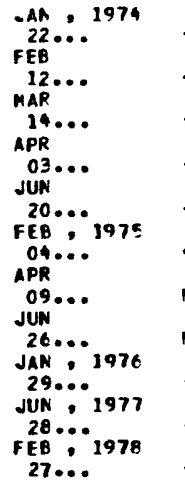

C2

--
--
--
$C 2$
--
NO
ND
NO
--
--
--
MOL YB-

$\begin{array}{ll}- & - \\ \ldots & - \\ - & 150 \\ - & 170 \\ - & 310 \\ - & 140 \\ 2 & -\end{array}$

MERCLIFY MOLYB-

SELE -

CCV- DIS- DIS-

SCLVED

(UGA IUEA (UGAL

SOL VED
PUG

AS SE)

-
-
-
-
-
-
-
-
--
--
250
SELE-
NIUH,
TOIAL
(UGSL
AS SEI

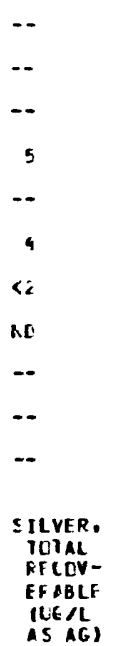

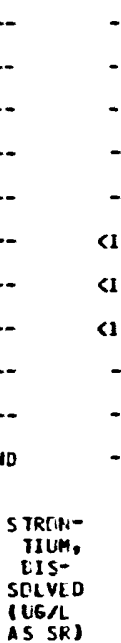

$\begin{array}{lllll}- & - & - & - & -\end{array}$

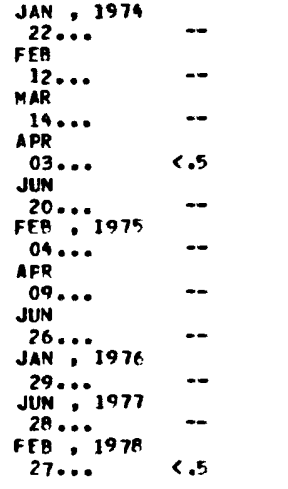

$\begin{array}{lll}-- & - \\ -- & - \\ -- & - \\ -- & - \\ -- & - \\ 1 & + \\ C 1 & \text { ND } \\ -- & -- \\ -- & -\end{array}$

$\bar{z}$
$\bar{z}$
$\bar{z}$
$\bar{z}$
$\bar{z}$

$\begin{array}{rrrrr}-- & - & - & -- & - \\ - & - & -- & -- & - \\ - & - & - & -- & - \\ - & -- & -- & 9 & - \\ -- & -- & -- & -- & - \\ - & - & 1.4 & <20 & - \\ -- & - & .0 & 20 & -- \\ - & 40 & -0 & <20 & -- \\ - & 140 & -- & -- & -- \\ <2 & 100 & -- & -- & -- \\ & 150 & -- & -- & <20\end{array}$ 
Site 22

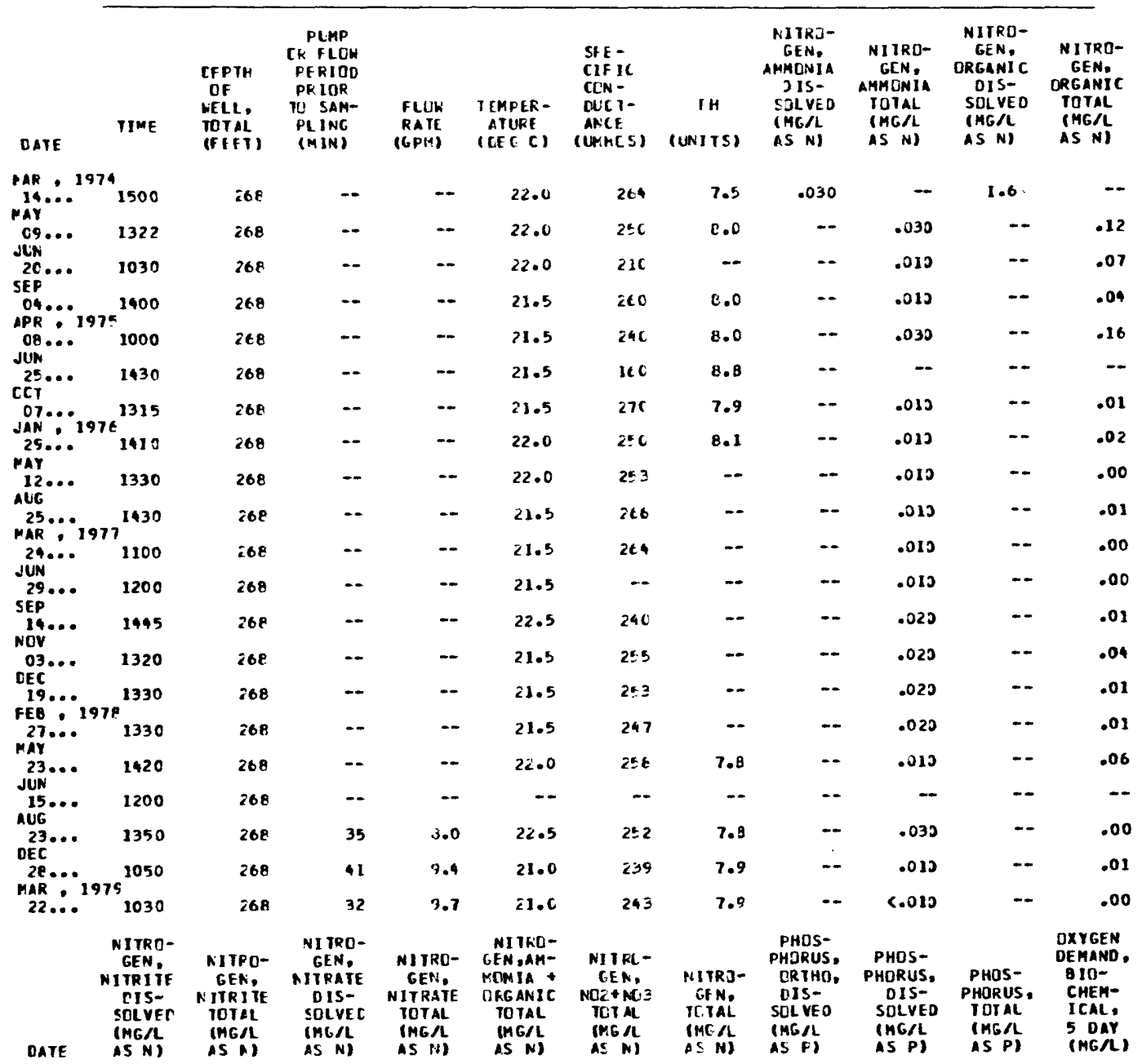

MAR, 1974

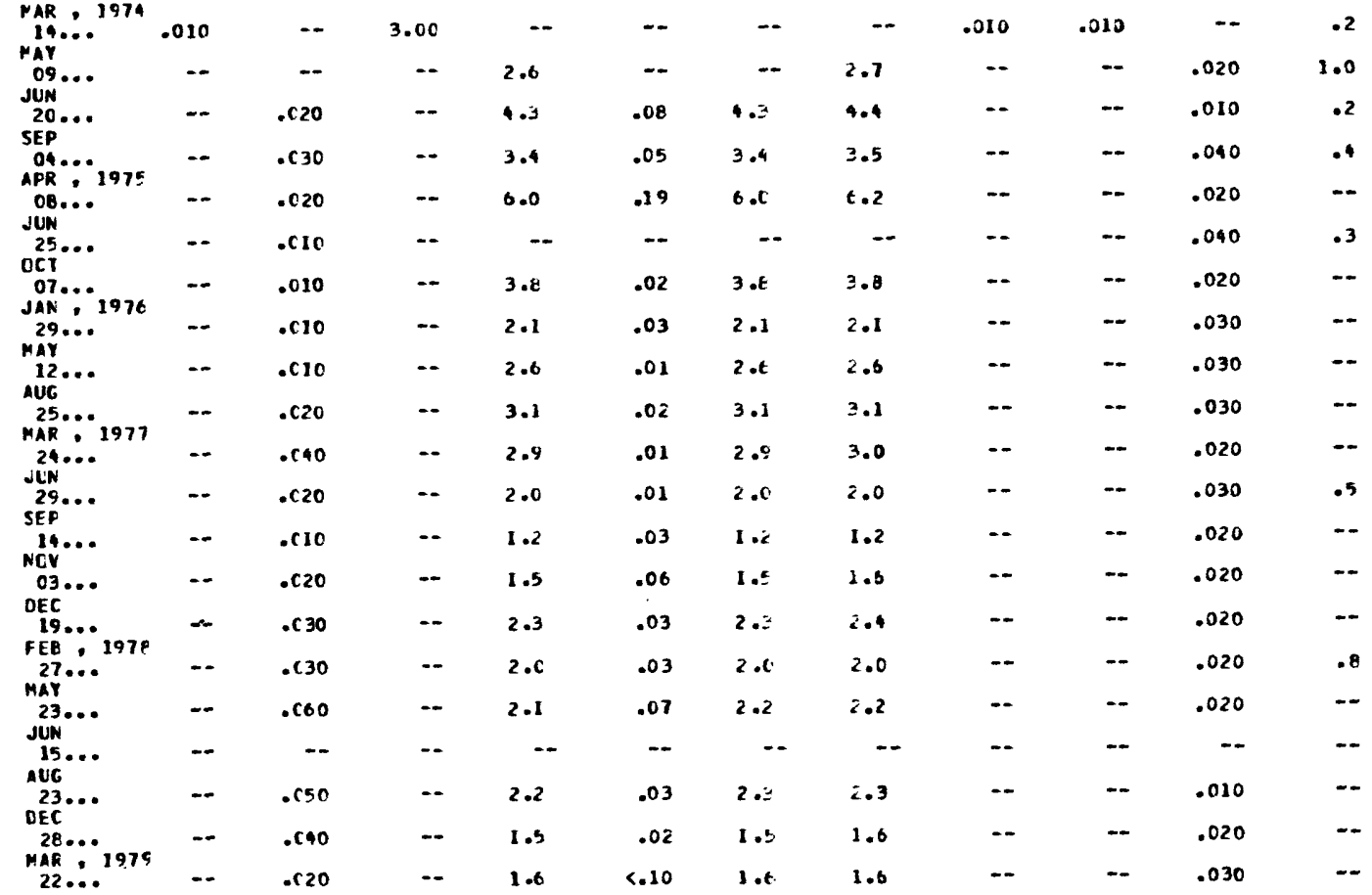




\begin{tabular}{|c|c|c|c|c|c|c|c|c|c|}
\hline \multicolumn{10}{|c|}{ Site 22--Continued } \\
\hline $\begin{array}{l}\text { DXYGEA } \\
\text { DEMANC. } \\
\text { CHEY- } \\
\text { ICAL } \\
\text { (HICH } \\
\text { LEVEL) } \\
\text { IFG IL) }\end{array}$ & $\begin{array}{l}\text { CAFFCN, } \\
\text { INCE- } \\
\text { GAAIC, } \\
\text { TOTAL } \\
\text { IMCA } \\
\text { AS CI }\end{array}$ & $\begin{array}{l}\text { CAKAON, } \\
\text { CRLANIC } \\
\text { TCTAL } \\
\text { (MG/L } \\
\text { AS CI) }\end{array}$ & $\begin{array}{l}\text { CARBUR, } \\
\text { IOTAL } \\
\text { IMC/L } \\
\text { AS C) }\end{array}$ & $\begin{array}{l}\text { ALKA- } \\
\text { LINIIY } \\
\text { FIELC } \\
\text { (FG/L } \\
\text { AS } \\
\text { CACC } 3)\end{array}$ & $\begin{array}{l}\text { HAFD- } \\
\text { AESSD } \\
\text { ACACAP- } \\
\text { ECNAIE } \\
\text { (FEAL } \\
\text { CACCB) }\end{array}$ & $\begin{array}{l}\text { HARn- } \\
\text { NESS } \\
\text { ( MGA } \\
\text { AS } \\
\text { CAC(B) }\end{array}$ & $\begin{array}{l}\text { CALCIUH } \\
\text { OIS- } \\
\text { SOLVEO } \\
\text { (HC/L } \\
\text { AS CA) }\end{array}$ & $\begin{array}{l}\text { YAGNE- } \\
\text { SI UM, } \\
\text { DIS- } \\
\text { SOL VED } \\
\text { (MG/L } \\
\text { IS MGI }\end{array}$ & $\begin{array}{l}\text { SODIUM, } \\
\text { DIS- } \\
\text { SOL VED } \\
\text { (MGAL } \\
\text { AS MA) }\end{array}$ \\
\hline
\end{tabular}

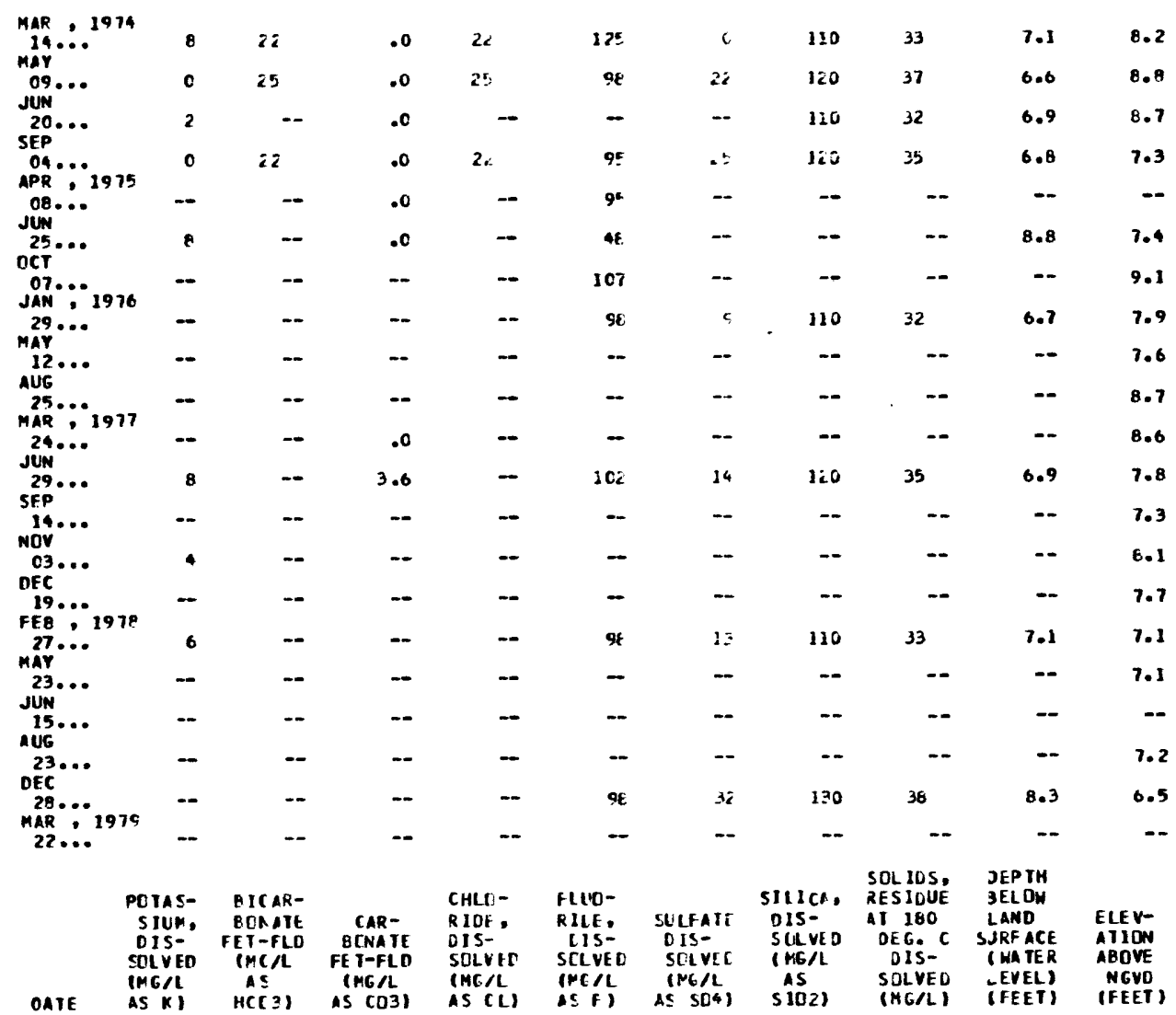

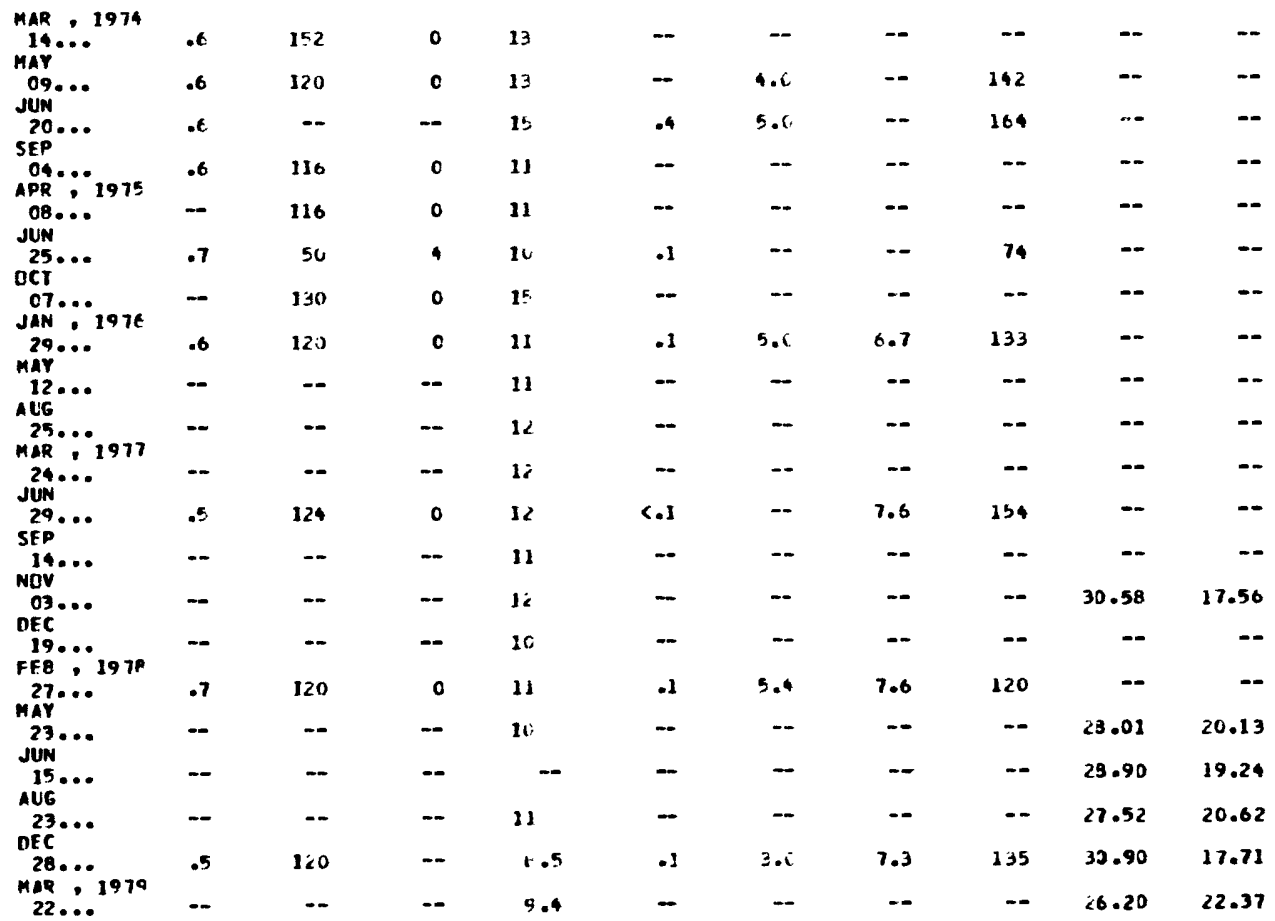


Site 22--Continued

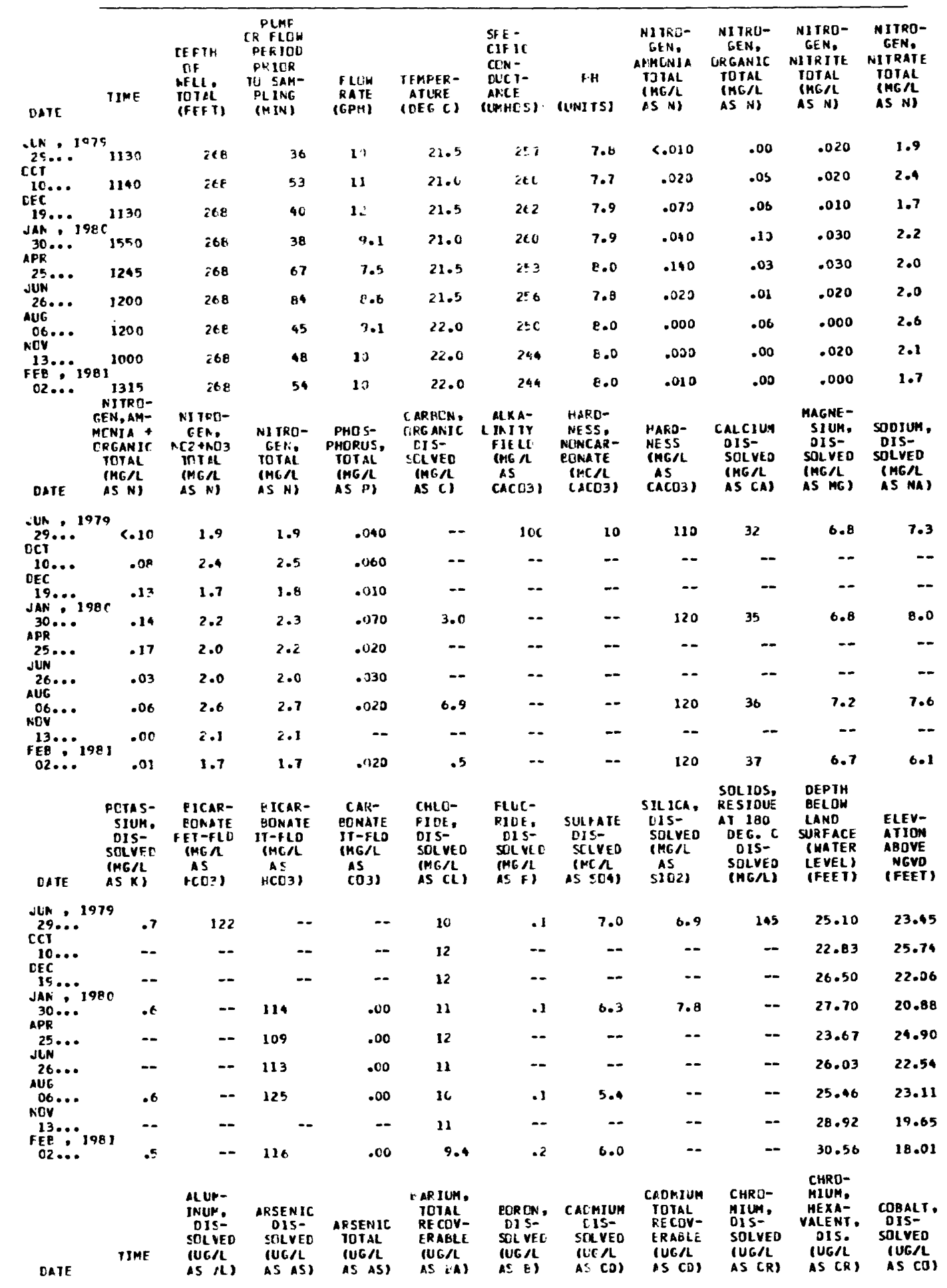

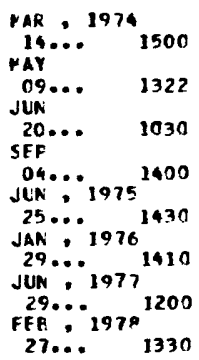

$\begin{array}{lll}-- & -- & - \\ -- & -- & -- \\ -- & <1 & -- \\ -- & -- & - \\ 20 & <1 & -- \\ -- & -- & - \\ -- & -- & -- \\ -- & -- & <1\end{array}$

$\begin{array}{cc}-- & 140 \\ -- & 30 \\ -- & 90 \\ -- & 160 \\ -- & -- \\ -- & - \\ -- & - \\ 100 & -\end{array}$ 
Site 22--Continued

\begin{tabular}{|c|c|c|c|c|c|c|c|c|c|c|c|}
\hline DATF & $\begin{array}{l}\text { COPP } \\
\text { OIS } \\
\text { SCL } \\
\text { IUG } \\
\text { AS }\end{array}$ & 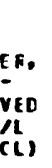 & $\begin{array}{l}\text { COPFFR. } \\
\text { TCTAL } \\
\text { RECTV- } \\
\text { ERAPLE } \\
\text { luC } \\
\text { AS (U) }\end{array}$ & $\begin{array}{l}\text { JVCN, } \\
\text { CIS- } \\
\text { SRLVED } \\
\text { (LGN } \\
\text { AS FE) }\end{array}$ & $\begin{array}{l}\text { IPGAN, } \\
\text { IOTAL } \\
\text { RECLV- } \\
\text { E RAELE } \\
\text { CUC/L } \\
\text { AS FE) }\end{array}$ & $\begin{array}{l}\text { LEAD, } \\
\text { OIS- } \\
\text { SCIVEC } \\
\text { EUCA } \\
\text { AS FEI }\end{array}$ & $\begin{array}{l}\text { IEAD. } \\
\text { TCIAL } \\
\text { GCCDV- } \\
\text { EF ABL } \\
\text { IUT } / 2 \\
\text { AS PES }\end{array}$ & $\begin{array}{l}\text { LIHIUM } \\
\text { DIS- } \\
\text { SULVIO } \\
\text { (IGA } \\
\text { AS II) }\end{array}$ & $\begin{array}{l}\text { MANGA- } \\
\text { NE SE, } \\
\text { TJTAL } \\
\text { RECOV- } \\
\text { ERAGLE } \\
\text { (UGAL } \\
\text { AS MN) }\end{array}$ & $\begin{array}{l}\text { MANGA- } \\
\text { VESL: } \\
\text { DIS- } \\
\text { SOL VEO } \\
\text { IUGA } \\
\text { AS MNI }\end{array}$ & $\begin{array}{l}\text { MERCURY } \\
\text { DIS- } \\
\text { SOLVED } \\
\text { (UGR } \\
\text { AS HG) }\end{array}$ \\
\hline MAR 1 & 74 & - & -- & - & $\cdots$ & - & $\cdots$ & $\cdots$ & -- & -- & - \\
\hline may & & $\cdots$ & $\cdots$ & - & - & $\cdots$ & - & $\cdots$ & - & $\cdots$ & - \\
\hline $\begin{array}{l}\operatorname{Jun}_{20} \\
\text { SEP }\end{array}$ & & $\cdots$ & - & -- & - & - & -- & - & $\cdots$ & -- & - \\
\hline SUN : ? & 75 & - & - & $\cdots$ & -- & - & - & $\cdots$ & -+ & $\cdots$ & $=$ \\
\hline & & 82 & - & $<10$ & - & $<2$ & - & $<: 0$ & - & 20 & <.5 \\
\hline $\begin{array}{c}\text { JAN }: 1 \\
29 . \cdots \\
\text { NWN: }\end{array}$ & 977 & -- & $\cdots$ & $\cdots$ & - & - & - & -- & -- & - & - \\
\hline FEA & 978 & - & $\cdots$ & $\cdots$ & - & - & - & $\cdots$ & $\cdots$ & $=$ & - \\
\hline $27 \ldots$ & & $\cdots$ & 2 & - & 670 & - & MK & $\cdots$ & $<10$ & - & - \\
\hline Date & $\begin{array}{l}\text { MERC } \\
\text { TET } \\
\text { REC } \\
\text { ERA } \\
\text { PUE } \\
\text { IS }\end{array}$ & $\begin{array}{l}\text { UEY } \\
\text { AL } \\
\text { OV- } \\
\text { Bet } \\
\text { HE) } \\
\text { HES }\end{array}$ & $\begin{array}{l}\text { MCL YB- } \\
\text { OERLM. } \\
\text { DIS- } \\
\text { SEI VEO } \\
\text { (UC A } \\
\text { AS MDS }\end{array}$ & $\begin{array}{l}\text { NI CKEL, } \\
\text { D IS- } \\
\text { SCLVED } \\
\text { (UGIL } \\
\text { AS NI) }\end{array}$ & $\begin{array}{l}\text { SELF- } \\
\text { NIUH, } \\
\text { DIS- } \\
\text { SOVEO } \\
\text { CUE/L } \\
\text { AS SEl }\end{array}$ & $\begin{array}{l}\text { SCLE- } \\
\text { NHUH, } \\
\text { TOTAL } \\
\text { (LCPL } \\
\text { AS SE) }\end{array}$ & 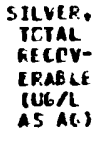 & $\begin{array}{l}\text { S IKON- } \\
\text { IIUM. } \\
\text { DIS- } \\
\text { SOLVLO } \\
\text { IUGA } \\
\text { AS SFI. }\end{array}$ & $\begin{array}{l}\text { YANA- } \\
\text { OI UH. } \\
\text { DIS- } \\
\text { SULVEO } \\
\text { (UG/L } \\
\text { AS V) }\end{array}$ & $\begin{array}{l}\text { ZINC. } \\
\text { DIS- } \\
\text { SOL VEO } \\
\text { (USR } \\
\text { IS ZN) }\end{array}$ & $\begin{array}{l}\text { ZINC, } \\
\text { TOTAL } \\
\text { RECON- } \\
\text { ERABLE } \\
\text { (UG/L } \\
\text { AS ZN) }\end{array}$ \\
\hline MAR & 974 & - & - & 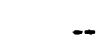 & - & - & $\cdots$ & -- & $-\infty$ & $-\infty$ & $\cdots$ \\
\hline $\begin{array}{l}\text { MAY } \\
\text { g9. . ... }\end{array}$ & & $\cdots$ & - & - & $\cdots$ & - & - & -- & $\cdots$ & $\cdots$ & - \\
\hline SEP... & & $-\infty$ & - & - & - & - & - & $\cdots$ & -- & $\cdots$ & - \\
\hline & & - & - & -- & - & $\infty$ & $\cdots$ & -- & -- & - & - \\
\hline 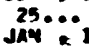 & & $\infty$ & 2 & $\mathbf{M D}$ & 1 & - & - & 40 & .0 & no & - \\
\hline & & $-\infty$ & $\cdots$ & - & $\cdots$ & - & $\cdots$ & 120 & - & $\infty$ & $\cdots$ \\
\hline 1 & & - & $\cdots$ & $\cdots$ & - & $\infty$ & - & 100 & $\cdots$ & - & - \\
\hline 27 & & $<.5$ & $\cdots$ & - & - & 4 & $<\hat{a}$ & $1: 0$ & $\cdots$ & $\infty$ & No \\
\hline
\end{tabular}

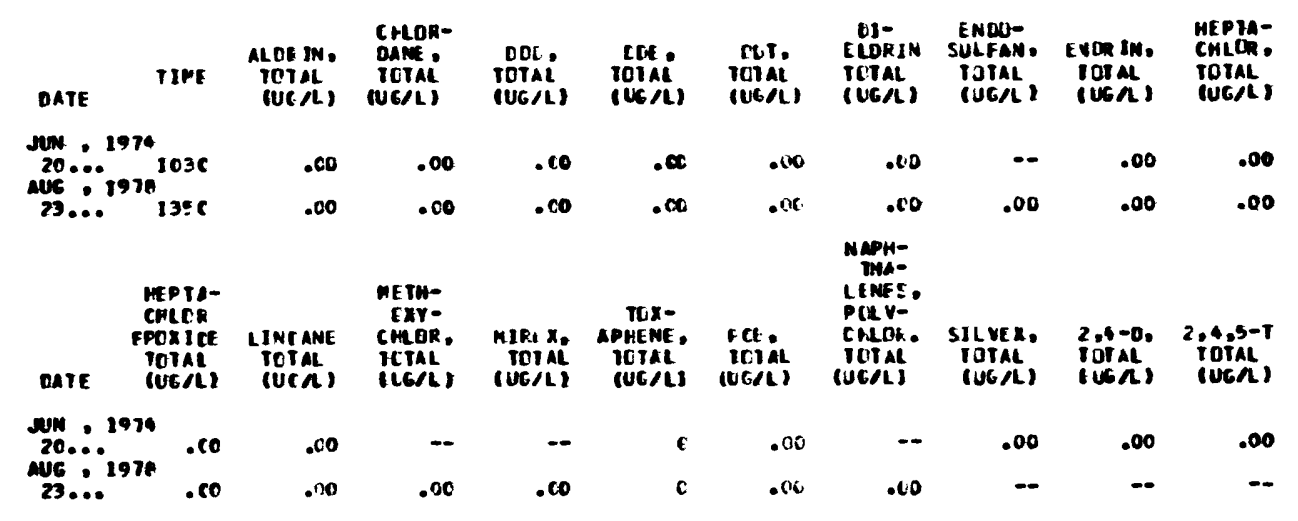


Site 23 near 4-big-gun area

\begin{tabular}{|c|c|c|c|c|c|c|c|c|c|c|}
\hline PATE & TINE & $\begin{array}{l}\text { DEFIH } \\
\text { OF } \\
\text { WFIL, } \\
\text { TYTAL } \\
\text { (FEET) }\end{array}$ & $\begin{array}{l}\text { TC PPE E- } \\
\text { A IURE } \\
\text { (DEG C) }\end{array}$ & $\begin{array}{l}\text { SFE- } \\
\text { CIFIC } \\
\text { CON- } \\
\text { DUCT- } \\
\text { ANCI } \\
\text { (UMHÉS) }\end{array}$ & $\begin{array}{l}\text { NITFC- } \\
\text { CEN. } \\
\text { AMMCN IA } \\
\text { TCTAL. } \\
\text { (MC/L } \\
\text { AS N) }\end{array}$ & $\begin{array}{l}\text { MIIRO- } \\
\text { CEN, } \\
\text { EFEANIC } \\
\text { ICIAL } \\
\text { (MESL } \\
\text { AS N) }\end{array}$ & $\begin{array}{l}\text { NITRO- } \\
\text { GEN, } \\
\text { NI IRITE } \\
\text { TOTAL } \\
\text { (MG/L } \\
\text { AS N) }\end{array}$ & $\begin{array}{l}\text { NITKO- } \\
\text { GEN, } \\
\text { NIIRAIE } \\
\text { TOTAL } \\
\text { (KG/L } \\
\text { AS N) }\end{array}$ & $\begin{array}{l}\text { PHOS- } \\
\text { PHORUS, } \\
\text { IOIAL } \\
\text { (MG } \\
\text { IS P) }\end{array}$ & $\begin{array}{l}\text { CHLO- } \\
\text { RIDE, } \\
\text { DIS- } \\
\text { SOLVE } \\
\text { IMGA } \\
\text { AS CL }\end{array}$ \\
\hline \multicolumn{11}{|c|}{ JUN, 1974} \\
\hline $\begin{array}{l}13 \ldots \\
14 \ldots\end{array}$ & $\begin{array}{l}1000 \\
0700\end{array}$ & $\begin{array}{l}42 \\
65\end{array}$ & $=$ & $\begin{array}{l}<35 \\
150\end{array}$ & $\begin{array}{l}.016 \\
.030\end{array}$ & $=$ & $\begin{array}{l}.010 \\
.010\end{array}$ & 20 & $\begin{array}{l}.020 \\
.010\end{array}$ & $\begin{array}{l}38 \\
14\end{array}$ \\
\hline $14 \ldots$ & $073 \mathrm{C}$ & 75 & - & 148 & $.06 C$ & - & .010 & 3.7 & .020 & $\begin{array}{l}14 \\
16\end{array}$ \\
\hline $14 \ldots$ & CBCC & 85 & -- & 210 & $.02 \mathrm{C}$ & -- & .030 & 9.1 & $\begin{array}{r}.010 \\
.010\end{array}$ & $\begin{array}{l}26 \\
22\end{array}$ \\
\hline $14 \ldots$ & OSOC & 95 & - & 188 & $.01 \mathrm{C}$ & - & .010 & 8.2 & $\begin{array}{r}.010 \\
.020\end{array}$ & $\begin{array}{l}22 \\
27\end{array}$ \\
\hline $14 \ldots$ & $13 \mathrm{CC}$ & 125 & 22.0 & $=20$ & $.02 \mathrm{C}$ & - & .010 & 12 & .010 & 30 \\
\hline $14 \ldots$ & $140 \mathrm{C}$ & 135 & 22.5 & $<30$ & .060 & -- & .010 & 12 & .020 & 30 \\
\hline $14 \ldots$ & 1600 & 337 & - & 210 & .120 & .64 & .100 & $\begin{array}{l}26 \\
20\end{array}$ & $\begin{array}{l}.020 \\
.020\end{array}$ & $\begin{array}{l}22 \\
51\end{array}$ \\
\hline $15 \ldots$ & 1200 & 150 & 22.5 & 390 & $\begin{array}{l}.080 \\
.060\end{array}$ & .0 & .010 & 19 & .020 & 39 \\
\hline $16 \ldots$ & $080 \mathrm{C}$ & 165 & 22.0 & $\begin{array}{l}3<0 \\
420\end{array}$ & $\begin{array}{l}.060 \\
.840\end{array}$ & - & .430 & 32 & .020 & $\begin{array}{l}39 \\
46\end{array}$ \\
\hline $\begin{array}{l}17 \ldots \\
17 \ldots\end{array}$ & $\begin{array}{l}080 C \\
1200\end{array}$ & $\begin{array}{l}185 \\
190\end{array}$ & $\begin{array}{l}21.5 \\
22.5\end{array}$ & $\begin{array}{l}420 \\
370\end{array}$ & $.12 \mathrm{C}$ & .10 & .070 & 20 & .100 & 44 \\
\hline $17 \ldots$ & 1400 & 200 & 22.0 & 420 & .490 & $\because$ & .490 & 21 & .070 & 46 \\
\hline $18 \ldots$ & 0900 & 215 & 22.0 & 350 & 2.60 & - & 1.70 & 18 & .020 & $\begin{array}{l}44 \\
45\end{array}$ \\
\hline $18 \ldots$ & $14 \mathrm{CC}$ & $\begin{array}{l}220 \\
240\end{array}$ & 22.0 & $4 \mathrm{CO}$ & .611 & $=$ & $\begin{array}{l}.740 \\
.110\end{array}$ & 23 & $\begin{array}{r}.020 \\
.020\end{array}$ & $\begin{array}{l}45 \\
47\end{array}$ \\
\hline $19 \ldots$ & $12 c \mathrm{C}$ & $\begin{array}{l}240 \\
250\end{array}$ & $\because$ & 50 & .140 & $=$ & $\begin{array}{l}.110 \\
.060\end{array}$ & $\begin{array}{l}24 \\
20\end{array}$ & .050 & 48 \\
\hline $\begin{array}{l}20 \ldots \ldots \\
20 \ldots\end{array}$ & $\begin{array}{l}080 \mathrm{C} \\
12 \mathrm{CC}\end{array}$ & $\begin{array}{l}250 \\
260\end{array}$ & $=$ & $\begin{array}{l}470 \\
470\end{array}$ & $\begin{array}{l}160 \\
.260\end{array}$ & $=$ & .060 & 26 & .050 & 48 \\
\hline 20. & $140 \mathrm{C}$ & 270 & - & 470 & .290 & -- & .270 & 25 & .040 & 50 \\
\hline
\end{tabular}

\begin{tabular}{|c|c|c|c|c|c|c|c|c|c|c|}
\hline \multicolumn{11}{|c|}{ Site 24} \\
\hline $\begin{array}{l}\text { OEPTH } \\
\text { OF } \\
\text { WELL, } \\
\text { TOTAL } \\
\text { (FEET) }\end{array}$ & $\begin{array}{l}\text { TERFER- } \\
\text { ATURE } \\
\text { (DEC C) }\end{array}$ & $\begin{array}{l}\text { SPF- } \\
\text { CIFIC } \\
\text { CON- } \\
\text { DUCT- } \\
\text { ANCE } \\
\text { (UNHOS) }\end{array}$ & $\begin{array}{c}\text { Ph } \\
\text { (UNIIS) }\end{array}$ & $\begin{array}{l}\text { NJIKO- } \\
\text { GEN, } \\
\text { ARMONIA } \\
\text { DIS- } \\
\text { SCLVEL } \\
\text { (RE/L } \\
\text { AS N) }\end{array}$ & $\begin{array}{l}\text { NITRO- } \\
\text { GEN, } \\
\text { AMMENIA } \\
\text { TUTAL } \\
\text { (NG/L } \\
\text { AS N) }\end{array}$ & $\begin{array}{l}\text { NITPQ- } \\
\text { GENE } \\
\text { ORGANIC } \\
\text { DIS- } \\
\text { SQRVD } \\
\text { (MG/L } \\
\text { AS N) }\end{array}$ & $\begin{array}{l}\text { NIIRD- } \\
\text { GEN, } \\
\text { ORGANIC } \\
\text { TUIAL } \\
\text { (MG/L } \\
\text { AS N) }\end{array}$ & $\begin{array}{l}\text { NITRO- } \\
\text { GEN, } \\
\text { NI IRIIE } \\
\text { DI S- } \\
\text { SJLVED } \\
\text { (MG } \\
\text { AS N) }\end{array}$ & $\begin{array}{l}\text { NITRO- } \\
\text { GEN, } \\
\text { MITRITE } \\
\text { TOTAL } \\
\text { (MGR } \\
\text { AS N) }\end{array}$ & $\begin{array}{l}\text { NITRC- } \\
\text { GEN, } \\
\text { NIIRAIE } \\
\text { DIS- } \\
\text { SOLVED } \\
\text { (MGGL } \\
\text { AS N) }\end{array}$ \\
\hline
\end{tabular}

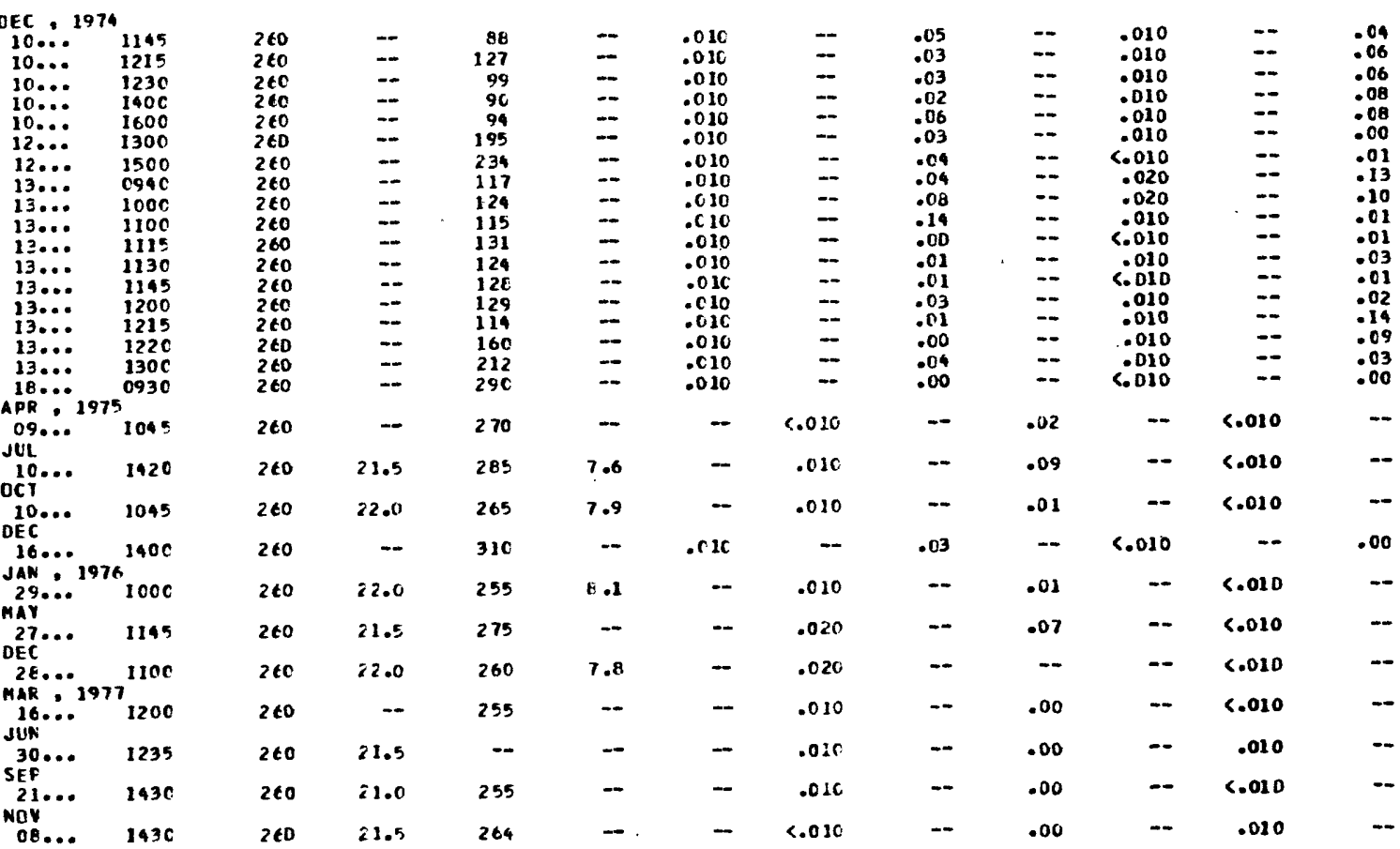


Site 24--Continued

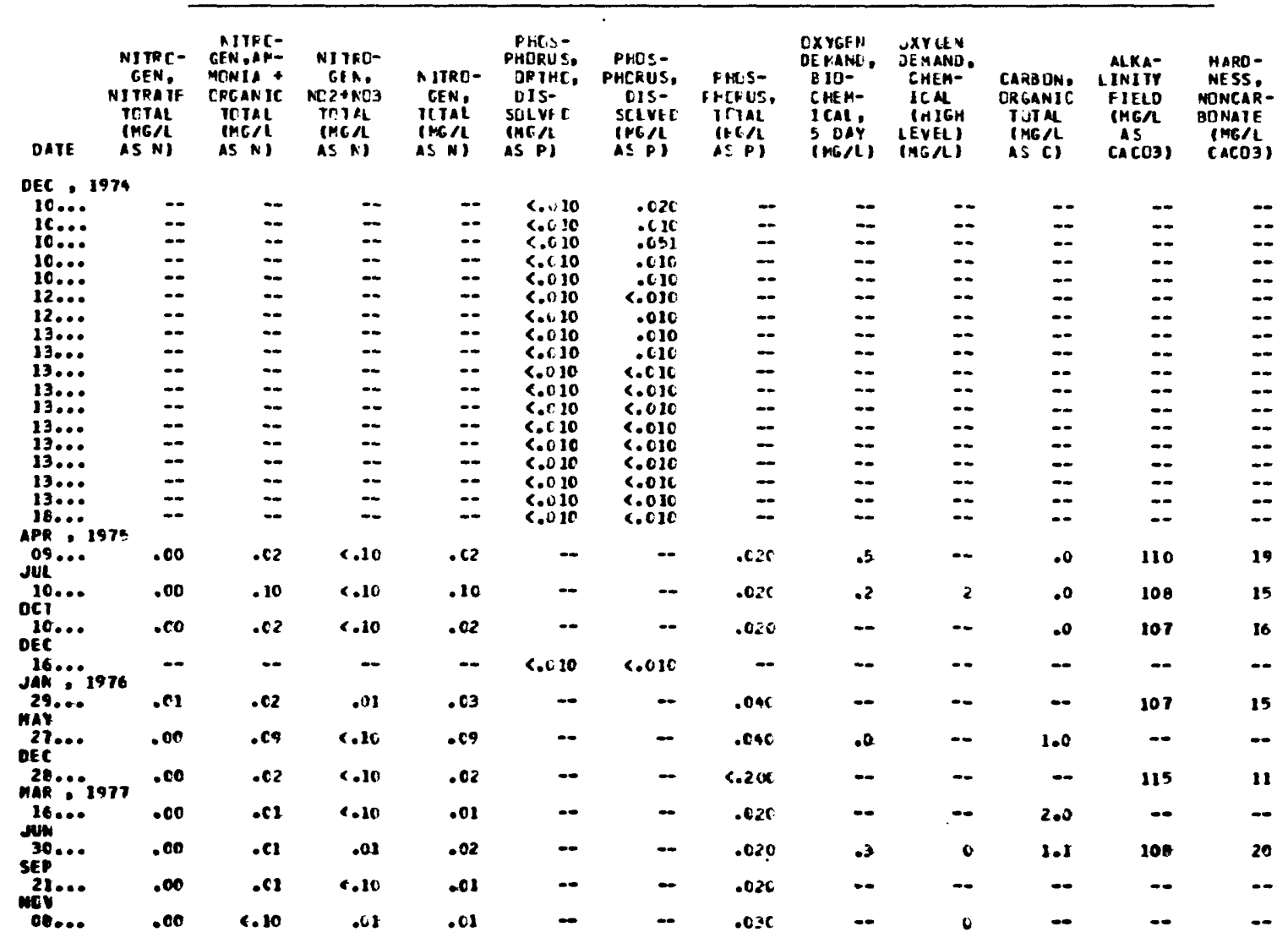

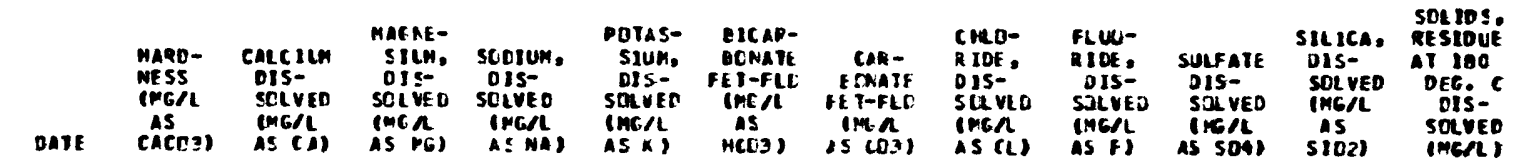

\begin{tabular}{|c|c|c|c|c|c|c|c|c|c|c|c|}
\hline DEC . 1979 & -- & - & $\cdots$ & -- & - & -- & - & 1.8 & -. & - & $\cdots$ \\
\hline & $\because$ & $\because$ & $\because$ & $\because$ & $=$ & $=$ & $=$ & 2.3 & $\cdots$ & $\cdots$ & $\cdots$ \\
\hline & $=$ & $\because$ & $\ldots$ & $=$ & $=$ & $=$ & $=$ & .6 & $\because$ & $\because$ & $=$ \\
\hline & $\cdots$ & - & $=$ & - & -- & $=$ & $=$ & .6 & $\because$ & 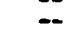 & $\ldots$ \\
\hline & 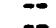 & $=$ & $=$ & 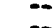 & - & - & - & 7.9 & $\cdots$ & $\cdots$ & $\cdots$ \\
\hline & $=$ & $=$ & $=$ & $\because$ & $=$ & $=$ & $=$ & 3.3 & $\because$ & - & $=$ \\
\hline & - & - & - & $\cdots$ & - & - & - & $\begin{array}{l}1.9 \\
3.9\end{array}$ & 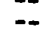 & $\because$ & $\ldots$ \\
\hline $23 . .$. & $\cdots$ & - & - & $\because$ & 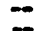 & $\infty$ & $=$ & 3.1 & $\cdots$ & - & $\cdots$ \\
\hline & 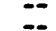 & $=$ & $=$ & $=$ & $\Xi$ & $=$ & $\bar{z}$ & 1.6 & $=$ & $\because$ & $\because$ \\
\hline & $\ldots$ & $=$ & $=$ & $\therefore$ & $=$ & - & .. & 1.9 & $\because$ & $=$ & 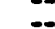 \\
\hline $3 \ldots$ & $\cdots$ & - & - & $\cdots$ & - & -- & - & 1.7 & -. & $\because$ & $\cdots$ \\
\hline$\because$ & $=$ & - & - & $\cdots$ & - & - & - & 1.3 & -- & -- & $\cdots$ \\
\hline & $=$ & $\ddot{B}$ & $=$ & $\because$ & $=$ & $=$ & $\bar{z}$ & 3.1 & $=$ & $\because$ & $=$ \\
\hline$\because \cdots$ & -. & - & $\cdots$ & $\ldots$ & - & - & - & 7.8 & -- & - & - \\
\hline & 130 & 32 & 12 & 4.4 & .4 & 134 & - & 0.9 & .2 & 17 & 9.1 \\
\hline & 120 & 31 & 11 & 4.4 & .5 & $13 i$ & $r$ & 0.0 & .3 & 19 & 8.6 \\
\hline & 120 & 32 & 10 & 4.6 & .5 & 136 & $i$ & 9.0 & .2 & 17 & 8.3 \\
\hline 1976 & $\cdots$ & - & - & $-\cdot$ & -- & - & - & 7.4 & - & $\cdots$ & -- \\
\hline & 120 & 32 & IC & 9.5 & .7 & $13 c$ & 6 & 6.6 & .2 & 18 & 8.2 \\
\hline & -- & - & $\cdots$ & 4.6 & - & $\cdots$ & - & 8.4 & $\cdots$ & $\cdots$ & $\cdots$ \\
\hline 1977 & 130 & 32 & 11 & 4.8 & .5 & 140 & $r$ & 0.7 & .2 & $\cdots$ & 8.6 \\
\hline$\bullet$ & $\cdots$ & $\cdots$ & $\cdots$ & 4.7 & $\cdots$ & - & - & 6.0 & $\cdots$ & $\cdots$ & -- \\
\hline 3 & 130 & 33 & 11 & 4.4 & .4 & 132 & 6 & 7.1 & .1 & 10 & 9.3 \\
\hline 2 & -- & - & - & 3.7 & -- & - & - & 0.6 & $\cdots$ & - & -. \\
\hline & -- & - & - & A.t & - & -- & - & 7.8 & -. & .. & $\cdots$ \\
\hline
\end{tabular}




\begin{tabular}{|c|c|c|c|c|c|c|c|c|c|c|c|}
\hline & \multicolumn{11}{|c|}{ Site 24--Continued } \\
\hline DATE & TIME & $\begin{array}{l}\text { TEFIH } \\
\text { OF } \\
\text { WELL, } \\
\text { IOTAL } \\
\text { (FEFI) }\end{array}$ & $\begin{array}{l}\text { PLMF } \\
\text { CR FLOW } \\
\text { PE RIDO } \\
\text { PRIDR } \\
\text { TO SAM- } \\
\text { PLING } \\
\text { (MIN) }\end{array}$ & $\begin{array}{l}\text { FLiK } \\
\text { RAYE } \\
\text { (GPM) }\end{array}$ & $\begin{array}{l}\text { TEMPER- } \\
\text { ATURE } \\
\text { (DFG C) }\end{array}$ & $\begin{array}{l}\text { SFI- } \\
\text { CIFIC } \\
\text { CEN- } \\
\text { CUCT- } \\
\text { ANCE } \\
\text { (URIHCS) }\end{array}$ & $\begin{array}{c}\text { IH } \\
\text { (UNITS) }\end{array}$ & $\begin{array}{l}\text { NI IRJ- } \\
\text { GEN, } \\
\text { AMMCNIA } \\
\text { TOIAL } \\
\text { (MG/L } \\
\text { AS N) }\end{array}$ & $\begin{array}{l}\text { NI IRO- } \\
\text { GEN. } \\
\text { ORGANIE } \\
\text { TOTAL } \\
\text { (MC/L } \\
\text { AS N) }\end{array}$ & $\begin{array}{l}\text { NIIRO- } \\
\text { GEN, } \\
\text { NITRIIE } \\
\text { IOTAL } \\
\text { (MG/L } \\
\text { AS N) }\end{array}$ & $\begin{array}{l}\text { NIIRO- } \\
\text { GEN, } \\
\text { NITRATE } \\
\text { TOTAL } \\
\text { (MG/L } \\
\text { AS N) }\end{array}$ \\
\hline SEP . & ${ }^{78} 0925$ & 260 & 45 & 7.5 & 22.0 & $2 ! 5$ & 7.9 & $<.010$ & .00 & $<.010$ & .00 \\
\hline $\begin{array}{l}\text { DEE } \\
2 E \ldots . . \\
\text { YAR }\end{array}$ & 0915 & 260 & 37 & 7.5 & 21.3 & 208 & e.2 & $<.010$ & .02 & $<.010$ & .00 \\
\hline $22 \ldots$ & 17905 & 260 & 30 & 7.9 & 21.5 & $2: 9$ & 7.9 & $<.010$ & .00 & $<.010$ & .00 \\
\hline & 1640 & 260 & 35 & 3.1 & 23.5 & $2 t E$ & 7.9 & $<.010$ & .00 & $<.010$ & .00 \\
\hline MAR & $8 \mathrm{C}^{1245}$ & .00 & 33 & 8.3 & 21.5 & $26 z$ & 7.7 & - & -- & .000 & .00 \\
\hline JUN $13 \ldots$ & 1540 & 260 & $3 e$ & 5.0 & 21.5 & $2 C 3$ & 7.9 & .010 & .07 & .000 & .00 \\
\hline rci $_{11 . . .}$ & 1555 & 260 & 97 & 3.2 & 21.5 & $26 \mathrm{C}$ & 7.9 & .020 & .00 & .000 & .00 \\
\hline$\underset{\text { JAN }}{16}$ & $981^{1515}$ & 260 & 67 & 3.9 & 22.0 & 240 & -- & .030 & .00 & .000 & .00 \\
\hline $09 \ldots$ & 1625 & 260 & 58 & 4.5 & $2 z \cdot 0$ & $2 e z$ & e.1 & .020 & .11 & .000 & .00 \\
\hline DATE & $\begin{array}{l}\text { NITRD- } \\
\text { GEN,AM- } \\
\text { MCNIA + } \\
\text { ORGANIF } \\
\text { TOIAL } \\
\text { (MGAL } \\
\text { AS N) }\end{array}$ & $\begin{array}{l}\text { AITRD- } \\
\text { GEN, } \\
\text { NCZ N NO3 } \\
\text { IOTAL } \\
\text { (MF/L } \\
\text { ASN) }\end{array}$ & $\begin{array}{l}\text { NI IRO- } \\
\text { GLN, } \\
\text { TOTAL } \\
\text { (MG/L } \\
\text { AS N) }\end{array}$ & $\begin{array}{l}\text { PHOS- } \\
\text { PHDRUS, } \\
\text { TOTAL. } \\
\text { (MGA } \\
\text { AS P) }\end{array}$ & $\begin{array}{l}\text { C AREUN, } \\
\text { ORGANIC } \\
\text { CIS- } \\
\text { SCLVED } \\
\text { (MERL } \\
\text { AS C) }\end{array}$ & $\begin{array}{l}\text { ALXA- } \\
\text { LINI TY } \\
\text { FIELI } \\
\text { IMG } / 1 \\
\text { AS } \\
\text { CACC3) }\end{array}$ & $\begin{array}{l}\text { HAFQ- } \\
\text { NESS, } \\
\text { NONCAR- } \\
\text { PONATE } \\
\text { (MC/L } \\
\text { CACO3) }\end{array}$ & $\begin{array}{l}\text { HAED- } \\
\text { NESS } \\
\text { (NGA } \\
\text { AS } \\
\text { CACO3) }\end{array}$ & $\begin{array}{l}\text { CALCIUH } \\
\text { DIS- } \\
\text { SOLVED } \\
\text { (MG/L } \\
\text { AS CA) }\end{array}$ & $\begin{array}{l}\text { MAGNE- } \\
\text { SIUM, } \\
\text { DIS- } \\
\text { SOLVED } \\
\text { (MGRL } \\
\text { AS MG) }\end{array}$ & $\begin{array}{l}\text { SOOIUH. } \\
\text { DIS- } \\
\text { SOLYED } \\
\text { (MGAL } \\
\text { AS NAS }\end{array}$ \\
\hline
\end{tabular}

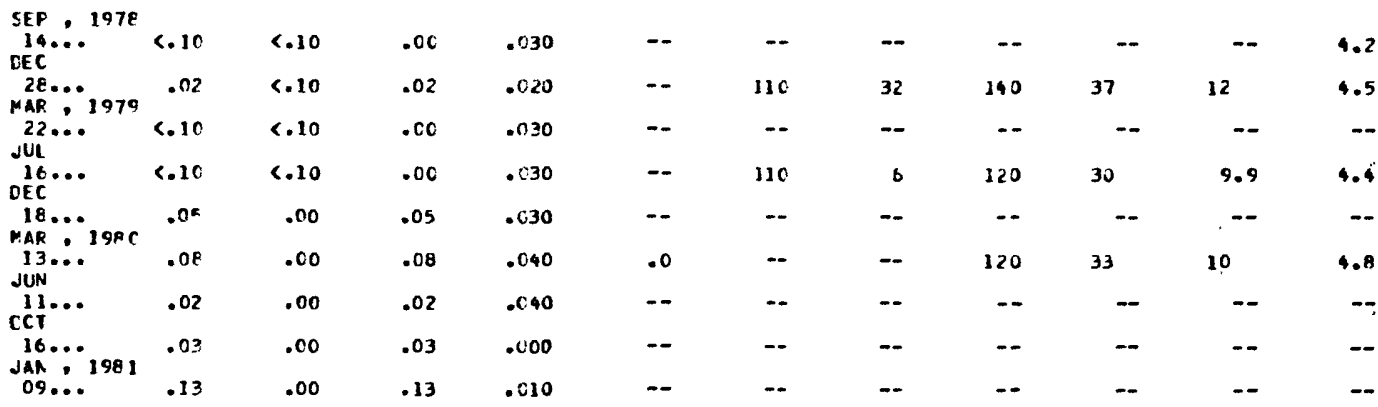

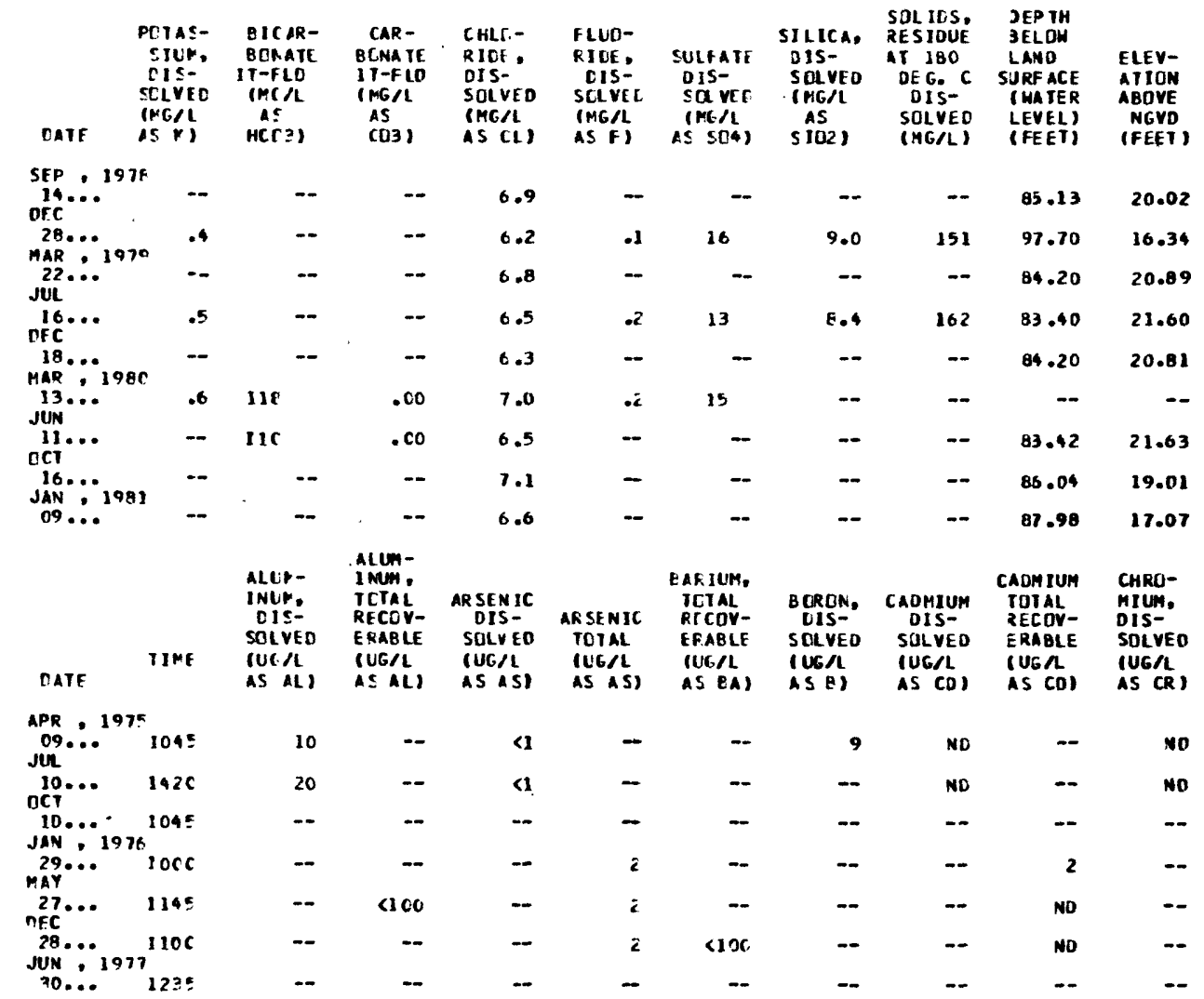


Table 6.--Physical and chemical characteristics of well and water at sites 1 through 70--Continued

Site 24--Continued

\begin{tabular}{|c|c|c|c|c|c|c|c|c|c|c|}
\hline & $\begin{array}{l}\text { CHRC- } \\
\text { MIUP, } \\
\text { HEXA- } \\
\text { VALENT, } \\
\text { DIS: } \\
\text { PUEAL }\end{array}$ & $\begin{array}{l}\text { CHFC- } \\
\text { MILH. } \\
\text { IOTAL } \\
\text { RCCCV- } \\
\text { ERARLE } \\
\text { FUC /L }\end{array}$ & $\begin{array}{l}\text { COBALT, } \\
\text { DIS- } \\
\text { SOLVED } \\
\text { CUGIL }\end{array}$ & $\begin{array}{l}\text { COBALT, } \\
\text { TOTAL } \\
\text { RECIV- } \\
\text { ERABLL } \\
\text { CUGA }\end{array}$ & $\begin{array}{l}\text { COPFEF, } \\
\text { CIS- } \\
\text { SALVE, } \\
\text { luC } / 1\end{array}$ & $\begin{array}{l}\text { COTFER, } \\
\text { ICIAL } \\
\text { KECEV- } \\
\text { EFABLE } \\
\text { ILC L }\end{array}$ & $\begin{array}{l}\text { IFON, } \\
\text { DIS- } \\
\text { SULVED } \\
\text { PUG }\end{array}$ & $\begin{array}{l}\text { IRON, } \\
\text { IJTAL } \\
\text { RECOV- } \\
\text { ERAGLE } \\
\text { (UG/L }\end{array}$ & $\begin{array}{l}\text { EAD, } \\
\text { DI S- } \\
\text { SOL VEO } \\
\text { ( US } / L\end{array}$ & $\begin{array}{l}\text { LEAO, } \\
\text { IOTAL } \\
\text { RECOV- } \\
\text { ERABLE } \\
\text { IUGA } \\
\text { US PB }\end{array}$ \\
\hline & AS (F) & $A S(R)$ & $A \div(C)$ & $A S(C)$ & AS (U) & $A \leq(1)$ & AS F(I) & AS FEl & As $P B$ I & \\
\hline
\end{tabular}

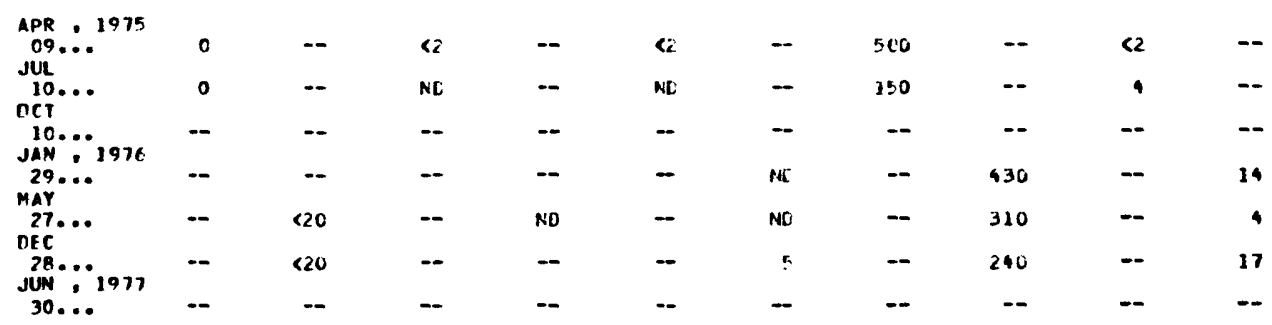

\begin{tabular}{|c|c|c|c|c|c|c|c|c|c|c|}
\hline DATE & $\begin{array}{l}11 \\
\text { sc } \\
\text { it }\end{array}$ & $\begin{array}{l}\text { IIUM } \\
\text { IS- } \\
\text { VEO } \\
\text { II } \\
\text { III }\end{array}$ & $\begin{array}{l}\text { LITHIUH } \\
\text { UOTAL } \\
\text { RECOV- } \\
\text { ERABLE } \\
\text { CUG/L } \\
\text { AS II) }\end{array}$ & $\begin{array}{l}\text { MANGA- } \\
\text { NESE, } \\
\text { IOTAL } \\
\text { RECEV- } \\
\text { ERALLE } \\
\text { TUGA } \\
\text { AS MNI }\end{array}$ & $\begin{array}{l}\text { MANGA- } \\
\text { NE SE, } \\
\text { DIS- } \\
\text { SOLVEU } \\
\text { (UEA } \\
\text { AS MN) }\end{array}$ & $\begin{array}{c}\text { MEFCLEY } \\
\text { ClS- } \\
\text { SEL VEC } \\
\text { IUG } \\
\text { AS HC) }\end{array}$ & $\begin{array}{l}\text { MEFCURY } \\
\text { TCIAL } \\
\text { RECCV- } \\
\text { EKAELE } \\
\text { (UC./L } \\
\text { AS HG) }\end{array}$ & $\begin{array}{l}\text { MDLYE- } \\
\text { DERUH, } \\
\text { DIS- } \\
\text { SOLVEO } \\
\text { IUC/L } \\
\text { AS MOI }\end{array}$ & $\begin{array}{l}\text { MOLYG- } \\
\text { DENUM, } \\
\text { TOTAL } \\
\text { RECUV- } \\
\text { ERAOLE } \\
\text { (UCAL } \\
\text { AS MOS }\end{array}$ & $\begin{array}{l}\text { NICKEL, } \\
\text { DIS- } \\
\text { SOLVEO } \\
\text { IUG/L } \\
\text { AS NII) }\end{array}$ \\
\hline \multicolumn{11}{|c|}{$\triangle P R, 1975$} \\
\hline Juh & & 10 & & & 30 & ... & - & 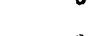 & & No \\
\hline$\underset{\text { CCT }}{10 .}$ & & $<10$ & -- & -- & 20 & c.5 & -- & $B$ & $=$ & NO \\
\hline JAN $10 \ldots$ & 976 & -- & -- & -- & - & -- & -- & -- & - & $\cdots$ \\
\hline 290 & & -- & - & (10 & - & -- & C.5 & -- & - & -- \\
\hline${ }_{\text {DEC }}^{27 \cdots}$ & & -- & $<10$ & $<30$ & -- & $=-$ & $<.5$ & -- & 5 & -- \\
\hline $\begin{array}{l}28 \ldots \\
\text { JUN }\end{array}$ & 977 & - & -- & $<10$ & -- & -- & $<.5$ & -- & -- & $\cdots$ \\
\hline $30 \ldots$ & & -- & -- & - & -- & - & $\cdots$ & - & - & -- \\
\hline DATE & $\begin{array}{l}\text { NI } \\
\text { II } \\
\text { RE } \\
\text { ER } \\
\text { II }\end{array}$ & $\begin{array}{l}\text { CEL. } \\
\text { CL } \\
\text { CV- } \\
\text { ELE } \\
\text { NII } \\
\text { Al) }\end{array}$ & $\begin{array}{l}\text { SELE- } \\
\text { NIUH, } \\
\text { DIS- } \\
\text { SOLVED } \\
\text { (UG/L } \\
\text { AS SE) }\end{array}$ & $\begin{array}{l}\text { SELE- } \\
\text { NIUIV. } \\
\text { TCTAL } \\
\text { (UG/L } \\
\text { IS SE) }\end{array}$ & $\begin{array}{l}\text { SILVER. } \\
\text { TOTAL } \\
\text { RECCV- } \\
\text { ER ABLE } \\
\text { (UC/L } \\
\text { AS AG) }\end{array}$ & $\begin{array}{l}\text { STR CN- } \\
\text { TILN, } \\
\text { CIS- } \\
\text { SCL VEC } \\
\text { (UE A } \\
\text { AS SF) }\end{array}$ & $\begin{array}{l}\text { VAHA- } \\
\text { DIUP: } \\
\text { CIS- } \\
\text { SCLVEO } \\
\text { CCC A } \\
\text { AS V) }\end{array}$ & $\begin{array}{l}\text { VANA- } \\
\text { OILM, } \\
\text { TOVAL } \\
\text { (UG } \\
\text { AS VI) }\end{array}$ & $\begin{array}{l}\text { ZINE. } \\
\text { DIS- } \\
\text { SOLUED } \\
\text { (UG/L } \\
\text { AS IN) }\end{array}$ & $\begin{array}{l}\text { ZINC, } \\
\text { IOTAL } \\
\text { RECQV- } \\
\text { ERABLE } \\
\text { (UG/L } \\
\text { AS IN) }\end{array}$ \\
\hline APR & 975 & & & & & & & & & \\
\hline JUL & & - & -- & -- & -- & -- & .0 & -- & 20 & -- \\
\hline $\begin{array}{l}10 . . \\
\text { LCT }\end{array}$ & & -- & (1) & - & - & $17 \mathrm{C}$ & 6.0 & -- & ND & -- \\
\hline JAN? & & -- & -- & - & -- & 230 & -- & -- & -- & - \\
\hline MAY & & -- & -- & -- & - & 250 & -- & -- & - & 20 \\
\hline $\operatorname{CEC}_{27}^{27} \cdots$ & & 7 & -- & $<1$ & -- & -- & -- & 8.9 & - & $<20$ \\
\hline JUN & & -- & -- & \&1 & ND & $\mu \mathrm{C}$ & $\cdots$ & $\cdots$ & -- & $<20$ \\
\hline $30 \ldots$ & & $\cdots$ & -- & -- & -- & 220 & -- & -- & - & $=-$ \\
\hline
\end{tabular}




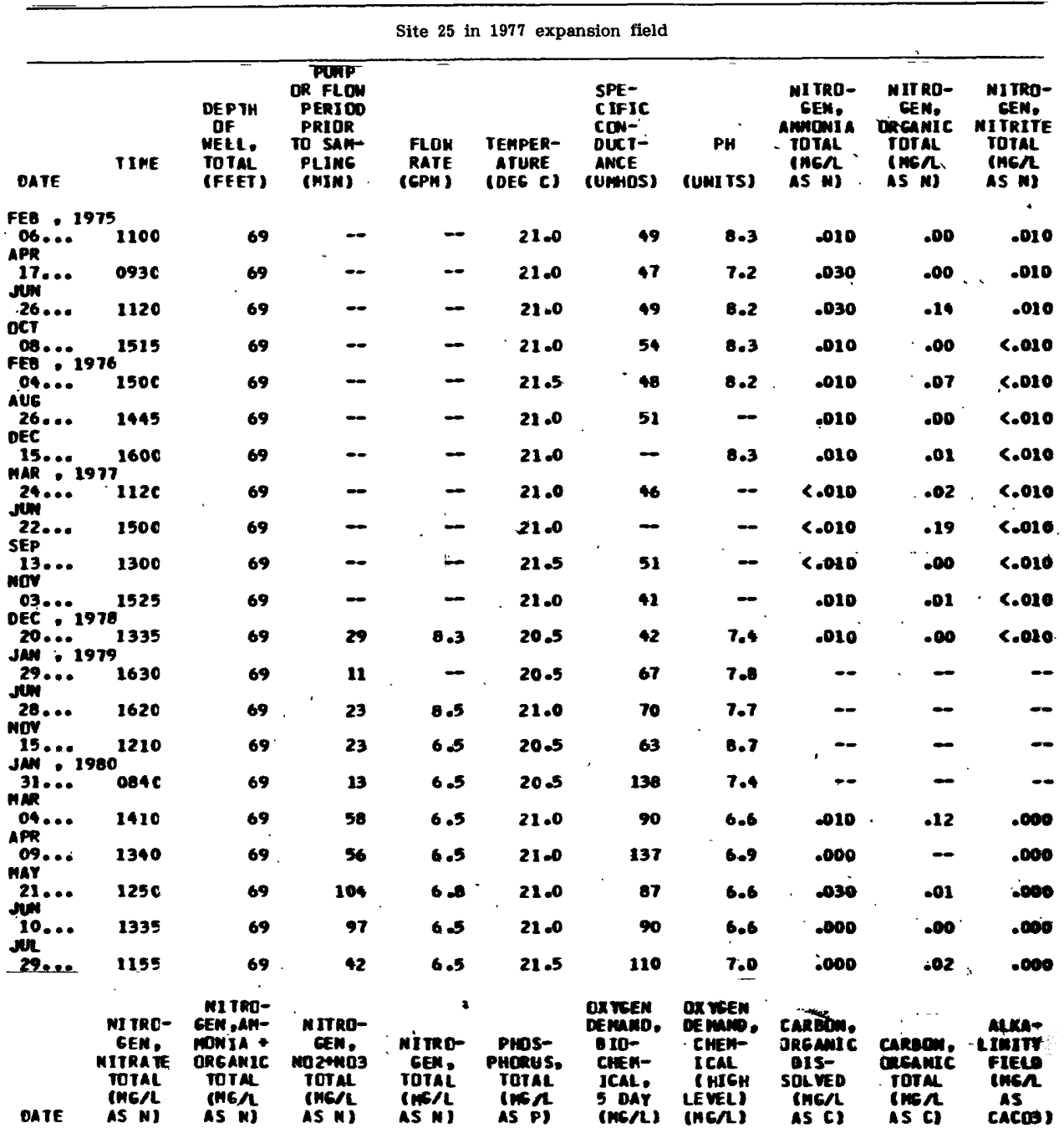

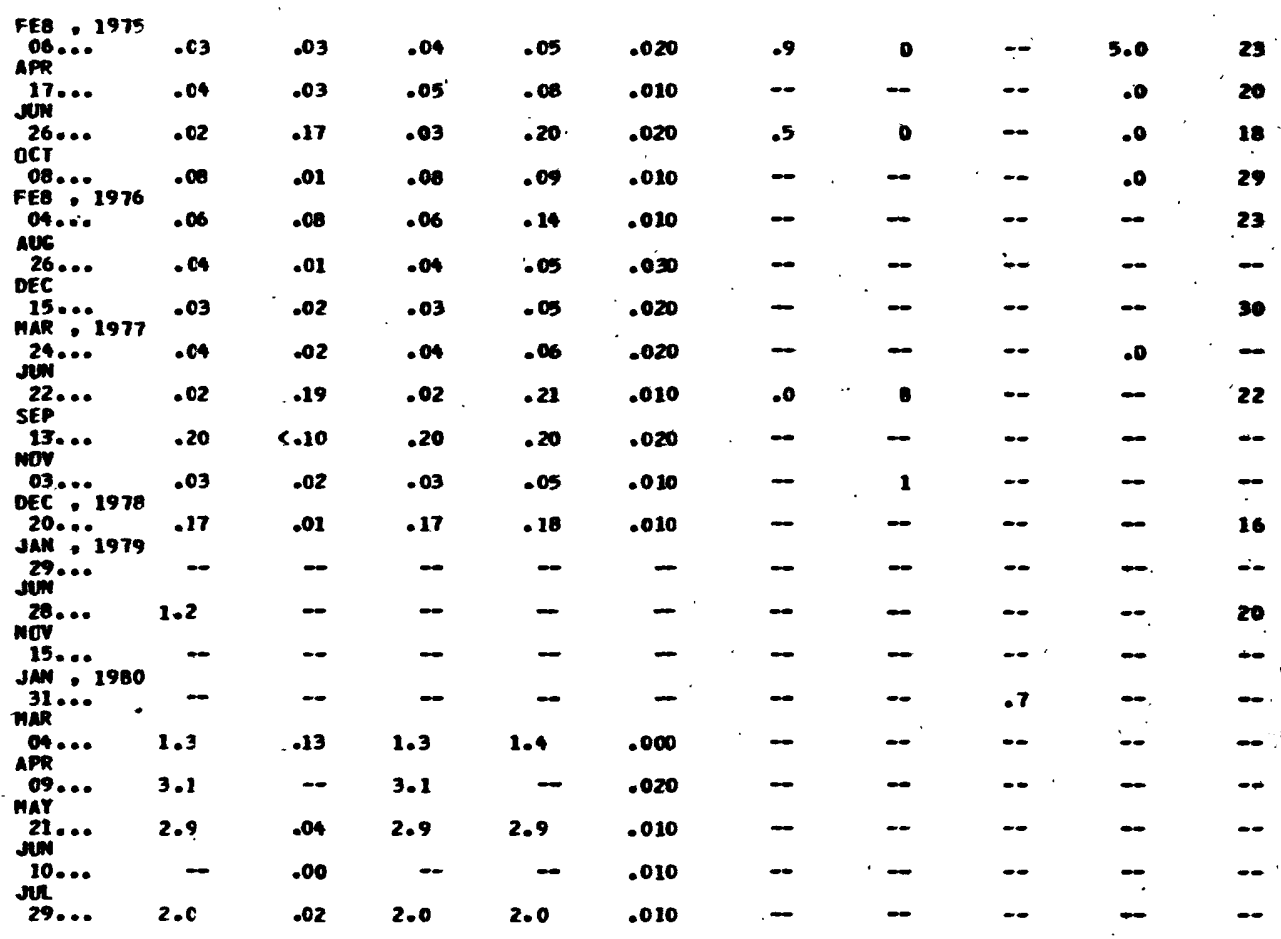


Site 25 in 1977 expansion field--Continued

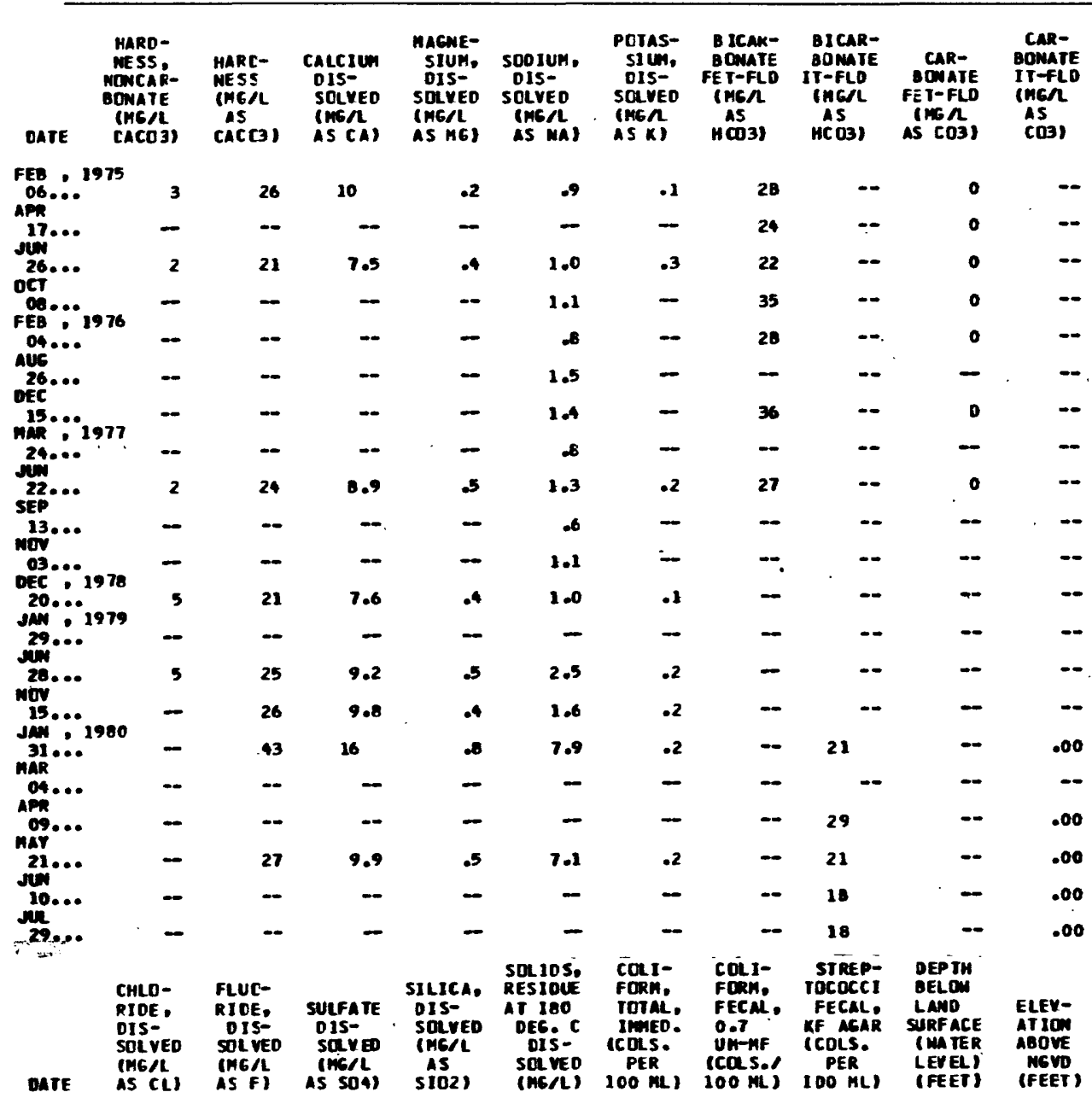

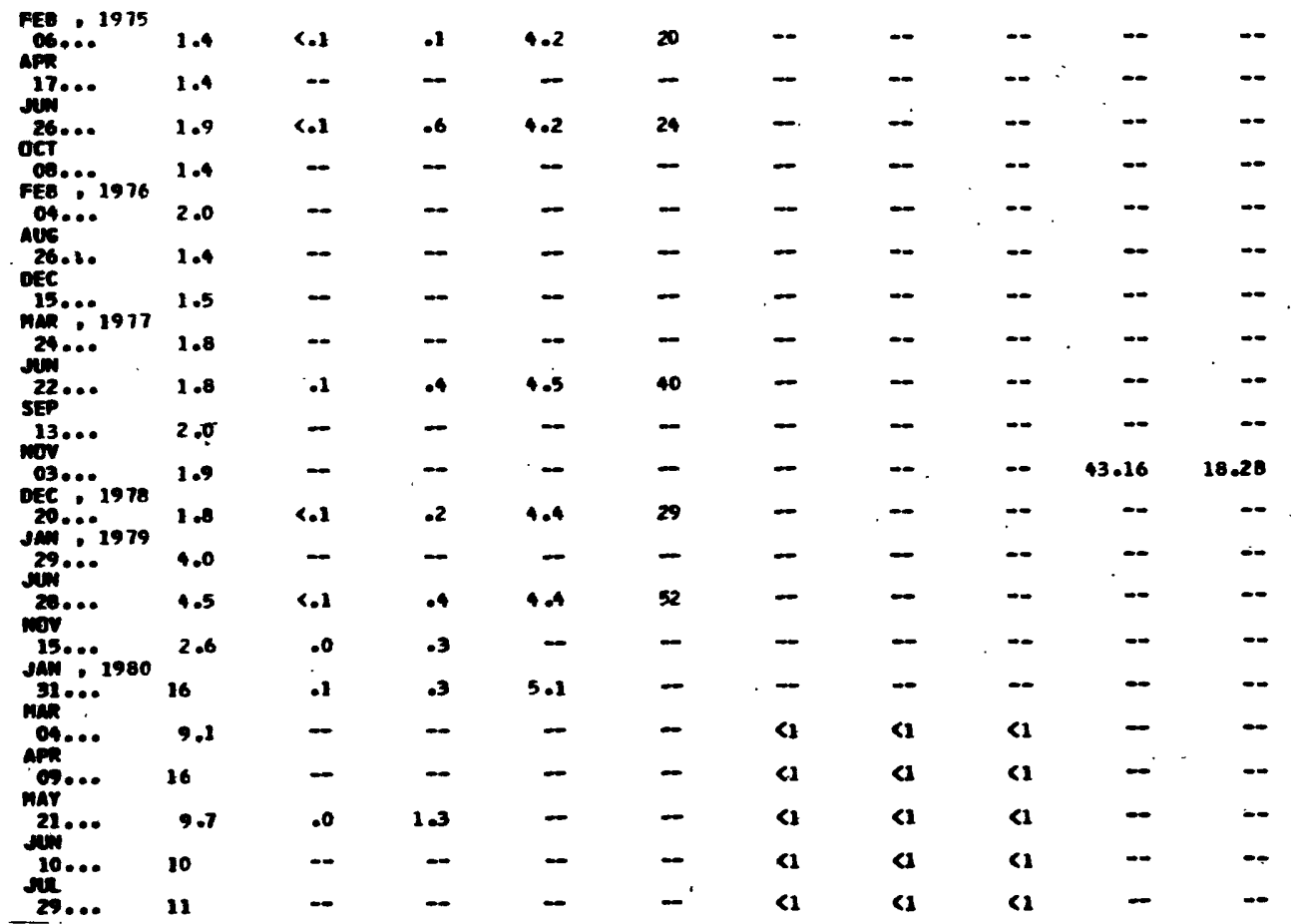


Site 25 in 1977 expansion field--Continued

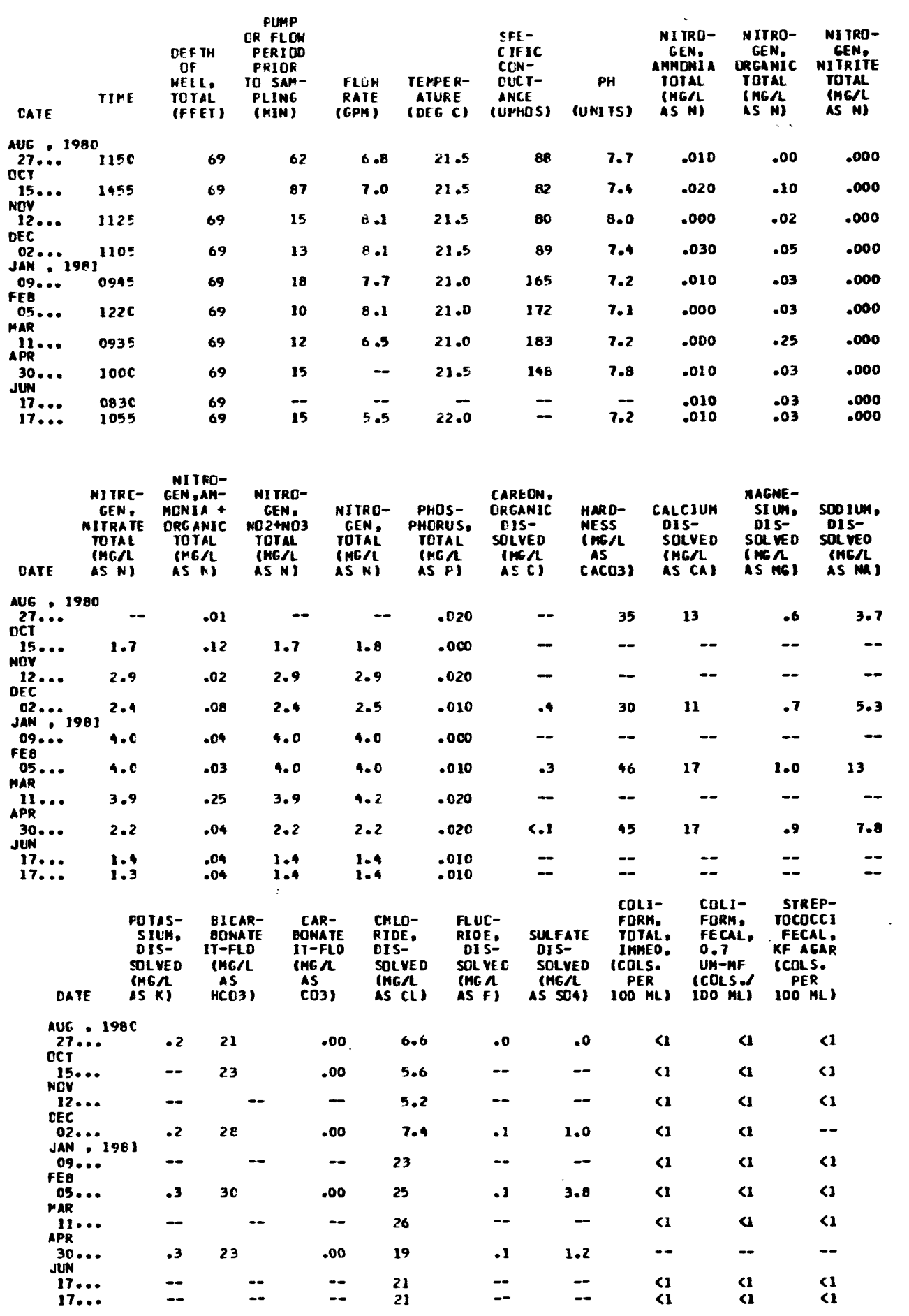


Site 25 in 1977 expansion field--Continued

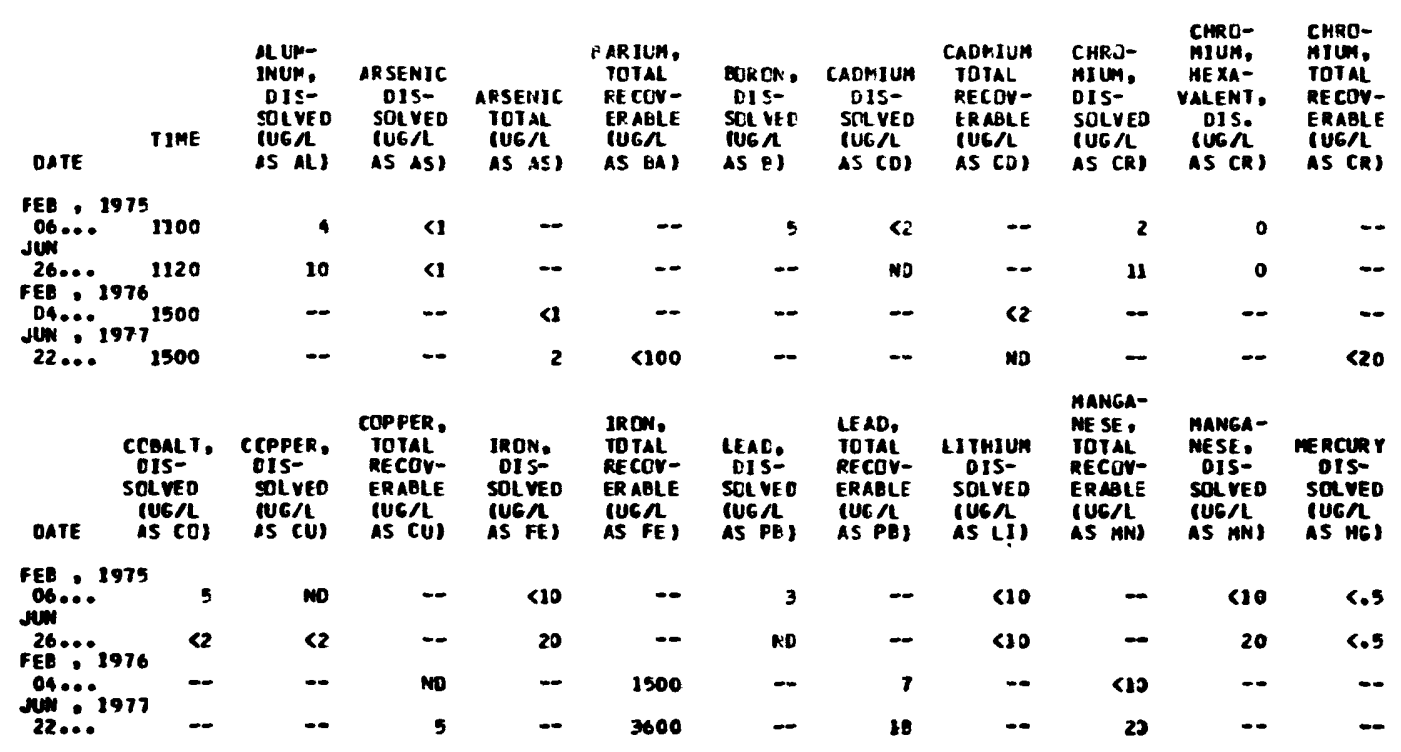

\begin{tabular}{|c|c|c|c|c|c|c|c|c|c|c|}
\hline DATE & $\begin{array}{l}\text { MERCURY } \\
\text { TOTAL } \\
\text { RE COV- } \\
\text { ERABLE } \\
\text { TUG/L } \\
\text { AS HES }\end{array}$ & 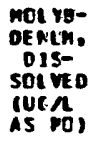 & $\begin{array}{l}\text { NI CXEL. } \\
\text { D IS- } \\
\text { SOLVED } \\
\text { IUS/L } \\
\text { AS NII }\end{array}$ & $\begin{array}{l}\text { SELE- } \\
\text { WIUW. } \\
\text { DIS- } \\
\text { SOLVEO } \\
\text { IUG/L } \\
\text { AS SES }\end{array}$ & $\begin{array}{l}\text { SELE- } \\
\text { NIUN, } \\
\text { rotAL } \\
\text { (UGCL } \\
\text { AS SE) }\end{array}$ & $\begin{array}{l}\text { SILVER. } \\
\text { TOTAL } \\
\text { RECoY- } \\
\text { ERALLE } \\
\text { (UGR } \\
\text { AS AG) }\end{array}$ & 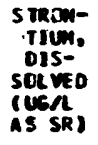 & $\begin{array}{l}\text { YAM- } \\
\text { DI UN. } \\
\text { OIS- } \\
\text { SOLVE } \\
\text { IUGA } \\
\text { AS VI }\end{array}$ & 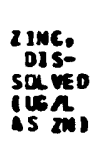 & 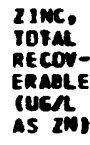 \\
\hline & $\cdots$ & < & 3 & - & - & - & $-\infty$ & .7 & 6 & $\cdots$ \\
\hline & - & (1) & wo & $\mathbf{a}$ & $\infty$ & - & 120 & .0 & mo & $\cdots$ \\
\hline & C.S & - & - & - & $\infty$ & - & $\infty$ & - & $\infty$ & \\
\hline 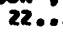 & $<.5$ & - & - & - & $\mathbf{A}$ & no & 30 & - & - & 100 \\
\hline
\end{tabular}


Site 26

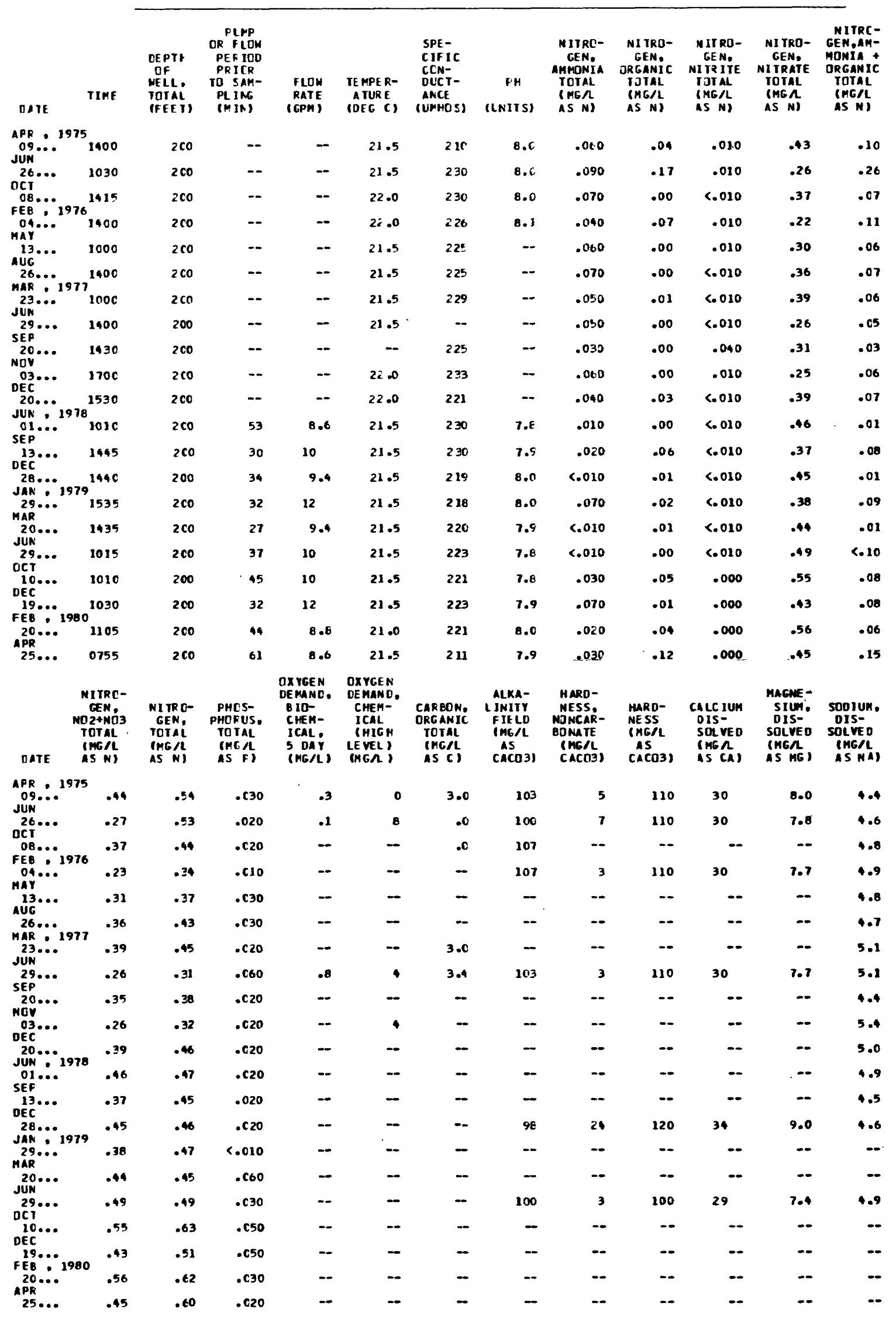


Site 26--Continued

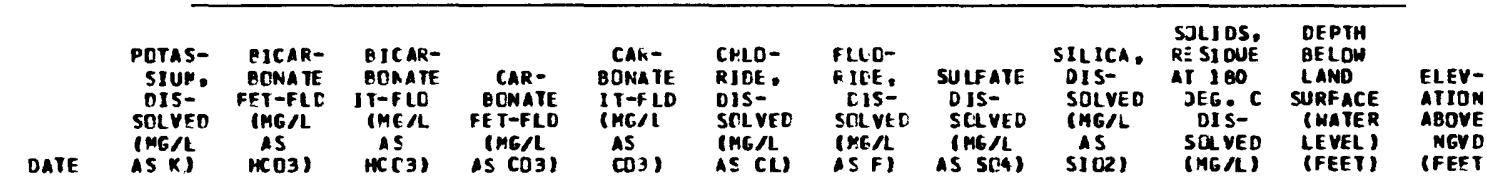

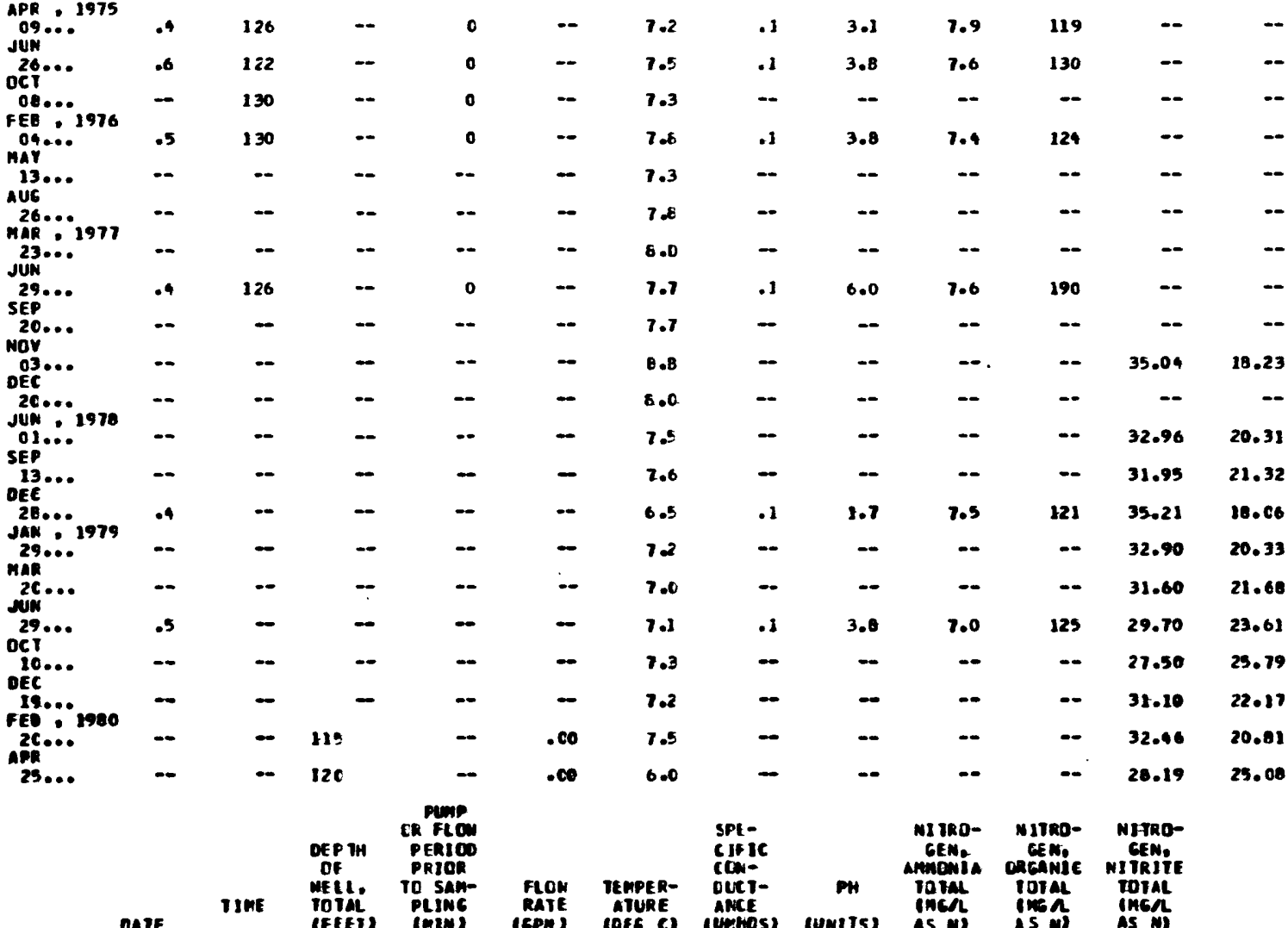

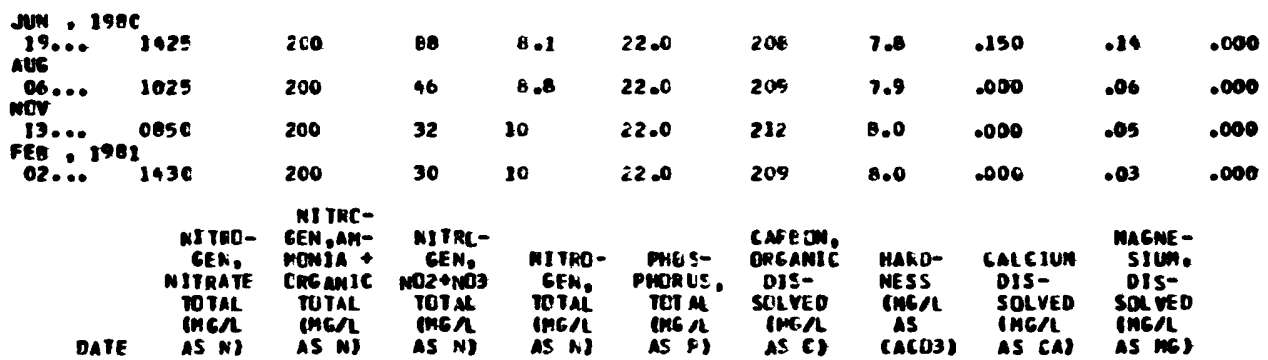

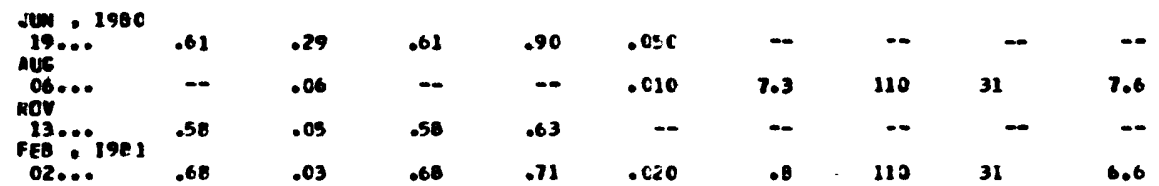




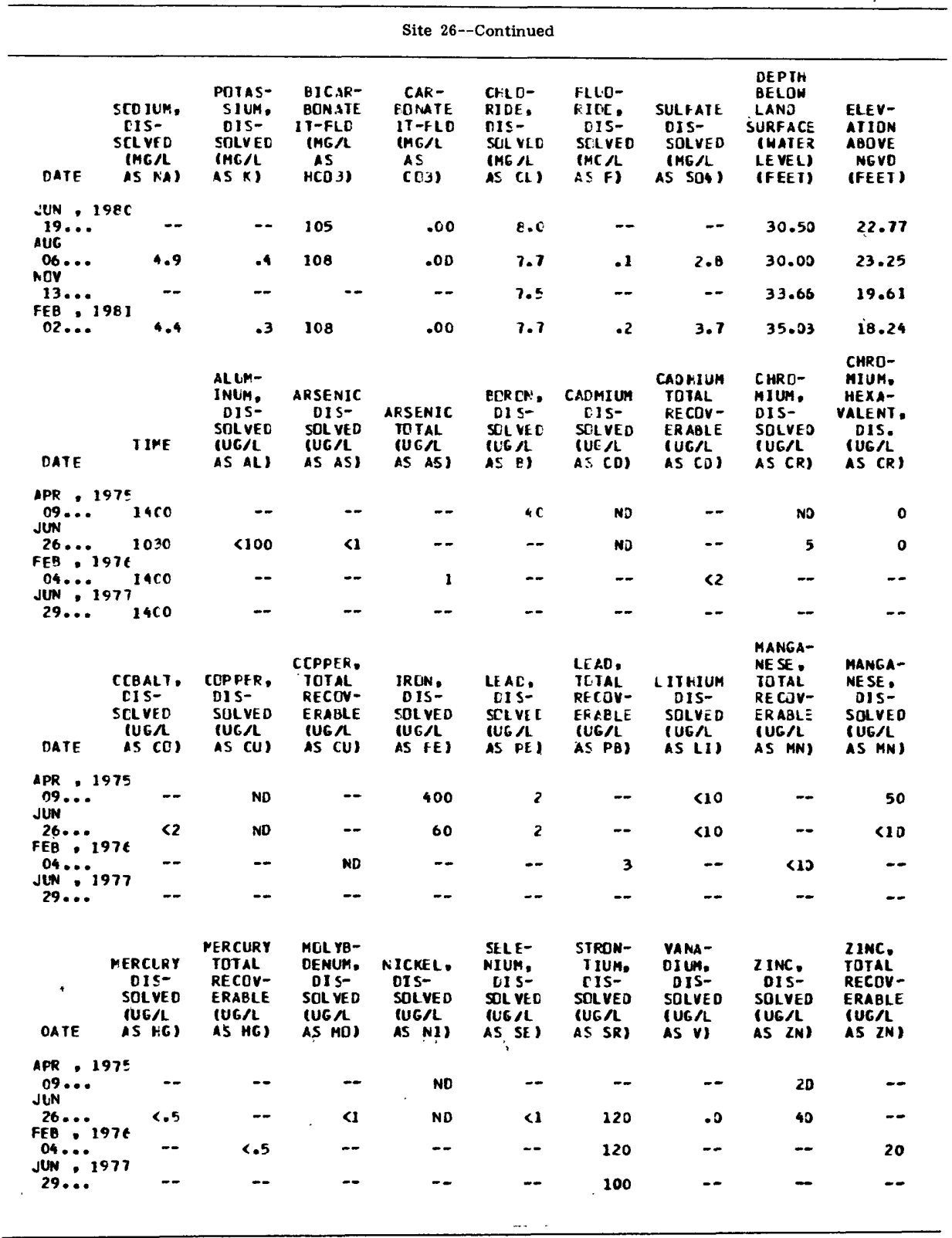

Site 27 in 4-big-gun area

\begin{tabular}{|c|c|c|c|c|c|c|c|}
\hline DATE & TIME & $\begin{array}{l}\text { OEPTH } \\
\text { OF } \\
\text { WELL. } \\
\text { TOTAL } \\
\text { (FEET) }\end{array}$ & $\begin{array}{l}\text { TEMPER- } \\
\text { ATURE } \\
\text { IOE C C }\end{array}$ & $\begin{array}{l}\text { SFE- } \\
\text { CIF IC } \\
\text { CON- } \\
\text { DUC I- } \\
\text { ANCE } \\
\text { (UNHOS) }\end{array}$ & $\begin{array}{l}\text { NITRO- } \\
\text { CEN, } \\
\text { AMMOMIA } \\
\text { TOTAL } \\
\text { (MC } \Omega \\
\text { AS N) }\end{array}$ & $\begin{array}{l}\text { N1TRD- } \\
\text { GEN, } \\
\text { DRGANIC } \\
\text { TOTAL } \\
\text { (MG/L } \\
\text { AS N) }\end{array}$ & $\begin{array}{l}\text { NITRO- } \\
\text { GEN, } \\
\text { NITRITE } \\
\text { TOTAL } \\
\text { ING/L } \\
\text { AS NI }\end{array}$ \\
\hline \multicolumn{8}{|c|}{ MOV , 1575} \\
\hline $\begin{array}{l}03 \ldots \ldots \\
04 \ldots \ldots \\
04 \ldots \ldots \\
04 \ldots \ldots \\
04 \ldots \ldots \\
05 \ldots \ldots \\
05 \ldots \ldots \\
05 \ldots \ldots \\
05 \ldots \ldots \\
05 \ldots \ldots \\
05 \ldots \ldots \\
05 \ldots \ldots\end{array}$ & $\begin{array}{l}1200 \\
0800 \\
0900 \\
1200 \\
1400 \\
0800 \\
1000 \\
1200 \\
1230 \\
1300 \\
1400 \\
1500\end{array}$ & $\begin{array}{r}30 \\
60 \\
72 \\
75 \\
80 \\
90 \\
100 \\
108 \\
120 \\
130 \\
140 \\
150\end{array}$ & 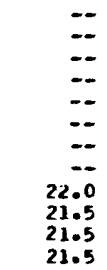 & $\begin{array}{l}725 \\
328 \\
290 \\
298 \\
262 \\
328 \\
436 \\
425 \\
422 \\
425 \\
450 \\
412\end{array}$ & $\begin{array}{l}.050 \\
.470 \\
.780 \\
.100 \\
.040 \\
.060 \\
.110 \\
.030 \\
.040 \\
.040 \\
.020 \\
.380\end{array}$ & $\begin{array}{l}.01 \\
.07 \\
.08 \\
.31 \\
.16 \\
.30 \\
.11 \\
.03 \\
.05 \\
.05 \\
.24 \\
.07\end{array}$ & $\begin{array}{l}.045 \\
.185 \\
.643 \\
.220 \\
.290 \\
.485 \\
.290 \\
.135 \\
.045 \\
.025 \\
.095 \\
.340\end{array}$ \\
\hline
\end{tabular}




\begin{tabular}{|c|c|c|c|c|c|c|c|}
\hline \multicolumn{8}{|c|}{ Site 27 in 4-big-gun area--Continued } \\
\hline DAIE & $\begin{array}{l}\text { NITRO- } \\
\text { GEN, } \\
\text { PITRATE } \\
\text { IOTAL } \\
\text { (MGAL } \\
\text { AS N) }\end{array}$ & $\begin{array}{l}\text { NITPE- } \\
\text { GEN,AAM- } \\
\text { MENIA } \\
\text { ORGANIC } \\
\text { TOTAL } \\
\text { IMGN } \\
\text { AS NI }\end{array}$ & $\begin{array}{l}\text { NITRD- } \\
\text { GEN, } \\
\text { NC2+NO3 } \\
\text { IOTAL } \\
\text { (ME } \\
\text { AS N) }\end{array}$ & $\begin{array}{l}\text { N1JKL- } \\
\text { GEA. } \\
\text { TEJAL } \\
\text { (WGA } \\
\text { AS NS }\end{array}$ & $\begin{array}{l}\text { PrES- } \\
\text { PHCRUS, } \\
\text { ICIAL } \\
\text { (rI/L } \\
\text { AS P) }\end{array}$ & $\begin{array}{l}\text { SODIUH, } \\
\text { UIS- } \\
\text { SOLVED } \\
\text { (ME N } \\
\text { AS NA) }\end{array}$ & $\begin{array}{l}\text { CHLO- } \\
\text { RIDE. } \\
\text { DIS- } \\
\text { SULVEO } \\
\text { (MGIL } \\
\text { AS CL) }\end{array}$ \\
\hline 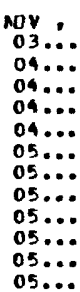 & $\begin{array}{c}75 \\
23 \\
7.8 \\
5.3 \\
6.3 \\
6.2 \\
7.5 \\
12 \\
12 \\
12 \\
12 \\
12 \\
9.6\end{array}$ & $\begin{array}{l}.06 \\
.54 \\
.86 \\
.41 \\
.20 \\
.36 \\
.22 \\
.06 \\
.09 \\
.09 \\
.26 \\
.45\end{array}$ & $\begin{array}{c}23 \\
8.0 \\
5.9 \\
6.5 \\
6.5 \\
8.0 \\
12 \\
12 \\
12 \\
12 \\
12 \\
9.9\end{array}$ & $\begin{array}{c}23 \\
8.5 \\
6.8 \\
6.9 \\
6.7 \\
8.2 \\
13 \\
12 \\
12 \\
12 \\
12 \\
10\end{array}$ & $\begin{array}{l}.040 \\
.070 \\
.010 \\
.010 \\
.030 \\
.020 \\
.020 \\
.020 \\
.040 \\
.040 \\
.020 \\
.020\end{array}$ & $\begin{array}{l}39 \\
16 \\
6.1 \\
5.8 \\
4.4 \\
6.3 \\
20 \\
17 \\
17 \\
17 \\
20 \\
17\end{array}$ & $\begin{array}{l}34 \\
20 \\
24 \\
24 \\
21 \\
26 \\
33 \\
32 \\
31 \\
32 \\
33 \\
32\end{array}$ \\
\hline
\end{tabular}

Site 28 in 4 -big-gun area

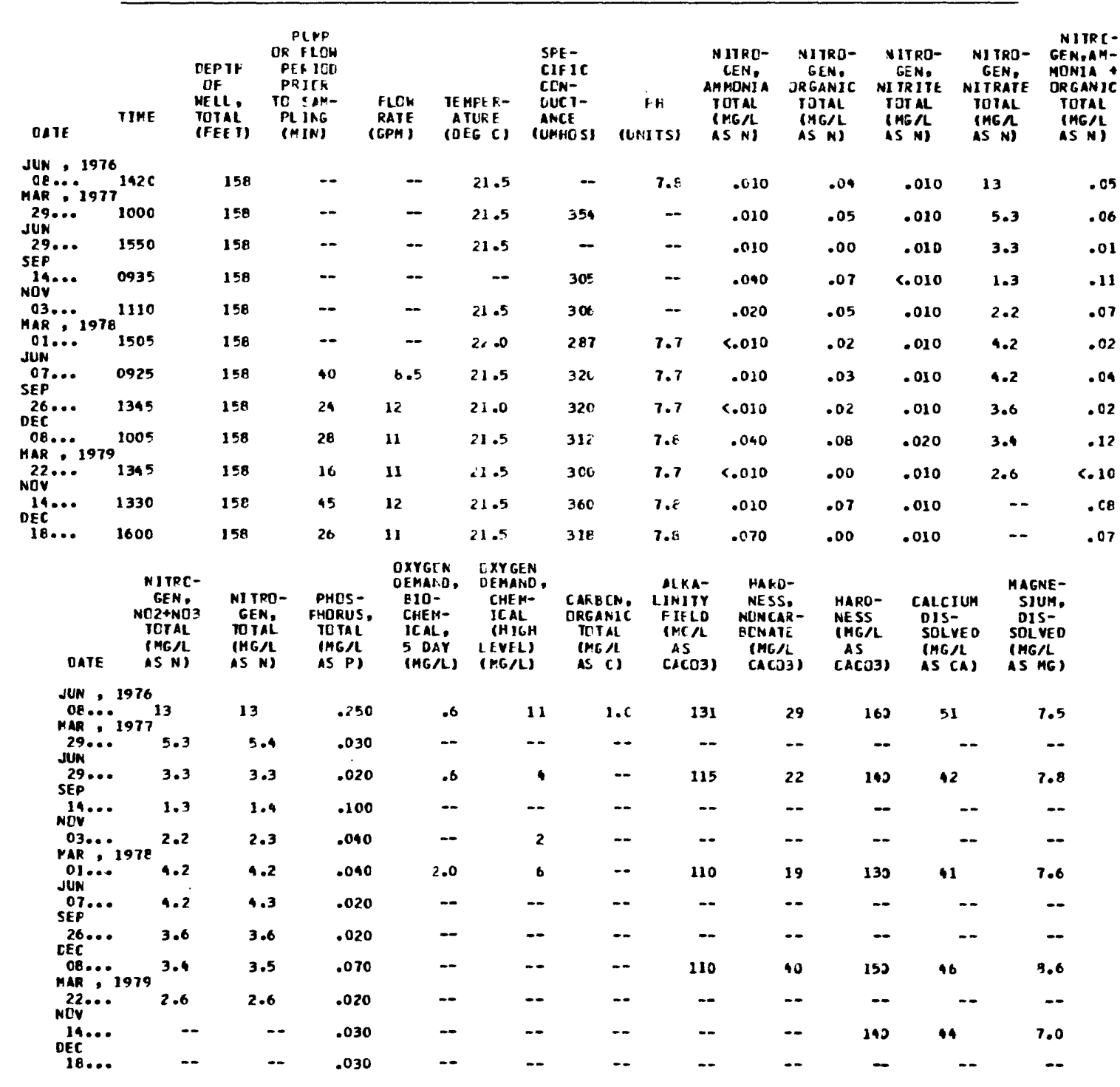


Site 28 in 4 -big-gun area--Continued

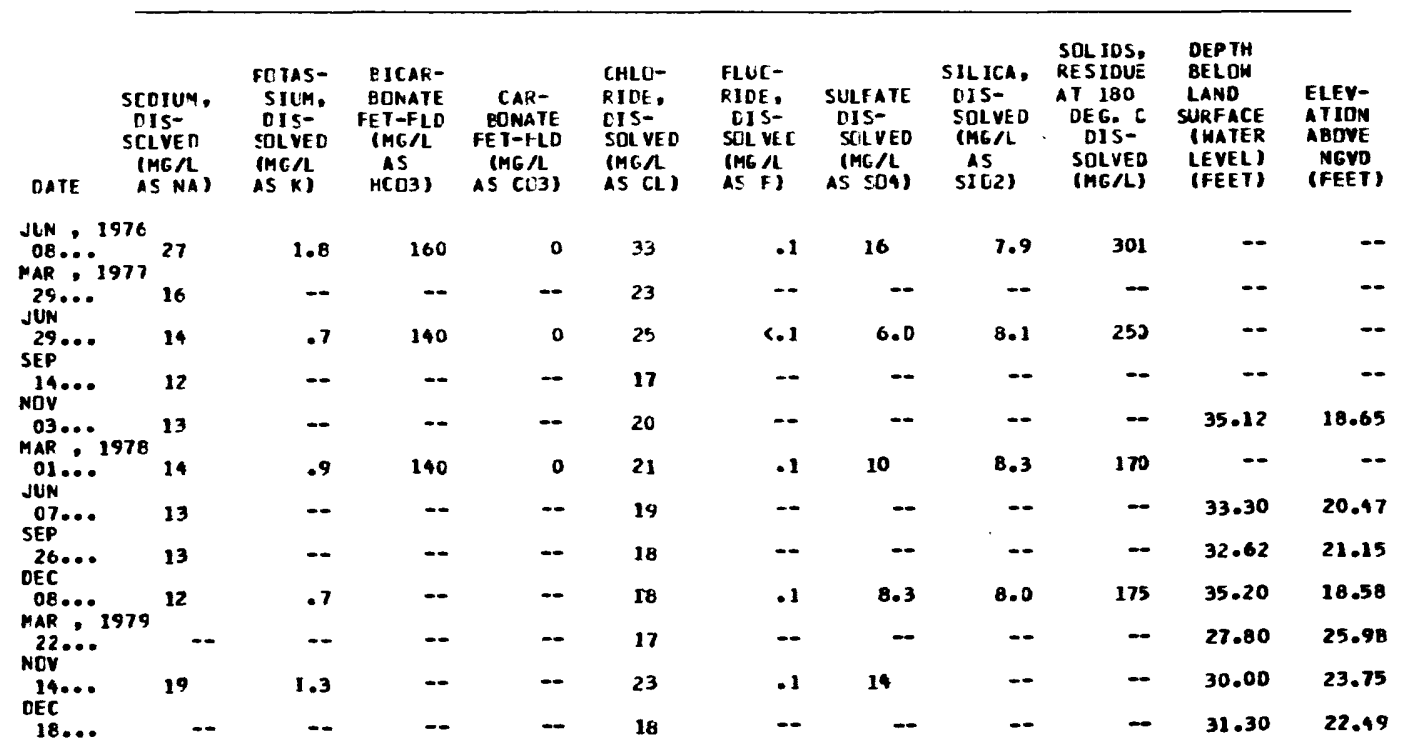

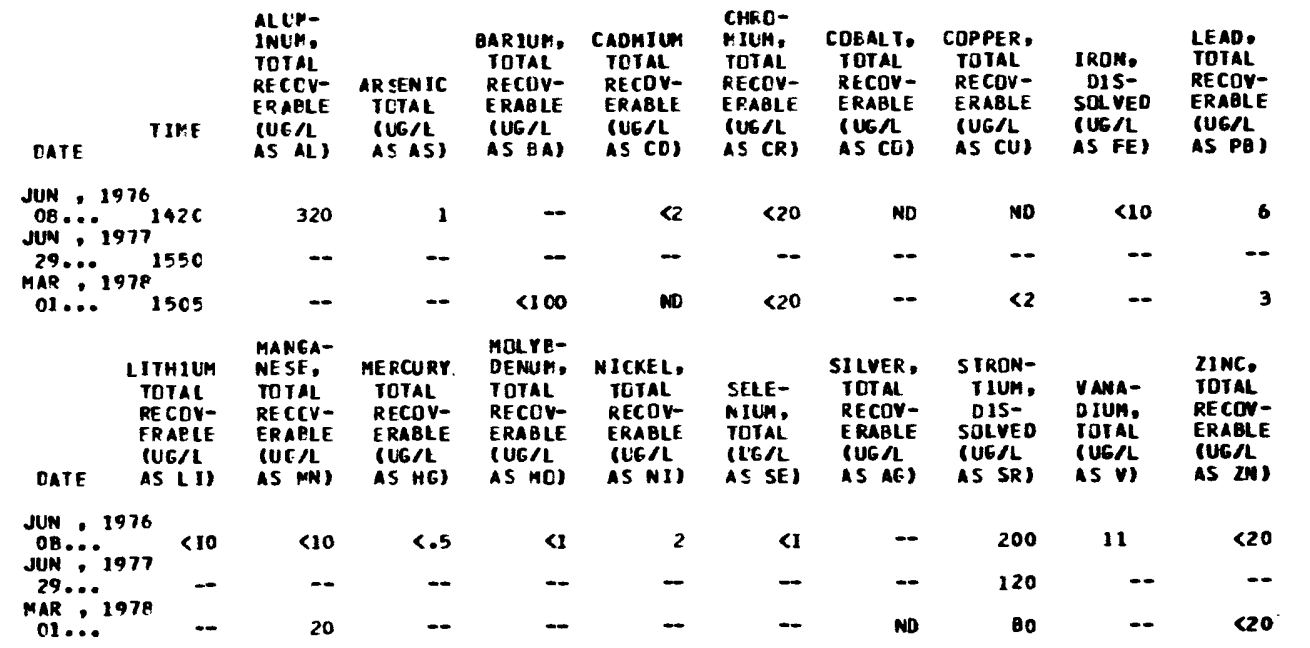


Table 6.--physical and chemical characteristics of well and water at sites 1 through $70--$ Continued

Site 29 in 4 -big-gun area

\begin{tabular}{|c|c|c|c|c|c|c|c|c|c|c|}
\hline IME & $\begin{array}{l}\text { TEPTH } \\
\text { DF } \\
\text { WELL, } \\
\text { TOTAL } \\
\text { (FEET) }\end{array}$ & $\begin{array}{l}\text { PLMP } \\
\text { CR FLOW } \\
\text { PERIOD } \\
\text { PRIDR } \\
\text { TO SAM- } \\
\text { PLING } \\
\text { (MIN) }\end{array}$ & $\begin{array}{l}\text { FLUW } \\
\text { RATE } \\
\text { (GPM) }\end{array}$ & $\begin{array}{l}\text { TEHPER - } \\
\text { AT URE } \\
\text { (DEG C) }\end{array}$ & $\begin{array}{l}\text { SFE - } \\
\text { CIF IC } \\
\text { CON- } \\
\text { DUC 1- } \\
\text { ANCE } \\
\text { (UMHCS) }\end{array}$ & $\begin{array}{c}\text { PH } \\
\text { (UNITS) }\end{array}$ & $\begin{array}{l}\text { NITRO- } \\
\text { GEN. } \\
\text { AMHENIA } \\
\text { TOTAL } \\
\text { (ME/L } \\
\text { AS N) }\end{array}$ & $\begin{array}{l}\text { NITRO- } \\
\text { GEN, } \\
\text { URGANIE } \\
\text { TOTAL } \\
\text { (MGNL } \\
\text { IS N) }\end{array}$ & $\begin{array}{l}\text { MITRO- } \\
\text { GEN. } \\
\text { NITRITE } \\
\text { TOTAL } \\
\text { (AG/L } \\
\text { AS N) }\end{array}$ & $\begin{array}{l}\text { NITRO- } \\
\text { GFN, } \\
\text { NITRATE } \\
\text { IOTAL } \\
\text { (MGRL } \\
\text { AS N) }\end{array}$ \\
\hline
\end{tabular}

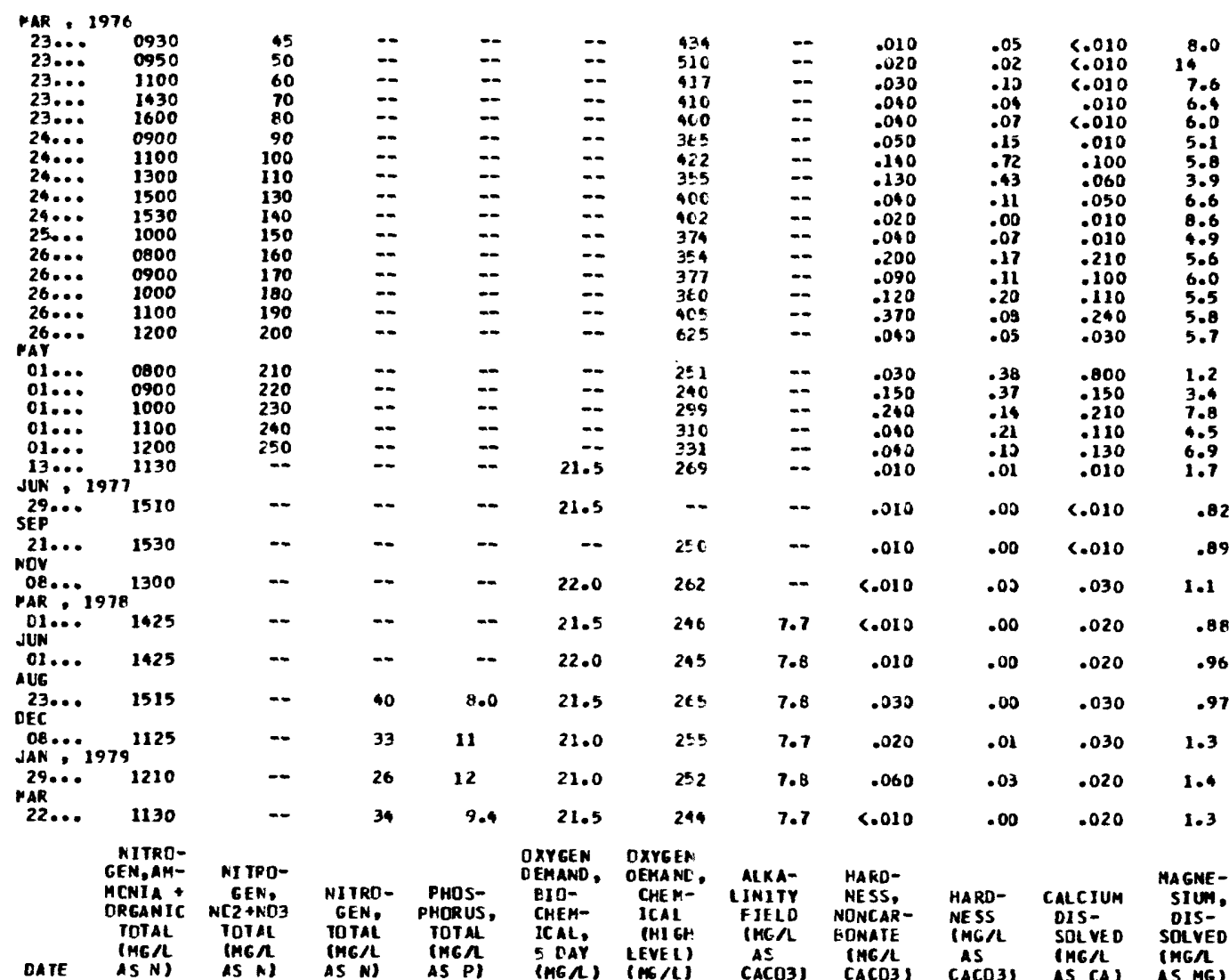

MAR , 1976

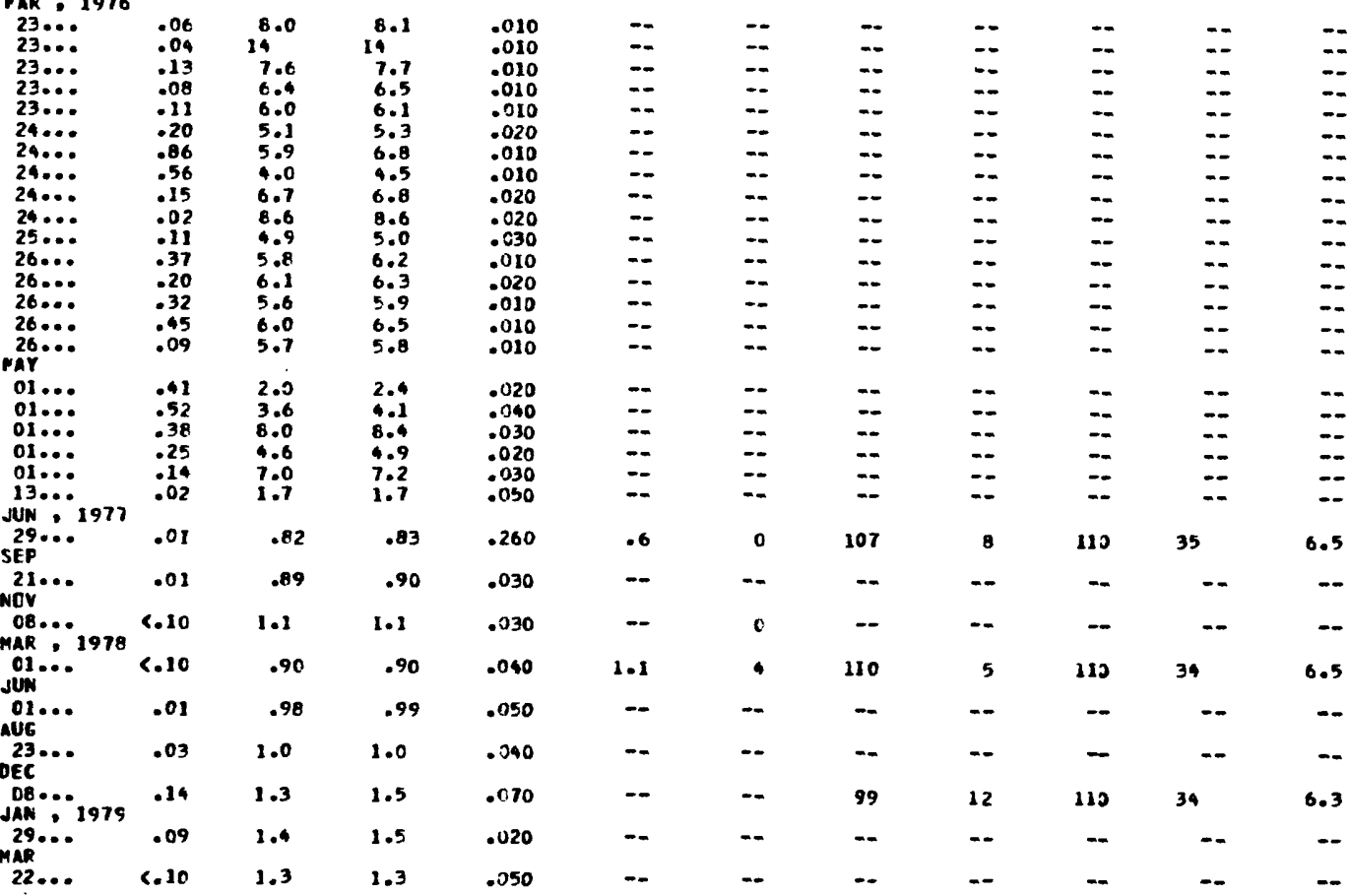


Site 29 in 4-big-gun area--Continued

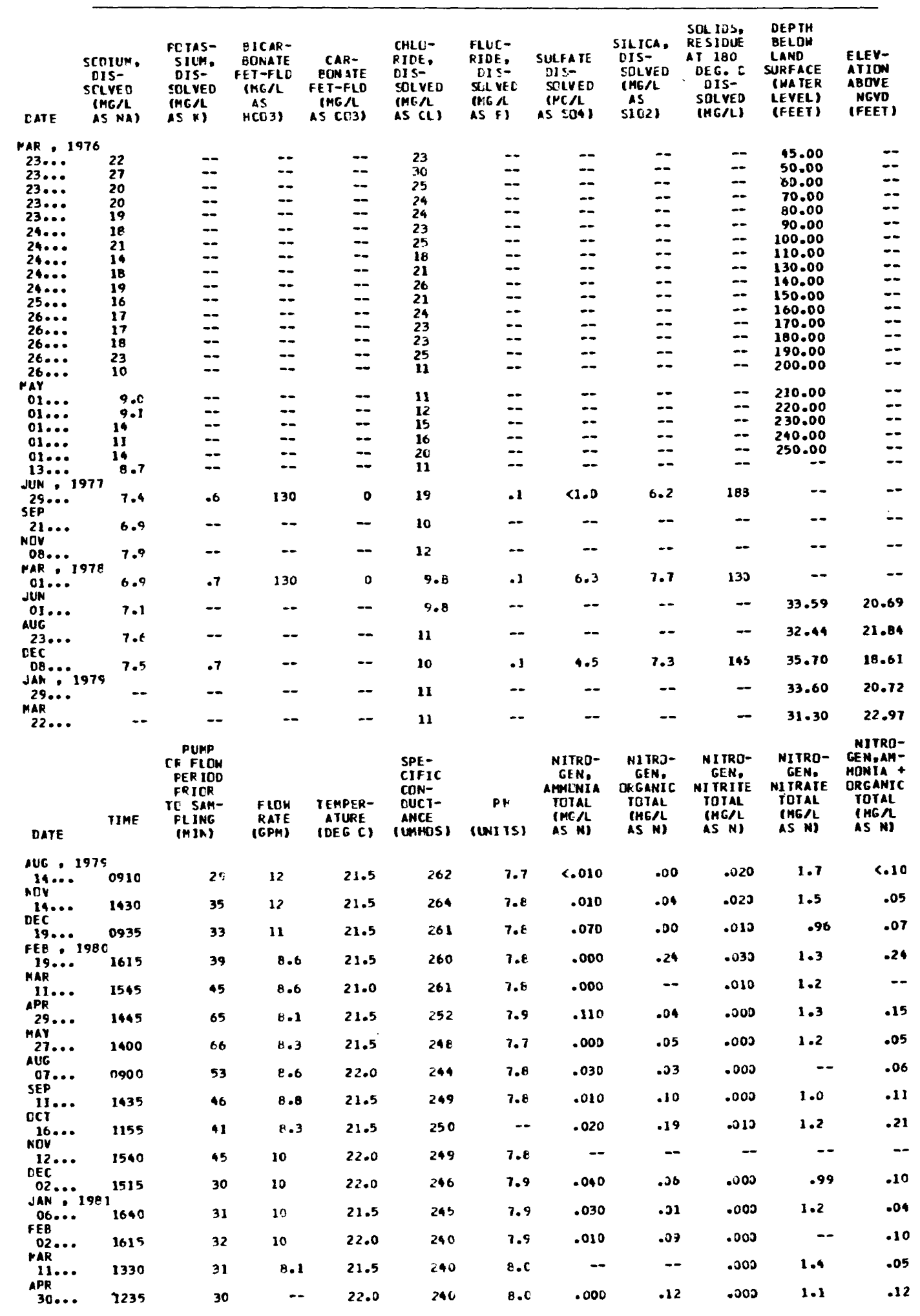


Site 29 in 4-big-gun area--Continued

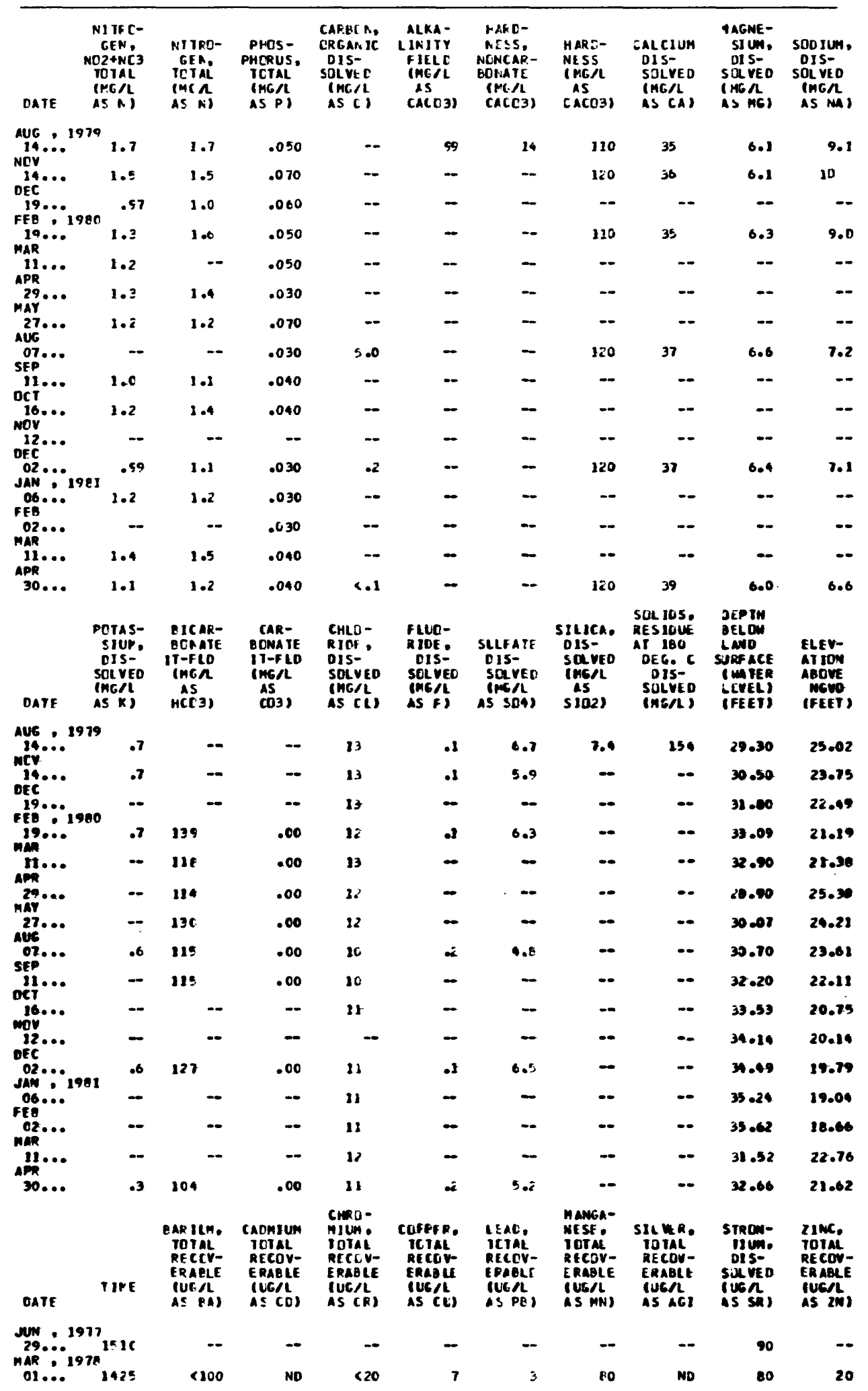




\begin{tabular}{|c|c|c|c|c|c|c|c|c|c|c|c|c|c|c|c|c|}
\hline DATE & TIYE & \multicolumn{2}{|c|}{$\begin{array}{c}\text { ALRRIN, } \\
\text { TOIAL } \\
\text { (UG/L) }\end{array}$} & \multicolumn{2}{|c|}{$\begin{array}{l}\text { CHLUR- } \\
\text { OANE, } \\
\text { IOTAL } \\
\text { IUG/L) }\end{array}$} & \multicolumn{2}{|c|}{$\begin{array}{l}\text { DOD, } \\
\text { DTAL } \\
\text { (UGA) }\end{array}$} & \multicolumn{2}{|c|}{$\begin{array}{l}\text { DUE, } \\
\text { TUIAL } \\
\text { (UG/L) }\end{array}$} & \multicolumn{2}{|c|}{$\begin{array}{l}\text { OCT, } \\
\text { TOTAL } \\
\text { (UG } / \mathrm{L} \text { ) }\end{array}$} & \multicolumn{2}{|c|}{$\begin{array}{l}\text { OI- } \\
\text { ELDRIN } \\
\text { TOTAL } \\
\text { (UG/L) }\end{array}$} & \multicolumn{2}{|c|}{$\begin{array}{l}\text { ENDO- } \\
\text { SULFAN, } \\
\text { TUIAL } \\
\text { (UGAL) }\end{array}$} & $\begin{array}{c}\text { ENDRIN, } \\
\text { TOTAL } \\
\text { (UG/L) }\end{array}$ \\
\hline \multicolumn{17}{|c|}{ AUE , 1978} \\
\hline $23 . \ldots$ & 3515 & & .00 & &.$c 0$ & & .00 & & .00 & & .00 & & .00 & & .00 & .00 \\
\hline DATE & & $\begin{array}{l}\text { HEPTA- } \\
\text { CHICR. } \\
\text { TOTAL } \\
\text { (UGA) }\end{array}$ & $\begin{array}{c}\text { HE } \\
\text { Ch } \\
\text { EPD } \\
T \\
\text { IU } \\
\text { IU }\end{array}$ & $\begin{array}{l}\text { PTA- } \\
\text { LOR } \\
\text { XIDE } \\
\text { TAL } \\
\text { G/I. }\end{array}$ & II & ALE & & $\begin{array}{l}\mathrm{H}- \\
\text { Y- } \\
\text { OR, } \\
\text { AL } \\
\text { iti }\end{array}$ & & $\begin{array}{l}E x, \\
\text { IAi } \\
\text { ani }\end{array}$ & $\begin{array}{r}\pi \\
\text { ApH } \\
\text { TO } \\
\text { iu }\end{array}$ & 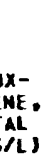 & $\begin{array}{l}P C \\
\text { ID } \\
\text { IJG }\end{array}$ & \multicolumn{3}{|c|}{$\begin{array}{l}\text { VAPH- } \\
\text { IHA- } \\
\text { LENES. } \\
\text { POLY- } \\
\text { CHLOR. } \\
\text { IOTAL } \\
\text { (UG/L) }\end{array}$} \\
\hline AUG & & .00 & & .00 & & .co & &. $\mathrm{co}$ & & .00 & & 0 & & .00 & & .00 \\
\hline
\end{tabular}

Site 30

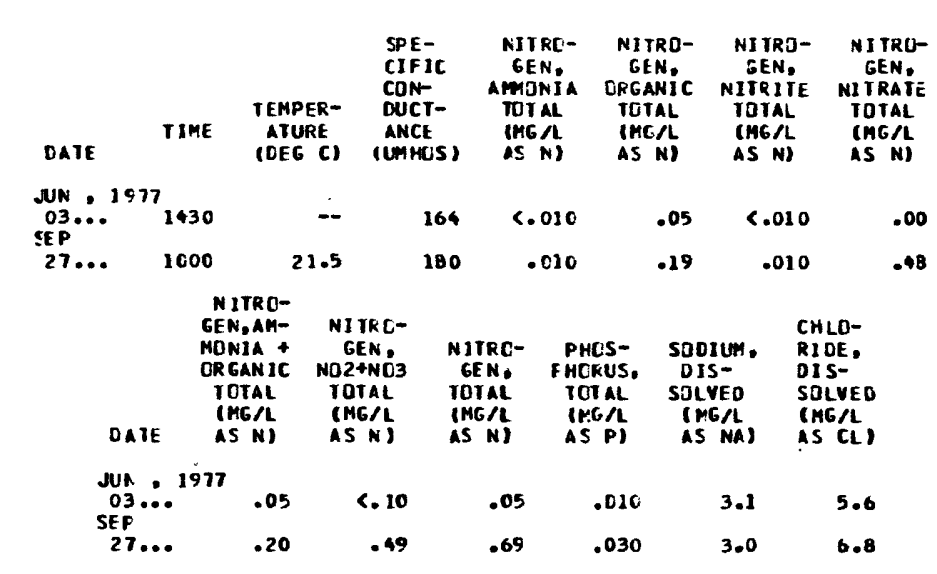


Site 31 in 4-big-gun area

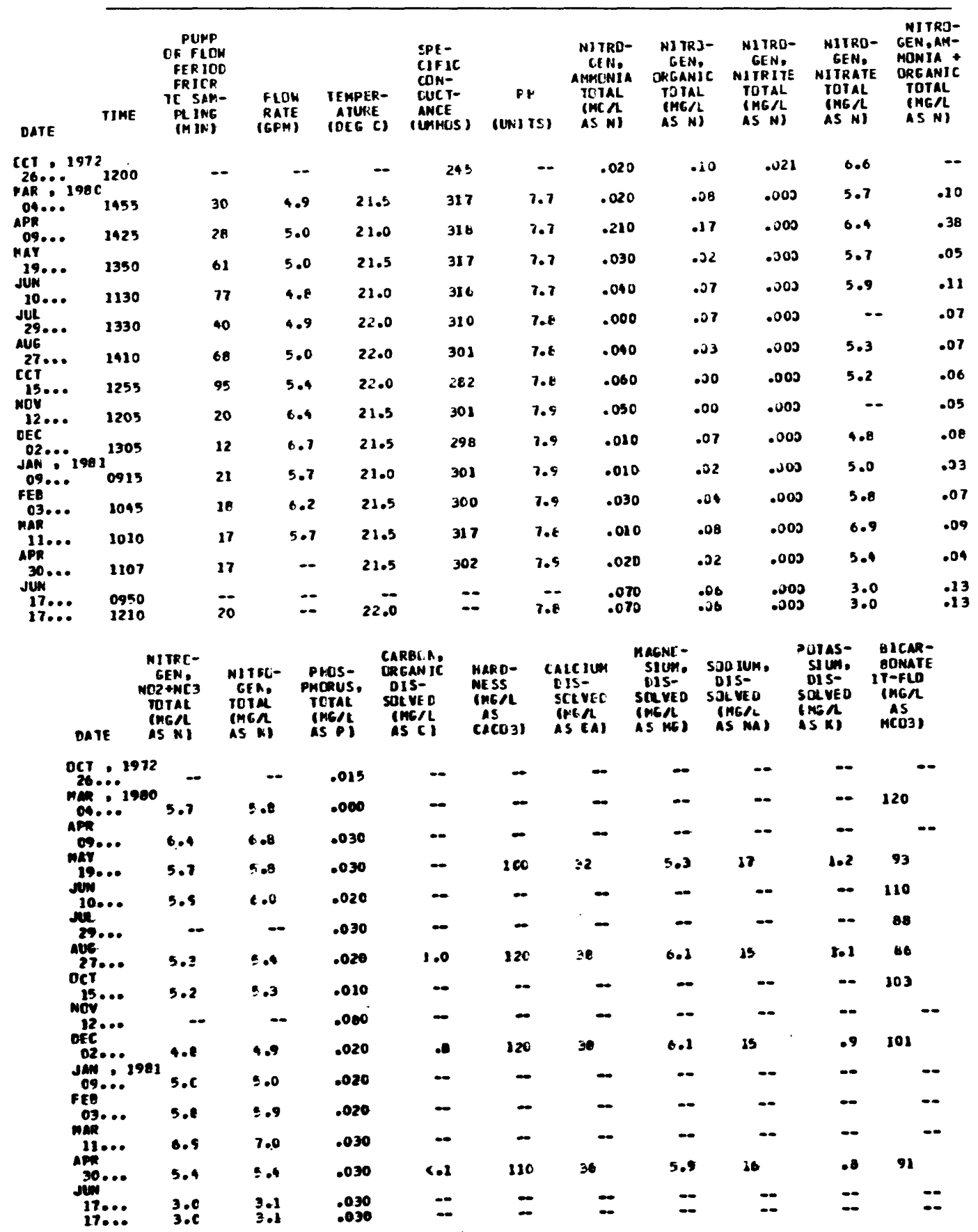


Site 31 in 4-big-gun area--Continued

\begin{tabular}{|c|c|c|c|c|c|c|c|c|c|c|}
\hline $\begin{array}{l}6 \\
\text { FC } \\
11 \\
\text { IN }\end{array}$ & $\begin{array}{l}\text { CAF- } \\
\text { CNATE } \\
T-F L O \\
M G / L \\
\text { ME } \\
\text { CCBI }\end{array}$ & $\begin{array}{l}\text { CHIC- } \\
\text { FIf } \\
\text { DIS- } \\
\text { SELVLO } \\
\text { (MCA } \\
\text { AS CL) }\end{array}$ & $\begin{array}{l}\text { FLUO- } \\
\text { RIDE. } \\
\text { LIS- } \\
\text { STLVEL } \\
\text { IMGAL } \\
\text { AS FI) }\end{array}$ & $\begin{array}{l}\text { SULFA TE } \\
\text { OIS- } \\
\text { SULVER } \\
\text { (MCIL } \\
\text { AS SLi4) }\end{array}$ & $\begin{array}{l}\text { SILICA, } \\
\text { DIS- } \\
\text { SCIVEE } \\
\text { (HCAL } \\
\text { AS } \\
\text { SIIL) }\end{array}$ & $\begin{array}{l}\text { CCLI- } \\
\text { FCAF, } \\
\text { ICIAL, } \\
\text { IPHEC: } \\
\text { ICCLS. } \\
\text { FHR } \\
\text { JOC NLS }\end{array}$ & $\begin{array}{l}\text { CQLI- } \\
\text { FCRM, } \\
\text { FICAL, } \\
0.7 \\
\text { JH-MF } \\
\text { (CLLS.' } \\
100 \mathrm{ML} \text { ) }\end{array}$ & $\begin{array}{c}\text { SIKEP- } \\
\text { IOCOCCI } \\
\text { FECAL, } \\
\text { KF AGAR } \\
\text { ICOLS. } \\
\text { PER } \\
100 \mathrm{ML} \text {, }\end{array}$ & $\begin{array}{l}\text { OEPIH } \\
\text { BELON } \\
\text { LAND } \\
\text { SURFACE } \\
\text { (WATER } \\
\text { LLVEL) } \\
\text { (FEET) }\end{array}$ & $\begin{array}{l}\text { ELEV- } \\
\text { ATION } \\
\text { ABOVE } \\
\text { NGVD } \\
\text { (FEET) }\end{array}$ \\
\hline 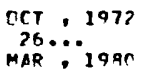 & -- & 30 & -- & - & T.E & -- & $\cdots$ & $\cdots$ & -- & -- \\
\hline$\underset{A R R}{B A . . .}$ & . $\mathrm{CO}$ & $:^{\circ}$ & $\cdots$ & -- & - & <1 & $<i$ & <1 & -- & - \\
\hline MAY & -- & 24 & -- & -- & -- & C1 & <1 & (1) & -- & - \\
\hline JUN & .00 & 22 & .1 & $1 /$ & - & CI & $<1$ & <1 & $\cdots$ & - \\
\hline $\operatorname{JIL}_{\text {JiL }}^{10 . . .}$ & . $\mathrm{CC}$ & $i$ & $\cdots$ & $\cdots$ & $\cdots$ & 1 & $<1$ & 2 & 325 & 2270 \\
\hline avg & .60 & 23 & -- & - & - & CI & $<1$ & $<1$ & 32.45 & 22.70 \\
\hline nci 27. & .80 & 21 & .1 & 34 & - & Ci & <1 & $<1$ & $32 \cdot 30$ & 22.80 \\
\hline${ }_{\text {NDV }}^{15} \cdots$ &. $\mathrm{CO}$ & 21 & $\cdots$ & -- & - & - & -- & -- & 34.33 & 20.83 \\
\hline${ }_{\text {DFC }}^{12} \cdots$ & - & aC & -- & -- & - & $<i$ & 1 & (1) & 35.10 & 20.06 \\
\hline JAN 19211 & $\cdot \mathrm{rO}$ & is & .1 & $1:$ & -- & $<1$ & <1 & (1) & 35.40 & 19.76 \\
\hline FEA & $\cdots$ & ar & -- & -- & - & CI & (I) & $<1$ & 35.14 & 19.02 \\
\hline$\underset{\operatorname{MAR}}{03 . . .}$ & - & $: 2$ & -- & -- & - & <1 & $<1$ & (1) & 36.62 & 18.54 \\
\hline APR $\cdots$ & - & 21 & -- & -- & -- & <1 & $<1$ & $<1$ & 32.48 & 22.68 \\
\hline JUN $30 .$. &. $\mathrm{co}$ & $: 3$ & .1 & $1^{*}$ & -- & $\rightarrow$ & - & $\cdots$ & 33.53 & 21.63 \\
\hline $17 \ldots$ & $=$ & $\begin{array}{l}22 \\
22\end{array}$ & 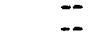 & $=$ & $\overline{-}$ & $<1$ & $\begin{array}{l}<1 \\
<1\end{array}$ & $\begin{array}{r}K 20 \\
2\end{array}$ & $35 . \overline{15}$ & $=$ \\
\hline
\end{tabular}

\begin{tabular}{|c|c|c|c|c|c|c|c|c|c|c|c|c|}
\hline \multirow[b]{3}{*}{ DA IE } & \multicolumn{11}{|c|}{ Site 32 in 1977 expansion field } & \multirow[b]{2}{*}{$\begin{array}{l}\text { MITRC- } \\
\text { GEN } \\
\text { NO2+NC3 } \\
\text { TOTAL } \\
\text { IMGRL }\end{array}$} \\
\hline & TIMF & $\begin{array}{l}\text { PUFF } \\
\text { OF FLCW } \\
\text { PEFICD } \\
\text { FRICK } \\
\text { TO SAF- } \\
\text { PLING }\end{array}$ & $\begin{array}{l}\text { FICW } \\
\text { RATE }\end{array}$ & $\begin{array}{l}\text { TEMPE F- } \\
\text { ATURE }\end{array}$ & $\begin{array}{l}\text { SPE- } \\
\text { r.IFI } \\
\text { CON- } \\
\text { DUCI- } \\
\text { ANCL }\end{array}$ & PH & $\begin{array}{c}\text { NIIRE- } \\
\text { GFN, } \\
\text { AMMENIA } \\
\text { TrTAL } \\
\text { IMGAL }\end{array}$ & $\begin{array}{l}\text { N JTRE- } \\
\text { GEN, } \\
\text { ORCANIC } \\
\text { TOTAL } \\
\text { THGAL }\end{array}$ & $\begin{array}{l}\text { NITKD- } \\
\text { SLN. } \\
\text { NITRIIE } \\
\text { IOTAL } \\
\text { IMGLL }\end{array}$ & $\begin{array}{l}\text { NIIRO- } \\
\text { GEN, } \\
\text { NI IRAIE } \\
\text { I DTAL } \\
\text { I MGR }\end{array}$ & $\begin{array}{l}\text { NI TRQ- } \\
\text { GEN,AM- } \\
\text { MONIA } \\
\text { ORGANIC } \\
\text { TOTAL } \\
\text { IMGN }\end{array}$ & \\
\hline & & (MIN) & (GFN) & (DEG C) & (UMHLCS) & (UNITS) & $A S N)$ & AS N) & AS N) & is $N$ ) & $A S N)$ & AS NS \\
\hline OC1,.. & -- & -- & -- & - & 50 & - & -- & - & -- & - & $\cdots$ & $\cdots$ \\
\hline $26 \ldots$ & $3^{1200}$ & -- & -- & -- & 58 & -- & .020 & .16 & .002 & .09 & -- & - \\
\hline DEC : 1 & - & - & -- & $-\infty$ & to & - & -- & - & -- & - & $\cdots$ & - \\
\hline JAA $\because 1$ & $9^{1545}$ & 38 & 5.6 & 21.0 & 53 & $9 . \mathrm{C}$ & .010 & .01 & $<.010$ & .18 & .02 & .10 \\
\hline $\begin{array}{l}25 \ldots \\
\text { JUL }\end{array}$ & 1255 & 22 & 5.7 & $21 \cdot c$ & $: 3$ & 9.0 & .020 & .02 & $<.010$ & .39 & .04 & - 39 \\
\hline Nov & 1120 & 24 & 5.3 & 21.5 & 74 & 8.9 & .010 & $.0 D$ & $\cdots$ & $1 \cdot 3$ & $<.10$ & 1.3 \\
\hline MAK $14 \ldots$ & $0^{1625}$ & 50 & 5.3 & $21 \cdot 0$ & 64 & 8.9 & .000 & .02 & .000 & 1.7 & .02 & 1.7 \\
\hline OAPR & 1245 & 50 & 4.3 & 20.5 & 96 & $\varepsilon$ & .020 & .07 & .000 & $1 \cdot \theta$ & .09 & 1.8 \\
\hline May & 1150 & 49 & 4.7 & 21.0 & 92 & - & .000 & .29 & .000 & 2.4 & .29 & 2.4 \\
\hline JUR $19 . \cdots$ & 1155 & 83 & 4.3 & $21 \cdot 5$ & so & B.F & .010 & .110 & .000 & 1.5 & .01 & 1.5 \\
\hline $\begin{array}{l}10 \ldots \\
\text { sue }\end{array}$ & $094 \mathrm{C}$ & 56 & 4.2 & $21 \cdot 0$ & 92 & 6.6 & .000 & .03 & .000 & 1.7 & .03 & 1.8 \\
\hline 25... & 1045 & 52 & 4.1 & 22.0 & .5 & 6.9 & $.00 \mathrm{C}$ & .02 & .000 & 2.6 & .02 & 2.6 \\
\hline oct & 1028 & 53 & 4.4 & $22 \cdot c$ & 98 & E.9 & $.02 \mathrm{C}$ & .01 & .000 & 1.6 & .03 & 1.6 \\
\hline NeV & 1200 & 50 & $4 . E$ & 22.0 & 105 & 6.9 & $.03 c$ & .16 & -OCO & 1.5 & .19 & 1.5 \\
\hline $\begin{array}{l}12 \ldots \\
\text { DEC } \\
C 2 \ldots\end{array}$ & $105 C$ & 41 & 4.3 & 21.5 & 112 & 9.0 & $.00 x$ & .00 & .000 & - & .00 & 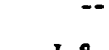 \\
\hline $\begin{array}{r}C 2 \ldots \\
\text { JAR } \\
09 . .\end{array}$ & $121^{*}$ & 24 & 5.7 & 21.5 & 106 & 9.0 & .050 & .27 & .000 & 1.9 & .22 & 1.9 \\
\hline $\begin{array}{l}09 \cdots \\
\text { FEe } \\
03 \ldots \ldots\end{array}$ & 1025 & 28 & 4.9 & 21.0 & 110 & $9 . \mathrm{C}$ & .010 & .00 & .000 & 1.8 & .01 & 1.8 \\
\hline MAK & $112^{*}$ & $2 p$ & 5.0 & 21.5 & 120 & 8.9 & .000 & .02 & .010 & 2.2 & .02 & 2.2 \\
\hline APF & $090 \mathrm{C}$ & 31 & 3.3 & 21.5 & $1 \equiv 5$ & $\varepsilon .9$ & .000 & .12 & .000 & 2.0 & .10 & 2.0 \\
\hline $\begin{array}{l}\text { jua } \\
\text { je... }\end{array}$ & 1045 & 30 & $\cdots$ & 20.6 & 140 & $8 \cdot \varepsilon$ & .010 & .60 & .000 & 1.6 & .01 & 1.7 \\
\hline 17 & 1140 & 30 & -- & 22.0 & - & 8.6 & $.07 \mathrm{c}$ & $\cdots$ & .000 & 12 & .03 & 12 \\
\hline
\end{tabular}


Table 6.--Physical and chemical characteristics of well and water at sites 1 through 70--Continued

Site 32 in 1977 expansion field--Continued

\begin{tabular}{|c|c|c|c|c|c|c|c|c|c|c|}
\hline $\begin{array}{l}\text { A ITPC- } \\
\text { GEN, } \\
\text { TRTAL } \\
\text { IMG/L } \\
\text { AS NI }\end{array}$ & $\begin{array}{l}\text { FHCS- } \\
\text { PFERES, } \\
\text { ICTAL } \\
\text { (MC / } \\
\text { AS F) }\end{array}$ & $\begin{array}{l}\text { CAFELN, } \\
\text { CPCANIC } \\
\text { LIS- } \\
\text { SDLVO } \\
\text { IRCAL } \\
\text { AS C) }\end{array}$ & $\begin{array}{l}\text { CARB'N, } \\
\text { ORGANIC } \\
\text { TLT IL } \\
\text { IYG/L } \\
\text { AS CI }\end{array}$ & $\begin{array}{l}\text { ALAA- } \\
\text { INIIY } \\
\text { FIELD } \\
\text { IHG/L } \\
\text { AS } \\
\text { CACLZ) }\end{array}$ & 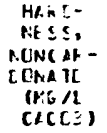 & $\begin{array}{l}\text { Hil } 0- \\
\text { HE } \leq S \\
\text { (PC/L } \\
\text { AS } \\
\text { CACCB) }\end{array}$ & $\begin{array}{l}\text { CILCIUM } \\
\text { LISE } \\
\text { SOLVED } \\
\text { IMER } \\
\text { AS CAS }\end{array}$ & $\begin{array}{l}\text { MAGUE - } \\
\text { SIJM, } \\
\text { ULS- } \\
\text { SOLVED } \\
\text { (MG/L } \\
\text { AS YG) }\end{array}$ & $\begin{array}{l}\text { SUDIUM, } \\
\text { DIS- } \\
\text { SOLVED } \\
\text { (MG/L } \\
\text { AS NA) }\end{array}$ & $\begin{array}{l}\text { POIAS- } \\
\text { SIUM. } \\
\text { OIS- } \\
\text { SOLVEO } \\
\text { (MG/L } \\
\text { AS K) }\end{array}$ \\
\hline
\end{tabular}

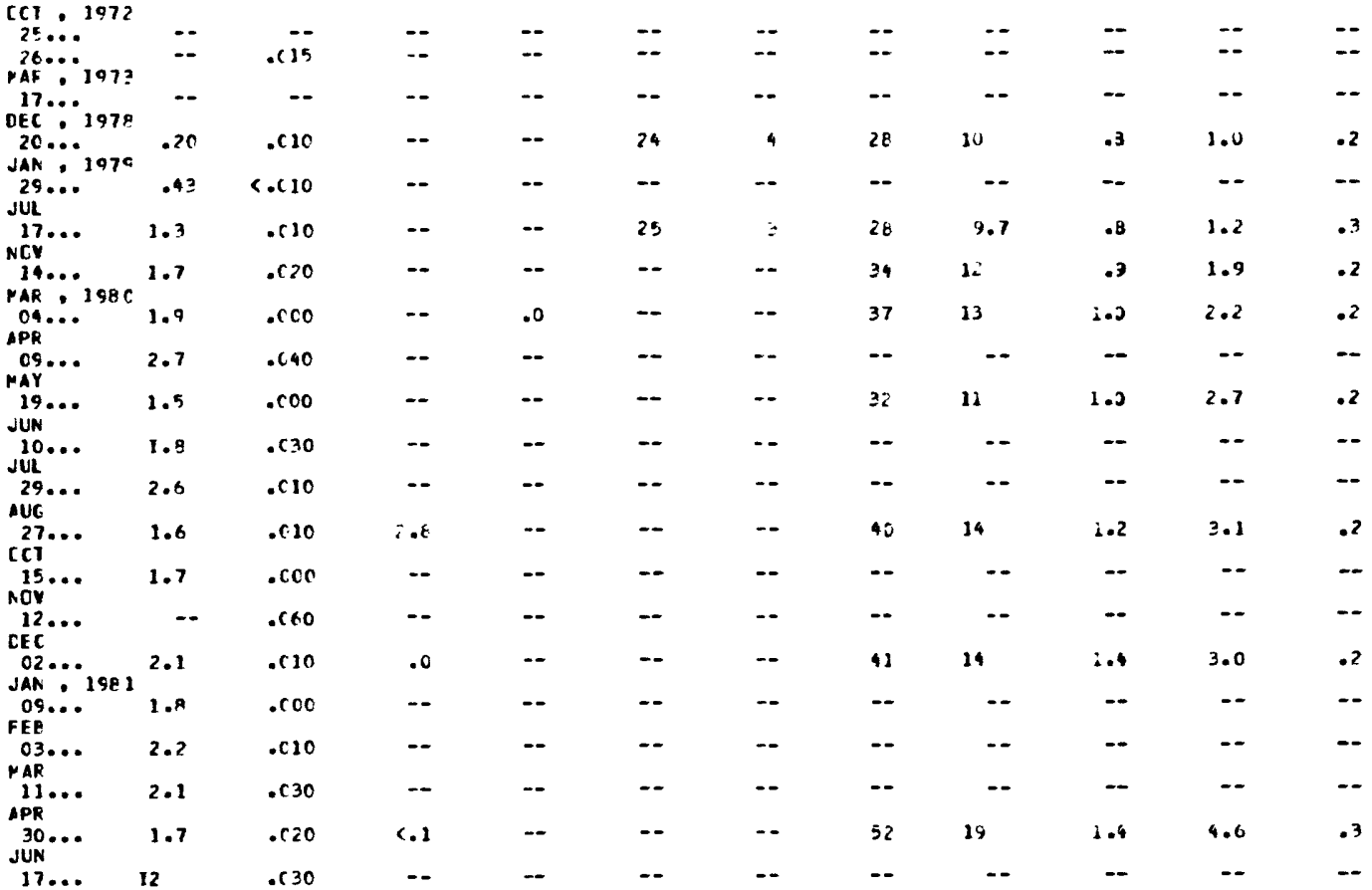

\begin{tabular}{|c|c|c|c|c|c|c|c|c|c|c|c|}
\hline & $\begin{array}{l}\text { PICAR- } \\
\text { PONATF } \\
\text { IT-FLO } \\
\text { (MG/L } \\
\text { AS } \\
\text { HCO3) }\end{array}$ & $\begin{array}{l}\text { CAF- } \\
\text { RONAIE } \\
\text { IT-FLO } \\
\text { CHG/L } \\
A S \\
\text { CC } 3)\end{array}$ & $\begin{array}{l}\text { CHLO- } \\
\text { KIOE. } \\
\text { DIS- } \\
\text { SOLVED } \\
\text { SMG/L }\end{array}$ & $\begin{array}{l}\text { FLUO- } \\
\text { RJOL, } \\
\text { DIS- } \\
\text { SULVED } \\
\text { (MGA }\end{array}$ & $\begin{array}{l}\text { SLLFAIE } \\
\text { DIS- } \\
\text { SOLVEO } \\
\text { IMGAL }\end{array}$ & $\begin{array}{l}\text { SHICA, } \\
\text { DIS- } \\
\text { SOLVEL } \\
\text { IMEA } \\
\text { AS } \\
\text { SICA) }\end{array}$ & $\begin{array}{l}\text { SUL IOS, } \\
\text { RCSIOUL } \\
\text { AT } 180 \\
\text { PC C C } \\
\text { DIS- } \\
\text { SILVED } \\
\text { IHC/L) }\end{array}$ & $\begin{array}{l}\text { CELI- } \\
\text { FOKM, } \\
\text { TOIAL, } \\
\text { IMMEJ. } \\
\text { PEJLS. } \\
\text { PEK } \\
100 \mathrm{ML} \text {. }\end{array}$ & $\begin{array}{l}\text { COLI- } \\
\text { FORIA, } \\
\text { FECAL, } \\
0.7 \\
\text { UK-HF } \\
\text { (CULS.I } \\
\text { IOOML) }\end{array}$ & $\begin{array}{l}\text { SIREP- } \\
\text { TOCOCC1 } \\
\text { FECAL. } \\
\text { KF AGAR } \\
\text { (COLS. } \\
\text { PER } \\
100 \mathrm{ML})\end{array}$ & $\begin{array}{l}\text { OEPTH } \\
\text { BELAH } \\
\text { LANO } \\
\text { SURFACE } \\
\text { (WATER } \\
\text { LEVEL) } \\
\text { (FEET) }\end{array}$ \\
\hline & $\mu(03)$ & $(C, 3)$ & & & & a) & L) & $100 \mathrm{ML} . \mathrm{s}$ & ) & $100 \mathrm{MLI}$ & \\
\hline
\end{tabular}

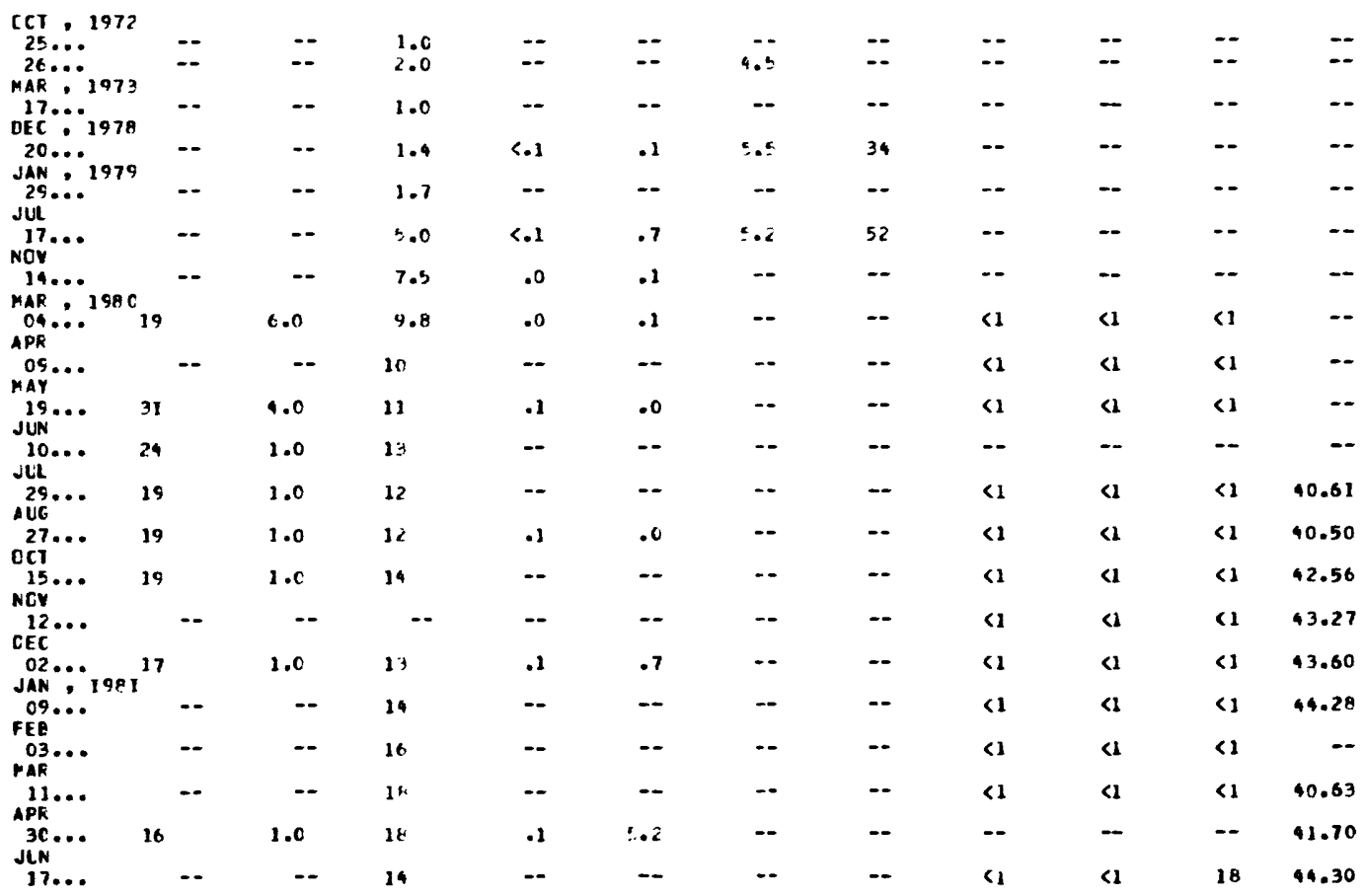


Table 6..-Physical and chemical characteristics of well and water at sites 1 through 70--Continued

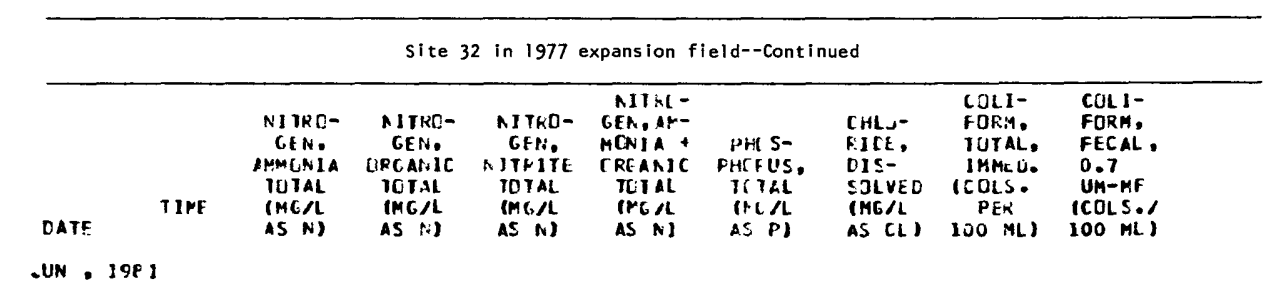

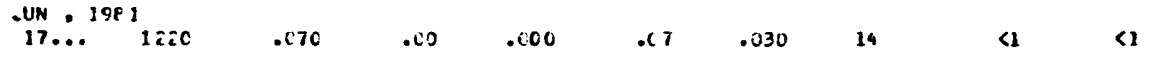

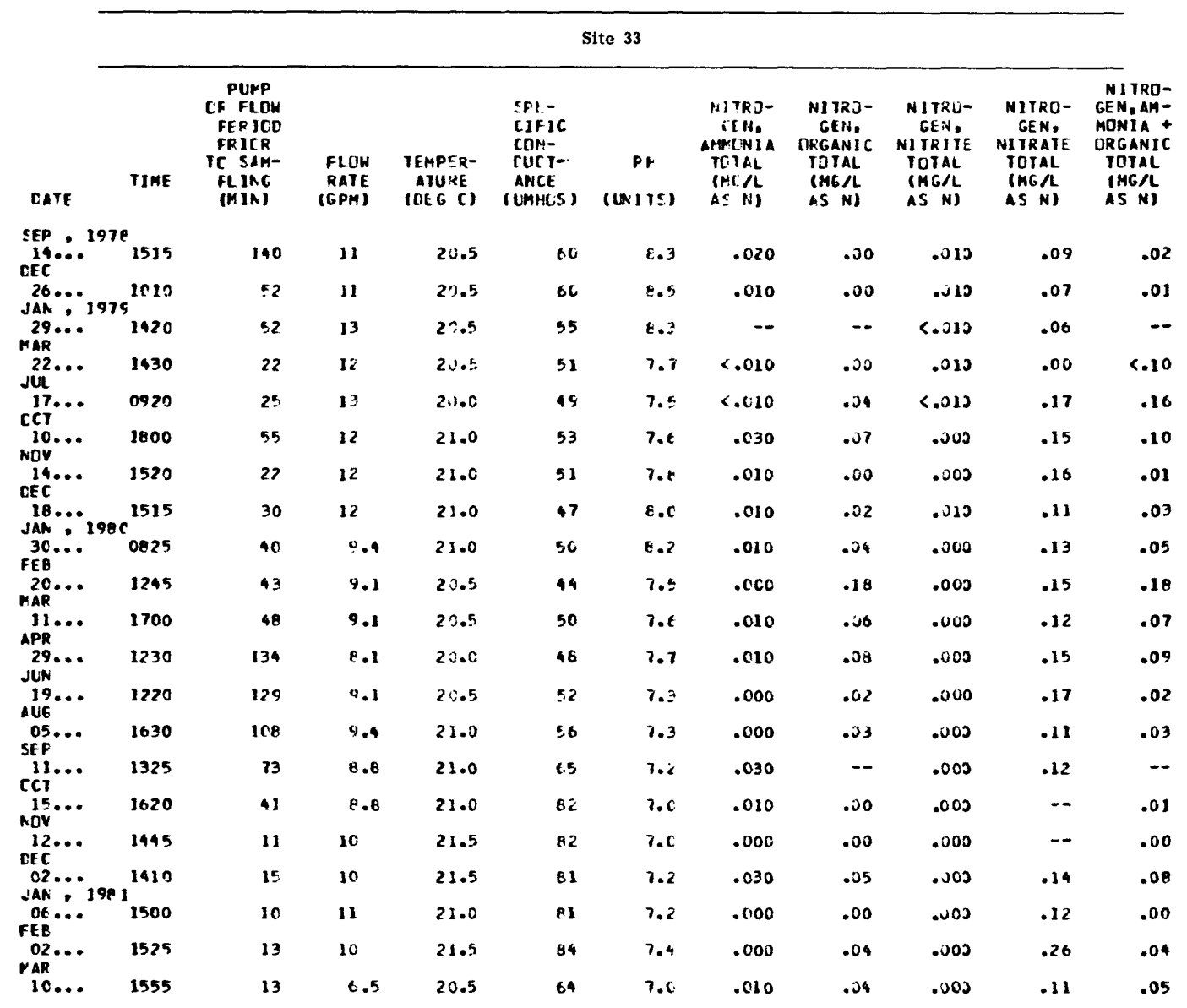


Site 33--Continued

\begin{tabular}{|c|c|c|c|c|c|c|c|c|c|c|}
\hline CAT & 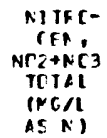 & $\begin{array}{l}\text { NIFO- } \\
\text { GIN, } \\
\text { INIAL } \\
\text { INCA } \\
\text { AS MI }\end{array}$ & $\begin{array}{l}\text { FHOS- } \\
\text { PHCRUS, } \\
\text { ICTAL } \\
\text { (MG/L } \\
\text { ASP) }\end{array}$ & $\begin{array}{l}\text { CAFEIA, } \\
\text { CRGAF IC } \\
\text { DIS- } \\
\text { SOLVER } \\
\text { (MCAL } \\
\text { AS (I) }\end{array}$ & $\begin{array}{l}\text { ALKA- } \\
\text { LNITY } \\
\text { IIELD } \\
\text { IAC I } \\
\text { AS } \\
\text { CACI.3) }\end{array}$ & 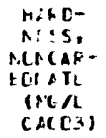 & $\begin{array}{l}\text { HAR?- } \\
\text { NISS } \\
\text { (MG/L } \\
\text { AS } \\
\text { (ACOS) }\end{array}$ & $\begin{array}{l}\text { CALCIUM } \\
\text { DIS- } \\
\text { SOLLEO } \\
\text { (MG/L } \\
\text { AS CR) }\end{array}$ & $\begin{array}{l}\text { YACNE- } \\
\text { SIUM, } \\
\text { DIS- } \\
\text { SULVEO } \\
\text { ( YL IL } \\
\text { A MG) }\end{array}$ & $\begin{array}{l}\text { SULILM, } \\
\text { DIS- } \\
\text { SULVED } \\
\text { (KG/L } \\
\text { AS NAI) }\end{array}$ \\
\hline
\end{tabular}

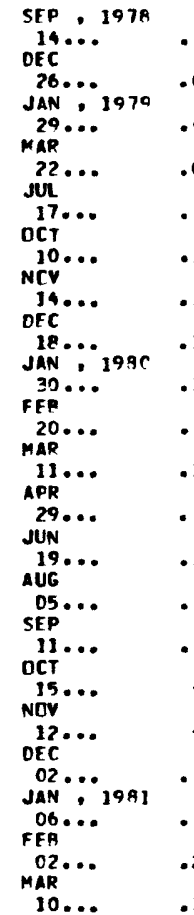

\section{.18}$$
10
$$

$12 \quad .00$ .09 $-$ .01

.33

.25

.17

.15

.16

.33

.19

.24

.19

.14

$--$

$-$

.020
.$- \quad .080$

.22

.12

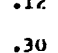

10..

$$
\begin{aligned}
& \begin{array}{l}
\text { POIAS- BICAR- } \\
\text { SILM, BCIATL } \\
\text { CIS- IT- } \\
\text { SCLVEO IMCA } \\
\text { CMG }
\end{array} \\
& \text { (MG/ A! }
\end{aligned}
$$

HC( 3$)$

$$
\text { cec -- }
$$$$
-
$$$$
-
$$$$
\begin{aligned}
& .030 \\
& .040
\end{aligned}
$$$$
.040
$$$$
.050
$$$$
\begin{array}{r}
.050 \\
.130
\end{array}
$$$$
.060
$$$$
.060
$$$$
.050
$$$$
.030
$$$$
.100
$$$$
.030
$$$$
.040
$$$$
.090
$$$$
.020
$$$$
\begin{aligned}
& .080 \\
& .070
\end{aligned}
$$$$
.070
$$$$
.090
$$

SEP , $107 \mathrm{~F}$
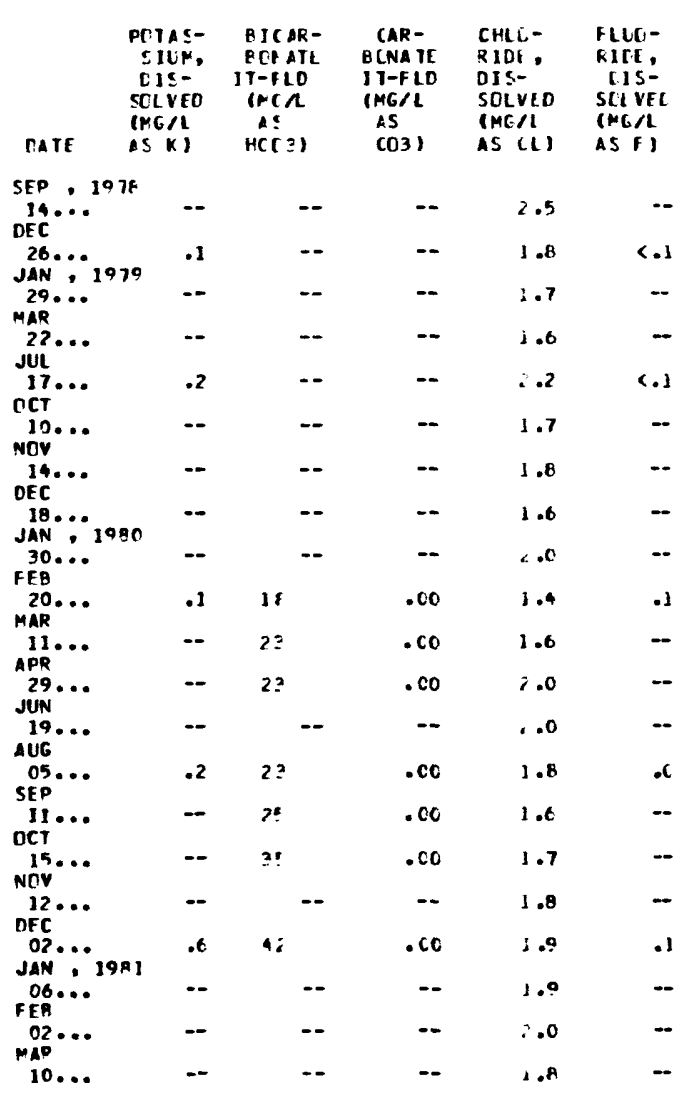

$$
\text { (C3) }
$$


Table 6.--Physical and chemical characteristics of well and water at sites 1 through 70--Continued

Site 33--Continued

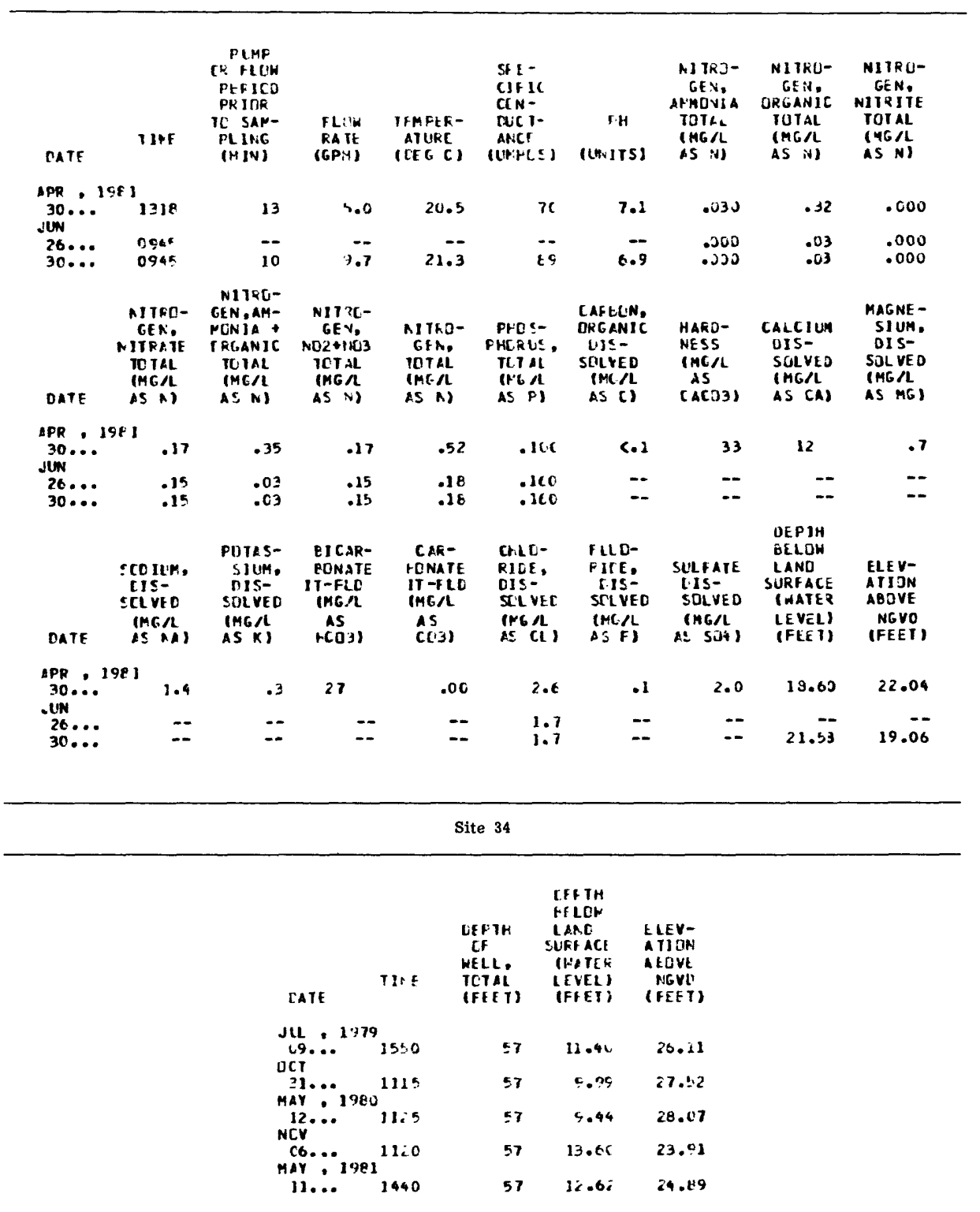


Site 35

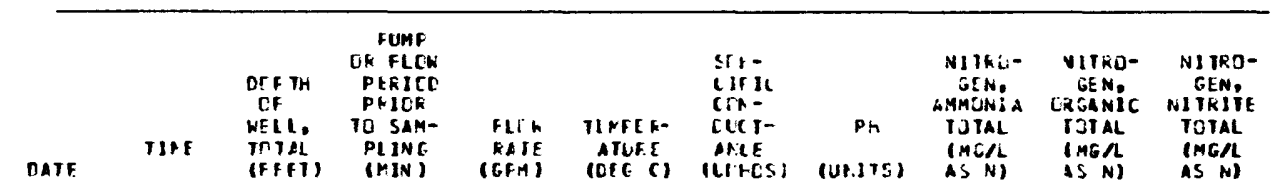

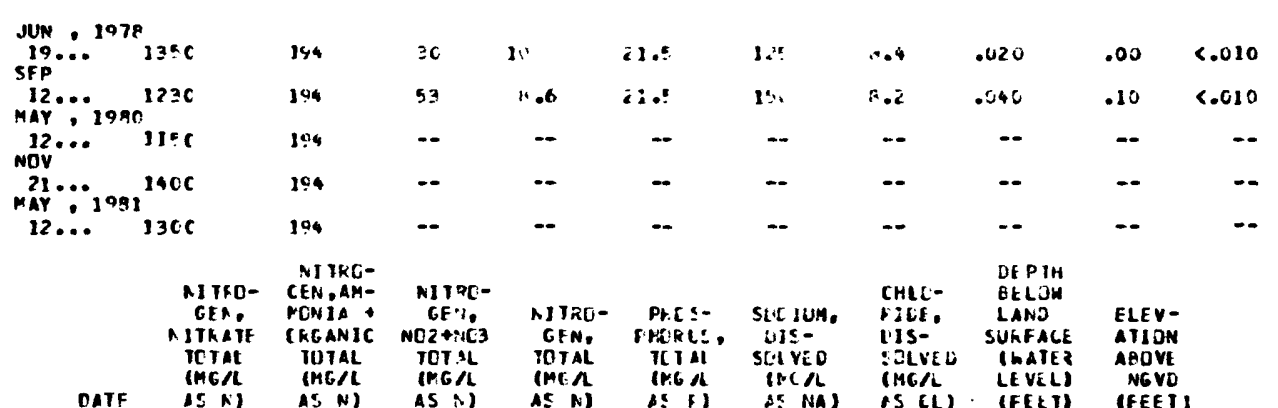
IMGA (HG/ IMG/L IMEA IMGA IMCA IMG/L LEVEL) NGVO

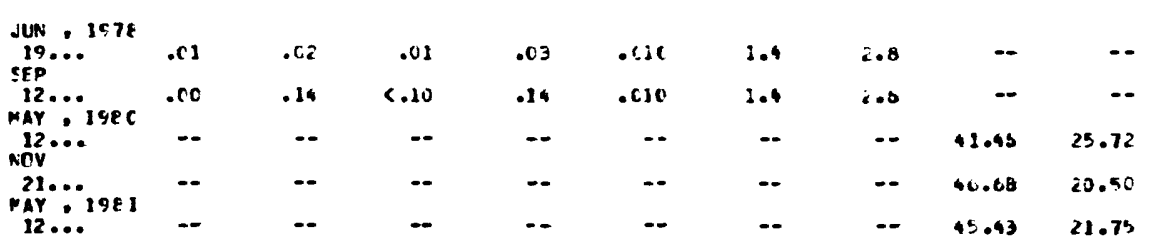

Site 36

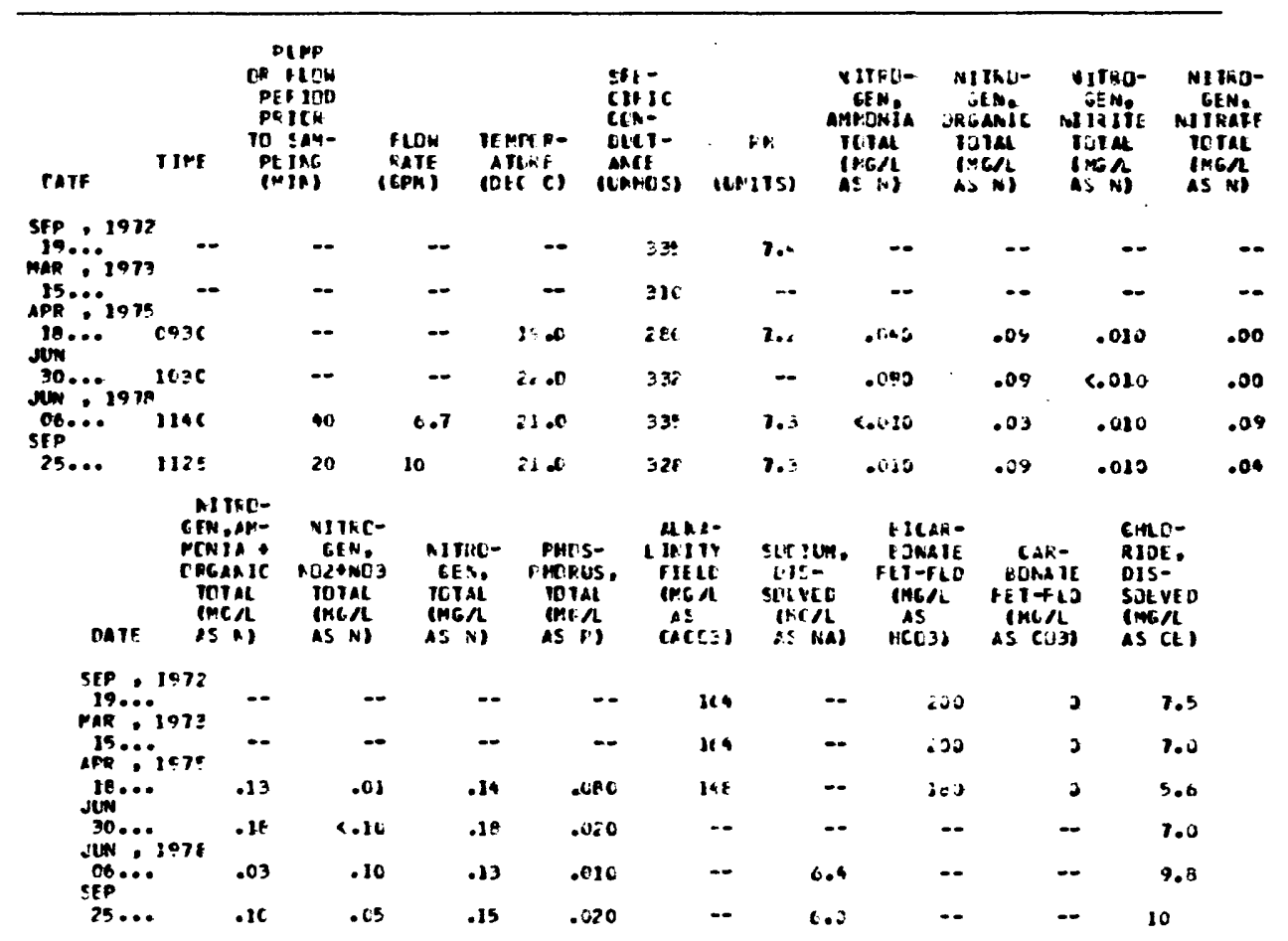


Site 37

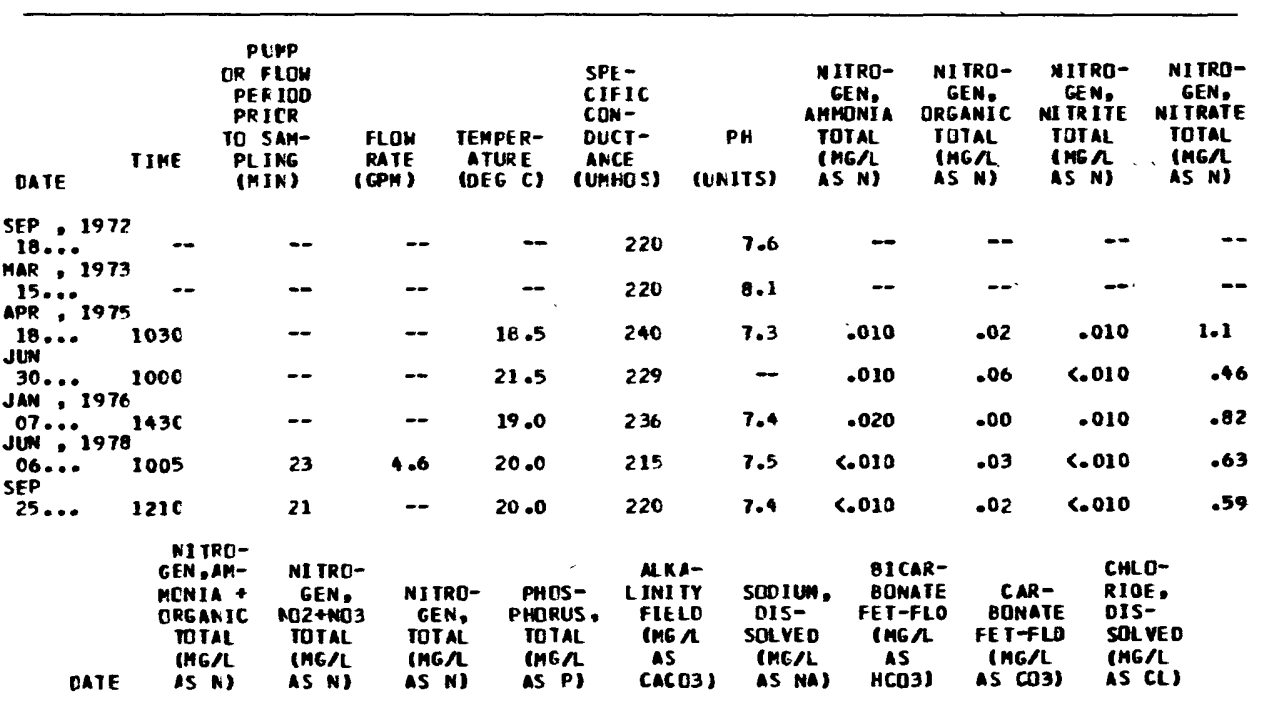

\begin{tabular}{|c|c|c|c|c|c|c|c|c|c|}
\hline SEP 1972 & -- & $-\infty$ & -- & $=-$ & 107 & - & 130 & 0 & 5.0 \\
\hline APR 1975 & - & - & - & -- & 115 & $\cdots$ & 140 & 0 & 5.0 \\
\hline JUW & .03 & 1.1 & 1.1 & .020 & 115 & - & 140 & 0 & 5.8 \\
\hline JAM $\because 1976$ & .07 & .46 & .53 & .020 & -- & -- & - & - & 6.5 \\
\hline JUn: 1978 & .02 & .83 & .85 & .090 & 107 & 4.5 & 130 & 0 & 6.2 \\
\hline SEP & .03 & .63 & .66 & .020 & - & 3.8 & - & - & 5.5 \\
\hline $25 \ldots$ & .02 & .59 & .61 & .020 & - & 3.3 & - & $\infty$ & 5.4 \\
\hline
\end{tabular}

Site 38

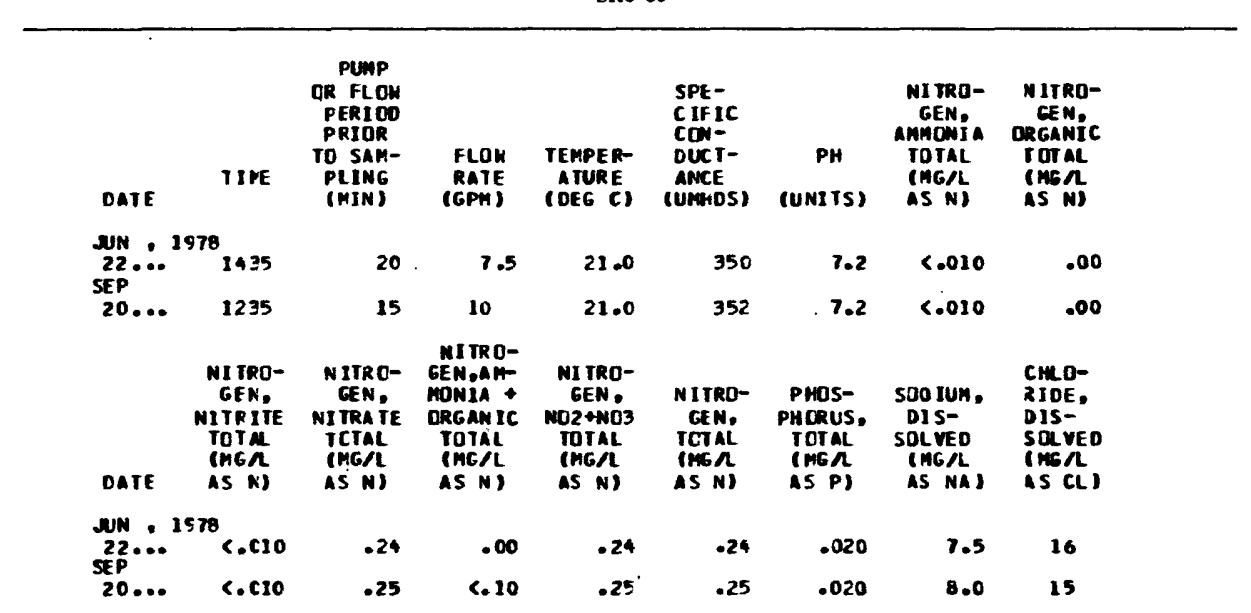


Site 39

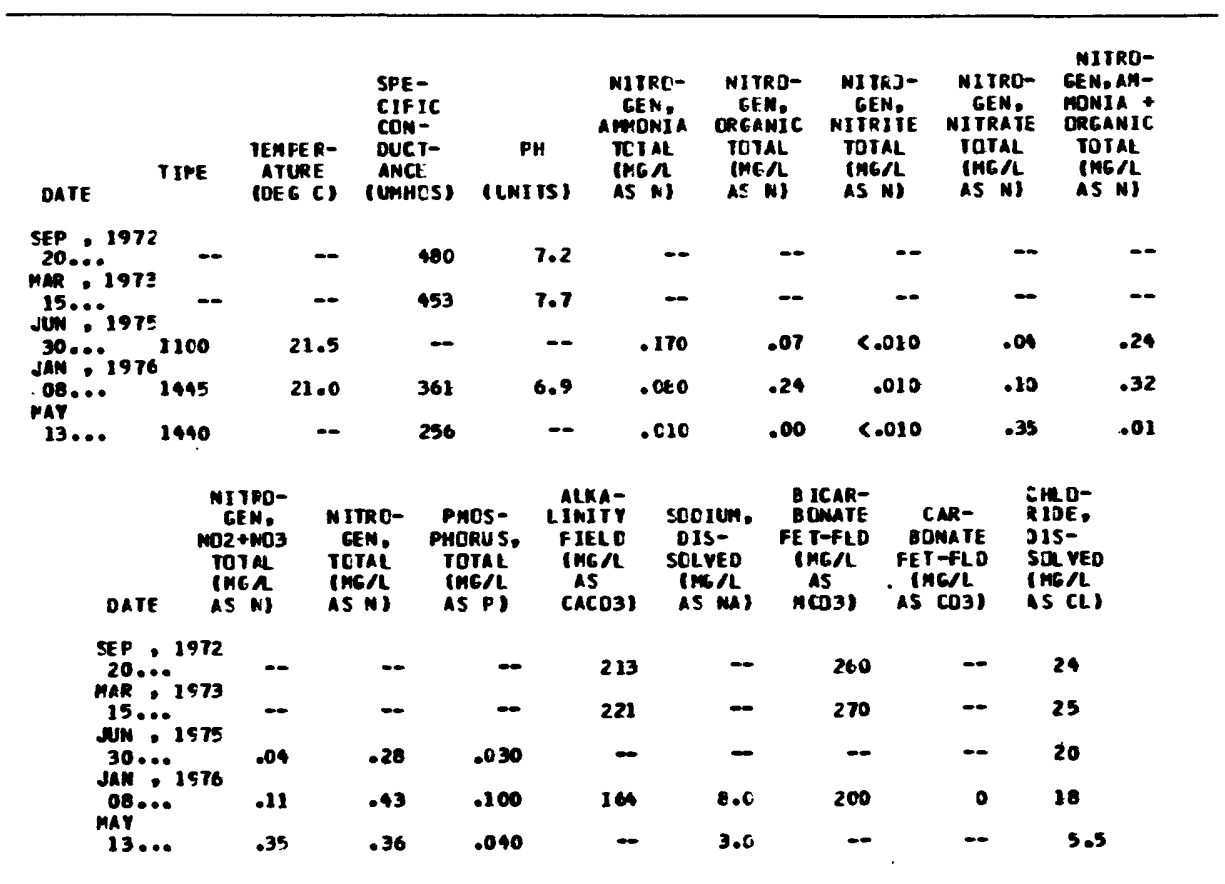

Site 40

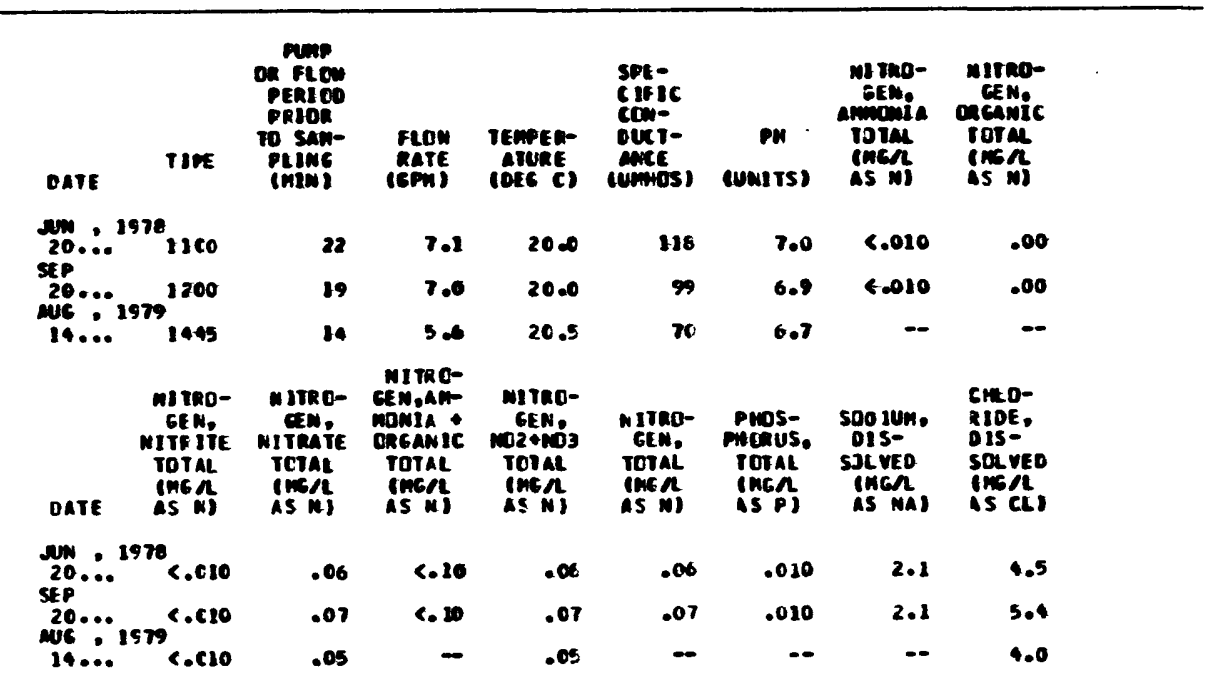


Site 41

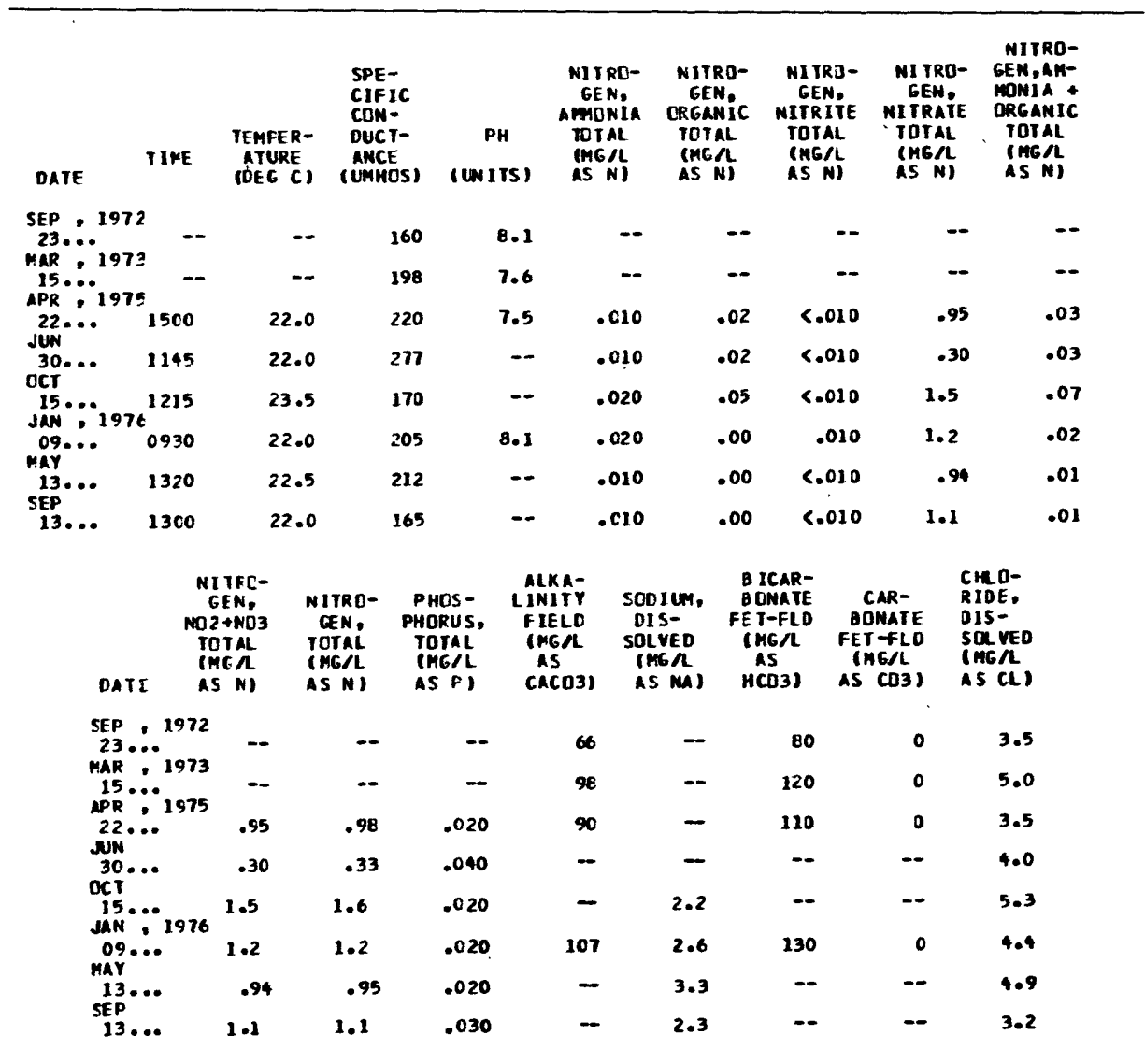

Site 42

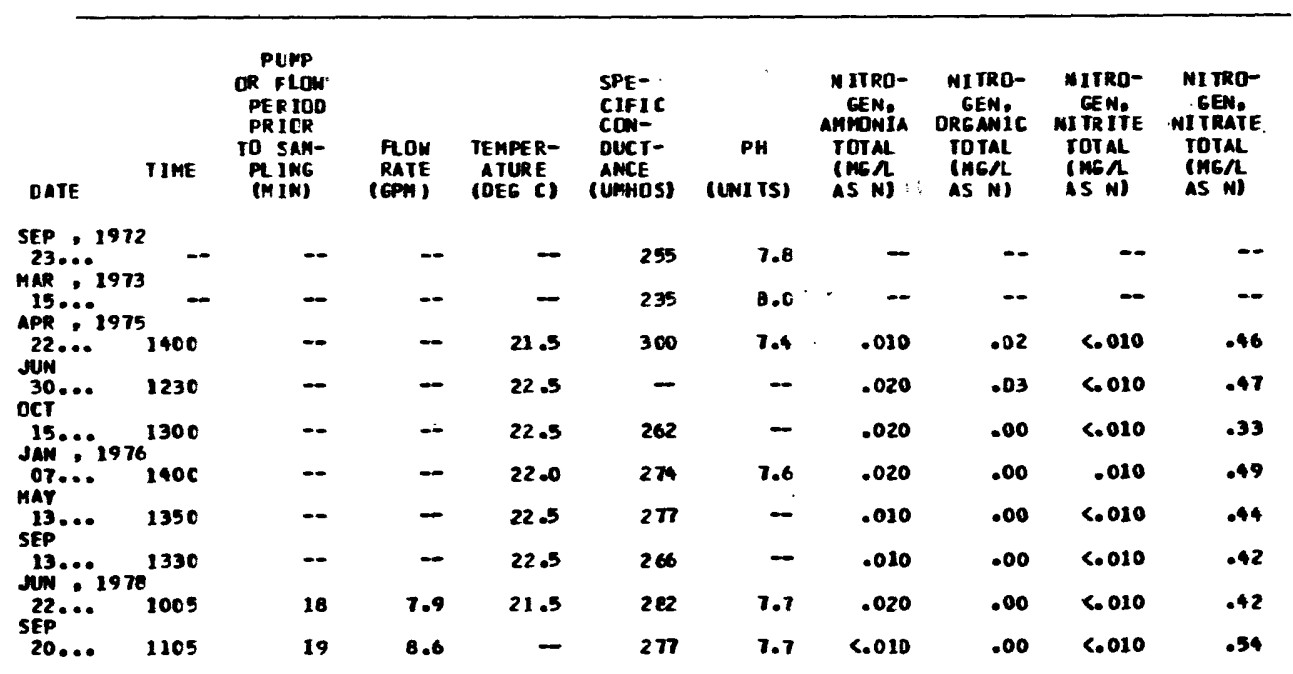


Table 6.--Physical and chemical characteristics of well and water at sites 1 through 70--Continued

\begin{tabular}{|c|c|c|c|c|c|c|c|c|c|c|}
\hline \multicolumn{11}{|c|}{ Site 42--Continued } \\
\hline DATE & $\begin{array}{l}\text { NI } \\
\text { GEN } \\
\text { MCN } \\
\text { ORG } \\
\text { ID } \\
\text { IN } \\
\text { IS }\end{array}$ & $\begin{array}{l}\text { TRC- } \\
\text { OAM- } \\
\text { IA } \\
\text { ANIC } \\
\text { TAL } \\
\text { GA } \\
\text { NI }\end{array}$ & $\begin{array}{l}\text { NITRC- } \\
\text { GEN, } \\
\text { NOZ +NC3 } \\
\text { TOTAL } \\
\text { (MG/L } \\
\text { AS N) }\end{array}$ & $\begin{array}{l}\text { MIIRE- } \\
\text { GEN, } \\
\text { TOIAL } \\
\text { (MGA } \\
\text { AS N) }\end{array}$ & $\begin{array}{l}\text { PHOS- } \\
\text { PHORUS } \\
\text { TOTAL } \\
\text { (MG/L } \\
\text { AS P) }\end{array}$ & $\begin{array}{l}\text { MLKA- } \\
\text { LINITY } \\
\text { FIELO } \\
\text { TMG } \\
\text { AS } \\
\text { CACOZ) }\end{array}$ & $\begin{array}{l}\text { SOC IUN, } \\
\text { DIS- } \\
\text { SOL VED } \\
\text { (MCAL } \\
\text { AS NA) }\end{array}$ & $\begin{array}{l}\text { BICAR- } \\
\text { PDNATE } \\
\text { FET-FLO } \\
\text { (HG/L } \\
\text { AS } \\
\text { HCO3) }\end{array}$ & $\begin{array}{c}\text { CAR- } \\
\text { BDNATE } \\
\text { FET-FLD } \\
\text { (HG/L } \\
\text { AS COB) }\end{array}$ & $\begin{array}{l}\text { CHLO- } \\
\text { RIDE. } \\
\text { OIS- } \\
\text { SDLVED } \\
\text { (HG } \\
\text { AS CL) }\end{array}$ \\
\hline SEP 23. & 972 & -- & - & -- & -- & 123 & -- & 150 & - & 5.0 \\
\hline APR & & -- & -- & - & - & 123 & - & 150 & -- & 5.0 \\
\hline $22 \ldots$ & & .03 & .46 & .49 & .040 & -- & - & $=$ & -- & 4.4 \\
\hline $\begin{array}{l}30 \ldots . . . \\
\text { OCT }\end{array}$ & & .05 & .47 & .52 & .040 & -- & $\cdots$ & -- & -- & 5.5 \\
\hline JAN & 976 & .02 & .33 & .35 & .040 & - & 3.1 & - & - & 6.2 \\
\hline nay & & .02 & .50 & .52 & .030 & 131 & 3.3 & 160 & 3 & 5.4 \\
\hline$\underset{\text { SEP }}{13} \cdots$ & & .01 & .44 & .45 & .040 & - & 3.5 & -- & -- & 5.5 \\
\hline JUN & 978 & .01 & .42 & .43 & .040 & -- & 3.0 & -- & - & 5.0 \\
\hline SEP & & .02 & .42 & .44 & .050 & -- & 3.0 & -- & - & 5.2 \\
\hline $20 \ldots$ & & C.10 & .54 & .54 & .040 & -- & 3.1 & $=-$ & - & 5.4 \\
\hline
\end{tabular}

Site 43

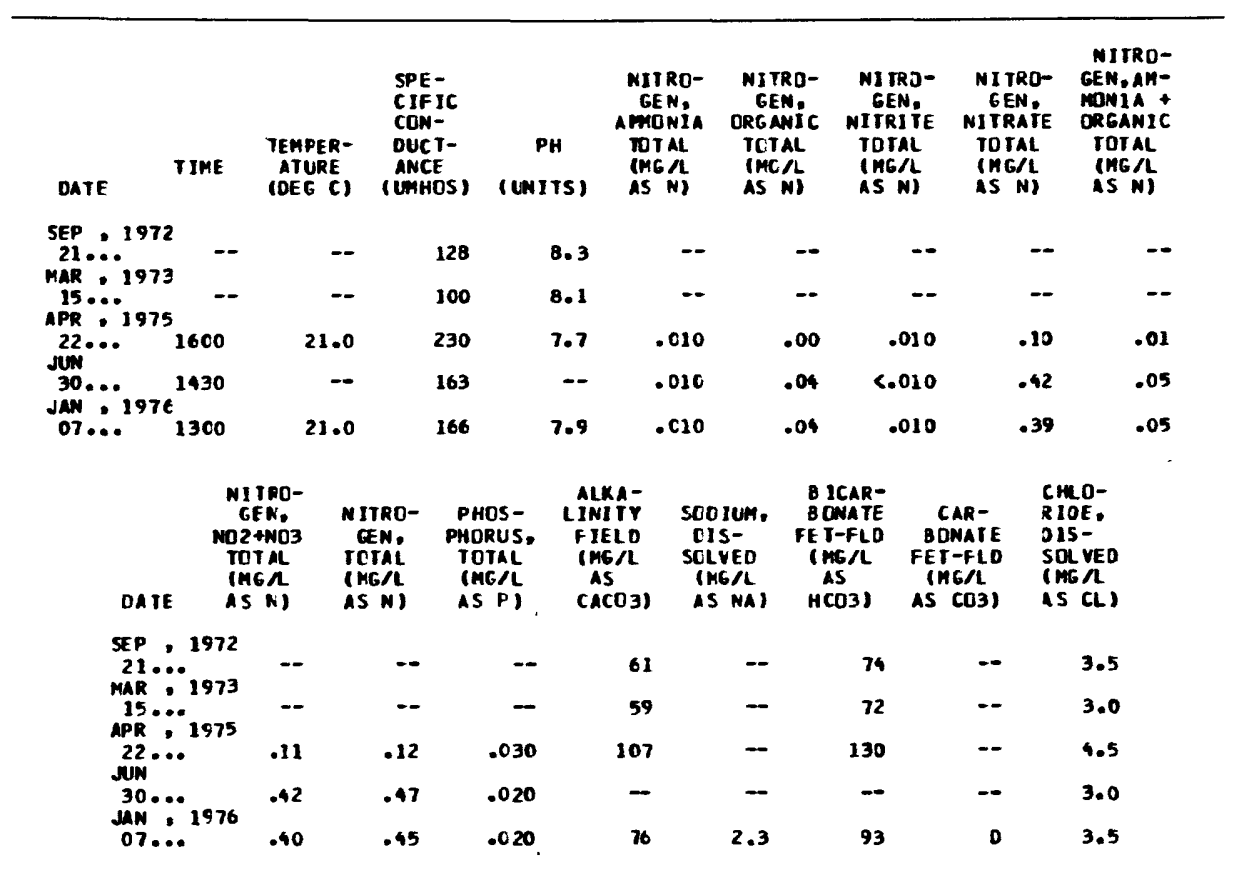




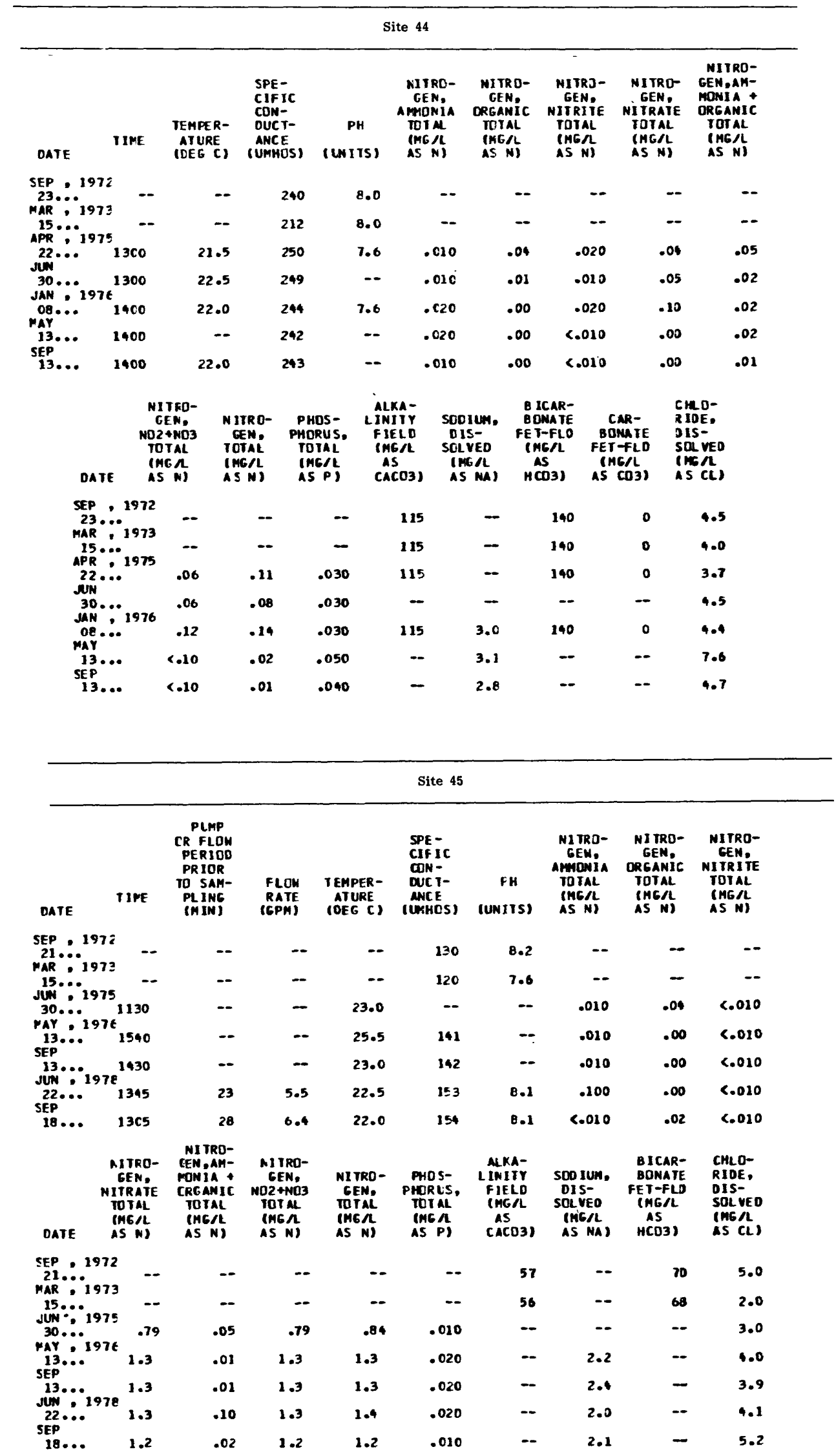


Site 46

\begin{tabular}{|c|c|c|c|c|c|c|c|c|c|c|c|c|}
\hline & \multicolumn{11}{|c|}{ Site 46} & \\
\hline 0115 & . TIME & $\begin{array}{l}\text { SPE - } \\
\text { CIFIC } \\
\text { CON- } \\
\text { DUCI- } \\
\text { ANCE } \\
\text { (UMHOS) }\end{array}$ & $\begin{array}{l}\text { NITRO- } \\
\text { GEN, } \\
\text { AMMCAIA } \\
\text { TOTAL } \\
\text { (MCR } \\
\text { ASA) }\end{array}$ & $\begin{array}{l}\text { NITRO- } \\
\text { GEN, } \\
\text { ORGANIC } \\
\text { TOTAL } \\
\text { (MG/L } \\
\text { AS N) }\end{array}$ & $\begin{array}{l}\text { NITRE- } \\
\text { GEN } \\
\text { NITRI IE } \\
\text { TOTAL } \\
\text { (MG/L } \\
\text { ASN) }\end{array}$ & $\begin{array}{l}\text { MITRO- } \\
\text { GFN } \\
\text { NITRAIE } \\
\text { TOTAL } \\
\text { (MGAL } \\
\text { AS N) }\end{array}$ & $\begin{array}{l}\text { NITRQ- } \\
\text { GEN,AH- } \\
\text { MGNIA + } \\
\text { CRGANIC } \\
\text { TCTAL } \\
\text { (MGAL } \\
\text { AS N) }\end{array}$ & $\begin{array}{l}\text { NITRO- } \\
\text { GEN, } \\
\text { M2+NO3 } \\
\text { TOTAL } \\
\text { (MG/L } \\
\text { AS N) }\end{array}$ & $\begin{array}{l}\text { NITRO- } \\
\text { GEN, } \\
\text { TOTAL } \\
\text { (HGAL } \\
\text { IS NI) }\end{array}$ & $\begin{array}{l}\text { PHOS- } \\
\text { PHORUS, } \\
\text { IOTAL } \\
\text { (MGA } \\
\text { IS PI }\end{array}$ & $\begin{array}{l}\text { SODIUR, } \\
\text { DIS- } \\
\text { SOLVED } \\
\text { (MGR } \\
\text { AS NA) }\end{array}$ & $\begin{array}{l}\text { CHLO- } \\
\text { RIDE, } \\
\text { OIS- } \\
\text { SOLVEC } \\
\text { (MG/L } \\
\text { AS CL) }\end{array}$ \\
\hline $\operatorname{mar}_{13 . .}$ & ${ }^{76}{ }_{1500}$ & 168 & .010 & .00 & C.c10 & 1.8 & .01 & 1.8 & $1 \cdot B$ & .020 & 0.7 & 8.4 \\
\hline
\end{tabular}

Site 47

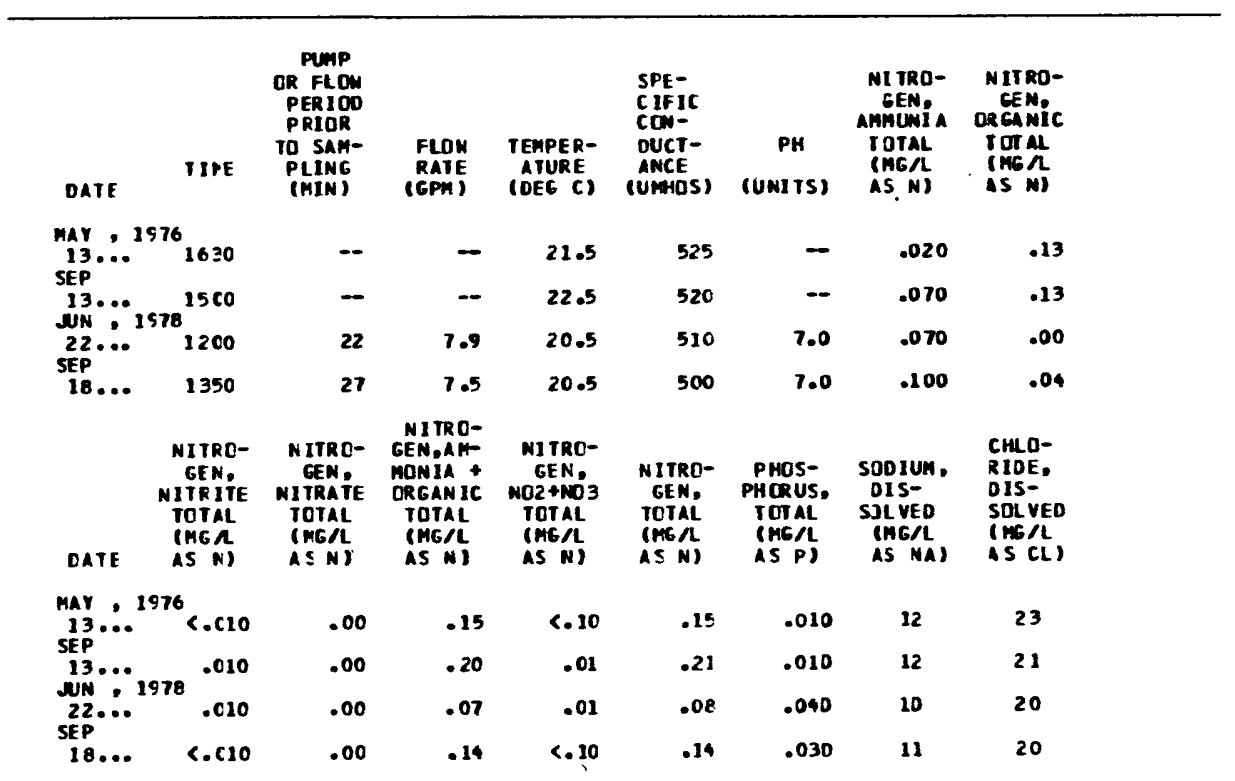

Site 48

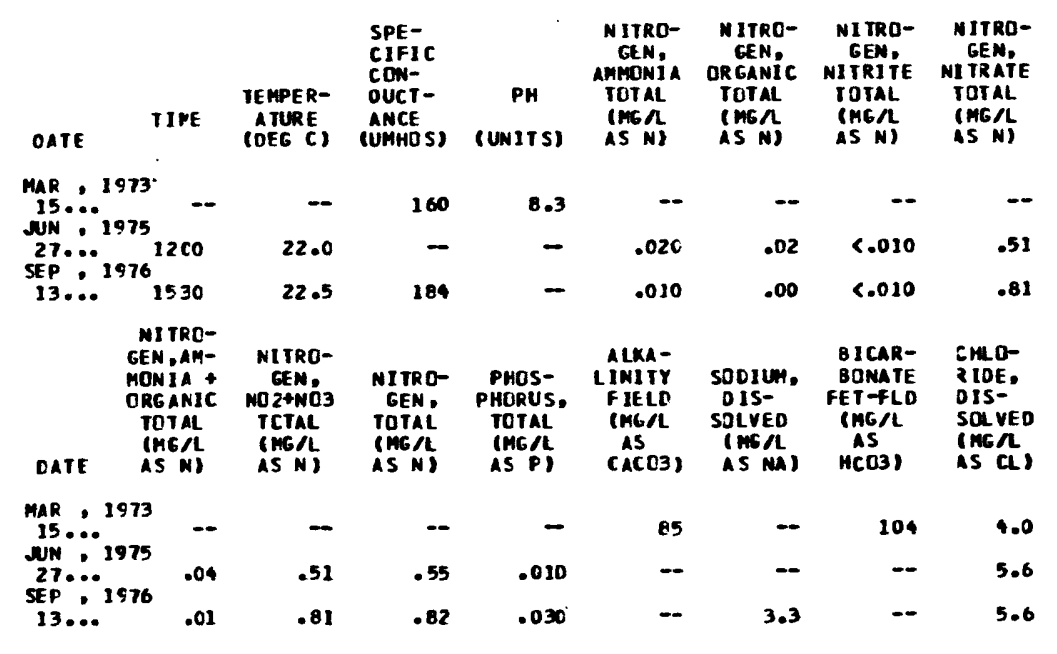


Site 49

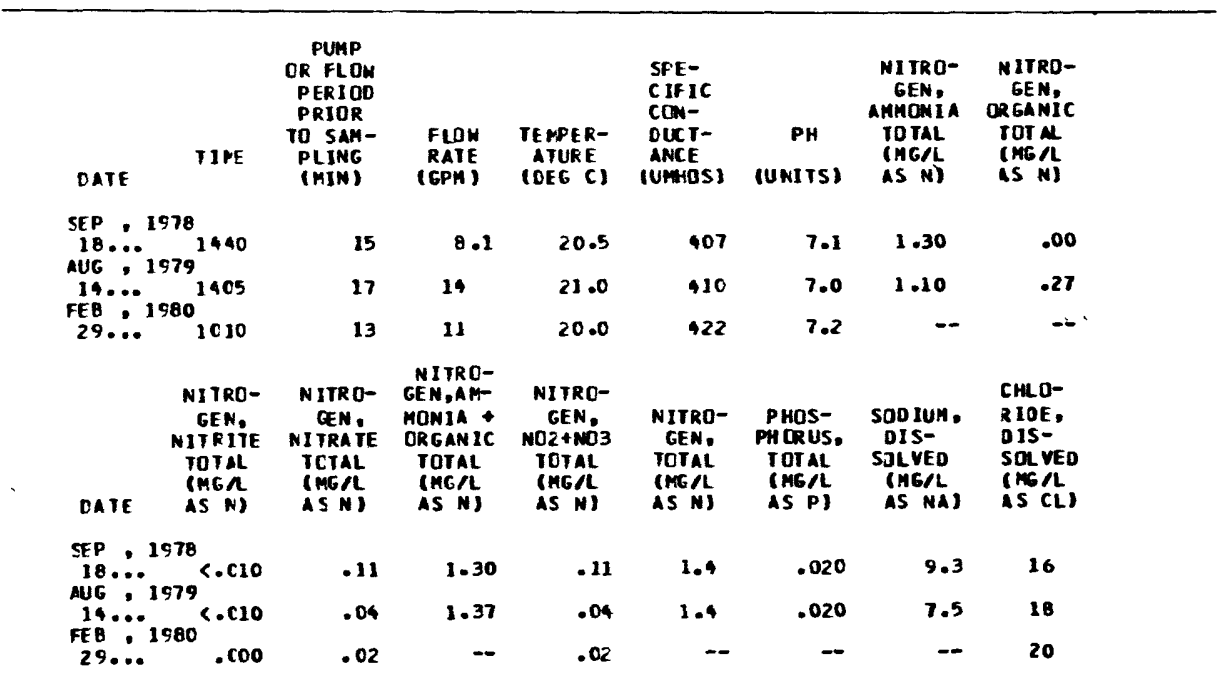

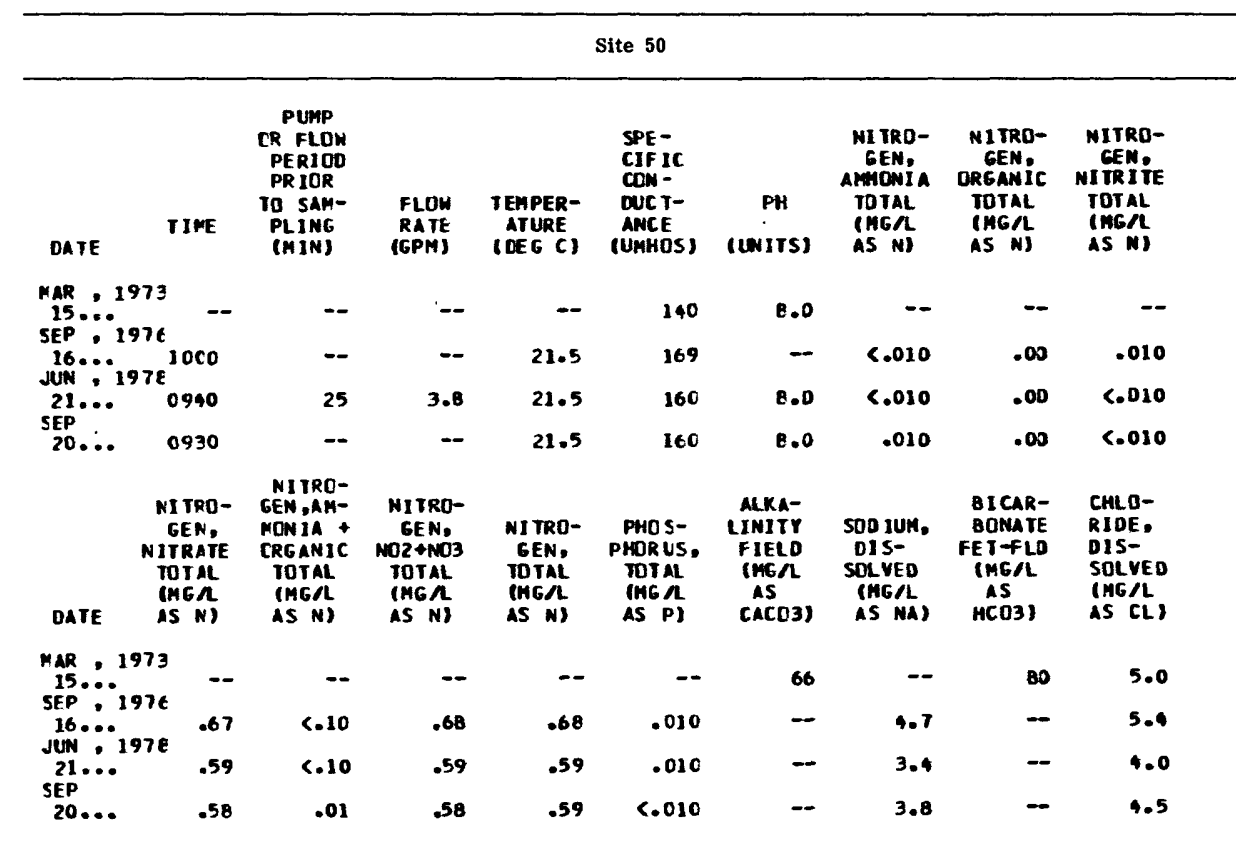


Table 6.--Physical and chemical characteristics of well and water at sites 1 through 70--Continued

Site 51

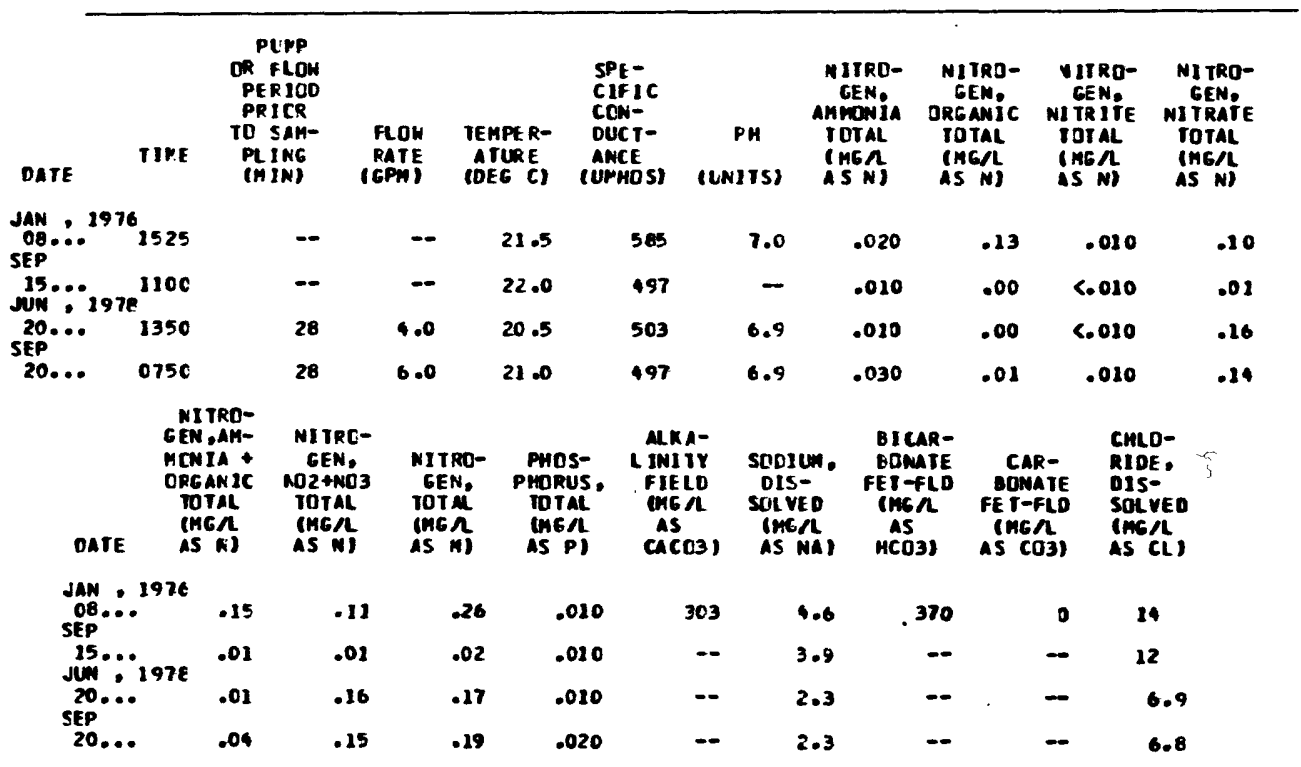

8te $\mathbf{5 2}$

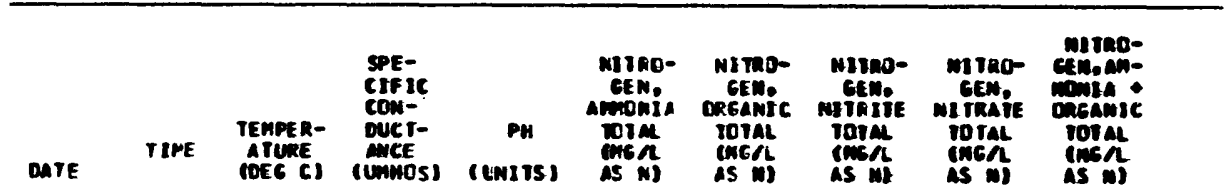

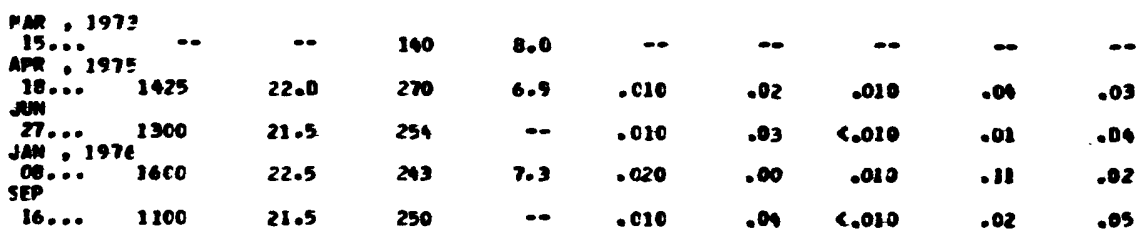

\begin{tabular}{|c|c|c|c|c|c|c|c|c|}
\hline DATE & 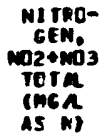 & 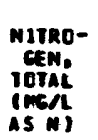 & $\begin{array}{l}\text { Pwos- } \\
\text { PuORuS, } \\
\text { rotal } \\
\text { (mefl } \\
\text { as p) }\end{array}$ & 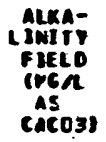 & $\begin{array}{l}\text { sccive, } \\
\text { ols- } \\
\text { solveo } \\
\text { iven } \\
\text { is was }\end{array}$ & 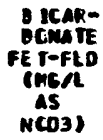 & 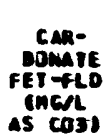 & 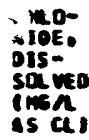 \\
\hline & $\cdots$ & - & - & 71 & - & 07 & 0 & 5.0 \\
\hline 18. & .05 & .08 & .010 & iss & - & 140 & e & 4.4 \\
\hline 27. & .01 & .05 & .010 & $\infty$ & - & $\infty$ & $\infty$ & 4.0 \\
\hline$\underset{\text { Sf }}{08}$ & .12 & .14 & .010 & 123 & 5.2 & 150 & 0 & 5.5 \\
\hline $16 \ldots$ & .02 & .07 & .020 & - & 3.8 & $-\infty$ & $\infty$ & 5.0 \\
\hline
\end{tabular}


Sìte 53

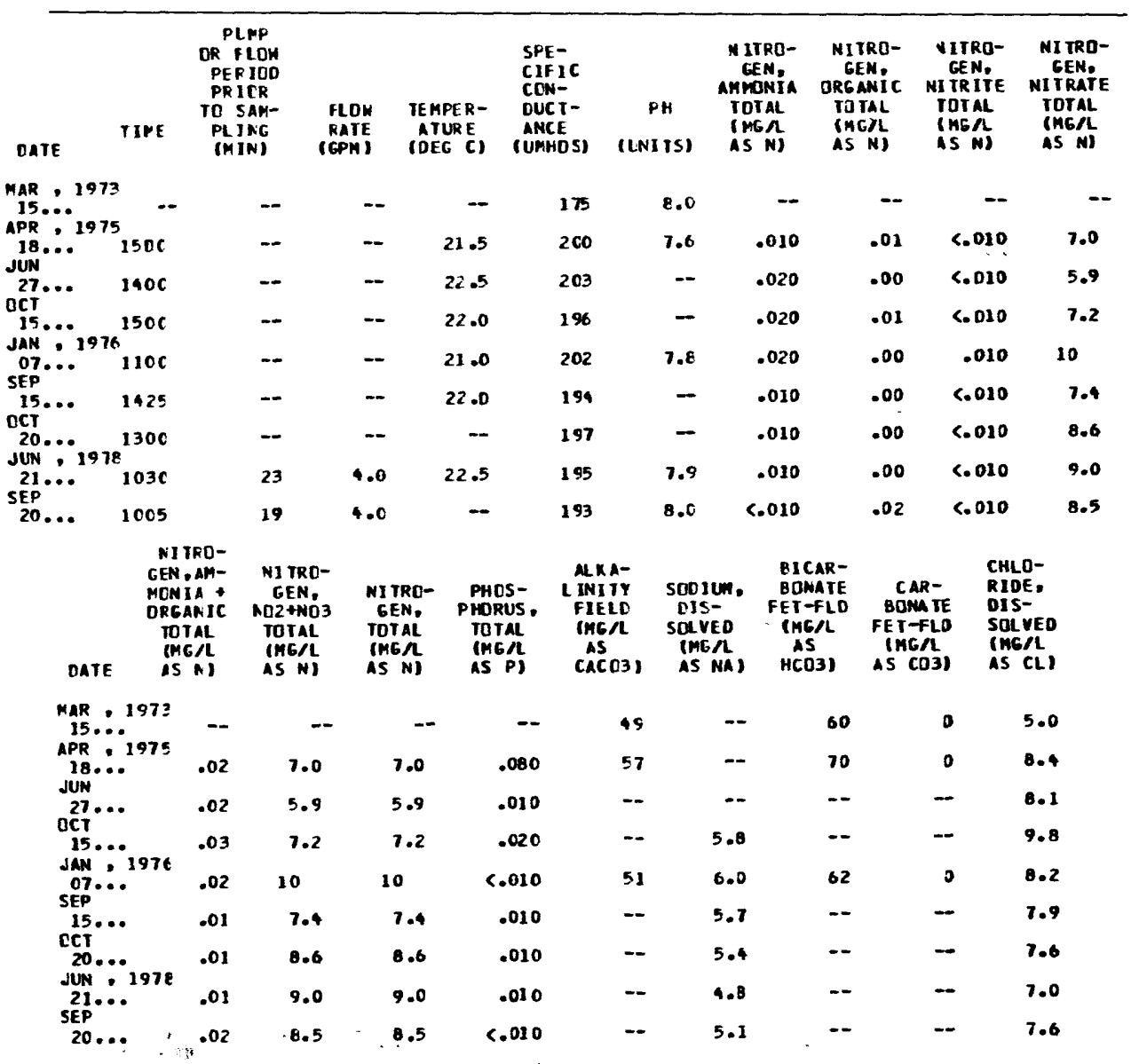

\begin{tabular}{|c|c|c|c|c|c|c|c|c|c|c|c|c|}
\hline \multirow[b]{2}{*}{$111 \mathrm{E}$} & \multirow[b]{2}{*}{ TIME } & \multicolumn{11}{|c|}{ Site 54} \\
\hline & & $\begin{array}{l}\text { TEMPE F- } \\
\text { ATURE } \\
\text { (DEG C) }\end{array}$ & $\begin{array}{l}\text { SPE - } \\
\text { CIF 1C } \\
\text { CON- } \\
\text { DUC I- } \\
\text { ANCE } \\
\text { (UNHCS) }\end{array}$ & $\begin{array}{c}\text { PH } \\
\text { (UNITS) }\end{array}$ & $\begin{array}{c}\text { MITPO- } \\
\text { GER: } \\
\text { AMHONIA } \\
\text { DIS- } \\
\text { SOLVEO } \\
\text { IMC/L } \\
\text { AS AI }\end{array}$ & $\begin{array}{l}\text { NITRC- } \\
\text { GEN, } \\
\text { AHMENIA } \\
\text { TOTAL } \\
\text { (HC/L } \\
\text { AS N) }\end{array}$ & $\begin{array}{l}\text { AITRO- } \\
\text { GEN: } \\
\text { CRGANIC } \\
\text { OIS- } \\
\text { SCLVES } \\
\text { (REA } \\
\text { IS N) }\end{array}$ & $\begin{array}{l}\text { NITRO- } \\
\text { GEN, } \\
\text { ORGANIC } \\
\text { TCTAL } \\
\text { (MGRL } \\
\text { AS N) }\end{array}$ & $\begin{array}{l}\text { NITRO- } \\
\text { GEN, } \\
\text { NITRITE } \\
\text { DIS- } \\
\text { SOLVED } \\
\text { (MGA } \\
\text { AS N) }\end{array}$ & $\begin{array}{l}\text { NITRO- } \\
\text { GEN, } \\
\text { NITRITE } \\
\text { TOT NL } \\
\text { (NG/2 } \\
\text { IS N) }\end{array}$ & $\begin{array}{l}\text { MIIRO- } \\
\text { GEN, } \\
\text { MIIRATE } \\
\text { DIS- } \\
\text { SOLYYO } \\
\text { (MGLL } \\
\text { AS N). }\end{array}$ & $\begin{array}{l}\text { MITRC- } \\
\text { GEN, } \\
\text { NITRA TE } \\
\text { TOTAL } \\
\text { (MG/L } \\
\text { AS N) }\end{array}$ \\
\hline $\begin{array}{c}\text { MAR D } \\
21 \ldots .\end{array}$ & ${ }^{2} 150 \mathrm{C}$ & 20.0 & 200 & 7.0 & -- & - & $\cdots$ & -- & .000 & .000 & .03 & .03 \\
\hline $\begin{array}{l}\text { JAN } \\
21 \ldots \\
\text { APR }\end{array}$ & $3080 c$ & $\cdots$ & 180 & 7.4 & -- & .030 & - & .24 & -- & .005 & -- & .01 \\
\hline & OBOC & 21.0 & 190 & $\therefore 7.7$ & - & .020 & -- & .29 & -- & .005 & -- & .08 \\
\hline$\underset{\text { SEP }}{27} \cdots$ & 1500 & -- & 189 & 7.5 & -- & .020 & -- & .39 & -- & .009 & -- & .05 \\
\hline $\operatorname{DEC}^{28} \cdots$ & 1500 & 23.0 & - & 7.7 & - & - & - & -- & -- & - & -- & - \\
\hline APR $21 \ldots$ & $133 \mathrm{C}$ & 21.0 & 205 & 8.0 & .620 & -- & - 25 & -- & $<.010$ & - & .00 & - \\
\hline $\operatorname{JUN}_{\text {Jun }}$ & $130 \mathrm{C}$ & 21.0 & 195 & 7.7 & .030 & - & .35 & - & $<.010$. & - & .03 & - \\
\hline 26 & $150 \mathrm{C}$ & 21.0 & - & - & .040 & - & .07 & $-\infty$ & .010 & - & .10 & 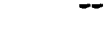 \\
\hline
\end{tabular}


Table 6.-- Physical and chemical characteristics of well and water at sites 1 through 70--Continued

Site 54--Continued

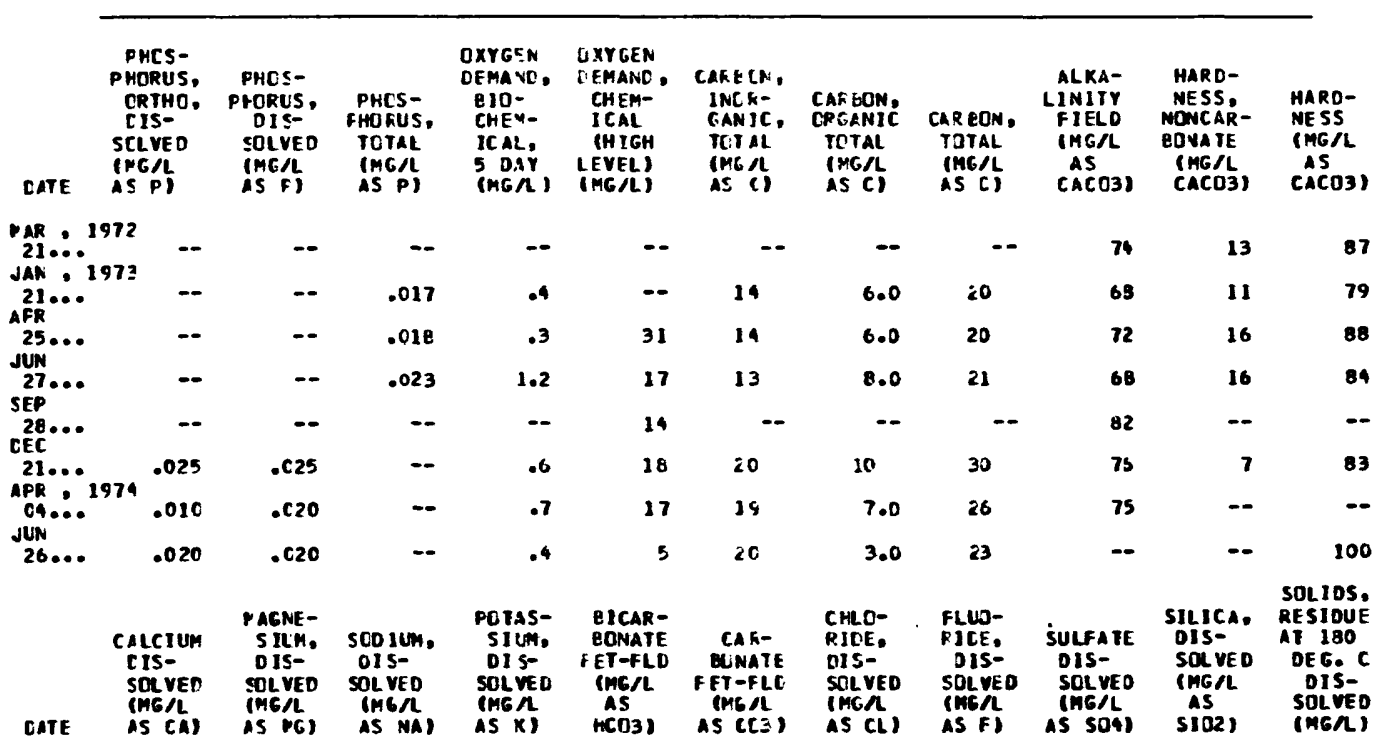

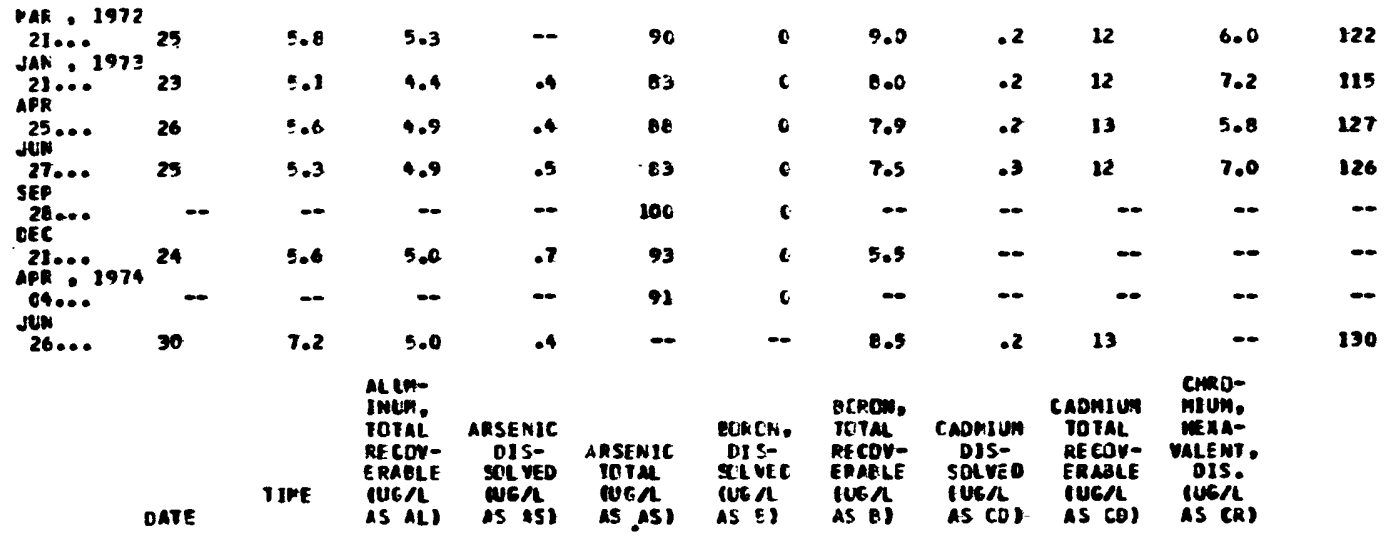

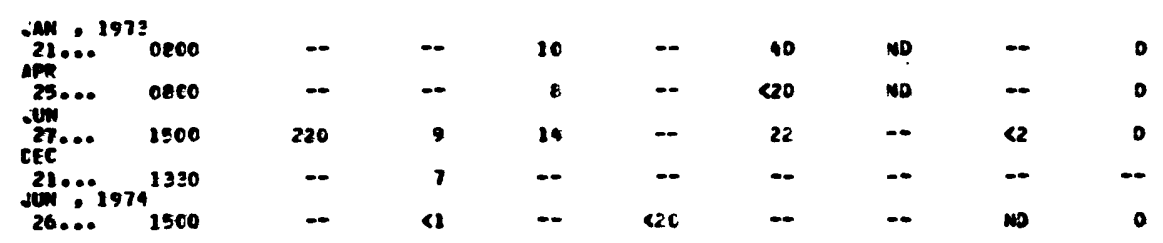


Site 54--Continued

\begin{tabular}{|c|c|c|c|c|c|c|c|c|c|}
\hline ATE & $\begin{array}{l}\text { CHRC- } \\
\text { PIUP, } \\
\text { YOTAL } \\
\text { RFCCV- } \\
\text { ERAELE } \\
\text { (UGRL } \\
\text { AS CR) }\end{array}$ & $\begin{array}{l}\text { CUB ALT. } \\
\text { TOYAL } \\
\text { RECOV- } \\
\text { ERABLE } \\
\text { (UG/L } \\
\text { AS CC) }\end{array}$ & $\begin{array}{l}\text { COPPER, } \\
\text { CIS- } \\
\text { SOL VED } \\
\text { (UG N } \\
\text { AS (U) }\end{array}$ & $\begin{array}{l}\text { CEPPER } \\
\text { TOTAL } \\
\text { RECLV- } \\
\text { ER ABLE } \\
\text { (UG/L } \\
\text { AS CU) }\end{array}$ & $\begin{array}{l}\text { IFCA, } \\
\text { CIS- } \\
\text { SCLVEC } \\
\text { (UE AL } \\
\text { AS FE) }\end{array}$ & $\begin{array}{l}\text { IRUN, } \\
\text { TCTAL } \\
\text { RECDV- } \\
\text { EFABLE } \\
\text { IUE } \\
\text { AS FE I }\end{array}$ & $\begin{array}{l}\text { LEAD. } \\
\text { DIS- } \\
\text { SJLVED } \\
\text { (UGRL } \\
\text { AS PB) }\end{array}$ & $\begin{array}{l}\text { LEAD, } \\
\text { TOTAL } \\
\text { RE COV- } \\
\text { ERABLE } \\
\text { (UG/L } \\
\text { AS PB) }\end{array}$ & $\begin{array}{l}\text { LITHIUM } \\
\text { TDTAL } \\
\text { RECOV- } \\
\text { ERABLE } \\
\text { (UG/L } \\
\text { AS LI) }\end{array}$ \\
\hline
\end{tabular}

$\begin{aligned} & \text { AN } \\ & 21 . \ldots\end{aligned}$
$\begin{aligned} & \text { APR } \\ & 25 . \ldots\end{aligned}$

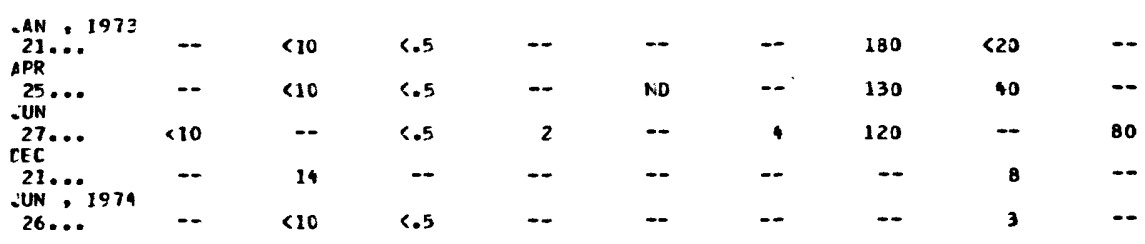

\begin{tabular}{|c|c|c|c|c|c|c|c|c|c|c|}
\hline \multicolumn{11}{|c|}{ Site 55} \\
\hline DATE & TIPE & $\begin{array}{l}\text { TEMFER- } \\
\text { ATURE } \\
\text { (DEC C) }\end{array}$ & $\begin{array}{l}\text { SPE- } \\
\text { CIFIC } \\
\text { CON- } \\
\text { DUCT- } \\
\text { ANCE } \\
\text { (UPHOS) }\end{array}$ & $\begin{array}{c}\text { PH } \\
\text { (UNITS) }\end{array}$ & $\begin{array}{l}\text { NIIRC- } \\
\text { GEN, } \\
\text { AMMON IA } \\
\text { DIS- } \\
\text { SOLVED } \\
\text { (KG/L } \\
\text { AS N) }\end{array}$ & $\begin{array}{l}\text { NITRE- } \\
\text { GEN, } \\
\text { AFMONIA } \\
\text { TCTAL } \\
\text { (MG/L } \\
\text { AS N) }\end{array}$ & $\begin{array}{c}\text { NITRE- } \\
\text { GEN, } \\
\text { ORGANIC } \\
\text { DIS- } \\
\text { SOLVED } \\
\text { (MS/L } \\
\text { AS N) }\end{array}$ & $\begin{array}{l}\text { NITRO- } \\
\text { GEN, } \\
\text { ORGANIC } \\
\text { TOTAL } \\
\text { ING/L } \\
\text { AS NS }\end{array}$ & $\begin{array}{l}\text { NIIRO- } \\
\text { GEN, } \\
\text { NIIRIIE } \\
\text { DIS- } \\
\text { SOL VED } \\
\text { (MG } \\
\text { IS N) }\end{array}$ & $\begin{array}{l}\text { MITRO- } \\
\text { GEN, } \\
\text { NITRITE } \\
\text { TOTAL } \\
\text { (MGRL } \\
\text { AS M) }\end{array}$ \\
\hline MAR . & 21600 & $2 C .0$ & 155 & 7.4 & -- & -- & $\cdots$ & $\cdots$ & -- & .003 \\
\hline 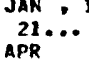 & $30 c c$ & -- & 180 & 7.4 & -- & .030 & - & .52 & - & .003 \\
\hline JUN 25 & I OCC & 21.0 & 189 & 7.5 & - & .020 & -- & .28 & - & .002 \\
\hline${ }_{S F P}^{27} \cdots$ & $130 \mathrm{C}$ & $-\infty$ & $18 B$ & 7.5 & - & .010 & -- & .24 & -- & $=.007$ \\
\hline DEC & $140 \mathrm{C}$ & - & 205 & 7.2 & - & .020 & -- & .32 & - & .005 \\
\hline${ }_{A P R}^{21} \cdots$ & $4^{1430}$ & 21.0 & 209 & 7.6 & .020 & -- & .19 & $\cdots$ & $<.010$ & $\cdots$ \\
\hline JUN... & $120 c$ & $=1.0$ & 197 & 7.7 & .010 & $\because$ & - & $-\infty$ & C.010 & -- \\
\hline $\begin{array}{l}26 \\
\text { AUG }\end{array}$ & $140 \mathrm{C}$ & 21.0 & - & -- & .020 & -- & .03 & -- & .010 & -- \\
\hline JAN 28, & $5^{120 C}$ & 21.5 & 200 & 7.1 & -- & .020 & -- & .32 & -- & .010 \\
\hline $16 \ldots$ & 1200 & 19.0 & 130 & 7.1 & .020 & - & .23 & -- & .010 & -- \\
\hline$\underset{\mathrm{JtL}}{18}$ & $133 C$ & $2 \mathrm{C} .0$ & 100 & 6.8 & - & .050 & - & .35 & -- & .020 \\
\hline${ }_{\text {OCT }}^{02}$ & $133 \mathrm{C}$ & 21.0 & 200 & 7.4 & -- & $.02 \mathrm{C}$ & -- & .08 & $\cdots$ & .010 \\
\hline JAN 15 & $6^{1430}$ & 21.0 & 189 & -- & $\therefore$ & .030 & -- & .14 & -- & $<.010$ \\
\hline$\underset{\operatorname{sep}}{22} \cdots$ & $143 c$ & 21.0 & 175 & 7.4 & -- & .020 & $-\infty$ & .09 & $\cdots$ & .020 \\
\hline JUN $15 .$. & $7^{1330}$ & 22.0 & 180 & -- & -- & .020 & -- & .40 & -- & .010 \\
\hline $09 .$. & $130 \mathrm{C}$ & -- & 216 & 7.8 & -- & $<.010$ & -- & .01 & -- & .010 \\
\hline
\end{tabular}


Site 55--Continued

\begin{tabular}{|c|c|c|c|c|c|c|c|c|c|c|}
\hline DATE & $\begin{array}{r}\text { MI } \\
\text { G } \\
\text { II } \\
0 \\
\text { S0 } \\
\text { IM } \\
\text { IS }\end{array}$ & $\begin{array}{l}\text { IRO- } \\
\text { N. } \\
\text { IATE } \\
\text { IS- } \\
\text { VED } \\
\text { A } \\
\text { AI }\end{array}$ & $\begin{array}{l}\text { NITEC- } \\
\text { GEN, } \\
\text { AITEATE } \\
\text { TOTAL } \\
\text { (HCNL } \\
\text { ASN) }\end{array}$ & $\begin{array}{l}\text { NITRU- } \\
\text { GENOAM- } \\
\text { MONIA } \\
\text { ORGANIC } \\
\text { TOTAL } \\
\text { (MGA } \\
\text { AS V) }\end{array}$ & $\begin{array}{l}\text { NI TKO - } \\
\text { GEN, } \\
\text { AO2 +NBJ } \\
\text { TOTAL } \\
\text { GG/L } \\
\text { AS NI }\end{array}$ & 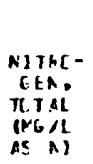 & 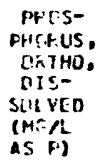 & $\begin{array}{l}\text { PHCS- } \\
\text { FHOKUS, } \\
\text { DIS- } \\
\text { SOLVED } \\
\text { (MG/L } \\
\text { AS PI }\end{array}$ & $\begin{array}{l}\text { PHOS- } \\
\text { PHORUS, } \\
\text { IOTAL } \\
\text { (MCAL } \\
\text { AS PJ }\end{array}$ & $\begin{array}{l}\text { DXYGEN } \\
\text { DEMAND, } \\
\text { BIO- } \\
\text { CHEM- } \\
\text { ICAL, } \\
5 \text { DAY } \\
\text { (MGAL) }\end{array}$ \\
\hline $\begin{array}{r}\text { MAR } \\
21 . . .\end{array}$ & & -- & .04 & -- & - & -- & -- & -- & - & -- \\
\hline 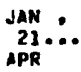 & 972 & -- & .03 & -- & -- & -- & -- & $\sim$ & .012 & .5 \\
\hline JUN $_{25} \cdots$ & & -- & .06 & 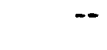 & $=$ & $\cdots$ & -- & -- & .015 & .3 \\
\hline $\operatorname{SEP}_{\text {SEP }}^{27}$ & & -- & .07 & -- & - & $\cdots$ & - & $=$ & .045 & .7 \\
\hline IEC & & -- & .05 & $\cdots$ & -- & -- & - & - & .240 & .4 \\
\hline$\underset{A P R}{21} \cdots$ & 974 & .03 & $\cdots$ & $\cdots$ & $\cdots$ & -- & .025 & .027 & -- & .9 \\
\hline O4 UN & & .04 & - & - & -- & -- & .020 & .020 & -- & .4 \\
\hline NUG & & .10 & $\cdots$ & $\cdots$ & $=$ & -- & .020 & .050 & -- & . B \\
\hline JAN & 975 & $-\infty$ & .09 & .34 & .10 & .44 & -- & $\cdots$ & .060 & .3 \\
\hline${ }_{A P R}^{16} \cdots$ & & .03 & -- & -- & - & -- & .010 & .020 & - & .6 \\
\hline$\underset{\text { JUL }}{18} \cdots$ & & - & .03 & .40 & .05 & .45 & -- & -- & .020 & -- \\
\hline Сटт $\cdots$ & & -- & .07 & .10 & .08 & $.1 E$ & -- & -- & .020 & .0 \\
\hline IS... & 976 & $\sim$ & .07 & .17 & .07 & .<4 & -- & -- & .030 & -- \\
\hline SEP $_{\text {SE. }}^{22}$ & & -- & .10 & .11 & .12 & .23 & - & -- & .020 & -- \\
\hline JUN... & & -- & .05 & .42 & .06 & $.4 F$ & - & $\cdots$ & .025 & -- \\
\hline 08. & & -- & .05 & .01 & .06 & to & - & -- & .020 & .0 \\
\hline
\end{tabular}

\begin{tabular}{|c|c|c|c|c|c|c|c|c|c|}
\hline 1 & $\begin{array}{l}\text { CYYCEN } \\
\text { DEMAND, } \\
\text { CHEM- } \\
\text { ICAL } \\
\text { (HIGH } \\
\text { LEVEL) } \\
\text { (YG/L) }\end{array}$ & $\begin{array}{l}\text { CAREON, } \\
\text { INCR- } \\
\text { GANIC, } \\
\text { TOTAL } \\
\text { (MGAL } \\
\text { AS C) }\end{array}$ & $\begin{array}{l}\text { CARBON, } \\
\text { DRGANIC } \\
\text { TOIAL } \\
\text { (MGA } \\
\text { AS C) }\end{array}$ & $\begin{array}{l}\text { CAR BON, } \\
\text { TOTAL } \\
\text { (MG/L } \\
\text { AS C) }\end{array}$ & $\begin{array}{l}\text { ALKA- } \\
\text { L INITY } \\
\text { FIELC } \\
\text { IMG } A \\
\text { AS } \\
\text { CACC3) }\end{array}$ & $\begin{array}{l}\text { HARD- } \\
\text { NESS, } \\
\text { NONCAR- } \\
\text { BONATE } \\
\text { (MG/L } \\
\text { CACO3) }\end{array}$ & $\begin{array}{l}\text { HARD- } \\
\text { NESS } \\
\text { (MG/L } \\
\text { AS } \\
\text { CACD3) }\end{array}$ & $\begin{array}{l}\text { CALCIUH } \\
\text { DIS- } \\
\text { SOLVED } \\
\text { (MG/L } \\
\text { AS CA) }\end{array}$ & $\begin{array}{l}\text { SIUH, } \\
\text { DI S- } \\
\text { SOL VEO } \\
\text { (ME/L } \\
\text { AS MG) }\end{array}$ \\
\hline
\end{tabular}

\begin{tabular}{|c|c|c|c|c|c|c|c|c|c|}
\hline $\begin{array}{l}\text { MR } 21972 \\
21 \cdots 1973\end{array}$ & $\cdots$ & -- & $\cdots$ & $\cdots$ & 74 & 13 & 87 & 25 & $\cdots$ \\
\hline$\underset{A P R}{21} \cdots$ & $\sim$ & 14 & 5.0 & 20 & $\in E$ & 10 & 79 & 23 & 5.1 \\
\hline$\underset{\text { JUN }}{25} \cdots$ & 29 & 15 & 4.0 & 19 & 72 & 16 & 88 & 26 & 5.6 \\
\hline SEP $27 \cdots$ & 16 & 13 & 10 & 23 & 74 & 11 & 34 & 25 & 5.3 \\
\hline OEC & 16 & 20 & 2.0 & 22 & 82 & - & $m$ & - & 6.3 \\
\hline$\underset{A P R}{21} \because 1974$ & 14 & 20 & 9.0 & 28 & 77 & 13 & 90 & 23 & B.o \\
\hline SUN & 16 & 18 & 7.0 & 25 & 73 & 12 & 35 & 25 & 5.5 \\
\hline$\underset{\text { AUG }}{26}$ & 7 & 20 & 5.0 & 25 & $\cdots$ & $\cdots$ & 100 & 30 & 7.2 \\
\hline $\begin{array}{l}28 \cdots 1975 \\
\text { JAN } 1975\end{array}$ & 18 & 18 & 9.0 & 26 & ES & 14 & 83 & 24 & 5.6 \\
\hline$\underset{A P R}{16} \cdots$ & 42 & 13 & 14 & 27 & 94 & 18 & 73 & 22 & 4.5 \\
\hline$\underset{\text { sul }}{18 \ldots}$ & $\cdots$ & B.C & 19 & 27 & $\equiv 3$ & -- & $\cdots$ & - & 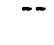 \\
\hline $\mathrm{CCl}^{\mathrm{C}} \mathrm{C}$ & - & 20 & 3.0 & 23 & EC & 13 & 92 & 27 & 6.0 \\
\hline JAN: 1597 & $\cdots$ & -- & $\cdots$ & - & $\cdots$ & $\cdots$ & $\cdots$ & $\cdots$ & $\cdots$ \\
\hline$\underset{\text { SEP }}{22} \cdots$ & $\cdots$ & $\cdots$ & - & $\cdots$ & $7 \tilde{z}$ & - & -- & - & $-\infty$ \\
\hline $\begin{array}{l}15 \ldots N \\
\text { JUE }\end{array}$ & - & - & 26 & $-\infty$ & - & $\cdots$ & 100 & 29 & - \\
\hline
\end{tabular}


Site 55--Continued

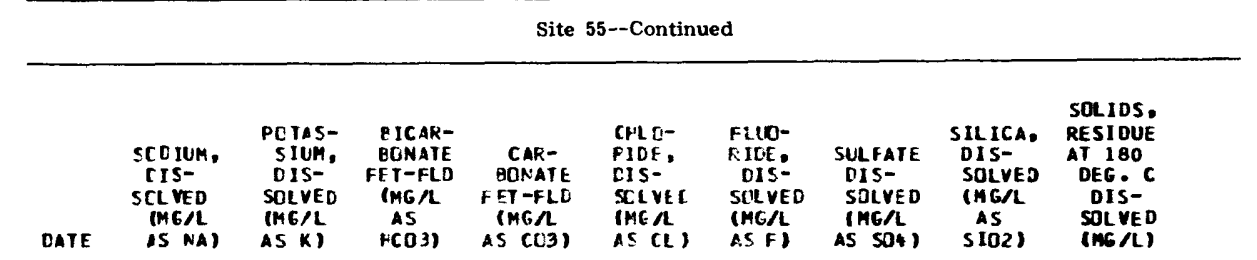

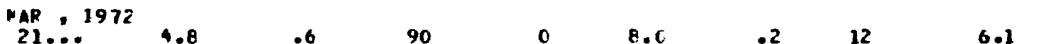

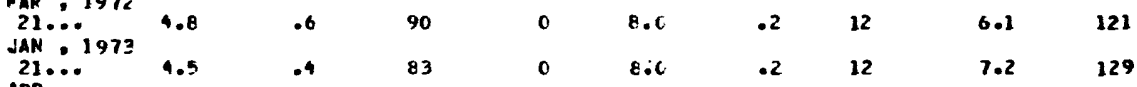$$
\begin{array}{llllllllll}
21 \ldots . .0 & 4.5 & .4 & 83 & 0 & 8.0 & .2 & 12 & 7.2 & 129 \\
25 \ldots & 4.6 & .4 & 98 & 0 & 7.7 & .3 & 11 & 5.7 & 123
\end{array}
$$

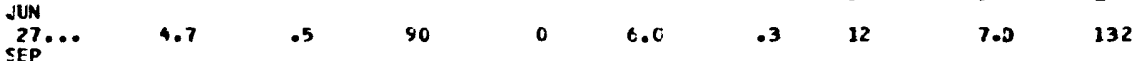

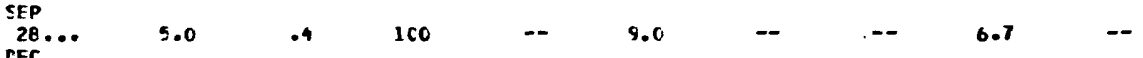

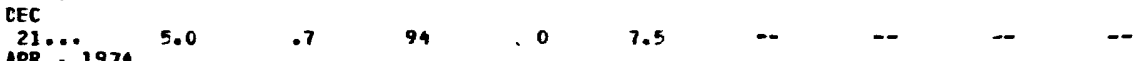

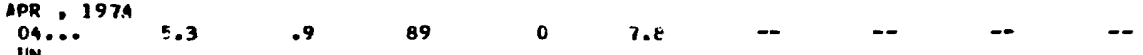$$
\begin{array}{llllllllll}
. \text { UN } \\
26 . . . & 5.1 & .4 & -- & -2 & 6.7 & .2 & 13 & -2 & 146
\end{array}
$$

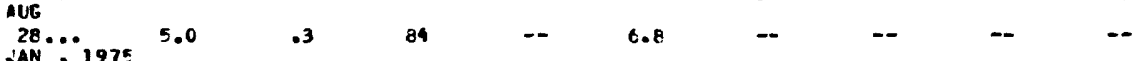

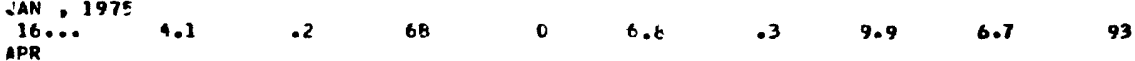

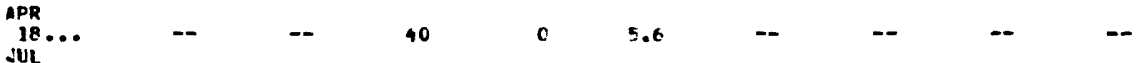$$
\begin{array}{rrrrrrrrrr}
02 \ldots . . & 4.9 & .5 & 97 & 0 & 7.2 & .1 & 12 & 6.6 & 130
\end{array}
$$

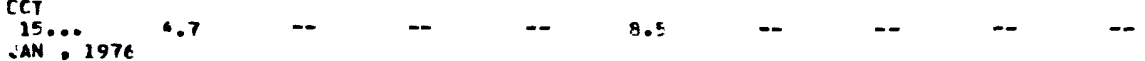

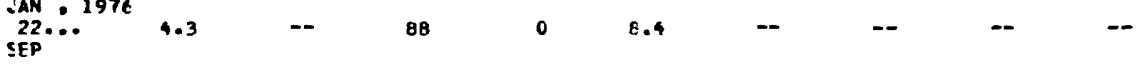

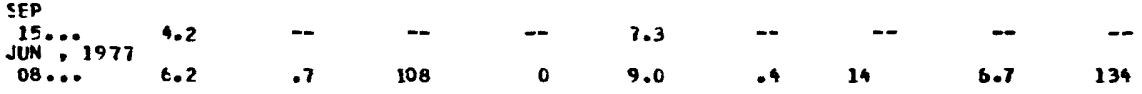

\begin{tabular}{|c|c|c|c|c|c|c|c|c|c|c|}
\hline CATE & TIFE & $\begin{array}{l}\text { ALUP- } \\
\text { INUY. } \\
\text { DIS- } \\
\text { SOEVEO } \\
\text { IUGA } \\
\text { AS ALI }\end{array}$ & $\begin{array}{l}\text { ALUH- } \\
\text { INUM, } \\
\text { IOTAL } \\
\text { RECOV- } \\
\text { ERABLE } \\
\text { (UGAL } \\
\text { AS AL) }\end{array}$ & $\begin{array}{l}\text { AR SENIC } \\
\text { DIS- } \\
\text { SOLVED } \\
\text { (UGN } \\
\text { ASAS) }\end{array}$ & $\begin{array}{l}\text { ARSEN IC } \\
\text { TOTAL } \\
\text { (UEAL } \\
\text { AS ASI }\end{array}$ & $\begin{array}{l}\text { BAFIUH, } \\
\text { TOTAL } \\
\text { RECEV- } \\
\text { EPAELE } \\
\text { (UE } / \text {. } \\
\text { AS EA) }\end{array}$ & $\begin{array}{l}\text { BQRON, } \\
\text { DIS- } \\
\text { SOLVED } \\
\text { (USA } \\
\text { AS B) }\end{array}$ & $\begin{array}{l}\text { BORON, } \\
\text { TOTAL } \\
\text { RECOY- } \\
\text { ERABLE } \\
\text { (UG/L } \\
\text { AS BI }\end{array}$ & $\begin{array}{l}\text { CADHIU } \\
\text { DIS- } \\
\text { SOL VED } \\
\text { (UE } n \\
\text { AS CD) }\end{array}$ & $\begin{array}{l}\text { CADMIUM } \\
\text { TOTAL } \\
\text { RE COY- } \\
\text { ERABLE } \\
\text { (UGRL } \\
\text { AS CD) }\end{array}$ \\
\hline \multicolumn{11}{|c|}{ JAN , 1973} \\
\hline${ }_{A P R}^{21} \cdots$ & $100 \mathrm{C}$ & -- & -- & -- & 10 & -- & -- & $<20$ & ND & -- \\
\hline JUN $25 .$. & 10ce & -- & -- & -- & $\varepsilon$ & -- & -- & $<20$ & ND & -- \\
\hline $\operatorname{SEP}_{S E}^{27} \cdots$ & $13 C c$ & -- & 220 & 13 & 13 & -- & -- & 22 & -- & $<2$ \\
\hline${ }_{D E C}^{28} \cdots$ & $140 \mathrm{C}$ & -- & -- & - & - & -- & - & 15 & - & - \\
\hline APR $21 \cdots$ & $143 c$ & -- & - & 6 & - & -- & -- & -- & -- & -- \\
\hline $\operatorname{sen}_{\text {Je... }}$ & $120 C$ & -- & -- & 2 & - & -- & $<20$ & -- & -- & MD \\
\hline${ }_{A U G}^{26} \cdots$ & $140 \mathrm{C}$ & $=$ & -- & <1 & - & -- & $<20$ & -- & -- & $<2$ \\
\hline JAN & $5^{120 C}$ & -- & - & -- & -- & -- & $<20$ & -- & $-\infty$ & -- \\
\hline $16 \ldots$ & $120 \mathrm{C}$ & 200 & - & 1 & - & -- & 100 & -- & ND & -- \\
\hline $\begin{array}{l}02 \ldots \\
\text { JUN }\end{array}$ & $7^{1330}$ & 60 & -- & $\mathbf{<}$ & - & -- & -- & -- & MD & -- \\
\hline $08 \ldots$ & $130 \mathrm{C}$ & -- & -- & - & $\hat{\imath}$ & $<100$ & -- & -- & -- & ND \\
\hline
\end{tabular}


Site 55--Continued

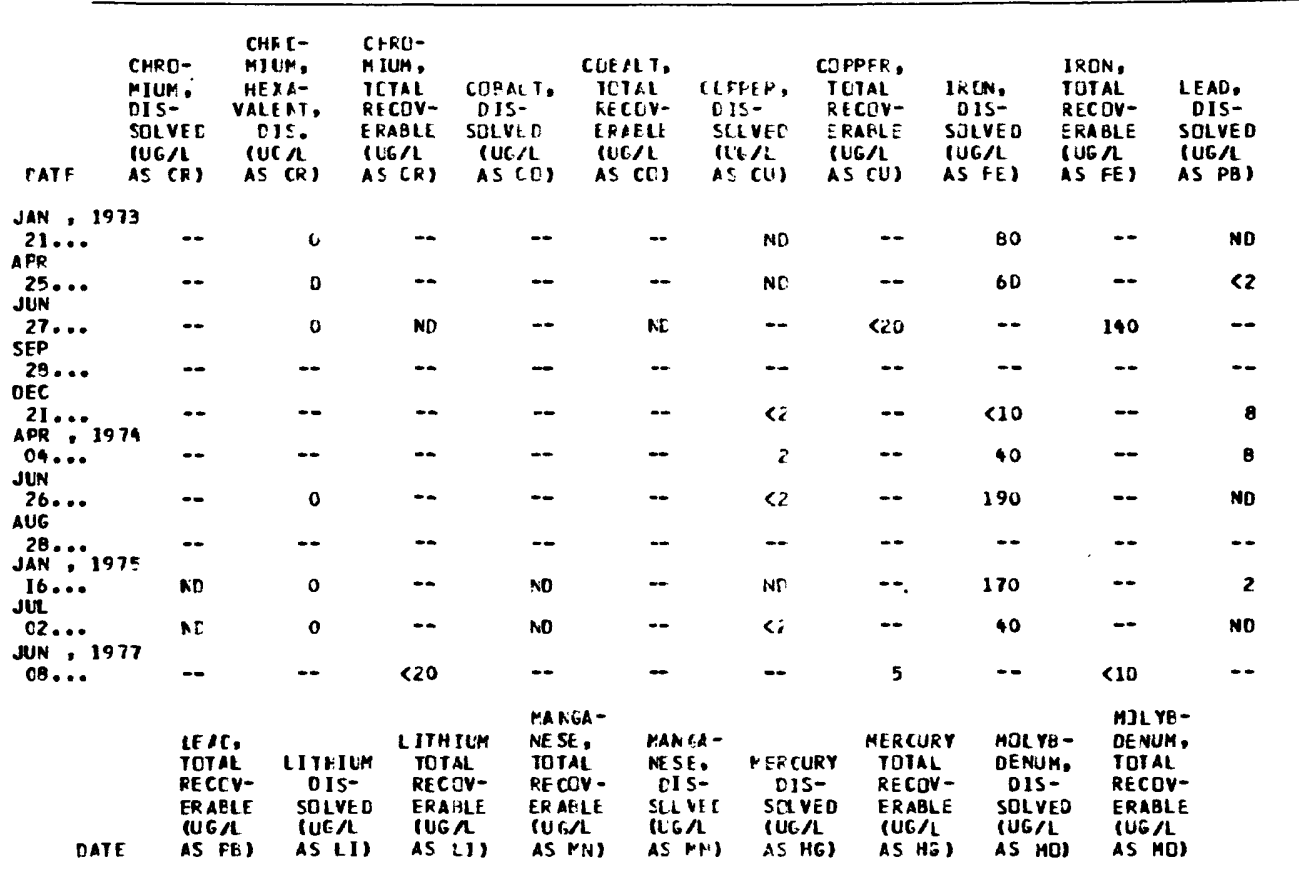

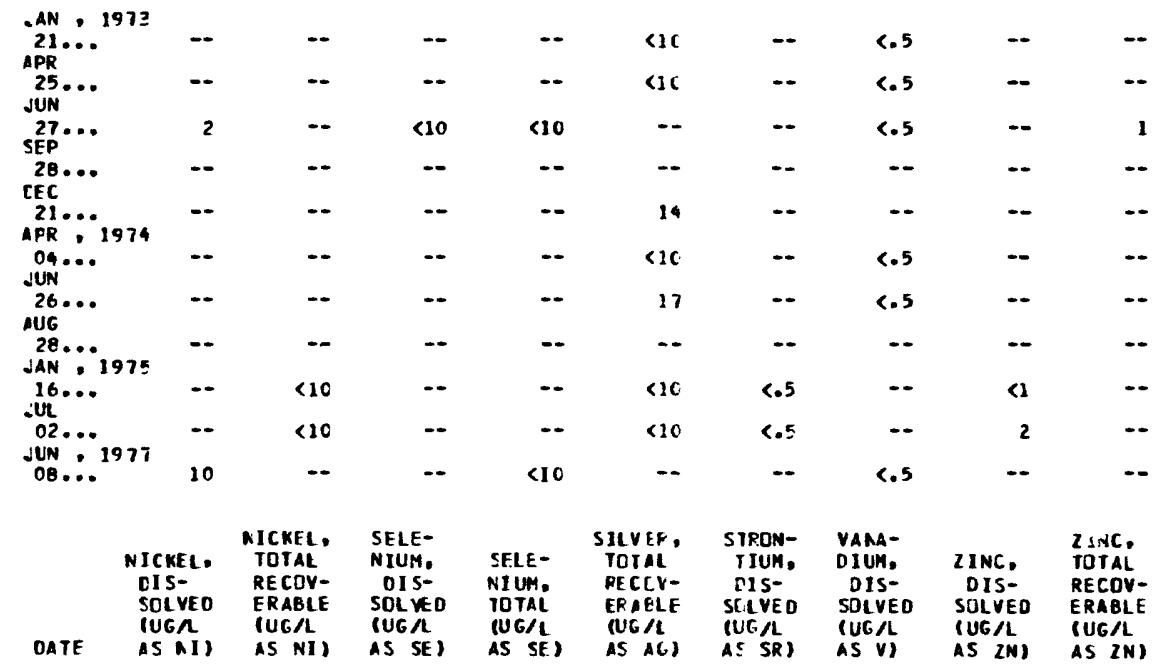

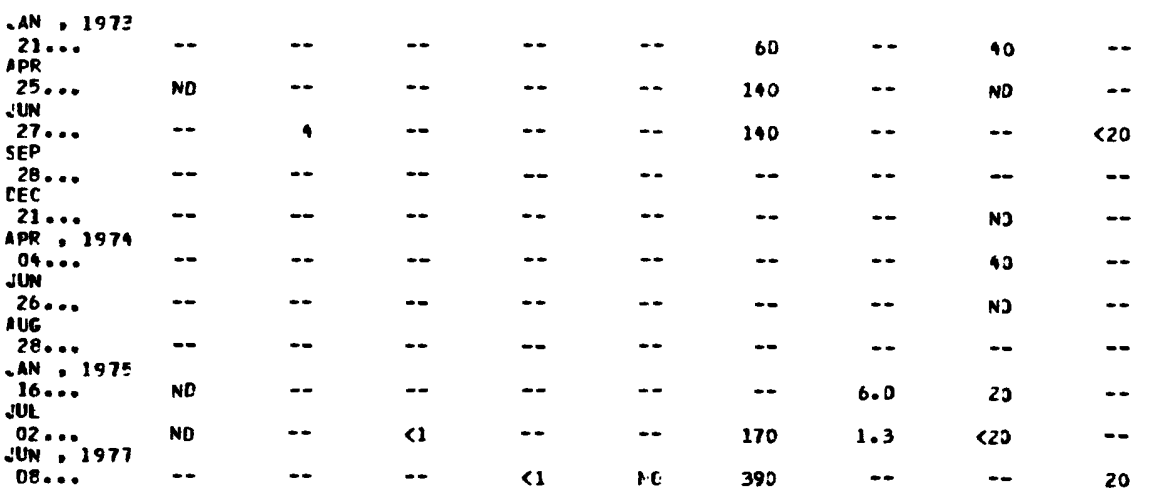


Site 56

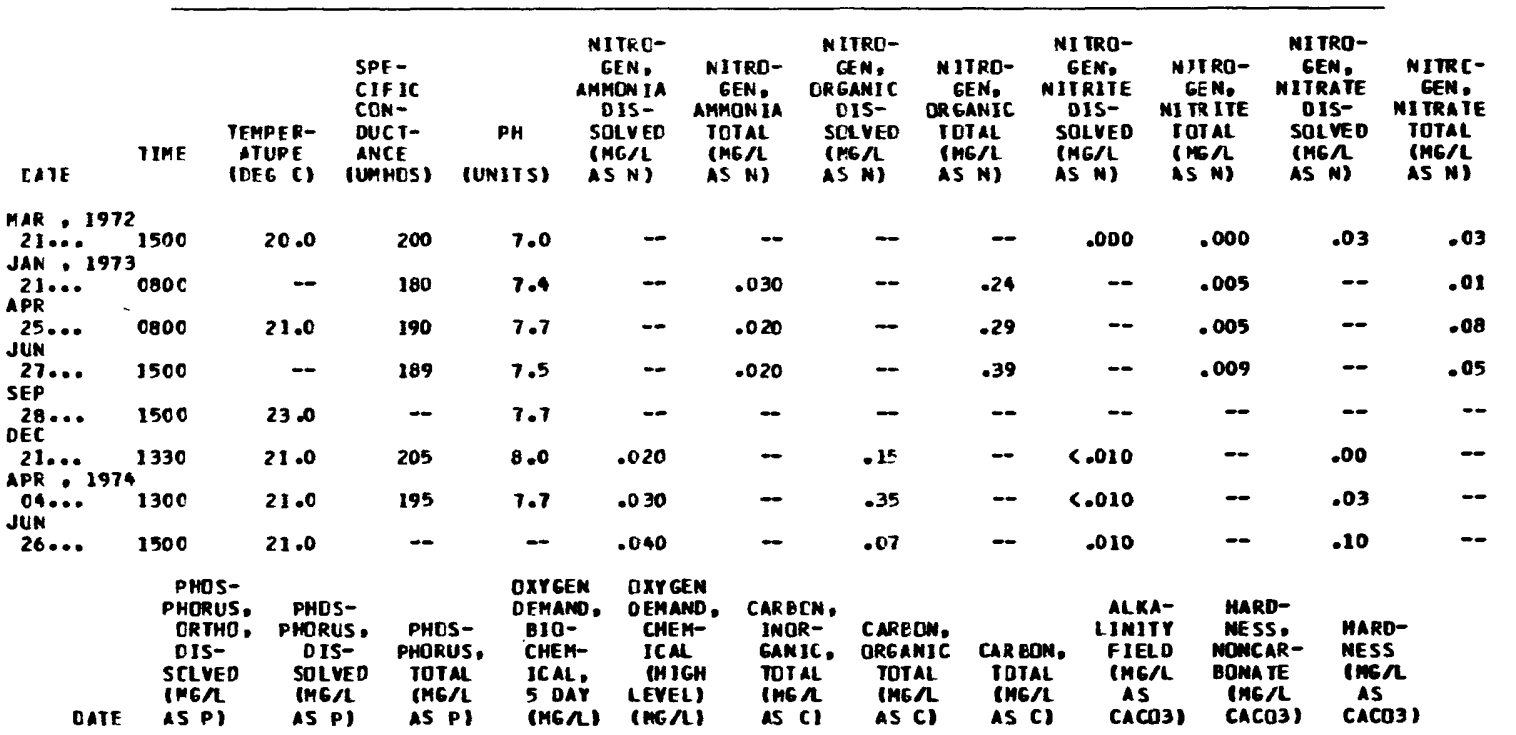

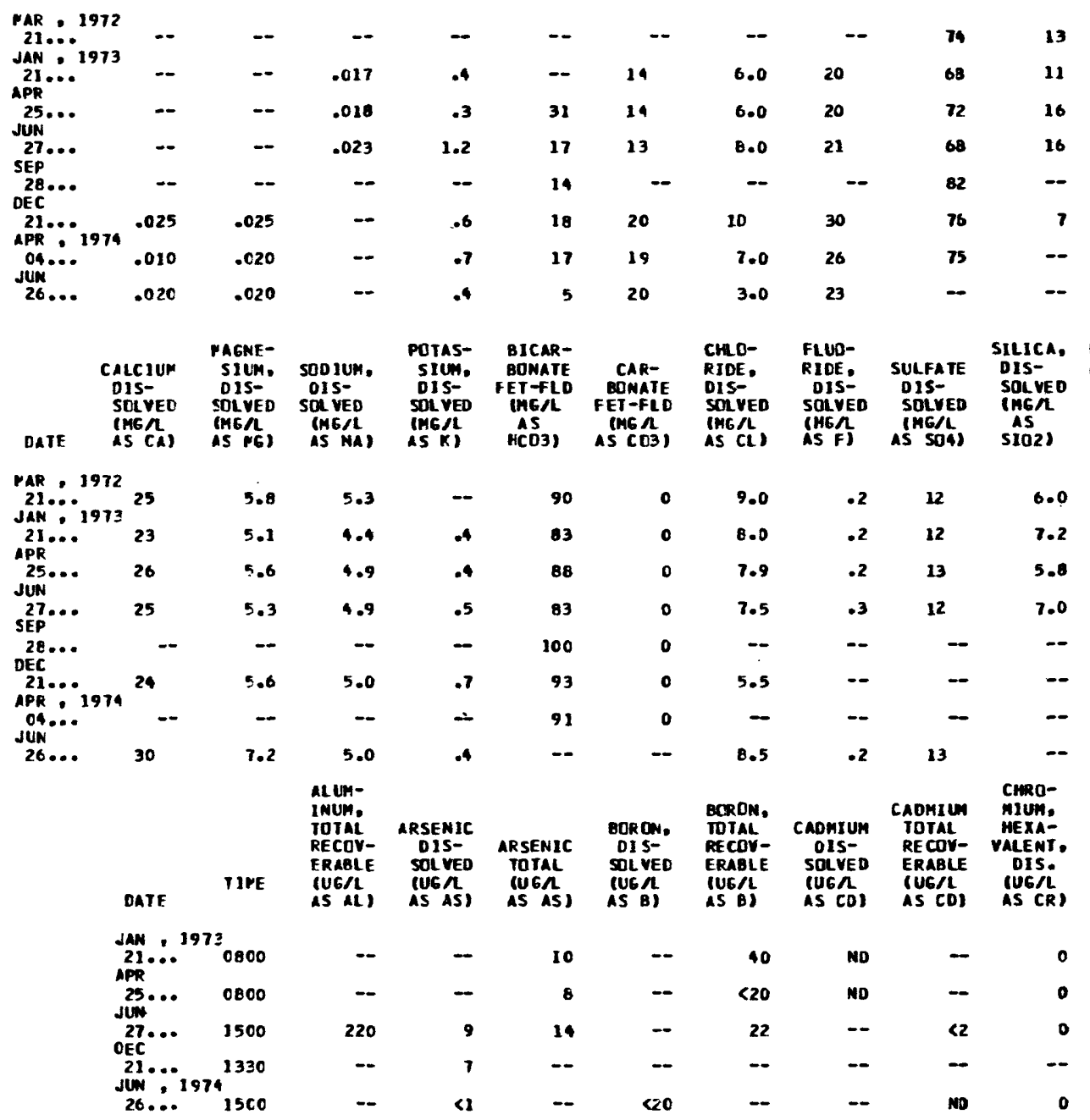


Site 56--Continued

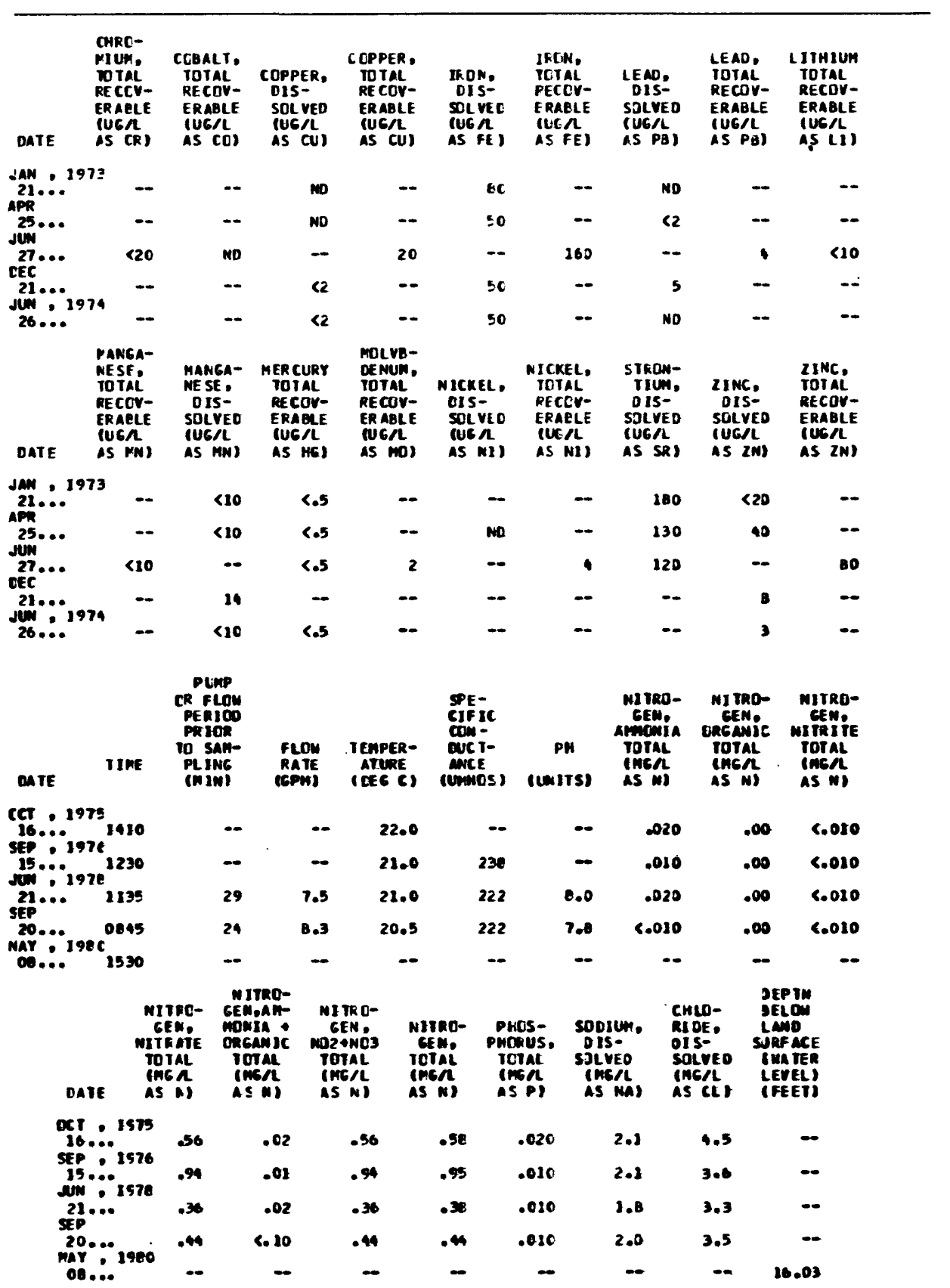


Table 6.-- Physical and chemical characteristics of well and water at sites 1 through 70--Continued

Site 57

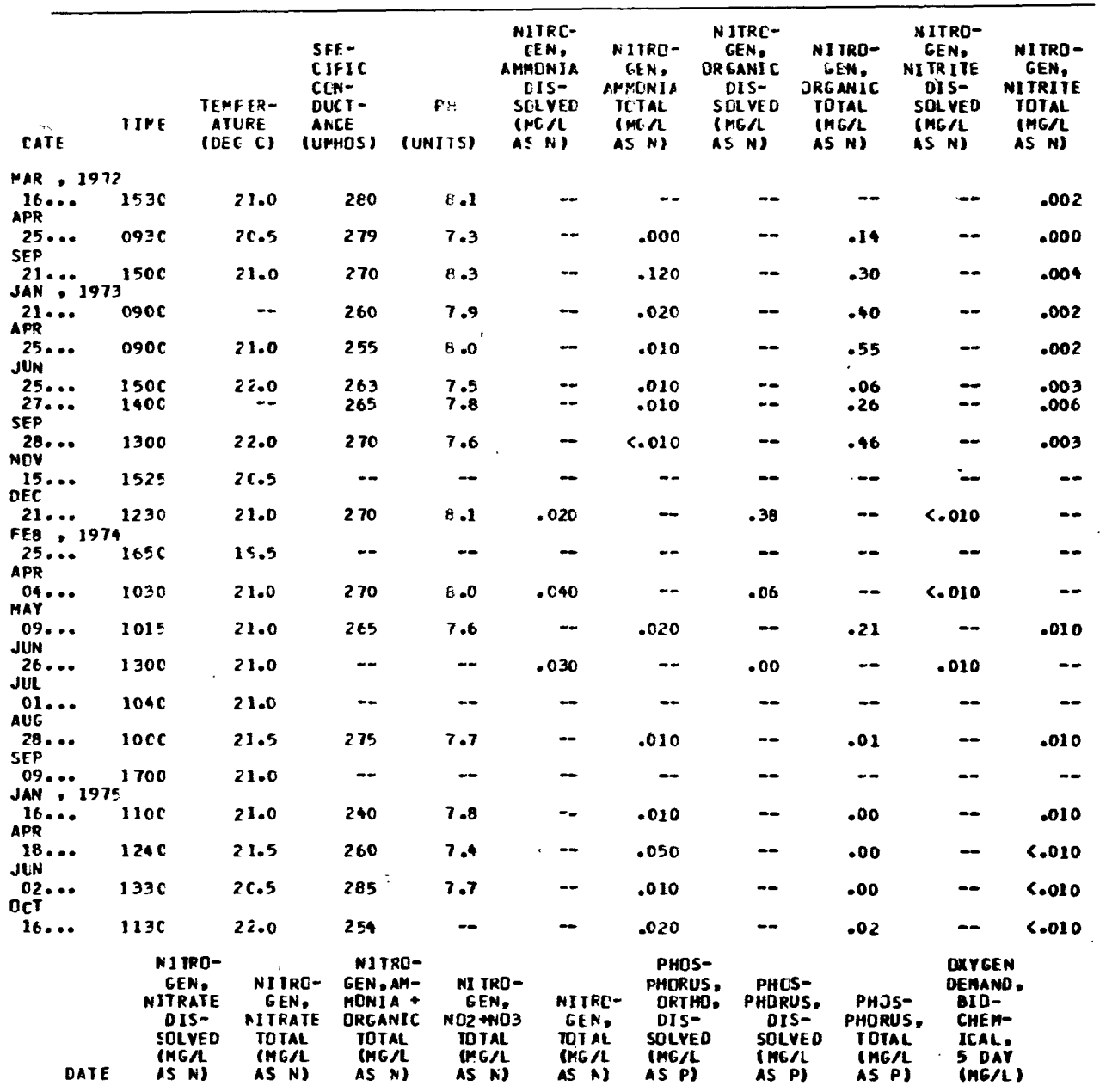

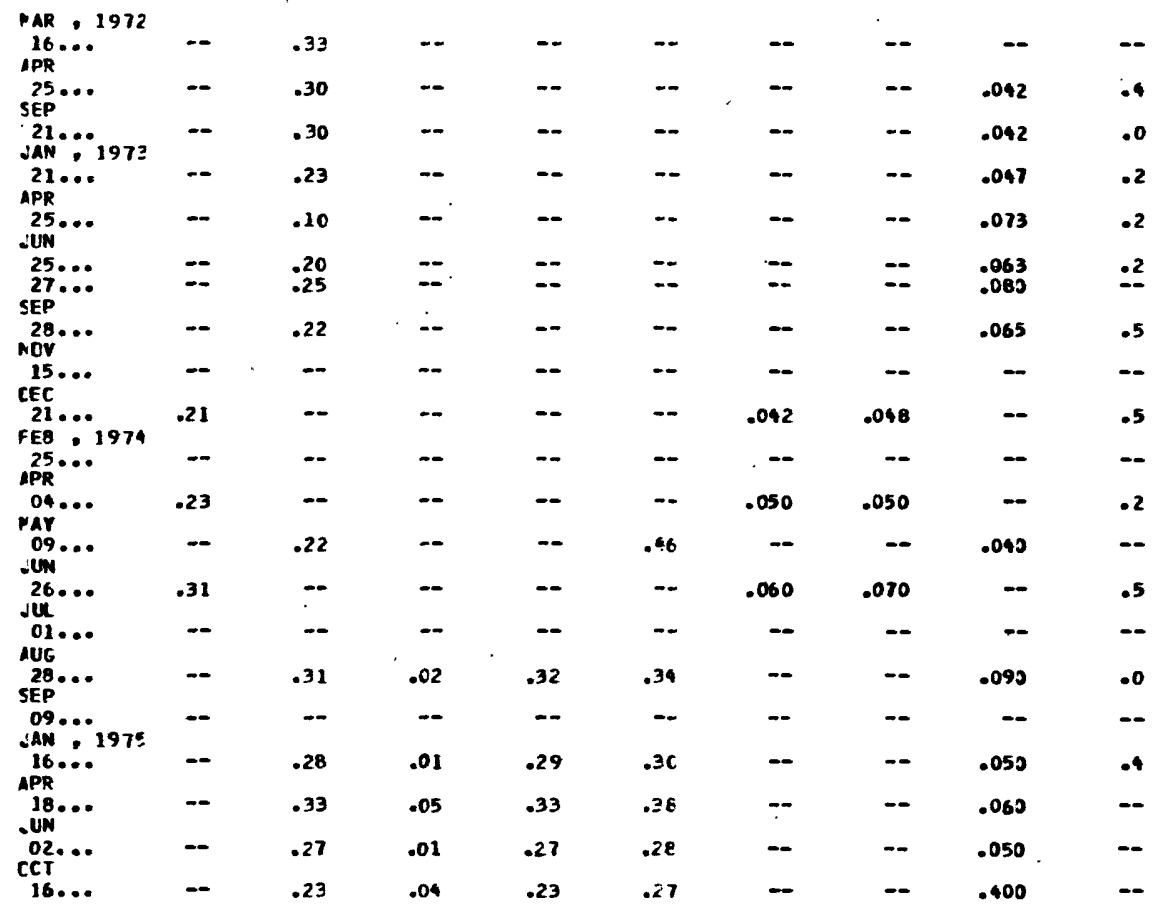


Site 57--Continued

\begin{tabular}{|c|c|c|c|c|c|c|c|c|c|}
\hline DATE & $\begin{array}{l}\text { CXYCEN } \\
\text { TERAND. } \\
\text { CHEP- } \\
\text { ICAL } \\
\text { (HICH } \\
\text { IEVEL) } \\
\text { (PGIL) }\end{array}$ & $\begin{array}{l}\text { CARFON, } \\
\text { INCR - } \\
\text { GAAIC, } \\
\text { IOTAL } \\
\text { IMGA } \\
\text { AS C) }\end{array}$ & $\begin{array}{l}\text { CARBON, } \\
\text { CRGANIC } \\
\text { TOTAL } \\
\text { (MGA } \\
\text { AS CI }\end{array}$ & $\begin{array}{l}\text { CAPFCN, } \\
\text { TOTAL } \\
\text { (MAGA } \\
\text { AS C) }\end{array}$ & $\begin{array}{l}\text { ALXA- } \\
\text { LAITY } \\
\text { FIELL } \\
\text { TEAL } \\
\text { AS } \\
C A C(3)\end{array}$ & $\begin{array}{l}\text { HAF D- } \\
\text { NFSS, } \\
\text { NURCAR- } \\
\text { BOAATE } \\
\text { (HG/L } \\
\text { CACD3) }\end{array}$ & $\begin{array}{l}\text { HARD- } \\
\text { NESS } \\
\text { (MG/L } \\
\text { AS } \\
\text { CACOB) }\end{array}$ & $\begin{array}{l}\text { CALCIUA } \\
\text { OIS- } \\
\text { SDLVED } \\
\text { (MGAL } \\
\text { AS CA) }\end{array}$ & $\begin{array}{l}\text { MAGME- } \\
\text { SIUM, } \\
\text { DIS- } \\
\text { SOLVED } \\
\text { (NGG } \\
\text { AS MG) }\end{array}$ \\
\hline
\end{tabular}

MAR , 1972

\begin{tabular}{|c|c|c|c|c|c|c|c|c|c|c|}
\hline$\underset{A P R}{16} \cdots$ & & -- & $\cdots$ & $\cdots$ & 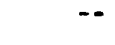 & 121 & 12 & 130 & 38 & 9.2 \\
\hline$\underset{S E P}{25 \ldots}$ & & - & 31 & .0 & 31 & $12 t$ & 8 & 130 & 39 & 8. 7 \\
\hline $\begin{array}{l}21 \cdots \\
\text { JAN } \because 1\end{array}$ & 1973 & -- & 30 & 1.0 & 31 & 116 & 9 & 130 & 36 & 8.9 \\
\hline APR $\cdots$ & & $\cdots$ & 22 & 5.0 & 27 & 121 & 9 & 130 & 38 & 8.6 \\
\hline JUW & & 15 & 14 & 6.0 & 20 & 115 & 11 & 130 & 38 & 7.6 \\
\hline $\begin{array}{l}25 \cdots \cdots \\
27 \cdots \cdots \\
\text { SEP }\end{array}$ & & 9 & $\begin{array}{l}20 \\
19\end{array}$ & $\begin{array}{l}7.0 \\
9.0\end{array}$ & $\begin{array}{l}27 \\
28\end{array}$ & $\begin{array}{l}11 E \\
121\end{array}$ & 11 & $\begin{array}{l}130 \\
130\end{array}$ & $\begin{array}{l}38 \\
37\end{array}$ & $\begin{array}{l}8.3 \\
8.4\end{array}$ \\
\hline $\begin{array}{r}28 \\
\text { NOV }\end{array}$ & & 14 & 31 & 1.0 & 32 & 120 & - & 130 & 38 & \\
\hline $\begin{array}{l}15 . \\
\text { CEC }\end{array}$ & & $\cdots$ & $\cdots$ & - & - & $\cdots$ & $-\infty$ & -- & - & \\
\hline${ }_{\text {FEB }}^{21} \cdots 1$ & 974 & 0 & 27 & 10 & 37 & 117 & 13 & 130 & 32 & 12 \\
\hline 25 & & - & - & - & - & $\cdots$ & $\cdots$ & - & - & \\
\hline MAY & & 4 & 30 & .0 & 30 & 117 & 13 & 130 & 37 & 8.9 \\
\hline 09 & & -- & 31 & 1.0 & 32 & 125 & 24 & 150 & 44 & 9.3 \\
\hline 26. & & 2 & 29 & 7.0 & 32 & $\cdots$ & $\cdots$ & 140 & 39 & 9.6 \\
\hline $01 . \cdots$ & & - & $\cdots$ & $\cdots$ & $\cdots$ & - & - & - & - & \\
\hline SEP & & 6 & 30 & 1.0 & 31 & 125 & 2 & 130 & 36 & 8.9 \\
\hline iAn $\because 1$ & 975 & $\cdots$ & - & - & $\cdots$ & $\cdots$ & - & -- & -- & \\
\hline apk & & 2 & 29 & .0 & 29 & I1e & 21 & 140 & 41 & 8.9 \\
\hline 18. & & $\cdots$ & - & 1.0 & - & 115 & - & - & - & \\
\hline ס2 & & -- & 30 & -0 & 30 & 127 & 5 & 130 & 33 & I \\
\hline $16 \ldots$ & & - & - & - & $\cdots$ & 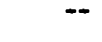 & -- & $\cdots$ & - & \\
\hline ATE & $\begin{array}{c}\text { sca } \\
\text { cI } \\
\text { sct } \\
\text { in } \\
\text { is }\end{array}$ & $\begin{array}{l}\text { W. } \\
\text { Eo } \\
\text { Nal }\end{array}$ & $\begin{array}{l}\text { Pojas- } \\
\text { siun, } \\
\text { ols- } \\
\text { sotveo } \\
\text { (HG/t } \\
\text { as KS }\end{array}$ & $\begin{array}{l}\text { EICAR- } \\
\text { BONATE } \\
\text { FET-FLD } \\
\text { AnGA } \\
\text { AS } \\
\text { nCo3s }\end{array}$ & $\begin{array}{l}\text { CAR- } \\
\text { Bonute } \\
\text { fET-FLo } \\
\text { men } \\
\text { AS co3s }\end{array}$ & 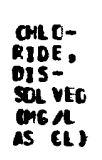 & $\begin{array}{l}\text { FLUD- } \\
\text { RIOE: } \\
\text { DIS- } \\
\text { SOVEO } \\
\text { ImbA } \\
\text { AS Fo }\end{array}$ & 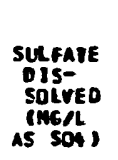 & $\begin{array}{l}\text { SILICA. } \\
\text { oIS- } \\
\text { SOLVE } \\
\text { Ins/L } \\
\text { AS } \\
\text { S1021 }\end{array}$ & 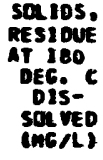 \\
\hline
\end{tabular}

\begin{tabular}{|c|c|c|c|c|c|c|c|c|c|}
\hline $16 \ldots$ & 4.7 & .6 & 198 & 0 & 7.6 & $\cdot 2$ & 10 & 11 & 155 \\
\hline & 3.7 & .3 & 154 & 0 & 3.4 & $\cdot 3$ & $17^{\circ}$ & 10 & 162 \\
\hline$\because 1972$ & $\bullet .0$ & .5 & 144 & 0 & $6 . c$ & .2 & 10 & 11 & 156 \\
\hline$\cdots$ & 1.1 & .6 & 248 & 0 & e.c & $\bullet 3$ & 11 & $\mathbf{H}$ & 154 \\
\hline $25 \ldots$ & $\bullet .3$ & .6 & 140 & 0 & 7.0 & $\bullet 3$ & 10 & $8 \cdot 2$ & 161 \\
\hline $\begin{array}{r}25 \\
27 \\
\text { SEP }\end{array}$ & $\begin{array}{l}4.0 \\
4.2\end{array}$ & .5 & $\begin{array}{l}104 \\
106\end{array}$ & 0 & $\begin{array}{l}8.0 \\
6.0\end{array}$ & .4 & $\begin{array}{l}10.2 \\
9.2\end{array}$ & $\begin{array}{l}12 \\
10\end{array}$ & $\begin{array}{l}150 \\
159\end{array}$ \\
\hline $\begin{array}{r}20 \\
100\end{array}$ & 3.9 & .5 & 146 & 0 & 5.0 & $-\infty$ & $\cdots$ & 10 & -- \\
\hline $15 \ldots$ & - & -- & $-\infty$ & -- & - & $-\infty$ & $-\infty$ & - & - \\
\hline 21:0. & 4.0 & .6 & 143 & 0 & 9.6 & - & -- & $\infty$ & - \\
\hline$\bullet$ & $\cdots$ & $\cdots$ & - & $-\infty$ & $\cdots$ & $\infty$ & -- & - & - \\
\hline$\bullet$ & 9.5 & $\bullet$ & 143 & 0 & 6.5 & $-\infty$ & - & $\cdots$ & - \\
\hline$\bullet$ & 3.9 & .5 & 152 & -- & 5.6 & .2 & 9.8 & 12 & 160 \\
\hline$\bullet$ & 3.7 & .5 & -- & -- & 5.9 & $\bullet 3$ & 9.9 & $-\infty$ & 178 \\
\hline & - & - & -- & -- & $-\infty$ & $\infty$ & $-\infty$ & $-\infty$ & - \\
\hline SE & 9.0 & $\bullet$ & 192 & -- & 4.0 & $\cdots$ & $-\infty$ & $-\infty$ & - \\
\hline JAN?:1975 & $-\infty$ & $\cdots$ & $\cdots$ & $\infty$ & - & $-\infty$ & $\cdots$ & $-\infty$ & -- \\
\hline 10 & 4.1 & .5 & 144 & 0 & 5.6 & .2 & 9.4 & 11 & 107 \\
\hline 1 & - & - & 140 & 0 & 5.6 & $-\infty$ & - & - & - \\
\hline cet & 4.0 & .5 & 155 & 0 & 5.7 & .1 & 8.3 & 11 & 148 \\
\hline & 3.6 & $\cdots$ & -- & $-\infty$ & 6.0 & $\cdots$ & $\cdots$ & - & - \\
\hline
\end{tabular}


Site 57--Continued

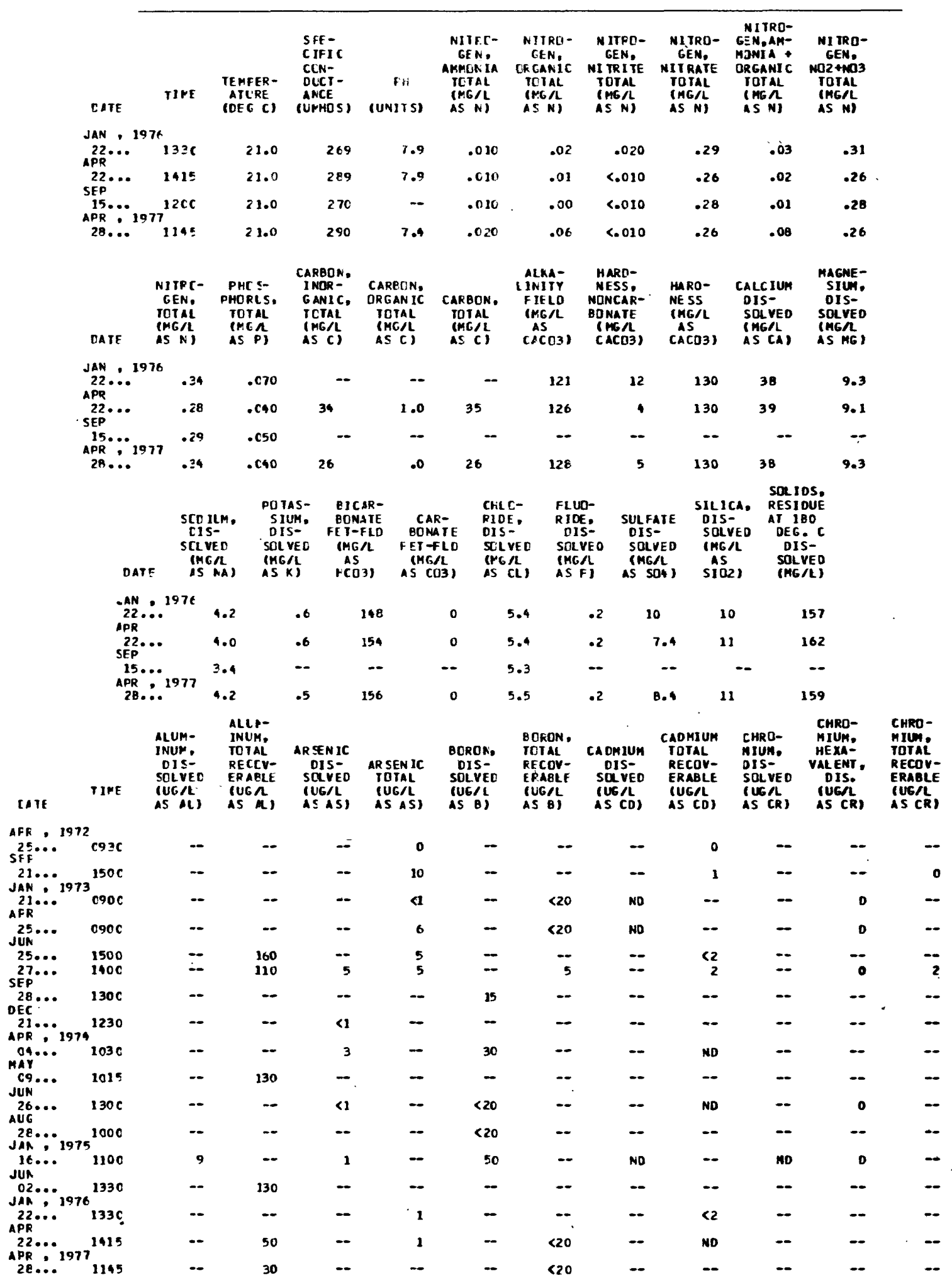


Site 57--Continued

\begin{tabular}{|c|c|c|c|c|c|c|c|c|c|c|c|c|}
\hline DATE & $\begin{array}{l}\text { CCEAL } \\
\text { OIS } \\
\text { SCLVE } \\
\text { IUG } \\
\text { IS }\end{array}$ & & $\begin{array}{l}\text { CCBALT, } \\
\text { TOTAL } \\
\text { RECCY- } \\
\text { ERAELE } \\
\text { IUGA } \\
\text { IS CE) }\end{array}$ & $\begin{array}{l}\text { COPFER, } \\
\text { OIS- } \\
\text { SOLVEO } \\
\text { (UER } \\
\text { AS CU) }\end{array}$ & $\begin{array}{l}\text { CRPPER, } \\
\text { TOIAL } \\
\text { RECTV- } \\
\text { EKA BLE } \\
\text { (UGA } \\
\text { AS CU) }\end{array}$ & $\begin{array}{l}\text { IPON, } \\
\text { DIS- } \\
\text { SOLVED } \\
\text { IUG/L } \\
\text { AS FEI }\end{array}$ & $\begin{array}{l}\text { IF.CA, } \\
\text { TCIAL } \\
\text { RE CCV- } \\
\text { EKAELE } \\
\text { (UG } \\
\text { AS FE) }\end{array}$ & $\begin{array}{l}\text { LEAD, } \\
\text { DIS- } \\
\text { SQL VEC } \\
\text { (UCA } \\
\text { AS PB) }\end{array}$ & $\begin{array}{l}\text { LEAD, } \\
\text { TOTAL } \\
\text { PECDV- } \\
\text { ERABLE } \\
\text { (UG/L } \\
\text { AS PB) }\end{array}$ & $\begin{array}{l}\text { LITHIUM } \\
\text { DIS- } \\
\text { SOLVED } \\
\text { IUG } \\
\text { AS LII }\end{array}$ & $\begin{array}{l}\text { LITHIUM } \\
\text { TOTAL } \\
\text { RECOY- } \\
\text { ERABLE } \\
\text { IUG } \\
\text { AS LIS }\end{array}$ & $\begin{array}{l}\text { MANGA- } \\
\text { NESE, } \\
\text { TOTAL } \\
\text { RECOY- } \\
\text { ERABLE } \\
\text { (UGR } \\
\text { AS MW I }\end{array}$ \\
\hline $\begin{array}{ll}A P R & 1 \\
25 . . . & \end{array}$ & 972 & -- & 0 & -- & 0 & 10 & 30 & 2 & 3 & -- & 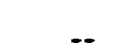 & 10 \\
\hline SEP & & - & 0 & $\cdots$ & 0 & 10 & 10 & 2 & $\bullet$ & - & $\cdots$ & 10 \\
\hline $\begin{array}{l}\text { JAN } 1 \\
21 \ldots . . \\
A P R\end{array}$ & 973 & - & $\cdots$ & ND & -- & $<10$ & - & $<2$ & -- & -- & $\cdots$ & $\cdots$ \\
\hline $25 \ldots$ & & $\cdots$ & - & ND & -- & 30 & - & $<2$ & - & -- & -- & -- \\
\hline $\begin{array}{l}25 \ldots \\
27 \ldots\end{array}$ & & $=$ & -- & ND & $-\bar{x}$ & $<10$ & $5 C$ & ND & -- & $\cdots$ & - & $\cdots$ \\
\hline $\begin{array}{l}27 \ldots \ldots \\
\text { SEP } \\
28 \ldots \ldots\end{array}$ & & $\cdots$ & NO & $\cdots$ & 20 & -- & 50 & $\cdots$ & 3 & -- & $<10$ & 3 \\
\hline $\begin{array}{l}28 \ldots \\
\text { DEC } \\
21 \ldots\end{array}$ & & $\cdots$ & -- & -- & - & -- & -- & -- & - & -- & -- & -- \\
\hline APR $21 \ldots$ & 974 & - & - & $<2$ & -- & $<10$ & $\cdots$ & - & $\cdots$ & -- & $\cdots$ & $\cdots$ \\
\hline C4... & & -- & $\cdots$ & $<2$ & - & 60 & -- & 11 & 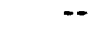 & $\cdots$ & -- & -- \\
\hline din & & - & - & 6 & - & 210 & 220 & ND & .3 & -- & -- & 40 \\
\hline AUG & & - & $\cdots$ & No & - & C10 & -- & NO & -- & $\cdots$ & -- & -- \\
\hline JAN $28 \cdots 1$ & 975 & - & - & $\cdots$ & -- & $\cdots$ & -- & -- & - & - & - & -- \\
\hline JUM & & ND & - & ND & -- & $<10$ & -- & $<2$ & -- & $<10$ & - & $\cdots$ \\
\hline JAN $\because 1$ & 976 & -- & $=$ & ND & -- & $<10$ & 20 & 6 & 6 & $\cdots$ & $\cdots$ & (10 \\
\hline APR & & - & - & -- & $<2$ & 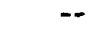 & \&IC & - & 14 & - & - & $<10$ \\
\hline $\begin{array}{r}22 \ldots \\
A P R\end{array}$ & & -- & $\cdots$ & ND & -- & $<10$ & 70 & NE & 3 & - & -- & $<10$ \\
\hline $2 E \ldots$ & & -- & -- & ND & $=$ & $<10$ & 26 & 7 & 10 & -- & -- & C10 \\
\hline DATE & $\begin{array}{l}\text { MAN } \\
\text { NES } \\
\text { DI } \\
\text { SOL. } \\
\text { fUG } \\
\text { IS }\end{array}$ & $\begin{array}{l}\text { GA- } \\
\text { ES } \\
\text { IS- } \\
\text { VED } \\
\text { MLI } \\
\text { MNI }\end{array}$ & $\begin{array}{c}\text { PERCLRY } \\
\text { DIS- } \\
\text { SOLVED } \\
\text { IUC/L } \\
\text { IS HG) }\end{array}$ & $\begin{array}{l}\text { MERCURY } \\
\text { TOTAL } \\
\text { RFCOV- } \\
\text { ERAELE } \\
\text { (UG } \\
\text { AS HG) }\end{array}$ & $\begin{array}{l}\text { MOLYE- } \\
\text { DENIH, } \\
\text { DIS- } \\
\text { SOLYED } \\
\text { (UG A } \\
\text { AS MD) }\end{array}$ & $\begin{array}{l}\text { HOLYE- } \\
\text { DENUM, } \\
\text { TO TAL } \\
\text { RECEV- } \\
\text { ERAELE } \\
\text { (UGAL } \\
\text { AS MO) }\end{array}$ & $\begin{array}{l}\text { NICKEL, } \\
\text { OIS- } \\
\text { STLVEC } \\
\text { (UGA } \\
\text { AS MI) }\end{array}$ & $\begin{array}{l}\text { NI CYEL. } \\
\text { TOTAL } \\
\text { RF COY- } \\
\text { ERABLE } \\
\text { (UG/L } \\
\text { AS NI) }\end{array}$ & $\begin{array}{l}\text { STKON- } \\
\text { TIUH. } \\
\text { DIS- } \\
\text { SOLVED } \\
\text { (UGN } \\
\text { AS SRI }\end{array}$ & $\begin{array}{l}\text { YANA- } \\
\text { DIUH, } \\
\text { DIS- } \\
\text { SOLVED } \\
\text { (UG/L } \\
\text { AS VI) }\end{array}$ & $\begin{array}{l}\text { ZINC, } \\
\text { DIS- } \\
\text { SOL YED } \\
\text { (UG L L } \\
\text { AS ZN) }\end{array}$ & $\begin{array}{l}\text { ZINC, } \\
\text { IOIAL } \\
\text { RECOV- } \\
\text { ERABLE } \\
\text { (UG } \Omega \\
\text { AS ZN) }\end{array}$ \\
\hline $\begin{array}{l}A P R, \\
25 \ldots \\
\text { SEP }\end{array}$ & & -- & $\cdots$ & .0 & -- & -- & -- & $\cdots$ & 110 & -- & - & 20 \\
\hline JAN $21 .$. & $97 ?$ & - & - & C.5 & $\cdots$ & $\cdots$ & $\cdots$ & - & 160 & $\cdots$ & -- & 0 \\
\hline${ }_{A P R}^{21} \cdots$ & & $<10$ & -- & $<.5$ & $\cdots$ & - & - & $\cdots$ & 150 & -- & $<20$ & -- \\
\hline $25 \ldots$ & & $<10$ & -- & C. 5 & -- & - & No & - & 120 & - & 20 & 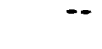 \\
\hline $\begin{array}{l}25 \ldots \\
27 \ldots\end{array}$ & & $<10$ & $\because$ & 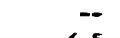 & -- & $=$ & $\because$ & - & 60 & $\cdots$ & $<20$ & $=$ \\
\hline $\begin{array}{c}27 \ldots \\
\text { SEF } \\
28 . . .\end{array}$ & & - & $\cdots$ & $<.5$ & - & 1 & $\cdots$ & 5 & 100 & $=$ & -- & $<20$ \\
\hline DEC & & $\cdots$ & - & -- & $\cdots$ & $\cdots$ & - & - & -- & - & -- & -- \\
\hline APR & 1974 & 14 & $\cdots$ & $\cdots$ & 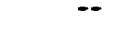 & -- & - & - & -- & $\cdots$ & 20 & -- \\
\hline $\operatorname{mar}^{04}$ & & (10 & $=$ & $<.5$ & -- & -- & $\cdots$ & - & -- & $\cdots$ & 5 & - \\
\hline Jum.... & & 20 & $\cdots$ & $\cdots$ & -- & $\cdots$ & -- & - & 140 & -- & 50 & - \\
\hline$\underset{A \cup G}{26} \ldots$ & & $<10$ & $\cdots$ & $<.5$ & -- & - & $\cdots$ & - & $\cdots$ & -- & ND & - \\
\hline JAK... & 1975 & -- & - & -- & - & - & -- & - & - & -- & -- & -- \\
\hline JUN & & (10 & $<.5$ & $\cdots$ & (1) & -- & ND & - & $\cdots$ & .3 .0 & 30 & -- \\
\hline JAN ? & 1976 & (10 & $\cdots$ & -- & - & $\cdots$ & - & - & 140 & -- & 30 & - \\
\hline APR. & & -- & -- & $<.5$ & -- & -- & -- & - & 140 & -- & -- & 20 \\
\hline 22... & 1977 & $<10$ & - & - & - & $\cdots$ & $=$ & NO & 100 & -- & ND & $=$ \\
\hline & & $<10$ & $=-$ & $\cdots$ & -- & - & -- & $\cdots$ & 100 & $\cdots$ & ND & - \\
\hline
\end{tabular}

\begin{tabular}{|c|c|c|c|c|c|c|c|c|}
\hline CATE & TIVE & $\begin{array}{l}\text { AL CRIN, } \\
\text { TCTAL } \\
\text { (UG/L) }\end{array}$ & $\begin{array}{l}\text { CHLCR- } \\
\text { DANE, } \\
\text { TOTAL } \\
\text { IUEAL }\end{array}$ & $\begin{array}{l}\text { COD, } \\
\text { TOTAL } \\
\text { (UG/L) }\end{array}$ & $\begin{array}{l}\text { CEE. } \\
\text { TDIAL } \\
\text { (UC/L) }\end{array}$ & $\begin{array}{l}\text { Dot, } \\
\text { TotaL } \\
\text { (UGri) }\end{array}$ & $\begin{array}{l}\text { DI- } \\
\text { ELORIN } \\
\text { IOTAL } \\
\text { (UG/L) }\end{array}$ & $\begin{array}{l}\text { ENDR IN, } \\
\text { IDTAL } \\
\text { (UG/L) }\end{array}$ \\
\hline $25 \ldots$ & 0920 & .00 & .00 &.$c 0$ & .00 & .00 & .00 & .00 \\
\hline
\end{tabular}


Site 57--Continued

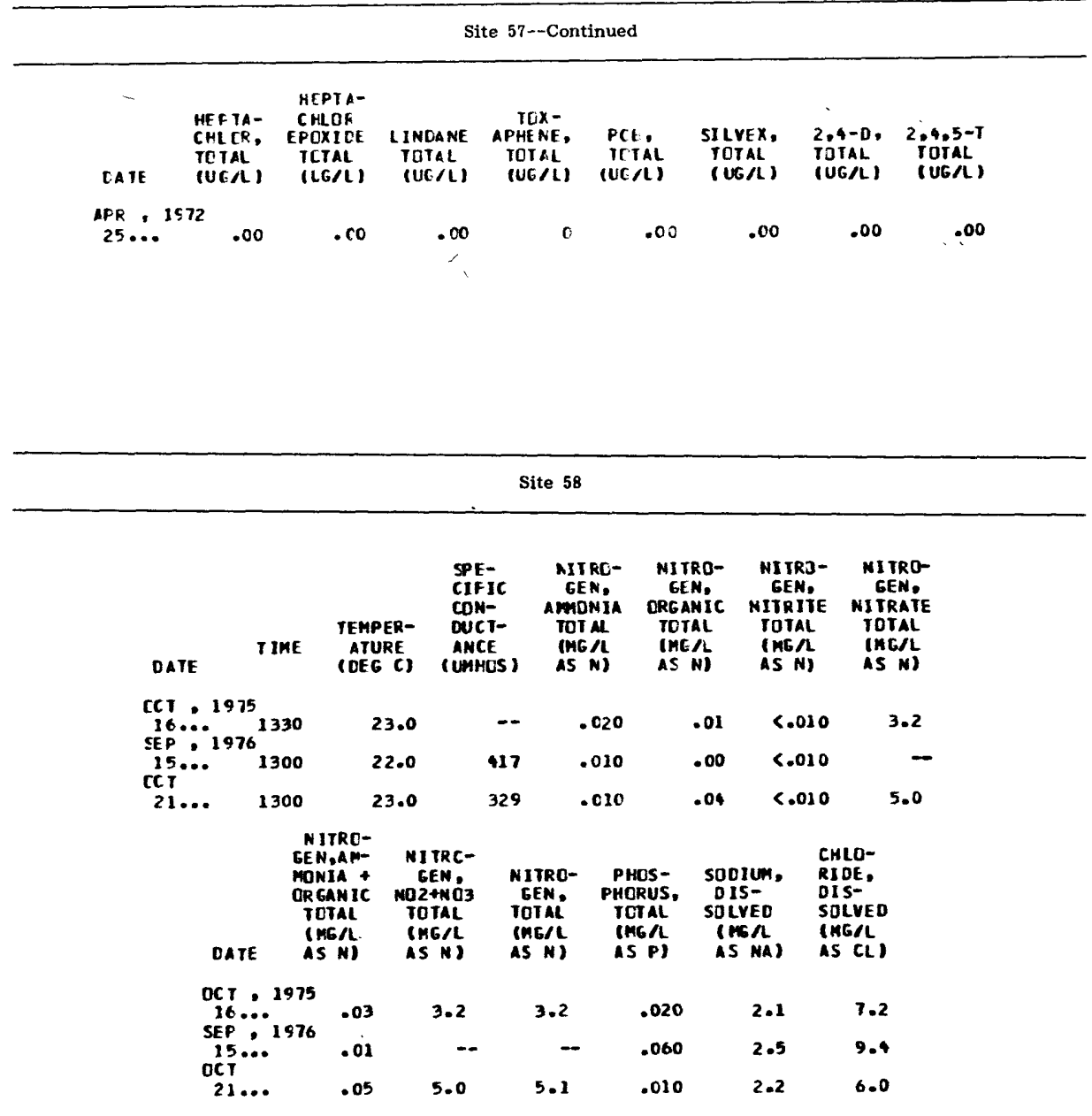

Site 59

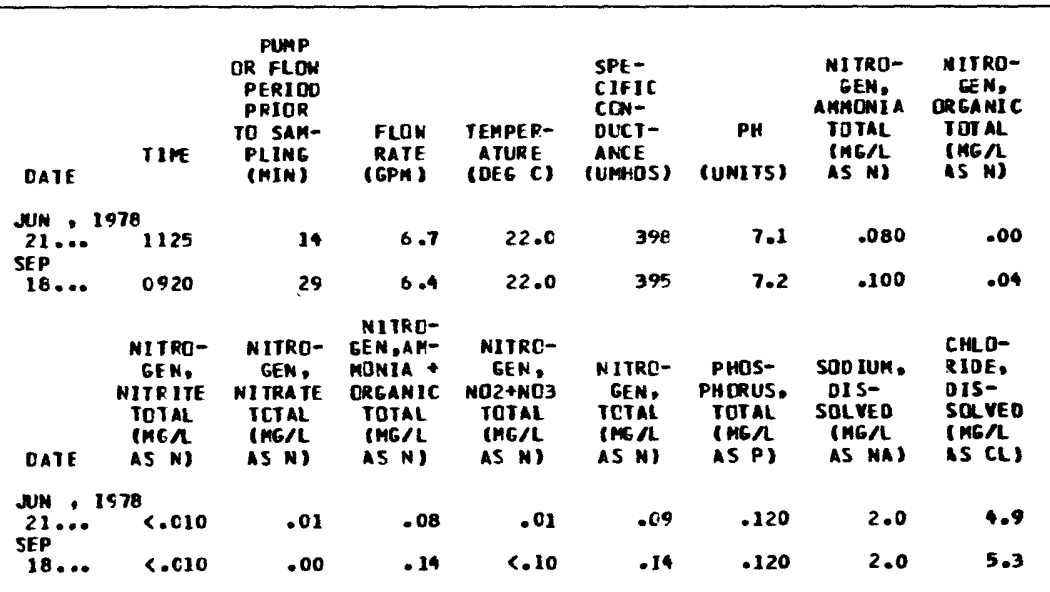


Site 61

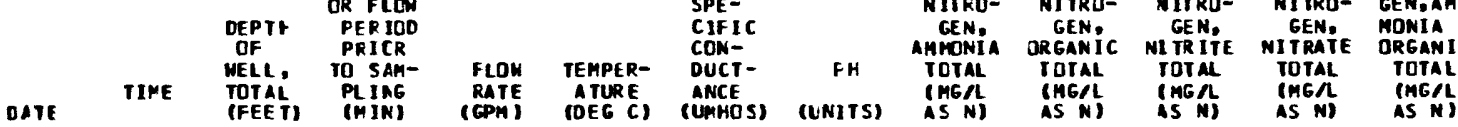

\begin{tabular}{|c|c|c|c|c|c|c|c|c|c|c|c|c|}
\hline $\begin{array}{l}\text { SEP } \\
27 . . .1978\end{array}$ & - & 80 & $\sim$ & - & 22.5 & - & -- & - & - & - & -- & -- \\
\hline $15 \ldots$ & - & 80 & - & - & - & 170 & e.c & - & - & -- & -- & - \\
\hline JUN 1974 & 1030 & 80 & $\sim$ & - & 22.0 & 225 & - & $\sim$ & $\cdots$ & - & $\cdots$ & $\sim$ \\
\hline JAN $\because 1975$ & $5^{1100}$ & 80 & -- & - & 22.0 & 200 & 8.1 & .010 & .02 & C.01D & 2.3 & .03 \\
\hline $16 \ldots$ & 1300 & 80 & - & - & 22.0 & 180 & 7.8 & .010 &.$D D$ & .010 & 2.0 & .01 \\
\hline JUN & 1230 & 80 & - & - & 22.0 & 220 & 7.5 & .010 & .02 & .010 & 1.2 & .03 \\
\hline${ }_{\text {OCT }}^{27 \cdots}$ & $143 \mathrm{C}$ & 80 & -- & - & 22.0 & 205 & - & .010 & .06 & $<.010$ & 1.5 & .07 \\
\hline JAN 1576 & 1430 & 80 & - & - & 22.0 & 212 & - & .020 & .00 & $<.010$ & 1.7 & .02 \\
\hline JUN & 1000 & 80 & -- & - & $22 \cdot 0$ & 190 & 7.6 & .010 & .01 & .010 & 2.2 & .02 \\
\hline SEP & 1300 & 80 & - & -- & 22.0 & 190 & - & .040 & .01 & C.D10 & 1.1 & .05 \\
\hline $16 \ldots$ & 1200 & 80 & -- & - & 22.5 & 189 & - & .010 & .00 & <. 010 & 2.0 & .01 \\
\hline $\operatorname{SEP}_{\mathrm{S}} 1 \ldots$ & 1415 & 80 & 15 & 6.7 & - & 197 & 8.0 & .170 & .00 & <.010 & 1.9 & .17 \\
\hline 18. & 1110 & 80 & 16 & 7.0 & - & 195 & 8.0 & $<.010$ & .02 & .010 & 2.0 & .02 \\
\hline TE & $\begin{array}{l}\text { NITRO- } \\
\text { GEN, } \\
\text { NO2+NO3 } \\
\text { TOTAL } \\
\text { (MGR } \\
\text { AS N) }\end{array}$ & $\begin{array}{l}\text { MI TRO - } \\
\text { GEN, } \\
\text { TOTAL } \\
\text { (MGA } \\
\text { AS N) }\end{array}$ & $\begin{array}{l}\text { PHOS- } \\
\text { FHORUS, } \\
\text { TOTAL } \\
\text { (HG/L } \\
\text { AS P) }\end{array}$ & $\begin{array}{l}\text { DXYGEN } \\
\text { DEMAND, } \\
\text { BID- } \\
\text { CHEK- } \\
\text { ICAL, } \\
5 \text { DAY } \\
\text { (MGAL) }\end{array}$ & $\begin{array}{l}\text { CXYGEN } \\
\text { OEHAND. } \\
\text { CHEM- } \\
\text { ICAL } \\
\text { (HIGH } \\
\text { LEVEL) } \\
\text { (HG/L) }\end{array}$ & $\begin{array}{l}\text { CARBCN, } \\
\text { INOR- } \\
\text { GANIC, } \\
\text { TDIAL } \\
\text { (NG AL } \\
\text { AS C) }\end{array}$ & $\begin{array}{l}\text { CAREON, } \\
\text { ORGANIC } \\
\text { TOTAL } \\
\text { (HC } \\
\text { AS C) }\end{array}$ & $\begin{array}{l}\text { CARBON, } \\
\text { TDTAL } \\
\text { (MGA } \\
\text { AS C) }\end{array}$ & $\begin{array}{l}\text { ALKA- } \\
\text { LINITY } \\
\text { FIELD } \\
\text { IAGA } \\
\text { AS } \\
\text { CACOBI }\end{array}$ & $\begin{array}{l}\text { HARO- } \\
\text { MESS, } \\
\text { MONCAR - } \\
\text { BONATE } \\
\text { (HG/L } \\
\text { CACO3) }\end{array}$ & $\begin{array}{l}\text { HARD- } \\
\text { MESS } \\
\text { (MGA } \\
\text { AS } \\
\text { CACD3) }\end{array}$ & \\
\hline
\end{tabular}

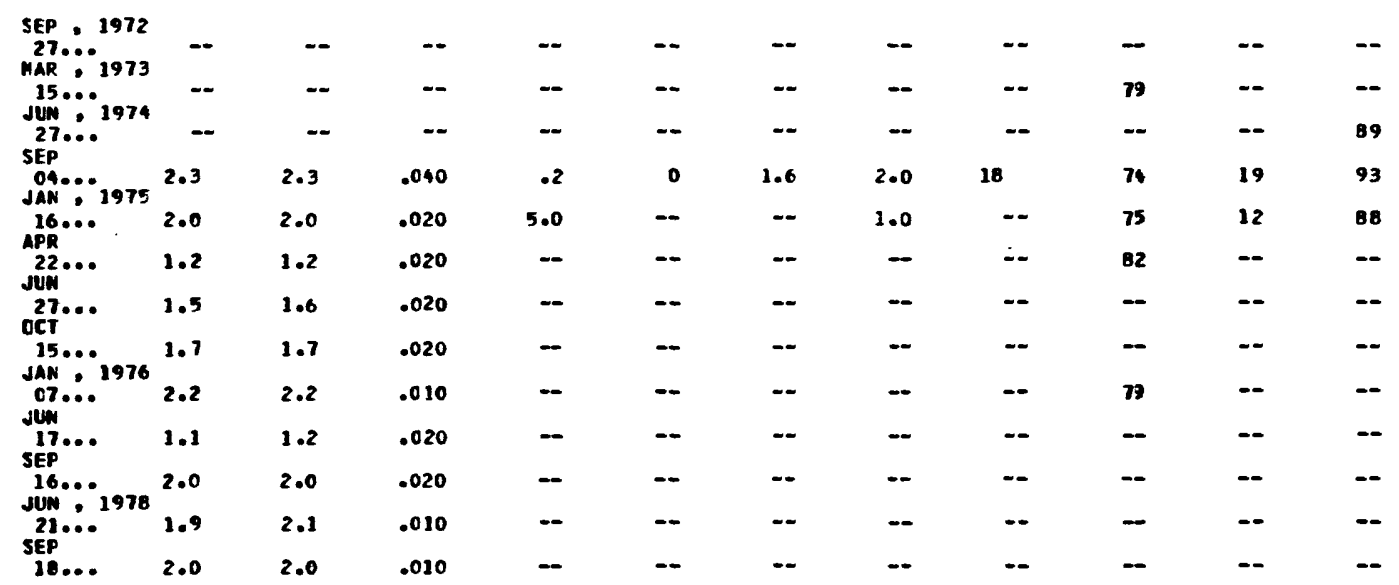




\begin{tabular}{|c|c|c|c|c|c|c|c|c|c|c|c|}
\hline DATE & $\begin{array}{l}\text { CALCIUM } \\
\text { OIS- } \\
\text { SOLVED } \\
\text { (HG/L } \\
\text { AS CAS }\end{array}$ & $\begin{array}{l}\text { VAGAE- } \\
\text { SIUM, } \\
\text { DIS- } \\
\text { SOLVED } \\
\text { (MGA } \\
\text { AS YG) }\end{array}$ & $\begin{array}{l}\text { SODIUM, } \\
\text { DIS- } \\
\text { SOLVED } \\
\text { (HGA } \\
\text { AS NA) }\end{array}$ & $\begin{array}{l}\text { POIAS- } \\
\text { SIUM, } \\
\text { DIS- } \\
\text { SOLVED } \\
\text { (MG/L } \\
\text { AS K) }\end{array}$ & $\begin{array}{l}\text { BICAR- } \\
\text { BONAIE } \\
\text { FET -FLO } \\
\text { TMEAL } \\
\text { AS } \\
\text { HCO3) }\end{array}$ & $\begin{array}{l}\text { CAR- } \\
\text { BONAIE } \\
\text { FET-FLD } \\
\text { (MG } R \\
\text { AS CCS) }\end{array}$ & $\begin{array}{l}\text { CHLO- } \\
\text { RIDE, } \\
\text { OIS- } \\
\text { SOLVED } \\
\text { (MGEL } \\
\text { AS (L) }\end{array}$ & $\begin{array}{l}\text { FLUO- } \\
\text { RIDE: } \\
\text { DIS: } \\
\text { SDLVED } \\
\text { (MG L } \\
\text { AS FI) }\end{array}$ & $\begin{array}{l}\text { SULFA TE } \\
\text { D IS- } \\
\text { SOLYES } \\
\text { (HG/L } \\
\text { AS SOQ) }\end{array}$ & $\begin{array}{l}\text { SILICA, } \\
\text { DIS- } \\
\text { SOLVEO } \\
\text { IHG/ } \\
\text { AS } \\
\text { SIOZ) }\end{array}$ & $\begin{array}{l}\text { SOL IOS, } \\
\text { RESIDUE } \\
\text { AT } 1 B 0 \\
\text { DEG. } \\
\text { DIS- } \\
\text { SOLVEO } \\
\text { (MG }\end{array}$ \\
\hline
\end{tabular}

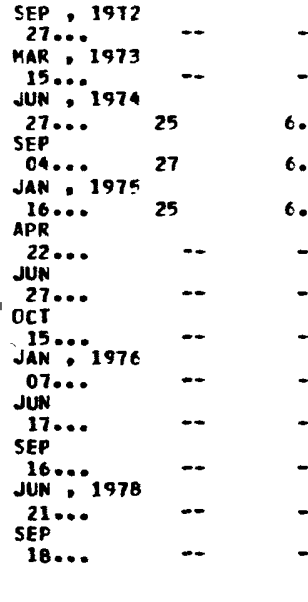

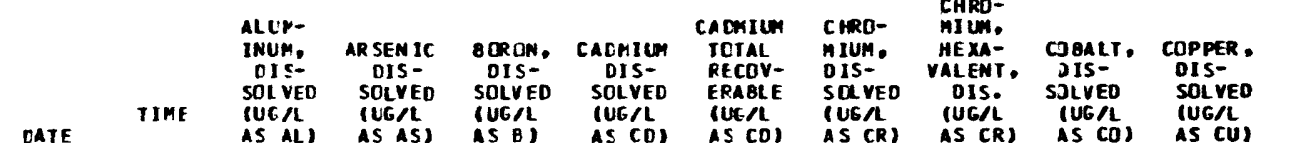

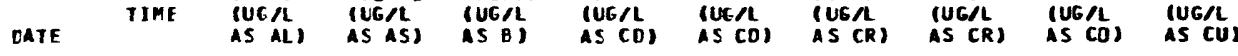

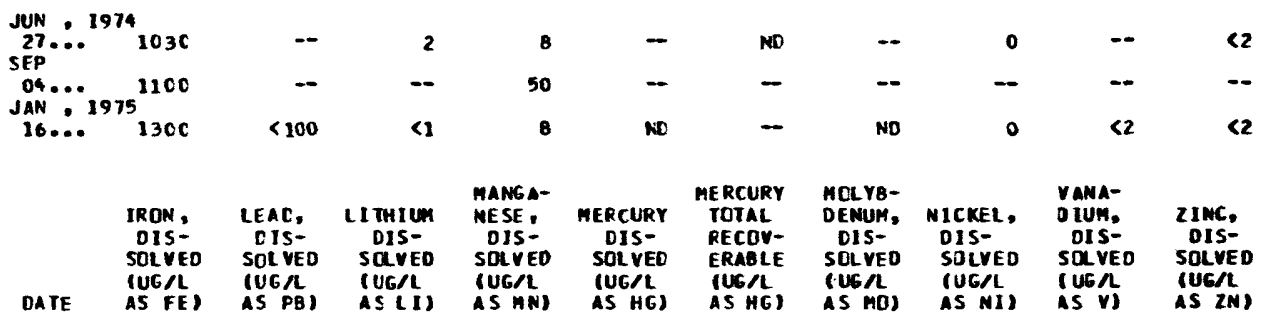

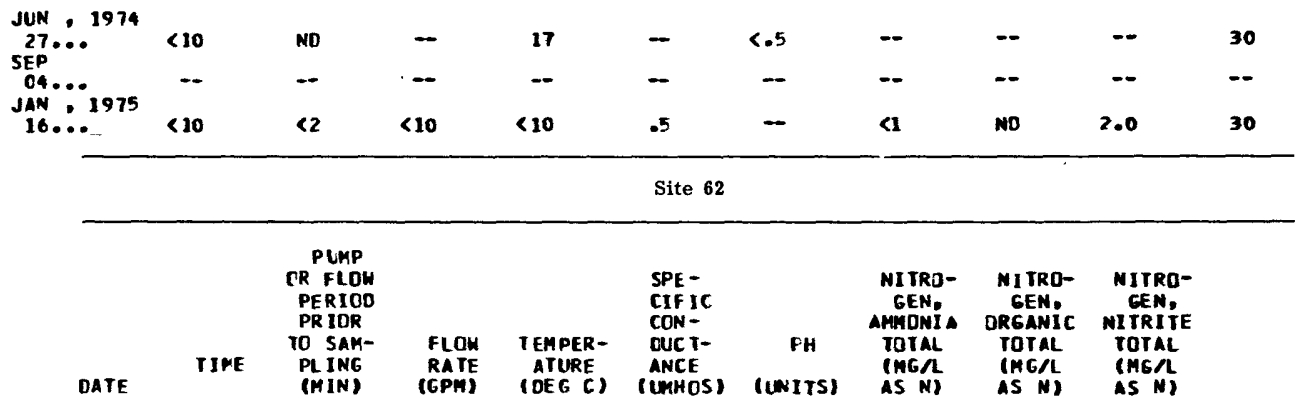

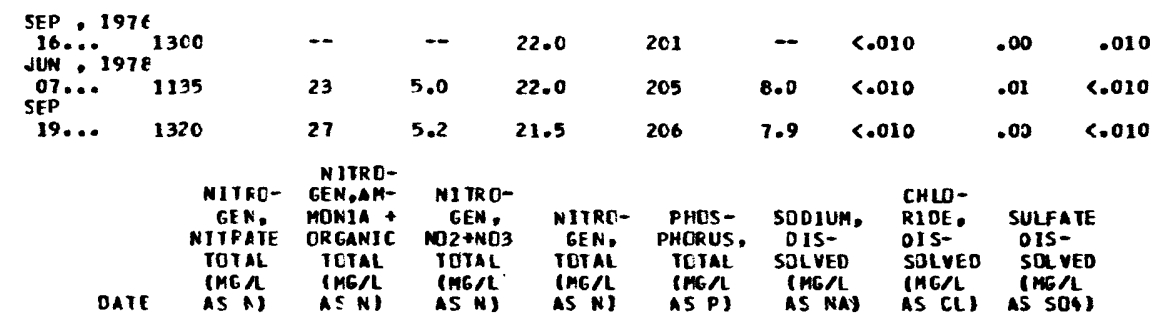

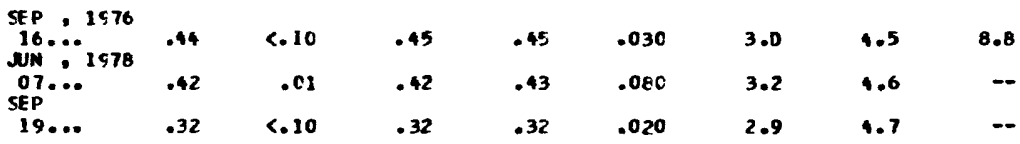




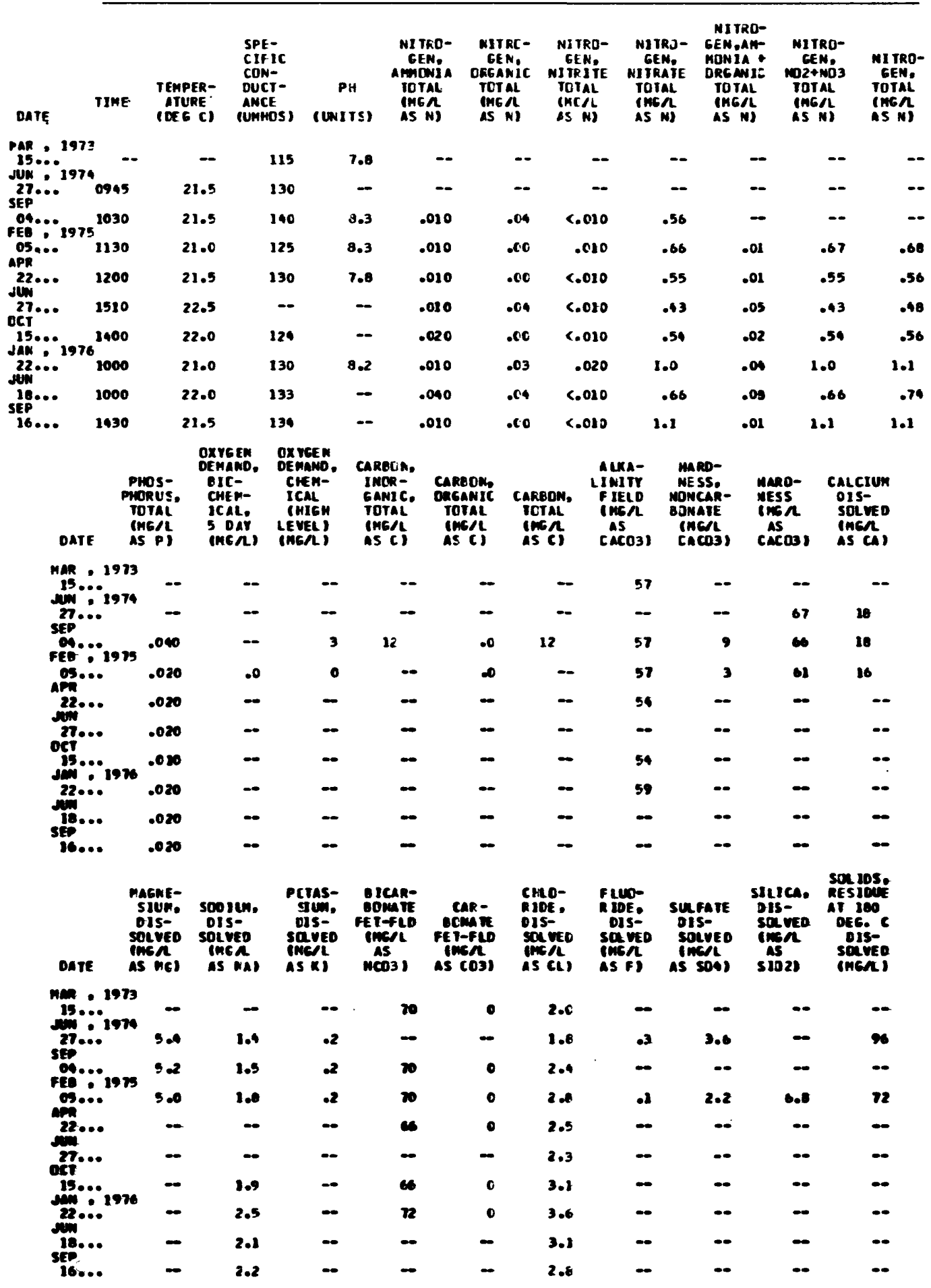


Table 6.--Physical and chemical characteristics of well and water at sites 1 through 70--Continued

Site 63--Continued

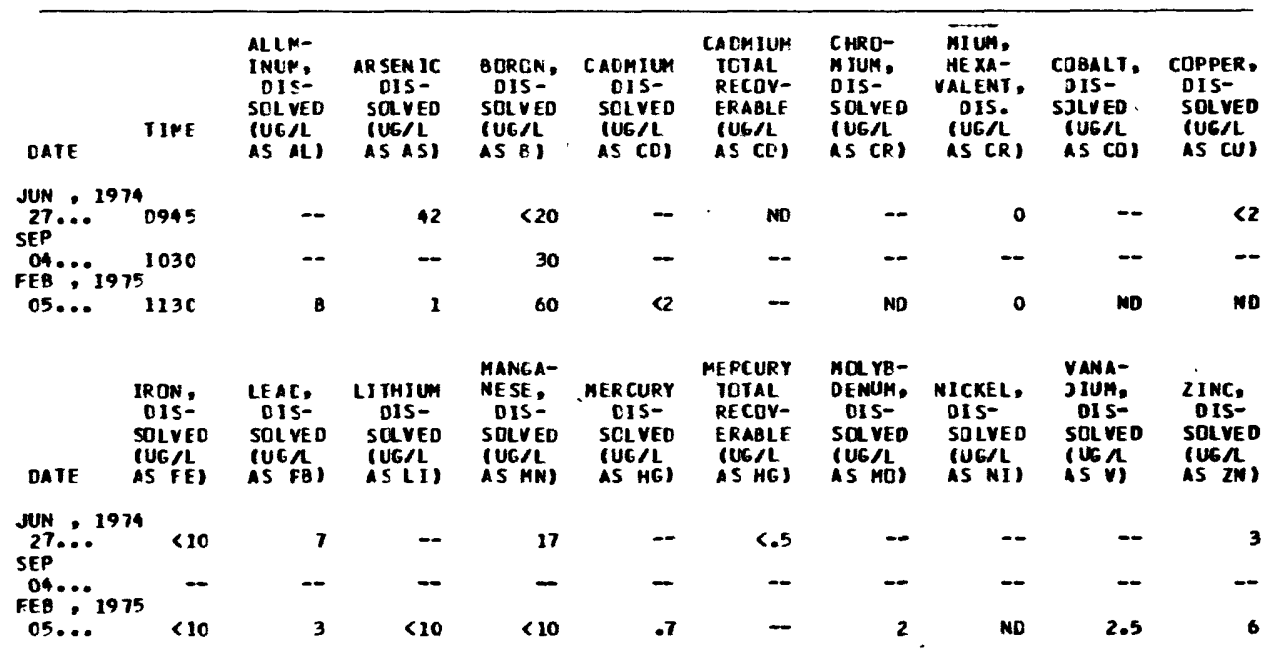

Site 64

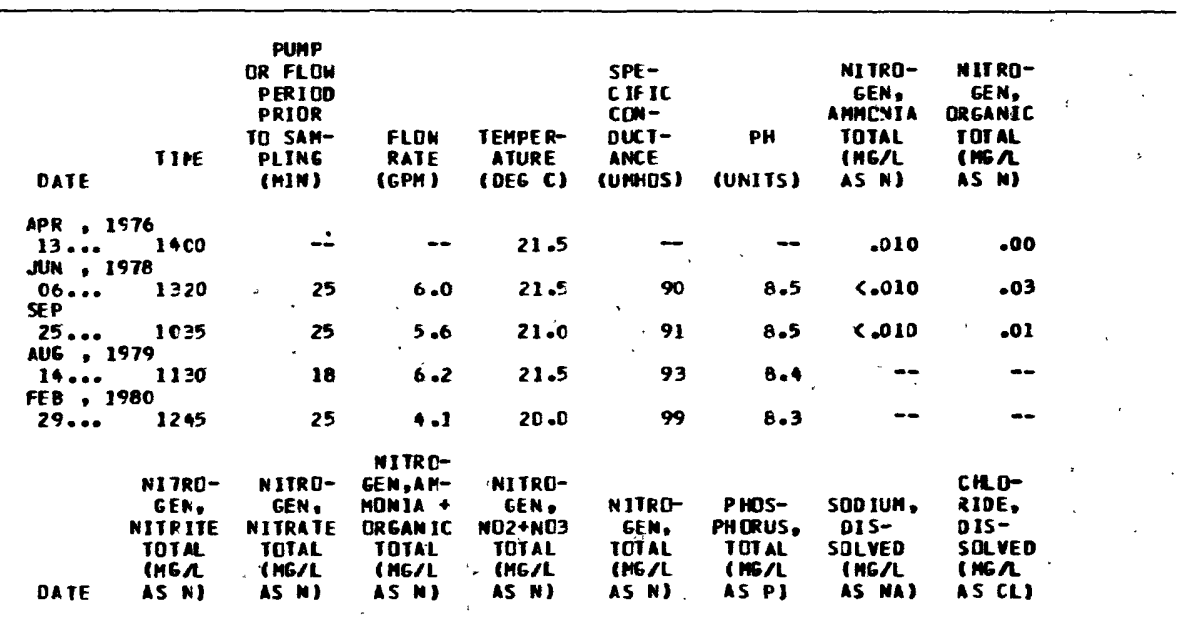

APR' 1976

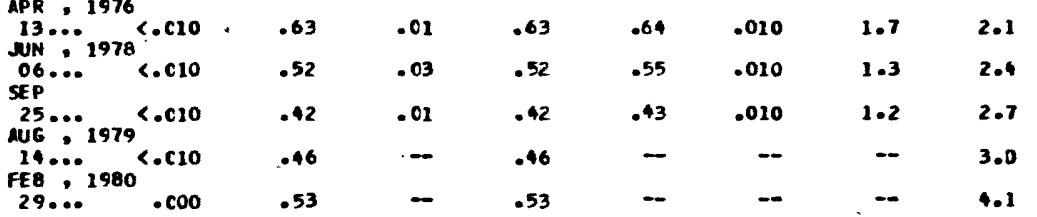


Site 65

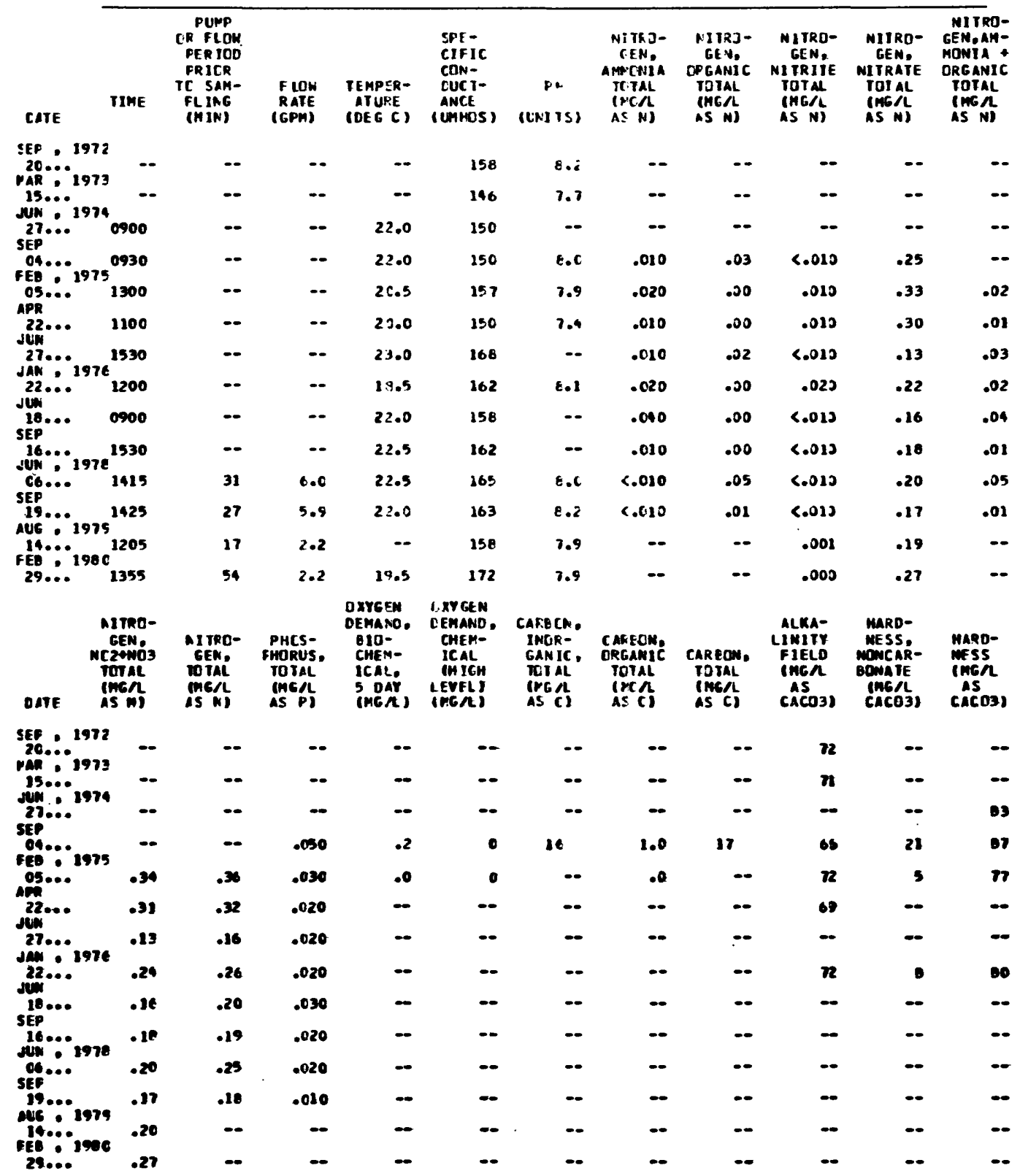


Site 65--Continued

\begin{tabular}{|c|c|c|c|c|c|c|c|c|c|c|c|}
\hline DRTE & $\begin{array}{l}\text { colcium } \\
\text { cIS- } \\
\text { solvED } \\
\text { (MG/L } \\
\text { is CA) }\end{array}$ & $\begin{array}{l}\text { PAGNE- } \\
\text { SICH. } \\
\text { DIS- } \\
\text { SCLVED } \\
\text { IMG/L } \\
\text { AS YGI }\end{array}$ & $\begin{array}{l}\text { SCD IUM, } \\
\text { DIS- } \\
\text { SOLYED } \\
\text { (MGA } \\
\text { AS NA) }\end{array}$ & $\begin{array}{l}\text { PUTAS- } \\
\text { SIUM. } \\
\text { DI C- } \\
\text { SOE VEO } \\
\text { IMG K } \\
\text { AS (I) }\end{array}$ & $\begin{array}{l}\text { BICAR- } \\
\text { BONATE } \\
\text { FETFLC } \\
\text { IMGA } \\
\text { AS } \\
\text { HCD3) }\end{array}$ & $\begin{array}{c}\text { CAR- } \\
\text { BCNATE } \\
\text { FET-FLE } \\
\text { (MGAL } \\
\text { AS CEZ) }\end{array}$ & $\begin{array}{l}\text { CHLO- } \\
\text { R.ICE, } \\
\text { UIS- } \\
\text { SCLVED } \\
\text { (MG/L } \\
\text { AS CL) }\end{array}$ & $\begin{array}{l}\text { FLUD- } \\
\text { RIOE, } \\
\text { DIS- } \\
\text { SOLVED } \\
\text { IMGA } \\
\text { AS FI }\end{array}$ & $\begin{array}{l}\text { SULFATE } \\
\text { DIS- } \\
\text { SOLVED } \\
\text { (MG/L } \\
\text { AS SO4) }\end{array}$ & $\begin{array}{l}\text { SILICA. } \\
\text { DIS- } \\
\text { SOLVED } \\
\text { (NG/L } \\
\text { AS } \\
\text { SIJ2) }\end{array}$ & $\begin{array}{l}\text { SOL IDS, } \\
\text { RESIDUE } \\
\text { AI I8O } \\
\text { OEG. C } \\
\text { DIS- } \\
\text { SOL VED } \\
\text { (MG } L \text { ) }\end{array}$ \\
\hline
\end{tabular}

SEP, 1972

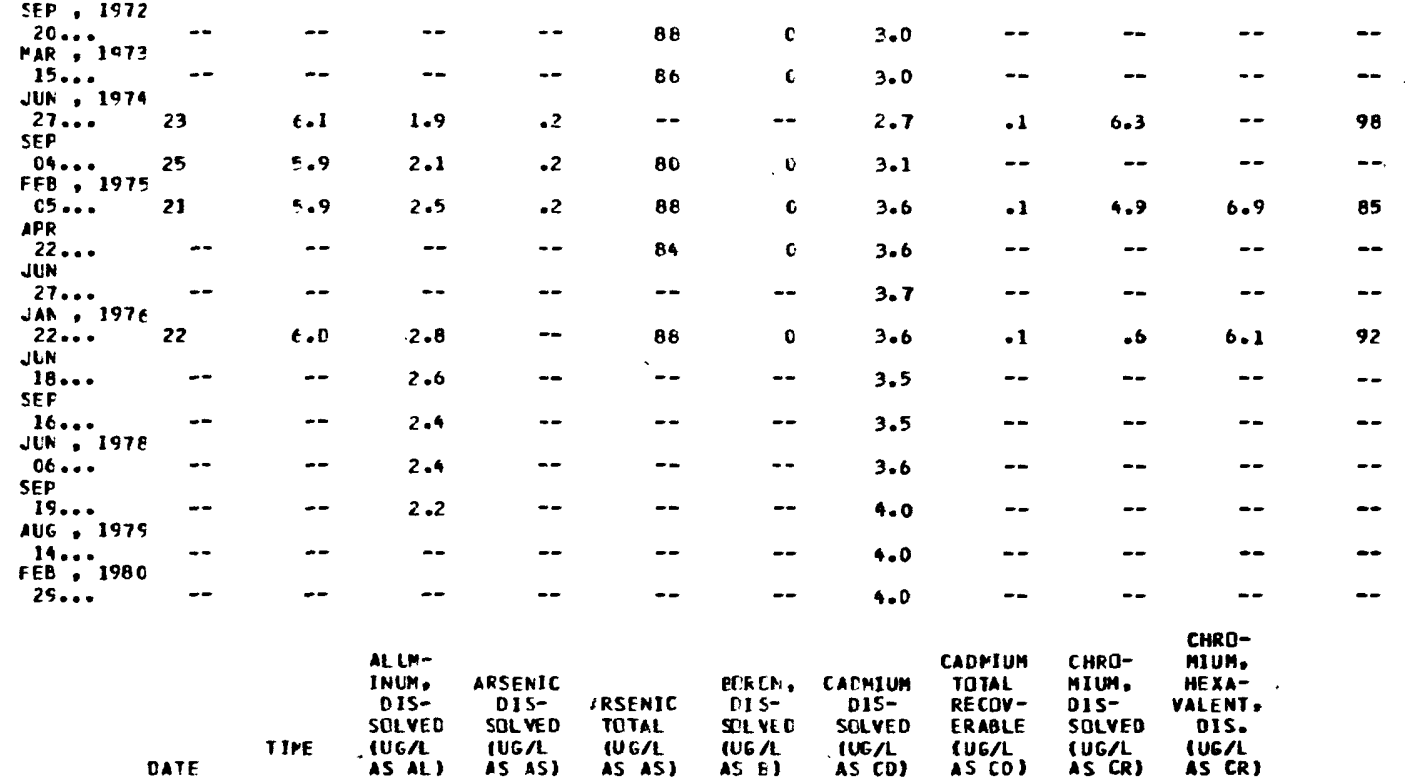

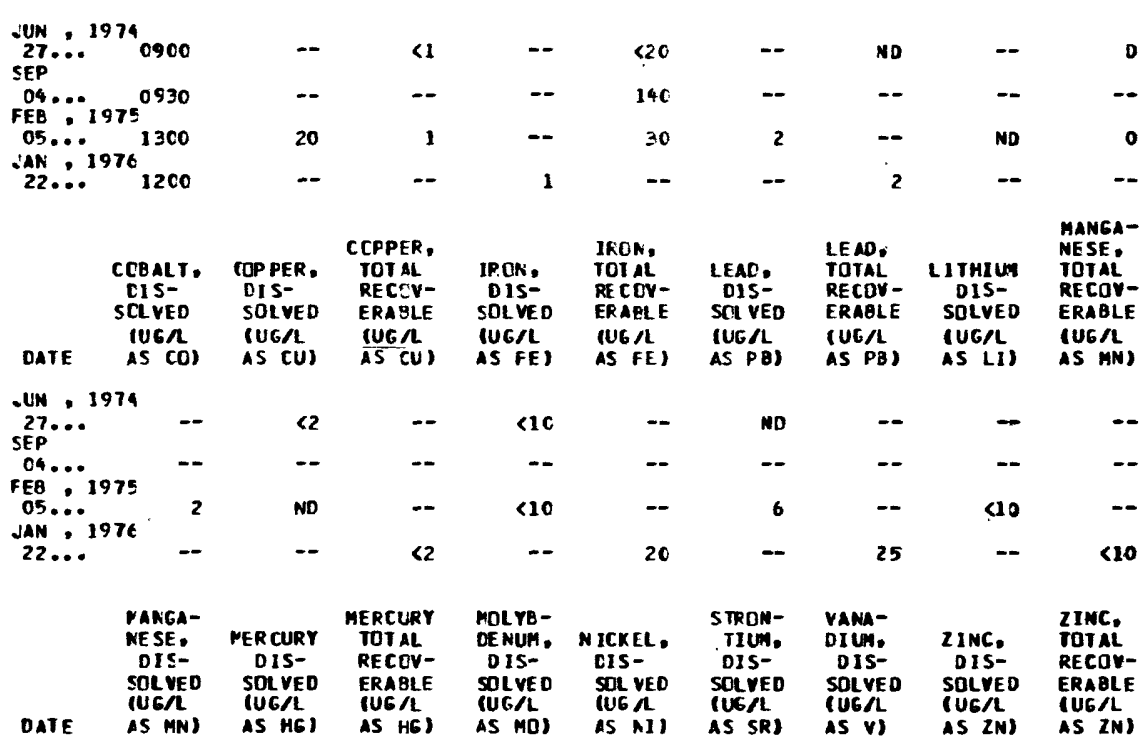

JUN $27 . \therefore 1974$
SEP
OA... 
Site 66

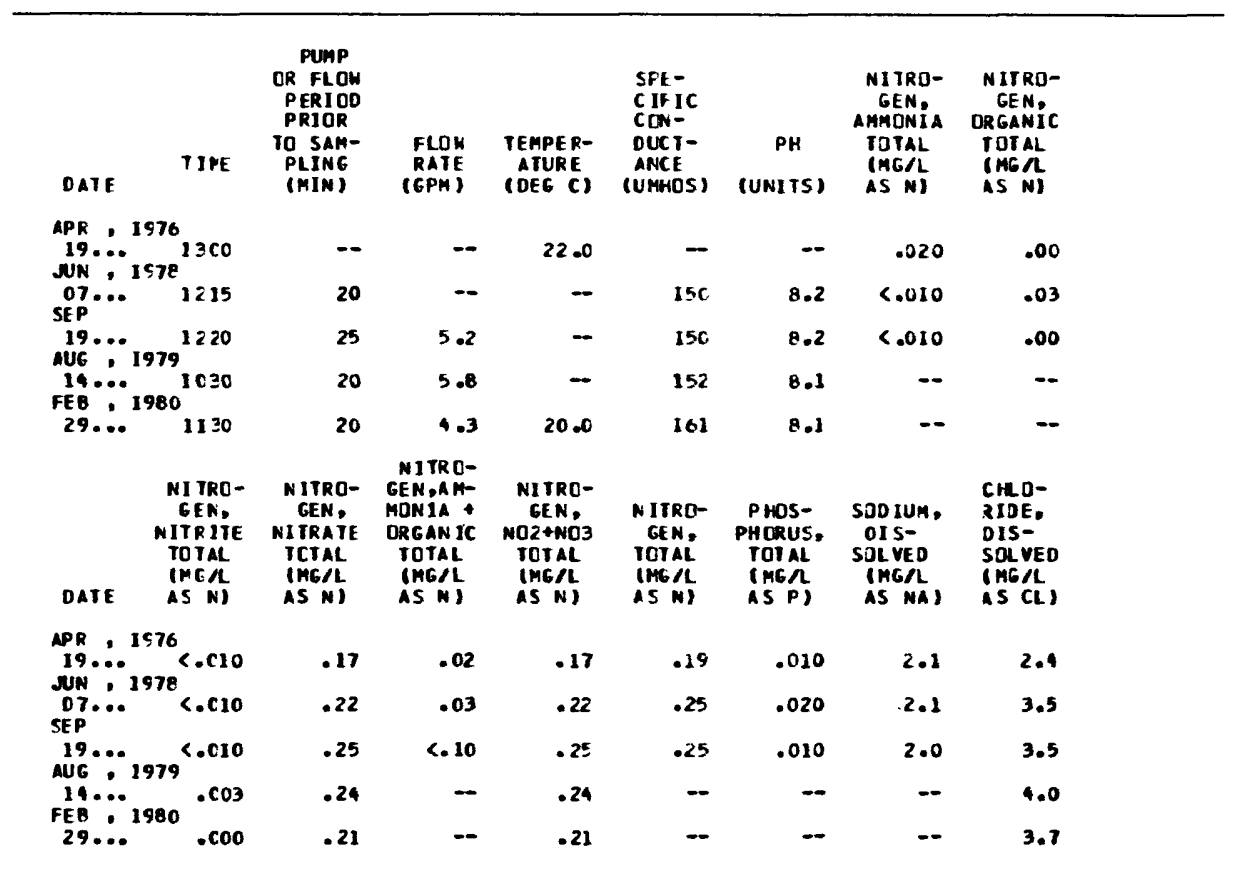

Site 67

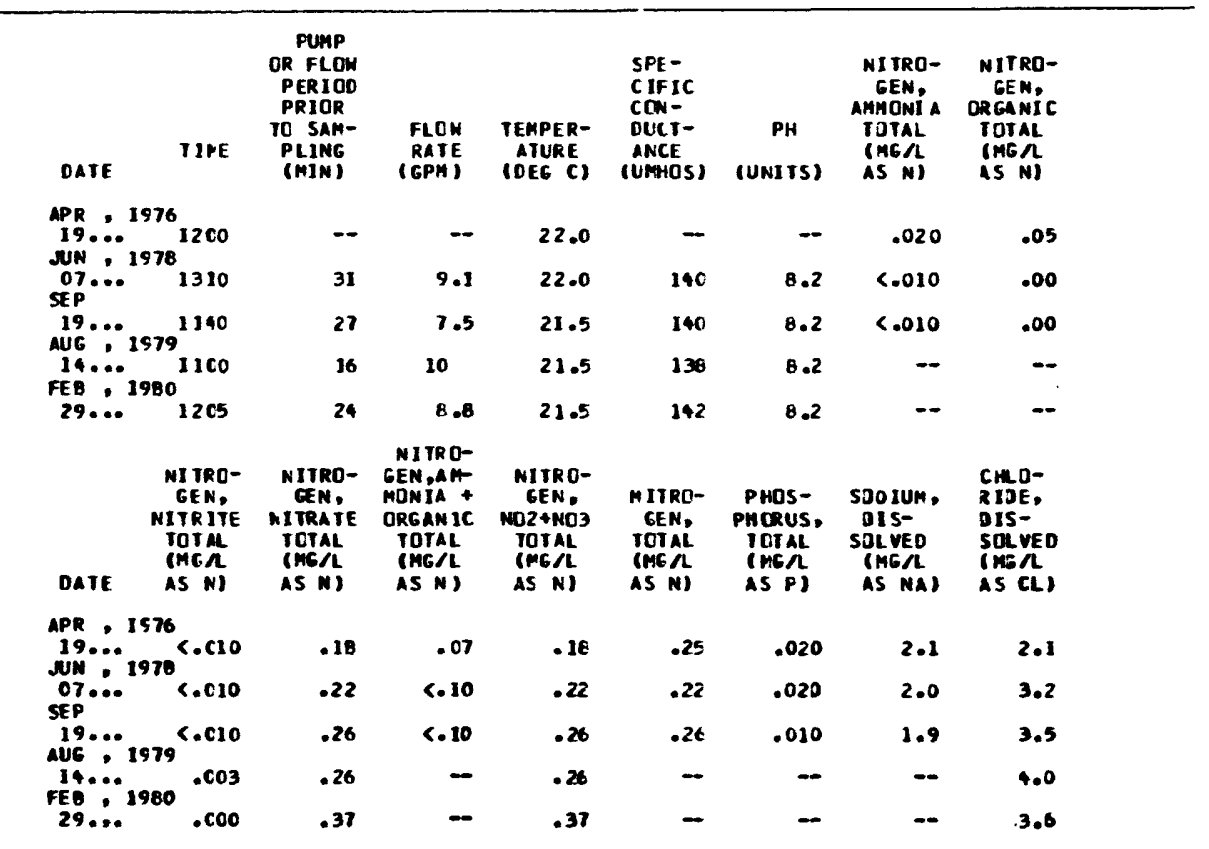


Site 68

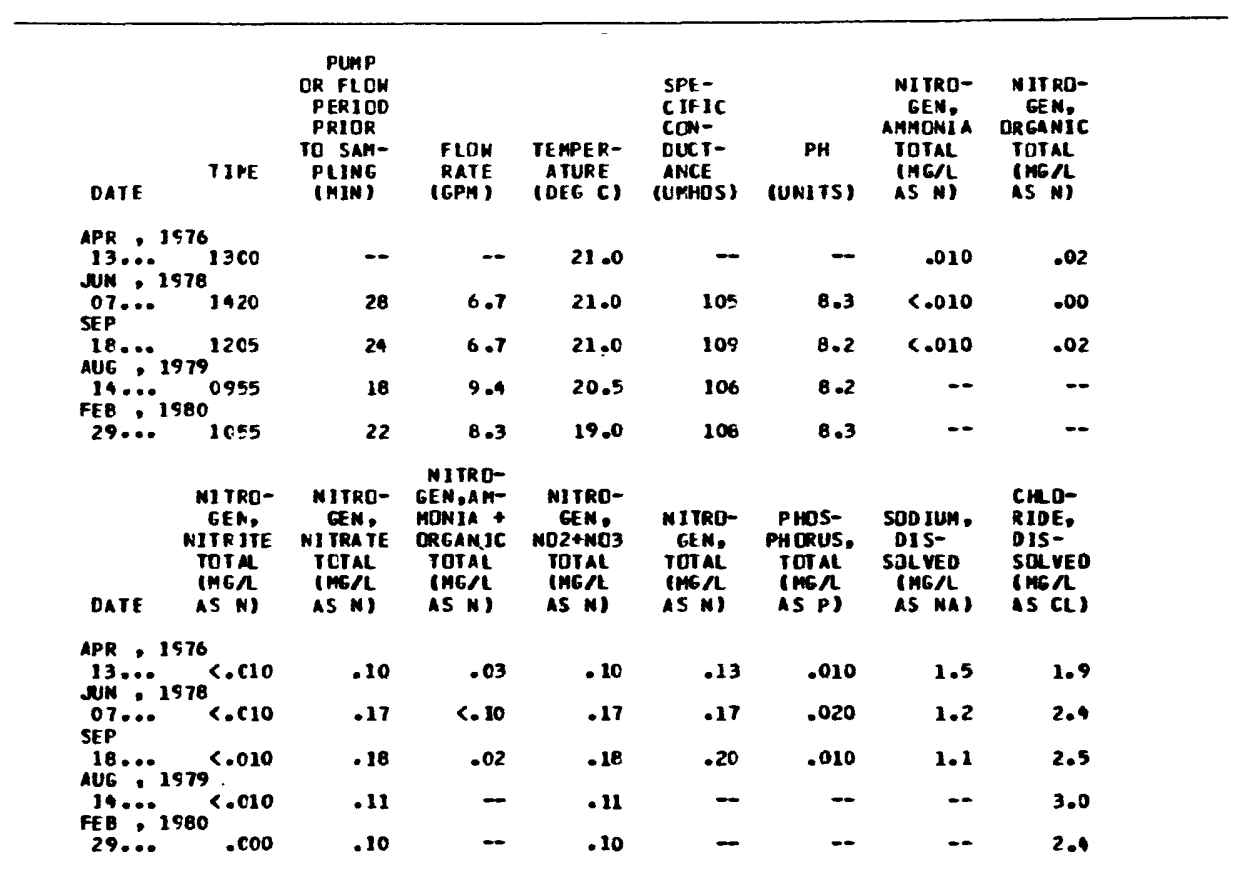

Site 69

\begin{tabular}{|c|c|c|c|c|c|c|c|c|}
\hline DATE & TIPE & $\begin{array}{l}\text { PUMP } \\
\text { OR FLON } \\
\text { PERIOD } \\
\text { PRIOR } \\
\text { IO SAM- } \\
\text { PLING } \\
\text { (MIN) }\end{array}$ & $\begin{array}{l}\text { FLOH } \\
\text { RATE } \\
\text { (GPH) }\end{array}$ & $\begin{array}{l}\text { TEMPER- } \\
\text { ATURE } \\
\text { (DEG C) }\end{array}$ & $\begin{array}{l}\text { SPE- } \\
\text { CIFIC } \\
\text { CON- } \\
\text { DUCI- } \\
\text { ANCE } \\
\text { (UNHOS) }\end{array}$ & $\begin{array}{c}\text { PH } \\
\text { (UNITS) }\end{array}$ & $\begin{array}{l}\text { NI TRD- } \\
\text { GEN, } \\
\text { AMHONIA } \\
\text { TOTAL } \\
\text { (NG/L } \\
\text { AS NI }\end{array}$ & $\begin{array}{l}\text { WITRO- } \\
\text { GEN, } \\
\text { ORCANIC } \\
\text { IOTAL } \\
\text { (MSNL } \\
\text { AS N) }\end{array}$ \\
\hline $\begin{array}{l}\text { SN } \\
20 \ldots \\
\text { SEP } \\
19 \ldots\end{array}$ & $\begin{array}{r}19781000 \\
1040\end{array}$ & 18 & $\begin{array}{l}8.6 \\
9.1\end{array}$ & $\begin{array}{l}21.0 \\
20.5\end{array}$ & $\begin{array}{l}245 \\
225\end{array}$ & $\begin{array}{l}7.7 \\
7.8\end{array}$ & $\begin{array}{l}<.010 \\
<.010\end{array}$ & $\begin{array}{l}.00 \\
.02\end{array}$ \\
\hline DATE & $\begin{array}{l}\text { NI IRD- } \\
\text { GER. } \\
\text { MITRITE } \\
\text { TOTML } \\
\text { IMEA } \\
\text { AS } N \text { ) }\end{array}$ & $\begin{array}{l}\text { NITRD- } \\
\text { GEN, } \\
\text { NITRATE } \\
\text { TETAL } \\
\text { (MG/L } \\
\text { AS M) }\end{array}$ & $\begin{array}{l}\text { MIIRO- } \\
\text { GEN,AM- } \\
\text { MONIA } \\
\text { ORGANIC } \\
\text { TOTAL } \\
\text { (MGSL } \\
\text { AS N) }\end{array}$ & $\begin{array}{l}\text { NI TRO- } \\
\text { GEN, } \\
\text { MO2+NO3 } \\
\text { TOTAL } \\
\text { (MG/L } \\
\text { AS N) }\end{array}$ & $\begin{array}{l}\text { NITRD- } \\
\text { GEN, } \\
\text { TOTAL } \\
\text { (MGA } \\
\text { AS N) }\end{array}$ & $\begin{array}{l}\text { PHOS- } \\
\text { PHORUS, } \\
\text { TOTAL } \\
\text { ( MS } \\
\text { AS P) }\end{array}$ & $\begin{array}{l}\text { SOD IUH, } \\
\text { DIS- } \\
\text { SDL VED } \\
\text { (MGA } \\
\text { AS NAI) }\end{array}$ & $\begin{array}{l}\text { CHLO- } \\
\text { RIDE, } \\
\text { DIS- } \\
\text { SOLVE } \\
\text { (MSAL } \\
\text { IS CL) }\end{array}$ \\
\hline $\begin{array}{l}\text { UN } \\
20 \ldots \\
\text { SEP } \\
19 \ldots\end{array}$ & $\begin{array}{r}1978<.010 \\
<. c 10\end{array}$ & $\begin{array}{l}2.2 \\
1.8\end{array}$ & $\begin{array}{r}<.10 \\
.02\end{array}$ & 2.2 & $\begin{array}{l}2.2 \\
1.8\end{array}$ & $\begin{array}{r}.010 \\
.020\end{array}$ & $\begin{array}{l}5.2 \\
5.1\end{array}$ & $\begin{array}{l}9.0 \\
6.9\end{array}$ \\
\hline
\end{tabular}




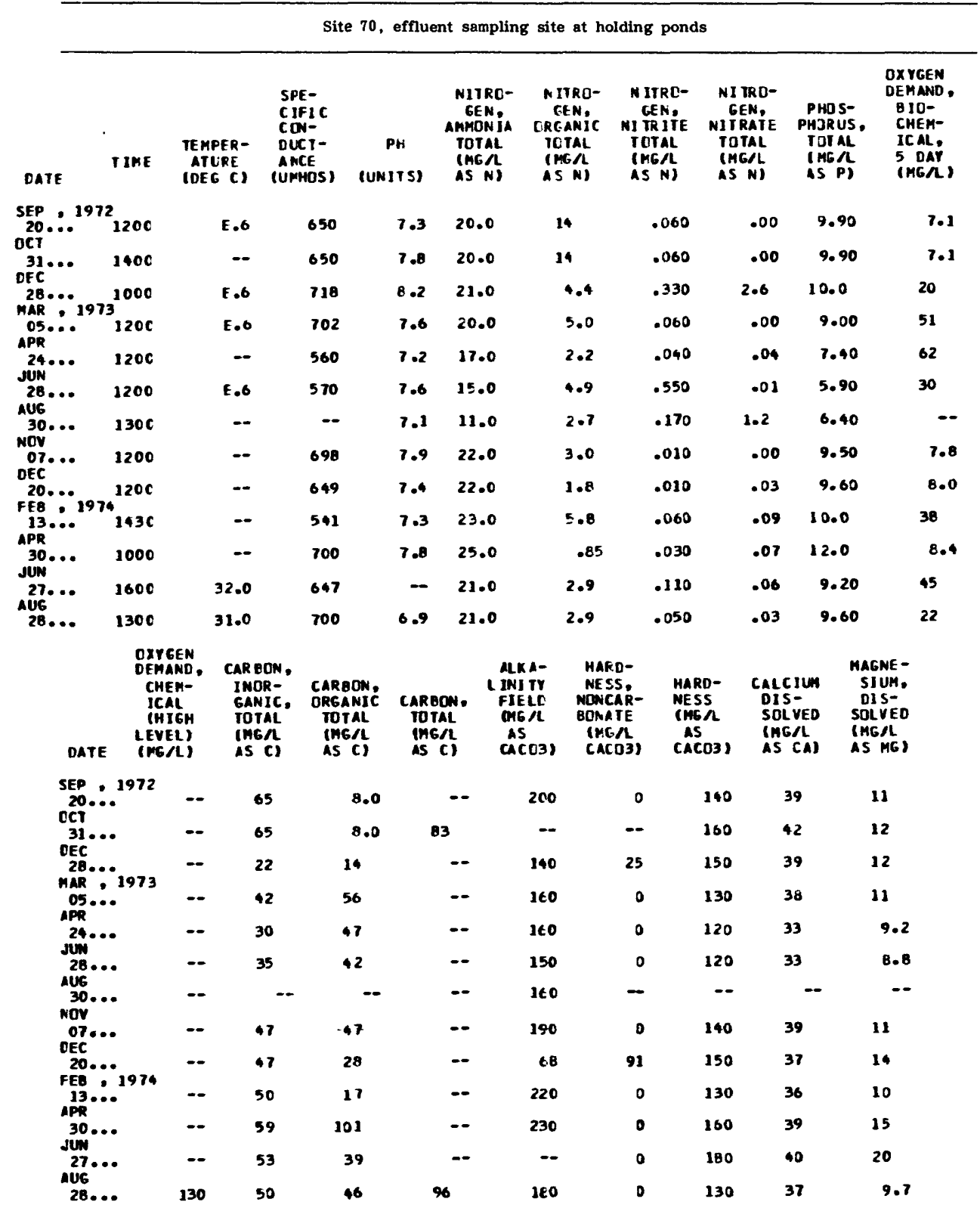


Site 70, effluent sampling site at holding ponds--Continued

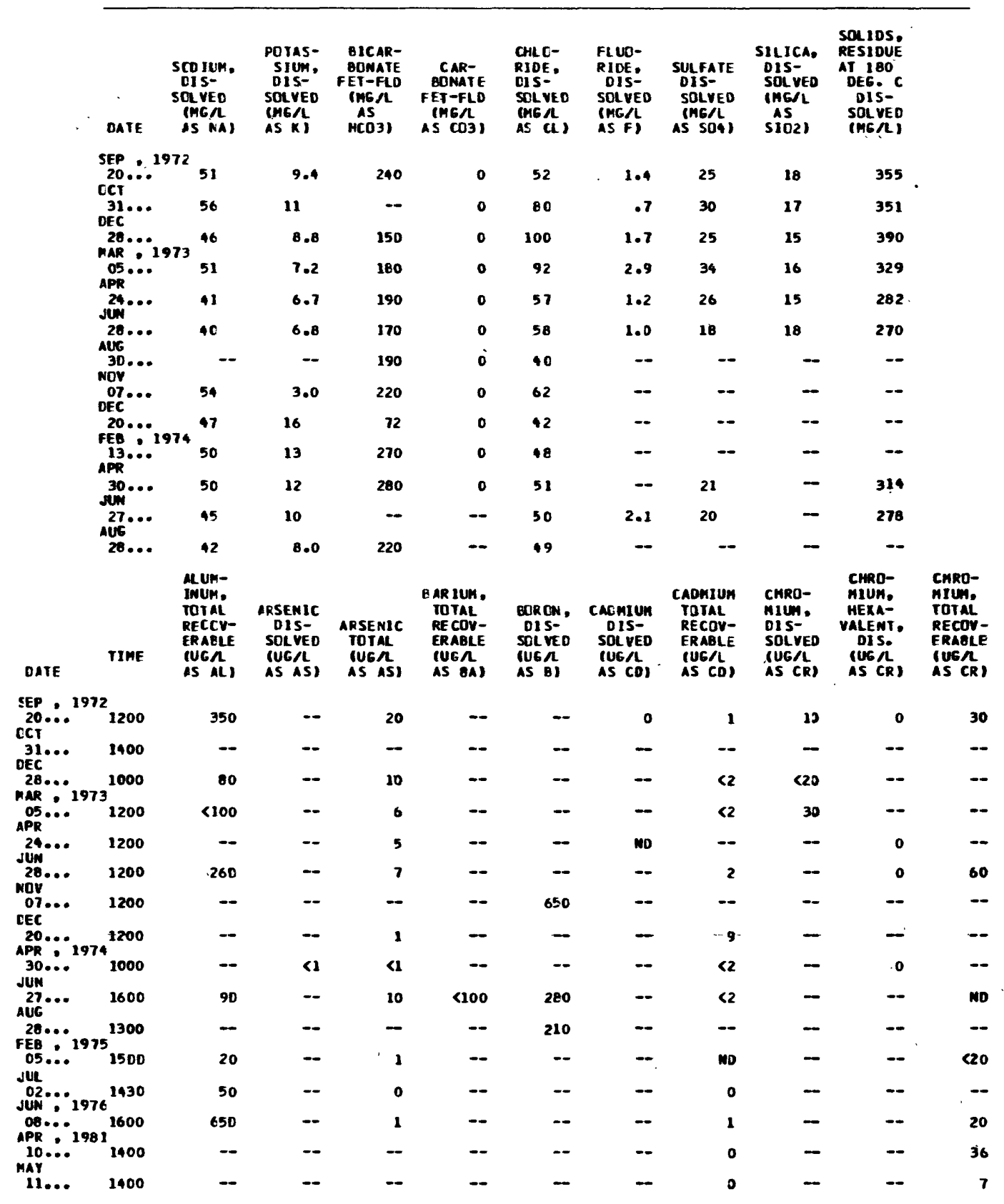


Table 6.--Physical and chemical characteristics of well and water at sites 1 through $70--$ Continued

Site 70 , effuent sampling ste st holding ponds--Continued

\begin{tabular}{|c|c|c|c|c|c|c|c|c|c|c|}
\hline 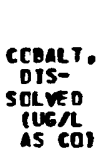 & 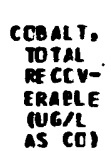 & $\begin{array}{l}\text { COPPER, } \\
\text { DI S- } \\
\text { SOL VED } \\
\text { (UG/R } \\
\text { AS CU) }\end{array}$ & $\begin{array}{l}\text { COPPER, } \\
\text { TOTAL } \\
\text { RECOV- } \\
\text { ERABLE } \\
\text { (UGA } \\
\text { AS CU) }\end{array}$ & $\begin{array}{l}\text { RRON, } \\
\text { DIS- } \\
\text { SOLVE } \\
\text { (U6R } \\
\text { AS FE) }\end{array}$ & $\begin{array}{l}\text { JROA, } \\
\text { TOTAL } \\
\text { RECEY- } \\
\text { ERAELE } \\
\text { TUGA } \\
\text { AS FE I }\end{array}$ & $\begin{array}{l}\text { LEAO, } \\
\text { CIS- } \\
\text { SOLVEO } \\
\text { (UG/L } \\
\text { AS PB) }\end{array}$ & $\begin{array}{l}\text { LEAD, } \\
\text { TOYAL } \\
\text { RECDY- } \\
\text { ERABLE } \\
\text { IUGR } \\
\text { AS PBI }\end{array}$ & $\begin{array}{l}\text { ITHIUA } \\
\text { OIS- } \\
\text { SOLVEO } \\
\text { IUG/L } \\
\text { AS LII }\end{array}$ & $\begin{array}{l}\text { LITHIUA } \\
\text { ToTAL } \\
\text { RECOV- } \\
\text { ERABLE } \\
\text { (UGSL } \\
\text { AS LI) }\end{array}$ & $\begin{array}{l}\text { MANGA- } \\
\text { MESE, } \\
\text { TOTAL } \\
\text { RECON- } \\
\text { ERABLE } \\
\text { IUGR } \\
\text { AS mis }\end{array}$ \\
\hline
\end{tabular}

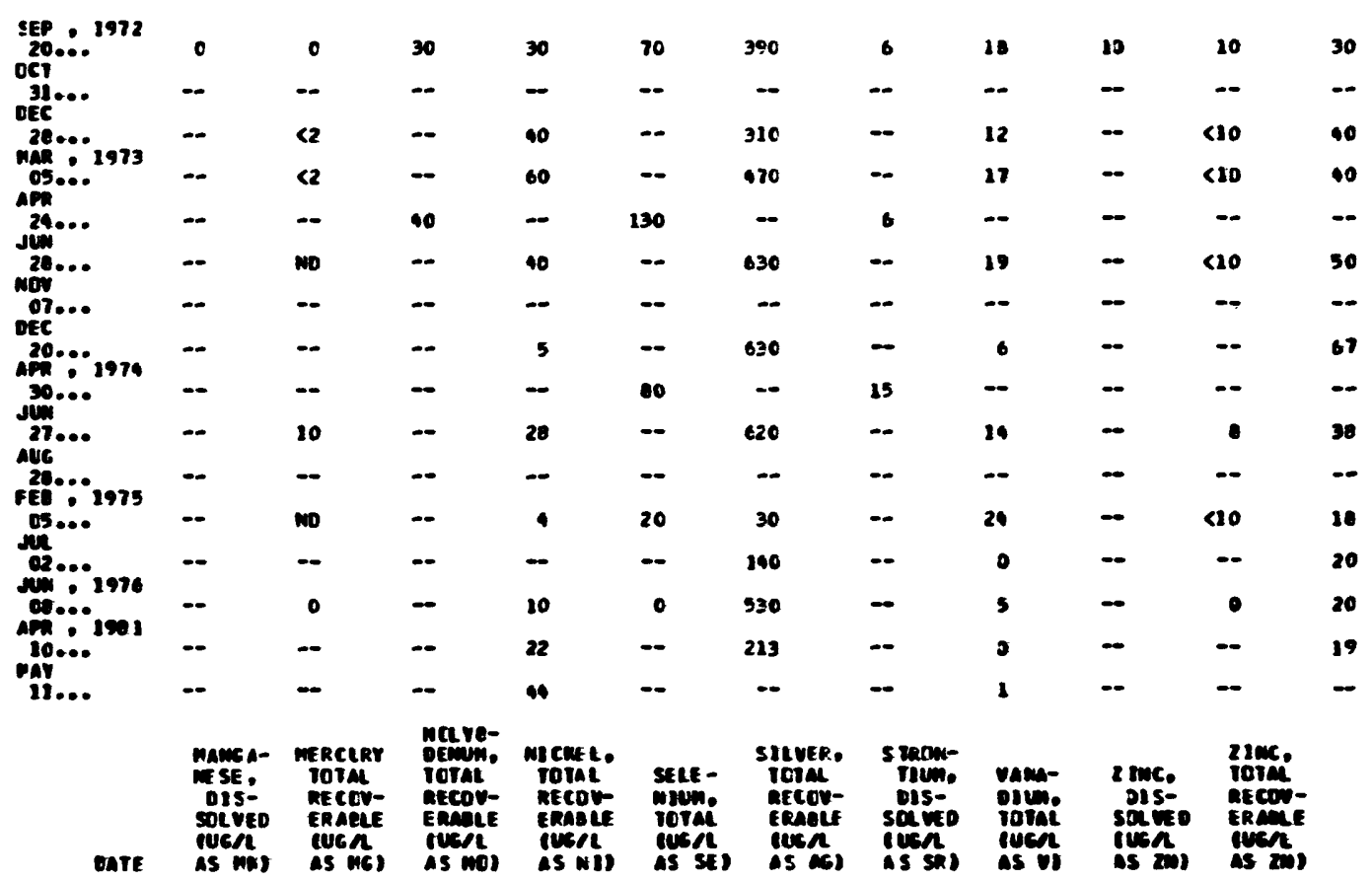

sT. 192

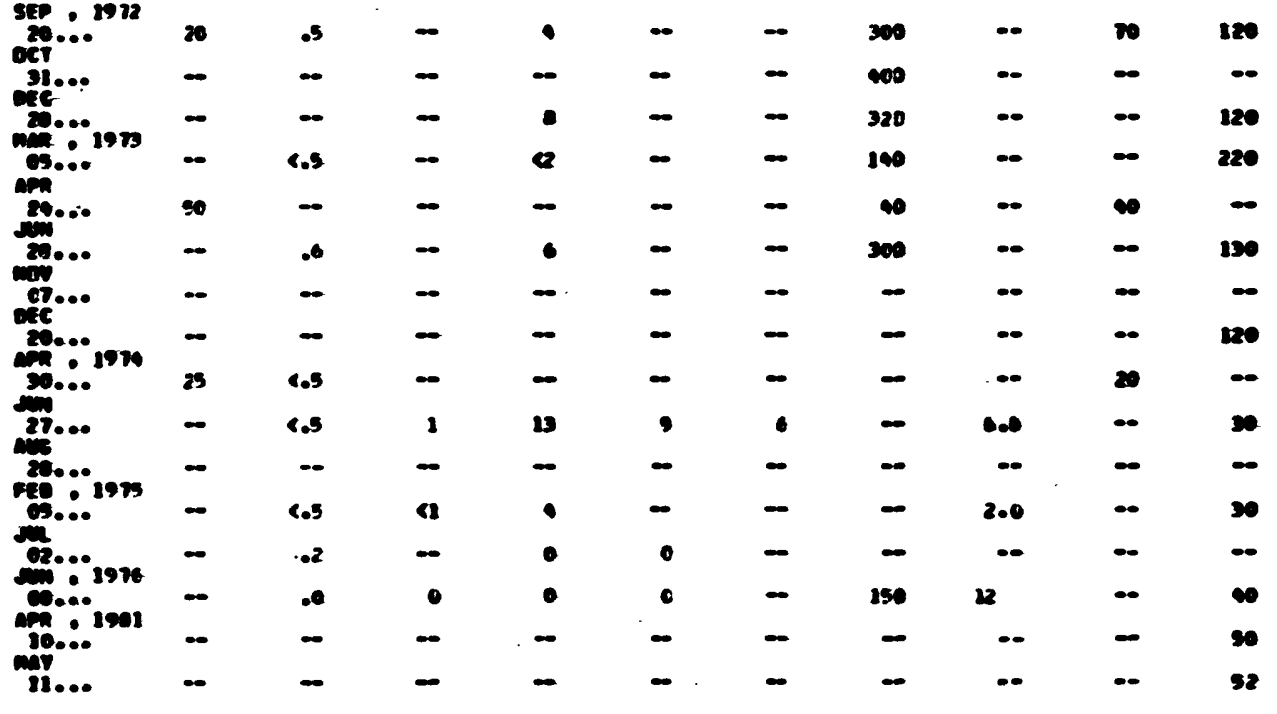

